\title{
The Cu II Spectrum
}

\author{
Alexander Kramida *, Gillian Nave and Joseph Reader \\ National Institute of Standards and Technology, Gaithersburg, MD 20899, USA; gillian.nave@nist.gov (G.N.); \\ joseph.reader@nist.gov (J.R.) \\ * Correspondence: alexander.kramida@nist.gov; Tel.: +1-301-975-8074; Fax: +1-301-975-5560 \\ Academic Editor: James Babb \\ Received: 16 December 2016; Accepted: 8 February 2017; Published: 24 February 2017
}

\begin{abstract}
New wavelength measurements in the vacuum ultraviolet (VUV), ultraviolet and visible spectral regions have been combined with available literature data to refine and extend the description of the spectrum of singly ionized copper ( $\mathrm{Cu}$ II). In the VUV region, we measured 401 lines using a concave grating spectrograph and photographic plates. In the UV and visible regions, we measured 276 lines using a Fourier-transform spectrometer. These new measurements were combined with previously unpublished data from the thesis of Ross, with accurate VUV grating measurements of Kaufman and Ward, and with less accurate older measurements of Shenstone to construct a comprehensive list of $\approx 2440$ observed lines, from which we derived a revised set of 379 optimized energy levels, complemented with 89 additional levels obtained using series formulas. Among the 379 experimental levels, 29 are new. Intensities of all lines observed in different experiments have been reduced to the same uniform scale by using newly calculated transition probabilities ( $A$-values). We combined our calculations with published measured and calculated $A$-values to provide a set of 555 critically evaluated transition probabilities with estimated uncertainties, 162 of which are less than $20 \%$.
\end{abstract}

Keywords: atomic spectra; singly ionized copper; energy levels; wavelengths; Ritz standards; transition probabilities; critical compilation

\section{Introduction}

The spectrum of singly ionized copper, belonging to the Ni isoelectronic sequence, has a long history of research. The most significant contributions to the analysis were made by Shenstone [1] in 1936 and by Ross [2] in 1969. Interest in this spectrum was mainly due to the fact that it has a large number of sharp distinct lines in the vacuum ultraviolet (VUV) region, as well as an equally large number of lines in the ultraviolet (UV), visible, and infrared (IR) regions, from which accurate wavelengths of the VUV lines can be established using the Ritz combination principle, thus providing a large set of lines usable as secondary VUV wavelength standards. This spectrum is also of considerable interest for astrophysics. Lines of $\mathrm{Cu}$ II were observed in the spectra of nebulae by Thackeray [3], Aller et al. [4], McKenna et al. [5], and by Wallerstein et al. [6], and also in interstellar H I clouds, as well as in Ap, Be, and Bp stars (Jaschek, and Jaschek [7], Danezis and Theodossiou [8]) and in the Sun (Samain [9]). $\mathrm{Cu}^{+}$was successfully used as an active lasing medium in various hollow cathodes. Continuous-wave or pulsed lasing has been reported for 28 lines of $\mathrm{Cu}$ II in a wide range from UV to IR (McNeil et al. [10,11], Jain [12], Zinchenko and Ivanov [13]). Since copper is an important impurity in tokamaks, the Cu II spectrum has potential applications in fusion research, where its lines can be used for diagnostic purposes.

For several decades after the original analysis by Shenstone [1] the VUV wavelengths of Cu II calculated from the energy levels (i.e., Ritz wavelengths) were widely used as auxiliary wavelength standards. After the refinements of the measured UV wavelengths by Reader et al. [14] and by Kaufman and Ward [15], and after improved and greatly extended measurements by Ross [2] in the UV and visible ranges, the quality of the VUV Ritz wavelengths of Cu II seemed to be so good that 
no further investigations of this spectrum were needed. The only inconvenience stemmed from the fact that Ross's thesis [2] was never published, although a large part of it was released with small modifications as a report of the Los Alamos National Laboratory, see Ross [16]. Energy levels and a few strongest lines from Ross [2] were included in the compilations by Sugar and Musgrove [17] and by Sansonetti and Martin [18], but the bulk of the wavelength and line intensity data remains nearly inaccessible. Thus, the initial goal of the present work was simply to digitize Ross's line lists and include them in the Atomic Spectra Database (ASD; see Kramida et al. [19]) of the National Institute of Standards and Technology (NIST) with consistent energy-level identifications. However, a close examination revealed several problems with Ross's data.

The first problem is with internal consistency of Ross's data. An important aspect of ASD is that it requires the observed wavelengths for an atom to be consistent with the energy levels tabulated for that atom. That is, the wavelengths derived from the energy levels (Ritz wavelengths) must agree with the observed wavelengths to within the stated uncertainties. However, in attempting to incorporate the data of Ross into ASD, we found that for a large number of lines the differences between observed and Ritz wavelengths were much greater than the uncertainties. We decided that, because of the importance of Ritz wavelengths for Cu II, this had to be investigated.

The second problem with Ross's data is the possible presence of systematic shifts in his wavelengths. Nave and Sansonetti [20] showed that Ritz wavelengths below $2400 \AA\left(41667 \mathrm{~cm}^{-1}\right)$ derived from Ross's Cu II energy levels are systematically too low, the mean relative deviation being about $4 \times 10^{-7}$. This error is significantly greater than the average relative uncertainty of Ritz wavenumbers given by Ross, $1.2 \times 10^{-7}$. Near $2000 \AA\left(50000 \mathrm{~cm}^{-1}\right)$, it corresponds to an error of $0.02 \mathrm{~cm}^{-1}$, compared to the mean stated uncertainty of $0.006 \mathrm{~cm}^{-1}$. This puts the usability of Ross's Ritz wavenumbers in the VUV into question.

The third problem is the absence of a consistent description of line intensities throughout the entire range of observed wavelengths. While Ross's own measurements cover the large range above $1980 \AA$, most of the observed intensities in the VUV region are furnished by old measurements of Shenstone [1], which were made with seven different instruments and with two different light sources. Additional measurements for some selections of strongest lines were made by Reader et al. [14] and by Kaufman and Ward [15], each with their own intensity scale.

Finally, Ross's theoretical interpretation of the energy levels, adopted by Sugar and Musgrove [17] and by Sansonetti and Martin [18], is inadequate. Ross used the LS coupling scheme in his analysis, as well as in his final level list. However, the observed fine-structure intervals indicate that the level structure of most configurations is best described by the $J_{1} l$ (a.k.a. $J K$ ) coupling scheme, similar to the isoelectronic Ni I spectrum interpreted by Litzén et al. [21].

Another problem that needs to be addressed is the scarcity of available critically evaluated data on radiative transition probabilities ( $A$-values). At present, the NIST ASD [19] includes only seven $A$-values of $\mathrm{Cu}$ II from the compilation of Wiese and Martin [22]. All of them have large estimated uncertainties ( $\leq 40 \%$ for six transitions and $\leq 50 \%$ for one transition).

The main purpose of the present work is to solve the above-mentioned problems and construct a self-consistent set of recommended energy levels and wavelengths of Cu II supplemented with a uniform description of line intensities. Re-interpretation of the energy levels, as well as the search for possible classifications of previously unidentified lines, required new calculations, which necessarily produce radiative transition probabilities ( $A$-values). Thus, we also critically evaluate all available data on $A$-values and extend them with our calculated ones found to be of sufficiently good accuracy.

The usability of the $\mathrm{Cu}$ II wavelengths as standards is limited by substantial hyperfine structure (HFS) splitting and presence of two stable isotopes, ${ }^{63} \mathrm{Cu}(69.15 \%)$ and ${ }^{65} \mathrm{Cu}(30.85 \%)$ in natural copper (Coursey et al. [23]). HFS and isotope shifts (IS) of a few tens of Cu II lines between $2218 \AA$ and $8096 \AA$ were first observed and roughly estimated by Shenstone [1] and then more accurately investigated by Elbel et al. [24,25]. The measured IS was in the range $(0.001$ to 0.101$) \mathrm{cm}^{-1}$. For singly-excited $3 \mathrm{~d}^{9} \mathrm{nl}$ configurations the IS is smaller than $0.05 \mathrm{~cm}^{-1}$, while for the doubly-excited $4 \mathrm{~d}^{8} 4 \mathrm{~s}^{2}$ configuration 
it is about $0.1 \mathrm{~cm}^{-1}$. The HFS constants $A_{\mathrm{hfs}}$ for several $\left[3 \mathrm{~d}^{9}\right] 4 \mathrm{~s}, 4 \mathrm{p}, 4 \mathrm{~d}, 5 \mathrm{~s}, 5 \mathrm{p}, 5 \mathrm{~d}, 6 \mathrm{~s}$ and $\left[3 \mathrm{~d}^{8}\right] 4 \mathrm{~s}^{2}$ and $4 \mathrm{~s} 5 \mathrm{p}$ levels were found to range from $0.022 \mathrm{~cm}^{-1}$ to $0.075 \mathrm{~cm}^{-1}$ except for one level, $3 \mathrm{~d}^{9} 4 \mathrm{~s}^{3} \mathrm{D}_{1}$, having a very small $A_{\mathrm{hfs}}=-0.004 \mathrm{~cm}^{-1}$. The nuclear spin $I$ of both ${ }^{63} \mathrm{Cu}$ and ${ }^{65} \mathrm{Cu}$ is $I=3 / 2$ [18]. Thus, depending on the total angular momentum $J$ of the levels, the width of the HFS varies between $0.016 \mathrm{~cm}^{-1}$ and $0.5 \mathrm{~cm}^{-1}$. The ionization energy of $\mathrm{Cu}^{+}$is $163669.2 \mathrm{~cm}^{-1}(20.29239 \mathrm{eV})$ (Ross [2]). Thus, excitation of this spectrum requires temperatures in excess of $6000 \mathrm{~K}(0.5 \mathrm{eV})$. Such temperatures lead to Doppler broadening $\Delta \sigma_{\text {Dop }} / \sigma>4 \times 10^{-6}$, resulting in line widths $>0.4 \mathrm{~cm}^{-1}$ at wavelengths near $1000 \AA, 0.2 \mathrm{~cm}^{-1}$ near $2000 \AA$, and $0.1 \mathrm{~cm}^{-1}$ near $4000 \AA$. As a result, most observed lines possess unresolved or partially resolved HFS and IS. The smallness of HFS and IS explains the scarcity of studies of these effects in $\mathrm{Cu}$ II. However, these effects should be relatively easy to observe in the infrared region. Such observations would be very valuable.

All wavelength measurements described in the present paper were made with natural copper samples. As we know, there is no such physical entity as an atom of natural copper. Thus, the energy levels and Ritz wavelengths derived from these measurements do not pertain to a real atom, but rather are empirical values that best describe the spectrum as normally observed.

\section{Wavelength Measurements}

The unfortunate consequence of the thorough analysis made by Ross $[2,16]$ is that, in the 46 years after his thesis, there were no studies devoted specifically to $\mathrm{Cu}$ II. Instead, his $\mathrm{Cu}$ II Ritz wavelengths were widely used as secondary standards for investigation of other species. In particular, the archive of the NIST Atomic Spectroscopy Group has several tens of photographic plates with VUV spectra obtained with the $10.7 \mathrm{~m}$ normal incidence vacuum spectrograph with a 1200 lines $/ \mathrm{mm}$ concave grating. These plates were recorded for studies of $\mathrm{Y}, \mathrm{Zr}, \mathrm{Ge}, \mathrm{La}$, and other elements. For calibration purposes, separate tracks were exposed on these plates with spectra of copper hollow cathodes. The latter, in addition to copper lines, contain lines of carrier gases (helium, neon, and argon), as well as hydrogen, carbon, oxygen, nitrogen, silicon, and germanium that were present as intrinsic or deliberately introduced impurities. These spectra were obtained in different years from 1969 to 1974. In addition to that, we have several high-resolution spectra recorded with two NIST vacuum Fourier transform spectrometers (FTS) in years 2002 to 2008 using $\mathrm{Cu} / \mathrm{Ge} / \mathrm{Pt} / \mathrm{Fe} / \mathrm{Ne}$ and $\mathrm{Cu} / \mathrm{Re} / \mathrm{Ar} / \mathrm{He}$ hollow cathodes. The "new" measurements described in the Abstract were all made with these old recordings. However, we carried out new reductions of the wavelengths and re-analyzed the uncertainties, so it is in this sense that the measurements can be considered as new. To obtain a consistent and comprehensive description of the $\mathrm{Cu}$ II spectrum, we combined these new measurements with the old published data from Shenstone [1], Kaufman and Ward [15], and Ross [2,16], which we re-analyzed to evaluate the wavelength calibration, measurement uncertainties, and observed line intensities. Since this re-analysis depends on our new measurements, we describe them first.

Table 1 lists the exposures used in the present analysis. Since exposures used in grating measurements were taken over a period of several years, several different lamp designs were employed. The design of these lamps was similar to the one described in detail by Reader and Davis [26], except that a special fitting was made to attach them to the NIST vacuum spectrograph. The cathode was solid copper with a cylindrical hole of about $7 \mathrm{~mm}$ in diameter. Following Kaufman and Ward [15], small pieces of Ge and $\mathrm{Si}$ were placed in the cathode to obtain good reference lines in regions that were not covered well by $\mathrm{Cu}$ II. The carrier gas was a combination of flowing helium at a pressure of about $0.5 \mathrm{kPa}$ (4 Torr) and neon or argon at about $70 \mathrm{~Pa}(0.5 \mathrm{Torr})$. The entrance slit of the spectrometer separated the lamp from the spectrometer chamber, which was maintained at a residual pressure of about $7 \times 10^{-3} \mathrm{~Pa}\left(5 \times 10^{-5}\right.$ Torr $)$. The demountable high-current lamp made at the University of Hannover, used several tens of years later in the FTS measurements, had a similar design described by Danzmann et al. [27]. Ar at a pressure of $200 \mathrm{~Pa}$ (1.5 Torr) with an addition of He at $70 \mathrm{~Pa}(0.5$ Torr) was used as a carrier gas in spectrum 14 , while $\mathrm{Ne}$ at a pressure of $270 \mathrm{~Pa}$ (2 Torr) was used in spectra 12 and 13. Pieces of $\mathrm{Ge}, \mathrm{Pt}, \mathrm{Fe}$, or Re were placed inside the cathode. The grating spectrograms covered the region (636 to 2682) $\AA$, while the FTS spectrograms covered the region (1792 to 11733) $\AA$. 
Table 1. Spectrograms used in the present measurements.

\begin{tabular}{|c|c|c|c|c|c|c|c|c|c|c|}
\hline Exposure & Track & Region $(\AA)$ & Hollow Cathode Used & Impurities & Equip. ${ }^{a}$ & Wavelength Standards ${ }^{b}$ & Track Label & Date & Fit Poly ${ }^{\mathrm{c}}$ & St. Dev. ${ }^{d}$ \\
\hline 1 & 1 & $636-827$ & $\mathrm{Cu} / \mathrm{He} / \mathrm{Ar} / \mathrm{Ne}$ & & NIVS & $\operatorname{Ar~II~(11),~Ne~I~(1),~Cu~II~(TW-1)~e~}$ & 418 & $4 / 3 / 1974$ & 2 & 0.0009 \\
\hline \multirow[t]{2}{*}{2} & 2 & $822-1000$ & $\mathrm{Cu} / \mathrm{Ge} / \mathrm{Si} / \mathrm{He} / \mathrm{Ar}$ & $\mathrm{H}, \mathrm{C}, \mathrm{O}$ & NIVS & H I (2), O I (9), O II (1), Ar II (2), Ge II (3) & 419_tr42 & 4/4/1974 & 2 & 0.0013 \\
\hline & 6 & 977-1166 & & $\mathrm{H}, \mathrm{C}, \mathrm{N}, \mathrm{O}$ & NIVS & C I (2), C II (1), N I (2), O I (7), Ar I (2), Si II (2), Ge II (3) & 419_tr42 & 4/4/1974 & 2 & 0.0013 \\
\hline 3 & 5 & $824-1020$ & $\mathrm{Cu} / \mathrm{He}$ & $\mathrm{H}, \mathrm{O}$ & NIVS & H I (1), O I (5), Cu II (KW66-4, TW-10) ${ }^{\mathrm{f}}$ & & $12 / 17 / 1969$ & 2 & 0.0012 \\
\hline \multirow[t]{2}{*}{4} & 7 & $1181-1360$ & $\mathrm{Cu} / \mathrm{Ge} / \mathrm{Si} / \mathrm{He} / \mathrm{Ar}$ & $\mathrm{H}, \mathrm{C}, \mathrm{N}, \mathrm{O}$ & NIVS, LiF & C I (6), N I (3), Si II (6), Ge II (1), Cu II (KW66-6, TW-1) g & X431_tr27 & $10 / 24 / 1974$ & 2 & 0.0024 \\
\hline & 8 & 1339-1527 & & & NIVS, LiF & C I (4), O I (1), Cu II (KW66-48) & X431_tr27 & $10 / 24 / 1974$ & $2,3^{\mathrm{k}}$ & 0.0021 \\
\hline 5 & 9 & 1399-1590 & $\mathrm{Cu} / \mathrm{He} / \mathrm{Ar}$ & $\mathrm{C}, \mathrm{N}$ & NIVS & C I (1), Cu II (KW66-62, TW-4) h & & $12 / 16 / 1969$ & $2,2^{1}$ & 0.0010 \\
\hline \multirow[t]{2}{*}{6} & 10 & 1398-1584 & $\mathrm{Cu} / \mathrm{Si} / \mathrm{He} / \mathrm{Ne}$ & $\mathrm{C}, \mathrm{N}$ & NIVS & C I (3), Si II (1), Cu II (KW66-44, TW-1) ${ }^{\mathrm{i}}$ & & $1 / 15 / 1970$ & 2 & 0.0012 \\
\hline & 17 & $1920-2106$ & & & NIVS & C I (1), Cu II (R69-20) & & $1 / 15 / 1970$ & 2 & 0.0021 \\
\hline \multirow[t]{2}{*}{7} & 11 & 1399-1587 & $\mathrm{Cu} / \mathrm{Ge} / \mathrm{Si} / \mathrm{He}$ & $\mathrm{C}, \mathrm{N}$ & NIVS & C I (3), N I (1), Si II (2), Ge II (2), Cu II (KW66-66) & X434_tr52 & $11 / 11 / 1974$ & 3 & 0.0014 \\
\hline & 14 & $1560-1743$ & & & NIVS & C I (5), N I (1), Ge II (3), Cu II (KW66-21) & X434_tr52 & $11 / 11 / 1974$ & 3 & 0.0016 \\
\hline \multirow[t]{3}{*}{8} & 12 & 1394-1584 & $\mathrm{Cu} / \mathrm{Ge} / \mathrm{Si} / \mathrm{He}$ & $\mathrm{C}, \mathrm{N}$ & NIVS & C I (3), Si II (2), Ge II (3), Cu II (KW66-52) & X434_tr27 & $11 / 11 / 1974$ & 2 & 0.0016 \\
\hline & 13 & $1559-1743$ & & & NIVS & C I (1), Si I (3), Ge II (1), Cu I (1), Cu II (KW66-14) & X434_tr27 & $11 / 11 / 1974$ & 2 & 0.0019 \\
\hline & 15 & 1751-1939 & & & NIVS & Si I (7), Si II (1), Cu I (1), Cu II (KW66-5) & X434_tr27 & $11 / 11 / 1974$ & 2 & 0.0017 \\
\hline 9 & 16 & 1790-1980 & $\mathrm{Cu} / \mathrm{Si} / \mathrm{He}$ & $\mathrm{C}$ & NIVS & C I (1), Cu I (1), Cu II (KW66-4, R69-1, TW-2) ${ }^{\mathrm{j}}$ & & $11 / 10 / 1969$ & 4 & 0.0012 \\
\hline \multirow[t]{4}{*}{10} & 18 & $1977-2162$ & $\mathrm{Cu} / \mathrm{Ge} / \mathrm{Si} / \mathrm{He}$ & $\mathrm{H}, \mathrm{C}, \mathrm{O}$ & NIVS & Si I (2), Ge I (6), Ge II (2), Cu I (I), Cu II (R69-11) & X433_tr27 & $11 / 7 / 1974$ & 2 & 0.0016 \\
\hline & 20 & 2139-2315 & & & NIVS & Si I (4), Ge I (1), Cu I (4), Cu II (R69-26) & X433_tr27 & $11 / 7 / 1974$ & 5 & 0.0021 \\
\hline & 21 & $2336-2517$ & & & NIVS & Si I (3), Cu I (1), Cu II (R69-13) & X433_tr27 & $11 / 7 / 1974$ & 3 & 0.0017 \\
\hline & 22 & $2490-2682$ & & & NIVS & O I (3), Si I (7), Ge I (3), Ge II (1), Cu II (KW66-2, R69-5) & X433_tr27 & $11 / 7 / 1974$ & 2 & 0.0011 \\
\hline 11 & 19 & $2117-2310$ & $\mathrm{Cu} / \mathrm{Ge} / \mathrm{Si} / \mathrm{He}$ & & NIVS & Si I (2), Ge I (1), Cu I (4), Cu II (R69-20) & E16-3 & $6 / 3 / 1969$ & 2 & 0.0019 \\
\hline 12 & - & $1792-3324$ & $\mathrm{Cu} / \mathrm{Ge} / \mathrm{Pt} / \mathrm{Fe} / \mathrm{Ne}$ & & FTS & Ge I-II (37) & & 2002 & 1 & $1.7 \times 10^{-8}$ \\
\hline 13 & - & $1825-3324$ & $\mathrm{Cu} / \mathrm{Ge} / \mathrm{Pt} / \mathrm{Fe} / \mathrm{Ne}$ & & FTS & Ge I-II (37) & & 2002 & 1 & $2.0 \times 10^{-8}$ \\
\hline 14 & - & $2769-11733$ & $\mathrm{Cu} / \mathrm{Re} / \mathrm{Ar} / \mathrm{He}$ & & FTS & Ar II (68) & & 2009 & 1 & $2.0 \times 10^{-9}$ \\
\hline
\end{tabular}

a Equipment used: NIVS = Normal Incidence Vacuum Spectrograph (NIST, $10.7 \mathrm{~m}$ grating, reciprocal linear dispersion 0.78 A); FTS = Fourier Transform Spectrometer (NIST, FT700 $0.2 \mathrm{~m}$ vacuum FTS for spectra 12 and 13, and a $2 \mathrm{~m}$ vacuum FTS for spectrum 14); LiF = a LiF window was used to remove higher diffraction orders;

$\mathrm{b}$ The number of spectral lines of each spectrum used as standards is given in parentheses. Unless otherwise indicated, the standard wavelengths used in grating measurements were the Ritz wavelengths taken from the ASD database [19]. For Cu II lines used as standards, the references are as follows: KW66-Kaufman and Ward [15]; R69—Ross [2]; TW—-this work. Standards used in the FTS measurements are described in the text;

c Power of the polynomial used to fit standard lines;

${ }^{\mathrm{d}}$ For grating spectra (exposures 1-11), standard deviation of the measured wavenumbers of the standard lines from the fitted polynomial ( $)$ ); For FTS spectra (exposures 12-14), standard deviation of the correction factor from the linear fit (dimensionless);

e Cu II line at $826.9946 \AA$ measured in $1^{\text {st }}$ order on track 2 was used as standard on track 1 ;

f $\mathrm{Ten} \mathrm{Cu}$ II lines measured in $1^{\text {st }}$ order on track 2 were used as standards on track 5;

g Cu II line at $1275.5713 \AA$ measured in $2^{\text {nd }}$ order on track 22 was used as standard on track 7

h Four $\mathrm{Cu}$ II lines measured in $1^{\text {st }}$ order on tracks 10-12 were used as standards on track 9;

i Cu II line at $1407.1688 \AA$ Å measured in $1^{\text {st }}$ order on tracks 8, 11, and 12 was used as standard on track 10;

j Two $\mathrm{Cu}$ II lines measured in 1st order on track 15 were used as standards on track 16 ;

k Long-wavelength end of track 8 was fitted separately with a cubic polynomial;

${ }^{1}$ Short-wavelength end of track 9 was fitted separately with a 2nd degree polynomial. 
The grating spectra were photographed on Kodak SWR plates and measured with a Grant semiautomatic comparator. (Commercial products are identified in this paper for adequate specification of the experimental procedure. This identification does not imply recommendation or endorsement by NIST.) Repeated measurements of the same plates were made, from which the measurement uncertainty of line positions was estimated to be $1.6 \mu \mathrm{m}$ for isolated well-resolved lines, corresponding to statistical wavelengths uncertainties of $0.0012 \AA$. Most of the lines were measured on two to six different tracks. Thirty-eight $\mathrm{Cu}$ II lines were measured in the second order of diffraction, and one (861.9932 $\AA$ ) in the third order, while the rest of them were measured in the first order. Below $1980 \AA$, most of the tracks had a sufficient number of impurity lines ( $\mathrm{H}, \mathrm{He}, \mathrm{C}, \mathrm{N}, \mathrm{O}, \mathrm{Ne}, \mathrm{Si}, \mathrm{Ar}, \mathrm{Ge})$, which were used as standards. Cu II lines accurately measured by Kaufman and Ward [15] were also used as standards in the region (860 to 1663) $\AA$. In the region above $1980 \AA$, we used Ross's [2] interferometric and grating measurements as standards. Although later we found that Ross's wavelengths are systematically too long (see below), the average error in the wavelengths we used as standards in the grating spectra was only $(-0.0004 \pm 0.0008) \AA$, well below our total measurement uncertainties. Therefore, we did not remove the systematic errors from Ross's wavelengths used at this stage. The systematic uncertainty of each measured wavelength on our plates was estimated as a combination in quadrature of the standard deviation of the fitted polynomial (given in Table 1) and a mean uncertainty of standard wavelengths in the vicinity of the measured line. These systematic uncertainties were combined in quadrature with the statistical uncertainty $(0.0012 \AA$ for sharp isolated lines and up to $0.005 \AA$ for blended or overexposed lines). Multiple measurements of the same line on different tracks were averaged with weights inversely proportional to squares of total uncertainties. The uncertainty of the mean was calculated as a combination in quadrature of the reduced statistical uncertainty and the straight average systematic uncertainty. In total, we have measured 1217 unique spectral lines in the grating spectra between $636 \AA$ and $2682 \AA$, of which 938 were either identified as Cu II lines or did not have any identification, and the rest were identified as belonging to impurities noted above. The wavelengths determined in the present work have uncertainties ranging from $0.0008 \AA$ to $0.010 \AA$. A number of lines reported by Kaufman and Ward [15] were overexposed on our plates, which led to significant systematic shifts in determining their positions. Such overexposed lines were not used in the current wavelength measurements.

Our FTS measurements were calibrated assuming a common calibration factor for all wavenumbers in each spectrogram. The value of the calibration factor was determined as a weighted mean of the ratio $\sigma / \sigma_{\text {std }}$, where $\sigma$ is the measured wavenumber, and $\sigma_{\text {std }}$ is the tabulated wavenumber of the reference line. For the Ar II and Ge I-II lines used as standards in the FTS spectra, we used the Ritz values from ASD as $\sigma_{\text {std }}$. We used reciprocal squared uncertainties of $\sigma_{\text {std }}$, combined in quadrature with our measurement uncertainties (see below), as weights in this averaging. Uncertainties of thus obtained calibration factors (given in Table 1) represent the systematic uncertainties in our FTS measurements. Positions of the line centers $\sigma$, line widths $W$, signal-to-noise ratios $S / N$, and total integrated intensities were evaluated using the XGREMLIN code by Nave et al. [28] either automatically, assuming a Lorentzian profile for isolated symmetrical lines, Gaussian profile for nearly symmetrical but visibly perturbed lines, or manually with a Gaussian profile with a line width fixed at a visually estimated value.

For well-resolved symmetrical lines, statistical uncertainties of FTS measurements can be approximated by Equation (1) of Brault [29]:

$$
\delta \sigma_{\text {stat }}=\frac{k}{\sqrt{N_{w}}} \cdot \frac{W}{S / N}
$$

where $\sigma$ is the wavenumber, $W$ is the full width at half maximum of the line, $N_{w}$ is the number of statistically-independent points in the line width, and $k$ is a constant depending on the line shape and the algorithm used for fitting the line. For Gaussian profiles, $k=0.693$. For an optimally sampled 
spectrum, the interferogram is recorded to a path difference such that $N_{w}$ is between 3 and 4 for the majority of the spectral lines. This gives the commonly-used approximation of

$$
\delta \sigma_{\text {stat }}=\frac{W}{2 \cdot S / N}
$$

Using $N_{w}=W / r$, where $r$ is the resolution of the spectrum in $\mathrm{cm}^{-1}$, Equation (1) can be re-written as:

$$
\delta \sigma_{\text {stat }}=\frac{k}{S / N} \sqrt{r W}
$$

Our spectra were taken with $r=0.015 \mathrm{~cm}^{-1}$ for the $\mathrm{Cu} / \mathrm{Re} / \mathrm{Ar} / \mathrm{He}$ spectrum and $r=0.13 \mathrm{~cm}^{-1}$ for the $\mathrm{Cu} / \mathrm{Ge} / \mathrm{Pt} / \mathrm{Fe} / \mathrm{Ne}$ spectra. Typical line widths of symmetrical lines in our spectra range from $0.04 \mathrm{~cm}^{-1}$ around $10000 \mathrm{~cm}^{-1}$ to $0.25 \mathrm{~cm}^{-1}$ around $30000 \mathrm{~cm}^{-1}$, giving values of $N_{w}$ between 2.5 and 3 . The instrumental line shape of the FTS comes from the finite length of the interferogram and is a sinc function of width $r$. When this is convolved with the Gaussian lines in our spectra, the increase in the width is negligible for a line with $N_{w}=3$ and only $0.2 \%$ for a line with $N_{w}=2$ and can thus be ignored in the calculation of the statistical uncertainty.

Many of the $\mathrm{Cu}$ lines are affected by HFS and cannot be adequately fitted using Gaussian profiles. For example, Figure 1 shows the profile of the $\mathrm{Cu}$ II line at $12852 \mathrm{~cm}^{-1}$.

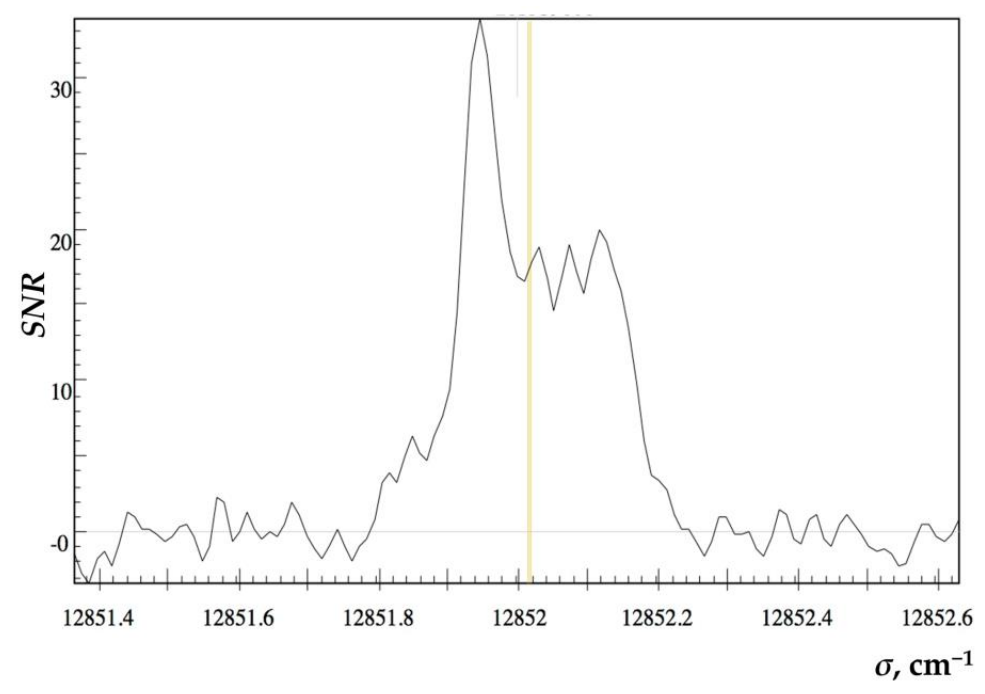

Figure 1. Profile of the $\mathrm{Cu}$ II line at $12852 \mathrm{~cm}^{-1}(7778.7 \AA)$ as measured in our Fourier transform spectrometers (FTS) spectrum. This line has a partially resolved hyperfine structure (HFS). The vertical line indicates the center of gravity.

The position of such lines was estimated using the center of gravity. For strong isolated lines $(S / N>150)$, where it is easy to estimate the range for calculation of the center of gravity, Equation (3) can be used to estimate the uncertainty using $k=1$ [29]. However, this uncertainty increases for weaker lines where it is not as easy to estimate the range of calculation for the center of gravity. The measurements are further complicated by the asymmetric HFS. Its precise shape and width is not studied so far for the $\mathrm{Cu}$ II spectrum. In our high-resolution $\mathrm{Cu} / \mathrm{Re} / \mathrm{Ar} / \mathrm{He}$ spectrogram, HFS patterns are partially resolved in 230 out of total $352 \mathrm{Cu}$ II lines. The average width of the HFS of those lines is $W_{\text {ave }}=0.14 \mathrm{~cm}^{-1}$. Since the shapes of the HFS are unknown, an additional uncertainty in the measured centers of gravity arises from the possible omission (or erroneous inclusion) of a weak HFS component at the far wing of the line profile. The possible error caused by this effect of noise is easy to estimate by calculating the shift of the center of gravity of the structure: 


$$
\delta \sigma_{\mathrm{hfs}}=0.5 W_{\mathrm{hfs}} I_{\text {noise }} /\left(I_{\mathrm{tot}}+I_{\text {noise }}\right),
$$

where $I_{\text {tot }}$ is the measured integrated intensity, $W_{\text {hfs }}$ is the maximum of $W_{\text {ave }}$ (see above) and the fitted width of the structure, and $I_{\text {noise }}$ is the estimated possible integrated intensity of a hypothetical missed or erroneously included HFS component, equal to the root of mean square of the noise amplitude in the vicinity of the structure multiplied by the average width of sharp Cu II lines (equal to $5 \times 10^{-6}$ times wavenumber for this spectrum).

The calibration of exposures 12 and 13, measured with a $\mathrm{Cu} / \mathrm{Ge} / \mathrm{Pt} / \mathrm{Ne}$ hollow cathode, was made using $37 \mathrm{Ge}$ I and II lines interferometrically measured by Kaufman and Andrew [30]. The reference wavenumbers from the latter paper have been decreased by 1.4 parts in $10^{8}$ to put them on the scale of a recent measurement of ${ }^{198} \mathrm{Hg}$ by Sansonetti and Veza [31], as described in Nave and Sansonetti [32]. Exposure 14, made with a $\mathrm{Cu} / \mathrm{Re} / \mathrm{Ar} / \mathrm{He}$ hollow cathode, was calibrated using $68 \mathrm{Ar}$ II standards from Whaling et al. [33]. Uncertainties of the calibration factors were determined as the sum in quadrature of the statistical uncertainty (coming from the different values of the calibration factor derived from different standard lines) and the mean uncertainty of the standard lines.

The total uncertainty of $\mathrm{Cu}$ II measurements was determined as the sum in quadrature of statistical and systematic uncertainties. The former was taken as the sum in quadrature of Equations (3) and (4), and the latter is the uncertainty in the calibration factor (given in Table 1) times the wavenumber. Total uncertainties of our Cu II wavelengths measured by FTS range from $0.000025 \AA$ to $0.023 \AA$.

Kaufman and Ward [15] photographed the VUV spectrum of a water-cooled hollow cathode discharge containing germanium and silicon in the first, second, and third orders of diffraction of the same $10.7 \mathrm{~m}$ Eagle-mounting vacuum grating spectrograph as used in the present work (designated as NIVS in Table 1). They reported 141 measured wavelengths of $\mathrm{Cu}$ II in the range (861 to 1663) $\AA$. As wavelength standards, they used lines of $\mathrm{Cu}$ II, Ge I, and Si I that were either calculated (Ritz) or interferometrically measured. The reciprocal linear dispersion was $0.78 \AA / \mathrm{mm}$ in the first order of diffraction. Many of the reported lines were measured on several (up to 11) spectrograms. We estimated their measurement uncertainties by comparing the measured wavelengths with the Ritz values (using Ross's [2] energy levels) separately for lines measured only in the first order $(0.003 \AA$ for a single measurement), measured several times in the 1st order, but only once in the 2nd and/or 3rd order $(0.0010 \AA)$, and for lines measured on several spectrograms in several orders of diffraction (varying from $0.0004 \AA$ to $0.0018 \AA$ ). These estimates agree well with those given explicitly by Kaufman and Ward for a few lines. Careful work was required to reconstruct the list of observed wavelengths, since some of the values given in Table III of Kaufman and Ward [15] are Ritz wavelengths, but many of them are also given in their Table I including the value of the residual $\lambda_{\text {Ritz }}-\lambda_{\text {obs }}$. Since there is no information regarding the wavelength standards actually used in various wavelength regions, all wavelengths reported by Kaufman and Ward [15] were adopted without corrections.

Ross [2,16] investigated the Cu II spectrum photographically from $1979 \AA$ to $11217 \AA$ using plane and concave grating spectrographs and Fabry-Perot interferometers. The light source was a water-cooled hollow cathode discharge with helium or neon as a buffer gas. Ross [2] noted that intensity of most $\mathrm{Cu}$ II lines was greatly enhanced when He at a pressure of about $0.9 \mathrm{kPa}$ (7 Torr) was used as the carrier gas, while if Ne is used, only lines below $3000 \AA$ arising from relatively low levels are excited. The standards for the Fabry-Perot interferograms were the lines of ${ }^{198} \mathrm{Hg}$ emitted by a water-cooled sealed electrodeless-discharge lamp containing argon carrier gas at a pressure of $33 \mathrm{~Pa}$ (0.25 Torr), with vacuum wavelengths 5462.27055 $\AA$ and 2537.2687 $\AA$ referred to Kaufman [34]. For grating measurements, a $9.2 \mathrm{~m}$ concave grating spectrograph with Paschen-Runge mounting was used. The grating had 600 lines $/ \mathrm{mm}$, was blazed at $10000 \AA$, and provided a reciprocal dispersion of $\approx 1.7 \AA / \mathrm{mm}$. For calibration, interferometrically measured Cu II lines were used as standards.

As noted in the Introduction, subsequent studies reported some systematic errors in Ross's short-wavelength measurements. This can be explained by the fact that below $2200 \AA$, Ross encountered severe technical difficulties in his interferometric measurements, caused by strong absorption in the 
crystalline quartz of the plates and windows. He partially solved the problem by removing the windows, which exposed the interferometer to spectroscopically non-standard air and did not permit thorough temperature control. In addition, Kaufman's values for the ${ }^{198} \mathrm{Hg}$ lines Ross used as standards, 5462.27046 $\AA$ and 2537.26877 $\AA$ [34], have since been re-evaluated. Their currently recommended vacuum wavelengths are 5462.27062(3) $\AA$ and 2537.268755(17) $\AA$ [35]. Additional errors could have been due to a possibly imperfect match between filling the interferometer's aperture with light from the hollow cathode and from the mercury lamp. To verify the calibration of Ross's wavelengths, we compared his reported wavelengths with those measured in our FTS spectra. Similar to FTS measurements, interferometric measurements of Ross can be corrected by a multiplicative factor [20]:

$$
\sigma_{\mathrm{c}}=\left(1+k_{\mathrm{eff}}\right) \sigma_{\mathrm{u}}
$$

where $\sigma_{\mathrm{c}}$ is the corrected wave number, $\sigma_{\mathrm{u}}$ is the uncorrected wave number, and the correction factor $k_{\text {eff }}$ is determined from one or more internal standard lines in the spectrum, in this case from our wavenumbers measured by FTS. Dependence of thus derived $k_{\text {eff }}$ on wavenumber is plotted in Figure 2 .

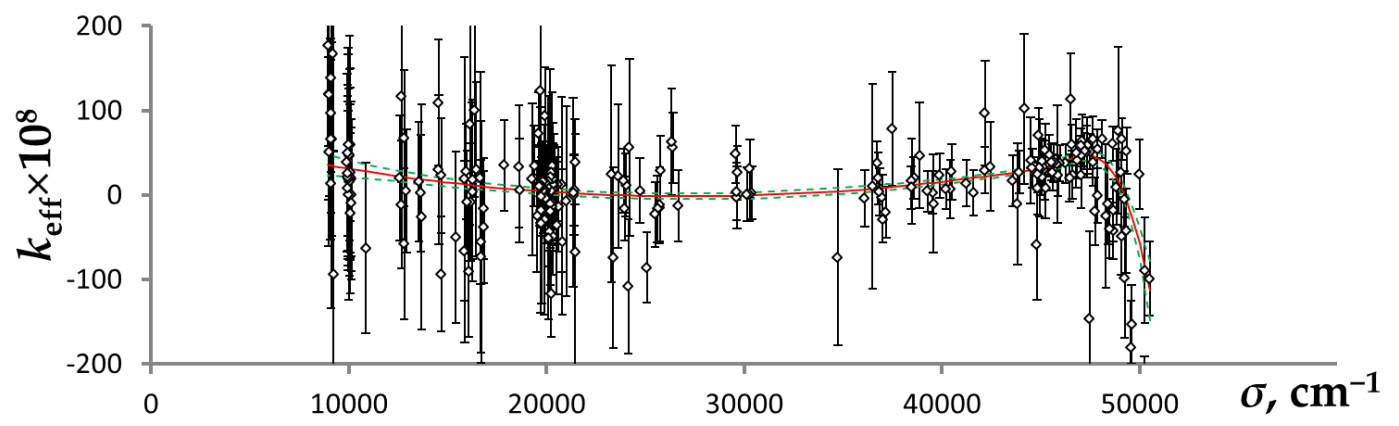

Figure 2. Dependence of calibration factor for interferometric measurements of Ross [2] on wavenumber. The error bars are measurement uncertainties of Ross [2] and our FTS data, combined in quadrature. The solid line is a weighted fit with two cubic polynomials stitched together at about $45,000 \mathrm{~cm}^{-1}$. The dashed lines are $68 \%$ confidence intervals ( \pm 1 standard deviation) of the fit.

It should be noted that Figure 2 includes all Ross's lines that were also measured in our FTS spectra. Many of those lines were weak in our FTS spectra, resulting in large error bars in Figure 2. However, there was a sufficiently large number of strong lines with small error bars, which explain the very small uncertainties of the fitted curve in Figure 2.

Unlike FTS measurements, where $k_{\text {eff }}$ is a constant over the entire range of measured lines, the calibration correction of Ross's measurements depends on wavenumber. This dependence is rather weak, and the value of $k_{\text {eff }}$ is small $\left(<10^{-7}\right)$ in the interval $(17000$ to 38000$) \mathrm{cm}^{-1}$ (6000 $\AA$ to $2700 \AA$ ), but $k_{\text {eff }}$ notably increases (up to $3.5 \times 10^{-7}$ ) for infrared lines, and varies rapidly for the far-UV lines below $2700 \AA$. The accuracy of the fitted $k_{\text {eff }}$ values varies from $3 \times 10^{-8}$ to $3.5 \times 10^{-7}$, depending on the measurement uncertainties in Ross [2] and in our FTS data. It determines the systematic uncertainties in the Ross's wavenumbers as now re-calibrated.

This re-calibration and increased uncertainty associated with it allowed us to explain only a small part of numerous lines strongly deviating from Ritz wavelengths in Ross's list, noted in the Introduction. To explain the remaining problematic lines, additional factors contributing to uncertainties, not accounted for by Ross, must be considered. One such factor can be pressure shifts caused by relatively high pressure of $\mathrm{He}$ in his discharge, $0.9 \mathrm{kPa}$ (7 Torr). Such shifts could cause quasi-random deviations of measured wavelengths in both directions from unperturbed values. Another contributing factor could be partially resolved HFS. The Doppler width reported by Ross for the sharp line at $2473 \AA\left(40419 \mathrm{~cm}^{-1}\right)$ was about $0.1 \mathrm{~cm}^{-1}$, which is of the same order of magnitude as most of the known HFS widths. He noted that partially-resolved HFS was indeed a problem in 
his wavelength measurements, and that his tabulated results are centers of gravity of observed HFS structures. However, he did not give any details about the method with which these centers of gravity were determined. Such determination depends on the measurement accuracy of the relative intensities of the HFS components, which might have been distorted by non-linearity of response of photographic plates used by Ross. The shift in the measured center-of-gravity wavenumber caused by errors in intensity measurements would be some fraction of the HFS width. We accounted for both types of such possible shifts (caused by pressure and HFS) by adding in quadrature a constant quantity to the wavenumber-measurement uncertainties of Ross [2]. The value of this constant was found empirically to be $0.007 \mathrm{~cm}^{-1}$. These increased uncertainties are statistically consistent with residuals $\sigma_{\mathrm{obs}}-\sigma_{\text {Ritz }}$ of the level-optimization procedure (see Section 3).

Shenstone [1] made the most comprehensive study of the $\mathrm{Cu}$ II spectrum prior to Ross [2]. He photographed spectra of a copper hollow cathode (Schuler tube) filled with helium or neon using several spectrographs: $6.4 \mathrm{~m}$ and $3 \mathrm{~m}$ normal incidence grating spectrographs and two Hilger prism spectrographs for the UV and visible regions, a $2 \mathrm{~m}$ normal incidence vacuum grating spectrograph for the VUV region, and another concave grating spectrograph (in NBS) for the infrared region. Since Ross could not observe lines shorter than $1979 \AA$ A, Shenstone's line list remained the only source of information about observed $\mathrm{Cu}$ II lines and their intensities in the VUV. We have re-measured many of VUV lines listed by Shenstone with much greater accuracy. Most of his lines in the UV, visible, and infrared regions were re-measured by Ross [2]. However, 94 lines from Shenstone [1] ranging from $836 \AA$ to $6870 \AA$ were not observed in our and Ross's spectra. For 15 of them, Shenstone did not give a measured wavelength (apparently, because low resolution did not permit accurate measurement), but gave the observed intensity. Wavelength calibration and uncertainties of Shenstone's measurements were investigated by comparing them with Ritz wavelengths calculated from Ross's [2] energy levels. Because of the relatively low resolution of Shenstone's measurements, the small differences of Ross's Ritz wavelengths from more accurate ones obtained from our final level optimization (see Section 3) did not have any effect on this analysis. Since most of Shenstone's lines used in the final line list were measured with the vacuum grating spectrograph, we compare his measurements made on this instrument with Ritz wavelength in Figure 3.

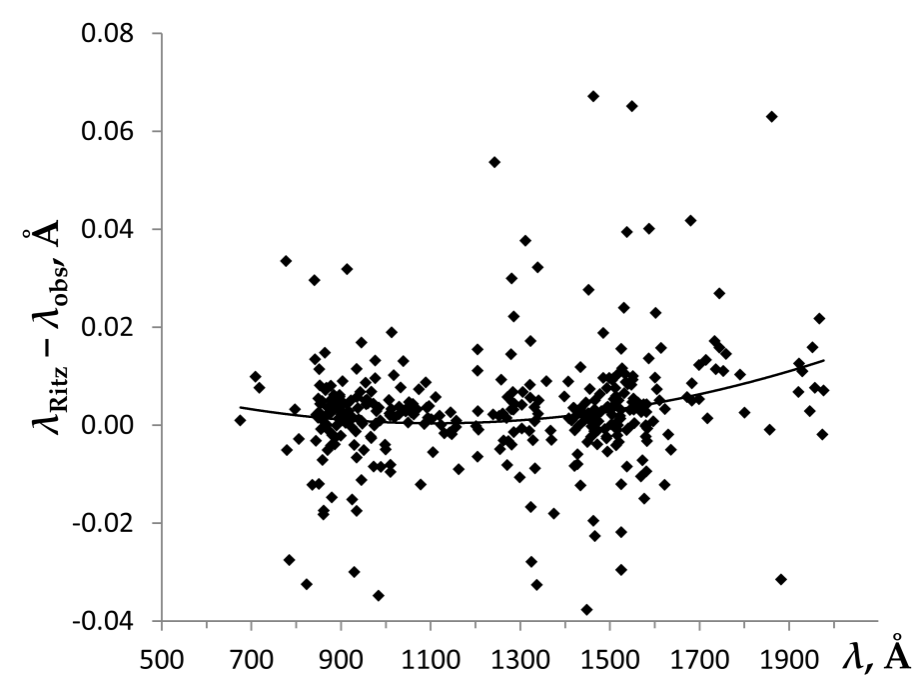

Figure 3. Deviations of vacuum ultraviolet (VUV) wavelengths measured by Shenstone [1] from Ritz values from Ross [2]. The solid line is a 2 nd degree polynomial fit.

Although Figure 3 includes all wavelengths reported by Shenstone (excluding a few lines with extremely large deviations), assessment of uncertainties was made separately for "good" wavelengths given with three figures after the decimal point (in $\AA$ ) and having no indication of blending or other 
perturbations, for wavelengths given with two decimal figures (apparently, deemed less accurate by Shenstone), and for lines indicated as blended or perturbed, or having multiple classifications. The trend indicated in Figure 3 by a solid line was derived from accurately measured lines only. It resulted in a correction to Shenstone's VUV wavelength varying from $+0.003 \AA$ for the shortest wavelengths near $700 \AA$ to $+0.010 \AA$ near $1980 \AA$. After this correction, the uncertainty for each category of lines was estimated as a root-mean-square (rms) of the residuals. These uncertainties were found to vary from $0.006 \AA$ for the best measurements to $0.07 \AA$ for the worst. A similar analysis was made separately for each spectrograph used by Shenstone.

For completeness, we included in our line list one line observed by Wagatsuma and Hirokawa [36] at $4485.3 \AA$, which was not reported by other observers. Since wavelengths reported by Wagatsuma and Hirokawa are the Ritz ones (owing to low precision of their measurements), we also give only the Ritz wavelength for this line.

In all the laboratory studies described above, only electric-dipole allowed (E1) lines could be observed, since densities in the discharge light sources are high enough to collisionally depopulate metastable levels, from which forbidden lines could emerge. However, four forbidden lines of $\mathrm{Cu}$ II were observed in other studies. The first observation known to us is that of Thackeray [3] who identified the line of $\mathrm{Cu}$ II at $3806.3 \AA$ as the electric-quadrupole (E2) $3 \mathrm{~d}^{10}{ }^{1} \mathrm{~S}_{0}-3 \mathrm{~d}^{9} 4 \mathrm{~s}^{1} \mathrm{D}_{2}$ transition in emission spectra of two nebulae, $\eta$ Carinae and RR Telescopii. Another E2 transition, $3 \mathrm{~d}^{10}{ }^{1} \mathrm{~S}_{0}-3 \mathrm{~d}^{9} 4 \mathrm{~s}^{3} \mathrm{D}_{2}$ at $4375.8 \AA$, as well as the hyperfine-induced $3 \mathrm{~d}^{10}{ }^{1} \mathrm{~S}_{0}-3 \mathrm{~d}^{9} 4 \mathrm{~s}^{3} \mathrm{D}_{3}$ transition at $4558.7 \AA$, were observed but not identified in emission of RR Telescopii by Aller et al. [4]. The above-mentioned E2 transition at $3806.3 \AA$, as well as the magnetic-dipole (M1) $3 \mathrm{~d}^{10}{ }^{1} \mathrm{~S}_{0}-3 \mathrm{~d}^{9} 4 \mathrm{~s}^{3} \mathrm{D}_{1}$ transition at $4165.7 \AA$, were observed and identified by McKenna et al. [5] in emission of the same nebula, RR Telescopii. In the laboratory, the two E2 transitions at $3806.3 \AA$ and $4375.8 \AA$ were observed (with an accuracy inferior to astrophysical observations) and their radiative decay rates were measured by Prior [37] in an electrostatic ion trap.

All observed and identified spectral lines of $\mathrm{Cu}$ II are collected in Appendix A, Table A1. In addition to observed lines, this table includes several predicted transitions, in particular, six M1 and E2 transitions between the levels of the first excited configuration, $3 \mathrm{~d}^{9} 4 \mathrm{~s}$. This table lists 2557 transitions corresponding to 2494 unique measured spectral lines, 677 of which were measured in this work, and includes 50 additional lines that are either predicted (not observed), observed but not measured, or were masked by stronger neighboring lines in observed spectra. In addition to observed wavelengths, Table A1 gives for each transition the Ritz wavelength with standard uncertainty obtained in the level optimization procedure (see Section 3), energy-level classification, intensity on a unified scale (see Section 5), and a reference to the source of the observed wavelength. For 555 transitions, we also give a critically evaluated transition probability ( $A$-value) with its uncertainty estimate and a reference. Lines on which laser action was reported in the literature are marked with " $L$ " in the Notes column.

In Table A1, wavelengths between $2000 \AA$ and $20000 \AA$ are given in standard air; outside of this region, they are in vacuum. Conversion from air to vacuum was made using the five-parameter formula from Peck and Reeder [38]. Ritz wavelengths and their uncertainties were obtained using the LOPT (level optimization) code [39] as described below in Section 3. Transition probabilities are either calculated in the present work or critically compiled from references [40-51] as described in Section 6.

\section{Energy Levels}

The precise positions of energy levels, as well as the Ritz wavelengths and their uncertainties, were derived from the identified lines listed in Table A1 using the least-squares level optimization code LOPT [39]. The resulting energy levels are listed in Table A2.

Of all 2557 transitions in Table A1, 113 were excluded from the level optimization procedure. Among those, there are 61 transitions that were either predicted (e.g., far-IR forbidden transitions), had poorly measured wavelengths (e.g., astrophysically observed forbidden lines), were severely 
blended, or were masked by much stronger nearby lines. The remaining 52 lines were excluded because their observed wavelengths deviated too much from the Ritz values. In total, we used 2443 observed transitions in our level-optimization procedure. For comparison, Ross [2,16] used 1691 observed transitions in his level optimization.

If one compares the presently found energy levels with those given by Ross [2,16], the average agreement is good. The mean difference $\left(E_{\mathrm{TW}}-E_{\mathrm{Ross}}\right)$ is only $0.007 \mathrm{~cm}^{-1}$ with a standard deviation of $0.016 \mathrm{~cm}^{-1}$ (the subscript "TW" means "this work"). However, a more detailed comparison shows that, of the 347 levels correctly identified by Ross, 156 deviate from those given in Table A2 by more than two (up to 11) combined uncertainties. Uncertainties of our level values are on average greater than those of Ross by a factor of 1.4, owing to our increased estimate of Ross's wavelength uncertainties. For only 32 levels our uncertainties are smaller than those of Ross (by up to a factor of eight). This improvement is due to our FTS measurements. Similar conclusions can be made for the Ritz wavelengths. Among about 500 Ritz VUV wavelengths listed by Ross, only a few are incorrect because of erroneous identifications described below. If these are excluded, the mean difference $\left(\sigma_{\mathrm{TW}}-\sigma_{\text {Ross }}\right)$ is $0.012 \mathrm{~cm}^{-1}$ with a standard deviation of $0.019 \mathrm{~cm}^{-1}$. However, for $273 \mathrm{VUV}$ lines (more than half of all given by Ross), Ross's Ritz wavelengths deviate from ours by more than twice the combined uncertainty. Our Table A1 includes 632 Ritz wavelengths for VUV lines below $2000 \AA$. Most of the new lines were observed and measured in this work.

The analysis that led to Tables A1 and A2 was made in an iterative manner. In the first step, after an initial list of all observed lines was constructed, it contained more than 600 unclassified lines, 143 of which were listed by Ross [2,16], 54 by Shenstone [1], one by Kaufman and Ward [15], and the rest were observed in our VUV grating spectra. Twenty-four of these lines (18 in Ross [2,16] and 6 in Shenstone [1]) were found to be due to previously identified transitions in $\mathrm{Cu}$ I. To find possible identifications for the remaining unknown lines and to find proper designations for the energy levels, we made a parametric analysis of the $\mathrm{Cu}$ II spectrum using Cowan's suite of atomic codes $\mathrm{RCN} / \mathrm{RCN} 2 / \mathrm{RCG} / \mathrm{RCE}$ [52] (A version of the codes adapted by A. Kramida for Windows-based personal computers is available online: http://das101.isan.troitsk.ru/COWAN). In this analysis, we included the following sets of configurations: $3 \mathrm{~d}^{10},\left[3 \mathrm{~d}^{9}\right](n \mathrm{~s}, n \mathrm{~d})(n=4-10), n \mathrm{~g}(n=5-10),\left[3 \mathrm{~d}^{8}\right]\left(4 \mathrm{~s}^{2}\right.$,

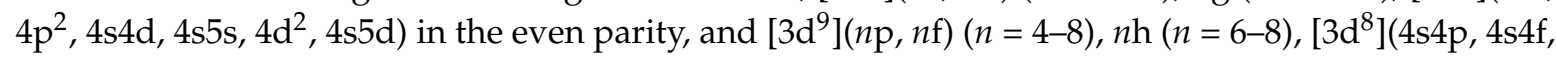
$4 \mathrm{p} 4 \mathrm{~d}, 4 \mathrm{~s} 5 \mathrm{p}, 4 \mathrm{~s} 5 \mathrm{f}$ ) in the odd parity, 25 configurations in total. All known energy levels from Sugar and Musgrove [17] were included in the least-squares parametric fitting (LSF) with the RCE code. The calculations were made in the relativistic mode (HFR), including Breit corrections and correlation term (explained in the RCN manual). The fitted parameters were substituted as input for the RCG code to obtain a list of predicted lines with calculated $A$-values. The calculated $A$-values and energy levels, as well as known experimental levels, were used in the input files for the visual line-identification code IDEN1 originally designed by Azarov [53] and later programmed for Windows-based computers by one of the present authors (AK). Using this tool, we identified 117 previously unclassified lines with transitions between known energy levels, revised four levels and found 28 new energy levels. The revised and newly found energy levels explained 79 previously unclassified lines and involved revised classifications of 24 lines previously identified by Ross [2] and Shenstone [1].

At this stage of the analysis, it was also found that two levels listed by Ross [2] and included in the compilation of Sugar and Musgrove [17] had to be rejected. One of them, an undesignated odd-parity level with $J=1$ at $144240.6 \mathrm{~cm}^{-1}$, was retained by Ross from Shenstone's level list. It was identified by one line measured by Shenstone at $823.802 \AA$, which we re-measured to be at $823.8361(18) \AA$ and identified with a transition between other previously known levels, $3 d^{9} 4 s^{1} D_{2}-3 d^{9}\left({ }^{2} D_{5 / 2}\right) 7 p^{2}[5 / 2]^{\circ}$. It was also found that there are no possible unknown odd-parity levels with $J=1$ sufficiently close to the value given by Ross to explain this level. Another rejected level is also of odd parity, designated by Ross as $3 \mathrm{~d}^{9} 6 \mathrm{p}^{3} \mathrm{P}_{0}^{\circ}$ (at $141154.164 \mathrm{~cm}^{-1}$ ). It was based by Ross on two lines, one observed by Shenstone at $853.56 \AA$ and identified by Ross as a transition from this level to $3 \mathrm{~d}^{9} 4 \mathrm{~s}^{3} \mathrm{D}_{1}$, and another observed by Ross at $3217.641 \AA$, which he interpreted as a transition from this level to $3 d^{9}\left({ }^{2} \mathrm{D}_{3 / 2}\right) 5 \mathrm{~s}$ 
${ }^{2}[3 / 2]_{1}$ (to be consistent with our tables, we use our new JK designations). Both these transitions were predicted to be extremely weak, certainly much weaker than other possible transitions from this level. We re-measured the first line to be at 853.544(2) $\AA$ and re-classified both lines as transitions from other levels. In addition, the energy given by Ross deviates too much from that predicted by our parametric fitting.

Two levels listed by Ross as $3 \mathrm{~d}^{9} 6 \mathrm{p}^{3} \mathrm{~F}^{\circ}{ }_{2}$ and ${ }^{3} \mathrm{D}^{\circ}{ }_{1}$ at $141244.576 \mathrm{~cm}^{-1}$ and $141734.167 \mathrm{~cm}^{-1}$, respectively, were each based by Ross on several transitions to lower-lying even levels with $J=1$ or 2 . Most of the corresponding lines were previously observed and similarly identified by Shenstone. However, we found that observed line intensities agree much better with those predicted if assignments of these two levels are interchanged. Further proof for this revision was provided by our identification of two transitions from the lower of these two levels down to levels with $J=3$. One of the corresponding lines was newly observed in our VUV spectrum, and another one was present among Ross's unclassified lines.

Following Shenstone [1], Ross interpreted the level at $137212.765 \mathrm{~cm}^{-1}$ as $3 \mathrm{~d}^{8} 4 \mathrm{~s} 4 \mathrm{p}^{1} \mathrm{P}^{\circ}{ }_{1}$. Sugar and Musgrove [17] gave a more specific designation, $3 \mathrm{~d}^{8}\left({ }^{3} \mathrm{P}\right) 4 \mathrm{~s} 4 \mathrm{p}\left({ }^{3} \mathrm{P}^{\circ}\right){ }^{1} \mathrm{P}^{\circ}{ }_{1}$ based on a parametric calculation made by Roth [54]. We found three transitions connecting this level with even $J=3$ levels. Since other combining levels have $J=1$ and 2, the only possible $J$ value for this level is 2 . We labeled this strongly mixed level as $3 \mathrm{~d}^{8}\left({ }^{1} \mathrm{G}\right) 4 \mathrm{~s} 4 \mathrm{p}\left({ }^{3} \mathrm{P}^{\circ}\right){ }^{3} \mathrm{~F}^{\circ}$, although it represents only $36 \%$ of the percentage composition; an equal contribution is from $3 \mathrm{~d}^{8}\left({ }^{3} \mathrm{~F}\right) 4 \mathrm{~s} 4 \mathrm{p}\left({ }^{1} \mathrm{P}^{\circ}\right){ }^{3} \mathrm{~F}^{\circ}{ }_{2}$, followed by $14 \%$ of $3 \mathrm{~d}^{8}\left({ }^{3} \mathrm{P}\right) 4 \mathrm{~s} 4 \mathrm{p}\left({ }^{3} \mathrm{P}^{\circ}\right){ }^{1} \mathrm{D}^{\circ}{ }_{2}$.

Ross found the level at $147491.888 \mathrm{~cm}^{-1}$, which he designated as $3 \mathrm{~d}^{9} 7 \mathrm{p}^{3} \mathrm{~F}_{3}^{\circ}$ based on three weak lines at $3360.9941 \AA, 9310.353 \AA$, and $9569.11 \AA$, identified as transitions from this level down to $3 d^{9} 4 d^{3} G_{3}, 3 d^{9} 5 d{ }^{3} P_{2}$, and $3 d^{9} 5 d^{3} F_{4}$. Although these three lines indeed perfectly satisfy the Ritz combination principle, our calculations indicate that their intensities should be negligibly small, while much stronger transitions to other even-parity levels should occur, but were not observed by Ross. The strongest predicted transitions from this level are in the VUV region inaccessible to Ross. We have found three lines in our VUV exposures at $824.663 \AA$, $1609.342 \AA$, and $1924.548 \AA$, which have wavelengths and intensities consistent with this level, placing it at our new revised position, $147525.93 \mathrm{~cm}^{-1}$. All other odd-parity levels with $J=3$ predicted to occur within $\pm 2000 \mathrm{~cm}^{-1}$ from this energy are experimentally known, which leads us to conclude that the combination of three lines observed by Ross is spurious. We note that the density of observed lines in the UV, visible, and IR regions is sufficiently high to produce several tens of spurious combinations of two, and a few of three lines, which we observed in the combined line list. We could not find alternate identifications for the three lines assigned previously by Ross to this revised level.

Three other levels in Ross's list were found to be questionable, either because they were based on only one or two weak lines, or because the energy strongly disagrees with our parametric calculation. These levels are at $154838.963 \mathrm{~cm}^{-1}, 155244.833 \mathrm{~cm}^{-1}$, and $156958.096 \mathrm{~cm}^{-1}$, designated by Ross as $3 \mathrm{~d}^{9} 8 \mathrm{~d}^{3} \mathrm{P}_{1},{ }^{3} \mathrm{P}_{0}$, and ${ }^{3} \mathrm{~F}_{2}$, respectively.

After the new identifications were made, the new experimental energies were incorporated in the LSF procedure, and the questionable levels removed. We also inserted in the LSF several tens of levels belonging to the $\left[3 \mathrm{~d}^{9}\right] 9 \mathrm{~d}, 10 \mathrm{~d}, 8 \mathrm{~g}, 9 \mathrm{~g}, 10 \mathrm{~g}, 7 \mathrm{f}, 8 \mathrm{f}, 6 \mathrm{~h}, 7 \mathrm{~h}$, and $8 \mathrm{~h}$ levels whose positions were accurately extrapolated by Ritz-type quantum defect or polarization formulas (see Section 4). These levels were found to be sufficiently pure, unperturbed by interactions with other configurations, and thus the series formulas provided dependable results.

In this final LSF, 218 known even-parity levels were fitted with 38 free parameters with a standard deviation of $40 \mathrm{~cm}^{-1}$, and 241 known odd-parity levels were fitted with 35 free parameters with a standard deviation of $72 \mathrm{~cm}^{-1}$. The fitted (LSF) and ab initio Hartree-Fock (HF) values of parameters are given in Table A3. In both parities, the $\zeta_{3 \mathrm{~d}}$ parameters of the $3 \mathrm{~d}^{n}$ core were linked together in one group for all configurations, so that their LSF/HF ratio was the same for all members of the group, but was allowed to vary in the fitting. Other parameters, similar in different configurations, such as the 
effective parameter $\alpha_{3 \mathrm{~d}}$, electrostatic parameter $F^{2}(3 \mathrm{~d}, 3 \mathrm{~d})$ of the $3 \mathrm{~d}^{8}$ core, and configuration-interaction parameters, were also linked in similar groups, which decreased the number of free parameters and made the fitting more stable.

Using the transformation procedures implemented in Cowan's RCE code, eigenvector compositions were calculated in several coupling schemes. Similar to the spectrum of neutral nickel analyzed by Litzén et al. [21], most of the $3 \mathrm{~d}^{9} \mathrm{nl}$ configurations were found to be best described in the $J_{1} l$ (otherwise known as $J K$ or $J_{\mathrm{c}} K$ ) coupling scheme, in which the total angular momentum of the core $\left(3 \mathrm{~d}^{9}\right.$ in this case) is combined with the orbital momentum of the valence electron to produce the $K$ quantum number, which is then combined with the spin of the valence electron to obtain the final total angular momentum $J$. In Ni I, Litzén et al. [21] found the lowest [ $\left.3 \mathrm{~d}^{9}\right] n l$ configurations, $4 \mathrm{~s}, 4 \mathrm{p}$, and $5 p$, to be better described in the LS coupling scheme, which is also the best for the $3 d^{8} 4 s^{2}$ and other $3 \mathrm{~d}^{8} n n^{\prime} l^{\prime}$ configurations. In Cu II, our findings are similar, except that the $3 \mathrm{~d}^{9} 5 \mathrm{p}$ configuration is better described by $J_{1} l$ coupling. The $J_{1} l$ purity of the $3 \mathrm{~d}^{9} \mathrm{nl}$ configurations increases from $65 \%$ for $3 \mathrm{~d}^{9} 6 \mathrm{p}$ to $100 \%$ for $3 \mathrm{~d}^{9} n s, n h(n \geq 6)$, and $n g(n \geq 6)$, and generally increases with increasing $n$ and $l$. This general trend is disrupted in $3 \mathrm{~d}^{9} 6 \mathrm{p}$. This configuration strongly interacts with $3 \mathrm{~d}^{8} 4 \mathrm{~s} 4 \mathrm{p}$, unlike $3 d^{9} 5 p$, for which this interaction is somewhat weaker, resulting in the average $J_{1} l$ purity of $75 \%$. For the $3 \mathrm{~d}^{8} 4 \mathrm{~s} 4 \mathrm{p}$ configuration, in agreement with the previous analysis by Roth [54], we found that the best description is obtained by first combining the quantum numbers of the $4 \mathrm{~s}$ and $4 \mathrm{p}$ electrons with each other to produce an intermediate $L S J$ term $\left({ }^{3} \mathrm{P}^{\circ}\right.$ or $\left.{ }^{1} \mathrm{P}^{\circ}\right)$ of the $4 \mathrm{~s} 4 \mathrm{p}$ valence shell, and then combine it with $L S J$ of the $3 \mathrm{~d}^{8}$ core. Although the average purity of $3 \mathrm{~d}^{8} 4 \mathrm{~s} 4 \mathrm{p}$ in this coupling scheme is rather high, $73 \%$, many of its levels are strongly mixed with other configurations, rendering assignment of single-configuration labels arbitrary. Several levels attributed to this configuration have a leading percentage of the composition smaller than $30 \%$. In a few cases, such as the levels at $139331.149 \mathrm{~cm}^{-1}$ $(J=3)$ and $139710.491 \mathrm{~cm}^{-1}(J=2)$, we assigned labels corresponding to the second leading term in the composition, in order to preserve uniqueness of the labels within the $J$ manifolds. The labels assigned to the levels do not fully describe their physical nature; percentage compositions given in Table A2 are somewhat better in this regard.

The high $J_{1} l$ purity of the $3 \mathrm{~d}^{9} n$ g configurations and very small values of parameters describing interactions between $3 \mathrm{~d}^{9}$ and $n \mathrm{~g}$ shells allows for accurate prediction of the levels that were missing in Ross's levels list. This permitted us to find some of these levels from the lines left unclassified in his analysis.

For each level, Table A2 gives a number of observed lines on which this level is based. Most of the levels are based on more than two observed lines. However, 71 of them are derived from only one or two observed lines. Most of them are firmly identified, because the observed lines are the strongest predicted ones, and the level positions are confirmed by trends along series. Five of such levels are marked as questionable, because the corresponding series are strongly perturbed, or because the observed lines are not the strongest predicted to occur from these levels. The absence of predicted stronger lines could be explained by different registration sensitivity in exposures used in different spectral regions.

We identified the previously missing $3 \mathrm{~d}^{8} 4 \mathrm{~s}^{2}{ }^{1} \mathrm{~S}_{0}$ level based on one line observed at $1807.8535 \AA$ in two exposures in our VUV spectra and at $1807.84 \AA$ by Shenstone [1]. We assigned this line to the strongest predicted transition from this level, terminating at $3 \mathrm{~d}^{9} 4 \mathrm{p}^{1} \mathrm{P}^{\circ}{ }_{1}$. Although this line is observed to be about 20 times stronger than predicted, this was the only unidentified line within the interval of $\pm 3000 \mathrm{~cm}^{-1}$, and it places the $3 \mathrm{~d}^{8} 4 \mathrm{~s}^{2}{ }^{2} \mathrm{~S}_{0}$ level within $25 \mathrm{~cm}^{-1}$ of its predicted position. Therefore, we believe that this identification is correct.

Five odd-parity levels are given in Table A2 with revised designations. The level with $J=3$ at $121524.8509 \mathrm{~cm}^{-1}$ was previously labeled by Sugar and Musgrove [17] as $3 \mathrm{~d}^{9} 5 \mathrm{p}{ }^{3} \mathrm{D}^{\circ}$ with a $53 \%$ contribution of this term in the composition, as given by Roth [54]. Although our calculation yields the same leading term in the composition of this level, the total contribution from the $3 \mathrm{~d}^{8} 4 \mathrm{~s} 4 \mathrm{p}$ configuration is calculated to be greater than from $3 \mathrm{~d}^{9} 5 \mathrm{p}$. Therefore, we designated this level by the second largest 
component, $3 \mathrm{~d}^{8}\left({ }^{3} \mathrm{~F}\right) 4 \mathrm{~s} 4 \mathrm{p}\left({ }^{3} \mathrm{P}^{\circ}\right){ }^{1} \mathrm{~F}^{\circ}$. A marginally larger contribution of the $3 \mathrm{~d}^{9} 5 \mathrm{p}^{3} \mathrm{D}^{\circ}$ term was found in the $J=3$ level at $121079.1501 \mathrm{~cm}^{-1}$, which we attributed to this configuration. The $J_{1} l$-coupling designation gives a better description of this level, the leading term being $54 \%$ of $3 d^{9}\left({ }^{2} D_{5 / 2}\right) 5 p^{2}[5 / 2]^{\circ}$. In effect, the identifications of these two $J=3$ levels at $121079.1501 \mathrm{~cm}^{-1}$ and $121524.8509 \mathrm{~cm}^{-1}$ have been interchanged. This revision is supported by better agreement of observed and calculated line intensities.

The $J=4$ level at $134742.863 \mathrm{~cm}^{-1}$ was labeled by Sugar and Musgrove [17] as $3 \mathrm{~d}^{9} 6 \mathrm{p}^{3} \mathrm{~F}^{\circ}$, and its composition was given as $49 \%$ of this term and $39 \%$ of $3 \mathrm{~d}^{8}\left({ }^{1} \mathrm{G}\right) 4 \mathrm{~s} 4 \mathrm{p}\left({ }^{3} \mathrm{P}^{\circ}\right){ }^{3} \mathrm{~F}^{\circ}$. We found the leading term to be $50 \%$ of $3 \mathrm{~d}^{8}\left({ }^{3} \mathrm{~F}\right) 4 \mathrm{~s} 4 \mathrm{p}\left({ }^{1} \mathrm{P}^{\circ}\right)$, which we use as a revised label. We note that Sugar and Musgrove gave the same configuration and term label to another $J=4$ level at $139395.786 \mathrm{~cm}^{-1}$ with almost the same percentage composition. We found for the latter level $59 \%$ contribution from $3 \mathrm{~d}^{9} 6 \mathrm{p}^{3} \mathrm{~F}^{\circ}$, but designated it in $J_{1} l$-coupling as $3 \mathrm{~d}^{9}\left({ }^{2} \mathrm{D}_{5 / 2}\right) 6 \mathrm{p}^{2}[7 / 2]^{\circ}$.

Another $J=4$ level at $137938.904 \mathrm{~cm}^{-1}$ was designated by Sugar and Musgrove [17] as $3 \mathrm{~d}^{8}\left({ }^{3} \mathrm{~F}\right) 4 \mathrm{~s} 4 \mathrm{p}\left({ }^{1} \mathrm{P}^{\circ}\right){ }^{3} \mathrm{~F}^{\circ}$ with $53 \%$ of this term in the composition. We found the leading terms to be $49 \%$ of $3 d^{8}\left({ }^{1} \mathrm{G}\right) 4 \mathrm{~s} 4 \mathrm{p}\left({ }^{3} \mathrm{P}^{\circ}\right){ }^{3} \mathrm{~F}^{\circ}, 39 \%$ of $3 \mathrm{~d}^{9} 6 \mathrm{p}^{3} \mathrm{~F}^{\circ}$, and only $5 \%$ of the term given as label by Sugar and Musgrove. We changed the label to the leading term indicated by our calculation.

The $J=3$ level at $139331.149 \mathrm{~cm}^{-1}$ was designated by Sugar and Musgrove [17] as $3 \mathrm{~d}^{9} 6 \mathrm{p}^{3} \mathrm{D}^{\circ}$ with $56 \%$ of this term in the composition. In our calculation, the leading $L S$ term is found to be $46 \%$ of $3 \mathrm{~d}^{9} 6 \mathrm{p}{ }^{1} \mathrm{~F}^{\circ}$. However, in $J_{1} l$-coupling adopted for the $3 \mathrm{~d}^{9} 6 \mathrm{p}$ configuration, the leading term is $32 \%$ of $3 \mathrm{~d}^{9}\left({ }^{2} \mathrm{D}_{5 / 2}\right) 6 \mathrm{p}^{2}[7 / 2]^{\circ}$, which is used to label another level having $62 \%$ of this term. Thus, we labeled the level at $139331.149 \mathrm{~cm}^{-1}$ by the second leading $L S$ term of its composition, $3 \mathrm{~d}^{8}\left({ }^{1} \mathrm{G}\right) 4 \mathrm{~s} 4 \mathrm{p}\left({ }^{3} \mathrm{P}^{\circ}\right){ }^{3} \mathrm{~F}^{\circ}$.

\section{Ionization Energy}

Ross [2] derived the value for the ionization limit by extrapolation of quantum defects along 12 series of the type $3 \mathrm{~d}^{9} n l$ (two $n \mathrm{~s}$, nine $n \mathrm{~d}$, and one $n f$, with $n$ ranging from 4 to 10 for the $n \mathrm{~s} J=3$ series, 4 to 9 for the $n \mathrm{~s} J=1$ series, and 4 to 8 for the rest). Although Ross used LS designations for all the levels involved, the series he chose are almost pure in $J_{1} l$ coupling, resulting in smooth behavior of quantum defects along the series. Using the RITZPL computer code by Sansonetti [55], we repeated Ross's derivation with our more accurate level values and obtained slightly different values for the series limits. In the derivation of the limit, we added two more 5 -member $3 d^{9} n d$ series, in which we found some new identifications. The weighted average of the limits obtained closely agrees with the average value adopted by Ross. In addition, series limits obtained from six three-member series with an exact fit of the polarization formula (Sansonetti [56]) produced values in close agreement with this average. Thus, we adopted the value for the ionization limit given by Ross [2], 163669.2(5) $\mathrm{cm}^{-1}$ or $20.29239(6) \mathrm{eV}$, which is now confirmed.

Table A2 includes predicted energies for $89\left[3 \mathrm{~d}^{9}\right] n \mathrm{~d}, n \mathrm{f}, n \mathrm{~g}$, and $n \mathrm{~h}$ levels for which no reliable identification could be found in the observed spectra. These levels are not necessarily those that produce the strongest predicted lines. Rather, they are those that are easier to accurately predict, because the corresponding series have relatively small perturbations. In this derivation, we used predicted positions for centers of gravity of groups of closely located levels along series, using the RITZPL (quantum-defect formula) and POLAR (polarization-formula) extrapolation codes by Sansonetti [55,56], which we combined with fine-structure intervals predicted by the LSF (described in Section 3). Uncertainties assigned to these levels in Table A2 are weighted means of the "observed-calculated" residuals of the LSF, calculated separately for known levels with $J_{1} l$ purities of $\geq 99 \%$, (97 to 98 ) \%, and (92 to 96 ) \%. Some of the remaining unclassified lines observed by Ross [2,16], Shenstone [1], and by us may be due to these levels. However, we could not reliably identify them because of lack of observed Ritz combinations. 


\section{Line Intensities}

Procedures used in the present work to adjust observed line intensities to a common scale were described in detail by Kramida [57,58]. In this derivation, line intensities observed in each experiment are roughly modeled as described by Boltzmann level populations with certain effective excitation temperature $T_{\text {eff }}$. This temperature is found from a Boltzmann plot built using calculated transition probabilities. The ratios of predicted Boltzmann-population intensities are plotted against wavelengths to derive the response function of the registration equipment, which is then removed from the observed intensities to obtain an improved fit for $T_{\text {eff }}$ from the Boltzmann plot. For photographic registration, an additional correction of non-linearity of intensity registration with exposure is deduced from a plot of ratios of calculated and observed intensities versus observed intensity. This procedure is repeated iteratively until convergence is achieved. Then it is easy to scale the reduced intensities observed in experiments with different $T_{\text {eff }}$ and different sensitivity to a common value of $T_{\text {eff }}$. In this way, we combined intensities observed in our 22 VUV and 3 FTS spectrograms listed in Table 1 with those reported by Shenstone [1], Kaufman and Ward [15], and Ross [2,16] and reduced them to the scale derived from Ross's observed intensities to produce the average values given in Table A1. This scale corresponds to $T_{\text {eff }}=1.5 \mathrm{eV}$, which is about average for all experiments included in the analysis. For different experiments, the fitted value of $T_{\text {eff }}$ varied between $0.6 \mathrm{eV}$ in some of our VUV exposures to $11 \mathrm{eV}$ in observations of Kaufman and Ward [15]. It should be noted that in most experiments analyzed here populations of highly excited levels were enhanced by resonant transfer from excited levels of helium or other rare gases used in the discharges. This population transfer is the mechanism leading to population inversion and producing laser action observed in hollow-cathode discharges. Thus, it should be expected and is indeed observed that level populations deviate from the Boltzmann distribution by a factor of three on average and strongly vary depending on discharge conditions. Line intensities are given in Table A1 in terms of total energy flux under the line contour.

As noted in Section 2, only E1-allowed lines were observed in laboratory discharges. Thus, the intensity scale adopted for E1-forbidden lines is different from the allowed lines. It is based on relative intensities observed in nebulae spectra by Thackeray [3], Aller et al. [4], and McKenna et al. [5], modified in such a way that the relative intensities of lines from a common upper level are consistent for different observations.

\section{Transition Probabilities}

Transition probabilities of $\mathrm{Cu}$ II were reported in 42 papers, the full list of which can be found in the NIST Atomic Probability Bibliographic Database (Kramida and Fuhr [59]). In addition, we have calculated them using Cowan's codes [52] using our LSF parameters described in Section 3. To assess the uncertainties of all available data sets, we used evaluation procedures described by Kramida [57].

The initial reference data set was constructed of $41 A$-values determined by Ortiz et al. [50] from branching fractions measured using laser-induced breakdown spectroscopy and lifetimes calculated using the Cowan suite of codes [52], modified by inclusion of core-polarization effects. The high accuracy of theoretical lifetimes used in that work was confirmed by excellent agreement with accurate measurements made by Pinnington et al. [51] and Cederquist et al. [43], as well as with other advanced calculations.

This initial selection was expanded by inclusion of theoretical $A$-values from Dong and Fritzsche [45] obtained in a large-scale multiconfiguration Dirac-Fock calculation accounting for relaxation effects in the orbitals. Uncertainties of these theoretical data were estimated by comparing the results obtained by those authors in the Babushkin (length) and Coulomb (velocity) gauges. This comparison indicated that the stronger lines with line strength $S>10^{-6}$ a.u. were calculated with uncertainties in $A$-values of about $12 \%$ on average, while for weaker lines the average uncertainty was much larger, 60\%. These conclusions are supported by good agreement (less than 10\%) of calculated lifetimes with measurements. For 18 transitions the $A$-values reported by Dong and 
Fritzsche [45] were normalized to lifetimes of the upper levels measured by Pinnington et al. [51] and by Cederquist et al. [43].

For three transitions representing the sole allowed decay channels of their upper levels, the lifetimes measured by Pinnington et al. [51] and by Cederquist et al. [43] were directly converted into $A$-values and also added to the reference data set. Further expansion of the reference data set was provided by results of Crespo López-Urrutia et al. [44] obtained by combining their own measurements of emission branching fractions with radiative lifetimes measured in other studies. From this work, the results obtained using the lifetimes reliably measured by Cederquist et al. [43] and by Kono and Hattori [48] were used without adjustments. However, for several transitions we re-normalized their results to lifetimes more accurately measured by Pinnington et al. [51]. Uncertainties of Crespo López-Urrutia et al. [44] were estimated by combining in quadrature systematic contributions from the uncertainties of the lifetimes and statistical uncertainties, which we estimated to vary from less than $5 \%$ for the strongest lines to $90 \%$ for the weakest lines, which we assumed to have a signal-to-noise ratio about 1.

Kono and Hattori [48] measured radiative lifetimes of some of the $3 d^{9} 4 p$ and $3 d^{9} 5$ s levels by a delayed coincidence technique and combined them with branching fractions, some of which were measured in a specially designed discharge tube, and some of which were obtained in a single-configuration LSF-adjusted intermediate-coupling calculation. These authors did not report separately their measured branching fractions, which impedes assessment of their results. Instead, they used all of their data, both experimental and theoretical, together, and adjusted the resulting $A$-values to satisfy the $J$-file sum rule. We renormalized several of their reported $A$-values to radiative lifetimes measured more accurately by Brown et al. [42], Pinnington et al. [51], and Cederquist et al. [43] and estimated the uncertainties by a method similar to the one described above in evaluation of results of Crespo López-Urrutia et al. [44].

Assessment of measurements made by Neger and Jäger [49] and by Hefferlin et al. [47] (hereafter referred to as N88 and H71) presented the greatest difficulties, since they grossly disagree with each other. N88 reported relative transition probabilities ( $A$-values) of seven $\mathrm{Cu}$ II lines measured by using an axial discharge type of an exploding copper wire. The rather vague description of these measurements does not include the temperature value used in the data reduction. However, Neger and Jäger compare their results with those reported by Lux [60], obtained with similar equipment, and note that Lux determined the plasma temperature to be $26500(3500) \mathrm{K}(2.3(3) \mathrm{eV})$ from a Boltzmann plot using several $\mathrm{Cu}$ I lines with known transition probabilities. The relative $A$-values reported by N88 (using the line at $4555.92 \AA$ as reference) have uncertainties ranging from $18 \%$ to $25 \%$ and perfectly agree with those of Lux [60]. Thus, we assumed that they used Lux's temperature value in their data reduction and restored the observed line intensities from the reported line ratios and this temperature. Two of the lines reported in N88 are in common with more accurate measurements of Ortiz et al. [50].

Hefferlin et al. [47] (H71) determined relative intensities of $11 \mathrm{Cu}$ II lines from photoelectric radiance measurements on the Burnout $\mathrm{V}$ experimental magnetic-confinement fusion reactor at the Oak Ridge National Laboratory. To derive the relative $\log (g f)$ values from these measurements, they assumed the electron temperature to be $67 \mathrm{eV}( \pm 50 \%)$. Neger and Jäger [49] blamed this large uncertainty in the temperature value for the discrepancy of the H71 results with theirs. We note that $\mathrm{Cu}^{+}$should be completely ionized at this high temperature. Thus, an obvious path would be to dismiss the results of H71 as erroneous. However, their reported relative $\log (g f)$ values appear to be internally consistent and agree well with other independent estimates. Thus, we restored the observed line intensities from them, using the $67 \mathrm{eV}$ temperature as reported in H71, and attempted to reconcile the two sets of relative intensities (from H71 and N88) by using the following procedure.

As a first step, to determine initial estimates of the excitation temperatures in H71 and N88, we used Boltzmann plots with reference $A$-values for the lines at $4043.48 \AA$ and $4227.94 \AA$ from Ortiz et al. [50], and for the line at $4909.73 \AA$ from Cederquist et al. [43], while for all other lines we used our Cowan-code calculations. As a second step, using the temperatures derived from these 
Boltzmann plots, we determined the adjusted $A$-values from the H71 and N88 observed line intensities. Then we replaced the Cowan-code $A$-values in the reference set with logarithmic means of thus obtained H71 and N88 adjusted $A$-values and found adjusted temperature values from the Boltzmann plots. The second step was repeated iteratively until reasonable convergence, i.e., until the change in the temperature values between iterations decreased below $0.001 \mathrm{eV}$. The final Boltzmann plots for H71 and N88 are shown in Figure 4a,b, respectively.
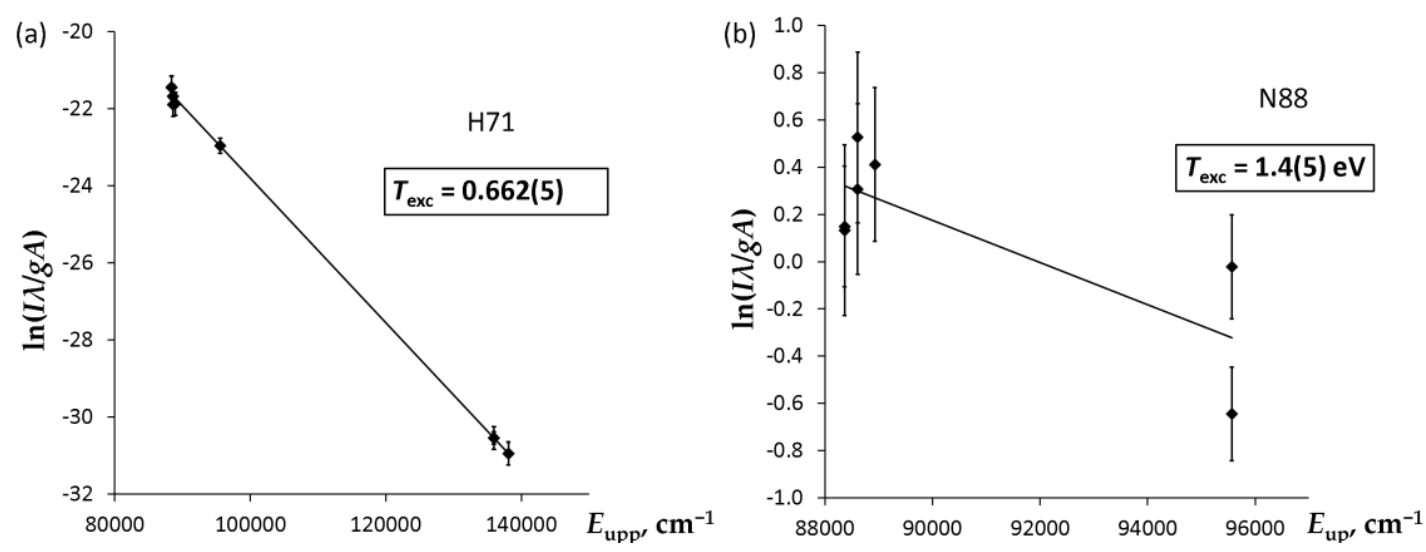

Figure 4. Boltzmann plots for relative intensities of observed lines: (a) Measurements of Hefferlin et al. [47] (H71); (b) Measurements of Neger and Jäger [49] (N88). The $A$-values used in these plots are the final adopted values given in Table A1. The error bars are combined uncertainties of $A$-values and relative intensities (see text).

The excitation temperatures determined for the $\mathrm{H} 71$ and $\mathrm{N} 88$ data sets are $0.662(5) \mathrm{eV}$ and $1.4(5) \mathrm{eV}$, respectively. The latter is somewhat lower than the Lux [60] value used in N88, 2.3(3) eV. The final adjusted $A$-values deduced from the H71 and N88 data are compared with the adopted $A$-values in Figure 5. In this plot, the reference $A$-values used for the two leftmost lines (4043.48 $\AA$ and $4227.94 \AA$ ) and for the rightmost line at $4909.73 \AA$ are from Ortiz et al. [50] and Cederquist et al. [43], while the rest of the lines use the mean of $\mathrm{H} 71$ and N88. The error bars are combined uncertainties of the measured line ratios and reference $A$-values. This plot shows that the adjusted $A$-values from H71 and N88 are consistent with each other, as well as with the adopted reference $A$-values.

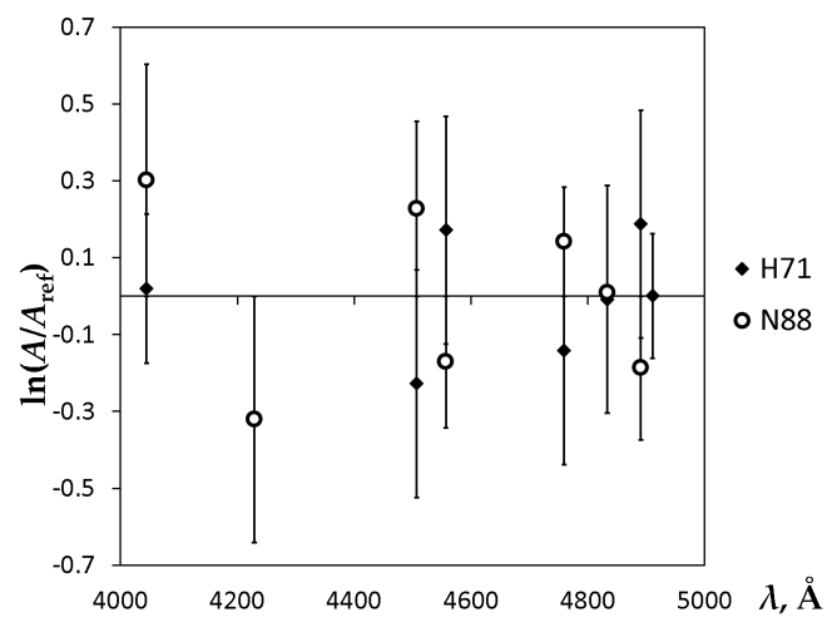

Figure 5. Comparison of adjusted $A$-values deduced from the line ratios reported by Hefferlin et al. [47] (H71) and by Neger and Jäger [49] (N88). The error bars are combined uncertainties of the measured line ratios and adopted reference $A$-values. 
With the reference data set constructed from data described above, assessment of uncertainties in the theoretical study by Biémont et al. [41] was relatively easy to do by plotting the ratio of the calculated values to the reference ones against line strength $S$ and calculating standard deviations for different ranges of $S$. This procedure resulted in estimated uncertainties of $9 \%$ for $S \geq 8.5$ atomic units (a.u.), $22 \%$ for $S=(0.2$ to 8.5$)$ a.u., and $120 \%$ for $S<0.2$ a.u.

Assessment procedures described above resulted in 210 reference $A$-values with uncertainties less than $25 \%$ covering a wide range of line strengths from 0.06 a.u. to 155 a.u., after which it became possible to evaluate the uncertainties of our LSF calculations. Comparison of our calculated $A$-values with the reference ones is illustrated in Figure 6.

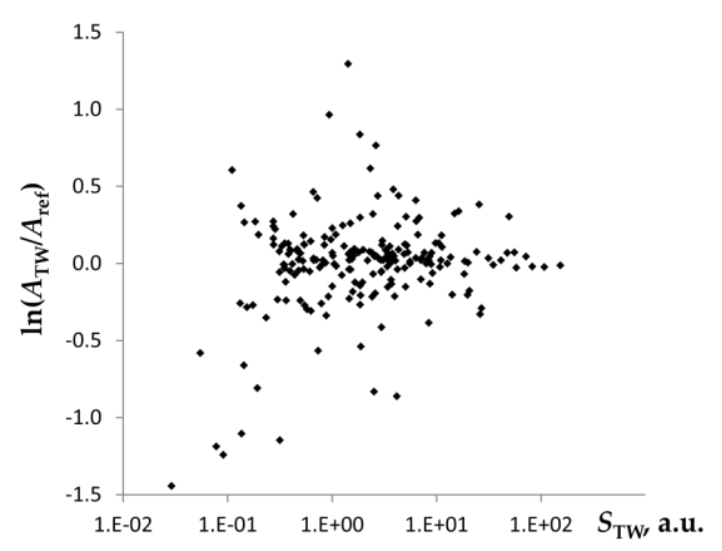

Figure 6. Comparison of transition probabilities calculated in this work $\left(A_{\mathrm{TW}}\right)$ with reference values ( $A_{\text {ref }}$, see text). Natural logarithm of the ratio $A_{\mathrm{TW}} / A_{\text {ref }}$ is plotted against our calculated line strength, $S_{\mathrm{TW}}$ (in atomic units).

This plot displays a typical behavior of theoretical transition probabilities. Namely, the strongest lines exhibit the smallest discrepancies from the reference values, while for weaker lines the magnitude of discrepancies grows. A similar comparison was made between $A$-values produced in our two LSFs (the final and preliminary ones, described in Section 3). From these comparisons, we estimated the uncertainties of our $A$-values to be $\leq 10 \%$ for $S>200$ a.u., $\leq 15 \%$ for $S=(50$ to 200 ) a.u., $\leq 30 \%$ for $S=(4.3$ to 50$)$ a.u., and $\geq 50 \%$ for smaller $S$. We included 79 of our best calculated $A$-values in Table A1.

Studies of E1-forbidden transition probabilities of Cu II are very scarce. Garstang [46] calculated $A$-values for several M1 and E2 transitions from the first excited configuration, $3 \mathrm{~d}^{9} 4 \mathrm{~s}$, down to lower-lying levels of the same configuration and to the ground state, $3 \mathrm{~d}^{101} \mathrm{~S}_{0}$. He used the pseudo-relativistic Hartree-Fock method with superposition of configurations and LSF-adjusted Slater parameters, similar to the one implemented in Cowan's codes [52] used in the present work, but limited by inclusion of only three low-lying configurations of even parity. Beck [61] made an ab initio restricted non-relativistic multiconfiguration Hartree-Fock calculation for the $3 d^{10}{ }^{1} S_{0}-3 d^{9} 4 s$ ${ }^{1,3} \mathrm{D}_{2}$ E2 transition and obtained $A=2.33 \mathrm{~s}^{-1}$, somewhat larger than the sum of Garstang's values for transitions from ${ }^{1} \mathrm{D}_{2}, 1.7 \mathrm{~s}^{-1}$, and ${ }^{3} \mathrm{D}_{2}, 0.12 \mathrm{~s}^{-1}$. Prior [37] measured the $A$-value for the $3 \mathrm{~d}^{10}{ }^{1} \mathrm{~S}_{0}-3 \mathrm{~d}^{9} 4 \mathrm{~s}$ ${ }^{1} \mathrm{D}_{2}$ transition at $3806.3 \AA$ to be $1.60(24) \mathrm{s}^{-1}$, in excellent agreement with Garstang's semiempirical value. The most recent multiconfiguration Dirac-Hartree-Fock calculation of Andersson et al. [40] gave $A=1.937 \mathrm{~s}^{-1}$ for this transition. However, their calculated wavelength is $3724.7 \AA$, significantly shorter than experimental. Adjustment to the experimental wavelength yields $A_{\text {adj }}=1.74 \mathrm{~s}^{-1}$, in good agreement with Prior [37] and Garstang [46]. A similar adjustment of the value of Andersson et al. [40] for the $3 \mathrm{~d}^{10}{ }^{1} \mathrm{~S}_{0}-3 \mathrm{~d}^{9} 4 \mathrm{~s}^{3} \mathrm{D}_{2}$ transition at $4375.8 \AA$ (which Andersson et al. [40] calculated to be at $4275.8 \AA$ ) gives $A_{\text {adj }}=0.093 \mathrm{~s}^{-1}$, about $30 \%$ lower than Garstang's result. For these two E2 transitions, our $A$-values calculated with Cowan's codes are too high by a factor of about 1.7 . We assume that our $A$-value for the predicted $3 \mathrm{~d}^{9} 4 \mathrm{~s}^{3} \mathrm{D}_{3}-{ }^{3} \mathrm{D}_{1} \mathrm{E} 2$ transition at $48318 \AA, 9 \times 10^{-8} \mathrm{~s}^{-1}$, has a similar 
low accuracy. The $3 \mathrm{~d}^{10}{ }^{1} \mathrm{~S}_{0}-3 \mathrm{~d}^{9} 4 \mathrm{~s}^{1} \mathrm{D}_{2}$ transition at $3806.3 \AA$ was observed by Thackeray [3] and by McKenna et al. [5] in emission spectra of nebulae.

It should be noted that, in addition to the two E2 transitions mentioned above, Andersson et al. [40] give calculated $A$-values for the magnetic-octupole (M3) and hyperfine-induced $3 \mathrm{~d}^{10}{ }^{1} \mathrm{~S}_{0}-3 \mathrm{~d}^{9} 4 \mathrm{~s}^{3} \mathrm{D}_{3}$ transitions at $4559 \AA$. The latter are different for various HFS components of the two isotopes $\left({ }^{63} \mathrm{Cu}\right.$ and $\left.{ }^{65} \mathrm{Cu}\right)$ and are typically on the order of a few times $10^{-9} \mathrm{~s}^{-1}$, three orders of magnitude greater than for the M3 transition. This transition, as well as the $3 \mathrm{~d}^{10}{ }^{1} \mathrm{~S}_{0}-3 \mathrm{~d}^{9} 4 \mathrm{~s}^{3} \mathrm{D}_{2}$ E2 transition at $4375.8 \AA$, was observed by Aller et al. [4] in the spectrum of the RR Telescopii nebula.

$A$-values for M1 transitions are relatively easier to calculate than E1 and E2 transitions, since their calculation does not involve radial integrals, but only amplitudes of eigenvector components. We compared our calculated $A$-values for M1 transitions with those of Garstang [46] and found that all of them agree to better than $15 \%$. We included in Table A1 our $A$-value for the $3 \mathrm{~d}^{10}{ }^{1} \mathrm{~S}_{0}-3 \mathrm{~d}^{9} 4 \mathrm{~s}$ ${ }^{3} \mathrm{D}_{1} \mathrm{M} 1$ transition at $4165.7 \AA$, which was not given by Garstang [46]. It turned out to be extremely small, $2.1 \times 10^{-12} \mathrm{~s}^{-1}$. Our calculation indicates that there is no E2 contribution to the total decay rate of the $3 \mathrm{~d}^{9} 4 \mathrm{~s}^{3} \mathrm{D}_{1}$ level. This makes the identification of the line observed at this wavelength by McKenna et al. [5] with this transition questionable. Possible explanations for this observation could be (1) our calculated M1 $A$-value is greatly underestimated; (2) there is a considerable contribution of E2 transition to this line, which for unknown reason is computed as negligibly small by Cowan's codes; (3) hyperfine-induced transition significantly increases the total radiative rate; (4) the population of the $3 \mathrm{~d}^{9} 4 \mathrm{~s}^{3} \mathrm{D}_{1}$ level in RR Telescopii is many orders of magnitude greater than that of ${ }^{3} \mathrm{D}_{2}$ and ${ }^{1} \mathrm{D}_{2}$; or (5) the identification is incorrect, and the observed line is possibly due to some other species. As explained above, the first explanation is rather unlikely. To confirm or disprove the second and third explanations, more extensive atomic calculations are needed, while checking the fourth one requires population-kinetics modeling.

\section{Conclusions}

The present work provides a comprehensive list of all observed, classified, and predicted spectral lines of $\mathrm{Cu}$ II, which includes 2557 transitions with wavelengths from $675 \AA$ to $10.9 \mu \mathrm{m}$. Over 600 of them were measured in this work using grating and Fourier-transform spectrometers. Experimental wavelengths of 2443 transitions were used in a least-squares level optimization procedure to produce optimized values for 379 energy levels, of which 29 are newly identified, and nine are revised. The previous analysis of Ross $[2,16]$ is largely confirmed. However, our extended base for the levels optimization results in more dependable level values and Ritz wavelengths that can be used as secondary standards in the vacuum ultraviolet region. An improved theoretical interpretation of the energy levels was made by a parametric least-squares fitting with Cowan's atomic codes, which included all experimental levels. The fitted Slater parameters were used to calculate radiative transition probabilities for electric-dipole, magnetic-dipole, and electric-quadrupole transitions, which were critically evaluated together with other published data to construct a list of recommended $A$-values for 555 transitions.

Acknowledgments: This work was partially supported by the National Aeronautics and Space Administration (NASA) under interagency agreement NNH10AN38I.

Author Contributions: All authors contributed equally to this work.

Conflicts of Interest: The authors declare no conflict of interest. 


\section{Appendix A}

Table A1. Spectral lines of Cu II.

\begin{tabular}{|c|c|c|c|c|c|c|c|c|c|c|c|c|c|c|}
\hline$\lambda_{\text {obs }}{ }^{\text {a }}(\AA)$ & $\sigma_{\mathrm{obs}} \mathrm{b}\left(\mathrm{cm}^{-1}\right)$ & $\lambda_{\text {Ritz }}{ }^{\mathrm{c}}(\AA)$ & $\begin{array}{c}\Delta \lambda_{\text {obs-Ritz }} \\
\stackrel{(\AA)}{(A)}\end{array}$ & $\begin{array}{c}I_{\text {obs }}{ }^{d} \\
\text { (arb.u.) }\end{array}$ & Char ${ }^{\mathrm{e}}$ & Low & evel & & Upper Level & $A\left(\mathrm{~s}^{-1}\right)$ & $\operatorname{Acc}{ }^{f}$ & Line Ref. $\mathrm{g}$ & TP Ref. $\mathrm{g}$ & Notes $^{h}$ \\
\hline 675.5994(20) & $148016.7(4)$ & $675.60192(8)$ & -0.0025 & 73000 & & $\mathrm{~d}^{10}$ & ${ }^{1} S_{0}$ & $5 \mathrm{f}$ & $(3 / 2)^{2}[3 / 2]^{\circ}{ }_{1}$ & & & TW & & \\
\hline $677.675(3)$ & $147563.3(6)$ & 677.67816(8) & -0.003 & 29000 & & $\mathrm{~d}^{10}$ & ${ }^{1} S_{0}$ & $7 \mathrm{p}$ & $(5 / 2)^{2}[3 / 2]^{\circ}{ }_{1}$ & & & TW & & \\
\hline $685.1377(20)$ & 145956.1(4) & $685.14058(8)$ & -0.0029 & 240000 & & $\mathrm{~d}^{10}$ & ${ }^{1} \mathrm{~S}_{0}$ & $5 \mathrm{f}$ & $(5 / 2)^{2}[3 / 2]^{\circ}{ }_{1}$ & & & TW & & \\
\hline 685.3922(20) & $145901.9(4)$ & 685.39671(8) & -0.0045 & 45000 & & $\mathrm{~d}^{10}$ & ${ }^{1} \mathrm{~S}_{0}$ & $5 f$ & $(5 / 2)^{2}[1 / 2]^{\circ}{ }_{1}$ & & & TW & & \\
\hline $709.3098(20)$ & 140982.1(4) & $709.31287(9)$ & -0.0031 & 250000 & & $\mathrm{~d}^{10}$ & ${ }^{1} \mathrm{~S}_{0}$ & $6 \mathrm{p}$ & $(3 / 2)^{2}[1 / 2]^{\circ}{ }_{1}$ & & & TW & & \\
\hline $718.1766(20)$ & $139241.5(4)$ & 718.17860(9) & -0.0020 & 310000 & & $\mathrm{~d}^{10}$ & ${ }^{1} \mathrm{~S}_{0}$ & $6 \mathrm{p}$ & $(5 / 2)^{2}[3 / 2]^{\circ}{ }_{1}$ & & & TW & & \\
\hline $724.4881(20)$ & $138028.5(4)$ & $724.48867(9)$ & -0.0006 & 490000 & & $\mathrm{~d}^{10}$ & ${ }^{1} \mathrm{~S}_{0}$ & $4 \mathrm{f}$ & $(3 / 2)^{2}[3 / 2]^{\circ}{ }_{1}$ & & & TW & & \\
\hline 735.5215(11) & 135957.95(20) & 735.52023(9) & 0.0013 & 780000 & & $\mathrm{~d}^{10}$ & ${ }^{1} \mathrm{~S}_{0}$ & $4 \mathrm{f}$ & $(5 / 2)^{2}[3 / 2]^{\circ}{ }_{1}$ & & & TW & & \\
\hline $736.0331(8)$ & $135863.46(16)$ & $736.03185(9)$ & 0.0012 & 870000 & & $\mathrm{~d}^{10}$ & ${ }^{1} \mathrm{~S}_{0}$ & $4 \mathrm{f}$ & $(5 / 2)^{2}[1 / 2]^{\circ}{ }_{1}$ & & & TW & & \\
\hline $763.2658(20)$ & 131016.0(3) & $763.2692(3)$ & -0.0034 & 140000 & & $4 \mathrm{~s}$ & ${ }^{3} \mathrm{D}_{3}$ & $\mathrm{sp}$ & $\left({ }^{3} \mathrm{P}\right)^{1} \mathrm{P}^{\circ}{ }^{3} \mathrm{P}_{2}^{\circ}$ & & & TWn & & \\
\hline 768.4821(20) & $130126.6(3)$ & $768.48565(19)$ & -0.0035 & 150000 & & $4 \mathrm{~s}$ & ${ }^{3} \mathrm{D}_{3}$ & $8 \mathrm{p}$ & $(5 / 2)^{2}[3 / 2]^{\circ} 2$ & & & TWn & & \\
\hline 776.484(3) & $128785.6(5)$ & 776.4877(3) & -0.004 & 58000 & & $4 \mathrm{~s}$ & ${ }^{3} \mathrm{D}_{1}$ & $\mathrm{sp}$ & $\left({ }^{3} \mathrm{P}\right)^{1} \mathrm{P}^{\circ}{ }^{3} \mathrm{P}_{0}^{\circ}$ & & & TWn & & \\
\hline $777.739(3)$ & $128577.8(5)$ & 777.74333(3) & -0.004 & 56000 & & $4 \mathrm{~s}$ & ${ }^{3} \mathrm{D}_{2}$ & $8 \mathrm{p}$ & $(5 / 2)^{2}[3 / 2]^{\circ}{ }_{1}$ & & & TW & & \\
\hline $779.2932(20)$ & 128321.4(3) & 779.29473(5) & -0.0015 & 590000 & & $4 \mathrm{~s}$ & ${ }^{3} \mathrm{D}_{3}$ & $\mathrm{sp}$ & $\left({ }^{1} \mathrm{D}\right)^{1} \mathrm{P}^{\circ}{ }^{1} \mathrm{D}_{2}^{\circ}$ & & & TW & & \\
\hline $779.328(3)$ & $128315.6(5)$ & $779.33660(4)$ & -0.008 & 480000 & & $4 \mathrm{~s}$ & ${ }^{3} \mathrm{D}_{2}$ & 6f & $(5 / 2)^{2}[1 / 2]^{\circ}{ }_{1}$ & & & TWn & & \\
\hline $784.9098(20)$ & 127403.2(3) & $784.91229(5)$ & -0.0025 & 69000 & & $4 \mathrm{~s}$ & ${ }^{3} \mathrm{D}_{2}$ & $\mathrm{sp}$ & $\left({ }^{1} \mathrm{D}\right)^{1} \mathrm{P}^{\circ}{ }^{1} \mathrm{D}_{2}^{\circ}$ & & & TW & & \\
\hline 786.392(3) & 127163.1(5) & $786.39222(4)$ & -0.000 & 21000 & * & $4 \mathrm{~s}$ & ${ }^{3} \mathrm{D}_{1}$ & $6 \mathrm{f}$ & $(5 / 2)^{2}[1 / 2]^{\circ}{ }_{1}$ & & & TWn & & \\
\hline $786.392(3)$ & 127163.1(5) & 786.39795(4) & -0.006 & 21000 & * & $4 \mathrm{~s}$ & ${ }^{1} \mathrm{D}_{2}$ & $6 \mathrm{f}$ & $(3 / 2)^{2}[3 / 2]^{\circ} 2$ & & & TW & & \\
\hline $787.907(3)$ & $126918.5(5)$ & $787.9056(3)$ & 0.001 & 33000 & & $4 \mathrm{~s}$ & ${ }^{3} \mathrm{D}_{2}$ & $7 \mathrm{p}$ & $(3 / 2)^{2}[3 / 2]^{\circ}{ }_{1}$ & & & TWn & & \\
\hline 788.012(3) & 126901.6(5) & $788.01778(20)$ & -0.006 & 45000 & & $4 \mathrm{~s}$ & ${ }^{1} \mathrm{D}_{2}$ & $\mathrm{sp}$ & $\left({ }^{1} \mathrm{D}\right){ }^{1} \mathrm{P}^{\circ}{ }^{1} \mathrm{P}_{1}^{\circ}{ }_{1}$ & & & TWn & & \\
\hline $789.3937(20)$ & $126679.5(3)$ & 789.3933(3) & 0.0004 & 100000 & * & $4 \mathrm{~s}$ & ${ }^{1} \mathrm{D}_{2}$ & $\mathrm{sp}$ & $\left({ }^{3} \mathrm{P}\right)^{1} \mathrm{P}^{\circ}{ }^{3} \mathrm{P}_{2}^{\circ}$ & & & TWn & & \\
\hline $789.3937(20)$ & $126679.5(3)$ & $789.3977(3)$ & -0.0040 & 100000 & * & $4 \mathrm{~s}$ & ${ }^{3} \mathrm{D}_{2}$ & $7 \mathrm{p}$ & $(3 / 2)^{2}[5 / 2]^{\circ}{ }_{2}$ & & & TWn & & \\
\hline $789.6575(20)$ & 126637.2(3) & 789.65867(5) & -0.0011 & 59000 & & $4 \mathrm{~s}$ & ${ }^{3} \mathrm{D}_{2}$ & $7 \mathrm{p}$ & $(3 / 2)^{2}[1 / 2]^{\circ}{ }_{1}$ & & & TWn & & \\
\hline $794.9719(20)$ & $125790.6(3)$ & $794.97430(20)$ & -0.0024 & 56000 & & $4 \mathrm{~s}$ & ${ }^{1} \mathrm{D}_{2}$ & $8 \mathrm{p}$ & $(5 / 2)^{2}[3 / 2]^{\circ} 2$ & & & TWn & & \\
\hline $797.4530(20)$ & $125399.2(3)$ & 797.45499(4) & -0.0020 & 560000 & & $4 \mathrm{~s}$ & ${ }^{3} \mathrm{D}_{3}$ & $7 \mathrm{p}$ & $(5 / 2)^{2}[3 / 2]^{\circ} 2$ & & & TW & & \\
\hline 797.6193(20) & 125373.1(3) & $797.6218(5)$ & -0.0025 & 35000 & & $4 \mathrm{~s}$ & ${ }^{3} \mathrm{D}_{1}$ & $7 \mathrm{p}$ & $(3 / 2)^{2}[1 / 2]_{0}^{\circ}$ & & & TWn & & \\
\hline 798.977(3) & $125160.0(5)$ & $798.97928(3)$ & -0.002 & 23000 & & $4 \mathrm{~s}$ & ${ }^{1} \mathrm{D}_{2}$ & $8 \mathrm{p}$ & $(5 / 2)^{2}[3 / 2]^{\circ}$ & & & TW & & \\
\hline $800.6592(20)$ & 124897.1(3) & $800.66084(5)$ & -0.0017 & 26000 & & $4 \mathrm{~s}$ & ${ }^{1} \mathrm{D}_{2}$ & 6f & $(5 / 2)^{2}[1 / 2]^{\circ}{ }_{1}$ & & & TWn & & \\
\hline $801.8229(20)$ & $124715.8(3)$ & $801.82460(4)$ & -0.0017 & 84000 & & $4 \mathrm{~s}$ & ${ }^{3} \mathrm{D}_{2}$ & $7 \mathrm{p}$ & $(5 / 2)^{2}[3 / 2]^{\circ}{ }_{1}$ & & & TW & & \\
\hline $803.3370(20)$ & $124480.8(3)$ & $803.33841(4)$ & -0.0014 & 77000 & & $4 \mathrm{~s}$ & ${ }^{3} \mathrm{D}_{2}$ & $7 \mathrm{p}$ & $(5 / 2)^{2}[3 / 2]_{2}^{\circ}$ & & & TW & & \\
\hline $806.5454(20)$ & $123985.6(3)$ & $806.54699(5)$ & -0.0016 & 180000 & & $4 \mathrm{~s}$ & ${ }^{1} \mathrm{D}_{2}$ & $\mathrm{sp}$ & $\left({ }^{1} \mathrm{D}\right)^{1} \mathrm{P}^{\circ}{ }^{1} \mathrm{D}_{2}^{\circ}$ & & & TW & & \\
\hline $809.2942(20)$ & $123564.5(3)$ & $809.29524(4)$ & -0.0011 & 17000 & & $4 \mathrm{~s}$ & ${ }^{3} \mathrm{D}_{1}$ & $7 p$ & $(5 / 2)^{2}[3 / 2]^{\circ}{ }_{1}$ & & & TW & & \\
\hline $809.706(3)$ & $123501.6(5)$ & $809.7079(3)$ & -0.002 & 12000 & & $4 \mathrm{~s}$ & $\begin{array}{l}{ }^{1} \mathrm{D}_{2} \\
\end{array}$ & $7 p$ & $(3 / 2)^{2}[3 / 2]^{\circ}$ & & & TWn & & \\
\hline $809.9630(20)$ & $123462.4(3)$ & $809.9649(3)$ & -0.0019 & 45000 & & $4 \mathrm{~s}$ & ${ }^{1} \mathrm{D}_{2}$ & $7 \mathrm{p}$ & $(3 / 2)^{2}[3 / 2]^{0}{ }_{2}$ & & & TWn & & \\
\hline $810.9987(20)$ & 123304.8(3) & 810.99829(11) & 0.0004 & 520000 & & $\mathrm{~d}^{10}$ & ${ }^{1} \mathrm{~S}_{0}$ & $5 p$ & $(3 / 2)^{2}[3 / 2]^{\circ}$ & & & TW & & \\
\hline $810.6365(20)$ & $123359.8(3)$ & $810.6361(3)$ & 0.0005 & 86000 & & $4 \mathrm{~s}$ & ${ }^{1} \mathrm{D}_{2}$ & $7 \mathrm{p}$ & $(3 / 2)^{2}[5 / 2]_{3}^{\circ}$ & & & TWn & & \\
\hline
\end{tabular}


Table A1. Cont.

\begin{tabular}{|c|c|c|c|c|c|c|c|c|c|c|c|c|c|c|}
\hline$\lambda_{\text {obs }}{ }^{a}(\AA)$ & $\sigma_{\text {obs }}{ }^{\mathrm{b}}\left(\mathrm{cm}^{-1}\right)$ & $\lambda_{\text {Ritz }}{ }^{\mathrm{c}}(\AA)$ & $\begin{array}{c}\Delta \lambda_{\text {obs-Ritz }} \\
\text { (A) }\end{array}$ & $\begin{array}{c}I_{\text {obs }} \mathrm{d} \\
\text { (arb. u.) }\end{array}$ & Char ${ }^{\mathrm{e}}$ & Low & evel & & pper Level & $A\left(\mathrm{~s}^{-1}\right)$ & $\operatorname{Acc}^{f}$ & Line Ref. $\mathrm{g}$ & TP Ref. $\mathrm{g}$ & Notes $^{h}$ \\
\hline $811.2843(20)$ & 123261.4(3) & $811.2839(4)$ & 0.0004 & 110000 & & $4 \mathrm{~s}$ & ${ }^{1} \mathrm{D}_{2}$ & $7 p$ & $(3 / 2)^{2}[5 / 2]^{\circ}{ }_{2}$ & & & TWn & & \\
\hline $811.5559(20)$ & 123220.1(3) & $811.55946(5)$ & -0.0036 & 13000 & & $4 \mathrm{~s}$ & ${ }^{1} \mathrm{D}_{2}$ & $7 p$ & $(3 / 2)^{2}[1 / 2]^{\circ}{ }_{1}$ & & & TWn & & \\
\hline $813.882(3)$ & $122867.9(4)$ & $813.88328(11)$ & -0.001 & 760000 & & $\mathrm{~d}^{10}$ & ${ }^{1} \mathrm{~S}_{0}$ & $5 p$ & $(3 / 2)^{2}[1 / 2]^{\circ}$ & & & TW & & \\
\hline $823.059(3)$ & $121497.9(4)$ & $823.05584(4)$ & 0.004 & 2000 & & $4 \mathrm{~s}$ & ${ }^{1} \mathrm{D}_{2}$ & $7 p$ & $(5 / 2)^{2}[5 / 2]_{3}^{\circ}$ & & & TW & & \\
\hline 823.8361(18) & 121383.4(3) & $823.83758(6)$ & -0.0015 & 90000 & & $4 \mathrm{~s}$ & ${ }^{1} \mathrm{D}_{2}$ & $7 p$ & $(5 / 2)^{2}[5 / 2]_{2}^{\circ}$ & & & TW & & \\
\hline $824.4127(20)$ & $121298.5(3)$ & $824.41507(4)$ & -0.0024 & 6100 & & $4 \mathrm{~s}$ & ${ }^{1} \mathrm{D}_{2}$ & $7 \mathrm{p}$ & $(5 / 2)^{2}[3 / 2]^{\circ}{ }_{1}$ & & & TW & & \\
\hline $824.6630(19)$ & $121261.7(3)$ & $824.6649(4)$ & -0.0019 & 90000 & & $4 \mathrm{~s}$ & ${ }^{1} \mathrm{D}_{2}$ & $7 \mathrm{p}$ & $(5 / 2)^{2}[7 / 2]^{\circ}{ }_{3}$ & & & TWn & & \\
\hline $826.014(3)$ & $121063.3(5)$ & $826.01546(5)$ & -0.001 & 4400 & & $4 \mathrm{~s}$ & ${ }^{1} \mathrm{D}_{2}$ & $7 \mathrm{p}$ & $(5 / 2)^{2}[3 / 2]_{2}^{\circ}$ & & & TW & & \\
\hline $826.9946(22)$ & 120919.8(3) & $826.99598(12)$ & -0.0014 & 1100000 & & $\mathrm{~d}^{10}$ & ${ }^{1} \mathrm{~S}_{0}$ & $5 p$ & $(5 / 2)^{2}[3 / 2]^{\circ}{ }_{1}$ & & & TW & & \\
\hline $829.360(5)$ & 120575.0(8) & $829.35106(14)$ & 0.008 & 7400 & & $4 \mathrm{~s}$ & ${ }^{3} \mathrm{D}_{2}$ & $\mathrm{sp}$ & $\left({ }^{1} \mathrm{G}\right)^{3} \mathrm{P}^{\circ}{ }^{3} \mathrm{G}^{\circ}{ }_{3}$ & & & TWn & & \\
\hline $836.042(20)$ & 119611(3) & $836.02765(3)$ & 0.014 & 27000 & & $4 \mathrm{~s}$ & ${ }^{3} \mathrm{D}_{3}$ & $6 \mathrm{p}$ & $(3 / 2)^{2}[3 / 2]_{2}^{\circ}$ & & & S36c & & \\
\hline $841.136(3)$ & $118886.8(4)$ & $841.13445(3)$ & 0.002 & 30000 & & $4 \mathrm{~s}$ & ${ }^{3} \mathrm{D}_{2}$ & $6 p$ & $(3 / 2)^{2}[3 / 2]^{\circ}{ }_{1}$ & & & TW & & \\
\hline $842.4973(24)$ & 118694.7(3) & $842.49630(3)$ & 0.0010 & 66000 & & $4 \mathrm{~s}$ & ${ }^{3} \mathrm{D}_{2}$ & $6 \mathrm{p}$ & $(3 / 2)^{2}[3 / 2]^{\circ}{ }_{2}$ & & & TW & & \\
\hline $844.6125(21)$ & $118397.5(3)$ & $844.61287(4)$ & -0.0003 & 88000 & & $4 \mathrm{~s}$ & ${ }^{3} \mathrm{D}_{2}$ & $6 p$ & $(3 / 2)^{2}[5 / 2]_{2}^{\circ}$ & & & TW & & \\
\hline 844.9124(21) & $118355.5(3)$ & $844.91208(4)$ & 0.0003 & 410000 & & $4 \mathrm{~s}$ & ${ }^{3} \mathrm{D}_{2}$ & $6 p$ & $(3 / 2)^{2}[5 / 2]_{3}^{\circ}$ & & & TW & & \\
\hline $848.8062(20)$ & $117812.5(3)$ & 848.80734(3) & -0.0011 & 920000 & & $4 \mathrm{~s}$ & ${ }^{3} \mathrm{D}_{3}$ & $6 p$ & $(5 / 2)^{2}[5 / 2]_{3}^{\circ}$ & & & TW & & \\
\hline 849.3599(19) & 117735.7(3) & $849.35931(3)$ & 0.0006 & 240000 & & $4 \mathrm{~s}$ & ${ }^{3} \mathrm{D}_{1}$ & $6 \mathrm{p}$ & $(3 / 2)^{2}[3 / 2]^{\circ}{ }_{1}$ & & & TW & & \\
\hline $850.7481(19)$ & $117543.6(3)$ & $850.74794(3)$ & 0.0001 & 140000 & & $4 \mathrm{~s}$ & ${ }^{3} \mathrm{D}_{1}$ & $6 \mathrm{p}$ & $(3 / 2)^{2}[3 / 2]_{2}^{\circ}$ & & & TW & & \\
\hline $851.3012(16)$ & $117467.24(23)$ & $851.30253(3)$ & -0.0013 & 1100000 & & $4 \mathrm{~s}$ & ${ }^{3} \mathrm{D}_{3}$ & $6 \mathrm{p}$ & $(5 / 2)^{2}[7 / 2]^{\circ} 4$ & & & TW & & \\
\hline $851.776(3)$ & $117401.8(4)$ & $851.77122(3)$ & 0.004 & 21000 & & $4 \mathrm{~s}$ & ${ }^{3} \mathrm{D}_{3}$ & $\mathrm{sp}$ & $\left({ }^{1} \mathrm{G}\right)^{3} \mathrm{P}^{\circ}{ }^{3} \mathrm{~F}^{\circ}{ }_{3}$ & & & TW & & \\
\hline 852.9074(21) & $117246.0(3)$ & $852.90622(4)$ & 0.0012 & 370000 & & $4 \mathrm{~s}$ & ${ }^{3} \mathrm{D}_{1}$ & $6 \mathrm{p}$ & $(3 / 2)^{2}[5 / 2]_{2}^{\circ}$ & & & TW & & \\
\hline $853.5444(24)$ & $117158.5(3)$ & $853.54260(15)$ & 0.0018 & 45000 & & $4 \mathrm{~s}$ & ${ }^{1} \mathrm{D}_{2}$ & $\mathrm{sp}$ & $\left({ }^{1} \mathrm{G}\right)^{3} \mathrm{P}^{\circ} 3^{3} \mathrm{G}_{3}^{\circ}$ & & & TWn & & \\
\hline $855.4770(19)$ & 116893.9(3) & $855.476052(24)$ & 0.0009 & 270000 & & $4 \mathrm{~s}$ & ${ }^{3} \mathrm{D}_{2}$ & $6 \mathrm{p}$ & $(5 / 2)^{2}[5 / 2]^{\circ}{ }_{3}$ & & & TW & & \\
\hline 855.6994(21) & $116863.5(3)$ & $855.70010(3)$ & -0.0007 & 720000 & & $4 \mathrm{~s}$ & ${ }^{3} \mathrm{D}_{2}$ & $\mathrm{sp}$ & $\left({ }^{3} \mathrm{~F}\right)^{1} \mathrm{P}^{\circ}{ }^{3} \mathrm{~F}^{\circ}{ }_{2}$ & & & TW & & \\
\hline 858.4844(20) & 116484.4(3) & $858.486771(24)$ & -0.0024 & 990000 & & $4 \mathrm{~s}$ & ${ }^{3} \mathrm{D}_{2}$ & $\mathrm{sp}$ & $\left({ }^{1} \mathrm{G}\right)^{3} \mathrm{P}^{\circ}{ }^{3} \mathrm{~F}^{\circ}{ }_{3}$ & & & TW & & \\
\hline $858.5639(20)$ & $116473.6(3)$ & $858.566433(20)$ & -0.0025 & 890000 & & $4 \mathrm{~s}$ & ${ }^{3} \mathrm{D}_{3}$ & $6 \mathrm{p}$ & $(5 / 2)^{2}[7 / 2]^{\circ}{ }_{3}$ & & & TW & & \\
\hline $859.1522(24)$ & $116393.8(3)$ & $859.15072(3)$ & 0.0014 & 14000 & & $4 \mathrm{~s}$ & ${ }^{3} \mathrm{D}_{2}$ & $6 \mathrm{p}$ & $(5 / 2)^{2}[3 / 2]^{\circ}{ }_{1}$ & & & TW & & \\
\hline $860.7214(24)$ & 116181.6(3) & $860.72158(4)$ & -0.0001 & 22000 & & $4 \mathrm{~s}$ & ${ }^{3} \mathrm{D}_{2}$ & $6 \mathrm{p}$ & $(5 / 2)^{2}[5 / 2]^{\circ}{ }_{2}$ & & & TW & & \\
\hline 861.9932(11) & $116010.19(14)$ & $861.99338(6)$ & -0.0002 & 1900000 & & $4 \mathrm{~s}$ & ${ }^{3} \mathrm{D}_{3}$ & $\mathrm{sp}$ & $\left({ }^{1} \mathrm{G}\right)^{3} \mathrm{P}^{\circ}{ }^{3} \mathrm{~F}^{\circ}{ }_{4}$ & & & TW & & \\
\hline $862.8221(20)$ & $115898.7(3)$ & $862.82245(4)$ & -0.0003 & 78000 & & $4 \mathrm{~s}$ & ${ }^{3} \mathrm{D}_{2}$ & $6 \mathrm{p}$ & $(5 / 2)^{2}[3 / 2]_{2}^{\circ}$ & & & TW & & \\
\hline $864.163(4)$ & $115718.9(5)$ & $864.15435(3)$ & 0.009 & 130000 & & $4 \mathrm{~s}$ & ${ }^{3} \mathrm{D}_{3}$ & $\mathrm{sp}$ & $\left({ }^{3} \mathrm{P}\right)^{3} \mathrm{P}^{\circ}{ }^{1} \mathrm{D}^{\circ}{ }_{2}$ & & & TW & & \\
\hline $864.214(3)$ & $115712.0(4)$ & $864.21371(3)$ & 0.001 & 670000 & & $4 \mathrm{~s}$ & ${ }^{3} \mathrm{D}_{1}$ & $\mathrm{sp}$ & $\left({ }^{3} \mathrm{~F}\right)^{1} \mathrm{P}^{\circ}{ }^{3} \mathrm{~F}^{\circ}{ }_{2}$ & & & TW & & \\
\hline $865.3907(8)$ & $115554.74(11)$ & $865.389986(23)$ & 0.0007 & 1600000 & & $4 \mathrm{~s}$ & ${ }^{3} \mathrm{D}_{2}$ & $6 \mathrm{p}$ & $(5 / 2)^{2}[7 / 2]_{3}^{\circ}$ & & & K66 & & \\
\hline $866.4430(20)$ & 115414.4(3) & $866.442524(13)$ & 0.0005 & 64000 & & $4 \mathrm{~s}$ & ${ }^{3} \mathrm{D}_{2}$ & $4 \mathrm{f}$ & $(3 / 2)^{2}[7 / 2]^{\circ}{ }_{3}$ & & & TW & & \\
\hline $867.7321(21)$ & $115242.9(3)$ & $867.73348(3)$ & -0.0014 & 320000 & & $4 \mathrm{~s}$ & ${ }^{3} \mathrm{D}_{1}$ & $6 p$ & $(5 / 2)^{2}[3 / 2]^{\circ}{ }_{1}$ & & & TW & & \\
\hline 869.0634(18) & $115066.40(24)$ & $869.06394(3)$ & -0.0005 & 250000 & & $4 \mathrm{~s}$ & ${ }^{3} \mathrm{D}_{2}$ & $\mathrm{sp}$ & $\left({ }^{3} \mathrm{~F}\right){ }^{1} \mathrm{P}^{\circ}{ }^{3} \mathrm{D}^{\circ}{ }_{1}$ & & & TW & & \\
\hline $869.3338(24)$ & $115030.6(3)$ & 869.33592(4) & -0.0021 & 1200000 & & $4 \mathrm{~s}$ & ${ }^{3} \mathrm{D}_{1}$ & $6 p$ & $(5 / 2)^{2}[5 / 2]^{\circ}{ }_{2}$ & & & TW & & \\
\hline $870.5390(18)$ & $114871.37(24)$ & $870.538673(24)$ & 0.0003 & 240000 & & $4 \mathrm{~s}$ & ${ }^{3} \mathrm{D}_{3}$ & $\mathrm{sp}$ & $\left({ }^{3} \mathrm{~F}\right)^{1} \mathrm{P}^{\circ}{ }^{3} \mathrm{D}^{\circ}{ }_{2}$ & & & TW & & \\
\hline 871.0674(18) & $114801.68(24)$ & $871.06737(3)$ & 0.0000 & 130000 & & $4 \mathrm{~s}$ & ${ }^{3} \mathrm{D}_{2}$ & $\mathrm{sp}$ & $\left({ }^{3} \mathrm{P}\right)^{3} \mathrm{P}^{\circ}{ }^{1} \mathrm{D}^{\circ}{ }_{2}$ & & & TW & & \\
\hline
\end{tabular}


Table A1. Cont.

\begin{tabular}{|c|c|c|c|c|c|c|c|c|c|c|c|c|c|c|}
\hline$\lambda_{\text {obs }}{ }^{a}(\AA)$ & $\sigma_{\mathrm{obs}}{ }^{\mathrm{b}}\left(\mathrm{cm}^{-1}\right)$ & $\lambda_{\text {Ritz }}{ }^{c}(\AA)$ & $\begin{array}{c}\Delta \lambda_{\text {obs-Ritz }} \\
\text { (A) }\end{array}$ & $\begin{array}{c}I_{\text {obs }} \mathrm{d} \\
\text { (arb. u.) }\end{array}$ & Char ${ }^{\mathrm{e}}$ & \multicolumn{2}{|c|}{ Lower Level } & \multicolumn{2}{|r|}{ Upper Level } & $A\left(\mathrm{~s}^{-1}\right)$ & $\operatorname{Acc}^{f}$ & Line Ref. $\mathrm{g}$ & TP Ref. $\mathrm{g}$ & Notes ${ }^{h}$ \\
\hline $873.2629(21)$ & 114513.0(3) & $873.262654(21)$ & 0.0003 & 310000 & & $4 \mathrm{~s}$ & ${ }^{3} \mathrm{D}_{3}$ & $\mathrm{sp}$ & $\left({ }^{3} \mathrm{~F}\right)^{1} \mathrm{P}^{\circ}{ }^{3} \mathrm{~F}_{3}^{\circ}$ & & & TW & & \\
\hline $876.7226(20)$ & 114061.2(3) & $876.722436(13)$ & 0.0002 & 830000 & & $4 \mathrm{~s}$ & ${ }^{3} \mathrm{D}_{3}$ & $4 \mathrm{f}$ & $(5 / 2)^{2}[7 / 2]^{\circ}{ }_{3}$ & & & TW & & \\
\hline $876.973(5)$ & $114028.6(7)$ & $876.962109(24)$ & 0.011 & 420000 & & $4 \mathrm{~s}$ & ${ }^{3} \mathrm{D}_{1}$ & $4 \mathrm{f}$ & $(3 / 2)^{2}[3 / 2]^{\circ}{ }_{1}$ & & & TW & & \\
\hline $877.0107(24)$ & $114023.7(3)$ & $877.01184(4)$ & -0.0012 & 890000 & & $4 \mathrm{~s}$ & ${ }^{3} \mathrm{D}_{3}$ & $\mathrm{sp}$ & $\left({ }^{3} \mathrm{P}\right)^{3} \mathrm{P}^{\circ}{ }^{5} \mathrm{~S}^{\circ}{ }_{2}$ & & & TW & & \\
\hline $877.5531(20)$ & 113953.2(3) & $877.554632(23)$ & -0.0015 & 820000 & & $4 \mathrm{~s}$ & ${ }^{3} \mathrm{D}_{2}$ & $\mathrm{sp}$ & $\left({ }^{3} \mathrm{~F}\right){ }^{1} \mathrm{P}^{\circ}{ }^{3} \mathrm{D}^{\circ}{ }_{2}$ & & & TW & & \\
\hline $877.8469(21)$ & 113915.1(3) & $877.84692(3)$ & 0.0000 & 610000 & & $4 \mathrm{~s}$ & ${ }^{3} \mathrm{D}_{1}$ & $\mathrm{sp}$ & $\left({ }^{3} \mathrm{~F}\right)^{1} \mathrm{P}^{\circ}{ }^{3} \mathrm{D}^{\circ}{ }_{1}$ & & & TW & & \\
\hline $878.6984(8)$ & $113804.69(10)$ & 878.69833(3) & 0.0001 & 1500000 & & $4 \mathrm{~s}$ & ${ }^{3} \mathrm{D}_{3}$ & $\mathrm{sp}$ & $\left({ }^{3} \mathrm{~F}\right){ }^{1} \mathrm{P}^{\circ}{ }^{3} \mathrm{D}^{\circ}{ }_{3}$ & & & K66 & & \\
\hline $879.8897(24)$ & $113650.6(3)$ & $879.89110(3)$ & -0.0014 & 21000 & & $4 \mathrm{~s}$ & ${ }^{3} \mathrm{D}_{1}$ & $\mathrm{sp}$ & $\left({ }^{3} \mathrm{P}\right)^{3} \mathrm{P}^{\circ}{ }^{1} \mathrm{D}^{\circ}{ }_{2}$ & & & TW & & \\
\hline $880.3225(24)$ & 113594.7(3) & $880.322766(20)$ & -0.0003 & 38000 & & $4 \mathrm{~s}$ & ${ }^{3} \mathrm{D}_{2}$ & $\mathrm{sp}$ & $\left({ }^{3} \mathrm{~F}\right)^{1} \mathrm{P}^{\circ}{ }^{3} \mathrm{~F}_{3}^{\circ}$ & & & TW & & \\
\hline $881.473(3)$ & $113446.4(4)$ & $881.47711(3)$ & -0.004 & 3100 & & $4 \mathrm{~s}$ & ${ }^{1} \mathrm{D}_{2}$ & $\mathrm{sp}$ & $\left({ }^{3} \mathrm{~F}\right)^{1} \mathrm{P}^{\circ}{ }^{3} \mathrm{~F}^{\circ}{ }_{2}$ & & & TW & & \\
\hline $883.2800(20)$ & $113214.4(3)$ & 883.27981(3) & 0.0002 & 41000 & & $4 \mathrm{~s}$ & ${ }^{3} \mathrm{D}_{1}$ & $\mathrm{sp}$ & $\left({ }^{1} \mathrm{G}\right)^{3} \mathrm{P}^{\circ}{ }^{3} \mathrm{~F}^{\circ}{ }_{2}$ & & & TW,S36r & & \\
\hline $883.8404(20)$ & $113142.6(3)$ & $883.838830(10)$ & 0.0016 & 55000 & & $4 \mathrm{~s}$ & ${ }^{3} \mathrm{D}_{2}$ & $4 \mathrm{f}$ & $(5 / 2)^{2}[7 / 2]^{\circ}{ }_{3}$ & & & TW & & \\
\hline $884.1340(21)$ & 113105.0(3) & $884.13295(4)$ & 0.0011 & 290000 & & $4 \mathrm{~s}$ & ${ }^{3} \mathrm{D}_{2}$ & $\mathrm{sp}$ & $\left({ }^{3} \mathrm{P}\right)^{3} \mathrm{P}^{\circ}{ }^{5} \mathrm{~S}^{\circ}{ }_{2}$ & & & TW & & \\
\hline $884.4346(18)$ & 113066.58(23) & $884.43449(3)$ & 0.0001 & 420000 & & $4 \mathrm{~s}$ & ${ }^{1} \mathrm{D}_{2}$ & $\mathrm{sp}$ & $\left({ }^{1} \mathrm{G}^{3}\right)^{3} \mathrm{P}^{\circ}{ }^{3} \mathrm{~F}^{\circ}{ }_{3}$ & & & TW & & \\
\hline $884.8262(20)$ & $113016.5(3)$ & $884.826019(13)$ & 0.0002 & 34000 & & $4 \mathrm{~s}$ & ${ }^{3} \mathrm{D}_{2}$ & $4 \mathrm{f}$ & $(5 / 2)^{2}[1 / 2]^{\circ} 1$ & & & TW & & \\
\hline $885.8463(20)$ & 112886.4(3) & $885.846966(24)$ & -0.0006 & 770000 & & $4 \mathrm{~s}$ & ${ }^{3} \mathrm{D}_{2}$ & $\mathrm{sp}$ & $\left({ }^{3} \mathrm{~F}\right)^{1} \mathrm{P}^{\circ}{ }^{3} \mathrm{D}^{\circ}{ }_{3}$ & & & TW & & \\
\hline $886.4163(24)$ & $112813.8(3)$ & $886.413782(20)$ & 0.0025 & 25000 & & $4 \mathrm{~s}$ & ${ }^{3} \mathrm{D}_{3}$ & sp & $\left({ }^{3} \mathrm{~F}\right)^{1} \mathrm{P}^{\circ}{ }^{3} \mathrm{~F}^{\circ}{ }_{4}^{\circ}$ & & & TW & & \\
\hline $886.5113(20)$ & $112801.7(3)$ & $886.510950(24)$ & 0.0004 & 280000 & & $4 \mathrm{~s}$ & ${ }^{3} \mathrm{D}_{1}$ & sp & $\left({ }^{3} \mathrm{~F}\right){ }^{1} \mathrm{P}^{\circ}{ }^{3} \mathrm{D}^{\circ}{ }_{2}$ & & & TW & & \\
\hline $886.9435(8)$ & $112746.75(10)$ & $886.94322(3)$ & 0.0003 & 1300000 & & $4 \mathrm{~s}$ & ${ }^{3} \mathrm{D}_{3}$ & $\mathrm{sp}$ & $\left({ }^{3} \mathrm{P}\right)^{3} \mathrm{P}^{\circ}{ }^{3} \mathrm{D}_{2}^{\circ}$ & & & K66 & & \\
\hline $890.5677(6)$ & $112287.93(8)$ & $890.56671(3)$ & 0.0010 & 1300000 & & $4 \mathrm{~s}$ & ${ }^{3} \mathrm{D}_{2}$ & $\mathrm{sp}$ & $\left({ }^{3} \mathrm{P}\right)^{3} \mathrm{P}^{\circ}{ }^{3} \mathrm{P}^{\circ}{ }_{1}$ & & & K66 & & \\
\hline $891.7636(24)$ & $112137.3(3)$ & 891.76309(3) & 0.0005 & 23000 & & $4 \mathrm{~s}$ & ${ }^{1} \mathrm{D}_{2}$ & $6 \mathrm{p}$ & $(5 / 2)^{2}[7 / 2]^{\circ}{ }_{3}$ & & & TW & & \\
\hline $892.4154(6)$ & $112055.44(8)$ & $892.41418(3)$ & 0.0012 & 1100000 & & $4 \mathrm{~s}$ & ${ }^{3} \mathrm{D}_{3}$ & $\mathrm{sp}$ & $\left({ }^{3} \mathrm{P}\right)^{3} \mathrm{P}^{\circ}{ }^{3} \mathrm{D}_{3}^{\circ}$ & & & K66 & & \\
\hline $893.634(5)$ & $111902.7(7)$ & 893.624985(19) & 0.009 & 1100000 & & $4 \mathrm{~s}$ & ${ }^{3} \mathrm{D}_{1}$ & $4 \mathrm{f}$ & $(5 / 2)^{2}[1 / 2]^{\circ} 0$ & & & TW & & \\
\hline $893.6787(6)$ & $111897.04(8)$ & $893.67746(3)$ & 0.0012 & 940000 & & $4 \mathrm{~s}$ & ${ }^{3} \mathrm{D}_{3}$ & $\mathrm{sp}$ & $\left({ }^{3} \mathrm{P}\right)^{3} \mathrm{P}^{\mathrm{o}}{ }^{3} \mathrm{P}^{\circ}{ }_{2}$ & & & K66 & & \\
\hline $894.2260(20)$ & $111828.56(25)$ & $894.227192(24)$ & -0.0012 & 860000 & & $4 \mathrm{~s}$ & ${ }^{3} \mathrm{D}_{2}$ & $\mathrm{sp}$ & $\left({ }^{3} \mathrm{P}\right)^{3} \mathrm{P}^{\circ}{ }^{3} \mathrm{D}^{\circ}{ }_{2}$ & & & TW & & \\
\hline $896.7583(15)$ & $111512.77(19)$ & $896.758608(23)$ & -0.0003 & 1000000 & & $4 \mathrm{~s}$ & ${ }^{3} \mathrm{D}_{2}$ & $\mathrm{sp}$ & $\left({ }^{3} \mathrm{P}\right)^{3} \mathrm{P}^{\circ}{ }^{3} \mathrm{D}^{\circ}{ }_{1}$ & & & TW & & \\
\hline $896.9741(20)$ & $111485.94(25)$ & $896.97600(3)$ & -0.0019 & 870000 & & $4 \mathrm{~s}$ & ${ }^{3} \mathrm{D}_{1}$ & $\mathrm{sp}$ & $\left({ }^{3} \mathrm{P}\right)^{3} \mathrm{P}^{\circ}{ }^{3} \mathrm{P}_{0}^{\circ}{ }_{0}$ & & & TW & & \\
\hline $897.7927(18)$ & $111384.29(22)$ & $897.79300(3)$ & -0.0003 & 410000 & & $4 \mathrm{~s}$ & ${ }^{1} \mathrm{D}_{2}$ & sp & $\left({ }^{3} \mathrm{P}\right)^{3} \mathrm{P}^{\circ}{ }^{1} \mathrm{D}^{\circ}{ }_{2}$ & & & TW & & \\
\hline 899.7904(18) & $111136.99(22)$ & $899.78867(3)$ & 0.0018 & 910000 & * & $4 \mathrm{~s}$ & ${ }^{3} \mathrm{D}_{2}$ & sp & $\left({ }^{3} \mathrm{P}\right)^{3} \mathrm{P}^{\circ}{ }^{3} \mathrm{D}^{\circ}{ }_{3}$ & & & TW & & \\
\hline 899.7904(18) & $111136.99(22)$ & $899.79200(3)$ & -0.0016 & 910000 & * & $4 \mathrm{~s}$ & ${ }^{3} \mathrm{D}_{1}$ & $\mathrm{sp}$ & $\left({ }^{3} \mathrm{P}\right)^{3} \mathrm{P}^{\circ}{ }^{3} \mathrm{P}_{1}^{\circ}$ & & & TW & & \\
\hline $901.0757(16)$ & $110978.47(20)$ & $901.07654(7)$ & -0.0009 & 660000 & * & $4 \mathrm{~s}$ & ${ }^{1} \mathrm{D}_{2}$ & $\mathrm{sp}$ & $\left({ }^{3} \mathrm{P}\right)^{3} \mathrm{P}^{\circ}{ }^{1} \mathrm{P}^{\circ}{ }_{1}$ & & & TW,S36r & & \\
\hline $901.0757(16)$ & 110978.47(20) & 901.07292(3) & 0.0027 & 660000 & * & $4 \mathrm{~s}$ & ${ }^{3} \mathrm{D}_{2}$ & sp & $\left({ }^{3} \mathrm{P}\right)^{3} \mathrm{P}^{\circ}{ }^{3} \mathrm{P}^{\circ}{ }_{2}$ & & & TW & & \\
\hline $901.322(3)$ & $110948.2(4)$ & $901.32128(3)$ & 0.000 & 25000 & & $4 \mathrm{~s}$ & ${ }^{1} \mathrm{D}_{2}$ & $\mathrm{sp}$ & $\left({ }^{1} \mathrm{G}\right)^{3} \mathrm{P}^{\circ}{ }^{3} \mathrm{~F}^{\circ}{ }_{2}$ & & & TWn & & \\
\hline $903.522(6)$ & $110678.0(7)$ & $903.52887(3)$ & -0.007 & 16000 & & $4 \mathrm{~s}$ & ${ }^{3} \mathrm{D}_{1}$ & $\mathrm{sp}$ & $\left({ }^{3} \mathrm{P}\right)^{3} \mathrm{P}^{\circ}{ }^{3} \mathrm{D}^{\circ}{ }_{2}$ & & & S36c & & \\
\hline $906.1130(20)$ & $110361.52(24)$ & $906.11330(3)$ & -0.0003 & 670000 & & $4 \mathrm{~s}$ & ${ }^{3} \mathrm{D}_{1}$ & $\mathrm{sp}$ & $\left({ }^{3} \mathrm{P}\right)^{3} \mathrm{P}^{\circ}{ }^{3} \mathrm{D}^{\circ}{ }_{1}$ & & & TW & & \\
\hline $910.5183(21)$ & $109827.56(25)$ & 910.51831(3) & -0.0000 & 240000 & & $4 \mathrm{~s}$ & ${ }^{3} \mathrm{D}_{1}$ & $\mathrm{sp}$ & $\left({ }^{3} \mathrm{P}\right)^{3} \mathrm{P}^{\circ}{ }^{3} \mathrm{P}_{2}^{\circ}$ & & & TW & & \\
\hline 911.630 & 109693.6 & $911.629867(16)$ & & 15000 & : & $4 \mathrm{~s}$ & ${ }^{1} \mathrm{D}_{2}$ & $4 \mathrm{f}$ & $(5 / 2)^{2}[3 / 2]^{\circ}{ }_{1}$ & & & S36c & & \\
\hline 911.679 & 109687.7 & $911.67901(4)$ & & 15000 & : & $4 \mathrm{~s}$ & ${ }^{1} \mathrm{D}_{2}$ & $\mathrm{sp}$ & $\left({ }^{3} \mathrm{P}\right)^{3} \mathrm{P}^{\circ}{ }^{5} \mathrm{~S}^{\circ}{ }_{2}$ & & & S36c & & \\
\hline 912.025 & 109646.2 & $912.024525(12)$ & & 7400 & : & $4 \mathrm{~s}$ & ${ }^{1} \mathrm{D}_{2}$ & $4 \mathrm{f}$ & $(5 / 2)^{2}[3 / 2]^{\circ}{ }_{2}$ & & & S36c & & \\
\hline $912.4142(24)$ & 109599.3(3) & $912.415956(16)$ & -0.0018 & 15000 & & $4 \mathrm{~s}$ & ${ }^{1} \mathrm{D}_{2}$ & $4 \mathrm{f}$ & $(5 / 2)^{2}[1 / 2]^{\circ} 1$ & & & TW & & \\
\hline
\end{tabular}


Table A1. Cont.

\begin{tabular}{|c|c|c|c|c|c|c|c|c|c|c|c|c|c|c|}
\hline$\lambda_{\text {obs }}{ }^{a}(\AA)$ & $\sigma_{\text {obs }}{ }^{\mathrm{b}}\left(\mathrm{cm}^{-1}\right)$ & $\lambda_{\text {Ritz }}{ }^{c}(\AA)$ & $\Delta \lambda_{\text {obs-Ritz }}$ & $\begin{array}{c}I_{\text {obs }}{ }^{d} \\
\text { (arb. u.) }\end{array}$ & Char $^{\mathrm{e}}$ & \multicolumn{2}{|c|}{ Lower Level } & \multicolumn{2}{|c|}{ Upper Level } & $A\left(\mathrm{~s}^{-1}\right)$ & $\operatorname{Acc}^{f}$ & Line Ref. $\mathrm{g}$ & TP Ref. $\mathrm{g}$ & Notes $^{h}$ \\
\hline 913.47(4) & $109472(5)$ & $913.50160(3)$ & -0.03 & 7300 & & $4 \mathrm{~s}$ & ${ }^{1} \mathrm{D}_{2}$ & $\mathrm{sp}$ & $\left({ }^{3} \mathrm{~F}\right)^{1} \mathrm{P}^{\circ}{ }^{3} \mathrm{D}_{3}^{\circ}$ & & & S36c & & \\
\hline $914.2128(6)$ & 109383.72(7) & 914.21305(11) & -0.0003 & 830000 & & $4 \mathrm{~s}$ & ${ }^{3} \mathrm{D}_{3}$ & $\mathrm{sp}$ & $\left({ }^{3} \mathrm{P}\right)^{3} \mathrm{P}^{\circ}{ }^{5} \mathrm{D}^{\circ}{ }_{4}$ & & & K66 & & \\
\hline $917.3051(20)$ & $109014.98(23)$ & $917.30556(8)$ & -0.0004 & 380000 & & $4 \mathrm{~s}$ & ${ }^{3} \mathrm{D}_{3}$ & $\mathrm{sp}$ & $\left({ }^{3} \mathrm{P}\right)^{3} \mathrm{P}^{\circ}{ }^{5} \mathrm{D}^{\circ}{ }_{2}$ & & & TW & & \\
\hline $922.0188(8)$ & $108457.66(9)$ & $922.01869(14)$ & 0.0001 & 520000 & & $4 \mathrm{~s}$ & ${ }^{3} \mathrm{D}_{3}$ & $\mathrm{sp}$ & $\left({ }^{1} \mathrm{D}\right)^{3} \mathrm{P}^{\circ}{ }^{3} \mathrm{P}^{\circ}{ }_{2}$ & & & K66 & & \\
\hline $922.4141(16)$ & 108411.18(19) & $922.41586(3)$ & -0.0018 & 350000 & & $4 \mathrm{~s}$ & ${ }^{1} \mathrm{D}_{2}$ & $\mathrm{sp}$ & $\left({ }^{3} \mathrm{P}\right)^{3} \mathrm{P}^{\circ}{ }^{3} \mathrm{D}_{2}^{\circ}$ & & & TW & & \\
\hline 924.2381(8) & 108197.23(9) & $924.23840(7)$ & -0.0003 & 550000 & & $4 \mathrm{~s}$ & ${ }^{3} \mathrm{D}_{2}$ & $\mathrm{sp}$ & $\left({ }^{3} \mathrm{P}\right)^{3} \mathrm{P}^{\circ}{ }^{5} \mathrm{D}^{\circ}{ }_{3}$ & & & K66 & & \\
\hline 925.119(10) & 108094.2(12) & $925.09896(8)$ & 0.020 & 510000 & * & $4 \mathrm{~s}$ & ${ }^{3} \mathrm{D}_{2}$ & $\mathrm{sp}$ & $\left({ }^{3} \mathrm{P}\right)^{3} \mathrm{P}^{\circ}{ }^{5} \mathrm{D}_{2}^{\circ}$ & & & TW & & \\
\hline 925.119(10) & 108094.2(12) & $925.10963(3)$ & 0.009 & 510000 & * & $4 \mathrm{~s}$ & ${ }^{1} \mathrm{D}_{2}$ & $\mathrm{sp}$ & $\left({ }^{3} \mathrm{P}\right)^{3} \mathrm{P}^{\circ}{ }^{3} \mathrm{D}^{\circ}{ }_{1}$ & & & TW & & \\
\hline 925.119(10) & 108094.2(12) & $925.12612(7)$ & -0.007 & 510000 & * & $4 \mathrm{~s}$ & ${ }^{3} \mathrm{D}_{2}$ & $\mathrm{sp}$ & $\left.\left({ }^{3} \mathrm{P}\right)\right)^{3} \mathrm{P}^{\circ}{ }^{5} \mathrm{D}^{\circ}{ }_{1}$ & & & TW & & \\
\hline $929.702(3)$ & 107561.4(3) & 929.70175(3) & 0.000 & 18000 & & $4 \mathrm{~s}$ & ${ }^{1} \mathrm{D}_{2}$ & $\mathrm{sp}$ & $\left({ }^{3} \mathrm{P}\right)^{3} \mathrm{P}^{\circ}{ }^{3} \mathrm{P}_{2}^{\circ}$ & & & TW & & \\
\hline $929.8940(20)$ & $107539.14(23)$ & 929.89272(14) & 0.0013 & 18000 & & $4 \mathrm{~s}$ & ${ }^{3} \mathrm{D}_{2}$ & $\mathrm{sp}$ & $\left({ }^{1} \mathrm{D}\right)^{3} \mathrm{P}^{\circ}{ }^{3} \mathrm{P}^{\circ}{ }_{2}$ & & & TW & & \\
\hline $932.9398(8)$ & 107188.05(9) & 932.93989(10) & -0.0001 & 590000 & & $4 \mathrm{~s}$ & ${ }^{3} \mathrm{D}_{3}$ & $\mathrm{sp}$ & $\left({ }^{1} \mathrm{D}\right)^{3} \mathrm{P}^{\circ}{ }^{3} \mathrm{D}^{\circ}{ }_{3}$ & $1.7 \mathrm{e}+08$ & $\mathrm{C}$ & K66 & B00 & \\
\hline 934.9761(18) & 106954.61(21) & 934.97102(14) & 0.0050 & 280000 & ? & $4 \mathrm{~s}$ & ${ }^{3} \mathrm{D}_{1}$ & $\mathrm{sp}$ & $\left({ }^{3} \mathrm{P}\right)^{3} \mathrm{P}^{\circ}{ }^{5} \mathrm{D}^{\circ}{ }_{0}$ & & & TWn & & \\
\hline \multirow[t]{2}{*}{$935.0564(14)$} & 106945.42(17) & $935.05755(8)$ & -0.0012 & 380000 & & $4 \mathrm{~s}$ & ${ }^{3} \mathrm{D}_{1}$ & $\mathrm{sp}$ & $\left({ }^{3} \mathrm{P}\right)^{3} \mathrm{P}^{\circ}{ }^{5} \mathrm{D}_{2}^{\circ}$ & & & TW & & \\
\hline & & $935.08529(7)$ & & & $\mathrm{m}$ & $4 \mathrm{~s}$ & ${ }^{3} \mathrm{D}_{1}$ & $\mathrm{sp}$ & $\left({ }^{3} \mathrm{P}\right)^{3} \mathrm{P}^{\circ}{ }^{5} \mathrm{D}^{\circ}{ }_{1}$ & & & S36c & & \\
\hline $935.2307(22)$ & 106925.49(25) & $935.23232(7)$ & -0.0016 & 450000 & & $4 \mathrm{~s}$ & ${ }^{3} \mathrm{D}_{3}$ & $\mathrm{sp}$ & $\left({ }^{1} \mathrm{D}\right)^{3} \mathrm{P}^{\circ}{ }^{3} \mathrm{D}^{\circ}{ }_{2}$ & & & TW & & \\
\hline 935.3408(18) & $106912.90(20)$ & 935.34317(5) & -0.0023 & 360000 & & $4 \mathrm{~s}$ & ${ }^{3} \mathrm{D}_{2}$ & $\mathrm{sp}$ & $\left({ }^{1} \mathrm{D}\right)^{3} \mathrm{P}^{\circ}{ }^{3} \mathrm{P}^{\circ}{ }_{1}$ & & & TW & & \\
\hline 935.892(3) & 106849.9(3) & 935.89753(15) & -0.006 & 620000 & & $4 \mathrm{~s}$ & ${ }^{3} \mathrm{D}_{3}$ & $\mathrm{sp}$ & $\left({ }^{1} \mathrm{D}\right)^{3} \mathrm{P}^{\circ}{ }^{3} \mathrm{~F}_{4}^{\circ}$ & $1.12 \mathrm{e}+08$ & C & TW & B00 & \\
\hline $937.815(6)$ & $106630.8(7)$ & $937.81726(7)$ & -0.002 & 48000 & & $4 \mathrm{~s}$ & ${ }^{3} \mathrm{D}_{3}$ & sp & $\left({ }^{1} \mathrm{D}\right)^{3} \mathrm{P}^{\circ}{ }^{3} \mathrm{~F}^{\circ}{ }_{3}$ & & & S36c & & \\
\hline 939.5218(15) & $106437.12(16)$ & $939.52288(6)$ & -0.0010 & 190000 & & $4 \mathrm{~s}$ & ${ }^{3} \mathrm{D}_{3}$ & $\mathrm{sp}$ & $\left({ }^{1} \mathrm{D}\right)^{3} \mathrm{P}^{\circ}{ }^{3} \mathrm{~F}_{2}^{\circ}$ & & & TW & & \\
\hline 943.3333(18) & $106007.07(20)$ & $943.33466(7)$ & -0.0014 & 580000 & & $4 \mathrm{~s}$ & ${ }^{3} \mathrm{D}_{2}$ & $\mathrm{sp}$ & $\left({ }^{1} \mathrm{D}\right)^{3} \mathrm{P}^{\circ}{ }^{3} \mathrm{D}^{\circ}{ }_{2}$ & $1.4 \mathrm{e}+08$ & $\mathrm{C}$ & TW & B00 & \\
\hline 945.5223(17) & 105761.65(19) & $945.52474(6)$ & -0.0025 & 510000 & & $4 \mathrm{~s}$ & ${ }^{3} \mathrm{D}_{1}$ & $\mathrm{sp}$ & $\left({ }^{1} \mathrm{D}\right)^{3} \mathrm{P}^{\circ}{ }^{3} \mathrm{P}^{\circ}{ }_{1}$ & & & TW & & \\
\hline $945.8771(21)$ & $105721.98(24)$ & $945.87663(7)$ & 0.0005 & 400000 & & $4 \mathrm{~s}$ & ${ }^{3} \mathrm{D}_{2}$ & $\mathrm{sp}$ & $\left({ }^{1} \mathrm{D}\right)^{3} \mathrm{P}^{\circ}{ }^{3} \mathrm{D}^{\circ}{ }_{1}$ & & & TW & & \\
\hline 945.9614(21) & 105712.56(24) & $945.96464(7)$ & -0.0033 & 460000 & & $4 \mathrm{~s}$ & ${ }^{3} \mathrm{D}_{2}$ & $\mathrm{sp}$ & $\left({ }^{1} \mathrm{D}\right)^{3} \mathrm{P}^{\circ}{ }^{3} \mathrm{~F}_{3}^{\circ}$ & $1.1 \mathrm{e}+08$ & C & TW & B00 & \\
\hline 947.6989(17) & 105518.74(19) & $947.70005(6)$ & -0.0011 & 14000 & & $4 \mathrm{~s}$ & ${ }^{3} \mathrm{D}_{2}$ & $\mathrm{sp}$ & $\left({ }^{1} \mathrm{D}\right)^{3} \mathrm{P}^{\circ}{ }^{3} \mathrm{~F}^{\circ}{ }_{2}$ & & & TW & & \\
\hline $951.4068(15)$ & 105107.51(16) & 951.40774(8) & -0.0010 & 24000 & & $4 \mathrm{~s}$ & ${ }^{3} \mathrm{D}_{1}$ & $\mathrm{sp}$ & $\left.\left({ }^{1} \mathrm{D}\right)\right)^{3} \mathrm{P}^{\circ}{ }^{3} \mathrm{P}^{\circ}{ }_{0}$ & & & TW & & \\
\hline 954.3815(15) & 104779.91(16) & $954.38279(7)$ & -0.0013 & 270000 & & $4 \mathrm{~s}$ & ${ }^{1} \mathrm{D}_{2}$ & $\mathrm{sp}$ & $\left({ }^{3} \mathrm{P}\right)^{3} \mathrm{P}^{\circ}{ }^{5} \mathrm{D}_{3}^{\circ}$ & & & TW & & \\
\hline 955.306(5) & $104678.5(6)$ & $955.30043(8)$ & 0.005 & 9900 & & $4 \mathrm{~s}$ & ${ }^{1} \mathrm{D}_{2}$ & $\mathrm{sp}$ & $\left({ }^{3} \mathrm{P}\right)^{3} \mathrm{P}^{\circ}{ }^{5} \mathrm{D}^{\circ}{ }_{2}$ & & & TW & & \\
\hline $955.320(5)$ & $104677.0(6)$ & $955.32939(7)$ & -0.009 & 9900 & & $4 \mathrm{~s}$ & ${ }^{1} \mathrm{D}_{2}$ & $\mathrm{sp}$ & $\left({ }^{3} \mathrm{P}\right)^{3} \mathrm{P}^{\circ}{ }^{5} \mathrm{D}^{\circ}{ }_{1}$ & & & TW & & \\
\hline 956.2871(17) & 104571.11(19) & $956.29010(7)$ & -0.0030 & 300000 & & $4 \mathrm{~s}$ & ${ }^{3} \mathrm{D}_{1}$ & $\mathrm{sp}$ & $\left({ }^{1} \mathrm{D}\right)^{3} \mathrm{P}^{\circ}{ }^{3} \mathrm{D}^{\circ}{ }_{1}$ & $1.8 \mathrm{e}+08$ & C & TW & B00 & \\
\hline 958.1503(17) & 104367.76(19) & $958.15393(6)$ & -0.0037 & 380000 & & $4 \mathrm{~s}$ & ${ }^{3} \mathrm{D}_{1}$ & $\mathrm{sp}$ & $\left({ }^{1} \mathrm{D}\right)^{3} \mathrm{P}^{\circ}{ }^{3} \mathrm{~F}_{2}^{\circ}$ & $1.6 \mathrm{e}+08$ & C & TW & B00 & \\
\hline 960.4115(17) & 104122.04(19) & $960.41317(15)$ & -0.0017 & 270000 & & $4 \mathrm{~s}$ & ${ }^{1} \mathrm{D}_{2}$ & sp & $\left({ }^{1} \mathrm{D}\right)^{3} \mathrm{P}^{\circ}{ }^{3} \mathrm{P}^{\circ}{ }_{2}$ & & & TW & & \\
\hline $966.2283(21)$ & 103495.21(23) & $966.22839(6)$ & -0.0001 & 11000 & & $4 \mathrm{~s}$ & ${ }^{1} \mathrm{D}_{2}$ & $\mathrm{sp}$ & $\left({ }^{1} \mathrm{D}\right)^{3} \mathrm{P}^{\circ}{ }^{3} \mathrm{P}^{\circ}{ }_{1}$ & & & TW & & \\
\hline $967.8711(21)$ & $103319.54(23)$ & $967.87255(7)$ & -0.0015 & 4800 & & $4 \mathrm{~s}$ & ${ }^{3} \mathrm{D}_{3}$ & $\mathrm{sp}$ & $\left({ }^{3} \mathrm{P}\right)^{3} \mathrm{P}^{\circ}{ }^{5} \mathrm{P}_{2}^{\circ}$ & & & TW & & \\
\hline 968.0392(17) & 103301.60(18) & $968.04176(16)$ & -0.0025 & 250000 & & $4 \mathrm{~s}$ & ${ }^{3} \mathrm{D}_{3}$ & $\mathrm{sp}$ & $\left({ }^{3} \mathrm{P}\right)^{3} \mathrm{P}^{\circ}{ }^{5} \mathrm{P}_{3}^{\circ}{ }_{3}$ & & & TW & & \\
\hline 972.2703(19) & 102852.06(20) & $972.26872(10)$ & 0.0016 & 24000 & & $4 \mathrm{~s}$ & ${ }^{1} \mathrm{D}_{2}$ & $\mathrm{sp}$ & $\left({ }^{1} \mathrm{D}\right)^{3} \mathrm{P}^{\circ}{ }^{3} \mathrm{D}_{3}^{\circ}$ & & & TW & & \\
\hline 973.509(10) & 102721.2(11) & 973.49928(20) & 0.010 & 18000 & & $4 \mathrm{~s}$ & ${ }^{3} \mathrm{D}_{2}$ & $\mathrm{sp}$ & $\left({ }^{3} \mathrm{P}\right)^{3} \mathrm{P}^{\circ}{ }^{5} \mathrm{P}^{\circ}{ }_{1}$ & & & S36c & & \\
\hline 974.7581(15) & $102589.56(15)$ & 974.75876(8) & -0.0007 & 200000 & & $4 \mathrm{~s}$ & ${ }^{1} \mathrm{D}_{2}$ & $\mathrm{sp}$ & $\left({ }^{1} \mathrm{D}\right)^{3} \mathrm{P}^{\circ}{ }^{3} \mathrm{D}_{2}^{\circ}$ & & & TW & & \\
\hline 976.5524(15) & 102401.06(15) & $976.55293(7)$ & -0.0005 & 110000 & & $4 \mathrm{~s}$ & ${ }^{3} \mathrm{D}_{2}$ & $\mathrm{sp}$ & $\left({ }^{3} \mathrm{P}\right)^{3} \mathrm{P}^{\circ}{ }^{5} \mathrm{P}^{\circ}{ }_{2}$ & & & TW & & \\
\hline $976.7238(17)$ & 102383.09(18) & 976.72519(17) & -0.0014 & 35000 & & $4 \mathrm{~s}$ & ${ }^{3} \mathrm{D}_{2}$ & $\mathrm{sp}$ & $\left({ }^{3} \mathrm{P}\right)^{3} \mathrm{P}^{\mathrm{o}}{ }^{5} \mathrm{P}^{\mathrm{o}}{ }_{3}$ & & & TW & & \\
\hline
\end{tabular}


Table A1. Cont.

\begin{tabular}{|c|c|c|c|c|c|c|c|c|c|c|c|c|c|c|}
\hline$\lambda_{\text {obs }}{ }^{\text {a }}(\AA)$ & $\sigma_{\mathrm{obs}}{ }^{\mathrm{b}}\left(\mathrm{cm}^{-1}\right)$ & $\lambda_{\mathrm{Ritz}}{ }^{\mathrm{c}}(\AA)$ & $\underset{\substack{\Delta \lambda_{\text {obs-Ritz }} \\
(\AA)}}{ }$ & $\begin{array}{c}I_{\text {obs }}{ }^{d} \\
\text { (arb. u.) }\end{array}$ & Char ${ }^{\mathrm{e}}$ & & evel & & Jpper Level & $A\left(\mathrm{~s}^{-1}\right)$ & $\operatorname{Acc}^{f}$ & Line Ref. $\mathrm{g}$ & TP Ref. $\mathrm{g}$ & Notes ${ }^{h}$ \\
\hline $977.5662(13)$ & $102294.86(14)$ & $977.56714(7)$ & -0.0009 & 190000 & & $4 \mathrm{~s}$ & ${ }^{1} \mathrm{D}_{2}$ & $\mathrm{sp}$ & $\left({ }^{1} \mathrm{D}\right)^{3} \mathrm{P}^{\circ}{ }^{3} \mathrm{~F}_{3}^{\circ}$ & & & TW & & \\
\hline $979.4200(15)$ & 102101.25(16) & $979.42055(6)$ & -0.0006 & 12000 & & $4 \mathrm{~s}$ & ${ }^{1} \mathrm{D}_{2}$ & $\mathrm{sp}$ & $\left({ }^{1} \mathrm{D}\right)^{3} \mathrm{P}^{\circ}{ }^{3} \mathrm{~F}^{\circ}{ }_{2}$ & & & TW & & \\
\hline $984.02(4)$ & 101624(4) & $983.979887(19)$ & 0.04 & 9100 & & $4 \mathrm{~s}$ & ${ }^{3} \mathrm{D}_{3}$ & $5 p$ & $(3 / 2)^{2}[3 / 2]_{2}^{\circ}$ & & & S36c & & \\
\hline 984.5331(15) & $101570.98(15)$ & 984.53339(21) & -0.0003 & 88000 & & $4 \mathrm{~s}$ & ${ }^{3} \mathrm{D}_{1}$ & $\mathrm{sp}$ & $\left({ }^{3} \mathrm{P}\right)^{3} \mathrm{P}^{\circ}{ }^{5} \mathrm{P}_{1}^{\circ}$ & & & TW & & \\
\hline 987.6568(12) & $101249.75(12)$ & $987.65677(8)$ & -0.0000 & 61000 & & $4 \mathrm{~s}$ & ${ }^{3} \mathrm{D}_{1}$ & $\mathrm{sp}$ & $\left({ }^{3} \mathrm{P}\right)^{3} \mathrm{P}^{\circ}{ }^{\circ} \mathrm{P}^{\circ}{ }_{2}$ & & & TW & & \\
\hline $989.2364(12)$ & $101088.07(12)$ & $989.236270(17)$ & 0.0002 & 30000 & & $4 \mathrm{~s}$ & ${ }^{3} \mathrm{D}_{3}$ & $5 p$ & $(3 / 2)^{2}[5 / 2]_{3}^{\circ}$ & & & TW & & \\
\hline $992.9530(13)$ & $100709.70(13)$ & $992.952929(16)$ & 0.0001 & 210000 & & $4 \mathrm{~s}$ & ${ }^{3} \mathrm{D}_{2}$ & $5 \mathrm{p}$ & $(3 / 2)^{2}[3 / 2]^{\circ}{ }_{2}$ & & & TW & & \\
\hline 998.3067(14) & $100169.62(14)$ & $998.305876(14)$ & 0.0008 & 40000 & & $4 \mathrm{~s}$ & ${ }^{3} \mathrm{D}_{2}$ & $5 \mathrm{p}$ & $(3 / 2)^{2}[5 / 2]^{\circ}{ }_{3}$ & & & TW & & \\
\hline 999.797(3) & $100020.3(3)$ & 999.793812(15) & 0.003 & 7600 & & $4 \mathrm{~s}$ & ${ }^{3} \mathrm{D}_{2}$ & $5 \mathrm{p}$ & $(3 / 2)^{2}[1 / 2]^{\circ}{ }_{1}$ & & & TW & & \\
\hline $1001.0129(19)$ & 99898.81(19) & $1001.012710(14)$ & 0.0002 & 22000 & & $4 \mathrm{~s}$ & ${ }^{3} \mathrm{D}_{2}$ & $5 p$ & $(3 / 2)^{2}[5 / 2]^{\circ}{ }_{2}$ & & & TW & & \\
\hline $1004.0540(17)$ & 99596.24(17) & $1004.055195(18)$ & -0.0012 & 330000 & & $4 \mathrm{~s}$ & ${ }^{3} \mathrm{D}_{3}$ & $\mathrm{sp}$ & $\left({ }^{3} \mathrm{~F}\right)^{3} \mathrm{P}^{\circ}{ }^{1} \mathrm{~F}_{3}^{\circ}$ & & & TW & & \\
\hline $1006.980(6)$ & $99306.8(6)$ & $1006.983925(18)$ & -0.004 & 13000 & & $4 \mathrm{~s}$ & ${ }^{3} \mathrm{D}_{1}$ & $5 p$ & $(3 / 2)^{2}[3 / 2]^{\circ}{ }_{1}$ & & & S36c & & \\
\hline 1008.5674(17) & $99150.54(16)$ & $1008.568623(17)$ & -0.0012 & 290000 & & $4 \mathrm{~s}$ & ${ }^{3} \mathrm{D}_{3}$ & $5 p$ & $(5 / 2)^{2}[5 / 2]^{\circ}{ }_{3}$ & & & TW & & \\
\hline $1008.7274(17)$ & 99134.81(16) & 1008.728151(15) & -0.0008 & 310000 & & $4 \mathrm{~s}$ & ${ }^{3} \mathrm{D}_{2}$ & $\mathrm{sp}$ & $\left({ }^{3} \mathrm{~F}\right)^{3} \mathrm{P}^{\circ}{ }^{1} \mathrm{D}_{2}^{\circ}$ & & & TW & & \\
\hline $1010.2680(17)$ & $98983.64(16)$ & $1010.26867(8)$ & -0.0007 & 320000 & & $4 \mathrm{~s}$ & ${ }^{1} \mathrm{D}_{2}$ & $\mathrm{sp}$ & $\left({ }^{3} \mathrm{P}\right)^{3} \mathrm{P}^{\circ}{ }^{5} \mathrm{P}_{2}^{\circ}$ & & & TW & & \\
\hline 1010.4520(18) & 98965.61(18) & $1010.45303(18)$ & -0.0011 & 48000 & & $4 \mathrm{~s}$ & ${ }^{1} \mathrm{D}_{2}$ & sp & $\left({ }^{3} \mathrm{P}\right)^{3} \mathrm{P}^{\circ}{ }^{5} \mathrm{P}_{3}^{\circ}$ & & & TW & & \\
\hline $1010.640(3)$ & 98947.2(3) & $1010.639186(19)$ & 0.001 & 18000 & & $4 \mathrm{~s}$ & ${ }^{3} \mathrm{D}_{3}$ & $5 p$ & $(5 / 2)^{2}[5 / 2]^{\circ}{ }_{2}$ & & & TW & & \\
\hline 1011.436 & 98869.4 & 1011.435606(17) & & 29000 & : & $4 \mathrm{~s}$ & ${ }^{3} \mathrm{D}_{1}$ & $5 p$ & $(3 / 2)^{2}[1 / 2]_{1}^{\circ}$ & & & S36c & & \\
\hline 1012.5956(18) & 98756.11(18) & $1012.596906(18)$ & -0.0013 & 290000 & & $4 \mathrm{~s}$ & ${ }^{3} \mathrm{D}_{3}$ & $5 \mathrm{p}$ & $(5 / 2)^{2}[7 / 2]^{\circ}{ }_{3}$ & & & TW & & \\
\hline 1012.6813(18) & $98747.75(18)$ & $1012.683073(16)$ & -0.0017 & 50000 & & $4 \mathrm{~s}$ & ${ }^{3} \mathrm{D}_{1}$ & $5 p$ & $(3 / 2)^{2}[5 / 2]_{2}^{\circ}$ & & & TW & & \\
\hline $1013.382(20)$ & 98679.5(19) & $1013.399849(15)$ & -0.018 & 15000 & & $4 \mathrm{~s}$ & ${ }^{3} \mathrm{D}_{2}$ & $\mathrm{sp}$ & $\left({ }^{3} \mathrm{~F}\right)^{3} \mathrm{P}^{\circ}{ }^{1} \mathrm{~F}_{3}^{\circ}$ & & & S36c & & \\
\hline $1017.9976(18)$ & $98232.06(18)$ & 1017.997872(13) & -0.0002 & 70000 & & $4 \mathrm{~s}$ & ${ }^{3} \mathrm{D}_{2}$ & $5 \mathrm{p}$ & $(5 / 2)^{2}[5 / 2]^{\circ}{ }_{3}$ & & & TW & & \\
\hline 1018.0628(19) & 98225.77(18) & $1018.064039(22)$ & -0.0013 & 180000 & & $4 \mathrm{~s}$ & ${ }^{3} \mathrm{D}_{1}$ & $5 p$ & $(3 / 2)^{2}[1 / 2]_{0}^{\circ}$ & & & TW & & \\
\hline $1018.7031(24)$ & $98164.03(23)$ & 1018.707024(17) & -0.0039 & 550000 & & $4 \mathrm{~s}$ & ${ }^{3} \mathrm{D}_{3}$ & $5 \mathrm{p}$ & $(5 / 2)^{2}[3 / 2]^{\circ}{ }_{2}$ & & & TW & & \\
\hline 1019.6525(18) & $98072.62(17)$ & $1019.654307(13)$ & -0.0018 & 240000 & & $4 \mathrm{~s}$ & ${ }^{3} \mathrm{D}_{2}$ & $5 p$ & $(5 / 2)^{2}[3 / 2]^{\circ}{ }_{1}$ & & & TW & & \\
\hline 1020.1070(18) & 98028.93(18) & $1020.107372(16)$ & -0.0004 & 280000 & & $4 \mathrm{~s}$ & ${ }^{3} \mathrm{D}_{2}$ & $5 \mathrm{p}$ & $(5 / 2)^{2}[5 / 2]^{\circ}{ }_{2}$ & & & TW & & \\
\hline 1022.102 & 97837.6 & 1022.101982(13) & & 91000 & : & $4 \mathrm{~s}$ & ${ }^{3} \mathrm{D}_{2}$ & $5 p$ & $(5 / 2)^{2}[7 / 2]^{\circ}{ }_{3}$ & & & S36c & & \\
\hline $1027.825(3)$ & $97292.8(3)$ & $1027.830823(19)$ & -0.006 & 1800000 & & $4 \mathrm{~s}$ & ${ }^{1} \mathrm{D}_{2}$ & $5 p$ & $(3 / 2)^{2}[3 / 2]^{\circ}{ }_{2}$ & $1.7 \mathrm{e}+08$ & C & TW & B00 & \\
\hline $1028.3251(18)$ & 97245.51(17) & 1028.327701(13) & -0.0026 & 670000 & & $4 \mathrm{~s}$ & ${ }^{3} \mathrm{D}_{2}$ & $5 p$ & $(5 / 2)^{2}[3 / 2]_{2}^{\circ}$ & & & TW & & \\
\hline 1029.7493(18) & 97111.01(17) & 1029.750492(24) & -0.0012 & 290000 & & $4 \mathrm{~s}$ & ${ }^{3} \mathrm{D}_{3}$ & $\mathrm{sp}$ & $\left({ }^{3} \mathrm{~F}\right)^{3} \mathrm{P}^{\circ}{ }^{3} \mathrm{~F}^{\circ}{ }_{2}$ & & & TW & & \\
\hline 1030.2612(18) & 97062.76(17) & $1030.26297(7)$ & -0.0017 & 500000 & & $4 \mathrm{~s}$ & ${ }^{3} \mathrm{D}_{3}$ & $\mathrm{sp}$ & $\left({ }^{3} \mathrm{~F}\right)^{3} \mathrm{P}^{\circ}{ }^{1} \mathrm{G}^{\circ}{ }_{4}$ & & & TW & & \\
\hline 1031.7650(18) & $96921.30(17)$ & $1031.766015(15)$ & -0.0010 & 240000 & & $4 \mathrm{~s}$ & ${ }^{3} \mathrm{D}_{1}$ & $5 p$ & $(5 / 2)^{2}[3 / 2]^{\circ}{ }_{1}$ & & & TW & & \\
\hline 1033.5668(18) & 96752.33(17) & $1033.567510(18)$ & -0.0007 & 200000 & & $4 \mathrm{~s}$ & ${ }^{1} \mathrm{D}_{2}$ & $5 p$ & $(3 / 2)^{2}[5 / 2]^{\circ}{ }_{3}$ & & & TW & & \\
\hline 1035.161(3) & 96603.3(3) & 1035.162498(19) & -0.001 & 85000 & & $4 \mathrm{~s}$ & ${ }^{1} \mathrm{D}_{2}$ & $5 p$ & $(3 / 2)^{2}[1 / 2]^{\circ}{ }_{1}$ & & & TW & & \\
\hline $1036.465(3)$ & $96481.8(3)$ & $1036.469217(18)$ & -0.004 & 1800000 & & $4 \mathrm{~s}$ & ${ }^{1} \mathrm{D}_{2}$ & $5 p$ & $(3 / 2)^{2}[5 / 2]_{2}^{\circ}$ & $1.01 \mathrm{e}+08$ & $\mathrm{C}$ & TW & B00 & \\
\hline $1039.341(3)$ & $96214.8(3)$ & $1039.34743(3)$ & -0.006 & 2100000 & & $4 \mathrm{~s}$ & ${ }^{3} \mathrm{D}_{3}$ & $\mathrm{sp}$ & $\left({ }^{3} \mathrm{~F}\right)^{3} \mathrm{P}^{\circ}{ }^{3} \mathrm{~F}^{\circ}{ }_{3}$ & $2.0 \mathrm{e}+08$ & $\mathrm{C}$ & TW & B00 & \\
\hline 1039.579(3) & $96192.8(3)$ & $1039.581895(21)$ & -0.003 & 1900000 & & $4 \mathrm{~s}$ & ${ }^{3} \mathrm{D}_{2}$ & $\begin{array}{l}1 \\
\mathrm{sp}\end{array}$ & $\left({ }^{3} \mathrm{~F}\right)^{3} \mathrm{P}^{\circ}{ }^{3} \mathrm{~F}^{\circ}{ }_{2}$ & $2.0 \mathrm{e}+08$ & $\mathrm{C}$ & TW & B00 & \\
\hline $1044.5185(8)$ & $95737.89(7)$ & $1044.51849(6)$ & 0.0000 & 2300000 & & $4 \mathrm{~s}$ & ${ }^{3} \mathrm{D}_{3}$ & $\mathrm{sp}$ & $\left({ }^{3} \mathrm{~F}\right)^{3} \mathrm{P}^{\circ}{ }^{3} \mathrm{~F}^{\circ}{ }_{4}$ & $2.3 e+08$ & C & K66 & B00 & \\
\hline $1044.7437(8)$ & $95717.26(7)$ & $1044.743170(19)$ & 0.0005 & 2200000 & & $4 \mathrm{~s}$ & ${ }^{1} \mathrm{D}_{2}$ & $\mathrm{sp}$ & $\left({ }^{3} \mathrm{~F}\right)^{3} \mathrm{P}^{\circ}{ }^{1} \mathrm{D}^{\circ}{ }_{2}$ & $4.8 \mathrm{e}+08$ & C & K66 & B00 & \\
\hline 1049.3642(18) & $95295.80(17)$ & $1049.363833(22)$ & 0.0004 & 770000 & & $4 \mathrm{~s}$ & ${ }^{3} \mathrm{D}_{2}$ & sp & $\left({ }^{3} \mathrm{~F}\right)^{3} \mathrm{P}^{\circ}{ }^{3} \mathrm{~F}_{3}^{\circ}$ & $1.11 \mathrm{e}+08$ & $\mathrm{C}$ & TW & B00 & \\
\hline
\end{tabular}


Table A1. Cont.

\begin{tabular}{|c|c|c|c|c|c|c|c|c|c|c|c|c|c|c|}
\hline \multirow{2}{*}{$\begin{array}{r}\lambda_{\text {obs }}{ }^{\text {a }}(\AA ̊) \\
1049.7551(6)\end{array}$} & \multirow{2}{*}{$\begin{array}{l}\sigma_{\text {obs }}{ }^{\mathbf{b}}\left(\mathbf{c m}^{-\mathbf{1}}\right) \\
95260.31(5)\end{array}$} & \multirow{2}{*}{ 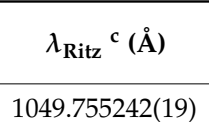 } & \multirow{2}{*}{$\begin{array}{c}\Delta \lambda_{\text {obs-Ritz }} \\
(\AA)\end{array}$} & \multirow{2}{*}{$\begin{array}{c}\begin{array}{c}I_{\text {obs }} \mathrm{d} \\
\text { (arb. u.) }\end{array} \\
2100000\end{array}$} & \multirow[t]{2}{*}{ Char ${ }^{\mathrm{e}}$} & \multicolumn{2}{|c|}{ Lower Level } & \multicolumn{2}{|c|}{ Upper Level } & \multirow{2}{*}{$\begin{array}{r}A\left(\mathrm{~s}^{-\mathbf{1}}\right) \\
1.04 \mathrm{e}+08\end{array}$} & \multirow{2}{*}{$\begin{array}{l}\operatorname{Acc}^{f} \\
C\end{array}$} & \multirow{2}{*}{$\begin{array}{l}\text { Line Ref. } \mathrm{g} \\
\text { K66 }\end{array}$} & \multirow{2}{*}{$\begin{array}{l}\text { TP Ref. } \mathrm{g} \\
\text { B00 }\end{array}$} & \multirow[t]{2}{*}{ Notes ${ }^{h}$} \\
\hline & & & & & & $4 \mathrm{~s}$ & ${ }^{1} \mathrm{D}_{2}$ & $\mathrm{sp}$ & $\left({ }^{3} \mathrm{~F}\right)^{3} \mathrm{P}^{\circ}{ }^{1} \mathrm{~F}_{3}^{\circ}$ & & & & & \\
\hline $1050.1523(18)$ & $95224.28(17)$ & $1050.15339(3)$ & -0.0010 & 530000 & & $4 \mathrm{~s}$ & ${ }^{3} \mathrm{D}_{2}$ & $\mathrm{sp}$ & $\left({ }^{3} \mathrm{~F}\right)^{3} \mathrm{P}^{\circ}{ }^{3} \mathrm{D}^{\circ}{ }_{1}$ & $1.4 \mathrm{e}+08$ & $\mathrm{C}$ & TW & B00 & \\
\hline 1050.4013(18) & 95201.71(17) & $1050.40243(3)$ & -0.0012 & 520000 & & $4 \mathrm{~s}$ & ${ }^{3} \mathrm{D}_{3}$ & $\mathrm{sp}$ & $\left({ }^{3} \mathrm{~F}\right)^{3} \mathrm{P}^{\circ}{ }^{3} \mathrm{D}^{\circ}{ }_{2}$ & $8.2 \mathrm{e}+07$ & $\mathrm{C}$ & TW & B00 & \\
\hline 1052.1742(18) & $95041.30(16)$ & $1052.174562(23)$ & -0.0004 & 1000000 & & $4 \mathrm{~s}$ & ${ }^{3} \mathrm{D}_{1}$ & $\mathrm{sp}$ & $\left({ }^{3} \mathrm{~F}\right)^{3} \mathrm{P}^{\circ}{ }^{3} \mathrm{~F}_{2}^{\circ}$ & $7.3 e+07$ & $\mathrm{C}$ & TW & B00 & \\
\hline 1054.6907(6) & $94814.53(5)$ & 1054.689891(17) & 0.0008 & 2800000 & & $4 \mathrm{~s}$ & ${ }^{1} \mathrm{D}_{2}$ & $5 p$ & $(5 / 2)^{2}[5 / 2]^{\circ} 3$ & $2.1 \mathrm{e}+08$ & C & K66 & B00 & \\
\hline 1055.792(3) & $94715.6(3)$ & $1055.79644(4)$ & -0.005 & 2500000 & & $4 \mathrm{~s}$ & ${ }^{3} \mathrm{D}_{3}$ & $\mathrm{sp}$ & $\left({ }^{3} \mathrm{~F}\right)^{3} \mathrm{P}^{\circ} 3^{3} \mathrm{G}^{\circ}{ }_{3}$ & $2.1 \mathrm{e}+08$ & C & TW & B00 & \\
\hline $1056.9549(6)$ & $94611.42(5)$ & $1056.954368(20)$ & 0.0005 & 2300000 & & $4 \mathrm{~s}$ & ${ }^{1} \mathrm{D}_{2}$ & $5 p$ & $(5 / 2)^{2}[5 / 2]^{\circ}{ }_{2}$ & $4.0 \mathrm{e}+08$ & C & K66 & B00 & \\
\hline $1058.798(3)$ & $94446.7(3)$ & $1058.79854(4)$ & -0.000 & 1800000 & & $4 \mathrm{~s}$ & ${ }^{3} \mathrm{D}_{3}$ & $\mathrm{sp}$ & $\left({ }^{3} \mathrm{~F}\right)^{3} \mathrm{P}^{\circ}{ }^{3} \mathrm{D}^{\circ}{ }_{3}$ & $1.9 \mathrm{e}+08$ & $\mathrm{C}$ & TW & B00 & \\
\hline 1059.0963(8) & $94420.12(7)$ & $1059.095825(18)$ & 0.0005 & 1400000 & & $4 \mathrm{~s}$ & ${ }^{1} \mathrm{D}_{2}$ & $5 p$ & $(5 / 2)^{2}[7 / 2]^{\circ}{ }_{3}$ & & & K66 & & \\
\hline 1060.631(3) & $94283.5(3)$ & $1060.63409(3)$ & -0.003 & 1700000 & & $4 \mathrm{~s}$ & ${ }^{3} \mathrm{D}_{2}$ & $\mathrm{sp}$ & $\left({ }^{3} \mathrm{~F}\right)^{3} \mathrm{P}^{\circ}{ }^{3} \mathrm{D}_{2}^{\circ}$ & $3.0 \mathrm{e}+08$ & C & TW & B00 & \\
\hline $1063.0027(18)$ & $94073.14(16)$ & 1063.00505(3) & -0.0024 & 1400000 & & $4 \mathrm{~s}$ & ${ }^{3} \mathrm{D}_{1}$ & $\mathrm{sp}$ & $\left({ }^{3} \mathrm{~F}\right)^{3} \mathrm{P}^{\circ}{ }^{3} \mathrm{D}^{\circ}{ }_{1}$ & $4.2 \mathrm{e}+08$ & C & TW & B00 & \\
\hline 1065.7837(18) & $93827.67(16)$ & $1065.781839(17)$ & 0.0019 & 560000 & & $4 \mathrm{~s}$ & ${ }^{1} \mathrm{D}_{2}$ & $5 p$ & $(5 / 2)^{2}[3 / 2]_{2}^{\circ}$ & & & TW & & \\
\hline 1066.1356(18) & $93796.70(16)$ & 1066.13397(4) & 0.0017 & 590000 & & $4 \mathrm{~s}$ & ${ }^{3} \mathrm{D}_{2}$ & $\mathrm{sp}$ & $\left({ }^{3} \mathrm{~F}\right)^{3} \mathrm{P}^{\circ} 3^{3} \mathrm{G}_{3}^{\circ}$ & & & TW & & \\
\hline 1069.1944(18) & $93528.36(16)$ & 1069.19523(4) & -0.0008 & 1000000 & & $4 \mathrm{~s}$ & ${ }^{3} \mathrm{D}_{2}$ & $\mathrm{sp}$ & $\left({ }^{3} \mathrm{~F}\right)^{3} \mathrm{P}^{\circ}{ }^{3} \mathrm{D}^{\circ}{ }_{3}$ & $1.03 e+08$ & C & TW & B00 & \\
\hline $1070.3134(18)$ & $93430.58(16)$ & $1070.31087(6)$ & 0.0025 & 380000 & & $4 \mathrm{~s}$ & ${ }^{3} \mathrm{D}_{3}$ & $\mathrm{sp}$ & $\left({ }^{3} \mathrm{~F}\right)^{3} \mathrm{P}^{\circ}{ }^{3} \mathrm{G}_{4}^{\circ}$ & & & TW & & \\
\hline 1073.7444(18) & $93132.04(16)$ & 1073.74516(3) & -0.0008 & 720000 & & $4 \mathrm{~s}$ & ${ }^{3} \mathrm{D}_{1}$ & sp & $\left({ }^{3} \mathrm{~F}\right)^{3} \mathrm{P}^{\circ}{ }^{3} \mathrm{D}_{2}^{\circ}$ & $1.5 \mathrm{e}+08$ & $\mathrm{C}$ & TW & B00 & \\
\hline 1077.889(20) & 92773.9(17) & $1077.87559(3)$ & 0.013 & 20000 & & $4 \mathrm{~s}$ & ${ }^{1} \mathrm{D}_{2}$ & sp & $\left({ }^{3} \mathrm{~F}\right)^{3} \mathrm{P}^{\circ}{ }^{3} \mathrm{~F}_{2}^{\circ}$ & & & S36c & & \\
\hline 1086.1134(18) & 92071.42(15) & $1086.10984(8)$ & 0.0036 & 72000 & & $4 \mathrm{~s}$ & ${ }^{3} \mathrm{D}_{3}$ & $\mathrm{sp}$ & $\left({ }^{3} \mathrm{~F}\right)^{3} \mathrm{P}^{\circ}{ }^{5} \mathrm{~F}^{\circ}{ }_{3}$ & & & TW & & \\
\hline 1088.405(5) & $91877.6(4)$ & $1088.39510(3)$ & 0.010 & 270000 & & $4 \mathrm{~s}$ & ${ }^{1} \mathrm{D}_{2}$ & $\mathrm{sp}$ & $\left({ }^{3} \mathrm{~F}\right)^{3} \mathrm{P}^{\circ}{ }^{3} \mathrm{~F}^{\circ}{ }_{3}$ & & & TW & & \\
\hline 1089.237(6) & 91807.4(5) & 1089.24451(3) & -0.007 & 39000 & & $4 \mathrm{~s}$ & ${ }^{1} \mathrm{D}_{2}$ & $\mathrm{sp}$ & $\left({ }^{3} \mathrm{~F}\right)^{3} \mathrm{P}^{\circ}{ }^{3} \mathrm{D}^{\circ}{ }_{1}$ & & & S36c & & \\
\hline 1091.293(3) & $91634.43(24)$ & $1091.29136(8)$ & 0.001 & 40000 & & $4 \mathrm{~s}$ & ${ }^{3} \mathrm{D}_{2}$ & $\mathrm{sp}$ & $\left({ }^{3} \mathrm{~F}\right)^{3} \mathrm{P}^{\circ}{ }^{5} \mathrm{~F}_{2}^{\circ}$ & & & TW & & \\
\hline 1094.399(3) & $91374.35(24)$ & $1094.40214(8)$ & -0.003 & 440000 & & $4 \mathrm{~s}$ & ${ }^{3} \mathrm{D}_{3}$ & $\mathrm{sp}$ & $\left({ }^{3} \mathrm{~F}\right)^{3} \mathrm{P}^{\circ}{ }^{5} \mathrm{~F}^{\circ}{ }_{4}$ & & & TW & & \\
\hline 1097.0512(18) & 91153.45(15) & $1097.05257(8)$ & -0.0014 & 320000 & & $4 \mathrm{~s}$ & ${ }^{3} \mathrm{D}_{2}$ & $\mathrm{sp}$ & $\left({ }^{3} \mathrm{~F}\right)^{3} \mathrm{P}^{\circ}{ }^{5} \mathrm{~F}^{\circ}{ }_{3}$ & & & TW & & \\
\hline $1100.523(3)$ & $90865.86(24)$ & $1100.52419(3)$ & -0.001 & 25000 & & $4 \mathrm{~s}$ & ${ }^{1} \mathrm{D}_{2}$ & $\mathrm{sp}$ & $\left({ }^{3} \mathrm{~F}\right)^{3} \mathrm{P}^{\circ}{ }^{3} \mathrm{D}^{\circ}{ }_{2}$ & & & TW & & \\
\hline $1101.836(6)$ & $90757.6(5)$ & $1101.83633(13)$ & -0.000 & 8200 & & $4 \mathrm{~s}$ & ${ }^{3} \mathrm{D}_{1}$ & $\mathrm{sp}$ & $\left({ }^{3} \mathrm{~F}\right)^{3} \mathrm{P}^{\circ}{ }^{5} \mathrm{~F}^{\circ}{ }_{1}$ & & & S36c & & \\
\hline 1105.1751(18) & $90483.40(15)$ & $1105.17629(8)$ & -0.0012 & 60000 & & $4 \mathrm{~s}$ & ${ }^{3} \mathrm{D}_{1}$ & $\mathrm{sp}$ & $\left({ }^{3} \mathrm{~F}\right)^{3} \mathrm{P}^{\circ}{ }^{5} \mathrm{~F}^{\circ}{ }_{2}$ & & & TW & & \\
\hline 1106.447 & 90379.4 & $1106.44670(4)$ & & 23000 & : & $4 \mathrm{~s}$ & ${ }^{1} \mathrm{D}_{2}$ & $\mathrm{sp}$ & $\left({ }^{3} \mathrm{~F}\right)^{3} \mathrm{P}^{\circ}{ }^{3} \mathrm{G}^{\circ}{ }_{3}$ & & & S36c & & \\
\hline 1109.744 & 90110.9 & $1109.74420(4)$ & & 7200 & : & $4 \mathrm{~s}$ & ${ }^{1} \mathrm{D}_{2}$ & $\mathrm{sp}$ & $\left({ }^{3} \mathrm{~F}\right)^{3} \mathrm{P}^{\circ}{ }^{3} \mathrm{D}^{\circ}{ }_{3}$ & & & S36c & & \\
\hline 1111.753(6) & $89948.0(5)$ & $1111.75742(11)$ & -0.004 & 3000 & & $4 \mathrm{~s}$ & ${ }^{3} \mathrm{D}_{3}$ & $\mathrm{sp}$ & $\left({ }^{3} \mathrm{~F}\right)^{3} \mathrm{P}^{\circ}{ }^{5} \mathrm{G}^{\circ}{ }_{3}$ & & & S36c & & \\
\hline $1119.9480(18)$ & $89289.86(15)$ & $1119.94659(11)$ & 0.0014 & 98000 & & $4 \mathrm{~s}$ & ${ }^{3} \mathrm{D}_{3}$ & $\mathrm{sp}$ & $\left({ }^{3} \mathrm{~F}\right)^{3} \mathrm{P}^{\circ}{ }^{5} \mathrm{G}^{\circ}{ }_{4}$ & & & TW & & \\
\hline $1123.2265(18)$ & $89029.24(14)$ & $1123.22580(11)$ & 0.0007 & 21000 & & $4 \mathrm{~s}$ & ${ }^{3} \mathrm{D}_{2}$ & $\mathrm{sp}$ & $\left({ }^{3} \mathrm{~F}\right)^{3} \mathrm{P}^{\circ}{ }^{5} \mathrm{G}_{3}^{\circ}$ & & & TW & & \\
\hline $1127.2516(18)$ & 88711.34(14) & & & 18000 & & $4 p$ & ${ }^{3} \mathrm{~F}_{2}^{\circ}$ & $10 \mathrm{~s}$ & $(3 / 2)^{2}[3 / 2]_{1}$ & & & TWn & & \\
\hline $1130.888(12)$ & $88426.1(9)$ & $1130.8852(3)$ & 0.003 & 4500 & * & $4 \mathrm{~s}$ & ${ }^{3} \mathrm{D}_{1}$ & $\mathrm{sp}$ & $\left({ }^{3} \mathrm{~F}\right)^{3} \mathrm{P}^{0}{ }^{5} \mathrm{G}^{\circ}{ }_{2}$ & & & S36c & & \\
\hline \multirow[t]{2}{*}{$1130.888(12)$} & $88426.1(9)$ & $1130.89779(11)$ & -0.010 & 4500 & * & $4 p$ & ${ }^{3} \mathrm{P}_{1}^{\circ}{ }_{1}$ & $9 \mathrm{~s}$ & $(3 / 2)^{2}[3 / 2]_{2}$ & & & S36c & & \\
\hline & & $1130.96216(16)$ & & & $\mathrm{m}$ & $4 p$ & ${ }^{3} \mathrm{P}^{\circ}{ }_{2}$ & $8 \mathrm{~d}$ & $(5 / 2)^{2}[3 / 2]_{1}$ & & & S36cn & & \\
\hline $1135.3657(18)$ & $88077.35(14)$ & $1135.34826(20)$ & 0.0174 & 8200 & $?$ & $4 \mathrm{p}$ & ${ }^{3} \mathrm{~F}^{\circ}{ }_{3}$ & $10 \mathrm{~s}$ & $(5 / 2)^{2}[5 / 2]_{2}$ & & & TW & & $x$ \\
\hline $1142.6393(18)$ & $87516.68(14)$ & $1142.64027(10)$ & -0.0009 & 73000 & & $4 \mathrm{~s}$ & ${ }^{3} \mathrm{D}_{2}$ & $\mathrm{sp}$ & $\left({ }^{3} \mathrm{~F}\right)^{3} \mathrm{P}^{0}{ }^{5} \mathrm{D}^{\circ}{ }_{2}$ & & & TW & & \\
\hline 1144.853(3) & $87347.48(22)$ & $1144.85531(8)$ & -0.003 & 120000 & & $4 \mathrm{~s}$ & ${ }^{3} \mathrm{D}_{3}$ & $\mathrm{sp}$ & $\left({ }^{3} \mathrm{~F}\right)^{3} \mathrm{P}^{\circ}{ }^{5} \mathrm{D}^{\circ}{ }_{3}$ & & & TW & & \\
\hline $1147.7628(18)$ & $87126.02(14)$ & & & 25000 & & $4 \mathrm{~s}$ & ${ }^{3} \mathrm{D}_{1}$ & $\mathrm{sp}$ & $\left({ }^{3} \mathrm{~F}\right)^{3} \mathrm{P}^{\circ}{ }^{5} \mathrm{D}^{\circ}{ }_{1}$ & & & TW & & \\
\hline $1157.0194(18)$ & $86428.97(14)$ & $1157.02043(8)$ & -0.0010 & 15000 & & $4 \mathrm{~s}$ & ${ }^{3} \mathrm{D}_{2}$ & sp & $\left({ }^{3} \mathrm{~F}\right)^{3} \mathrm{P}^{\circ}{ }^{5} \mathrm{D}_{3}^{\circ}$ & & & TW & & \\
\hline
\end{tabular}


Table A1. Cont.

\begin{tabular}{|c|c|c|c|c|c|c|c|c|c|c|c|c|c|c|}
\hline \multirow{3}{*}{$\frac{\lambda_{\text {obs }}{ }^{\text {a }}(\AA)}{1157.8714(18)}$} & \multirow{3}{*}{$\frac{\sigma_{\text {obs }}{ }^{\mathbf{b}}\left(\mathbf{c m}^{-1}\right)}{86365.38(14)}$} & \multirow{3}{*}{$\begin{array}{c}\lambda_{\text {Ritz }}{ }^{\mathrm{c}}(\AA) \\
1157.87172(10) \\
1157.88056(11)\end{array}$} & \multirow{3}{*}{$\begin{array}{c}\Delta \lambda_{\text {obs-Ritz }} \\
(\mathbf{A})\end{array}$} & \multirow{2}{*}{$\begin{array}{c}\begin{array}{c}I_{\text {obs }}{ }^{d} \\
(\text { arb. u. })\end{array} \\
19000\end{array}$} & \multirow{3}{*}{$\begin{array}{l}\text { Char }^{\mathrm{e}} \\
\mathrm{m}\end{array}$} & \multicolumn{2}{|c|}{ Lower Level } & \multicolumn{2}{|c|}{ Upper Level } & \multirow[t]{2}{*}{$A\left(\mathrm{~s}^{-1}\right)$} & \multirow[t]{2}{*}{$\operatorname{Acc}^{f}$} & \multirow{2}{*}{$\begin{array}{l}\text { Line Ref. } \mathbf{g} \\
\text { TW }\end{array}$} & \multirow[t]{2}{*}{ TP Ref. $\mathrm{g}$} & \multirow[t]{2}{*}{ Notes ${ }^{h}$} \\
\hline & & & & & & $4 \mathrm{~s}$ & ${ }^{3} \mathrm{D}_{1}$ & $\mathrm{sp}$ & $\left({ }^{3} \mathrm{~F}\right)^{3} \mathrm{P}^{\circ}{ }^{5} \mathrm{D}^{\circ}{ }_{2}$ & & & & & \\
\hline & & & & & & $4 p$ & ${ }^{3} \mathrm{P}^{\circ}{ }_{1}$ & $9 \mathrm{~s}$ & $(5 / 2)^{2}[5 / 2]_{2}$ & & & TW & & \\
\hline 1162.5991(18) & 86014.17(13) & $1162.60059(14)$ & -0.0015 & 6300 & & $4 \mathrm{~s}$ & ${ }^{3} \mathrm{D}_{3}$ & $\mathrm{sp}$ & $\left({ }^{3} \mathrm{~F}\right)^{3} \mathrm{P}^{\circ}{ }^{5} \mathrm{D}^{\circ}{ }_{4}$ & & & TW & & \\
\hline 1185.899 & 84324.2 & $1185.89903(5)$ & & 19000 & . & $4 p$ & ${ }^{3} \mathrm{P}_{2}^{\circ}$ & $8 \mathrm{~s}$ & $(5 / 2)^{2}[5 / 2]_{3}$ & & & S36c & & \\
\hline $1201.627(6)$ & $83220.5(4)$ & $1201.62566(8)$ & 0.001 & 18000 & & $4 p$ & ${ }^{3} \mathrm{~F}_{3}^{\circ}$ & $7 \mathrm{~d}$ & $(5 / 2)^{2}[9 / 2]_{4}$ & & & S36c & & \\
\hline $1204.643(20)$ & 83012.1(14) & $1204.61568(7)$ & 0.027 & 9100 & * & $4 p$ & ${ }^{3} \mathrm{~F}_{4}^{\circ}$ & $7 \mathrm{~d}$ & $(5 / 2)^{2}[7 / 2]_{4}$ & & & S36c & & \\
\hline $1204.643(20)$ & 83012.1(14) & $1204.63538(5)$ & 0.008 & 9100 & * & $4 p$ & ${ }^{3} \mathrm{~F}_{4}^{\circ}$ & $7 \mathrm{~d}$ & $(5 / 2)^{2}[7 / 2]_{3}$ & & & S36c & & \\
\hline $1204.643(20)$ & 83012.1(14) & $1204.65288(9)$ & -0.010 & 9100 & * & $4 \mathrm{~s}$ & ${ }^{1} \mathrm{D}_{2}$ & $\mathrm{sp}$ & $\left({ }^{3} \mathrm{~F}\right)^{3} \mathrm{P}^{\circ}{ }^{5} \mathrm{D}^{\circ}{ }_{3}$ & & & S36c & & \\
\hline $1205.180(12)$ & $82975.2(8)$ & $1205.14656(6)$ & 0.034 & 4800 & * & $4 p$ & ${ }^{3} \mathrm{~F}_{4}^{\circ}$ & $7 d$ & $(5 / 2)^{2}[5 / 2]_{3}$ & & & S36c & & \\
\hline 1205.180(12) & $82975.2(8)$ & $1205.19424(8)$ & -0.014 & 4800 & * & $4 \mathrm{p}$ & ${ }^{1} \mathrm{~F}_{3}^{\circ}$ & $7 \mathrm{~d}$ & $(3 / 2)^{2}[5 / 2]_{3}$ & & & S36c & & \\
\hline $1205.180(12)$ & 82975.2(8) & $1205.2024(3)$ & -0.022 & 4800 & * & $4 p$ & ${ }^{3} \mathrm{D}^{\circ}{ }_{2}$ & $9 \mathrm{~s}$ & $(3 / 2)^{2}[3 / 2]_{1}$ & & & S36c & & \\
\hline 1205.180(12) & $82975.2(8)$ & $1205.21874(7)$ & -0.039 & 4800 & * & $4 p$ & ${ }^{3} \mathrm{~F}_{2}^{\circ}$ & $8 \mathrm{~s}$ & $(3 / 2)^{2}[3 / 2]_{2}$ & & & S36cn & & \\
\hline $1205.901(6)$ & $82925.5(4)$ & $1205.90271(8)$ & -0.002 & 18000 & & $4 \mathrm{p}$ & ${ }^{3} \mathrm{~F}_{4}^{\circ}$ & $7 \mathrm{~d}$ & $(5 / 2)^{2}[9 / 2]_{5}$ & & & S36c & & \\
\hline 1206.771(6) & $82865.8(4)$ & $1206.76906(5)$ & 0.002 & 4400 & & $4 \mathrm{p}$ & ${ }^{3} \mathrm{P}^{\circ}{ }_{1}$ & $8 s$ & $(5 / 2)^{2}[5 / 2]_{2}$ & & & S36c & & \\
\hline 1214.540 & 82335.7 & 1214.53971(8) & & 9600 & : & $4 p$ & ${ }^{3} \mathrm{D}^{\circ}{ }_{3}$ & $9 \mathrm{~s}$ & $(5 / 2)^{2}[5 / 2]_{3}$ & & & S36c & & \\
\hline 1214.554 & 82334.7 & $1214.55446(5)$ & & 8600 & : & $4 p$ & ${ }^{3} \mathrm{~F}_{3}^{\circ}$ & $8 \mathrm{~s}$ & $(5 / 2)^{2}[5 / 2]_{2}$ & & & S36c & & \\
\hline 1219.334 & 82012.0 & $1219.33363(5)$ & & 8500 & $:$ & $4 \mathrm{p}$ & ${ }^{3} \mathrm{~F}_{4}^{\circ}$ & $8 \mathrm{~s}$ & $(5 / 2)^{2}[5 / 2]_{3}$ & & & S36c & & \\
\hline $1235.93(5)$ & $80911(3)$ & $1235.87291(5)$ & 0.06 & 4000 & & $4 \mathrm{p}$ & ${ }^{3} \mathrm{~F}_{2}^{\circ}$ & $8 \mathrm{~s}$ & $(5 / 2)^{2}[5 / 2]_{2}$ & & & S36c & & \\
\hline $1240.026(6)$ & $80643.5(4)$ & $1240.02707(5)$ & -0.001 & 7400 & & $4 p$ & ${ }^{3} \mathrm{P}^{\circ}{ }_{1}^{2}$ & $6 \mathrm{~d}$ & $(3 / 2)^{2}[3 / 2]_{2}$ & & & S36c & & \\
\hline 1241.964 & 80517.6 & $1241.96398(3)$ & & 14000 & : & $4 \mathrm{p}$ & ${ }^{3} \mathrm{P}_{2}^{\circ}$ & $7 \mathrm{~s}$ & $(3 / 2)^{2}[3 / 2]_{2}$ & & & $\mathrm{~S} 36 \mathrm{c}$ & & \\
\hline $1243.03(5)$ & $80448(3)$ & $1243.08557(6)$ & -0.05 & 7300 & & $4 \mathrm{p}$ & ${ }^{3} \mathrm{P}_{1}^{\circ}$ & $6 \mathrm{~d}$ & $(3 / 2)^{2}[1 / 2]_{1}$ & & & S36c & & \\
\hline $1248.796(3)$ & 80077.12(17) & $1248.79156(5)$ & 0.005 & 26000 & & $4 \mathrm{p}$ & ${ }^{3} \mathrm{P}_{2}^{\circ}$ & $6 \mathrm{~d}$ & $(5 / 2)^{2}[5 / 2]_{3}$ & & & TW & & \\
\hline $1250.058(4)$ & 79996.30(22) & $1250.04822(6)$ & 0.010 & 69000 & & $4 p$ & ${ }^{3} \mathrm{P}_{2}^{\circ}$ & $6 \mathrm{~d}$ & $(5 / 2)^{2}[3 / 2]_{2}$ & & & TW & & \\
\hline $1253.185(3)$ & 79796.67(17) & $1253.18074(7)$ & 0.004 & 25000 & & $4 \mathrm{p}$ & ${ }^{3} \mathrm{P}_{2}{ }_{2}$ & $6 \mathrm{~d}$ & $(5 / 2)^{2}[1 / 2]_{1}$ & & & TW & & \\
\hline 1255.163(6) & 79670.9(4) & $1255.15693(6)$ & 0.006 & 6800 & & $4 p$ & ${ }^{3} \mathrm{P}_{0}^{\circ}$ & $6 \mathrm{~d}$ & $(3 / 2)^{2}[3 / 2]_{1}$ & & & S36c & & \\
\hline $1257.675(6)$ & 79511.8(4) & $1257.68320(7)$ & -0.008 & 6700 & & $4 p$ & ${ }^{3} \mathrm{P}_{0}^{\circ}$ & $6 \mathrm{~d}$ & $(3 / 2)^{2}[1 / 2]_{1}$ & & & S36c & & \\
\hline $1261.214(6)$ & 79288.7(4) & $1261.21537(5)$ & -0.001 & 3500 & & $4 \mathrm{p}$ & ${ }^{1} \mathrm{D}^{\circ}{ }_{2}$ & $8 \mathrm{~s}$ & $(5 / 2)^{2}[5 / 2]_{2}$ & & & S36c & & \\
\hline $1262.929(6)$ & $79181.0(4)$ & $1262.92481(9)$ & 0.004 & 19000 & & $4 \mathrm{p}$ & ${ }^{3} \mathrm{P}^{\circ}{ }_{1}^{2}$ & $6 d$ & $(5 / 2)^{2}[1 / 2]_{0}$ & & & S36c & & \\
\hline $1265.510(3)$ & 79019.53(17) & $1265.50624(3)$ & 0.004 & 72000 & & $4 p$ & ${ }^{3} \mathrm{P}^{\circ}{ }_{1}$ & $7 \mathrm{~s}$ & $(3 / 2)^{2}[3 / 2]_{2}$ & & & TW & & \\
\hline $1266.313(3)$ & 78969.42(17) & $1266.30992(4)$ & 0.003 & 27000 & & $4 p$ & ${ }^{3} \mathrm{P}^{\circ}{ }_{1}^{1}$ & $7 \mathrm{~s}$ & $(3 / 2)^{2}[3 / 2]_{1}$ & & & TW & & \\
\hline 1268.71(5) & $78820(3)$ & $1268.66846(5)$ & 0.04 & 3300 & & $4 p$ & ${ }^{3} \mathrm{D}_{3}^{\circ}$ & $8 \mathrm{~s}$ & $(5 / 2)^{2}[5 / 2]_{3}$ & & & S36c & & \\
\hline $1269.446(6)$ & $78774.5(4)$ & $1269.44626(6)$ & 0.000 & 6200 & & $4 p$ & ${ }^{3} \mathrm{~F}_{2}^{\circ}$ & $6 \mathrm{~d}$ & $(3 / 2)^{2}[5 / 2]_{2}$ & & & S36c & & \\
\hline $1271.327(6)$ & 78658.0(4) & $1271.31771(4)$ & 0.010 & 12000 & & $4 p$ & ${ }^{3} \mathrm{P}^{\circ}{ }_{1}^{2}$ & $6 \mathrm{~d}$ & $(5 / 2)^{2}[5 / 2]_{2}$ & & & S36c & & \\
\hline $1272.043(3)$ & 78613.69(17) & $1272.04165(6)$ & 0.001 & 31000 & & $4 p$ & ${ }^{3} \mathrm{~F}_{2}^{\circ}$ & $6 \mathrm{~d}$ & $(3 / 2)^{2}[7 / 2]_{3}$ & & & TW & & \\
\hline $1273.705(6)$ & 78511.1(4) & $1273.70053(5)$ & 0.005 & 11000 & & $4 \mathrm{p}$ & ${ }^{3} \mathrm{P}^{\circ}{ }_{1}^{2}$ & $6 \mathrm{~d}$ & $(5 / 2)^{2}[3 / 2]_{1}$ & & & S36c & & \\
\hline 1274.071 & 78488.6 & $1274.07065(3)$ & & 17000 & : & $4 p$ & ${ }^{3} \mathrm{~F}_{3}^{\circ}$ & $7 \mathrm{~s}$ & $(3 / 2)^{2}[3 / 2]_{2}$ & & & S36c & & \\
\hline 1274.465 & 78464.3 & 1274.46493(3) & & 16000 & : & $4 p$ & ${ }^{3} \mathrm{P}_{2}^{\circ}$ & $7 \mathrm{~s}$ & $(5 / 2)^{2}[5 / 2]_{2}$ & & & S36c & & \\
\hline $1275.5713(15)$ & $78396.24(9)$ & $1275.57159(3)$ & -0.0003 & 75000 & & $4 p$ & ${ }^{3} \mathrm{P}_{2}^{\circ}$ & $7 \mathrm{~s}$ & $(5 / 2)^{2}[5 / 2]_{3}$ & & & TW & & \\
\hline $1279.948(20)$ & $78128.2(12)$ & $1279.96123(5)$ & -0.013 & 2700 & & $4 \mathrm{p}$ & ${ }^{3} \mathrm{~F}_{3}^{\circ}$ & $6 \mathrm{~d}$ & $(5 / 2)^{2}[5 / 2]_{2}$ & & & S36c & & \\
\hline
\end{tabular}


Table A1. Cont.

\begin{tabular}{|c|c|c|c|c|c|c|c|c|c|c|c|c|c|c|}
\hline \multirow{2}{*}{$\frac{\lambda_{\text {obs }}{ }^{\text {a }}(\AA ̊)}{1280.271(3)}$} & \multirow{2}{*}{$\begin{array}{l}\sigma_{\text {obs }}{ }^{\mathbf{b}}\left(\mathbf{c m}^{-1}\right) \\
78108.48(17)\end{array}$} & \multirow{2}{*}{$\frac{\lambda_{\text {Ritz }}{ }^{\mathrm{c}}(\AA ̊)}{1280.26800(4)}$} & \multirow{2}{*}{$\begin{array}{c}\begin{array}{c}\Delta \lambda_{\text {obs-Ritz }} \\
(\AA)\end{array} \\
0.003\end{array}$} & \multirow{2}{*}{$\begin{array}{c}\begin{array}{c}I_{\text {obs }} \mathrm{d} \\
(\text { arb. u. })\end{array} \\
30000\end{array}$} & \multirow[t]{2}{*}{ Char ${ }^{\mathrm{e}}$} & \multicolumn{2}{|c|}{ Lower Level } & \multicolumn{2}{|c|}{ Upper Level } & \multirow[t]{2}{*}{$A\left(\mathrm{~s}^{-1}\right)$} & \multirow[t]{2}{*}{$\operatorname{Acc}^{f}$} & \multirow{2}{*}{$\begin{array}{l}\text { Line Ref. } \mathrm{g} \\
\text { TW,S36r }\end{array}$} & \multirow[t]{2}{*}{ TP Ref. $\mathrm{g}$} & \multirow[t]{2}{*}{ Notes $^{\mathrm{h}}$} \\
\hline & & & & & & $4 p$ & ${ }^{3} \mathrm{~F}_{3}^{\circ}$ & $6 \mathrm{~d}$ & $(5 / 2)^{2}[7 / 2]_{3}$ & & & & & \\
\hline 1281.094(3) & $78058.29(17)$ & $1281.09394(9)$ & -0.000 & 10000 & & $s^{2}$ & ${ }^{3} \mathrm{~F}_{4}$ & $7 \mathrm{p}$ & $(5 / 2)^{2}[5 / 2]^{\circ}{ }_{3}$ & & & TW & & \\
\hline $1281.228(20)$ & $78050.1(12)$ & $1281.25682(6)$ & -0.029 & 16000 & & $4 p$ & ${ }^{3} \mathrm{~F}_{3}^{\circ}$ & $6 \mathrm{~d}$ & $(5 / 2)^{2}[5 / 2]_{3}$ & & & S36c & & \\
\hline $1281.462(3)$ & 78035.88(17) & $1281.46142(4)$ & 0.000 & 44000 & & $4 p$ & ${ }^{3} \mathrm{P}_{0}^{\circ}$ & $7 \mathrm{~s}$ & $(3 / 2)^{2}[3 / 2]_{1}$ & & & TW & & \\
\hline 1282.455(3) & 77975.44(17) & $1282.45466(5)$ & 0.000 & 72000 & & $4 p$ & ${ }^{3} \mathrm{~F}_{3}^{\circ}$ & $6 \mathrm{~d}$ & $(5 / 2)^{2}[9 / 2]_{4}$ & & & TW & & \\
\hline $1283.824(6)$ & $77892.3(4)$ & $1283.82966(10)$ & -0.005 & 5300 & & $\mathrm{~s}^{2}$ & ${ }^{3} \mathrm{~F}_{4}$ & $7 \mathrm{p}$ & $(5 / 2)^{2}[7 / 2]_{4}^{\circ}$ & & & S36c & & \\
\hline $1284.872(3)$ & $77828.74(16)$ & $1284.87116(5)$ & 0.001 & 36000 & & $4 p$ & ${ }^{3} \mathrm{~F}_{4}^{\circ}$ & $6 \mathrm{~d}$ & $(5 / 2)^{2}[7 / 2]_{4}$ & & & TW & & \\
\hline $1285.521(6)$ & 77789.5(4) & $1285.51841(5)$ & 0.003 & 5400 & & $4 p$ & ${ }^{1} \mathrm{~F}_{3}^{\circ}$ & $6 \mathrm{~d}$ & $(3 / 2)^{2}[5 / 2]_{3}$ & & & S36c & & \\
\hline \multirow[t]{2}{*}{ 1285.901(20) } & $77766.5(12)$ & $1285.92203(6)$ & -0.021 & 5100 & & $4 \mathrm{p}$ & ${ }^{3} \mathrm{~F}_{4}^{\circ}$ & $6 \mathrm{~d}$ & $(5 / 2)^{2}[5 / 2]_{3}$ & & & S36c & & \\
\hline & & $1287.43101(6)$ & & & $\mathrm{m}$ & $4 \mathrm{p}$ & ${ }^{1} \mathrm{~F}_{3}^{\circ}$ & $6 \mathrm{~d}$ & $(3 / 2)^{2}[7 / 2]_{4}$ & & & TW & & \\
\hline $1287.4690(8)$ & 77671.77(5) & $1287.46810(6)$ & 0.0009 & 53000 & & $4 \mathrm{p}$ & ${ }^{3} \mathrm{~F}_{4}^{\circ}$ & $6 \mathrm{~d}$ & $(5 / 2)^{2}[9 / 2]_{5}$ & & & K66 & & \\
\hline $1297.550(4)$ & 77068.31(21) & $1297.54980(3)$ & 0.000 & 4900 & & $4 p$ & ${ }^{3} \mathrm{~F}_{2}^{\circ}$ & $7 \mathrm{~s}$ & $(3 / 2)^{2}[3 / 2]_{2}$ & & & TW & & \\
\hline 1297.979(20) & $77042.8(12)$ & $1297.9922(9)$ & -0.013 & 4900 & & $s^{\frac{1}{2}}$ & ${ }^{3} \mathrm{~F}_{2}$ & $7 p$ & $(3 / 2)^{2}[3 / 2]^{\circ}{ }_{1}$ & & & S36cn & & \\
\hline 1298.3952(8) & 77018.15(5) & $1298.39471(4)$ & 0.0005 & 60000 & & $4 p$ & ${ }^{3} \mathrm{~F}_{2}^{\circ}$ & $7 \mathrm{~s}$ & $(3 / 2)^{2}[3 / 2]_{1}$ & & & K66 & & \\
\hline 1298.917(12) & $76987.2(7)$ & $1298.90527(6)$ & 0.012 & 4700 & & $4 p$ & ${ }^{1} \mathrm{D}_{2}^{\circ}$ & $6 \mathrm{~d}$ & $(3 / 2)^{2}[7 / 2]_{3}$ & & & S36c & & \\
\hline 1299.2684(8) & 76966.39(5) & $1299.26777(3)$ & 0.0006 & 44000 & & $4 p$ & ${ }^{3} \mathrm{P}^{\circ}{ }_{1}^{2}$ & $7 \mathrm{~s}$ & $(5 / 2)^{2}[5 / 2]_{2}$ & & & K66 & & \\
\hline $1303.661(4)$ & 76707.04(21) & $1303.66001(5)$ & 0.001 & 2900 & & $4 p$ & ${ }^{3} \mathrm{~F}_{2}^{\circ}$ & $6 \mathrm{~d}$ & $(5 / 2)^{2}[5 / 2]_{2}$ & & & TW & & \\
\hline $1303.978(3)$ & $76688.38(16)$ & $1303.97824(4)$ & 0.000 & 6400 & & $4 p$ & ${ }^{3} \mathrm{~F}_{2}^{\circ}$ & $6 \mathrm{~d}$ & $(5 / 2)^{2}[7 / 2]_{3}$ & & & TW & & \\
\hline 1305.562(3) & $76595.40(16)$ & $1305.56082(6)$ & 0.001 & 19000 & & $4 p$ & ${ }^{3} \mathrm{D}_{3}^{\circ}$ & $6 \mathrm{~d}$ & $(3 / 2)^{2}[7 / 2]_{4}$ & & & TW & & \\
\hline $1308.2982(6)$ & 76435.17(4) & $1308.29687(3)$ & 0.0013 & 99000 & & $4 p$ & ${ }^{3} \mathrm{~F}_{3}^{\circ}$ & $7 \mathrm{~s}$ & $(5 / 2)^{2}[5 / 2]_{2}$ & & & K66 & & \\
\hline 1309.464(3) & 76367.12(16) & $1309.46310(3)$ & 0.001 & 52000 & & $4 \mathrm{p}$ & ${ }^{3} \mathrm{~F}_{3}^{\circ}$ & $7 \mathrm{~s}$ & $(5 / 2)^{2}[5 / 2]_{3}$ & & & TW & & \\
\hline $1311.76(4)$ & $76233.5(23)$ & $1311.79458(10)$ & -0.04 & 3900 & & $s^{2}$ & ${ }^{3} \mathrm{~F}_{3}$ & $7 p$ & $(5 / 2)^{2}[5 / 2]_{3}^{\circ}$ & & & S36c & & \\
\hline $1314.1498(8)$ & 76094.83(5) & $1314.14936(4)$ & 0.0004 & 50000 & & $4 p$ & ${ }^{1} \mathrm{~F}_{3}^{\circ}$ & $7 \mathrm{~s}$ & $(3 / 2)^{2}[3 / 2]_{2}$ & & & K66 & & \\
\hline $1314.3371(6)$ & 76083.98(3) & $1314.33637(3)$ & 0.0007 & 150000 & & $4 \mathrm{p}$ & ${ }^{3} \mathrm{~F}_{4}^{\circ}$ & $7 \mathrm{~s}$ & $(5 / 2)^{2}[5 / 2]_{3}$ & & & K66 & & \\
\hline $1320.687(3)$ & 75718.15(16) & $1320.68581(5)$ & 0.002 & 30000 & & $4 \mathrm{p}$ & ${ }^{1} \mathrm{~F}_{3}^{\circ}$ & $6 \mathrm{~d}$ & $(5 / 2)^{2}[7 / 2]_{4}$ & & & TW & & \\
\hline $1321.798(3)$ & 75654.53(16) & 1321.79611(6) & 0.002 & 11000 & & $4 \mathrm{p}$ & ${ }^{1} \mathrm{~F}_{3}^{\circ}$ & $6 \mathrm{~d}$ & $(5 / 2)^{2}[5 / 2]_{3}$ & & & TW & & \\
\hline $1322.628(6)$ & $75607.0(3)$ & $1322.63248(13)$ & -0.004 & 21000 & & $4 \mathrm{p}$ & ${ }^{1} \mathrm{P}^{\circ}{ }_{1}$ & $6 \mathrm{~d}$ & $(3 / 2)^{2}[1 / 2]_{0}$ & & & S36c & & \\
\hline 1323.188(20) & 75575.0(11) & $1323.20408(6)$ & -0.016 & 9700 & & $4 \mathrm{p}$ & ${ }^{1} \mathrm{~F}_{3}^{\circ}{ }_{3}$ & $6 \mathrm{~d}$ & $(5 / 2)^{2}[3 / 2]_{2}$ & & & S36c & & \\
\hline 1323.812(20) & 75539.4(11) & $1323.79410(6)$ & 0.018 & 21000 & & $4 p$ & ${ }^{3} \mathrm{D}^{\circ}{ }_{1}$ & $6 \mathrm{~d}$ & $(3 / 2)^{2}[5 / 2]_{2}$ & & & S36c & & \\
\hline $1325.272(20)$ & 75456.2(11) & $1325.24199(5)$ & 0.030 & 3300 & & $4 p$ & ${ }^{3} \mathrm{D}^{\circ}{ }_{1}$ & $6 \mathrm{~d}$ & $(3 / 2)^{2}[3 / 2]_{2}$ & & & S36c & & \\
\hline $1325.515(3)$ & 75442.39(15) & $1325.51345(3)$ & 0.001 & 8300 & & $4 p$ & ${ }^{1} \mathrm{D}^{\circ}{ }_{2}$ & $7 \mathrm{~s}$ & $(3 / 2)^{2}[3 / 2]_{2}$ & & & TW & & \\
\hline $1326.396(3)$ & $75392.26(15)$ & $1326.39519(4)$ & 0.001 & 28000 & & $4 p$ & ${ }^{1} \mathrm{D}_{2}^{\circ}$ & $7 \mathrm{~s}$ & $(3 / 2)^{2}[3 / 2]_{1}$ & & & TW & & \\
\hline 1328.415(3) & 75277.66(15) & $1328.41289(5)$ & 0.002 & 12000 & & $4 p$ & ${ }^{3} \mathrm{D}^{\circ}{ }_{2}$ & $6 \mathrm{~d}$ & $(3 / 2)^{2}[5 / 2]_{3}$ & & & TW & & \\
\hline $1329.656(20)$ & 75207.4(11) & $1329.66943(6)$ & -0.014 & 3100 & & $4 p$ & ${ }^{3} \mathrm{D}^{\circ}{ }_{2}$ & $6 \mathrm{~d}$ & $(3 / 2)^{2}[3 / 2]_{2}$ & & & S36c & & \\
\hline 1331.892(3) & 75081.17(15) & $1331.89052(5)$ & 0.001 & 14000 & & $4 p$ & ${ }^{1} \mathrm{D}^{\circ}{ }_{2}$ & $6 \mathrm{~d}$ & $(5 / 2)^{2}[5 / 2]_{2}$ & & & TW & & \\
\hline $1332.224(3)$ & $75062.46(15)$ & $1332.22268(4)$ & 0.001 & 17000 & & $4 p$ & ${ }^{1} \mathrm{D}_{2}^{\circ}$ & $6 \mathrm{~d}$ & $(5 / 2)^{2}[7 / 2]_{3}$ & & & TW & & \\
\hline \multirow[t]{2}{*}{$1333.0457(8)$} & $75016.18(5)$ & 1333.04501(3) & 0.0007 & 58000 & & $4 p$ & ${ }^{3} \mathrm{D}^{\circ}{ }_{3}$ & $7 \mathrm{~s}$ & $(3 / 2)^{2}[3 / 2]_{2}$ & & & K66 & & \\
\hline & & $1333.06670(3)$ & & & $\mathrm{m}$ & $4 p$ & ${ }^{3} \mathrm{~F}_{2}^{\circ}$ & $7 \mathrm{~s}$ & $(5 / 2)^{2}[5 / 2]_{2}$ & & & TW & & \\
\hline $1334.56(4)$ & $74931.2(22)$ & $1334.50604(5)$ & 0.05 & 5500 & $\mathrm{p}$ & $4 p$ & ${ }^{1} \mathrm{D}_{2}^{\circ}$ & $6 \mathrm{~d}$ & $(5 / 2)^{2}[3 / 2]_{1}$ & & & S36c & & $x$ \\
\hline $1334.650(4)$ & 74926.02(20) & $1334.65445(7)$ & -0.005 & 5800 & & $\begin{array}{l}1 \\
4 \mathrm{p}\end{array}$ & ${ }^{1} \mathrm{P}^{\circ}{ }_{1}$ & $6 \mathrm{~d}$ & $(3 / 2)^{2}[3 / 2]_{1}$ & & & TW & & \\
\hline
\end{tabular}


Table A1. Cont.

\begin{tabular}{|c|c|c|c|c|c|c|c|c|c|c|c|c|c|c|}
\hline$\lambda_{\text {obs }}{ }^{a}(\AA)$ & $\sigma_{\mathrm{obs}}{ }^{\mathrm{b}}\left(\mathrm{cm}^{-1}\right)$ & $\lambda_{\text {Ritz }}{ }^{c}(\AA)$ & $\Delta \lambda_{\text {obs-Ritz }}$ & $\begin{array}{c}I_{\text {obs }} \mathrm{d} \\
\text { (arb. u.) }\end{array}$ & Char ${ }^{\mathrm{e}}$ & Lor & Level & & Jpper Level & $A\left(\mathrm{~s}^{-1}\right)$ & $\operatorname{Acc}^{f}$ & Line Ref. $\mathrm{g}$ & TP Ref. $\mathrm{g}$ & Notes ${ }^{h}$ \\
\hline $1334.686(6)$ & $74924.0(3)$ & 1334.68792(17) & -0.002 & 1500 & & $s^{2}$ & ${ }^{3} \mathrm{~F}_{2}$ & $7 \mathrm{p}$ & $(5 / 2)^{2}[5 / 2]^{\circ}{ }_{2}$ & & & TW & & \\
\hline $1337.55(4)$ & $74763.8(22)$ & 1337.51122(8) & 0.03 & 1400 & & $4 p$ & ${ }^{1} \mathrm{P}_{1}^{\circ}$ & $6 \mathrm{~d}$ & $(3 / 2)^{2}[1 / 2]_{1}$ & & & S36c & & \\
\hline 1339.46(4) & $74656.7(22)$ & $1339.49492(5)$ & -0.03 & 1300 & & $4 p$ & ${ }^{3} \mathrm{D}_{3}^{\circ}$ & $6 \mathrm{~d}$ & $(5 / 2)^{2}[5 / 2]_{2}$ & & & S36c & & \\
\hline $1339.7734(23)$ & $74639.49(13)$ & $1339.77126(5)$ & 0.0022 & 14000 & & $4 p$ & ${ }^{3} \mathrm{D}_{3}^{\circ}$ & $6 \mathrm{~d}$ & $(5 / 2)^{2}[7 / 2]_{4}$ & & & TW & & \\
\hline 1340.9161(23) & 74575.88(13) & $1340.91390(6)$ & 0.0022 & 6700 & & $4 p$ & ${ }^{3} \mathrm{D}_{3}^{\circ}$ & $6 \mathrm{~d}$ & $(5 / 2)^{2}[5 / 2]_{3}$ & & & TW & & \\
\hline $1350.5963(20)$ & 74041.37(11) & $1350.59358(4)$ & 0.0027 & 29000 & & $4 \mathrm{p}$ & ${ }^{1} \mathrm{~F}_{3}^{\circ}$ & $7 \mathrm{~s}$ & $(5 / 2)^{2}[5 / 2]_{2}$ & & & TW & & \\
\hline $1351.8384(8)$ & $73973.34(4)$ & $1351.83646(3)$ & 0.0019 & 53000 & & $4 p$ & ${ }^{1} \mathrm{~F}_{3}^{\circ}$ & $7 \mathrm{~s}$ & $(5 / 2)^{2}[5 / 2]_{3}$ & & & K66 & & \\
\hline $1355.3066(8)$ & 73784.04(4) & $1355.30507(4)$ & 0.0015 & 36000 & & $4 p$ & ${ }^{3} \mathrm{D}^{\circ}{ }_{1}$ & $7 \mathrm{~s}$ & $(3 / 2)^{2}[3 / 2]_{1}$ & & & K66 & & \\
\hline $1358.7736(6)$ & 73595.78(3) & $1358.7729(3)$ & 0.0007 & 15000000 & & $\mathrm{~d}^{10}$ & ${ }^{1} \mathrm{~S}_{0}$ & $4 \mathrm{p}$ & ${ }^{1} \mathrm{P}_{1}^{\circ}$ & $3.3 e+08$ & $\mathrm{C}+$ & K66 & B09 & \\
\hline $1359.0107(8)$ & 73582.94(4) & $1359.00914(3)$ & 0.0016 & 38000 & & $4 p$ & ${ }^{3} \mathrm{D}_{2}^{\circ}$ & $7 \mathrm{~s}$ & $(3 / 2)^{2}[3 / 2]_{2}$ & & & K66 & & \\
\hline $1359.9394(20)$ & $73532.69(11)$ & $1359.93602(4)$ & 0.0034 & 11000 & & $4 p$ & ${ }^{3} \mathrm{D}_{2}^{\circ}$ & $7 \mathrm{~s}$ & $(3 / 2)^{2}[3 / 2]_{1}$ & & & TW & & \\
\hline $1362.6004(6)$ & 73389.09(3) & $1362.59959(3)$ & 0.0008 & 49000 & & $4 p$ & ${ }^{1} \mathrm{D}^{\circ}{ }_{2}$ & $7 \mathrm{~s}$ & $(5 / 2)^{2}[5 / 2]_{2}$ & & & K66 & & \\
\hline $1363.506(2)$ & $73340.36(13)$ & $1363.50304(4)$ & 0.003 & 12000 & & $4 p$ & ${ }^{1} \mathrm{P}^{\circ}{ }_{1}$ & $7 \mathrm{~s}$ & $(3 / 2)^{2}[3 / 2]_{2}$ & & & TW & & \\
\hline $1367.9508(6)$ & $73102.04(3)$ & $1367.9509(3)$ & -0.0001 & 5800000 & & $\mathrm{~d}^{10}$ & ${ }^{1} \mathrm{~S}_{0}$ & $4 p$ & ${ }^{3} \mathrm{D}^{\circ}{ }_{1}$ & $8.3 e+07$ & $\mathrm{C}+$ & K66 & D05 & \\
\hline $1370.257(6)$ & $72979.0(3)$ & $1370.25181(6)$ & 0.005 & 2700 & & $4 p$ & ${ }^{1} \mathrm{P}^{\circ}{ }_{1}$ & $6 \mathrm{~d}$ & $(5 / 2)^{2}[5 / 2]_{2}$ & & & S36c & & \\
\hline $1370.561(3)$ & $72962.83(18)$ & $1370.55975(3)$ & 0.001 & 1800 & & $4 p$ & ${ }^{3} \mathrm{D}^{\circ}{ }_{3}$ & $7 \mathrm{~s}$ & $(5 / 2)^{2}[5 / 2]_{2}$ & & & TW & & \\
\hline $1371.8400(6)$ & $72894.80(3)$ & $1371.83967(3)$ & 0.0003 & 43000 & & $4 p$ & ${ }^{3} \mathrm{D}_{3}^{\circ}$ & $7 \mathrm{~s}$ & $(5 / 2)^{2}[5 / 2]_{3}$ & & & K66 & & \\
\hline $1375.522(20)$ & 72699.7(11) & $1375.50173(4)$ & 0.020 & 6900 & & $4 p$ & ${ }^{3} \mathrm{P}_{2}^{\circ}$ & $5 \mathrm{~d}$ & $(3 / 2)^{2}[5 / 2]_{3}$ & & & S36c & & \\
\hline 1393.129(2) & $71780.88(13)$ & 1393.12738(3) & 0.001 & 18000 & & $4 p$ & ${ }^{3} \mathrm{D}^{\circ}{ }_{1}$ & $7 \mathrm{~s}$ & $(5 / 2)^{2}[5 / 2]_{2}$ & & & TW & & \\
\hline $1398.6428(16)$ & 71497.88(8) & 1398.64196(11) & 0.0009 & 40000 & & $s^{\frac{1}{2}}$ & ${ }^{3} \mathrm{~F}_{4}$ & $6 \mathrm{p}$ & $(3 / 2)^{2}[5 / 2]^{\circ}{ }_{3}$ & & & TW & & \\
\hline 1399.3532(17) & 71461.59(9) & $1399.35262(3)$ & 0.0005 & 15000 & & $4 p$ & ${ }^{3} \mathrm{D}_{2}^{\circ}$ & $7 \mathrm{~s}$ & $(5 / 2)^{2}[5 / 2]_{3}$ & & & TW & & \\
\hline 1402.7785(8) & 71287.09(4) & $1402.77693(4)$ & 0.0016 & 98000 & & $4 p$ & ${ }^{1} \mathrm{P}^{\circ}{ }_{1}$ & $7 \mathrm{~s}$ & $(5 / 2)^{2}[5 / 2]_{2}$ & & & K66 & & \\
\hline 1403.985(3) & $71225.85(16)$ & 1403.99171(5) & -0.007 & 4900 & & $4 p$ & ${ }^{3} \mathrm{P}^{\circ}{ }_{1}$ & $5 \mathrm{~d}$ & $(3 / 2)^{2}[5 / 2]_{2}$ & & & TW & & \\
\hline $1407.1688(16)$ & $71064.68(8)$ & $1407.16862(5)$ & 0.0001 & 60000 & & $4 \mathrm{p}$ & ${ }^{3} \mathrm{P}_{1}^{\circ}$ & $5 d$ & $(3 / 2)^{2}[3 / 2]_{2}$ & & & TW & & \\
\hline $1408.8131(23)$ & 70981.74(12) & $1408.81230(5)$ & 0.0008 & 4900 & & $4 p$ & ${ }^{3} \mathrm{P}^{\circ}{ }_{1}$ & $5 \mathrm{~d}$ & $(3 / 2)^{2}[3 / 2]_{1}$ & & & TW & & \\
\hline $1414.4390(19)$ & $70699.41(10)$ & $1414.4371(4)$ & 0.0019 & 26000 & & $\mathrm{~s}^{2}$ & ${ }^{3} \mathrm{~F}_{2}$ & $\mathrm{sp}$ & $\left({ }^{1} \mathrm{G}\right)^{3} \mathrm{P}^{\circ}{ }^{3} \mathrm{G}^{\circ}{ }_{3}$ & & & TWn & & \\
\hline $1414.8980(15)$ & $70676.47(8)$ & $1414.89768(6)$ & 0.0003 & 49000 & & $4 p$ & ${ }^{3} \mathrm{P}_{1}^{\circ}{ }_{1}$ & $5 \mathrm{~d}$ & $(3 / 2)^{2}[1 / 2]_{1}$ & & & TW & & \\
\hline $1418.4250(14)$ & 70500.73(7) & $1418.42631(3)$ & -0.0014 & 140000 & & $4 p$ & ${ }^{3} \mathrm{P}_{2}{ }_{2}$ & $5 \mathrm{~d}$ & $(5 / 2)^{2}[5 / 2]_{3}$ & & & TW & & \\
\hline $1419.7465(20)$ & $70435.11(10)$ & $1419.74554(4)$ & 0.0010 & 7500 & & $4 \mathrm{p}$ & ${ }^{3} \mathrm{~F}_{3}^{\circ}$ & $5 d$ & $(3 / 2)^{2}[7 / 2]_{4}$ & & & TW & & \\
\hline $1421.3746(13)$ & 70354.43(7) & $1421.37346(5)$ & 0.0011 & 35000 & & $4 p$ & ${ }^{3} \mathrm{P}^{\circ}{ }_{2}$ & $5 \mathrm{~d}$ & $(5 / 2)^{2}[3 / 2]_{1}$ & & & TW & & \\
\hline 1421.7587(6) & 70335.42(3) & $1421.75866(4)$ & 0.0000 & 200000 & & $4 p$ & ${ }^{3} \mathrm{P}_{2}^{\circ}$ & $5 \mathrm{~d}$ & $(5 / 2)^{2}[3 / 2]_{2}$ & & & K66 & & \\
\hline $1427.5920(12)$ & $70048.03(6)$ & $1427.59106(6)$ & 0.0009 & 58000 & & $4 p$ & ${ }^{3} \mathrm{P}^{\circ}{ }_{0}$ & $5 \mathrm{~d}$ & $(3 / 2)^{2}[3 / 2]_{1}$ & & & TW & & \\
\hline $1427.8283(8)$ & $70036.43(4)$ & $1427.82905(7)$ & -0.0007 & 180000 & & $s^{2}$ & ${ }^{3} \mathrm{~F}_{4}$ & $6 \mathrm{p}$ & $(5 / 2)^{2}[5 / 2]^{\circ}{ }_{3}$ & & & K66 & & \\
\hline $1428.3572(8)$ & 70010.50(4) & 1428.35801(11) & -0.0008 & 190000 & & $s^{2}$ & ${ }^{3} \mathrm{~F}_{3}$ & $6 \mathrm{p}$ & $(3 / 2)^{2}[3 / 2]^{\circ}{ }_{2}$ & & & K66 & & \\
\hline $1430.2425(8)$ & 69918.21(4) & $1430.24252(5)$ & -0.0000 & 240000 & & $4 p$ & ${ }^{3} \mathrm{P}_{2}^{\circ}$ & $5 d$ & $(5 / 2)^{2}[1 / 2]_{1}$ & & & K66 & & \\
\hline $1433.8415(12)$ & $69742.72(6)$ & $1433.84011(7)$ & 0.0014 & 53000 & & $4 \mathrm{p}$ & ${ }^{3} \mathrm{P}_{0}^{\circ}$ & $5 \mathrm{~d}$ & $(3 / 2)^{2}[1 / 2]_{1}$ & & & TW & & \\
\hline $1434.452(3)$ & 69713.01(13) & $1434.45240(14)$ & -0.000 & 3600 & & $s^{2}$ & ${ }^{3} \mathrm{~F}_{3}$ & $6 p$ & $(3 / 2)^{2}[5 / 2]^{0}{ }_{2}$ & & & TWn & & \\
\hline $1434.7712(12)$ & 69697.52(6) & $1434.76999(8)$ & 0.0013 & 76000 & & $4 p$ & ${ }^{3} \mathrm{P}^{\circ}{ }_{1}$ & $5 \mathrm{~d}$ & $(5 / 2)^{2}[1 / 2]_{0}$ & & & TW & & \\
\hline $1434.9035(6)$ & 69691.10(3) & $1434.90377(9)$ & -0.0003 & 270000 & & $s^{2}$ & ${ }^{3} \mathrm{~F}_{4}$ & $6 \mathrm{p}$ & $(5 / 2)^{2}[7 / 2]^{\circ}{ }_{4}$ & & & K66 & & \\
\hline
\end{tabular}


Table A1. Cont.

\begin{tabular}{|c|c|c|c|c|c|c|c|c|c|c|c|c|c|c|}
\hline$\lambda_{\text {obs }}{ }^{a}(\AA)$ & $\sigma_{\mathrm{obs}}{ }^{\mathrm{b}}\left(\mathrm{cm}^{-1}\right)$ & $\lambda_{\text {Ritz }}{ }^{c}(\AA)$ & $\begin{array}{c}\Delta \lambda_{\text {obs-Ritz }} \\
\text { (A) }\end{array}$ & $\begin{array}{l}I_{\text {obs }} \mathrm{d} \\
\text { (arb. u.) }\end{array}$ & Char ${ }^{\mathrm{e}}$ & Low & Level & & Jpper Level & $A\left(\mathrm{~s}^{-1}\right)$ & $\operatorname{Acc}^{f}$ & Line Ref. $\mathrm{g}$ & TP Ref. $\mathrm{g}$ & Notes ${ }^{h}$ \\
\hline 1435.3153(13) & 69671.10(6) & $1435.31565(13)$ & -0.0004 & 110000 & & $\mathrm{~s}^{2}$ & ${ }^{3} \mathrm{~F}_{3}$ & $6 p$ & $(3 / 2)^{2}[5 / 2]^{\circ}{ }_{3}$ & & & TW & & \\
\hline 1436.2352(12) & $69626.48(6)$ & $1436.23585(7)$ & -0.0007 & 130000 & & $s^{2}$ & ${ }^{3} \mathrm{~F}_{4}$ & $\mathrm{sp}$ & $\left({ }^{1} \mathrm{G}\right)^{3} \mathrm{P}^{\circ}{ }^{3} \mathrm{~F}_{3}^{\circ}{ }_{3}$ & & & TW & & \\
\hline $1442.1398(12)$ & $69341.40(6)$ & $1442.13845(4)$ & 0.0014 & 66000 & & $4 p$ & ${ }^{3} \mathrm{P}_{2}^{\circ}$ & $6 s$ & $(3 / 2)^{2}[3 / 2]_{2}$ & & & TW & & \\
\hline $1443.5423(12)$ & 69274.04(6) & $1443.54170(6)$ & 0.0006 & 73000 & & $4 p$ & ${ }^{3} \mathrm{~F}_{2}^{\circ}$ & $5 d$ & $(3 / 2)^{2}[5 / 2]_{2}$ & & & TW & & \\
\hline $1444.1295(22)$ & 69245.87(10) & $1444.13017(4)$ & -0.0007 & 7700 & & $4 \mathrm{p}$ & ${ }^{3} \mathrm{P}_{2}^{\circ}$ & $6 s$ & $(3 / 2)^{2}[3 / 2]_{1}$ & & & TW & & \\
\hline $1445.9841(12)$ & 69157.05(6) & $1445.98335(4)$ & 0.0007 & 97000 & & $4 \mathrm{p}$ & ${ }^{3} \mathrm{P}^{\circ}{ }_{1}^{2}$ & $5 \mathrm{~d}$ & $(5 / 2)^{2}[5 / 2]_{2}$ & & & TW & & \\
\hline $1446.9008(22)$ & 69113.24(10) & $1446.90033(6)$ & 0.0005 & 4600 & & $4 \mathrm{p}$ & ${ }^{3} \mathrm{~F}_{2}^{\circ}$ & $5 \mathrm{~d}$ & $(3 / 2)^{2}[3 / 2]_{2}$ & & & TW & & \\
\hline $1448.6390(19)$ & 69030.31(9) & $1448.63819(6)$ & 0.0008 & 6100 & & $4 \mathrm{p}$ & ${ }^{3} \mathrm{~F}_{2}^{\circ}$ & $5 d$ & $(3 / 2)^{2}[3 / 2]_{1}$ & & & TW & & \\
\hline $1449.0578(8)$ & $69010.36(4)$ & $1449.05784(12)$ & -0.0000 & 180000 & & $\mathrm{~s}^{23} \mathrm{~F}$ & 2 & $6 p$ & $(3 / 2)^{2}[3 / 2]^{0}{ }_{1}$ & & & K66 & & \\
\hline $1450.3032(12)$ & $68951.10(6)$ & $1450.30363(4)$ & -0.0004 & 170000 & & $4 p$ & ${ }^{3} \mathrm{~F}_{2}^{\circ}$ & $5 \mathrm{~d}$ & $(3 / 2)^{2}[7 / 2]_{3}$ & & & TW & & \\
\hline $1452.2950(12)$ & 68856.53(6) & 1452.29331(5) & 0.0017 & 97000 & & $4 \mathrm{p}$ & ${ }^{3} \mathrm{P}_{1}^{\circ}$ & $5 \mathrm{~d}$ & $(5 / 2)^{2}[3 / 2]_{1}$ & & & TW & & \\
\hline $1452.670(20)$ & $68838.7(9)$ & $1452.69545(4)$ & -0.025 & 3600 & & $4 p$ & ${ }^{3} \mathrm{P}^{\circ}{ }_{1}^{1}$ & $5 \mathrm{~d}$ & $(5 / 2)^{2}[3 / 2]_{2}$ & & & S36c & & \\
\hline $1455.6643(13)$ & $68697.16(6)$ & $1455.66225(7)$ & 0.0021 & 22000 & & $s^{\frac{1}{2}}$ & ${ }^{3} \mathrm{~F}_{4}$ & $6 \mathrm{p}$ & $(5 / 2)^{2}[7 / 2]^{0}{ }_{3}$ & & & TW & & \\
\hline 1457.1778(12) & $68625.81(6)$ & 1457.17554(5) & 0.0023 & 55000 & & $4 p$ & ${ }^{3} \mathrm{~F}_{3}^{\circ}$ & $5 d$ & $(5 / 2)^{2}[5 / 2]_{2}$ & & & TW & & \\
\hline $1458.0022(6)$ & 68587.00(3) & $1458.00133(5)$ & 0.0009 & 150000 & & $4 \mathrm{p}$ & ${ }^{3} \mathrm{~F}_{3}^{\circ}$ & $5 \mathrm{~d}$ & $(5 / 2)^{2}[7 / 2]_{3}$ & & & K66 & & \\
\hline 1459.4131(6) & $68520.70(3)$ & $1459.41216(15)$ & 0.0009 & 220000 & & $s^{2}$ & ${ }^{3} \mathrm{~F}_{2}$ & $6 \mathrm{p}$ & $(3 / 2)^{2}[5 / 2]^{\circ}{ }^{\circ}$ & & & K66 & & \\
\hline $1460.3076(13)$ & 68478.72(6) & $1460.30573(14)$ & 0.0018 & 14000 & & $s^{2}$ & ${ }^{3} \mathrm{~F}_{2}$ & $6 \mathrm{p}$ & $(3 / 2)^{2}[5 / 2]^{\circ}{ }_{3}$ & & & TW & & \\
\hline $1460.4620(13)$ & $68471.48(6)$ & $1460.45917(4)$ & 0.0028 & 17000 & & $4 p$ & ${ }^{3} \mathrm{~F}_{3}^{\circ}$ & $5 \mathrm{~d}$ & $(5 / 2)^{2}[5 / 2]_{3}$ & & & TW & & \\
\hline 1461.5557(11) & $68420.25(5)$ & $1461.55369(5)$ & 0.0020 & 60000 & & $4 p$ & ${ }^{3} \mathrm{P}^{\circ}{ }_{1}^{\circ}$ & $5 d$ & $(5 / 2)^{2}[1 / 2]_{1}$ & & & TW & & \\
\hline $1462.8534(18)$ & $68359.55(9)$ & $1462.85242(6)$ & 0.0010 & 5100 & & $s^{2}$ & ${ }^{3} \mathrm{~F}_{4}$ & $4 \mathrm{f}$ & $(3 / 2)^{2}[9 / 2]^{\circ}$ & & & TW & & \\
\hline $1463.7512(6)$ & 68317.62(3) & $1463.75130(4)$ & -0.0001 & 350000 & & $4 p$ & ${ }^{3} \mathrm{~F}_{3}^{\circ}$ & $5 \mathrm{~d}$ & $(5 / 2)^{2}[9 / 2]_{4}$ & & & K66 & & \\
\hline $1463.8367(6)$ & 68313.63(3) & $1463.83785(5)$ & -0.0012 & 200000 & & $4 \mathrm{p}$ & ${ }^{3} \mathrm{~F}_{4}^{\circ}$ & $5 \mathrm{~d}$ & $(5 / 2)^{2}[7 / 2]_{4}$ & & & K66 & & \\
\hline 1463.9947(12) & $68306.26(6)$ & $1463.99219(5)$ & 0.0025 & 20000 & & $4 \mathrm{p}$ & ${ }^{3} \mathrm{~F}_{3}^{\circ}$ & $5 d$ & $(5 / 2)^{2}[3 / 2]_{2}$ & & & TW & & \\
\hline $1464.1173(22)$ & 68300.54(10) & $1464.11637(6)$ & 0.0009 & 4600 & & $4 \mathrm{p}$ & ${ }^{1} \mathrm{~F}_{3}^{\circ}$ & $5 \mathrm{~d}$ & $(3 / 2)^{2}[5 / 2]_{2}$ & & & TW & & \\
\hline 1464.6035(11) & $68277.86(5)$ & 1464.60141(5) & 0.0021 & 69000 & & $4 \mathrm{p}$ & ${ }^{1} \mathrm{~F}_{3}^{\circ}$ & $5 d$ & $(3 / 2)^{2}[5 / 2]_{3}$ & & & TW & & \\
\hline $1465.0339(18)$ & 68257.81(9) & $1465.03633(14)$ & -0.0024 & 4300 & & $s^{2}$ & ${ }^{3} \mathrm{~F}_{2}$ & $6 \mathrm{p}$ & $(3 / 2)^{2}[1 / 2]^{\circ}{ }_{1}$ & & & TW & & \\
\hline $1465.5404(6)$ & 68234.22(3) & $1465.54069(16)$ & -0.0003 & 170000 & & $\mathrm{~s}^{2}$ & ${ }^{3} \mathrm{~F}_{4}$ & $\mathrm{sp}$ & $\left({ }^{1} \mathrm{G}\right)^{3} \mathrm{P}^{\circ}{ }^{3} \mathrm{~F}^{\circ}{ }_{4}$ & & & K66 & & \\
\hline $1466.0705(6)$ & 68209.54(3) & $1466.07026(9)$ & 0.0002 & 280000 & & $\mathrm{~s}^{2}$ & ${ }^{3} \mathrm{~F}_{3}$ & $6 \mathrm{p}$ & $(5 / 2)^{2}[5 / 2]^{0}{ }_{3}$ & & & K66 & & \\
\hline $1466.5247(10)$ & 68188.42(5) & $1466.52373(4)$ & 0.0010 & 79000 & & $4 p$ & ${ }^{3} \mathrm{~F}^{\circ}{ }_{4}$ & $5 d$ & $(5 / 2)^{2}[5 / 2]_{3}$ & & & K66 & & \\
\hline $1466.7305(12)$ & $68178.85(6)$ & $1466.72839(10)$ & 0.0022 & 42000 & & $s^{\frac{1}{2}}$ & ${ }^{3} \mathrm{~F}_{3}$ & $\mathrm{sp}$ & $\left({ }^{3} \mathrm{~F}\right)^{1} \mathrm{P}^{\circ}{ }^{3} \mathrm{~F}^{\circ}{ }_{2}$ & & & TW & & \\
\hline $1467.574(5)$ & $68139.68(24)$ & $1467.57153(6)$ & 0.002 & 1400 & & $4 p$ & ${ }^{1} \mathrm{~F}_{3}^{\circ}$ & $5 d$ & $(3 / 2)^{2}[3 / 2]_{2}$ & & & TW & & \\
\hline $1469.6935(6)$ & 68041.40(3) & 1469.69291(5) & 0.0006 & 150000 & & $4 \mathrm{p}$ & ${ }^{1} \mathrm{~F}_{3}^{\circ}$ & $5 d$ & $(3 / 2)^{2}[7 / 2]_{4}$ & & & K66 & & \\
\hline $1469.8457(12)$ & $68034.35(5)$ & $1469.84329(5)$ & 0.0024 & 49000 & & $4 \mathrm{p}$ & ${ }^{3} \mathrm{~F}^{\circ}{ }_{4}^{\circ}$ & $5 d$ & $(5 / 2)^{2}[9 / 2]_{4}$ & & & TW & & \\
\hline 1470.6970(6) & 67994.97(3) & $1470.69711(4)$ & -0.0001 & 500000 & & $4 \mathrm{p}$ & ${ }^{3} \mathrm{~F}_{4}^{\circ}$ & $5 d$ & $(5 / 2)^{2}[9 / 2]_{5}$ & & & K66 & & \\
\hline $1471.072(5)$ & 67977.65(24) & $1471.07289(5)$ & -0.001 & 2700 & & $4 p$ & ${ }^{1} \mathrm{~F}_{3}^{\circ}{ }_{3}$ & $5 \mathrm{~d}$ & $(3 / 2)^{2}[7 / 2]_{3}$ & & & TW & & \\
\hline $1472.3946(6)$ & 67916.58(3) & $1472.3950(4)$ & -0.0004 & 6800000 & & $\mathrm{~d}^{10}$ & ${ }^{1} \mathrm{~S}_{0}$ & $4 p$ & ${ }^{3} \mathrm{P}^{\circ}{ }_{1}$ & $8 . e+06$ & E & K66 & D05se & \\
\hline $1473.5295(8)$ & 67864.27(4) & $1473.53001(10)$ & -0.0005 & 100000 & & $s^{2}$ & ${ }^{3} \mathrm{~F}_{3}$ & $6 \mathrm{p}$ & $(5 / 2)^{2}[7 / 2]^{\circ}{ }_{4}$ & & & K66 & & \\
\hline 1473.9786(6) & 67843.59(3) & $1473.97844(4)$ & 0.0002 & 190000 & & $4 p$ & ${ }^{3} \mathrm{P}^{\circ}{ }_{1}$ & $6 \mathrm{~s}$ & $(3 / 2)^{2}[3 / 2]_{2}$ & & & K66 & & \\
\hline $1474.9348(6)$ & 67799.61(3) & $1474.93480(9)$ & -0.0000 & 230000 & & $\mathrm{~s}^{2}$ & ${ }^{3} \mathrm{~F}_{3}$ & $\mathrm{sp}$ & $\left({ }^{1} \mathrm{G}\right)^{3} \mathrm{P}^{\circ}{ }^{3} \mathrm{~F}_{3}^{\circ}$ & & & K66 & & \\
\hline 1475.4361(22) & $67776.57(10)$ & $1475.4383(9)$ & -0.0023 & 23000 & & $s^{2}$ & ${ }^{1} \mathrm{D}_{2}$ & $\mathrm{sp}$ & $\left({ }^{1} \mathrm{D}\right){ }^{1} \mathrm{P}^{\circ}{ }^{1} \mathrm{P}^{\circ}{ }_{1}$ & & & TWn & & \\
\hline
\end{tabular}


Table A1. Cont.

\begin{tabular}{|c|c|c|c|c|c|c|c|c|c|c|c|c|c|c|}
\hline$\lambda_{\text {obs }}{ }^{a}(\AA)$ & $\sigma_{\mathrm{obs}}{ }^{\mathrm{b}}\left(\mathrm{cm}^{-1}\right)$ & $\lambda_{\text {Ritz }}{ }^{c}(\AA)$ & $\begin{array}{c}\Delta \lambda_{\text {obs-Ritz }} \\
\text { (A) }\end{array}$ & $\begin{array}{l}I_{\text {obs }} \mathrm{d} \\
\text { (arb. u.) }\end{array}$ & Char ${ }^{\mathrm{e}}$ & Low & Level & & Jpper Level & $A\left(\mathrm{~s}^{-1}\right)$ & $\operatorname{Acc}^{f}$ & Line Ref. $\mathrm{g}$ & TP Ref. $\mathrm{g}$ & Notes ${ }^{h}$ \\
\hline 1476.0596(6) & 67747.94(3) & $1476.05914(4)$ & 0.0005 & 150000 & & $4 p$ & ${ }^{3} \mathrm{P}^{\circ}{ }_{1}$ & $6 \mathrm{~s}$ & $(3 / 2)^{2}[3 / 2]_{1}$ & & & K66 & & \\
\hline $1478.2385(13)$ & $67648.08(6)$ & $1478.23606(6)$ & 0.0024 & 23000 & & $4 \mathrm{p}$ & ${ }^{1} \mathrm{D}_{2}^{\circ}$ & $5 \mathrm{~d}$ & $(3 / 2)^{2}[5 / 2]_{2}$ & & & TW & & \\
\hline $1481.5438(6)$ & 67497.16(3) & $1481.54375(12)$ & 0.0000 & 130000 & & $s^{2}$ & ${ }^{3} \mathrm{~F}_{3}$ & $6 p$ & $(5 / 2)^{2}[5 / 2]_{2}^{\circ}$ & & & K66 & & \\
\hline $1484.178(5)$ & 67377.37(24) & $1484.17557(13)$ & 0.002 & 1200 & & $s^{2}$ & ${ }^{3} \mathrm{~F}_{4}$ & $\mathrm{sp}$ & $\left({ }^{1} \mathrm{G}\right)^{3} \mathrm{P}^{\circ}{ }^{3} \mathrm{H}^{\circ}{ }_{5}$ & & & TW & & \\
\hline $1485.3282(8)$ & $67325.19(4)$ & $1485.32773(4)$ & 0.0005 & 110000 & & $4 \mathrm{p}$ & ${ }^{1} \mathrm{D}^{\circ}{ }_{2}$ & $5 d$ & $(3 / 2)^{2}[7 / 2]_{3}$ & & & K66 & & \\
\hline $1485.6143(13)$ & $67312.22(6)$ & $1485.60994(4)$ & 0.0043 & 53000 & & $4 \mathrm{p}$ & ${ }^{3} \mathrm{~F}_{3}^{\circ}$ & $6 s$ & $(3 / 2)^{2}[3 / 2]_{2}$ & & & TW & & \\
\hline $1485.6788(8)$ & $67309.30(4)$ & 1485.67761(4) & 0.0012 & 110000 & & $4 \mathrm{p}$ & ${ }^{3} \mathrm{P}_{2}^{\circ}$ & $6 \mathrm{~s}$ & $(5 / 2)^{2}[5 / 2]_{2}$ & & & K66 & & \\
\hline $1487.612(5)$ & 67221.81(24) & $1487.60927(6)$ & 0.003 & 1400 & & $4 p$ & ${ }^{3} \mathrm{D}_{3}^{\circ}$ & $5 \mathrm{~d}$ & $(3 / 2)^{2}[5 / 2]_{2}$ & & & TW & & \\
\hline $1487.7805(12)$ & 67214.22(5) & $1487.77920(12)$ & 0.0013 & 68000 & & $s^{2}$ & ${ }^{3} \mathrm{~F}_{3}$ & $6 p$ & $(5 / 2)^{2}[3 / 2]_{2}^{\circ}$ & & & TW & & \\
\hline $1487.9719(12)$ & $67205.57(5)$ & $1487.96988(5)$ & 0.0020 & 70000 & & $4 p$ & ${ }^{3} \mathrm{~F}_{2}^{\circ}$ & $5 d$ & $(5 / 2)^{2}[5 / 2]_{2}$ & & & TW & & \\
\hline $1488.1123(12)$ & 67199.23(5) & $1488.11000(4)$ & 0.0023 & 53000 & & $4 \mathrm{p}$ & ${ }^{3} \mathrm{D}_{3}^{\circ}$ & $5 \mathrm{~d}$ & $(3 / 2)^{2}[5 / 2]_{3}$ & & & TW & & \\
\hline $1488.2853(13)$ & 67191.42(6) & $1488.2853(12)$ & -0.0000 & 34000 & & $s^{2}$ & ${ }^{1} \mathrm{D}_{2}$ & $8 p$ & $(5 / 2)^{2}[5 / 2]_{2}^{\circ}$ & & & TWn & & \\
\hline $1488.6366(6)$ & 67175.56(3) & $1488.63692(4)$ & -0.0003 & 1100000 & & $4 p$ & ${ }^{3} \mathrm{P}_{2}^{\circ}$ & $6 \mathrm{~s}$ & $(5 / 2)^{2}[5 / 2]_{3}$ & & & K66 & & \\
\hline 1488.8319(12) & $67166.75(6)$ & 1488.83094(5) & 0.0009 & 120000 & & $4 \mathrm{p}$ & ${ }^{3} \mathrm{~F}_{2}^{\circ}$ & $5 \mathrm{~d}$ & $(5 / 2)^{2}[7 / 2]_{3}$ & & & TW & & \\
\hline 1491.178(5) & $67061.07(24)$ & $1491.17634(6)$ & 0.002 & 1400 & & $4 p$ & ${ }^{3} \mathrm{D}^{\circ}{ }_{3}$ & $5 \mathrm{~d}$ & $(3 / 2)^{2}[3 / 2]_{2}$ & & & TW & & \\
\hline $1491.384(6)$ & $67051.8(3)$ & $1491.39392(4)$ & -0.010 & 1200 & & $4 p$ & ${ }^{3} \mathrm{~F}_{2}^{\circ}$ & $5 \mathrm{~d}$ & $(5 / 2)^{2}[5 / 2]_{3}$ & & & TW & & \\
\hline $1492.1535(10)$ & 67017.23(4) & $1492.15247(11)$ & 0.0010 & 95000 & & $s^{\frac{1}{2}}$ & ${ }^{3} \mathrm{~F}_{2}$ & $6 p$ & $(5 / 2)^{2}[5 / 2]^{\circ}{ }_{3}$ & & & K66 & & \\
\hline $1492.6819(10)$ & 66993.51(4) & $1492.68146(13)$ & 0.0004 & 100000 & & $4 p$ & ${ }^{1} \mathrm{P}^{\circ}{ }_{1}$ & $5 \mathrm{~d}$ & $(3 / 2)^{2}[1 / 2]_{0}$ & & & K66 & & \\
\hline $1492.8346(6)$ & $66986.66(3)$ & $1492.83423(11)$ & 0.0004 & 330000 & & $\mathrm{~s}^{2}$ & ${ }^{3} \mathrm{~F}_{2}$ & $\mathrm{sp}$ & $\left({ }^{3} \mathrm{~F}\right)^{1} \mathrm{P}^{\circ}{ }^{3} \mathrm{~F}^{\circ}{ }_{2}$ & $5.5 \mathrm{e}+08$ & $\mathrm{D}+$ & K66 & TW & \\
\hline $1493.3675(6)$ & $66962.75(3)$ & $1493.36656(4)$ & 0.0009 & 180000 & & $4 p$ & ${ }^{3} \mathrm{D}^{\circ}{ }_{3}$ & $5 d$ & $(3 / 2)^{2}[7 / 2]_{4}$ & & & K66 & & \\
\hline 1494.661(6) & $66904.8(3)$ & $1494.65244(5)$ & 0.008 & 49000 & & $4 \mathrm{p}$ & ${ }^{3} \mathrm{~F}_{2}^{\circ}$ & $5 \mathrm{~d}$ & $(5 / 2)^{2}[3 / 2]_{1}$ & & & S36c & & \\
\hline $1494.7930(19)$ & $66898.89(8)$ & $1494.79138(4)$ & 0.0016 & 6700 & & $4 \mathrm{p}$ & ${ }^{3} \mathrm{D}^{\circ}{ }_{3}$ & $5 d$ & $(3 / 2)^{2}[7 / 2]_{3}$ & & & TW & & \\
\hline $1495.4296(6)$ & 66870.42(3) & $1495.42965(9)$ & -0.0001 & 200000 & & $\mathrm{~s}^{23} \mathrm{~F}$ & & $6 p$ & $(5 / 2)^{2}[7 / 2]^{\circ}{ }_{3}$ & & & K66 & & \\
\hline 1496.6862(6) & 66814.27(3) & $1496.68654(4)$ & -0.0003 & 260000 & & $4 p$ & ${ }^{3} \mathrm{P}_{0}^{\circ}$ & $6 s$ & $(3 / 2)^{2}[3 / 2]_{1}$ & & & K66 & & \\
\hline $1498.5779(14)$ & $66729.93(6)$ & $1498.57545(7)$ & 0.0024 & 18000 & & $s^{2}$ & ${ }^{3} \mathrm{~F}_{3}$ & $4 \mathrm{f}$ & $(3 / 2)^{2}[7 / 2]_{3}^{\circ}$ & & & TW & & \\
\hline $1499.5135(10)$ & 66688.30(4) & 1499.51299(7) & 0.0005 & 79000 & & $\mathrm{~s}^{2}$ & ${ }^{3} \mathrm{~F}_{3}$ & $4 \mathrm{f}$ & $(3 / 2)^{2}[7 / 2]_{4}^{\circ}$ & & & K66 & & \\
\hline $1500.016(3)$ & $66665.97(13)$ & $1500.0148(7)$ & 0.001 & 14000 & & $s^{2}$ & ${ }^{1} \mathrm{D}_{2}$ & $8 p$ & $(5 / 2)^{2}[3 / 2]_{2}^{\circ}$ & & & TWn & & \\
\hline $1501.3359(12)$ & $66607.35(5)$ & 1501.33622(11) & -0.0003 & 110000 & & $\mathrm{~s}^{2}$ & ${ }^{3} \mathrm{~F}_{2}$ & $\mathrm{sp}$ & $\left({ }^{1} \mathrm{G}\right)^{3} \mathrm{P}^{\circ}{ }^{3} \mathrm{~F}^{\circ}{ }_{3}$ & & & TW & & \\
\hline 1502.811(3) & 66541.96(13) & $1502.80934(8)$ & 0.002 & 2600 & & $s^{2}$ & ${ }^{3} \mathrm{~F}_{3}$ & $4 \mathrm{f}$ & $(3 / 2)^{2}[9 / 2]^{\circ}{ }_{4}$ & & & TW & & \\
\hline $1503.3675(6)$ & 66517.34(3) & 1503.36800(13) & -0.0005 & 150000 & & $s^{2}$ & ${ }^{3} \mathrm{~F}_{2}$ & $6 \mathrm{p}$ & $(5 / 2)^{2}[3 / 2]^{\circ}{ }_{1}$ & & & K66 & & \\
\hline 1504.7561(6) & 66455.95(3) & $1504.75691(4)$ & -0.0008 & 220000 & & $s^{2}$ & ${ }^{3} \mathrm{~F}_{4}$ & $4 \mathrm{f}$ & $(5 / 2)^{2}[9 / 2]_{5}^{\circ}$ & & & K66 & & \\
\hline 1505.3866(6) & 66428.12(3) & $1505.38756(4)$ & -0.0010 & 160000 & & $s^{2}$ & ${ }^{3} \mathrm{~F}_{4}$ & $4 \mathrm{f}$ & $(5 / 2)^{2}[7 / 2]^{\circ}{ }_{4}$ & & & K66 & & \\
\hline $1505.8576(12)$ & $66407.34(5)$ & $1505.85714(18)$ & 0.0005 & 60000 & & $s^{2}$ & ${ }^{3} \mathrm{~F}_{3}$ & $\mathrm{sp}$ & $\left({ }^{1} \mathrm{G}\right)^{3} \mathrm{P}^{\circ}{ }^{3} \mathrm{~F}_{4}^{\circ}$ & & & TW & & \\
\hline $1507.4705(22)$ & 66336.29(10) & $1507.4706(5)$ & -0.0000 & 9700 & & $s^{2}$ & ${ }^{3} \mathrm{P}_{2}$ & $7 \mathrm{f}$ & $(5 / 2)^{2}[5 / 2]^{\circ}{ }_{2}$ & & & TWn & & \\
\hline $1507.6008(22)$ & $66330.56(10)$ & $1507.59910(4)$ & 0.0017 & 3200 & & $s^{2}$ & ${ }^{3} \mathrm{~F}_{4}$ & $4 \mathrm{f}$ & $(5 / 2)^{2}[5 / 2]_{3}^{\circ}$ & & & TW & & \\
\hline $1508.1835(6)$ & 66304.93(3) & 1508.18443(13) & -0.0009 & 170000 & & $s^{2}$ & ${ }^{3} \mathrm{~F}_{2}$ & $6 p$ & $(5 / 2)^{2}[5 / 2]_{2}^{\circ}$ & & & K66 & & \\
\hline $1508.4845(22)$ & 66291.70(10) & $1508.4829(15)$ & 0.0016 & 6400 & & $s^{2}$ & ${ }^{3} \mathrm{P}_{2}$ & $7 \mathrm{f}$ & $(5 / 2)^{2}[3 / 2]_{1}^{\circ}$ & & & TWn & & \\
\hline $1508.6313(6)$ & 66285.25(3) & $1508.63203(5)$ & -0.0007 & 150000 & & $\mathrm{~s}^{2}$ & ${ }^{3} \mathrm{~F}_{4}$ & $4 \mathrm{f}$ & $(5 / 2)^{2}[7 / 2]^{\circ}{ }_{3}$ & & & K66 & & \\
\hline 1510.5051(6) & 66203.02(3) & $1510.50562(5)$ & -0.0005 & 210000 & & $4 p$ & ${ }^{1} \mathrm{~F}_{3}^{\circ}$ & $5 \mathrm{~d}$ & $(5 / 2)^{2}[7 / 2]_{4}$ & & & K66 & & \\
\hline $1510.724(3)$ & 66193.41(13) & $1510.72666(5)$ & -0.002 & 8900 & & $4 \mathrm{p}$ & ${ }^{1} \mathrm{~F}_{3}^{\circ}$ & $5 d$ & $(5 / 2)^{2}[7 / 2]_{3}$ & & & TW & & \\
\hline
\end{tabular}


Table A1. Cont.

\begin{tabular}{|c|c|c|c|c|c|c|c|c|c|c|c|c|c|c|}
\hline$\lambda_{\text {obs }}{ }^{a}(\AA)$ & $\sigma_{\mathrm{obs}}{ }^{\mathrm{b}}\left(\mathrm{cm}^{-1}\right)$ & $\lambda_{\text {Ritz }}{ }^{c}(\AA)$ & $\begin{array}{c}\Delta \lambda_{\text {obs-Ritz }} \\
\text { (A) }\end{array}$ & $\begin{array}{c}I_{\text {obs }} \mathrm{d} \\
\text { (arb. u.) }\end{array}$ & Char ${ }^{\mathrm{e}}$ & \multicolumn{2}{|c|}{ Lower Level } & \multicolumn{2}{|c|}{ Upper Level } & $A\left(\mathrm{~s}^{-1}\right)$ & $\operatorname{Acc}^{f}$ & Line Ref. $\mathrm{g}$ & TP Ref. $\mathrm{g}$ & Notes ${ }^{h}$ \\
\hline $1512.1738(12)$ & 66129.96(5) & $1512.17367(6)$ & 0.0002 & 98000 & & $\mathrm{~s}^{2}$ & ${ }^{3} \mathrm{~F}_{4}$ & $\mathrm{sp}$ & $\left({ }^{3} \mathrm{~F}\right)^{1} \mathrm{P}^{\circ}{ }^{3} \mathrm{G}_{4}^{\circ}$ & & & TW & & \\
\hline $1512.4640(8)$ & 66117.28(3) & $1512.46442(11)$ & -0.0004 & 97000 & & $s^{2}$ & ${ }^{3} F_{3}$ & $\mathrm{sp}$ & $\left({ }^{3} \mathrm{P}\right)^{3} \mathrm{P}^{\circ}{ }^{1} \mathrm{D}^{\circ}{ }_{2}$ & & & K66 & & \\
\hline 1512.5393(19) & 66113.98(8) & $1512.5391(6)$ & 0.0003 & 18000 & & $s^{2}$ & ${ }^{3} \mathrm{P}_{1}$ & $8 p$ & $(3 / 2)^{2}[1 / 2]^{\circ}$ & & & TWn & & \\
\hline $1513.0157(22)$ & 66093.17(9) & $1513.0151(5)$ & 0.0005 & 9800 & & $s^{2}$ & ${ }^{3} \mathrm{P}_{1}$ & $7 \mathrm{f}$ & $(5 / 2)^{2}[5 / 2]_{2}^{\circ}$ & & & TWn & & \\
\hline $1513.3651(6)$ & 66077.91(3) & $1513.36565(4)$ & -0.0005 & 140000 & & $4 p$ & ${ }^{1} \mathrm{~F}_{3}^{\circ}$ & $5 \mathrm{~d}$ & $(5 / 2)^{2}[5 / 2]_{3}$ & & & K66 & & \\
\hline $1513.9340(13)$ & $66053.08(6)$ & $1513.93287(14)$ & 0.0011 & 46000 & & $\mathrm{~s}^{2}$ & ${ }^{1} \mathrm{D}_{2}$ & $6 \mathrm{f}$ & $(5 / 2)^{2}[7 / 2]^{\circ}{ }_{3}$ & & & TW & & \\
\hline $1514.2339(8)$ & $66040.00(3)$ & $1514.23361(6)$ & 0.0003 & 80000 & & $4 p$ & ${ }^{3} \mathrm{D}^{\circ}{ }_{1}$ & $5 d$ & $(3 / 2)^{2}[5 / 2]_{2}$ & & & K66 & & \\
\hline $1514.3380(12)$ & 66035.46(5) & 1514.33770(11) & 0.0003 & 110000 & & $s^{2}$ & ${ }^{1} \mathrm{D}_{2}$ & $8 p$ & $(5 / 2)^{2}[3 / 2]^{\circ}{ }_{1}$ & & & TW & & \\
\hline 1514.4921(6) & 66028.74(3) & 1514.49222(8) & -0.0001 & 700000 & & $s^{2}$ & ${ }^{3} \mathrm{~F}_{4}$ & $\mathrm{sp}$ & $\left({ }^{3} \mathrm{~F}\right)^{1} \mathrm{P}^{\circ}{ }^{3} \mathrm{D}^{\circ}{ }_{3}$ & $4.8 \mathrm{e}+08$ & $\mathrm{D}+$ & K66 & TW & \\
\hline 1514.6461(13) & $66022.02(6)$ & 1514.64663(14) & -0.0005 & 48000 & & $\mathrm{~s}^{2}$ & ${ }^{3} \mathrm{~F}_{2}$ & $6 \mathrm{p}$ & $(5 / 2)^{2}[3 / 2]^{\circ} 2$ & & & TW & & \\
\hline $1514.805(5)$ & $66015.12(23)$ & $1514.80334(10)$ & 0.001 & 13000 & & $s^{2}$ & ${ }^{1} \mathrm{D}_{2}$ & $6 f$ & $(5 / 2)^{2}[5 / 2]_{2}^{\circ}$ & & & TW & & \\
\hline $1515.456(3)$ & $65986.75(12)$ & $1515.46044(10)$ & -0.005 & 8600 & & $s^{2}$ & ${ }^{1} \mathrm{D}_{2}$ & $6 \mathrm{f}$ & $(5 / 2)^{2}[3 / 2]^{\circ}{ }_{2}$ & & & TW & & \\
\hline $1515.491(5)$ & 65985.22(23) & $1515.49438(15)$ & -0.004 & 4300 & & $s^{2}$ & ${ }^{1} \mathrm{D}_{2}$ & $6 f$ & $(5 / 2)^{2}[3 / 2]^{\circ}{ }_{1}$ & & & TW & & \\
\hline $1516.9018(13)$ & $65923.84(6)$ & $1516.90090(5)$ & 0.0009 & 43000 & & $4 p$ & ${ }^{1} \mathrm{~F}_{3}^{\circ}$ & $5 \mathrm{~d}$ & $(5 / 2)^{2}[9 / 2]_{4}$ & & & TW & & \\
\hline $1517.1600(12)$ & 65912.63(5) & 1517.15960(5) & 0.0004 & 81000 & & $4 p$ & ${ }^{1} \mathrm{~F}_{3}^{\circ}$ & $5 \mathrm{~d}$ & $(5 / 2)^{2}[3 / 2]_{2}$ & & & TW & & \\
\hline $1517.6309(8)$ & $65892.17(3)$ & $1517.63100(4)$ & -0.0001 & 130000 & & $4 \mathrm{p}$ & ${ }^{3} \mathrm{~F}_{2}^{\circ}$ & $6 s$ & $(3 / 2)^{2}[3 / 2]_{2}$ & & & K66 & & \\
\hline 1517.9297(8) & $65879.20(3)$ & 1517.92967(6) & 0.0000 & 87000 & & $4 \mathrm{p}$ & ${ }^{3} \mathrm{D}^{\circ}{ }_{1}$ & $5 \mathrm{~d}$ & $(3 / 2)^{2}[3 / 2]_{2}$ & & & K66 & & \\
\hline $1519.4914(6)$ & $65811.49(3)$ & $1519.49170(4)$ & -0.0003 & 410000 & & $4 p$ & ${ }^{3} \mathrm{P}^{\circ}{ }_{1}$ & $6 \mathrm{~s}$ & $(5 / 2)^{2}[5 / 2]_{2}$ & & & K66 & & \\
\hline $1519.8366(6)$ & 65796.55(3) & $1519.83686(4)$ & -0.0003 & 540000 & & $4 \mathrm{p}$ & ${ }^{3} \mathrm{~F}_{2}^{\circ}$ & $6 \mathrm{~s}$ & $(3 / 2)^{2}[3 / 2]_{1}$ & & & K66 & & \\
\hline & & $1519.84246(6)$ & & & $\mathrm{m}$ & $4 p$ & ${ }^{3} \mathrm{D}^{\circ}{ }_{1}$ & $5 d$ & $(3 / 2)^{2}[3 / 2]_{1}$ & & & K66 & & \\
\hline $1519.923(3)$ & 65792.81(13) & $1519.9165(7)$ & 0.007 & 11000 & * & $\mathrm{s}^{\frac{1}{2}}$ & ${ }^{3} \mathrm{P}_{0}$ & $8 p$ & $(3 / 2)^{2}[1 / 2]^{\circ}{ }_{1}$ & & & TWn & & \\
\hline $1519.923(3)$ & 65792.81(13) & $1519.9261(7)$ & -0.003 & 11000 & * & $s^{2}$ & ${ }^{3} \mathrm{~F}_{2}$ & $\mathrm{sp}$ & $\left({ }^{3} \mathrm{P}\right)^{3} \mathrm{P}^{\circ}{ }^{3} \mathrm{~S}^{\circ}{ }_{1}$ & & & TWn & & \\
\hline $1520.0166(12)$ & $65788.75(5)$ & $1520.01664(6)$ & -0.0000 & 53000 & & $4 p$ & ${ }^{3} \mathrm{D}^{\circ}{ }_{2}$ & $5 \mathrm{~d}$ & $(3 / 2)^{2}[5 / 2]_{2}$ & & & TW & & \\
\hline $1520.3899(12)$ & $65772.60(5)$ & 1520.38979(17) & 0.0001 & 150000 & & $s^{2}$ & ${ }^{1} \mathrm{D}_{2}$ & $6 \mathrm{f}$ & $(5 / 2)^{2}[1 / 2]^{\circ}{ }_{1}$ & & & TWn & & \\
\hline $1520.5397(6)$ & 65766.12(3) & $1520.53944(5)$ & 0.0003 & 150000 & & $4 p$ & ${ }^{3} \mathrm{D}_{2}^{\circ}$ & $5 \mathrm{~d}$ & $(3 / 2)^{2}[5 / 2]_{3}$ & & & K66 & & \\
\hline 1521.4252(22) & $65727.84(9)$ & $1521.4270(16)$ & -0.0018 & 8000 & & $s^{2}$ & ${ }^{3} \mathrm{P}_{0}$ & $7 \mathrm{f}$ & $(5 / 2)^{2}[3 / 2]^{\circ}{ }_{1}$ & & & TWn & & \\
\hline $1522.5070(19)$ & 65681.14(8) & $1522.50482(12)$ & 0.0022 & 8900 & & $\mathrm{~s}^{2}$ & ${ }^{3} \mathrm{~F}_{3}$ & $\mathrm{sp}$ & $\left({ }^{1} \mathrm{G}\right)^{3} \mathrm{P}^{\circ}{ }^{3} \mathrm{~F}^{\circ}{ }_{2}$ & & & TWn & & \\
\hline $1522.5767(6)$ & 65678.14(3) & $1522.57664(11)$ & 0.0001 & 97000 & & $s^{2}$ & ${ }^{3} \mathrm{~F}_{2}$ & $6 \mathrm{p}$ & $(5 / 2)^{2}[7 / 2]^{\circ}{ }_{3}$ & & & K66 & & \\
\hline $1523.7415(8)$ & 65627.93(3) & $1523.74102(6)$ & 0.0005 & 76000 & & $4 p$ & ${ }^{3} \mathrm{D}_{2}^{\circ}$ & $5 \mathrm{~d}$ & $(3 / 2)^{2}[3 / 2]_{2}$ & & & K66 & & \\
\hline 1523.9231(15) & $65620.11(6)$ & $1523.9230(10)$ & 0.0001 & 31000 & & $s^{2}$ & ${ }^{3} \mathrm{P}_{1}$ & $8 p$ & $(3 / 2)^{2}[3 / 2]^{\circ}{ }_{1}$ & & & TWn & & \\
\hline $1524.8604(8)$ & $65579.77(3)$ & $1524.85998(5)$ & 0.0004 & 130000 & & $4 p$ & ${ }^{1} \mathrm{D}_{2}^{\circ}$ & $5 d$ & $(5 / 2)^{2}[5 / 2]_{2}$ & & & K66 & & \\
\hline $1525.656(20)$ & $65545.6(9)$ & $1525.63103(13)$ & 0.025 & 92000 & & $s^{\frac{1}{2}}$ & ${ }^{3} \mathrm{~F}_{3}$ & $\mathrm{sp}$ & $\left({ }^{3} \mathrm{~F}\right)^{1} \mathrm{P}^{\circ}{ }^{3} \mathrm{G}_{3}^{\circ}$ & & & S36c & & \\
\hline $1525.6433(13)$ & $65546.12(5)$ & $1525.64065(7)$ & 0.0026 & 73000 & & $4 p$ & ${ }^{1} \mathrm{P}_{1}^{\circ}$ & $5 \mathrm{~d}$ & $(3 / 2)^{2}[5 / 2]_{2}$ & & & TW & & \\
\hline $1525.656(20)$ & $65545.6(9)$ & $1525.66850(6)$ & -0.013 & 97000 & & $4 p$ & ${ }^{3} \mathrm{D}^{\circ}{ }_{2}$ & $5 \mathrm{~d}$ & $(3 / 2)^{2}[3 / 2]_{1}$ & & & S36c & & \\
\hline $1525.7649(6)$ & $65540.90(3)$ & $1525.76429(5)$ & 0.0006 & 150000 & & $4 \mathrm{p}$ & ${ }^{1} \mathrm{D}_{2}^{\circ}$ & $5 d$ & $(5 / 2)^{2}[7 / 2]_{3}$ & & & K66 & & \\
\hline $1525.8388(8)$ & 65537.72(3) & $1525.83781(9)$ & 0.0010 & 92000 & & $\mathrm{~s}^{2}$ & ${ }^{3} \mathrm{~F}_{2}$ & $4 \mathrm{f}$ & $(3 / 2)^{2}[7 / 2]^{\circ}{ }_{3}$ & & & K66 & & \\
\hline 1526.932(5) & 65490.79(21) & $1526.92724(7)$ & 0.005 & 32000 & & $4 p$ & ${ }^{3} \mathrm{D}^{\circ}{ }_{1}$ & $5 \mathrm{~d}$ & $(3 / 2)^{2}[1 / 2]_{1}$ & & & TW & & \\
\hline $1526.9944(7)$ & 65488.12(3) & $1526.9931(5)$ & 0.0013 & 130000 & & $\mathrm{~s}^{2}$ & ${ }^{3} \mathrm{P}_{2}$ & $\mathrm{sp}$ & ${ }^{3} \mathrm{D}_{3}^{\circ}$ & & & K66n & & \\
\hline $1527.8127(13)$ & $65453.05(6)$ & $1527.81239(9)$ & 0.0003 & 42000 & & $s^{2}$ & ${ }^{3} \mathrm{~F}_{2}$ & $4 \mathrm{f}$ & $(3 / 2)^{2}[5 / 2]^{\circ}{ }_{2}$ & & & TW & & \\
\hline $1528.4583(22)$ & $65425.40(9)$ & $1528.45612(3)$ & 0.0022 & 4700 & & $4 p$ & ${ }^{1} \mathrm{D}^{\circ}{ }_{2}$ & $5 \mathrm{~d}$ & $(5 / 2)^{2}[5 / 2]_{3}$ & & & TW & & \\
\hline
\end{tabular}


Table A1. Cont.

\begin{tabular}{|c|c|c|c|c|c|c|c|c|c|c|c|c|c|c|}
\hline$\lambda_{\text {obs }}{ }^{a}(\AA)$ & $\sigma_{\mathrm{obs}}{ }^{\mathrm{b}}\left(\mathrm{cm}^{-1}\right)$ & $\lambda_{\text {Ritz }}{ }^{c}(\AA)$ & $\begin{array}{c}\Delta \lambda_{\text {obs-Ritz }}(\AA) \\
\text { (A) }\end{array}$ & $\begin{array}{c}I_{\text {obs }}{ }^{d} \\
\text { (arb. u.) }\end{array}$ & Char $^{\mathrm{e}}$ & \multicolumn{2}{|c|}{ Lower Level } & \multicolumn{2}{|c|}{ Upper Level } & $A\left(\mathrm{~s}^{-1}\right)$ & $\operatorname{Acc}^{\mathrm{f}}$ & Line Ref. $\mathrm{g}$ & TP Ref. $\mathrm{g}$ & Notes $^{h}$ \\
\hline $1528.785(20)$ & $65411.4(9)$ & $1528.89495(9)$ & -0.110 & 19000 & ? & $\mathrm{s}^{2}$ & ${ }^{3} \mathrm{~F}_{2}$ & $4 \mathrm{f}$ & $(3 / 2)^{2}[5 / 2]^{\circ} 3$ & & & S36c & & $x$ \\
\hline $1529.3927(22)$ & 65385.43(9) & $1529.39267(7)$ & 0.0000 & 4900 & & $4 p$ & ${ }^{1} \mathrm{P}^{\circ}{ }_{1}^{-}$ & $5 \mathrm{~d}$ & $(3 / 2)^{2}[3 / 2]_{2}$ & & & TW & & \\
\hline $1531.2874(14)$ & 65304.53(6) & $1531.28647(11)$ & 0.0010 & 70000 & & $\mathrm{~s}^{2}$ & ${ }^{3} \mathrm{~F}_{2}$ & $4 \mathrm{f}$ & $(3 / 2)^{2}[3 / 2]^{\circ}{ }_{1}$ & & & TW & & \\
\hline $1531.3338(14)$ & $65302.55(6)$ & $1531.33448(7)$ & -0.0007 & 41000 & & $4 p$ & ${ }^{1} \mathrm{P}_{1}^{\circ}$ & $5 \mathrm{~d}$ & $(3 / 2)^{2}[3 / 2]_{1}$ & & & TW & & \\
\hline $1531.4120(14)$ & 65299.21(6) & 1531.4121(10) & -0.0002 & 60000 & & $s^{2}$ & ${ }^{3} \mathrm{P}_{0}$ & $8 p$ & $(3 / 2)^{2}[3 / 2]^{\circ}{ }_{1}$ & & & TWn & & \\
\hline \multirow[t]{2}{*}{$1531.8555(6)$} & 65280.31(3) & $1531.85562(4)$ & -0.0001 & 700000 & & $4 p$ & ${ }^{3} \mathrm{~F}_{3}^{\circ}$ & $6 s$ & $(5 / 2)^{2}[5 / 2]_{2}$ & & & K66 & & \\
\hline & & $1531.87878(5)$ & & & $\mathrm{m}$ & $4 \mathrm{p}$ & ${ }^{1} \mathrm{D}_{2}^{\circ}$ & $5 \mathrm{~d}$ & $(5 / 2)^{2}[3 / 2]_{1}$ & & & TW & & \\
\hline $1532.128(3)$ & $65268.70(13)$ & 1532.13042(10) & -0.003 & 390000 & & $\mathrm{~s}^{\frac{1}{2}}$ & ${ }^{3} \mathrm{~F}_{3}$ & sp & $\left({ }^{3} \mathrm{~F}\right)^{1} \mathrm{P}^{\circ}{ }^{3} \mathrm{D}^{\circ}{ }_{2}$ & $1.0 \mathrm{e}+09$ & $\mathrm{D}+$ & TW & TW & \\
\hline 1532.8083(15) & 65239.73(6) & $1532.80783(7)$ & 0.0004 & 17000 & & $4 p$ & ${ }^{3} \mathrm{D}_{2}^{\circ}$ & $5 \mathrm{~d}$ & $(3 / 2)^{2}[1 / 2]_{1}$ & & & TW & & \\
\hline 1533.9867(6) & 65189.61(3) & $1533.98624(12)$ & 0.0005 & 170000 & & $\mathrm{~s}^{2}$ & ${ }^{3} \mathrm{~F}_{2}$ & $\mathrm{sp}$ & $\left({ }^{3} \mathrm{~F}\right)^{1} \mathrm{P}^{\circ}{ }^{3} \mathrm{D}^{\circ}{ }_{1}$ & & & K66 & & \\
\hline $1534.6282(13)$ & $65162.37(6)$ & $1534.62719(10)$ & 0.0010 & 43000 & & $s^{2}$ & ${ }^{3} \mathrm{~F}_{3}$ & $\mathrm{sp}$ & $\left({ }^{1} \mathrm{G}\right)^{3} \mathrm{P}^{\circ}{ }^{3} \mathrm{H}^{\circ}{ }_{4}$ & & & TW & & \\
\hline 1535.0023(6) & 65146.48(3) & $1535.00194(4)$ & 0.0004 & 350000 & & $4 p$ & ${ }^{3} \mathrm{~F}_{3}^{\circ}$ & 6s & $(5 / 2)^{2}[5 / 2]_{3}$ & & & K66 & & \\
\hline 1535.5242(6) & 65124.34(3) & 1535.52352(4) & 0.0007 & 130000 & & $4 p$ & ${ }^{3} \mathrm{D}_{3}^{\circ}$ & $5 \mathrm{~d}$ & $(5 / 2)^{2}[7 / 2]_{4}$ & & & K66 & & \\
\hline $1536.193(5)$ & $65096.00(21)$ & $1536.19869(21)$ & -0.006 & 8200 & & $\mathrm{~s}^{2}$ & ${ }^{3} \mathrm{P}_{2}$ & $6 \mathrm{f}$ & $(3 / 2)^{2}[3 / 2]^{\circ}{ }_{1}$ & & & TW & & \\
\hline $1536.9317(16)$ & $65064.70(7)$ & $1536.93333(14)$ & -0.0016 & 24000 & & $s^{2}$ & ${ }^{3} \mathrm{P}_{2}$ & $6 \mathrm{f}$ & $(3 / 2)^{2}[3 / 2]_{2}^{\circ}$ & & & TW & & \\
\hline $1537.3218(12)$ & 65048.19(5) & $1537.32130(14)$ & 0.0005 & 120000 & & $s^{2}$ & ${ }^{3} \mathrm{P}_{2}$ & $6 \mathrm{f}$ & $(3 / 2)^{2}[5 / 2]^{\circ}{ }_{3}$ & & & TW & & \\
\hline 1537.5581(6) & 65038.19(3) & $1537.55884(8)$ & -0.0007 & 860000 & & $s^{2}$ & ${ }^{3} \mathrm{~F}_{4}$ & $\mathrm{sp}$ & $\left({ }^{3} \mathrm{~F}\right)^{1} \mathrm{P}^{\circ}{ }^{3} \mathrm{~F}_{4}^{\circ}$ & $1.4 \mathrm{e}+09$ & $\mathrm{D}+$ & K66 & TW & \\
\hline $1538.4795(6)$ & 64999.24(3) & $1538.47917(3)$ & 0.0003 & 96000 & & $4 p$ & ${ }^{3} \mathrm{D}_{3}^{\circ}$ & $5 \mathrm{~d}$ & $(5 / 2)^{2}[5 / 2]_{3}$ & & & K66 & & \\
\hline $1538.522(5)$ & 64997.45(21) & 1538.52706(8) & -0.005 & 40000 & & $4 p$ & ${ }^{1} \mathrm{P}^{\circ}{ }_{1}$ & $5 \mathrm{~d}$ & $(3 / 2)^{2}[1 / 2]_{1}$ & & & TW & & \\
\hline $1540.2392(6)$ & 64924.98(3) & $1540.23914(12)$ & 0.0001 & 120000 & & $s^{2}$ & ${ }^{3} \mathrm{~F}_{2}$ & $\mathrm{sp}$ & $\left({ }^{3} \mathrm{P}\right)^{3} \mathrm{P}^{\circ}{ }^{1} \mathrm{D}^{\circ}{ }_{2}$ & & & K66 & & \\
\hline $1540.3886(6)$ & 64918.68(3) & $1540.38850(4)$ & 0.0001 & 290000 & & $4 p$ & ${ }^{1} \mathrm{~F}_{3}^{\circ}$ & $6 \mathrm{~s}$ & $(3 / 2)^{2}[3 / 2]_{2}$ & & & K66 & & \\
\hline $1540.5879(6)$ & 64910.29(3) & $1540.58814(9)$ & -0.0002 & 600000 & & $\mathrm{~s}^{2}$ & ${ }^{3} \mathrm{~F}_{3}$ & $\mathrm{sp}$ & $\left({ }^{3} \mathrm{~F}\right)^{1} \mathrm{P}^{\circ}{ }^{3} \mathrm{~F}^{\circ}{ }_{3}$ & $9 . e+08$ & $\mathrm{D}+$ & K66 & TW & \\
\hline $1541.246(5)$ & $64882.59(22)$ & $1541.24593(13)$ & -0.000 & 5200 & & $s^{2}$ & ${ }^{3} \mathrm{P}_{1}$ & $6 \mathrm{f}$ & $(3 / 2)^{2}[5 / 2]_{2}^{\circ}$ & & & TW & & \\
\hline 1541.7007(22) & 64863.43(9) & 1541.70280(5) & -0.0021 & 860000 & & $4 p$ & ${ }^{3} \mathrm{~F}_{4}^{\circ}$ & $6 \mathrm{~s}$ & $(5 / 2)^{2}[5 / 2]_{3}$ & & & K66 & & \\
\hline 1541.7542(18) & 64861.18(8) & $1541.75580(18)$ & -0.0016 & 260000 & & $s^{\frac{1}{2}}$ & ${ }^{1} \mathrm{D}_{2}$ & $\mathrm{sp}$ & $\left({ }^{1} \mathrm{D}\right)^{1} \mathrm{P}^{\circ}{ }^{1} \mathrm{D}^{\circ}{ }_{2}$ & $1.4 \mathrm{e}+09$ & $\mathrm{D}+$ & K66 & TW & \\
\hline 1541.9565(22) & 64852.67(9) & 1541.95701(21) & -0.0005 & 11000 & & $\mathrm{~s}^{2}$ & ${ }^{3} \mathrm{P}_{1}$ & $6 \mathrm{f}$ & $(3 / 2)^{2}[3 / 2]^{\circ}{ }_{1}$ & & & TW & & \\
\hline $1542.4007(13)$ & $64834.00(6)$ & $1542.40025(4)$ & 0.0004 & 55000 & & $4 p$ & ${ }^{3} \mathrm{D}^{\circ}{ }_{3}$ & $5 \mathrm{~d}$ & $(5 / 2)^{2}[3 / 2]_{2}$ & & & TW & & \\
\hline 1542.6989(22) & $64821.46(9)$ & $1542.69717(14)$ & 0.0017 & 10000 & & $s^{\frac{1}{2}}$ & ${ }^{3} \mathrm{P}_{1}$ & $6 f$ & $(3 / 2)^{2}[3 / 2]_{2}^{\circ}$ & & & TW & & \\
\hline 1543.1328(13) & $64803.24(5)$ & $1543.1327(9)$ & 0.0001 & 64000 & & $\mathrm{~s}^{2}$ & ${ }^{3} \mathrm{P}_{2}$ & $\mathrm{sp}$ & $\left({ }^{1} \mathrm{D}\right)^{1} \mathrm{P}^{\circ}{ }^{1} \mathrm{P}_{1}^{\circ}{ }_{1}^{-}$ & & & TWn & & \\
\hline $1544.6764(6)$ & 64738.48(3) & $1544.67690(8)$ & -0.0005 & 430000 & & $s^{2}$ & ${ }^{3} F_{3}$ & $4 \mathrm{f}$ & $(5 / 2)^{2}[9 / 2]^{\circ}{ }_{4}$ & & & K66 & & \\
\hline 1547.9581(8) & 64601.23(3) & $1547.95800(7)$ & 0.0001 & 59000 & & $s^{2}$ & ${ }^{3} \mathrm{~F}_{3}$ & $4 \mathrm{f}$ & $(5 / 2)^{2}[7 / 2]^{\circ}{ }_{4}$ & & & K66 & & \\
\hline 1548.4169(12) & 64582.09(5) & $1548.4165(12)$ & 0.0004 & 200000 & & $s^{2}$ & ${ }^{3} \mathrm{P}_{2}$ & $\mathrm{sp}$ & $\left({ }^{3} \mathrm{P}\right)^{1} \mathrm{P}^{\circ}{ }^{3} \mathrm{P}^{\mathrm{o}}{ }_{2}$ & $1.0 \mathrm{e}+09$ & D+ & TWn & TW & \\
\hline $1548.942(3)$ & $64560.22(12)$ & $1548.9433(10)$ & -0.002 & 15000 & & $s^{2}$ & ${ }^{3} \mathrm{P}_{1}$ & $\mathrm{sp}$ & $\left({ }^{1} \mathrm{D}\right)^{1} \mathrm{P}^{\circ}{ }^{1} \mathrm{P}^{\circ}{ }_{1}$ & & & TWn & & \\
\hline $1549.56(7)$ & $64534(3)$ & $1549.62492(22)$ & -0.06 & 24000 & & $s^{2}$ & ${ }^{3} \mathrm{P}_{0}$ & $6 f$ & $(3 / 2)^{2}[3 / 2]^{\circ}{ }_{1}$ & & & S36nc & & \\
\hline 1550.0971(17) & 64512.09(7) & $1550.09682(9)$ & 0.0003 & 10000 & & $4 p$ & ${ }^{3} \mathrm{D}^{\circ}{ }_{1}$ & $5 \mathrm{~d}$ & $(5 / 2)^{2}[1 / 2]_{0}$ & & & TW & & \\
\hline $1550.2978(13)$ & 64503.73(5) & $1550.29648(7)$ & 0.0014 & 29000 & & $\mathrm{~s}^{\frac{1}{2}}$ & ${ }^{3} \mathrm{~F}_{3}$ & $4 \mathrm{f}$ & $(5 / 2)^{2}[5 / 2]_{3}^{\circ}$ & & & TW & & \\
\hline $1550.6528(6)$ & $64488.969(25)$ & $1550.65296(13)$ & -0.0002 & 180000 & & $s^{2}$ & ${ }^{3} \mathrm{~F}_{2}$ & $\mathrm{sp}$ & $\left({ }^{1} \mathrm{G}\right)^{3} \mathrm{P}^{\circ}{ }^{3} \mathrm{~F}_{2}^{\circ}$ & $7.3 e+08$ & $\mathrm{D}+$ & K66n & TW & \\
\hline $1551.3886(6)$ & $64458.383(25)$ & $1551.38877(7)$ & -0.0002 & 250000 & & $s^{2}$ & ${ }^{3} \mathrm{~F}_{3}$ & $4 \mathrm{f}$ & $(5 / 2)^{2}[7 / 2]^{\circ}{ }_{3}$ & & & K66 & & \\
\hline $1552.297(5)$ & $64420.66(22)$ & 1552.29519(14) & 0.002 & 1100 & & $\mathrm{~s}^{2}$ & ${ }^{3} \mathrm{~F}_{3}$ & $\mathrm{sp}$ & $\left({ }^{3} \mathrm{P}\right)^{3} \mathrm{P}^{\circ}{ }^{5} \mathrm{~S}^{\circ}{ }_{2}$ & & & TW & & \\
\hline $1552.6450(6)$ & $64406.223(25)$ & $1552.64631(10)$ & -0.0013 & 720000 & & $\mathrm{~s}^{2}$ & ${ }^{3} \mathrm{~F}_{4}$ & $\mathrm{sp}$ & $\left({ }^{3} \mathrm{~F}\right)^{1} \mathrm{P}^{\circ}{ }^{3} \mathrm{G}_{5}^{\circ}$ & $1.9 \mathrm{e}+09$ & D+ & K66 & TW & \\
\hline
\end{tabular}


Table A1. Cont.

\begin{tabular}{|c|c|c|c|c|c|c|c|c|c|c|c|c|c|c|}
\hline$\lambda_{\text {obs }}{ }^{a}(\AA)$ & $\sigma_{\mathrm{obs}}{ }^{\mathrm{b}}\left(\mathrm{cm}^{-1}\right)$ & $\lambda_{\text {Ritz }}{ }^{c}(\AA)$ & $\begin{array}{c}\Delta \lambda_{\text {obs-Ritz }} \\
\text { (A) }\end{array}$ & $\begin{array}{c}I_{\text {obs }} \mathrm{d} \\
\text { (arb. u.) }\end{array}$ & Char ${ }^{e}$ & & Level & & Jpper Level & $A\left(\mathrm{~s}^{-1}\right)$ & $\operatorname{Acc}^{f}$ & Line Ref. $\mathrm{g}$ & TP Ref. $\mathrm{g}$ & Notes ${ }^{h}$ \\
\hline $1553.3473(13)$ & 64377.11(5) & $1553.3472(13)$ & 0.0001 & 96000 & & $\mathrm{~s}^{2}$ & ${ }^{1} \mathrm{D}_{2}$ & $7 \mathrm{p}$ & $(3 / 2)^{2}[3 / 2]^{\circ}{ }_{1}$ & & & TWn & & \\
\hline 1553.8961(6) & 64354.367(25) & $1553.89596(14)$ & 0.0001 & 210000 & & $s^{2}$ & ${ }^{3} \mathrm{~F}_{2}$ & $\mathrm{sp}$ & $\left({ }^{3} \mathrm{~F}\right)^{1} \mathrm{P}^{\circ}{ }^{3} \mathrm{G}_{3}^{\circ}$ & $1.4 \mathrm{e}+09$ & D+ & K66 & TW & \\
\hline 1554.2933(12) & 64337.92(5) & $1554.2934(11)$ & -0.0001 & 120000 & & $s^{2}$ & ${ }^{1} \mathrm{D}_{2}$ & $7 \mathrm{p}$ & $(3 / 2)^{2}[3 / 2]^{\circ}{ }_{2}$ & & & TWn & & \\
\hline $1555.1336(6)$ & 64303.157(25) & $1555.13425(8)$ & -0.0007 & 810000 & & $s^{2}$ & ${ }^{3} \mathrm{~F}_{3}$ & $\mathrm{sp}$ & $\left({ }^{3} \mathrm{~F}\right)^{1} \mathrm{P}^{\circ}{ }^{3} \mathrm{G}_{4}^{\circ}$ & $1.3 e+09$ & D+ & K66 & TW & \\
\hline $1555.7018(6)$ & $64279.671(25)$ & $1555.70295(9)$ & -0.0012 & 880000 & & $s^{2}$ & ${ }^{3} \mathrm{~F}_{4}$ & $\mathrm{sp}$ & $\left({ }^{3} \mathrm{P}\right)^{3} \mathrm{P}^{\circ}{ }^{3} \mathrm{D}^{\circ}{ }_{3}$ & $6.8 \mathrm{e}+08$ & $\mathrm{D}+$ & K66 & TW & \\
\hline $1556.0269(8)$ & 64266.24(3) & $1556.02548(4)$ & 0.0014 & 40000 & & $4 p$ & ${ }^{1} \mathrm{D}_{2}^{\circ}$ & $6 \mathrm{~s}$ & $(3 / 2)^{2}[3 / 2]_{2}$ & & & K66 & & \\
\hline $1556.6836(21)$ & 64239.13(9) & $1556.6810(10)$ & 0.0027 & 19000 & & $s^{2}$ & ${ }^{3} \mathrm{P}_{0}$ & $\mathrm{sp}$ & $\left({ }^{1} \mathrm{D}\right)^{1} \mathrm{P}^{\circ}{ }^{1} \mathrm{P}^{\circ}{ }_{1}$ & & & TWn & & \\
\hline $1556.7668(12)$ & $64235.70(5)$ & $1556.7668(12)$ & 0.0001 & 180000 & & $s^{2}$ & ${ }^{1} \mathrm{D}_{2}$ & $7 \mathrm{p}$ & $(3 / 2)^{2}[5 / 2]^{\circ}{ }_{3}$ & & & TWn & & \\
\hline 1557.5867(6) & $64201.884(25)$ & $1557.58652(10)$ & 0.0002 & 110000 & & $s^{2}$ & ${ }^{3} \mathrm{~F}_{3}$ & $\mathrm{sp}$ & $\left({ }^{3} \mathrm{~F}\right)^{1} \mathrm{P}^{\circ}{ }^{3} \mathrm{D}^{\circ}{ }_{3}$ & & & K66 & & \\
\hline $1558.1590(13)$ & $64178.30(6)$ & 1558.1587(13) & 0.0003 & 62000 & & $\mathrm{~s}^{2}$ & ${ }^{3} \mathrm{P}_{1}$ & $\mathrm{sp}$ & $\left({ }^{3} \mathrm{P}\right)^{1} \mathrm{P}^{\circ}{ }^{3} \mathrm{P}^{\circ}{ }_{0}$ & & & TWn & & \\
\hline $1558.3446(6)$ & $64170.659(25)$ & $1558.34444(4)$ & 0.0002 & 180000 & & $4 p$ & ${ }^{1} \mathrm{D}_{2}^{\circ}$ & $6 \mathrm{~s}$ & $(3 / 2)^{2}[3 / 2]_{1}$ & & & K66 & & \\
\hline 1559.1587(14) & $64137.15(6)$ & 1559.1576(13) & 0.0011 & 210000 & & $s^{\frac{1}{2}}$ & ${ }^{1} \mathrm{D}_{2}$ & $7 p$ & $(3 / 2)^{2}[5 / 2]_{2}^{\circ}$ & & & TWn & & \\
\hline $1563.108(3)$ & 63975.10(12) & 1563.1083(13) & -0.000 & 9400 & & $s^{2}$ & ${ }^{3} \mathrm{P}_{1}$ & $8 \mathrm{p}$ & $(5 / 2)^{2}[5 / 2]_{2}^{\circ}$ & & & TWn & & \\
\hline $1563.1959(13)$ & 63971.51(5) & $1563.19353(5)$ & 0.0024 & 22000 & & $4 p$ & ${ }^{3} \mathrm{D}^{\circ}{ }_{1}$ & $5 \mathrm{~d}$ & $(5 / 2)^{2}[5 / 2]_{2}$ & & & TW & & \\
\hline $1565.9244(6)$ & $63860.043(24)$ & $1565.92414(4)$ & 0.0003 & 190000 & & $4 p$ & ${ }^{3} \mathrm{~F}_{2}^{\circ}$ & $6 \mathrm{~s}$ & $(5 / 2)^{2}[5 / 2]_{2}$ & & & K66 & & \\
\hline $1566.4148(4)$ & $63840.051(16)$ & $1566.41460(4)$ & 0.0002 & 220000 & & $4 \mathrm{p}$ & ${ }^{3} \mathrm{D}^{\circ}{ }_{3}$ & $6 s$ & $(3 / 2)^{2}[3 / 2]_{2}$ & & & K66 & & \\
\hline $1569.2124(8)$ & $63726.24(3)$ & $1569.21212(4)$ & 0.0003 & 40000 & & $4 \mathrm{p}$ & ${ }^{3} \mathrm{~F}_{2}^{\circ}$ & $6 s$ & $(5 / 2)^{2}[5 / 2]_{3}$ & & & K66 & & \\
\hline 1569.4154(11) & 63717.99(4) & $1569.41524(11)$ & 0.0001 & 42000 & & $s^{2}$ & ${ }^{3} F_{2}$ & $\mathrm{sp}$ & $\left({ }^{3} \mathrm{~F}\right)^{1} \mathrm{P}^{\circ}{ }^{3} \mathrm{~F}^{\circ}{ }_{3}$ & & & TW & & \\
\hline $1570.0365(11)$ & $63692.79(4)$ & $1570.0367(8)$ & -0.0002 & 94000 & & $s^{2}$ & ${ }^{3} \mathrm{P}_{2}$ & $8 p$ & $(5 / 2)^{2}[3 / 2]^{\circ} 2$ & & & TW,S36r & & \\
\hline $1570.5722(12)$ & 63671.06(5) & $1570.57051(5)$ & 0.0017 & 17000 & & $4 p$ & ${ }^{3} \mathrm{D}^{\circ}{ }_{1}$ & $5 \mathrm{~d}$ & $(5 / 2)^{2}[3 / 2]_{1}$ & & & TW & & \\
\hline $1573.1666(22)$ & 63566.06(9) & $1573.16667(4)$ & -0.0001 & 4000 & & $4 \mathrm{p}$ & ${ }^{3} \mathrm{D}_{2}^{\circ}$ & $5 \mathrm{~d}$ & $(5 / 2)^{2}[5 / 2]_{3}$ & & & TW & & \\
\hline $1575.3547(12)$ & $63477.77(5)$ & $1575.35310(6)$ & 0.0016 & 34000 & & $4 \mathrm{p}$ & ${ }^{1} \mathrm{P}_{1}^{\circ}$ & $5 d$ & $(5 / 2)^{2}[5 / 2]_{2}$ & & & TW & & \\
\hline $1576.0523(12)$ & $63449.67(5)$ & $1576.0520(8)$ & 0.0003 & 47000 & & $s^{2}$ & ${ }^{3} \mathrm{P}_{1}$ & $8 p$ & $(5 / 2)^{2}[3 / 2]^{\circ}{ }_{2}$ & & & TWn & & \\
\hline $1577.2693(22)$ & $63400.71(9)$ & $1577.26680(5)$ & 0.0025 & 4500 & & $4 p$ & ${ }^{3} \mathrm{D}^{\circ}{ }_{2}$ & $5 d$ & $(5 / 2)^{2}[3 / 2]_{2}$ & & & TW & & \\
\hline $1579.4926(8)$ & 63311.47(3) & $1579.49148(9)$ & 0.0011 & 64000 & & $\mathrm{~s}^{2}$ & ${ }^{3} \mathrm{~F}_{2}$ & $4 \mathrm{f}$ & $(5 / 2)^{2}[5 / 2]^{\circ}{ }_{3}$ & & & K66 & & \\
\hline $1580.0260(11)$ & 63290.10(4) & $1580.02469(9)$ & 0.0013 & 43000 & & $s^{2}$ & ${ }^{3} \mathrm{~F}_{2}$ & $4 \mathrm{f}$ & $(5 / 2)^{2}[5 / 2]_{2}^{\circ}$ & & & TW & & \\
\hline $1580.6258(6)$ & 63266.081(24) & $1580.62531(9)$ & 0.0005 & 71000 & & $\mathrm{~s}^{2}$ & ${ }^{3} \mathrm{~F}_{2}$ & $4 \mathrm{f}$ & $(5 / 2)^{2}[7 / 2]^{\circ}{ }_{3}$ & & & K66 & & \\
\hline $1581.4029(14)$ & 63234.99(6) & $1581.40630(5)$ & -0.0034 & 5300 & & $4 p$ & ${ }^{3} \mathrm{D}^{\circ}{ }_{1}$ & $5 d$ & $(5 / 2)^{2}[1 / 2]_{1}$ & & & TW & & \\
\hline 1581.420(12) & $63234.3(5)$ & 1581.41834(10) & 0.001 & 2300 & & $s^{\frac{1}{2}}$ & ${ }^{3} F_{2}$ & $4 \mathrm{f}$ & $(5 / 2)^{2}[3 / 2]^{\circ}{ }_{1}$ & & & S36c & & \\
\hline $1581.9962(6)$ & 63211.277(24) & 1581.99510(10) & 0.0011 & 82000 & & $s^{2}$ & ${ }^{3} \mathrm{~F}_{3}$ & $\mathrm{sp}$ & $\left({ }^{3} \mathrm{~F}\right)^{1} \mathrm{P}^{\circ}{ }^{3} \mathrm{~F}_{4}^{\circ}$ & & & K66 & & \\
\hline $1582.6074(20)$ & $63186.86(8)$ & $1582.60633(9)$ & 0.0011 & 6600 & & $s^{2}$ & ${ }^{3} \mathrm{~F}_{2}$ & $4 \mathrm{f}$ & $(5 / 2)^{2}[3 / 2]_{2}^{\circ}$ & & & TW & & \\
\hline 1582.8471(11) & 63177.30(4) & $1582.84558(6)$ & 0.0015 & 35000 & & $4 p$ & ${ }^{1} \mathrm{P}^{\circ}{ }_{1}$ & $5 \mathrm{~d}$ & $(5 / 2)^{2}[3 / 2]_{1}$ & & & TW & & \\
\hline $1583.6831(4)$ & $63143.946(16)$ & $1583.68224(10)$ & 0.0009 & 160000 & & $s^{2}$ & ${ }^{3} \mathrm{~F}_{3}$ & $\mathrm{sp}$ & $\left({ }^{3} \mathrm{P}\right)^{3} \mathrm{P}^{\circ}{ }^{3} \mathrm{D}^{\circ}{ }_{2}$ & & & K66 & & \\
\hline $1586.276(5)$ & 63040.74(21) & 1586.27882(10) & -0.003 & 4100 & & $s^{2}$ & ${ }^{3} \mathrm{P}_{2}$ & $6 \mathrm{f}$ & $(5 / 2)^{2}[5 / 2]^{\circ}{ }_{3}$ & & & TW & & \\
\hline $1587.0607(22)$ & 63009.56(9) & $1587.05935(12)$ & 0.0014 & 6100 & & $s^{2}$ & ${ }^{3} \mathrm{~F}_{2}$ & $\mathrm{sp}$ & $\left({ }^{3} \mathrm{~F}\right)^{1} \mathrm{P}^{\circ}{ }^{3} \mathrm{D}^{\circ}{ }_{3}$ & & & TW & & \\
\hline $1587.716(3)$ & 62983.56(11) & $1587.71486(5)$ & 0.001 & 2600 & & $4 p$ & ${ }^{3} \mathrm{D}^{\circ} 2$ & $5 \mathrm{~d}$ & $(5 / 2)^{2}[1 / 2]_{1}$ & & & TW & & \\
\hline 1590.1649(4) & 62886.560(16) & $1590.16460(4)$ & 0.0003 & 110000 & & $4 p$ & ${ }^{1} \mathrm{~F}_{3}^{\circ}$ & $6 s$ & $(5 / 2)^{2}[5 / 2]_{2}$ & & & K66 & & \\
\hline 1593.5559(4) & 62752.741(16) & $1593.55527(4)$ & 0.0006 & 190000 & & $4 \mathrm{p}$ & ${ }^{1} \mathrm{~F}_{3}^{\circ}$ & $6 \mathrm{~s}$ & $(5 / 2)^{2}[5 / 2]_{3}$ & & & K66 & & \\
\hline $1596.749(6)$ & $62627.26(24)$ & $1596.74558(10)$ & 0.003 & 5100 & & $s^{2}$ & ${ }^{1} \mathrm{D}_{2}$ & $5 f$ & $(3 / 2)^{2}[3 / 2]^{\circ}{ }_{1}$ & & & S36c & & \\
\hline $1598.4025(4)$ & 62562.465(16) & $1598.40212(4)$ & 0.0004 & 140000 & & $4 p$ & ${ }^{3} \mathrm{D}^{\circ}{ }_{1}$ & $6 \mathrm{~s}$ & $(3 / 2)^{2}[3 / 2]_{1}$ & & & K66 & & \\
\hline
\end{tabular}


Table A1. Cont.

\begin{tabular}{|c|c|c|c|c|c|c|c|c|c|c|c|c|c|c|}
\hline$\lambda_{\text {obs }}{ }^{a}(\AA)$ & $\sigma_{\mathrm{obs}}{ }^{\mathrm{b}}\left(\mathrm{cm}^{-1}\right)$ & $\lambda_{\text {Ritz }}{ }^{c}(\AA)$ & $\begin{array}{c}\Delta \lambda_{\text {obs-Ritz }} \\
\text { (A) }\end{array}$ & $\begin{array}{c}I_{\text {obs }} \mathrm{d} \\
\text { (arb. u.) }\end{array}$ & Char ${ }^{e}$ & Lor & Level & & Upper Level & $A\left(\mathrm{~s}^{-1}\right)$ & $\operatorname{Acc}^{f}$ & Line Ref. $\mathrm{g}$ & TP Ref. $\mathrm{g}$ & Notes ${ }^{h}$ \\
\hline 1601.211(3) & $62452.75(12)$ & $1601.20967(12)$ & 0.001 & 2800 & & $\mathrm{~s}^{2}$ & ${ }^{3} \mathrm{~F}_{3}$ & $\mathrm{sp}$ & $\left({ }^{3} \mathrm{P}\right)^{3} \mathrm{P}^{\circ}{ }^{3} \mathrm{D}_{3}^{\circ}$ & & & TW & & \\
\hline $1602.2739(8)$ & $62411.30(3)$ & $1602.27264(12)$ & 0.0013 & 28000 & & $s^{2}$ & ${ }^{3} \mathrm{~F}_{2}$ & $\mathrm{sp}$ & $\left({ }^{3} \mathrm{P}\right)^{3} \mathrm{P}^{\circ}{ }^{3} \mathrm{P}^{\circ}{ }_{1}$ & & & K66 & & \\
\hline $1602.3887(4)$ & $62406.831(16)$ & $1602.38797(4)$ & 0.0007 & 130000 & & $4 p$ & ${ }^{3} \mathrm{D}_{2}^{\circ}$ & $6 \mathrm{~s}$ & $(3 / 2)^{2}[3 / 2]_{2}$ & & & K66 & & \\
\hline $1603.2280(23)$ & 62374.16(9) & $1603.22651(14)$ & 0.0014 & 13000 & & $s^{2}$ & ${ }^{1} \mathrm{D}_{2}$ & $7 \mathrm{p}$ & $(5 / 2)^{2}[5 / 2]^{\circ}{ }_{3}$ & & & TW & & \\
\hline $1604.8475(4)$ & $62311.216(16)$ & $1604.84729(4)$ & 0.0002 & 56000 & & $4 p$ & ${ }^{3} \mathrm{D}_{2}^{\circ}$ & $6 \mathrm{~s}$ & $(3 / 2)^{2}[3 / 2]_{1}$ & & & K66 & & \\
\hline $1605.2810(4)$ & $62294.390(16)$ & $1605.28112(12)$ & -0.0001 & 150000 & & $s^{2}$ & ${ }^{3} \mathrm{~F}_{3}$ & $\mathrm{sp}$ & $\left({ }^{3} \mathrm{P}\right)^{3} \mathrm{P}^{\circ}{ }^{3} \mathrm{P}_{2}^{\circ}$ & & & K66 & & \\
\hline 1606.1963(17) & $62258.89(6)$ & $1606.19537(24)$ & 0.0009 & 76000 & & $s^{2}$ & ${ }^{1} \mathrm{D}_{2}$ & $7 \mathrm{p}$ & $(5 / 2)^{2}[5 / 2]^{\circ} 2$ & & & TW & & \\
\hline $1606.8338(4)$ & $62234.190(15)$ & $1606.83396(4)$ & -0.0002 & 170000 & & $4 p$ & ${ }^{1} \mathrm{D}_{2}^{\circ}$ & $6 s$ & $(5 / 2)^{2}[5 / 2]_{2}$ & & & K66 & & \\
\hline 1608.3931(17) & $62173.85(6)$ & $1608.39195(15)$ & 0.0012 & 87000 & & $s^{\frac{1}{2}}$ & ${ }^{1} \mathrm{D}_{2}$ & $7 p$ & $(5 / 2)^{2}[3 / 2]^{\circ} 1$ & & & TW & & \\
\hline $1608.6395(4)$ & 62164.332(15) & 1608.63928(6) & 0.0002 & 72000 & & $4 p$ & ${ }^{1} \mathrm{P}^{\circ}{ }_{1}$ & $6 \mathrm{~s}$ & $(3 / 2)^{2}[3 / 2]_{2}$ & & & K66 & & \\
\hline 1609.3421(23) & $62137.19(9)$ & $1609.3430(15)$ & -0.0009 & 10000 & & $s^{\frac{1}{2}}$ & ${ }^{1} \mathrm{D}_{2}$ & $7 p$ & $(5 / 2)^{2}[7 / 2]^{\circ} 3$ & & & TWn & & \\
\hline $1610.2967(8)$ & $62100.36(3)$ & $1610.29617(4)$ & 0.0005 & 21000 & & $4 p$ & ${ }^{1} \mathrm{D}_{2}^{\circ}$ & $6 \mathrm{~s}$ & $(5 / 2)^{2}[5 / 2]_{3}$ & & & K66 & & \\
\hline $1611.1185(20)$ & 62068.68(8) & $1611.11784(6)$ & 0.0006 & 12000 & & $4 p$ & ${ }^{1} \mathrm{P}^{\circ}{ }_{1}$ & $6 \mathrm{~s}$ & $(3 / 2)^{2}[3 / 2]_{1}$ & & & TW & & \\
\hline 1614.159(5) & 61951.76(21) & $1614.16058(12)$ & -0.001 & 2100 & & $\mathrm{~s}^{2}$ & ${ }^{3} \mathrm{~F}_{2}$ & $\mathrm{sp}$ & $\left({ }^{3} \mathrm{P}\right)^{3} \mathrm{P}^{0}{ }^{3} \mathrm{D}^{\circ}{ }_{2}$ & & & TW & & \\
\hline $1614.4945(18)$ & $61938.89(7)$ & 1614.49462(18) & -0.0002 & 75000 & & $s^{2}$ & ${ }^{1} \mathrm{D}_{2}$ & $7 \mathrm{p}$ & $(5 / 2)^{2}[3 / 2]^{\circ}{ }_{2}$ & & & TW & & \\
\hline $1617.9152(8)$ & 61807.94(3) & $1617.91504(4)$ & 0.0002 & 24000 & & $4 p$ & ${ }^{3} \mathrm{D}_{3}^{\circ}$ & $6 \mathrm{~s}$ & $(5 / 2)^{2}[5 / 2]_{2}$ & & & K66 & & \\
\hline $1621.4262(4)$ & $61674.099(15)$ & 1621.42522(4) & 0.0010 & 84000 & & $4 p$ & ${ }^{3} \mathrm{D}_{3}^{\circ}$ & $6 \mathrm{~s}$ & $(5 / 2)^{2}[5 / 2]_{3}$ & & & K66 & & \\
\hline $1622.4281(4)$ & $61636.013(15)$ & $1622.42766(12)$ & 0.0004 & 80000 & & $s^{2}$ & ${ }^{3} \mathrm{~F}_{2}$ & $\mathrm{sp}$ & $\left({ }^{3} \mathrm{P}\right)^{3} \mathrm{P}^{\circ}{ }^{3} \mathrm{D}^{\circ}{ }_{1}$ & & & K66 & & \\
\hline $1623.1726(6)$ & $61607.743(23)$ & $1623.1731(3)$ & -0.0005 & 36000 & & $s^{2}$ & ${ }^{3} \mathrm{~F}_{4}$ & $\mathrm{sp}$ & $\left({ }^{3} \mathrm{P}\right)^{3} \mathrm{P}^{\circ}{ }^{5} \mathrm{D}^{\circ}{ }_{4}$ & & & K66 & & \\
\hline $1629.603(3)$ & $61364.65(12)$ & $1629.6017(12)$ & 0.001 & 9500 & & $s^{2}$ & ${ }^{3} \mathrm{P}_{2}$ & $7 \mathrm{p}$ & $(3 / 2)^{2}[3 / 2]^{\circ}{ }_{2}$ & & & TWn & & \\
\hline $1630.2669(18)$ & $61339.65(7)$ & $1630.26799(21)$ & -0.0011 & 32000 & & $\mathrm{~s}^{2}$ & ${ }^{3} F_{4}$ & $\mathrm{sp}$ & $\left({ }^{3} \mathrm{P}\right)^{3} \mathrm{P}^{\circ}{ }^{5} \mathrm{D}_{3}^{\circ}$ & & & TW & & \\
\hline $1636.0706(23)$ & $61122.06(9)$ & $1636.06917(21)$ & 0.0014 & 19000 & & $s^{2}$ & ${ }^{3} \mathrm{P}_{2}$ & $7 \mathrm{p}$ & $(3 / 2)^{2}[1 / 2]^{\circ}{ }_{1}$ & & & TWn & & \\
\hline $1636.6049(20)$ & 61102.10(7) & $1636.60469(14)$ & 0.0002 & 11000 & & $s^{2}$ & ${ }^{3} \mathrm{~F}_{2}$ & $\mathrm{sp}$ & $\left({ }^{3} \mathrm{P}\right)^{3} \mathrm{P}^{\circ}{ }^{3} \mathrm{P}^{\circ}{ }_{2}$ & & & TW & & \\
\hline $1645.6583(23)$ & 60765.95(8) & $1645.6575(22)$ & 0.0008 & 12000 & & $s^{2}$ & ${ }^{3} \mathrm{P}_{1}$ & $7 \mathrm{p}$ & $(3 / 2)^{2}[1 / 2]_{0}^{\circ}$ & & & TWn & & \\
\hline $1649.4570(14)$ & 60626.01(5) & $1649.45739(4)$ & -0.0004 & 26000 & & $4 p$ & ${ }^{3} \mathrm{D}^{\circ}{ }_{1}$ & $6 s$ & $(5 / 2)^{2}[5 / 2]_{2}$ & & & K66 & & \\
\hline 1656.322 & 60374.74 & $1656.32175(4)$ & & 27000 & : & $4 p$ & ${ }^{3} \mathrm{D}_{2}^{\circ}$ & $6 \mathrm{~s}$ & $(5 / 2)^{2}[5 / 2]_{2}$ & & & S36c & & \\
\hline $1660.0022(14)$ & $60240.88(5)$ & $1660.00075(4)$ & 0.0014 & 18000 & & $4 p$ & ${ }^{3} \mathrm{D}_{2}^{\circ}$ & $6 \mathrm{~s}$ & $(5 / 2)^{2}[5 / 2]_{3}$ & & & K66 & & \\
\hline $1663.0029(14)$ & 60132.19(5) & $1663.00184(5)$ & 0.0011 & 31000 & & $4 p$ & ${ }^{1} \mathrm{P}^{\circ}{ }_{1}$ & $6 \mathrm{~s}$ & $(5 / 2)^{2}[5 / 2]_{2}$ & & & K66 & & \\
\hline $1672.7773(23)$ & $59780.82(8)$ & $1672.7755(4)$ & 0.0018 & 8600 & & $s^{2}$ & ${ }^{3} \mathrm{~F}_{3}$ & $\mathrm{sp}$ & $\left({ }^{3} \mathrm{P}\right)^{3} \mathrm{P}^{\circ}{ }^{5} \mathrm{D}^{\circ}{ }_{4}$ & & & TW & & \\
\hline $1680.27(4)$ & 59514.1(14) & $1680.31169(23)$ & -0.04 & 980 & & $s^{2}$ & ${ }^{3} \mathrm{~F}_{3}$ & $\mathrm{sp}$ & $\left({ }^{3} \mathrm{P}\right)^{3} \mathrm{P}^{\circ}{ }^{5} \mathrm{D}^{\circ}{ }_{3}$ & & & S36c & & \\
\hline 1683.152(5) & 59412.32(18) & $1683.1596(3)$ & -0.007 & 34000 & * & $s^{2}$ & ${ }^{3} \mathrm{~F}_{4}$ & $\mathrm{sp}$ & $\left({ }^{1} \mathrm{D}\right)^{3} \mathrm{P}^{\circ}{ }^{3} \mathrm{D}^{\circ}{ }_{3}^{\circ}$ & $1.1 \mathrm{e}+08$ & C & TW & B00 & \\
\hline $1683.152(5)$ & $59412.32(18)$ & 1683.1583(3) & -0.006 & 34000 & * & $\mathrm{s}^{2}$ & ${ }^{3} \mathrm{~F}_{3}$ & $\mathrm{sp}$ & $\left({ }^{3} \mathrm{P}\right)^{3} \mathrm{P}^{\circ}{ }^{5} \mathrm{D}^{\circ}{ }_{2}$ & & & TW & & \\
\hline $1695.9031(23)$ & $58965.63(8)$ & $1695.90238(20)$ & 0.0007 & 12000 & & $s^{2}$ & ${ }^{3} \mathrm{P}_{2}$ & $7 \mathrm{p}$ & $(5 / 2)^{2}[3 / 2]^{\circ}{ }_{2}$ & & & TW & & \\
\hline $1699.0958(20)$ & $58854.83(7)$ & 1699.0950(5) & 0.0008 & 22000 & * & $s^{2}$ & ${ }^{3} \mathrm{~F}_{3}$ & $\mathrm{sp}$ & $\left({ }^{1} \mathrm{D}\right)^{3} \mathrm{P}^{\circ}{ }^{3} \mathrm{P}^{\circ}{ }_{2}$ & & & TW & & \\
\hline $1699.0958(20)$ & $58854.83(7)$ & $1699.10217(23)$ & -0.0064 & 22000 & * & $s^{2}$ & ${ }^{3} \mathrm{~F}_{4}$ & $\mathrm{sp}$ & $\left({ }^{1} \mathrm{D}\right)^{3} \mathrm{P}^{\circ}{ }^{3} \mathrm{~F}_{3}^{\circ}$ & & & TW & & \\
\hline $1702.9236(23)$ & $58722.54(8)$ & $1702.92295(21)$ & 0.0006 & 8600 & & $s^{2}$ & ${ }^{3} \mathrm{P}_{1}$ & $7 \mathrm{p}$ & $(5 / 2)^{2}[3 / 2]^{\circ}{ }_{2}$ & & & TW & & \\
\hline $1714.655(20)$ & $58320.8(7)$ & 1714.6631(3) & -0.008 & 460 & & $s^{2}$ & ${ }^{3} \mathrm{~F}_{2}$ & $\mathrm{sp}$ & $\left({ }^{3} \mathrm{P}\right)^{3} \mathrm{P}^{\circ}{ }^{\circ} \mathrm{D}_{3}{ }_{3}$ & & & S36c & & \\
\hline $1717.7188(22)$ & $58216.75(7)$ & $1717.7210(3)$ & -0.0023 & 11000 & & $s^{2}$ & ${ }^{3} \mathrm{~F}_{2}$ & $\mathrm{sp}$ & $\left({ }^{3} \mathrm{P}\right)^{3} \mathrm{P}^{\circ}{ }^{5} \mathrm{D}^{\circ}{ }_{1}$ & & & TW & & \\
\hline $1734.2254(23)$ & 57662.63(8) & $1734.2267(5)$ & -0.0013 & 4200 & & $\mathrm{~s}^{2}$ & ${ }^{3} \mathrm{~F}_{2}$ & $\mathrm{sp}$ & $\left({ }^{1} \mathrm{D}\right)^{3} \mathrm{P}^{\circ}{ }^{3} \mathrm{P}_{2}^{\mathrm{O}}{ }_{2}$ & & & TW & & \\
\hline 1736.5531(22) & $57585.34(7)$ & $1736.5563(3)$ & -0.0032 & 8900 & & $s^{2}$ & ${ }^{3} \mathrm{~F}_{3}$ & sp & $\left({ }^{1} \mathrm{D}\right)^{3} \mathrm{P}^{\circ}{ }^{3} \mathrm{D}^{\circ}{ }_{3}$ & & & TW & & \\
\hline
\end{tabular}


Table A1. Cont.

\begin{tabular}{|c|c|c|c|c|c|c|c|c|c|c|c|c|c|c|}
\hline \multirow{2}{*}{$\frac{\lambda_{\text {obs }}{ }^{\mathrm{a}}(\AA ̊)}{1744.506(20)}$} & \multirow{2}{*}{$\begin{array}{l}\sigma_{\text {obs }}{ }^{\mathbf{b}}\left(\mathbf{c m}^{-1}\right) \\
57322.8(7)\end{array}$} & \multirow{2}{*}{$\begin{array}{r}\lambda_{\text {Ritz }}{ }^{\mathrm{c}}(\AA) \\
1744.5159(3)\end{array}$} & \multirow{2}{*}{$\begin{array}{c}\begin{array}{c}\Delta \lambda_{\text {obs-Ritz }} \\
(\AA)\end{array} \\
-0.010\end{array}$} & \multirow{2}{*}{$\begin{array}{c}\begin{array}{c}I_{\text {obs }}{ }^{d} \\
\text { (arb. u.) }\end{array} \\
22000\end{array}$} & \multirow[t]{2}{*}{ Char ${ }^{\mathrm{e}}$} & \multicolumn{2}{|c|}{ Lower Level } & \multicolumn{2}{|r|}{ Upper Level } & \multirow{2}{*}{$\begin{array}{c}A\left(\mathbf{s}^{-\mathbf{1}}\right) \\
5.9 \mathrm{e}+07\end{array}$} & \multirow{2}{*}{$\frac{\operatorname{Acc}^{\mathrm{f}}}{C}$} & \multirow{2}{*}{$\begin{array}{l}\text { Line Ref. } \mathbf{g} \\
\text { S36c }\end{array}$} & \multirow{2}{*}{$\begin{array}{l}\text { TP Ref. }{ }^{\mathrm{g}} \\
\text { B00 }\end{array}$} & \multirow[t]{2}{*}{ Notes ${ }^{h}$} \\
\hline & & & & & & $\mathrm{s}^{2}$ & ${ }^{3} \mathrm{~F}_{3}$ & $\mathrm{sp}$ & $\left({ }^{1} \mathrm{D}\right)^{3} \mathrm{P}^{\circ}{ }^{3} \mathrm{D}^{\circ}{ }_{2}$ & & & & & \\
\hline 1753.2791(21) & $57035.99(7)$ & $1753.28069(22)$ & -0.0016 & 21000 & & $s^{2}$ & ${ }^{3} \mathrm{~F}_{2}$ & $\mathrm{sp}$ & $\left({ }^{1} \mathrm{D}\right)^{3} \mathrm{P}^{\circ}{ }^{3} \mathrm{P}^{\circ}{ }_{1}$ & & & TW & & \\
\hline $1759.5030(21)$ & $56834.23(7)$ & $1759.50415(22)$ & -0.0012 & 3400 & & $s^{2}$ & ${ }^{3} \mathrm{~F}_{3}$ & $\mathrm{sp}$ & $\left({ }^{1} \mathrm{D}\right)^{3} \mathrm{P}^{\circ}{ }^{3} \mathrm{~F}_{2}^{\circ}$ & & & TW & & \\
\hline $1781.573(3)$ & $56130.16(10)$ & $1781.5715(3)$ & 0.002 & 4400 & & $s^{2}$ & ${ }^{3} \mathrm{~F}_{2}$ & sp & $\left({ }^{1} \mathrm{D}\right)^{3} \mathrm{P}^{\circ}{ }^{3} \mathrm{D}^{\circ}{ }_{2}$ & & & TW & & \\
\hline $1790.6606(21)$ & $55845.31(7)$ & 1790.6599(3) & 0.0007 & 33000 & & $\mathrm{~s}^{2}$ & ${ }^{3} \mathrm{~F}_{2}$ & $\mathrm{sp}$ & $\left({ }^{1} \mathrm{D}\right)^{3} \mathrm{P}^{\circ}{ }^{3} \mathrm{D}^{\circ}{ }_{1}$ & $4.5 \mathrm{e}+07$ & C & TW & B00 & \\
\hline $1800.9806(24)$ & $55525.31(7)$ & $1800.9789(6)$ & 0.0018 & 11000 & & $s^{2}$ & ${ }^{3} \mathrm{~F}_{4}$ & $\mathrm{sp}$ & $\left({ }^{3} \mathrm{P}\right)^{3} \mathrm{P}^{\circ}{ }^{5} \mathrm{P}^{\circ}{ }_{3}$ & & & TW & & \\
\hline $1807.8535(21)$ & $55314.22(7)$ & & & 160000 & & $4 p$ & ${ }^{1} \mathrm{P}^{\circ}{ }_{1}$ & $s^{\frac{1}{2}}$ & ${ }^{1} \mathrm{~S}_{0}$ & & & TW,S36r & & \\
\hline $1856.937(20)$ & $53852.1(6)$ & $1856.92890(17)$ & 0.009 & 14000 & & $s^{2}$ & ${ }^{1} \mathrm{D}_{2}$ & $6 p$ & $(5 / 2)^{2}[3 / 2]^{\circ} 1$ & & & S36c & & \\
\hline 1861.57(4) & $53718.2(12)$ & $1861.6226(3)$ & -0.06 & 10000 & & $s^{2}$ & ${ }^{3} \mathrm{~F}_{3}$ & $\mathrm{sp}$ & $\left({ }^{3} \mathrm{P}\right)^{3} \mathrm{P}^{\circ}{ }^{5} \mathrm{P}^{\circ}{ }_{2}$ & & & S36c & & \\
\hline 1874.166(5) & $53357.06(14)$ & $1874.16676(18)$ & -0.001 & 14000 & & $s^{2}$ & ${ }^{1} \mathrm{D}_{2}$ & $6 \mathrm{p}$ & $(5 / 2)^{2}[3 / 2]^{\circ}{ }_{2}$ & & & TW & & \\
\hline 1875.74638(18) & $53312.112(5)$ & 1875.74622(7) & 0.00016 & 20000 & & $\mathrm{~s}^{2}$ & ${ }^{3} \mathrm{~F}_{4}$ & $5 p$ & $(3 / 2)^{2}[5 / 2]^{\circ}{ }_{3}$ & & & $\mathrm{~F}$ & & \\
\hline $1882.253(3)$ & $53127.82(8)$ & $1882.2566(10)$ & -0.003 & 17000 & & $s^{2}$ & ${ }^{1} \mathrm{D}_{2}$ & $\mathrm{sp}$ & $\left({ }^{3} \mathrm{P}\right)^{3} \mathrm{P}^{\circ}{ }^{3} \mathrm{~S}^{\circ}{ }_{1}$ & & & TWn & & \\
\hline $1903.858(5)$ & $52524.93(15)$ & 1903.86698(15) & -0.009 & 410 & & $\mathrm{~s}^{2}$ & ${ }^{1} \mathrm{D}_{2}$ & $\mathrm{sp}$ & $\left({ }^{3} \mathrm{~F}\right)^{1} \mathrm{P}^{\circ}{ }^{3} \mathrm{D}^{\circ}{ }_{1}$ & & & TW & & \\
\hline 1920.6709(8) & $52065.141(22)$ & $1920.67133(7)$ & -0.0004 & 43000 & & $4 p$ & ${ }^{3} \mathrm{P}_{2}^{\circ}$ & $4 \mathrm{~d}$ & $(3 / 2)^{2}[5 / 2]_{3}$ & $1.5 \mathrm{e}+07$ & C & $\mathrm{F}$ & B00 & \\
\hline $1922.14229(17)$ & $52025.285(5)$ & 1922.14231(11) & -0.00003 & 94000 & & $\mathrm{~s}^{2}$ & ${ }^{3} \mathrm{~F}_{3}$ & $5 p$ & $(3 / 2)^{2}[3 / 2]^{\circ} 2$ & & & $\mathrm{~F}$ & & \\
\hline $1924.548(3)$ & $51960.26(9)$ & $1924.5458(22)$ & 0.002 & 5200 & & $s^{2}$ & ${ }^{1} \mathrm{G}_{4}$ & $7 \mathrm{p}$ & $(5 / 2)^{2}[7 / 2]^{\circ}{ }_{3}$ & & & TWn & & \\
\hline \multirow[t]{2}{*}{$1928.46(4)$} & $51854.9(11)$ & $1928.4863(3)$ & -0.03 & 46000 & & $s^{2}$ & ${ }^{1} \mathrm{D}_{2}$ & $\begin{array}{l}1 \\
\mathrm{sp}\end{array}$ & $\left({ }^{3} \mathrm{P}\right)^{3} \mathrm{P}^{\circ}{ }^{1} \mathrm{P}^{\circ}{ }_{1}$ & & & S36cn & & \\
\hline & & 1929.60764(17) & & & $\mathrm{m}$ & $\mathrm{s}^{2}$ & ${ }^{1} \mathrm{D}_{2}$ & $\mathrm{sp}$ & $\left({ }^{1} \mathrm{G}\right)^{3} \mathrm{P}^{\circ}{ }^{3} \mathrm{~F}^{\circ}{ }_{2}$ & & & S36cn & & \\
\hline 1929.75131(6) & $51820.1488(16)$ & $1929.75129(5)$ & 0.00002 & 340000 & & $s^{2}$ & ${ }^{3} \mathrm{~F}_{4}$ & sp & $\left({ }^{3} \mathrm{~F}\right)^{3} \mathrm{P}^{\circ}{ }^{1} \mathrm{~F}^{\circ}{ }_{3}$ & $2.1 \mathrm{e}+07$ & $\mathrm{C}$ & $\mathrm{F}$ & B00 & \\
\hline $1942.3030(7)$ & $51485.272(19)$ & 1942.30290(12) & 0.0001 & 31000 & & $s^{2}$ & ${ }^{3} \mathrm{~F}_{3}$ & $5 p$ & $(3 / 2)^{2}[5 / 2]^{\circ} 3$ & & & $\mathrm{~F}$ & & \\
\hline $1944.59596(14)$ & $51424.564(4)$ & $1944.59601(6)$ & -0.00005 & 6000000 & & $4 \mathrm{~s}$ & ${ }^{3} \mathrm{D}_{3}$ & $4 p$ & ${ }^{3} \mathrm{D}_{2}^{\circ}$ & $1.99 \mathrm{e}+07$ & $\mathrm{C}+$ & $\mathrm{F}$ & D05se & \\
\hline 1946.4928(3) & $51374.451(7)$ & 1946.49291(7) & -0.0001 & 130000 & & $s^{2}$ & ${ }^{3} \mathrm{~F}_{4}$ & $5 p$ & $(5 / 2)^{2}[5 / 2]^{\circ} 3$ & & & $\mathrm{~F}$ & & \\
\hline 1952.5792(18) & $51214.31(5)$ & 1952.57557(12) & 0.0037 & 97000 & & $s^{2}$ & ${ }^{3} \mathrm{~F}_{3}$ & $5 p$ & $(3 / 2)^{2}[5 / 2]^{\circ} 2$ & & & $\mathrm{~F}$ & & \\
\hline 1957.5176(3) & $51085.108(8)$ & 1957.51767(7) & -0.0000 & 190000 & & $s^{2}$ & ${ }^{3} \mathrm{~F}_{4}$ & $5 p$ & $(5 / 2)^{2}[7 / 2]^{\circ} 4$ & & & $\mathrm{~F}$ & & \\
\hline 1968.008(3) & $50812.79(8)$ & $1968.01129(8)$ & -0.003 & 9700 & & $4 p$ & ${ }^{3} \mathrm{P}^{\circ}{ }_{2}$ & $4 \mathrm{~d}$ & $(3 / 2)^{2}[1 / 2]_{1}$ & $1.8 \mathrm{e}+07$ & $\mathrm{C}$ & TW & $\mathrm{O} 07$ & \\
\hline 1970.4936(6) & 50748.707(15) & 1970.49395(7) & -0.0004 & 760000 & & $4 \mathrm{~s}$ & ${ }^{3} \mathrm{D}_{2}$ & $4 p$ & ${ }^{1} \mathrm{P}_{1}^{\circ}$ & $5.3 e+06$ & $\mathrm{C}+$ & $\mathrm{F}$ & K82cor & \\
\hline $1974.467(3)$ & $50646.57(8)$ & 1974.46773(8) & -0.000 & 2800 & & $\mathrm{~s}^{2}$ & ${ }^{1} \mathrm{D}_{2}$ & $4 \mathrm{f}$ & $(5 / 2)^{2}[5 / 2]^{\circ} 3$ & & & TW & & \\
\hline 1977.0266(3) & $50581.009(6)$ & 1977.02671(15) & -0.0001 & 80000 & & $s^{2}$ & ${ }^{3} \mathrm{~F}_{2}$ & $5 p$ & $(3 / 2)^{2}[3 / 2]^{\circ}{ }_{1}$ & & & $\mathrm{~F}$ & & \\
\hline $1979.95578(4)$ & $50506.1785(10)$ & 1979.95579(3) & -0.00002 & 18000000 & & $4 \mathrm{~s}$ & ${ }^{3} \mathrm{D}_{2}$ & $4 p$ & ${ }^{3} \mathrm{D}_{2}^{\circ}$ & $9.6 e+07$ & $\mathrm{C}+$ & $\mathrm{F}$ & D05se & \\
\hline $1982.1503(20)$ & $50450.26(5)$ & $1982.14827(12)$ & 0.0021 & 16000 & & $s^{2}$ & ${ }^{3} \mathrm{~F}_{3}$ & sp & $\left({ }^{3} \mathrm{~F}\right)^{3} \mathrm{P}^{\circ}{ }^{1} \mathrm{D}^{\circ}{ }_{2}$ & & & F & & \\
\hline 1984.763(3) & $50383.85(8)$ & 1984.76431(19) & -0.001 & 14000 & & $s^{2}$ & ${ }^{3} \mathrm{P}_{2}$ & $6 \mathrm{p}$ & $(5 / 2)^{2}[3 / 2]^{\circ} 2$ & & & TW & & \\
\hline 1986.307(3) & $50344.68(8)$ & 1986.30794(14) & -0.001 & 8100 & & $s^{2}$ & ${ }^{1} \mathrm{D}_{2}$ & $\begin{array}{l}1 \\
\mathrm{sp}\end{array}$ & $\left({ }^{3} \mathrm{~F}\right)^{1} \mathrm{P}^{\circ}{ }^{3} \mathrm{D}^{\circ}{ }_{3}$ & & & TW & & \\
\hline 1989.85478(3) & $50254.9236(9)$ & $1989.85480(3)$ & -0.00001 & 7400000 & & $4 \mathrm{~s}$ & ${ }^{3} \mathrm{D}_{2}$ & $4 p$ & ${ }^{3} \mathrm{D}^{\circ}{ }_{1}$ & $9.8 \mathrm{e}+07$ & $\mathrm{C}+$ & $\mathrm{F}$ & D05 & \\
\hline 1990.18000(19) & $50246.711(5)$ & 1990.18009(7) & -0.00009 & 670000 & & $4 p$ & ${ }^{3} \mathrm{P}^{\circ}{ }_{1}$ & $4 \mathrm{~d}$ & $(3 / 2)^{2}[3 / 2]_{2}$ & $1.3 e+08$ & $\mathrm{C}$ & F & B00 & \\
\hline 1993.8395(21) & 50154.49(5) & $1993.8395(12)$ & -0.0000 & 34000 & & $\mathrm{~s}^{2}$ & ${ }^{3} \mathrm{P}_{2}$ & $\mathrm{sp}$ & $\left({ }^{3} \mathrm{P}\right)^{3} \mathrm{P}^{\circ}{ }^{3} \mathrm{~S}^{\circ}{ }_{1}$ & & & TWn & & \\
\hline 1994.257(3) & $50143.98(8)$ & $1994.25958(15)$ & -0.002 & 17000 & & $s^{2}$ & ${ }^{3} \mathrm{~F}_{2}$ & $5 p$ & $(3 / 2)^{2}[1 / 2]^{\circ}{ }_{1}$ & & & TW & & \\
\hline 1998.5575(16) & $50036.09(4)$ & 1998.55790(7) & -0.0004 & 34000 & & $4 p$ & ${ }^{3} \mathrm{~F}_{3}^{\circ}$ & $4 \mathrm{~d}$ & $(3 / 2)^{2}[5 / 2]_{3}$ & & & $\mathrm{~F}$ & & \\
\hline 1999.5327(18) & $50011.69(5)$ & 1999.53361(8) & -0.0009 & 140000 & & $4 p$ & ${ }^{3} \mathrm{P}^{\circ}{ }_{1}$ & $4 \mathrm{~d}$ & $(3 / 2)^{2}[3 / 2]_{1}$ & $5.8 \mathrm{e}+07$ & C & TW & B00 & \\
\hline $1999.69752(6)$ & 49991.3640(16) & 1999.69754(5) & -0.00002 & 20000000 & & $4 \mathrm{~s}$ & ${ }^{3} \mathrm{D}_{3}$ & $4 p$ & ${ }^{3} \mathrm{D}_{3}$ & $2.13 e+08$ & B & $\mathrm{F}$ & $\mathrm{C} 94 \mathrm{c}$ & \\
\hline 2002.9033(20) & 49911.36(5) & 2002.9020(12) & 0.0013 & 73000 & & $s^{2}$ & ${ }^{3} \mathrm{P}_{1}$ & $\mathrm{sp}$ & $\left({ }^{3} \mathrm{P}\right)^{3} \mathrm{P}^{\circ}{ }^{3} \mathrm{~S}^{\circ}{ }_{1}$ & & & TWn & & \\
\hline
\end{tabular}


Table A1. Cont.

\begin{tabular}{|c|c|c|c|c|c|c|c|c|c|c|c|c|c|c|}
\hline \multirow{2}{*}{$\frac{\lambda_{\text {obs }}{ }^{\text {a }}(\AA)}{2009.32(15)}$} & \multirow{2}{*}{$\begin{array}{l}\sigma_{\text {obs }}{ }^{\mathbf{b}}\left(\mathbf{c m}^{-\mathbf{1}}\right) \\
49752(4)\end{array}$} & \multirow{2}{*}{$\begin{array}{c}\lambda_{\text {Ritz }}{ }^{c}(\AA) \\
2009.28239(8)\end{array}$} & \multirow{2}{*}{$\begin{array}{l}\begin{array}{c}\Delta \lambda_{\text {obs-Ritz }} \\
(\mathrm{A})\end{array} \\
0.04\end{array}$} & \multirow{2}{*}{$\begin{array}{c}\begin{array}{c}I_{\text {obs }}{ }^{d} \\
\text { (arb. u.) }\end{array} \\
6800\end{array}$} & \multirow[t]{2}{*}{ Char ${ }^{\mathrm{e}}$} & \multicolumn{2}{|c|}{ Lower Level } & \multicolumn{2}{|c|}{ Upper Level } & \multirow[t]{2}{*}{$A\left(\mathrm{~s}^{-1}\right)$} & \multirow[t]{2}{*}{$\operatorname{Acc}^{f}$} & \multirow{2}{*}{$\begin{array}{l}\text { Line Ref. } \mathrm{g} \\
\text { S36c }\end{array}$} & \multirow[t]{2}{*}{ TP Ref. $\mathrm{g}$} & \multirow[t]{2}{*}{ Notes $^{h}$} \\
\hline & & & & & & $4 p$ & ${ }^{3} \mathrm{~F}_{4}^{\circ}$ & $4 \mathrm{~d}$ & $(3 / 2)^{2}[5 / 2]_{3}$ & & & & & \\
\hline 2012.98037(14) & 49661.540(4) & 2012.98041(6) & -0.00004 & 1800000 & & $4 \mathrm{p}$ & ${ }^{3} \mathrm{P}^{\circ}{ }_{2}$ & $4 \mathrm{~d}$ & $(5 / 2)^{2}[5 / 2]_{3}$ & $2.5 e+08$ & C & $\mathrm{F}$ & B00 & \\
\hline 2015.5822(4) & 49597.442(10) & 2015.58222(8) & 0.0000 & 1400000 & & $4 \mathrm{~s}$ & ${ }^{3} \mathrm{D}_{1}$ & $4 p$ & ${ }^{1} \mathrm{P}^{\circ}{ }_{1}$ & $2.1 \mathrm{e}+07$ & $\mathrm{C}+$ & $\mathrm{F}$ & K82cor & \\
\hline $2015.8660(24)$ & $49590.46(6)$ & 2015.8649(12) & 0.0011 & 39000 & & $s^{2}$ & ${ }^{3} \mathrm{P}_{0}$ & $\mathrm{sp}$ & $\left({ }^{3} \mathrm{P}\right)^{3} \mathrm{P}^{\circ}{ }^{3} \mathrm{~S}^{\circ}{ }_{1}$ & & & TWn & & \\
\hline $2016.8964(6)$ & $49565.129(14)$ & 2016.89673(6) & -0.0003 & 1700000 & & $4 \mathrm{~s}$ & ${ }^{3} \mathrm{D}_{3}$ & $4 \mathrm{p}$ & ${ }^{1} \mathrm{D}_{2}^{\circ}$ & $8.1 \mathrm{e}+06$ & $\mathrm{C}+$ & $\mathrm{F}$ & D05se & \\
\hline 2017.6096(16) & 49547.61(4) & 2017.60977(12) & -0.0002 & 160000 & & $s^{2}$ & ${ }^{3} \mathrm{~F}_{3}$ & $5 p$ & $(5 / 2)^{2}[5 / 2]_{3}^{\circ}$ & & & F & & \\
\hline 2022.1913(16) & 49435.37(4) & $2022.19147(7)$ & -0.0002 & 170000 & & $4 p$ & ${ }^{3} \mathrm{~F}_{3}^{\circ}$ & $4 \mathrm{~d}$ & $(3 / 2)^{2}[7 / 2]_{4}$ & $1.14 \mathrm{e}+07$ & $\mathrm{C}+$ & $\mathrm{F}$ & $\mathrm{O} 07$ & \\
\hline $2025.48773(12)$ & 49354.924(3) & 2025.48775(5) & -0.00001 & 8100000 & & $\begin{array}{l}1 \\
4 \mathrm{~s}\end{array}$ & ${ }^{3} \mathrm{D}_{1}$ & $4 p$ & ${ }^{3} \mathrm{D}_{2}^{\circ}$ & $6.8 \mathrm{e}+07$ & $\mathrm{C}+$ & $\mathrm{F}$ & D05se & \\
\hline 2025.919(3) & $49344.43(8)$ & $2025.91683(13)$ & 0.002 & 25000 & & $s^{2}$ & ${ }^{3} \mathrm{~F}_{3}$ & $5 p$ & $(5 / 2)^{2}[5 / 2]_{2}^{\circ}$ & & & TW & & \\
\hline 2027.1336(6) & 49314.858(15) & 2027.13416(9) & -0.0005 & 610000 & & $4 p$ & ${ }^{3} \mathrm{P}^{\circ}{ }_{1}$ & $4 \mathrm{~d}$ & $(3 / 2)^{2}[1 / 2]_{1}$ & $1.3 e+08$ & C & $\mathrm{F}$ & O07se & \\
\hline $2029.471(3)$ & $49258.06(8)$ & $2029.47227(16)$ & -0.001 & 37000 & & $\mathrm{~s}^{2}$ & ${ }^{3} \mathrm{~F}_{2}$ & $\mathrm{sp}$ & $\left({ }^{3} \mathrm{~F}\right)^{3} \mathrm{P}^{\circ}{ }^{1} \mathrm{D}^{\circ}{ }_{2}$ & & & TW & & \\
\hline 2029.9491(7) & $49246.469(17)$ & $2029.94908(6)$ & 0.0000 & 560000 & & $4 p$ & ${ }^{3} \mathrm{P}^{\circ}{ }_{2}$ & $4 \mathrm{~d}$ & $(5 / 2)^{2}[3 / 2]_{1}$ & $8.0 \mathrm{e}+07$ & B & $\mathrm{F}$ & $\mathrm{O} 07$ & \\
\hline 2031.03579(16) & $49220.123(4)$ & 2031.03598(6) & -0.00020 & 2700000 & & $4 p$ & ${ }^{3} \mathrm{P}_{2}^{\circ}$ & $4 \mathrm{~d}$ & $(5 / 2)^{2}[3 / 2]_{2}$ & $4.5 \mathrm{e}+08$ & B & $\mathrm{F}$ & $\mathrm{O} 07$ & \\
\hline 2033.844(3) & 49152.18(8) & 2033.84242(8) & 0.001 & 62000 & & $4 p$ & ${ }^{3} \mathrm{~F}^{\circ} 4$ & $4 \mathrm{~d}$ & $(3 / 2)^{2}[7 / 2]_{4}$ & $8.9 e+06$ & $\mathrm{C}+$ & TW & $\mathrm{O} 07$ & \\
\hline 2035.85321(6) & 49103.6704(15) & $2035.85327(4)$ & -0.00006 & 19000000 & & $4 \mathrm{~s}$ & ${ }^{3} \mathrm{D}_{1}$ & $4 p$ & ${ }^{3} \mathrm{D}^{\circ}{ }_{1}$ & $3.7 \mathrm{e}+08$ & $\mathrm{C}+$ & $\mathrm{F}$ & D05 & \\
\hline 2036.9201(3) & 49077.954(8) & 2036.92001(9) & 0.0001 & 730000 & & $4 p$ & ${ }^{3} \mathrm{P}_{0}^{\circ}$ & $4 d$ & $(3 / 2)^{2}[3 / 2]_{1}$ & $1.9 \mathrm{e}+08$ & $\mathrm{C}$ & $\mathrm{F}$ & B00 & \\
\hline 2037.12672(4) & $49072.9776(9)$ & 2037.12668(3) & 0.00004 & 13000000 & & $4 \mathrm{~s}$ & ${ }^{3} \mathrm{D}_{2}$ & $4 \mathrm{p}$ & ${ }^{3} \mathrm{D}^{\circ}{ }_{3}$ & $1.36 e+08$ & B & $\mathrm{F}$ & C94c & \\
\hline 2043.8019(5) & 48912.725(11) & 2043.80148(7) & 0.0004 & 21000000 & & $4 \mathrm{~s}$ & ${ }^{3} \mathrm{D}_{3}$ & $4 \mathrm{p}$ & ${ }^{1} \mathrm{~F}_{3}^{\circ}$ & $1.42 \mathrm{e}+08$ & $\mathrm{C}+$ & R1c & D05se & \\
\hline $2047.67825(16)$ & $48820.143(4)$ & 2047.67825(11) & -0.00000 & 1300000 & & $4 p$ & ${ }^{1} \mathrm{P}^{\circ}{ }_{1}$ & $4 \mathrm{~d}$ & $(3 / 2)^{2}[1 / 2]_{0}$ & $5.32 \mathrm{e}+08$ & $\mathrm{~B}+$ & $\mathrm{F}$ & B09 & \\
\hline $2054.25259(21)$ & $48663.922(5)$ & 2054.25263(8) & -0.00003 & 810000 & & $4 p$ & ${ }^{3} \mathrm{~F}_{2}^{\circ}$ & $4 \mathrm{~d}$ & $(3 / 2)^{2}[5 / 2]_{2}$ & $1.4 \mathrm{e}+08$ & C & $\mathrm{F}$ & B00 & \\
\hline 2054.4171(3) & $48660.025(7)$ & $2054.41739(8)$ & -0.0003 & 960000 & & $4 p$ & ${ }^{3} \mathrm{P}^{\circ}{ }_{1}$ & $4 \mathrm{~d}$ & $(5 / 2)^{2}[1 / 2]_{0}$ & $6.7 \mathrm{e}+08$ & C & $\mathrm{F}$ & B00 & \\
\hline $2054.97836(4)$ & $48646.7370(10)$ & 2054.97836(3) & 0.00001 & 18000000 & & $4 \mathrm{~s}$ & ${ }^{3} \mathrm{D}_{2}$ & $4 p$ & ${ }^{1} \mathrm{D}^{\circ}{ }_{2}$ & $1.65 \mathrm{e}+08$ & $\mathrm{C}+$ & $\mathrm{F}$ & D05se & \\
\hline 2058.611(3) & $48560.91(8)$ & 2058.61377(13) & -0.003 & 49000 & & $s^{2}$ & ${ }^{3} \mathrm{~F}_{3}$ & $5 p$ & $(5 / 2)^{2}[3 / 2]^{\circ} 2$ & & & TW & & \\
\hline 2062.4201(4) & $48471.230(8)$ & $2062.42011(6)$ & -0.0000 & 1500000 & & $4 p$ & ${ }^{3} \mathrm{P}^{\circ}{ }_{1}$ & $4 d$ & $(5 / 2)^{2}[5 / 2]_{2}$ & $1.7 \mathrm{e}+08$ & $\mathrm{C}$ & $\mathrm{F}$ & B00 & \\
\hline 2066.2609(5) & 48381.143(12) & 2066.26109(10) & -0.0002 & 960000 & & $4 p$ & ${ }^{3} \mathrm{P}_{0}^{\circ}{ }_{0}$ & $4 \mathrm{~d}$ & $(3 / 2)^{2}[1 / 2]_{1}$ & $2.00 \mathrm{e}+08$ & $\mathrm{~B}+$ & $\mathrm{F}$ & O07 & \\
\hline 2067.368(3) & $48355.23(7)$ & 2067.36392(16) & 0.004 & 35000 & & $\mathrm{~s}^{2}$ & ${ }^{3} \mathrm{~F}_{2}$ & $5 p$ & $(5 / 2)^{2}[5 / 2]^{\circ}{ }_{3}$ & & & TW & & \\
\hline 2069.9356(13) & $48295.27(3)$ & $2069.93487(7)$ & 0.0007 & 240000 & & $4 p$ & ${ }^{3} \mathrm{~F}^{\circ}{ }_{2}$ & $4 \mathrm{~d}$ & $(3 / 2)^{2}[3 / 2]_{2}$ & $3.7 \mathrm{e}+07$ & C & $\mathrm{F}$ & B00 & \\
\hline \multirow[t]{2}{*}{ 2074.213(3) } & $48195.68(8)$ & 2074.20995(16) & 0.003 & 39000 & & $\mathrm{~s}^{2}$ & ${ }^{3} \mathrm{~F}_{2}$ & $5 p$ & $(5 / 2)^{2}[3 / 2]_{1}^{\circ}$ & & & TW & & \\
\hline & & $2074.24066(15)$ & & & $\mathrm{m}$ & $s^{2}$ & ${ }^{3} \mathrm{P}_{1}$ & $\mathrm{sp}$ & $\left({ }^{3} \mathrm{~F}\right)^{1} \mathrm{P}^{\circ}{ }^{3} \mathrm{D}_{2}^{\circ}$ & & & TW & & \\
\hline 2078.66147(9) & $48092.5549(21)$ & 2078.66153(6) & -0.00005 & 6700000 & & $4 p$ & ${ }^{3} \mathrm{P}_{2}^{\circ}$ & $4 \mathrm{~d}$ & $(5 / 2)^{2}[1 / 2]_{1}$ & $6.3 e+08$ & $\mathrm{~B}+$ & $\mathrm{F}$ & $\mathrm{O} 07$ & \\
\hline 2080.0593(13) & $48060.24(3)$ & 2080.05953(9) & -0.0002 & 210000 & & $4 p$ & ${ }^{3} \mathrm{~F}_{2}^{\circ}$ & $4 \mathrm{~d}$ & $(3 / 2)^{2}[3 / 2]_{1}$ & $4.9 \mathrm{e}+07$ & C & R1c & B00 & \\
\hline 2082.920(20) & 47994.2(5) & 2082.91535(6) & 0.005 & 56000 & & $4 \mathrm{~s}$ & ${ }^{3} \mathrm{D}_{2}$ & $4 p$ & ${ }^{1} \mathrm{~F}_{3}^{\circ}$ & $1.0 \mathrm{e}+06$ & $\mathrm{E}$ & S36c & D05se & \\
\hline $2084.3229(20)$ & 47961.94(5) & $2084.32369(22)$ & -0.0008 & 170000 & & $s^{2}$ & ${ }^{3} \mathrm{~F}_{4}$ & $\mathrm{sp}$ & $\left({ }^{3} \mathrm{~F}\right)^{3} \mathrm{P}^{\circ}{ }^{3} \mathrm{~F}_{4}^{\circ}$ & & & TW & & \\
\hline 2085.2735(9) & 47940.081(21) & 2085.27476(6) & -0.0012 & 860000 & & $4 p$ & ${ }^{3} \mathrm{~F}_{3}^{\circ}$ & $4 \mathrm{~d}$ & $(5 / 2)^{2}[5 / 2]_{2}$ & $6.7 \mathrm{e}+07$ & C & R1c & B00 & \\
\hline 2085.297(4) & 47939.55(9) & $2085.30956(7)$ & -0.013 & 2500000 & & $4 \mathrm{~s}$ & ${ }^{3} \mathrm{D}_{3}$ & $4 p$ & ${ }^{3} \mathrm{~F}_{2}^{\circ}$ & $4.1 \mathrm{e}+06$ & $\mathrm{C}+$ & TW & D05cor & \\
\hline 2087.91812(11) & $47879.3666(25)$ & 2087.91817(7) & -0.00005 & 3400000 & & $4 p$ & ${ }^{3} \mathrm{~F}_{2}^{\circ}$ & $4 \mathrm{~d}$ & $(3 / 2)^{2}[7 / 2]_{3}$ & $4.2 \mathrm{e}+08$ & B & $\mathrm{F}$ & B00 & \\
\hline $2087.96979(24)$ & 47878.182(5) & 2087.96987(6) & -0.00008 & 3700000 & & $4 p$ & ${ }^{3} \mathrm{~F}_{3}^{\circ}$ & $4 d$ & $(5 / 2)^{2}[7 / 2]_{3}$ & $2.6 \mathrm{e}+08$ & C & $\mathrm{F}$ & B00 & \\
\hline 2093.6366(5) & 47748.606(11) & 2093.63705(6) & -0.0004 & 2400000 & & $4 p$ & ${ }^{3} \mathrm{P}^{\circ}{ }_{1}$ & $4 \mathrm{~d}$ & $(5 / 2)^{2}[3 / 2]_{1}$ & $2.20 \mathrm{e}+08$ & B & R1c & $\mathrm{O} 07$ & \\
\hline $2094.7925(9)$ & $47722.261(21)$ & 2094.79321(6) & -0.0007 & 290000 & & $4 p$ & ${ }^{3} \mathrm{P}^{\circ}{ }_{1}$ & $4 \mathrm{~d}$ & $(5 / 2)^{2}[3 / 2]_{2}$ & $2.21 \mathrm{e}+07$ & $\mathrm{C}+$ & R1c & $\mathrm{O} 07$ & \\
\hline 2096.1891(14) & $47690.47(3)$ & 2096.19052(9) & -0.0014 & 120000 & & $4 p$ & ${ }^{1} \mathrm{~F}_{3}^{\circ}$ & $4 \mathrm{~d}$ & $(3 / 2)^{2}[5 / 2]_{2}$ & $1.6 \mathrm{e}+07$ & C & R1c & B00 & \\
\hline
\end{tabular}


Table A1. Cont.

\begin{tabular}{|c|c|c|c|c|c|c|c|c|c|c|c|c|c|c|}
\hline \multirow{2}{*}{$\begin{array}{r}\lambda_{\text {obs }}{ }^{\text {a }}(\AA ̊) \\
2098.3067(3)\end{array}$} & \multirow{2}{*}{$\begin{array}{l}\sigma_{\text {obs }}{ }^{\mathbf{b}}\left(\mathbf{c m}^{-\mathbf{1}}\right) \\
47642.348(7)\end{array}$} & \multirow{2}{*}{$\begin{array}{r}\lambda_{\text {Ritz }}{ }^{c}(\AA) \\
2098.30677(8)\end{array}$} & \multirow{2}{*}{$\begin{array}{c}\Delta \lambda_{\text {obs-Ritz }} \\
(\AA)\end{array}$} & \multirow{2}{*}{$\begin{array}{c}\begin{array}{c}I_{\text {obs }} \mathrm{d} \\
\text { (arb. u.) }\end{array} \\
1200000\end{array}$} & \multirow[t]{2}{*}{ Char ${ }^{\mathrm{e}}$} & \multicolumn{2}{|c|}{ Lower Level } & \multicolumn{2}{|c|}{ Upper Level } & \multirow{2}{*}{$\begin{array}{r}A\left(\mathrm{~s}^{-1}\right) \\
1.01 \mathrm{e}+08\end{array}$} & \multirow{2}{*}{$\begin{array}{l}\operatorname{Acc}^{\mathrm{f}} \\
\mathrm{C}\end{array}$} & \multirow{2}{*}{$\begin{array}{l}\text { Line Ref. } g \\
\text { F }\end{array}$} & \multirow{2}{*}{$\begin{array}{l}\text { TP Ref. }{ }^{\mathrm{g}} \\
\text { B00 }\end{array}$} & \multirow[t]{2}{*}{ Notes ${ }^{h}$} \\
\hline & & & & & & $4 p$ & ${ }^{1} \mathrm{~F}_{3}^{\circ}$ & $4 \mathrm{~d}$ & $(3 / 2)^{2}[5 / 2]_{3}$ & & & & & \\
\hline 2098.3971(2) & $47640.295(6)$ & 2098.39724(8) & -0.0001 & 5000000 & & $4 p$ & ${ }^{3} \mathrm{~F}_{4}^{\circ}$ & $4 \mathrm{~d}$ & $(5 / 2)^{2}[7 / 2]_{4}$ & $2.09 \mathrm{e}+08$ & $\mathrm{~B}$ & $\mathrm{~F}$ & $\mathrm{O} 07$ & \\
\hline 2098.7405(9) & $47632.502(21)$ & $2098.74108(6)$ & -0.0006 & 300000 & & $4 p$ & ${ }^{3} \mathrm{~F}_{3}^{\circ}$ & $4 \mathrm{~d}$ & $(5 / 2)^{2}[5 / 2]_{3}$ & $2.0 \mathrm{e}+07$ & $\mathrm{C}$ & R1c & B00 & \\
\hline $2100.397(3)$ & $47594.94(7)$ & $2100.39318(8)$ & 0.004 & 53000 & & $4 p$ & ${ }^{3} \mathrm{~F}_{4}^{\circ}$ & $4 \mathrm{~d}$ & $(5 / 2)^{2}[7 / 2]_{3}$ & & & TW & & \\
\hline 2104.79587(5) & $47495.4827(11)$ & 2104.79586(4) & 0.00002 & 62000000 & & $4 \mathrm{~s}$ & ${ }^{3} \mathrm{D}_{1}$ & $4 p$ & ${ }^{1} \mathrm{D}^{\circ}{ }_{2}$ & $7.7 \mathrm{e}+07$ & $\mathrm{C}+$ & $\mathrm{F}$ & D05se & \\
\hline $2106.3816(11)$ & $47459.73(3)$ & 2106.3791(3) & 0.0025 & 240000 & & $s^{2}$ & ${ }^{3} \mathrm{~F}_{3}$ & $\mathrm{sp}$ & $\left({ }^{3} \mathrm{~F}\right)^{3} \mathrm{P}^{\circ}{ }^{1} \mathrm{G}_{4}^{\circ}$ & & & $\mathrm{F}$ & & \\
\hline $2110.308(3)$ & $47371.45(7)$ & $2110.30833(15)$ & -0.001 & 90000 & & $s^{2}$ & ${ }^{3} \mathrm{P}_{2}$ & $\mathrm{sp}$ & $\left({ }^{3} \mathrm{~F}\right)^{1} \mathrm{P}^{\circ}{ }^{3} \mathrm{D}^{\circ}{ }_{3}$ & & & TW & & \\
\hline 2111.2934(4) & $47349.333(10)$ & $2111.29323(8)$ & 0.0001 & 860000 & & $4 p$ & ${ }^{3} \mathrm{~F}_{4}^{\circ}$ & $4 \mathrm{~d}$ & $(5 / 2)^{2}[5 / 2]_{3}$ & $6.6 \mathrm{e}+07$ & $\mathrm{C}$ & R1c & B00 & \\
\hline $2112.09930(19)$ & $47331.268(4)$ & $2112.09946(9)$ & -0.00016 & 55000000 & & $4 \mathrm{~s}$ & ${ }^{1} \mathrm{D}_{2}$ & $4 p$ & ${ }^{1} \mathrm{P}^{\circ}{ }_{1}$ & $3.5 \mathrm{e}+08$ & $\mathrm{C}+$ & $\mathrm{F}$ & D05se & \\
\hline $2112.5245(14)$ & 47321.74(3) & $2112.52195(8)$ & 0.0026 & 110000 & & $4 p$ & ${ }^{1} \mathrm{~F}_{3}^{\circ}$ & $4 \mathrm{~d}$ & $(3 / 2)^{2}[3 / 2]_{2}$ & $1.2 \mathrm{e}+07$ & $\mathrm{C}$ & R1c & B00 & \\
\hline 2117.30881(9) & $47214.8259(20)$ & 2117.30874(7) & 0.00006 & 12000000 & & $4 p$ & ${ }^{3} \mathrm{~F}_{3}^{\circ}$ & $4 \mathrm{~d}$ & $(5 / 2)^{2}[9 / 2]_{4}$ & $7.2 \mathrm{e}+08$ & B & $\mathrm{F}$ & $\mathrm{O} 07$ & \\
\hline $2118.3748(20)$ & 47191.07(5) & $2118.37484(7)$ & -0.0001 & 260000 & & $4 p$ & ${ }^{3} \mathrm{~F}_{3}^{\circ}$ & $4 \mathrm{~d}$ & $(5 / 2)^{2}[3 / 2]_{2}$ & $2.7 \mathrm{e}+07$ & B & TW & $\mathrm{O} 07$ & \\
\hline $2122.97861(13)$ & $47088.745(3)$ & $2122.97858(6)$ & 0.00003 & 71000000 & & $4 \mathrm{~s}$ & ${ }^{1} \mathrm{D}_{2}$ & $4 p$ & ${ }^{3} \mathrm{D}^{\circ}{ }_{2}$ & $2.2 \mathrm{e}+08$ & $\mathrm{C}+$ & $\mathrm{F}$ & D05se & \\
\hline $2125.10512(12)$ & 47041.631(3) & $2125.10507(7)$ & 0.00004 & 3800000 & & $4 p$ & ${ }^{1} \mathrm{~F}_{3}^{\circ}$ & $4 \mathrm{~d}$ & $(3 / 2)^{2}[7 / 2]_{4}$ & $2.28 \mathrm{e}+08$ & $\mathrm{~B}+$ & $\mathrm{F}$ & $\mathrm{O} 07$ & \\
\hline $2125.2670(5)$ & $47038.048(12)$ & $2125.26686(8)$ & 0.0001 & 610000 & & $4 p$ & ${ }^{1} \mathrm{D}_{2}^{\circ}$ & $4 \mathrm{~d}$ & $(3 / 2)^{2}[5 / 2]_{2}$ & $6.6 \mathrm{e}+07$ & $\mathrm{C}$ & $\mathrm{F}$ & B00 & \\
\hline $2126.04361(6)$ & $47020.8676(14)$ & $2126.04362(5)$ & -0.00001 & 74000000 & & $4 \mathrm{~s}$ & ${ }^{3} \mathrm{D}_{2}$ & $4 p$ & ${ }^{3} \mathrm{~F}_{2}^{\circ}$ & $1.41 \mathrm{e}+08$ & B & $\mathrm{F}$ & $\mathrm{C} 94 \mathrm{c}$ & \\
\hline $2130.0848(5)$ & $46931.669(10)$ & $2130.08459(9)$ & 0.0002 & 1000000 & & $4 p$ & ${ }^{3} \mathrm{~F}^{\circ}$ & $4 \mathrm{~d}$ & $(5 / 2)^{2}[9 / 2]_{4}$ & $4.4 \mathrm{e}+07$ & $\mathrm{~B}+$ & R1c & $\mathrm{O} 07$ & \\
\hline $2131.2548(6)$ & $46905.908(13)$ & $2131.25595(8)$ & -0.0011 & 190000 & & $4 p$ & ${ }^{1} \mathrm{~F}_{3}^{\circ}{ }_{3}^{4}$ & $4 \mathrm{~d}$ & $(3 / 2)^{2}[7 / 2]_{3}$ & $1.3 \mathrm{e}+07$ & $\mathrm{C}$ & R1c & B00 & \\
\hline $2134.3400(4)$ & $46838.113(9)$ & $2134.34030(8)$ & -0.0003 & 9500000 & & $4 p$ & ${ }^{3} \mathrm{~F}_{4}^{\circ}$ & $4 \mathrm{~d}$ & $(5 / 2)^{2}[9 / 2]_{5}$ & $7.7 \mathrm{e}+08$ & B & R1c & B00 & \\
\hline 2135.399 & 46814.89 & $2135.39887(8)$ & & & : & $4 p$ & ${ }^{3} \mathrm{P}_{0}^{\circ}{ }_{0}^{4}$ & $4 \mathrm{~d}$ & $(5 / 2)^{2}[3 / 2]_{1}$ & $4 . \mathrm{e}+07$ & $\mathrm{E}$ & & O07se & \\
\hline $2135.98008(7)$ & $46802.1541(16)$ & $2135.98004(6)$ & 0.00004 & 140000000 & & $4 \mathrm{~s}$ & ${ }^{3} \mathrm{D}_{3}$ & $4 p$ & ${ }^{3} \mathrm{~F}^{\circ}{ }_{4}$ & $4.59 \mathrm{e}+08$ & A+ & $\mathrm{F}$ & P97 & \\
\hline 2144.706(10) & $46611.76(22)$ & $2144.70358(8)$ & 0.002 & 160000 & * & $4 p$ & ${ }^{3} \mathrm{D}_{3}^{\circ}$ & $4 \mathrm{~d}$ & $(3 / 2)^{2}[5 / 2]_{2}$ & $1.5 \mathrm{e}+07$ & C & R1c & B00 & \\
\hline $2144.706(10)$ & $46611.76(22)$ & $2144.72206(16)$ & -0.016 & 160000 & * & $s^{2}$ & ${ }^{3} \mathrm{~F}_{3}$ & $\mathrm{sp}$ & $\left({ }^{3} \mathrm{~F}\right)^{3} \mathrm{P}^{\circ} 3^{3} \mathrm{~F}_{3}^{\circ}$ & & & R1c & & \\
\hline $2145.4920(4)$ & 46594.683(9) & $2145.49206(7)$ & -0.0001 & 1200000 & & $4 p$ & ${ }^{3} \mathrm{P}_{1}^{\circ}$ & $4 \mathrm{~d}$ & $(5 / 2)^{2}[1 / 2]_{1}$ & $1.14 \mathrm{e}+08$ & B & R1c & $\mathrm{O} 07$ & \\
\hline \multirow[t]{2}{*}{$2146.9187(4)$} & $46563.722(8)$ & $2146.91894(7)$ & -0.0002 & 1100000 & & $4 p$ & ${ }^{3} \mathrm{D}_{3}^{\circ}$ & $4 \mathrm{~d}$ & $(3 / 2)^{2}[5 / 2]_{3}$ & $1.04 \mathrm{e}+08$ & C & $\mathrm{F}$ & B00 & \\
\hline & & 2148.94587(6) & & & $\mathrm{m}$ & $4 p$ & ${ }^{3} \mathrm{~F}_{2}^{\circ}{ }_{2}^{3}$ & $4 \mathrm{~d}$ & $(5 / 2)^{2}[5 / 2]_{2}$ & $1.12 \mathrm{e}+08$ & $\mathrm{C}$ & S36c & B00 & \\
\hline 2148.9830(6) & $46518.999(13)$ & $2148.98283(8)$ & 0.0002 & 59000000 & & $4 \mathrm{~s}$ & ${ }^{3} \mathrm{D}_{3}$ & $4 p$ & ${ }^{3} \mathrm{~F}^{\circ}{ }_{3}$ & $8.8 \mathrm{e}+07$ & B & $\mathrm{R} 1 \mathrm{c}$ & C94c & \\
\hline 2151.8083(4) & 46457.927(9) & 2151.80815(7) & 0.0001 & 4200000 & & $4 p$ & ${ }^{3} \mathrm{~F}^{\circ}{ }_{2}$ & $4 \mathrm{~d}$ & $(5 / 2)^{2}[7 / 2]_{3}$ & $2.59 \mathrm{e}+08$ & $\mathrm{~B}$ & R1c & B00 & \\
\hline 2152.910(20) & $46434.2(4)$ & $2152.90029(9)$ & 0.010 & 23000 & & $4 \mathrm{p}$ & ${ }^{1} \mathrm{D}^{\circ}{ }_{2}$ & $4 \mathrm{~d}$ & $(3 / 2)^{2}[3 / 2]_{1}$ & & & S36c & & \\
\hline $2158.4108(10)$ & $46315.829(21)$ & $2158.41130(19)$ & -0.0005 & 60000 & & $s^{2}$ & ${ }^{3} \mathrm{~F}_{2}$ & $\mathrm{sp}$ & $\left({ }^{3} \mathrm{~F}\right)^{3} \mathrm{P}^{\circ} 3^{3} \mathrm{~F}^{\circ}{ }_{2}$ & & & R1c & & \\
\hline $2161.31978(23)$ & 46253.498(5) & $2161.31990(7)$ & -0.00012 & 4200000 & & $4 p$ & ${ }^{1} \mathrm{D}_{2}^{\circ}$ & $4 \mathrm{~d}$ & $(3 / 2)^{2}[7 / 2]_{3}$ & $3.1 \mathrm{e}+08$ & B & $\mathrm{F}$ & В00 & \\
\hline $2161.7998(10)$ & $46243.228(21)$ & $2161.80255(7)$ & -0.0027 & 58000 & & $4 p$ & ${ }^{3} \mathrm{D}_{3}{ }_{3}^{2}$ & $4 \mathrm{~d}$ & $(3 / 2)^{2}[3 / 2]_{2}$ & & & R1c & & \\
\hline $2166.850(20)$ & $46135.5(4)$ & $2166.8678(3)$ & -0.018 & 21000 & & $s^{2}$ & ${ }^{3} \mathrm{~F}_{3}$ & $\mathrm{sp}$ & $\left({ }^{3} \mathrm{~F}\right)^{3} \mathrm{P}^{\circ}{ }^{3} \mathrm{~F}^{\circ}{ }_{4}$ & & & S36c & & \\
\hline $2174.98119(9)$ & $45963.0038(18)$ & $2174.98126(6)$ & -0.00007 & 7500000 & & $4 p$ & ${ }^{3} \mathrm{D}_{3}^{\circ}$ & $4 \mathrm{~d}$ & $(3 / 2)^{2}[7 / 2]_{4}$ & $4.9 \mathrm{e}+08$ & B & $\mathrm{F}$ & $\mathrm{O} 07$ & \\
\hline $2179.41026(22)$ & $45869.606(5)$ & $2179.40994(6)$ & 0.00033 & 72000000 & & $4 \mathrm{~s}$ & ${ }^{3} \mathrm{D}_{1}$ & $4 p$ & ${ }^{3} \mathrm{~F}^{\circ}{ }_{2}$ & $2.15 \mathrm{e}+08$ & B & $\mathrm{F}$ & C $94 \mathrm{c}$ & \\
\hline $2180.7508(5)$ & $45841.413(11)$ & & & 680000 & & $s^{2}$ & ${ }^{3} \mathrm{~F}_{4}$ & $\mathrm{sp}$ & $\left({ }^{3} \mathrm{~F}\right)^{3} \mathrm{P}^{\circ}{ }^{3} \mathrm{G}^{\circ}{ }_{5}$ & & & R1c & & \\
\hline $2181.4243(4)$ & 45827.261(9) & $2181.42461(7)$ & -0.0003 & 290000 & & $4 p$ & ${ }^{3} \mathrm{D}_{3}^{\circ}$ & $4 \mathrm{~d}$ & $(3 / 2)^{2}[7 / 2]_{3}$ & $2.4 \mathrm{e}+07$ & $\mathrm{C}$ & R1c & B00 & \\
\hline $2182.8585(5)$ & 45797.154(11) & $2182.85780(7)$ & 0.0007 & 560000 & & $4 p$ & ${ }^{3} \mathrm{~F}_{2}^{\circ}$ & $4 \mathrm{~d}$ & $(5 / 2)^{2}[3 / 2]_{1}$ & $8.0 \mathrm{e}+07$ & $\mathrm{C}+$ & R1c & $\mathrm{O} 07$ & \\
\hline $2189.3693(10)$ & $45660.976(21)$ & $2189.36930(8)$ & 0.0000 & 240000 & & $4 \mathrm{p}$ & ${ }^{3} \mathrm{P}^{\circ}{ }_{0}^{2}$ & $4 \mathrm{~d}$ & $(5 / 2)^{2}[1 / 2]_{1}$ & $1.3 e+07$ & $\mathrm{C}$ & R1c & O07se & \\
\hline $2189.62967(9)$ & $45655.5468(18)$ & $2189.62971(6)$ & -0.00004 & 47000000 & & $4 \mathrm{~s}$ & ${ }^{1} \mathrm{D}_{2}$ & $4 p$ & ${ }^{3} \mathrm{D}^{\circ}{ }_{3}$ & $1.04 \mathrm{e}+08$ & B & $\mathrm{F}, \mathrm{R} 1 \mathrm{c}$ & $\mathrm{C} 94 \mathrm{c}$ & \\
\hline $2190.500(20)$ & $45637.4(4)$ & $2190.5192(3)$ & -0.019 & 57000 & & $\mathrm{~s}^{2}$ & ${ }^{1} \mathrm{G}_{4}$ & $6 \mathrm{p}$ & $(3 / 2)^{2}[5 / 2]^{\circ}{ }_{3}^{\circ}$ & & & S36c & & \\
\hline
\end{tabular}


Table A1. Cont.

\begin{tabular}{|c|c|c|c|c|c|c|c|c|c|c|c|c|c|c|}
\hline$\lambda_{\mathrm{obs}}{ }^{\mathrm{a}}(\AA)$ & $\sigma_{\mathrm{obs}}^{\mathrm{b}}\left(\mathrm{cm}^{-1}\right)$ & $\lambda_{\mathrm{Ritz}}{ }^{\mathrm{c}}(\AA)$ & $\Delta \lambda_{\text {obs-Ritz }}$ & $\begin{array}{c}I_{\text {obs }}{ }^{d} \\
\text { (arb. u.) }\end{array}$ & Char $^{e}$ & & Level & & pper Level & $A\left(\mathrm{~s}^{-1}\right)$ & $\operatorname{Acc}^{f}$ & Line Ref. $\mathrm{g}$ & TP Ref. ${ }^{g}$ & Notes ${ }^{h}$ \\
\hline 2192.26759(8) & $45600.6159(16)$ & $2192.26753(6)$ & 0.00007 & 73000000 & & $4 \mathrm{~s}$ & ${ }^{3} \mathrm{D}_{2}$ & $4 p$ & ${ }^{3} \mathrm{~F}_{3}^{\circ}$ & $2.8 \mathrm{e}+08$ & B & $\mathrm{F}$ & C94c & \\
\hline $2195.68180(17)$ & $45529.716(4)$ & 2195.68192(7) & -0.00012 & 4200000 & & $4 p$ & ${ }^{1} \mathrm{~F}_{3}^{\circ}$ & $4 \mathrm{~d}$ & $(5 / 2)^{2}[7 / 2]_{4}$ & $4.2 \mathrm{e}+08$ & B & $\mathrm{F}$ & $\mathrm{O} 07$ & \\
\hline 2197.8688(15) & $45484.42(3)$ & $2197.86726(7)$ & 0.0016 & 71000 & & $4 p$ & ${ }^{1} \mathrm{~F}_{3}^{\circ}$ & $4 \mathrm{~d}$ & $(5 / 2)^{2}[7 / 2]_{3}$ & & & R1c & & \\
\hline $2200.300(20)$ & $45434.2(4)$ & $2200.31543(20)$ & -0.015 & 47000 & & $s^{\frac{1}{2}}$ & ${ }^{3} \mathrm{P}_{0}$ & $\mathrm{sp}$ & $\left({ }^{3} \mathrm{P}\right)^{3} \mathrm{P}^{\circ}{ }^{3} \mathrm{D}^{\circ}{ }_{1}$ & & & S36c & & \\
\hline $2200.5078(3)$ & $45429.874(6)$ & $2200.50822(8)$ & -0.0004 & 1600000 & & $4 p$ & ${ }^{3} \mathrm{D}^{\circ}{ }_{1}$ & $4 \mathrm{~d}$ & $(3 / 2)^{2}[5 / 2]_{2}$ & $2.1 \mathrm{e}+08$ & C & $\mathrm{F}$ & $\mathrm{B} 00$ & \\
\hline $2201.000(20)$ & $45419.7(4)$ & $2201.02822(20)$ & -0.028 & 33000 & & $s^{\frac{1}{2}}$ & ${ }^{3} \mathrm{~F}_{2}$ & $\mathrm{sp}$ & $\left({ }^{3} \mathrm{~F}\right)^{3} \mathrm{P}^{\circ}{ }^{3} \mathrm{~F}^{\circ}{ }_{3}$ & & & S36c & & \\
\hline 2209.8049(4) & $45238.760(8)$ & $2209.80507(7)$ & -0.0002 & 1900000 & & $4 p$ & ${ }^{1} \mathrm{~F}^{\circ}{ }_{3}$ & $4 \mathrm{~d}$ & $(5 / 2)^{2}[5 / 2]_{3}$ & 2.1e+08 & C & R1c & B00 & \\
\hline 2210.26692(11) & $45229.3048(22)$ & $2210.26693(6)$ & -0.00001 & 56000000 & & $4 \mathrm{~s}$ & ${ }^{1} \mathrm{D}_{2}$ & $4 p$ & ${ }^{1} \mathrm{D}_{2}^{\circ}$ & $1.53 e+08$ & $\mathrm{C}+$ & $\mathrm{F}$ & D05se & \\
\hline $2212.7470(6)$ & $45178.617(12)$ & $2212.74730(8)$ & -0.0003 & 1100000 & & $4 p$ & ${ }^{3} \mathrm{D}^{\circ}{ }_{2}$ & $4 \mathrm{~d}$ & $(3 / 2)^{2}[5 / 2]_{2}$ & $1.4 \mathrm{e}+08$ & C & $\mathrm{F}$ & B00 & \\
\hline 2215.1054(3) & $45130.521(5)$ & $2215.10550(8)$ & -0.0001 & 3800000 & & $4 \mathrm{p}$ & ${ }^{3} \mathrm{D}^{\circ}{ }_{2}$ & $4 \mathrm{~d}$ & $(3 / 2)^{2}[5 / 2]_{3}$ & $4.9 \mathrm{e}+08$ & B & $\mathrm{F}$ & B00 & \\
\hline 2218.10770(6) & $45069.4401(12)$ & 2218.10772(5) & -0.00002 & 66000000 & & $4 \mathrm{~s}$ & ${ }^{3} \mathrm{D}_{2}$ & $4 p$ & ${ }^{3} \mathrm{P}^{\circ}{ }_{1}$ & $3.5 e+08$ & $\mathrm{C}+$ & $\mathrm{F}$ & D05se & \\
\hline $2218.5123(4)$ & $45061.222(9)$ & $2218.51209(8)$ & 0.0002 & 1900000 & & $4 p$ & ${ }^{3} \mathrm{D}^{\circ}{ }_{1}$ & $4 \mathrm{~d}$ & $(3 / 2)^{2}[3 / 2]_{2}$ & $2.6 e+08$ & $\mathrm{C}$ & R1c & B00 & \\
\hline 2221.649(3) & $44997.60(6)$ & 2221.6477(8) & 0.001 & 99000 & & $\mathrm{~s}^{2}$ & ${ }^{1} \mathrm{D}_{2}$ & $\mathrm{sp}$ & $\left({ }^{1} \mathrm{D}\right)^{3} \mathrm{P}^{\circ}{ }^{3} \mathrm{P}^{\circ}{ }_{2}$ & & & R1c & & \\
\hline $2224.6906(5)$ & $44936.092(10)$ & 2224.69062(11) & -0.0000 & 1300000 & & $4 p$ & ${ }^{1} \mathrm{P}^{\circ}{ }_{1}$ & $4 \mathrm{~d}$ & $(3 / 2)^{2}[5 / 2]_{2}$ & $1.3 e+08$ & C & R1c & B00 & \\
\hline $2226.7798(4)$ & $44893.936(8)$ & $2226.77999(6)$ & -0.0002 & 2200000 & & $4 \mathrm{p}$ & ${ }^{1} \mathrm{D}^{\circ}{ }_{2}$ & $4 \mathrm{~d}$ & $(5 / 2)^{2}[5 / 2]_{2}$ & $2.9 \mathrm{e}+08$ & C & $\mathrm{R} 1 \mathrm{c}$ & B00 & \\
\hline $2228.86748(6)$ & $44851.8904(11)$ & $2228.86745(6)$ & 0.00003 & 35000000 & & $4 \mathrm{~s}$ & ${ }^{3} \mathrm{D}_{1}$ & $4 p$ & ${ }^{3} \mathrm{P}_{0}^{\circ}$ & $3.88 \mathrm{e}+08$ & $\mathrm{~B}+$ & $\mathrm{F}$ & P97 & \\
\hline $2229.8529(4)$ & $44832.072(8)$ & $2229.85346(6)$ & -0.0006 & 1800000 & & $4 p$ & ${ }^{1} \mathrm{D}^{\circ}{ }_{2}$ & $4 d$ & $(5 / 2)^{2}[7 / 2]_{3}$ & $2.1 \mathrm{e}+08$ & C & R1c & B00 & \\
\hline $2230.1439(7)$ & $44826.223(15)$ & $2230.14604(9)$ & -0.0022 & 1900000 & & $4 p$ & ${ }^{3} \mathrm{D}^{\circ}{ }_{1}$ & $4 \mathrm{~d}$ & $(3 / 2)^{2}[3 / 2]_{1}$ & $1.9 \mathrm{e}+08$ & C & R1c & B00 & \\
\hline $2230.3979(7)$ & 44821.118(13) & $2230.39901(9)$ & -0.0011 & 440000 & & $4 p$ & ${ }^{1} \mathrm{~F}^{\circ}{ }_{3}$ & $4 \mathrm{~d}$ & $(5 / 2)^{2}[9 / 2]_{4}$ & $2.0 \mathrm{e}+07$ & C & R1c & O07se & \\
\hline $2230.9512(6)$ & $44810.003(12)$ & $2230.95279(8)$ & -0.0016 & 1600000 & & $4 p$ & ${ }^{3} \mathrm{D}^{\circ}{ }_{2}$ & $4 \mathrm{~d}$ & $(3 / 2)^{2}[3 / 2]_{2}$ & $2.2 \mathrm{e}+08$ & C & R1c & B00 & \\
\hline $2231.5817(4)$ & $44797.343(8)$ & $2231.58204(7)$ & -0.0003 & 1100000 & & $4 \mathrm{p}$ & ${ }^{1} \mathrm{~F}_{3}^{\circ}$ & $4 \mathrm{~d}$ & $(5 / 2)^{2}[3 / 2]_{2}$ & $1.14 \mathrm{e}+08$ & B & R1c & $\mathrm{O} 07$ & \\
\hline 2242.1424(11) & $44586.363(21)$ & $2242.14217(6)$ & 0.0003 & 240000 & & $4 p$ & ${ }^{1} \mathrm{D}^{\circ}{ }_{2}$ & $4 \mathrm{~d}$ & $(5 / 2)^{2}[5 / 2]_{3}$ & $1.07 \mathrm{e}+07$ & C & R1c & B00 & \\
\hline $2242.61775(10)$ & $44576.9146(20)$ & 2242.61764(7) & 0.00011 & 54000000 & & $4 \mathrm{~s}$ & ${ }^{1} \mathrm{D}_{2}$ & $4 p$ & ${ }^{1} \mathrm{~F}_{3}^{\circ}$ & $2.5 \mathrm{e}+08$ & $\mathrm{C}+$ & $\mathrm{F}$ & D05se & \\
\hline $2242.718(3)$ & 44574.93(5) & 2242.71791(9) & -0.000 & 100000 & & $4 p$ & ${ }^{3} \mathrm{D}^{\circ}{ }_{2}$ & $4 \mathrm{~d}$ & $(3 / 2)^{2}[3 / 2]_{1}$ & $5.8 \mathrm{e}+07$ & C & TW & B00 & \\
\hline $2243.0938(7)$ & 44567.454(14) & 2243.09395(10) & -0.0001 & 230000 & & $4 p$ & ${ }^{1} \mathrm{P}_{1}^{\circ}$ & $4 \mathrm{~d}$ & $(3 / 2)^{2}[3 / 2]_{2}$ & $4.0 \mathrm{e}+07$ & C & R1c & B00 & \\
\hline 2247.0017(5) & 44489.953(11) & 2247.00170(9) & -0.0000 & 66000000 & & $4 \mathrm{~s}$ & ${ }^{3} \mathrm{D}_{3}$ & $4 p$ & ${ }^{3} \mathrm{P}_{2}^{\circ}$ & $3.3 e+08$ & B & R1c & $\mathrm{C} 94 \mathrm{c}$ & \\
\hline $2248.9666(4)$ & $44451.086(8)$ & $2248.96667(6)$ & -0.0001 & 1800000 & & $4 p$ & ${ }^{3} \mathrm{D}_{3}^{\circ}$ & $4 \mathrm{~d}$ & $(5 / 2)^{2}[7 / 2]_{4}$ & $9.1 \mathrm{e}+07$ & B & R1c & $\mathrm{O} 07$ & \\
\hline 2251.8564(5) & $44394.047(9)$ & $2251.85601(8)$ & 0.0004 & 72000 & & $4 \mathrm{p}$ & ${ }^{3} \mathrm{D}^{\circ}{ }_{2}$ & $4 \mathrm{~d}$ & $(3 / 2)^{2}[7 / 2]_{3}$ & $5.8 \mathrm{e}+06$ & C & R1c & B00 & \\
\hline $2253.0326(16)$ & $44370.87(3)$ & 2253.0273(3) & 0.0053 & 51000 & & $s^{2}$ & ${ }^{1} \mathrm{D}_{2}$ & $\mathrm{sp}$ & $\left({ }^{1} \mathrm{D}\right)^{3} \mathrm{P}^{\circ}{ }^{3} \mathrm{P}^{\circ}{ }_{1}$ & & & R1c & & \\
\hline $2254.9880(4)$ & $44332.402(8)$ & $2254.98778(11)$ & 0.0002 & 620000 & & $4 p$ & ${ }^{1} \mathrm{P}^{\circ}{ }_{1}$ & $4 \mathrm{~d}$ & $(3 / 2)^{2}[3 / 2]_{1}$ & $1.9 \mathrm{e}+08$ & C & R1c & B00 & \\
\hline $2263.2130(4)$ & $44171.302(8)$ & $2263.21318(6)$ & -0.0002 & 580000 & & $4 p$ & ${ }^{1} \mathrm{D}^{\circ}{ }_{2}$ & $4 \mathrm{~d}$ & $(5 / 2)^{2}[3 / 2]_{1}$ & $1.54 \mathrm{e}+08$ & $\mathrm{~B}+$ & R1c & $\mathrm{O} 07$ & \\
\hline $2263.7857(4)$ & $44160.128(8)$ & $2263.78578(6)$ & -0.0000 & 1700000 & & $4 \mathrm{p}$ & ${ }^{3} \mathrm{D}_{3}^{\circ}$ & $4 \mathrm{~d}$ & $(5 / 2)^{2}[5 / 2]_{3}$ & $1.8 \mathrm{e}+08$ & $\mathrm{C}$ & R1c & B00 & \\
\hline 2264.5671(16) & $44144.89(3)$ & $2264.56423(6)$ & 0.0029 & 50000 & & $4 p$ & ${ }^{1} \mathrm{D}^{\circ}{ }_{2}$ & $4 \mathrm{~d}$ & $(5 / 2)^{2}[3 / 2]_{2}$ & $1.4 \mathrm{e}+06$ & E & R1c & O07se & \\
\hline $2265.3643(4)$ & $44129.358(8)$ & $2265.36424(10)$ & 0.0001 & 190000 & & $4 p$ & ${ }^{3} \mathrm{D}^{\circ}{ }_{1}$ & $4 \mathrm{~d}$ & $(3 / 2)^{2}[1 / 2]_{1}$ & $1.25 \mathrm{e}+08$ & B & R1c & $\mathrm{O} 07$ & \\
\hline $2274.7407(6)$ & 43947.476(11) & $2274.74107(8)$ & -0.0004 & 150000 & & $4 p$ & ${ }^{3} \mathrm{P}^{\circ}{ }_{2}$ & $5 s$ & $(3 / 2)^{2}[3 / 2]_{2}$ & 4.e+06 & E & R1c & C94 & \\
\hline $2276.2577(4)$ & 43918.191(8) & $2276.25797(7)$ & -0.0003 & 16000000 & & $4 \mathrm{~s}$ & ${ }^{3} \mathrm{D}_{1}$ & $4 p$ & ${ }^{3} \mathrm{P}^{\circ}{ }_{1}$ & $6.3 e+07$ & $\mathrm{C}+$ & R1c & D05se & \\
\hline $2278.3378(5)$ & $43878.098(10)$ & $2278.33737(10)$ & 0.0004 & 220000 & & $4 p$ & ${ }^{3} \mathrm{D}^{\circ}{ }_{2}$ & $4 d$ & $(3 / 2)^{2}[1 / 2]_{1}$ & $1.32 \mathrm{e}+08$ & $\mathrm{~B}+$ & R1c & $\mathrm{O} 07$ & \\
\hline $2280.9423(4)$ & $43827.999(8)$ & $2280.9428(3)$ & -0.0005 & 260000 & & $\mathrm{~s}^{2}$ & ${ }^{3} \mathrm{~F}_{3}$ & $\mathrm{sp}$ & $\left({ }^{3} \mathrm{~F}\right)^{3} \mathrm{P}^{\circ}{ }^{3} \mathrm{G}^{\circ}{ }_{4}$ & & & R1c & & \\
\hline $2284.202(4)$ & $43765.46(7)$ & $2284.19835(22)$ & 0.004 & 17000 & & $s^{2}$ & ${ }^{1} \mathrm{G}_{4}$ & $\mathrm{sp}$ & $\left({ }^{1} \mathrm{G}\right)^{3} \mathrm{P}^{\circ}{ }^{3} \mathrm{~F}^{\circ}{ }_{3}$ & & & R1c & & \\
\hline
\end{tabular}


Table A1. Cont.

\begin{tabular}{|c|c|c|c|c|c|c|c|c|c|c|c|c|c|c|}
\hline$\lambda_{\text {obs }}{ }^{\text {a }}(\AA)$ & $\sigma_{\mathrm{obs}}{ }^{\mathrm{b}}\left(\mathrm{cm}^{-1}\right)$ & $\lambda_{\text {Ritz }}{ }^{c}(\AA)$ & $\begin{array}{c}\Delta \lambda_{\text {obs-Ritz }} \\
\text { (A) }\end{array}$ & $\begin{array}{c}I_{\text {obs }} \mathrm{d} \\
\text { (arb. u.) }\end{array}$ & Char ${ }^{e}$ & & wer Level & & pper Level & $A\left(\mathrm{~s}^{-1}\right)$ & $\operatorname{Acc}^{f}$ & Line Ref. $\mathrm{g}$ & TP Ref. $\mathrm{g}$ & Notes $^{h}$ \\
\hline $2286.6447(4)$ & $43718.712(8)$ & $2286.64494(6)$ & -0.0002 & 810000 & & $4 p$ & ${ }^{3} \mathrm{D}_{3}^{\circ}$ & $4 \mathrm{~d}$ & $(5 / 2)^{2}[3 / 2]_{2}$ & $1.52 \mathrm{e}+08$ & $\mathrm{~B}+$ & R1c & O07 & \\
\hline $2289.4160(4)$ & 43665.797(8) & $2289.41619(8)$ & -0.0002 & 170000 & & $4 p$ & ${ }^{3} \mathrm{P}^{\circ}{ }_{2}$ & $5 s$ & $(3 / 2)^{2}[3 / 2]_{1}$ & 7.e+06 & $\mathrm{D}$ & $\mathrm{R} 1 \mathrm{c}$ & C94 & \\
\hline $2290.1606(7)$ & $43651.600(14)$ & $2290.16119(24)$ & -0.0006 & 49000 & & $\mathrm{~s}^{\frac{1}{2}}$ & ${ }^{3} \mathrm{~F}_{2}$ & $\mathrm{sp}$ & $\left({ }^{3} \mathrm{~F}\right)^{3} \mathrm{P}^{\circ}{ }^{3} \mathrm{D}^{\circ}{ }_{3}$ & & & R1c & & \\
\hline 2291.0018(4) & $43635.575(8)$ & $2291.00110(12)$ & 0.0007 & 550000 & & $4 p$ & ${ }^{1} \mathrm{P}^{\circ}{ }_{1}$ & $4 \mathrm{~d}$ & $(3 / 2)^{2}[1 / 2]_{1}$ & $1.76 \mathrm{e}+08$ & $\mathrm{~B}+$ & $\mathrm{R} 1 \mathrm{c}$ & $\mathrm{O} 07$ & \\
\hline $2292.6896(6)$ & 43603.454(11) & $2292.69060(8)$ & -0.0010 & 19000 & & $4 \mathrm{~s}$ & ${ }^{1} \mathrm{D}_{2}$ & $4 p$ & ${ }^{3} \mathrm{~F}_{2}^{\circ}$ & $4 . e+05$ & $\mathrm{E}$ & R1c & D05cor & \\
\hline $2292.9699(6)$ & 43598.124(11) & $2292.9700(3)$ & -0.0002 & 20000 & & $\mathrm{~s}^{2}$ & ${ }^{3} \mathrm{~F}_{4}$ & $\mathrm{sp}$ & $\left({ }^{3} \mathrm{~F}\right)^{3} \mathrm{P}^{\circ}{ }^{5} \mathrm{~F}_{4}^{\circ}$ & & & R1c & & \\
\hline $2294.3674(4)$ & 43571.571(8) & $2294.36758(7)$ & -0.0002 & 15000000 & & $4 \mathrm{~s}$ & ${ }^{3} \mathrm{D}_{2}$ & $4 p$ & ${ }^{3} \mathrm{P}^{\circ}{ }_{2}$ & $3.4 \mathrm{e}+07$ & $\mathrm{C}+$ & $\mathrm{R} 1 \mathrm{c}$ & $\mathrm{C} 94 \mathrm{c}$ & \\
\hline 2299.4885(5) & $43474.542(9)$ & 2299.48891(10) & -0.0004 & 140000 & & $4 p$ & ${ }^{3} \mathrm{D}^{\circ}{ }_{1}$ & $4 \mathrm{~d}$ & $(5 / 2)^{2}[1 / 2]_{0}$ & $1.07 \mathrm{e}+08$ & $\mathrm{C}$ & R1c & B00 & \\
\hline 2309.5189(4) & $43285.747(8)$ & $2309.51890(6)$ & -0.0000 & 200000 & & $4 p$ & ${ }^{3} \mathrm{D}^{\circ}{ }_{1}$ & $4 \mathrm{~d}$ & $(5 / 2)^{2}[5 / 2]_{2}$ & $1.02 \mathrm{e}+07$ & C & R1c & B00 & \\
\hline $2315.681(5)$ & $43170.57(10)$ & $2315.6826(4)$ & -0.002 & 17000 & & $\mathrm{~s}^{2}$ & ${ }^{1} \mathrm{D}_{2}$ & $\mathrm{sp}$ & $\left({ }^{1} \mathrm{D}\right)^{3} \mathrm{P}^{\circ}{ }^{3} \mathrm{~F}^{\circ}$ & & & R1c & & \\
\hline 2323.0039(5) & $43034.495(9)$ & $2323.00409(6)$ & -0.0002 & 200000 & & $4 p$ & ${ }^{3} \mathrm{D}_{2}{ }_{2}$ & $4 \mathrm{~d}$ & $(5 / 2)^{2}[5 / 2]_{2}$ & & & R1c & & \\
\hline $2323.9280(6)$ & 43017.383(11) & 2323.92802(7) & 0.0000 & 86000 & & $4 p$ & ${ }^{1} \mathrm{D}^{\circ}{ }_{2}$ & $4 \mathrm{~d}$ & $(5 / 2)^{2}[1 / 2]_{1}$ & & & $\mathrm{R} 1 \mathrm{c}$ & & \\
\hline $2325.9100(17)$ & $42980.73(3)$ & $2325.90832(12)$ & 0.0017 & 25000 & & $4 p$ & ${ }^{1} \mathrm{P}^{\circ}{ }_{1}$ & $4 \mathrm{~d}$ & $(5 / 2)^{2}[1 / 2]_{0}$ & & & R1c & & \\
\hline $2327.5669(6)$ & 42950.137(11) & 2327.5672(4) & -0.0003 & 12000 & & $\mathrm{~s}^{2}$ & ${ }^{3} \mathrm{~F}_{3}$ & $\mathrm{sp}$ & $\left({ }^{3} \mathrm{~F}\right)^{3} \mathrm{P}^{\circ}{ }^{5} \mathrm{~F}^{\circ}{ }_{2}$ & & & R1c & & \\
\hline 2333.743(3) & $42836.49(6)$ & $2333.75088(22)$ & -0.008 & 23000 & & $s^{2}$ & ${ }^{1} \mathrm{G}_{4}$ & $6 \mathrm{p}$ & $(5 / 2)^{2}[7 / 2]^{\circ} 3$ & & & R1c & & \\
\hline $2336.1707(4)$ & $42791.971(8)$ & $2336.17057(10)$ & 0.0001 & 230000 & & $4 p$ & ${ }^{1} \mathrm{P}_{1}^{\circ}$ & $4 \mathrm{~d}$ & $(5 / 2)^{2}[5 / 2]_{2}$ & $4.9 \mathrm{e}+07$ & C & $\mathrm{R} 1 \mathrm{c}$ & B00 & \\
\hline 2339.7275(5) & $42726.924(10)$ & $2339.72732(6)$ & 0.0002 & 68000 & & $4 \mathrm{p}$ & ${ }^{3} \mathrm{D}_{2}^{\circ}$ & $4 \mathrm{~d}$ & $(5 / 2)^{2}[5 / 2]_{3}$ & & & R1c & & \\
\hline $2341.3713(12)$ & $42696.930(21)$ & & & 17000 & & $\mathrm{~s}^{\frac{1}{2}}$ & ${ }^{3} \mathrm{~F}_{4}$ & $\mathrm{sp}$ & $\left({ }^{3} \mathrm{~F}\right)^{3} \mathrm{P}^{\circ}{ }^{5} \mathrm{~F}^{\circ}{ }_{5}$ & & & R1c & & \\
\hline $2342.1723(17)$ & $42682.33(3)$ & $2342.1734(4)$ & -0.0011 & 55000 & & $\mathrm{~s}^{2}$ & ${ }^{3} \mathrm{P}_{2}$ & $\mathrm{sp}$ & $\left({ }^{3} \mathrm{P}\right)^{3} \mathrm{P}^{\circ}{ }^{5} \mathrm{D}_{3}^{\circ}$ & & & R1c & & \\
\hline $2347.890(20)$ & 42578.4(4) & $2347.8850(4)$ & 0.005 & 9000 & * & $s^{2}$ & ${ }^{3} \mathrm{P}_{2}$ & $\mathrm{sp}$ & $\left({ }^{3} \mathrm{P}\right)^{3} \mathrm{P}^{\circ}{ }^{5} \mathrm{D}^{\circ}{ }_{1}$ & & & $\mathrm{~S} 36 \mathrm{c}$ & & \\
\hline $2347.890(20)$ & $42578.4(4)$ & 2347.914(3) & -0.024 & 9000 & * & $5 \mathrm{~s}$ & $(3 / 2)^{2}[3 / 2]_{2}$ & $\mathrm{sp}$ & $\left({ }^{3} \mathrm{P}\right)^{1} \mathrm{P}^{\circ}{ }^{3} \mathrm{P}^{\circ}{ }_{2}$ & & & S36cn & & \\
\hline $2348.7330(4)$ & $42563.115(8)$ & $2348.73311(7)$ & -0.0001 & 120000 & & $4 p$ & ${ }^{3} \mathrm{D}^{\circ}{ }_{1}$ & $4 \mathrm{~d}$ & $(5 / 2)^{2}[3 / 2]_{1}$ & $2.8 \mathrm{e}+07$ & B & R1c & $\mathrm{O} 07$ & \\
\hline 2350.1902(8) & $42536.727(15)$ & $2350.18820(6)$ & 0.0020 & 14000 & & $4 \mathrm{p}$ & ${ }^{3} \mathrm{D}^{\circ}{ }_{1}$ & $4 \mathrm{~d}$ & $(5 / 2)^{2}[3 / 2]_{2}$ & & & R1c & & \\
\hline 2352.2911(12) & $42498.740(21)$ & $2352.29447(19)$ & -0.0034 & 30000 & & $\mathrm{~s}^{\frac{1}{2}}$ & ${ }^{1} \mathrm{G}_{4}$ & $4 \mathrm{f}$ & $(3 / 2)^{2}[9 / 2]^{\circ} 5$ & & & R1c & & \\
\hline $2353.9437(5)$ & $42468.905(9)$ & $2353.9434(4)$ & 0.0003 & 27000 & & $\mathrm{~s}^{2}$ & ${ }^{3} \mathrm{~F}_{3}$ & $\mathrm{sp}$ & $\left({ }^{3} \mathrm{~F}\right)^{3} \mathrm{P}^{0}{ }^{5} \mathrm{~F}^{\circ}{ }_{3}$ & & & R1c & & \\
\hline $2355.0143(6)$ & $42449.600(11)$ & $2355.01447(8)$ & -0.0001 & 610000 & & $4 p$ & ${ }^{3} \mathrm{P}^{\circ}{ }_{1}$ & $5 \mathrm{~s}$ & $(3 / 2)^{2}[3 / 2]_{2}$ & $2.5 \mathrm{e}+07$ & $\mathrm{C}+$ & R1c & K82 & \\
\hline $2356.6402(5)$ & $42420.316(9)$ & $2356.64033(9)$ & -0.0001 & 1400000 & & $4 \mathrm{~s}$ & ${ }^{3} \mathrm{D}_{1}$ & $4 p$ & ${ }^{3} \mathrm{P}_{2}^{\circ}$ & $3.0 \mathrm{e}+06$ & $\mathrm{C}+$ & R1c & C94c & \\
\hline $2360.6389(9)$ & $42348.465(16)$ & 2360.6391(9) & -0.0002 & 9200 & & $\mathrm{~s}^{2}$ & ${ }^{3} \mathrm{P}_{1}$ & $\mathrm{sp}$ & $\left({ }^{3} \mathrm{P}\right)^{3} \mathrm{P}^{\circ}{ }^{5} \mathrm{D}_{0}^{\circ}$ & & & $\mathrm{R} 2 \mathrm{nc}$ & & \\
\hline 2361.1901(12) & $42338.579(21)$ & $2361.1910(5)$ & -0.0008 & 37000 & & $\mathrm{~s}^{2}$ & ${ }^{3} \mathrm{P}_{1}$ & $\mathrm{sp}$ & $\left({ }^{3} \mathrm{P}\right){ }^{3} \mathrm{P}^{\circ}{ }^{5} \mathrm{D}^{\circ}{ }_{2}$ & & & $\mathrm{R} 1 \mathrm{c}$ & & \\
\hline $2362.6809(9)$ & $42311.867(16)$ & $2362.68143(7)$ & -0.0005 & 26000 & & $4 p$ & ${ }^{3} \mathrm{D}^{\circ}{ }_{2}$ & $4 \mathrm{~d}$ & $(5 / 2)^{2}[3 / 2]_{1}$ & $6.3 e+06$ & $\mathrm{C}+$ & R1c & $\mathrm{O} 07$ & \\
\hline $2364.1538(6)$ & $42285.508(10)$ & $2364.15386(7)$ & -0.0000 & 57000 & & $4 p$ & ${ }^{3} \mathrm{D}_{2}^{\circ}$ & $4 \mathrm{~d}$ & $(5 / 2)^{2}[3 / 2]_{2}$ & $3.4 \mathrm{e}+06$ & B & R1c & $\mathrm{O} 07$ & \\
\hline $2366.980(3)$ & $42235.03(5)$ & $2366.9785(4)$ & 0.001 & 8700 & & $5 s$ & $(5 / 2)^{2}[5 / 2]_{3}$ & $\mathrm{sp}$ & $\left({ }^{1} \mathrm{D}\right)^{1} \mathrm{P}^{\circ}{ }^{1} \mathrm{D}^{\circ}{ }_{2}$ & & & R1c & & \\
\hline $2369.8893(5)$ & $42183.179(9)$ & $2369.88902(9)$ & 0.0003 & 12000000 & & $4 \mathrm{~s}$ & ${ }^{1} \mathrm{D}_{2}$ & $4 p$ & ${ }^{3} \mathrm{~F}_{3}^{\circ}$ & $5.3 e+07$ & B & R1c & C94c & \\
\hline $2370.7464(5)$ & $42167.930(8)$ & $2370.74698(8)$ & -0.0006 & 490000 & & $4 p$ & ${ }^{3} \mathrm{P}^{\circ}{ }_{1}$ & $5 s$ & $(3 / 2)^{2}[3 / 2]_{1}$ & $2.4 \mathrm{e}+07$ & $\mathrm{C}+$ & R1c & K82 & \\
\hline 2376.3030(4) & $42069.335(8)$ & $2376.30277(10)$ & 0.0003 & 330000 & & $4 p$ & ${ }^{1} \mathrm{P}^{\circ}{ }_{1}$ & $4 \mathrm{~d}$ & $(5 / 2)^{2}[3 / 2]_{1}$ & $1.61 \mathrm{e}+08$ & $\mathrm{C}+$ & R1c & O07se & \\
\hline $2377.7920(6)$ & 42042.994(10) & 2377.79223(10) & -0.0003 & 12000 & & $4 \mathrm{p}$ & ${ }^{1} \mathrm{P}_{1}^{\circ}$ & $4 \mathrm{~d}$ & $(5 / 2)^{2}[3 / 2]_{2}$ & $3.5 e+06$ & $\mathrm{D}+$ & $\mathrm{R} 1 \mathrm{c}$ & $\mathrm{O} 07$ & \\
\hline $2378.4047(7)$ & $42032.163(13)$ & $2378.4063(6)$ & -0.0015 & 19000 & & $s^{2}$ & ${ }^{3} \mathrm{~F}_{2}$ & $\mathrm{sp}$ & $\left({ }^{3} \mathrm{~F}\right)^{3} \mathrm{P}^{\circ}{ }^{\circ} \mathrm{F}_{1}$ & & & R1c & & \\
\hline 2378.8442(12) & $42024.399(21)$ & $2378.8439(9)$ & 0.0002 & 220000 & & $s^{2}$ & ${ }^{3} \mathrm{P}_{2}$ & $\mathrm{sp}$ & $\left({ }^{1} \mathrm{D}\right)^{3} \mathrm{P}^{\circ}{ }^{3} \mathrm{P}^{\circ}{ }_{2}$ & & & R1c & & \\
\hline 2379.4048(12) & $42014.499(21)$ & $2379.4055(5)$ & -0.0007 & 53000 & & $s^{2}$ & ${ }^{3} \mathrm{P}_{0}$ & $\mathrm{sp}$ & $\left({ }^{3} \mathrm{P}\right)^{3} \mathrm{P}^{\circ}{ }^{5} \mathrm{D}^{\circ}{ }_{1}$ & & & R1c & & \\
\hline $2384.80(6)$ & $41919.5(11)$ & $2384.85893(9)$ & -0.06 & 49000 & & $4 p$ & ${ }^{3} \mathrm{~F}_{3}^{\circ}$ & $5 s$ & $(3 / 2)^{2}[3 / 2]_{2}$ & $6 . e+05$ & E & S36c & K82cal & \\
\hline
\end{tabular}


Table A1. Cont.

\begin{tabular}{|c|c|c|c|c|c|c|c|c|c|c|c|c|c|c|}
\hline$\lambda_{\text {obs }}{ }^{\text {a }}(\AA)$ & $\sigma_{\mathrm{obs}}{ }^{\mathrm{b}}\left(\mathrm{cm}^{-1}\right)$ & $\lambda_{\text {Ritz }}{ }^{c}(\AA)$ & $\begin{array}{c}\Delta \lambda_{\text {obs-Ritz }} \\
\text { (A) }\end{array}$ & $\begin{array}{c}I_{\text {obs }} \mathrm{d} \\
\text { (arb. u.) }\end{array}$ & Char ${ }^{\mathrm{e}}$ & & wer Level & & Upper Level & $A\left(\mathrm{~s}^{-1}\right)$ & $\operatorname{Acc}^{f}$ & Line Ref. $\mathrm{g}$ & TP Ref. $\mathrm{g}$ & Notes $^{h}$ \\
\hline $2384.9439(4)$ & $41916.926(8)$ & $2384.94408(9)$ & -0.0002 & 100000 & & $4 p$ & ${ }^{3} \mathrm{P}_{2}^{\circ}$ & $5 \mathrm{~s}$ & $(5 / 2)^{2}[5 / 2]_{2}$ & $7 . e+06$ & E & R1c & K82cal & \\
\hline 2385.0951(18) & $41914.27(3)$ & $2385.0945(4)$ & 0.0006 & 7400 & & $5 s$ & $(5 / 2)^{2}[5 / 2]_{2}$ & $\mathrm{sp}$ & $\left({ }^{1} \mathrm{D}\right){ }^{1} \mathrm{P}^{\circ}{ }^{1} \mathrm{D}_{2}^{\circ}{ }_{2}$ & & & R1c & & \\
\hline 2392.6852(18) & 41781.32(3) & 2392.6858(9) & -0.0006 & 6900 & & $s^{2}$ & ${ }^{3} \mathrm{P}_{1}$ & $\mathrm{sp}$ & $\left({ }^{1} \mathrm{D}\right)^{3} \mathrm{P}^{\circ}{ }^{3} \mathrm{P}^{\circ}{ }_{2}$ & & & R1c & & \\
\hline $2393.2599(5)$ & $41771.287(9)$ & 2393.2602(4) & -0.0003 & 23000 & & $\mathrm{~s}^{2}$ & ${ }^{3} \mathrm{~F}_{3}$ & $\mathrm{sp}$ & $\left({ }^{3} \mathrm{~F}\right)^{3} \mathrm{P}^{\circ} 5^{5} \mathrm{~F}_{4}^{\circ}$ & & & R1c & & \\
\hline 2394.0296(5) & $41757.858(9)$ & $2394.0296(4)$ & -0.0000 & 20000 & & $s^{2}$ & ${ }^{3} \mathrm{~F}_{2}$ & $\mathrm{sp}$ & $\left({ }^{3} \mathrm{~F}\right)^{3} \mathrm{P}^{\circ} 5^{5} \mathrm{~F}^{\circ}{ }_{2}$ & & & R1c & & \\
\hline $2400.1140(7)$ & $41652.008(12)$ & $2400.11404(9)$ & -0.0001 & 3700000 & & $4 \mathrm{~s}$ & ${ }^{1} \mathrm{D}_{2}$ & $4 \mathrm{p}$ & ${ }^{3} \mathrm{P}_{1}^{\circ}$ & $6.9 \mathrm{e}+06$ & $\mathrm{C}+$ & R1c & D05se & \\
\hline 2403.3373(5) & $41596.149(8)$ & $2403.33713(9)$ & 0.0002 & 2200000 & & $4 p$ & ${ }^{3} \mathrm{P}_{2}^{\circ}$ & $5 s$ & $(5 / 2)^{2}[5 / 2]_{3}$ & $1.06 \mathrm{e}+08$ & $\mathrm{C}+$ & $\mathrm{F}$ & $\mathrm{C} 94$ & \\
\hline 2414.1881(5) & $41409.205(9)$ & $2414.18856(8)$ & -0.0004 & 36000 & & $4 \mathrm{p}$ & ${ }^{3} \mathrm{D}^{\circ}{ }_{1}$ & $4 \mathrm{~d}$ & $(5 / 2)^{2}[1 / 2]_{1}$ & 2.e+06 & E & R1c & O07cal & \\
\hline $2414.8568(5)$ & $41397.740(9)$ & $2414.8563(4)$ & 0.0005 & 37000 & & $\mathrm{~s}^{\frac{1}{2}}$ & ${ }^{3} \mathrm{P}_{2}$ & $\mathrm{sp}$ & $\left({ }^{1} \mathrm{D}\right)^{3} \mathrm{P}^{\circ}{ }^{3} \mathrm{P}^{\circ}{ }_{1}$ & & & R1c & & \\
\hline $2421.9424(6)$ & $41276.637(11)$ & $2421.9425(4)$ & -0.0001 & 6700 & & $\mathrm{~s}^{2}$ & ${ }^{3} \mathrm{~F}_{2}$ & $\mathrm{sp}$ & $\left({ }^{3} \mathrm{~F}\right)^{3} \mathrm{P}^{\circ} 5^{5} \mathrm{~F}_{3}^{\circ}$ & & & R1c & & \\
\hline $2424.4338(3)$ & $41234.223(5)$ & $2424.43430(9)$ & -0.0005 & 1100000 & & $4 p$ & ${ }^{3} \mathrm{P}_{0}^{\circ}$ & $5 s$ & $(3 / 2)^{2}[3 / 2]_{1}$ & $5.0 \mathrm{e}+07$ & $\mathrm{C}+$ & $\mathrm{F}$ & C94 & \\
\hline $2426.558(5)$ & 41198.13(8) & $2426.5614(9)$ & -0.003 & 10000 & & $4 \mathrm{~d}$ & $(5 / 2)^{2}[9 / 2]_{5}$ & $8 \mathrm{f}$ & $(5 / 2)^{2}[11 / 2]^{\circ} 6$ & & & $\mathrm{R} 1 \mathrm{c}$ & & \\
\hline $2426.996(9)$ & $41190.69(15)$ & $2427.016(3)$ & -0.019 & 5000 & & $5 s$ & $(5 / 2)^{2}[5 / 2]_{2}$ & $7 p$ & $(3 / 2)^{2}[5 / 2]^{\circ}{ }_{2}^{\circ}$ & & & $\mathrm{R} 2 \mathrm{nc}$ & & \\
\hline 2428.9274(5) & 41157.944(8) & $2428.92746(8)$ & -0.0000 & 38000 & & $4 p$ & ${ }^{3} \mathrm{D}_{2}^{\circ}$ & $4 \mathrm{~d}$ & $(5 / 2)^{2}[1 / 2]_{1}$ & $1.93 e+07$ & $\mathrm{C}+$ & R1c & $\mathrm{O} 07$ & \\
\hline 2430.6772(13) & $41128.318(21)$ & $2430.67651(24)$ & 0.0007 & 5600 & & $\mathrm{~s}^{\frac{1}{2}}$ & ${ }^{1} \mathrm{G}_{4}$ & $\mathrm{sp}$ & $\left({ }^{1} \mathrm{G}\right)^{3} \mathrm{P}^{\circ}{ }^{3} \mathrm{H}^{\circ}{ }_{4}$ & & & R1c & & \\
\hline $2432.420(3)$ & $41098.86(5)$ & $2432.4183(5)$ & 0.001 & 4800 & & $4 \mathrm{~d}$ & $(5 / 2)^{2}[9 / 2]_{4}$ & $8 \mathrm{f}$ & $(5 / 2)^{2}[11 / 2]^{\circ}{ }_{5}^{*}$ & & & R1c & & \\
\hline $2442.6646(5)$ & 40926.494(9) & & & 90000 & & $\mathrm{~s}^{2}$ & ${ }^{3} \mathrm{~F}_{4}$ & $\mathrm{sp}$ & $\left({ }^{3} \mathrm{~F}\right)^{3} \mathrm{P}^{\circ}{ }^{5} \mathrm{G}^{\circ}{ }_{5}$ & & & R1c & & \\
\hline 2443.3256(5) & 40915.423(8) & $2443.32555(11)$ & 0.0001 & 44000 & & $4 p$ & ${ }^{1} \mathrm{P}_{1}^{\circ}$ & $4 \mathrm{~d}$ & $(5 / 2)^{2}[1 / 2]_{1}$ & $2.00 \mathrm{e}+07$ & B & R1c & $\mathrm{O} 07$ & \\
\hline $2448.2143(13)$ & 40833.727(21) & $2448.2131(4)$ & 0.0012 & 46000 & & $s^{2}$ & ${ }^{3} \mathrm{P}_{0}$ & $\mathrm{sp}$ & $\left({ }^{1} \mathrm{D}\right)^{3} \mathrm{P}^{\circ}{ }^{3} \mathrm{P}^{\circ}{ }_{1}$ & & & R1c & & \\
\hline $2452.951(6)$ & $40754.88(10)$ & $2452.9576(7)$ & -0.006 & 3900 & & $\mathrm{~s}^{2}$ & ${ }^{3} \mathrm{P}_{2}$ & $\mathrm{sp}$ & $\left({ }^{1} \mathrm{D}\right)^{3} \mathrm{P}^{\circ}{ }^{3} \mathrm{D}^{\circ}{ }_{3}$ & & & R1c & & \\
\hline $2456.008(4)$ & 40704.16(7) & 2455.99461(18) & 0.013 & 7600 & & $s^{2}$ & ${ }^{1} \mathrm{G}_{4}$ & $4 \mathrm{f}$ & $(5 / 2)^{2}[9 / 2]^{\circ}{ }_{4}$ & & & R1c & & \\
\hline $2462.6140(19)$ & 40594.98(3) & $2462.61266(17)$ & 0.0014 & 11000 & & $\mathrm{~s}^{2}$ & ${ }^{1} \mathrm{G}_{4}$ & $4 \mathrm{f}$ & $(5 / 2)^{2}[9 / 2]^{7}$ & & & R1c & & \\
\hline $2464.307(5)$ & 40567.10(8) & $2464.30282(17)$ & 0.004 & 3500 & & $s^{2}$ & ${ }^{1} \mathrm{G}_{4}$ & $4 \mathrm{f}$ & $(5 / 2)^{2}[7 / 2]^{\circ}{ }_{4}$ & & & R1c & & \\
\hline $2468.3475(8)$ & $40500.690(13)$ & $2468.3480(6)$ & -0.0005 & 33000 & & $s^{2}$ & ${ }^{3} \mathrm{P}_{1}$ & $\mathrm{sp}$ & $\left({ }^{1} \mathrm{D}\right)^{3} \mathrm{P}^{\circ} 3^{3} \mathrm{P}_{0}^{\circ}{ }_{0}$ & & & R1c & & \\
\hline $2468.5000(5)$ & 40498.187(8) & $2468.50111(9)$ & -0.0011 & 170000 & & $4 p$ & ${ }^{3} \mathrm{~F}_{2}^{\circ}$ & $5 s$ & $(3 / 2)^{2}[3 / 2]_{2}$ & $1.30 \mathrm{e}+07$ & $\mathrm{C}+$ & R1c & K82 & \\
\hline 2473.3333(4) & $40419.053(7)$ & $2473.33345(9)$ & -0.0002 & 570000 & & $4 \mathrm{p}$ & ${ }^{3} \mathrm{P}_{1}^{\circ}$ & $5 s$ & $(5 / 2)^{2}[5 / 2]_{2}$ & $5.9 e+07$ & $\mathrm{C}+$ & R1c & C94 & \\
\hline $2476.4439(7)$ & 40368.287(11) & $2476.44459(19)$ & -0.0007 & 15000 & & $\mathrm{~s}^{\frac{1}{2}}$ & ${ }^{1} \mathrm{G}_{4}$ & $4 \mathrm{f}$ & $(5 / 2)^{2}[11 / 2]^{\circ}{ }_{5}$ & & & R1c & & \\
\hline $2483.784(5)$ & $40249.00(8)$ & $2483.7876(5)$ & -0.003 & 2900 & & $\mathrm{~s}^{2}$ & ${ }^{3} \mathrm{P}_{1}$ & $\mathrm{sp}$ & $\left({ }^{1} \mathrm{D}\right)^{3} \mathrm{P}^{\circ}{ }^{3} \mathrm{D}^{\circ}{ }_{2}$ & & & R1c & & \\
\hline 2485.7919(5) & $40216.489(8)$ & $2485.79176(9)$ & 0.0002 & 1000000 & & $4 p$ & ${ }^{3} \mathrm{~F}_{2}^{\circ}$ & $5 s$ & $(3 / 2)^{2}[3 / 2]_{1}$ & $1.42 \mathrm{e}+08$ & $\mathrm{C}+$ & R1c & C94 & $\mathrm{L}$ \\
\hline $2489.6523(6)$ & 40154.136(10) & $2489.65236(11)$ & -0.0001 & 660000 & & $4 \mathrm{~s}$ & ${ }^{1} \mathrm{D}_{2}$ & $4 p$ & ${ }^{3} \mathrm{P}_{2}^{\circ}$ & $1.0 \mathrm{e}+06$ & $\mathrm{D}+$ & R1c & C94c & \\
\hline $2499.001(8)$ & 40003.94(12) & 2499.0132(4) & -0.013 & 4900 & & $\mathrm{~s}^{2}$ & ${ }^{3} \mathrm{P}_{2}$ & $\mathrm{sp}$ & $\left({ }^{1} \mathrm{D}\right)^{3} \mathrm{P}^{\circ}{ }^{3} \mathrm{~F}_{2}^{\circ}{ }_{2}$ & & & R1c & & \\
\hline 2501.4933(13) & $39964.076(21)$ & $2501.4947(5)$ & -0.0014 & 4800 & & $s^{2}$ & ${ }^{3} \mathrm{P}_{1}^{-}$ & $\mathrm{sp}$ & $\left({ }^{1} \mathrm{D}\right)^{3} \mathrm{P}^{\circ}{ }^{3} \mathrm{D}^{\circ}{ }_{1}$ & & & R1c & & \\
\hline $2506.2727(3)$ & $39887.871(5)$ & $2506.27258(10)$ & 0.0001 & 1000000 & & $4 p$ & ${ }^{3} \mathrm{~F}_{3}^{\circ}$ & $5 s$ & $(5 / 2)^{2}[5 / 2]_{2}$ & $2.0 \mathrm{e}+08$ & $\mathrm{C}+$ & $\mathrm{F}$ & C94 & $\mathrm{L}$ \\
\hline 2514.2919(10) & $39760.659(16)$ & 2514.2931(4) & -0.0012 & 4200 & & $\mathrm{~s}^{2}$ & ${ }^{3} \mathrm{P}_{1}$ & $\mathrm{sp}$ & $\left({ }^{1} \mathrm{D}\right)^{3} \mathrm{P}^{\circ} 3^{3} \mathrm{~F}_{2}{ }_{2}$ & & & R1c & & \\
\hline $2515.0822(7)$ & 39748.166(11) & 2515.0831(3) & -0.0008 & 8400 & & $5 s$ & $(5 / 2)^{2}[5 / 2]_{3}$ & $7 \mathrm{p}$ & $(5 / 2)^{2}[5 / 2]^{\circ}{ }_{3}$ & & & R1c & & \\
\hline 2518.9484(5) & $39687.164(8)$ & $2518.9484(5)$ & -0.0001 & 34000 & & $s^{2}$ & ${ }^{3} \mathrm{~F}_{3}$ & $\mathrm{sp}$ & $\left({ }^{3} \mathrm{~F}\right)^{3} \mathrm{P}^{\circ}{ }^{5} \mathrm{G}^{\circ}{ }_{4}$ & & & R1c & & \\
\hline $2526.3277(14)$ & $39571.246(21)$ & $2526.3234(4)$ & 0.0043 & 1900 & & $\mathrm{~s}^{2}$ & ${ }^{3} \mathrm{~F}_{4}$ & $\mathrm{sp}$ & $\left({ }^{3} \mathrm{~F}\right)^{3} \mathrm{P}^{\circ}{ }^{5} \mathrm{D}^{\circ}{ }_{3}$ & & & R1c & & \\
\hline 2526.5923(5) & $39567.103(7)$ & $2526.59232(10)$ & -0.0000 & 500000 & & $4 p$ & ${ }^{3} \mathrm{~F}_{3}^{\circ}$ & $5 s$ & $(5 / 2)^{2}[5 / 2]_{3}$ & $2.7 \mathrm{e}+07$ & $\mathrm{C}+$ & R1c & K82 & \\
\hline $2529.30395(21)$ & 39524.686(3) & $2529.30385(10)$ & 0.00010 & 940000 & & $4 p$ & ${ }^{1} \mathrm{~F}_{3}^{\circ}$ & $5 s$ & $(3 / 2)^{2}[3 / 2]_{2}$ & $1.06 \mathrm{e}+08$ & $\mathrm{C}+$ & $\mathrm{F}$ & C94 & \\
\hline $2542.9345(8)$ & $39312.840(12)$ & 2542.9357(4) & -0.0012 & 3200 & & $5 \mathrm{~s}$ & $(5 / 2)^{2}[5 / 2]_{3}$ & $7 \mathrm{p}$ & $(5 / 2)^{2}[3 / 2]^{2}{ }_{2}$ & & & R1c & & \\
\hline $2544.80509(20)$ & $39283.945(3)$ & $2544.80482(11)$ & 0.00027 & 1200000 & & $4 p$ & ${ }^{3} \mathrm{~F}_{4}^{\circ}$ & $5 s$ & $(5 / 2)^{2}[5 / 2]_{3}$ & $1.94 \mathrm{e}+08$ & B & $\mathrm{F}$ & B00 & \\
\hline
\end{tabular}


Table A1. Cont.

\begin{tabular}{|c|c|c|c|c|c|c|c|c|c|c|c|c|c|c|}
\hline$\lambda_{\text {obs }}{ }^{\text {a }}(\AA)$ & $\sigma_{\mathrm{obs}}{ }^{\mathrm{b}}\left(\mathrm{cm}^{-1}\right)$ & $\lambda_{\text {Ritz }}{ }^{c}(\AA)$ & $\begin{array}{c}\Delta \lambda_{\text {obs-Ritz }} \\
\text { (A) }\end{array}$ & $\begin{array}{l}I_{\text {obs }} \mathrm{d} \\
\text { (arb. u.) }\end{array}$ & Char ${ }^{e}$ & & wer Level & & Upper Level & $A\left(\mathrm{~s}^{-1}\right)$ & $\operatorname{Acc}^{f}$ & Line Ref. $\mathrm{g}$ & TP Ref. $\mathrm{g}$ & Notes $^{h}$ \\
\hline $2553.3430(5)$ & $39152.595(8)$ & $2553.3430(5)$ & -0.0000 & 14000 & & $\mathrm{~s}^{2}$ & ${ }^{3} \mathrm{~F}_{2}$ & $\mathrm{sp}$ & $\left({ }^{3} \mathrm{~F}\right)^{3} \mathrm{P}^{\circ}{ }^{5} \mathrm{G}^{\circ}{ }_{3}$ & & & R1c & & \\
\hline $2556.3698(9)$ & $39106.241(13)$ & 2556.3691(3) & 0.0007 & 2800 & & $4 \mathrm{~d}$ & $(5 / 2)^{2}[9 / 2]_{5}$ & $7 f$ & $(5 / 2)^{2}[9 / 2]^{\circ}{ }_{5}$ & & & R1c & & \\
\hline 2558.2130(5) & $39078.067(8)$ & 2558.2134(4) & -0.0004 & 5400 & & $4 \mathrm{~d}$ & $(5 / 2)^{2}[9 / 2]_{5}$ & $7 \mathrm{f}$ & $(5 / 2)^{2}[11 / 2]^{\circ}{ }_{6}$ & & & R1c & & \\
\hline 2559.4301(14) & $39059.485(21)$ & $2559.4301(13)$ & -0.0000 & 2700 & & $4 \mathrm{~d}$ & $(5 / 2)^{2}[3 / 2]_{2}$ & $7 f$ & $(5 / 2)^{2}[5 / 2]^{\circ}{ }_{2}$ & & & $\mathrm{R} 2 \mathrm{nc}$ & & \\
\hline 2559.7925(20) & 39053.96(3) & 2559.7938(18) & -0.0012 & 1300 & & $4 \mathrm{~d}$ & $(5 / 2)^{2}[3 / 2]_{1}$ & $8 p$ & $(3 / 2)^{2}[1 / 2]^{\circ} 1$ & & & $\mathrm{R} 2 \mathrm{nc}$ & & \\
\hline 2564.7257(6) & $38978.841(9)$ & $2564.7264(4)$ & -0.0007 & 5100 & & $4 \mathrm{~d}$ & $(5 / 2)^{2}[9 / 2]_{4}$ & $7 \mathrm{f}$ & $(5 / 2)^{2}[11 / 2]^{\circ}{ }_{5}$ & & & R1c & & \\
\hline $2565.0459(7)$ & $38973.975(10)$ & 2565.0471(5) & -0.0011 & 2500 & & $4 \mathrm{~d}$ & $(3 / 2)^{2}[7 / 2]_{3}$ & $7 \mathrm{f}$ & $(3 / 2)^{2}[9 / 2]^{\circ} 4$ & & & $\mathrm{R} 1 \mathrm{c}$ & & \\
\hline 2568.9061(20) & 38915.41(3) & $2568.9075(4)$ & -0.0014 & 2400 & & $4 \mathrm{~d}$ & $(5 / 2)^{2}[1 / 2]_{1}$ & $6 f$ & $(3 / 2)^{2}[3 / 2]^{0}{ }_{2}$ & & & R1c & & \\
\hline 2571.7551(6) & $38872.306(9)$ & $2571.75556(8)$ & -0.0004 & 220000 & & $4 p$ & ${ }^{1} \mathrm{D}^{\circ}{ }_{2}$ & $5 \mathrm{~s}$ & $(3 / 2)^{2}[3 / 2]_{2}$ & $1.10 \mathrm{e}+07$ & $\mathrm{C}+$ & R1c & K82 & \\
\hline 2574.4124(7) & $38832.185(11)$ & $2574.4125(5)$ & -0.0001 & 2100 & & $\mathrm{~s}^{2}$ & ${ }^{3} \mathrm{~F}_{3}$ & $\mathrm{sp}$ & $\left({ }^{3} \mathrm{~F}\right)^{3} \mathrm{P}^{\circ}{ }^{5} \mathrm{D}_{2}{ }_{2}$ & & & R1c & & \\
\hline 2574.6371(7) & $38828.796(11)$ & 2574.6371(5) & -0.0000 & 4600 & & $4 \mathrm{~d}$ & $(3 / 2)^{2}[7 / 2]_{4}$ & $7 \mathrm{f}$ & $(3 / 2)^{2}[9 / 2]^{\circ}{ }_{5}$ & & & R1c & & \\
\hline 2590.4012(9) & $38592.515(14)$ & 2590.3994(4) & 0.0019 & 990 & & $4 \mathrm{~d}$ & $(5 / 2)^{2}[5 / 2]_{3}$ & $7 \mathrm{f}$ & $(5 / 2)^{2}[7 / 2]^{\circ} 4$ & & & $\mathrm{R} 1 \mathrm{c}$ & & \\
\hline $2590.52825(17)$ & $38590.6226(25)$ & $2590.52826(8)$ & -0.00001 & 440000 & & $4 p$ & ${ }^{1} \mathrm{D}^{\circ}{ }_{2}$ & $5 \mathrm{~s}$ & $(3 / 2)^{2}[3 / 2]_{1}$ & $6.0 \mathrm{e}+07$ & $\mathrm{C}+$ & $\mathrm{F}$ & C94 & $\mathrm{L}$ \\
\hline $2598.8126(6)$ & $38467.613(9)$ & $2598.81194(10)$ & 0.0006 & 400000 & & $4 p$ & ${ }^{3} \mathrm{~F}_{2}^{\circ}$ & $5 \mathrm{~s}$ & $(5 / 2)^{2}[5 / 2]_{2}$ & $4.2 \mathrm{e}+07$ & $\mathrm{C}+$ & R1c & K82 & $\mathrm{L}$ \\
\hline 2600.26992(14) & $38446.0554(20)$ & $2600.26973(8)$ & 0.00018 & 500000 & & $4 p$ & ${ }^{3} \mathrm{D}_{3}^{\circ}$ & $5 \mathrm{~s}$ & $(3 / 2)^{2}[3 / 2]_{2}$ & $1.01 \mathrm{e}+08$ & $\mathrm{C}+$ & $\mathrm{F}$ & C94 & \\
\hline $2604.5253(21)$ & $38383.24(3)$ & $2604.5089(11)$ & 0.0164 & 870 & ? & $\mathrm{sp}$ & $\left({ }^{3} \mathrm{~F}\right)^{3} \mathrm{P}^{\circ}{ }^{3} \mathrm{~F}^{\circ}{ }_{3}$ & $10 \mathrm{~s}$ & $(5 / 2)^{2}[5 / 2]_{2}$ & & & R1c & & $\mathrm{x}$ \\
\hline $2606.5804(14)$ & $38352.984(21)$ & $2606.5819(5)$ & -0.0015 & 1700 & & $4 \mathrm{~d}$ & $(5 / 2)^{2}[7 / 2]_{3}$ & $7 \mathrm{f}$ & $(5 / 2)^{2}[7 / 2]^{\circ}{ }_{3}$ & & & R1c & & \\
\hline $2606.8761(8)$ & $38348.634(12)$ & $2606.8759(4)$ & 0.0001 & 3400 & & $4 \mathrm{~d}$ & $(5 / 2)^{2}[7 / 2]_{3}$ & $7 \mathrm{f}$ & $(5 / 2)^{2}[9 / 2]^{\circ} 4$ & & & R1c & & \\
\hline $2606.9973(10)$ & $38346.850(15)$ & $2606.9973(4)$ & 0.0001 & 2500 & & $4 \mathrm{~d}$ & $(5 / 2)^{2}[7 / 2]_{3}$ & $7 f$ & $(5 / 2)^{2}[7 / 2]^{\circ} 4$ & & & R1c & & \\
\hline 2607.041 & 38346.21 & $2607.0410(4)$ & & & : & $4 \mathrm{~d}$ & $(5 / 2)^{2}[7 / 2]_{3}$ & $7 \mathrm{f}$ & $(5 / 2)^{2}[5 / 2]^{\circ}{ }_{3}$ & & & & & \\
\hline $2609.9088(6)$ & $38304.075(9)$ & 2609.9094(3) & -0.0006 & 8400 & & $4 \mathrm{~d}$ & $(5 / 2)^{2}[7 / 2]_{4}$ & $7 f$ & $(5 / 2)^{2}[9 / 2]^{\circ}{ }_{5}$ & & & R1c & & \\
\hline $2610.7937(14)$ & $38291.094(21)$ & $2610.7940(5)$ & -0.0003 & 1600 & & $4 \mathrm{~d}$ & $(5 / 2)^{2}[5 / 2]_{2}$ & $7 \mathrm{f}$ & $(5 / 2)^{2}[7 / 2]^{\circ}{ }_{3}$ & & & R1c & & \\
\hline 2611.2544(8) & $38284.338(11)$ & $2611.2546(4)$ & -0.0002 & 810 & & $4 \mathrm{~d}$ & $(5 / 2)^{2}[5 / 2]_{2}$ & $7 \mathrm{f}$ & $(5 / 2)^{2}[5 / 2]^{\circ}{ }_{3}$ & & & R1c & & \\
\hline $2614.4127(7)$ & 38238.092(11) & $2614.4126(7)$ & 0.0001 & 15000 & & $s^{2}$ & ${ }^{3} \mathrm{~F}_{4}$ & $\mathrm{sp}$ & $\left({ }^{3} \mathrm{~F}\right)^{3} \mathrm{P}^{\circ}{ }^{5} \mathrm{D}^{\circ}{ }_{4}$ & & & R1c & & \\
\hline 2619.2104(5) & $38168.054(8)$ & $2619.21044(15)$ & -0.0000 & 8000 & & $s^{2}$ & ${ }^{1} \mathrm{D}_{2}$ & $5 p$ & $(3 / 2)^{2}[3 / 2]^{0}{ }_{2}$ & & & $\mathrm{R} 1 \mathrm{c}$ & & \\
\hline $2620.6656(5)$ & $38146.862(7)$ & $2620.66627(10)$ & -0.0007 & 56000 & & $4 p$ & ${ }^{3} \mathrm{~F}_{2}^{\circ}$ & $5 \mathrm{~s}$ & $(5 / 2)^{2}[5 / 2]_{3}$ & $1.3 e+06$ & E & R1c & $\mathrm{K} 82 \mathrm{cal}$ & \\
\hline $2636.6187(6)$ & $37916.065(8)$ & $2636.61965(16)$ & -0.0010 & 2200 & & $\mathrm{~s}^{\frac{1}{2}}$ & ${ }^{1} \mathrm{D}_{2}$ & $5 p$ & $(3 / 2)^{2}[3 / 2]^{\circ} 1$ & & & R1c & & \\
\hline $2648.6056(7)$ & $37744.477(9)$ & $2648.6059(4)$ & -0.0003 & 3000 & & $\mathrm{~s}^{2}$ & ${ }^{3} \mathrm{~F}_{3}$ & $\mathrm{sp}$ & $\left({ }^{3} \mathrm{~F}\right)^{3} \mathrm{P}^{\circ}{ }^{5} \mathrm{D}_{3}{ }_{3}$ & & & $\mathrm{R} 1 \mathrm{c}$ & & \\
\hline $2655.9644(7)$ & $37639.906(9)$ & $2655.9642(5)$ & 0.0002 & 840 & & $s^{2}$ & ${ }^{3} \mathrm{~F}_{2}$ & $\mathrm{sp}$ & $\left({ }^{3} \mathrm{~F}\right)^{3} \mathrm{P}^{\circ}{ }^{5} \mathrm{D}^{\circ}{ }_{2}$ & & & R1c & & \\
\hline 2666.2906(5) & $37494.140(8)$ & $2666.29047(11)$ & 0.0001 & 230000 & & $4 p$ & ${ }^{1} \mathrm{~F}_{3}^{\circ}$ & $5 s$ & $(5 / 2)^{2}[5 / 2]_{2}$ & $2.0 \mathrm{e}+07$ & $\mathrm{C}+$ & R1c & K82 & \\
\hline 2667.4229(15) & $37478.224(21)$ & $2667.4250(4)$ & -0.0021 & 990 & & $5 s$ & $(3 / 2)^{2}[3 / 2]_{1}$ & $7 p$ & $(5 / 2)^{2}[3 / 2]^{\circ}$ & & & R1c & & \\
\hline $2676.0660(6)$ & $37357.184(8)$ & $2676.06654(15)$ & -0.0005 & 3100 & & $s^{2}$ & ${ }^{1} \mathrm{D}_{2}$ & $5 p$ & $(3 / 2)^{2}[5 / 2]^{\circ}{ }_{2}$ & & & R1c & & \\
\hline 2681.4966(15) & $37281.533(21)$ & $2681.4957(7)$ & 0.0008 & 2100 & & $5 \mathrm{~s}$ & $(3 / 2)^{2}[3 / 2]_{2}$ & $7 p$ & $(5 / 2)^{2}[5 / 2]^{\circ}$ & & & R1c & & \\
\hline $2682.7484(8)$ & $37264.137(11)$ & $2682.75015(9)$ & -0.0017 & 440 & & $4 p$ & ${ }^{3} \mathrm{D}^{\circ}{ }_{1}$ & $5 s$ & $(3 / 2)^{2}[3 / 2]_{2}$ & $9 . e+05$ & $\mathrm{E}$ & R1c & K82cal & \\
\hline 2689.2993(5) & $37173.370(7)$ & 2689.29932(11) & 0.0000 & 410000 & & $4 p$ & ${ }^{1} \mathrm{~F}_{3}^{\circ}$ & $5 \mathrm{~s}$ & $(5 / 2)^{2}[5 / 2]_{3}$ & $5.9 \mathrm{e}+07$ & $\mathrm{C}+$ & R1c & C94 & \\
\hline 2692.4978(15) & 37129.213(21) & $2692.4942(4)$ & 0.0036 & 3200 & & $4 \mathrm{~d}$ & $(5 / 2)^{2}[7 / 2]_{3}$ & $6 f$ & $(3 / 2)^{2}[9 / 2]^{\circ}$ & & & $\mathrm{R} 1 \mathrm{c}$ & & \\
\hline $2700.96242(17)$ & $37012.8599(24)$ & $2700.96252(9)$ & -0.00011 & 520000 & & $4 p$ & ${ }^{3} \mathrm{D}_{2}^{\circ}$ & $5 \mathrm{~s}$ & $(3 / 2)^{2}[3 / 2]_{2}$ & $1.02 \mathrm{e}+08$ & $\mathrm{C}+$ & $\mathrm{F}$ & C94 & \\
\hline 2703.18424(13) & $36982.4398(18)$ & 2703.18448(8) & -0.00024 & 540000 & & $4 \mathrm{p}$ & ${ }^{3} \mathrm{D}^{\circ}{ }_{1}$ & $5 \mathrm{~s}$ & $(3 / 2)^{2}[3 / 2]_{1}$ & $1.17 \mathrm{e}+08$ & $\mathrm{C}+$ & $\mathrm{F}$ & C94 & \\
\hline $2704.5194(23)$ & $36964.18(3)$ & $2704.5157(16)$ & 0.0037 & 1100 & & $\mathrm{~s}^{\frac{1}{2}}$ & ${ }^{3} \mathrm{P}_{1}$ & $\mathrm{sp}$ & $\left({ }^{3} \mathrm{P}\right)^{3} \mathrm{P}^{\circ}{ }^{5} \mathrm{P}^{\circ}{ }_{1}$ & & & R1c & & \\
\hline $2708.2695(8)$ & 36913.003(11) & 2708.2695(3) & -0.0000 & 7200 & & $4 \mathrm{~d}$ & $(5 / 2)^{2}[1 / 2]_{1}$ & $8 p$ & $(5 / 2)^{2}[3 / 2]^{\circ}{ }_{1}$ & & & $\mathrm{R} 1 \mathrm{c}$ & & \\
\hline $2709.7592(6)$ & $36892.711(8)$ & 2709.7597(3) & -0.0006 & 1600 & & $4 \mathrm{~d}$ & $(5 / 2)^{2}[1 / 2]_{1}$ & $6 \mathrm{f}$ & $(5 / 2)^{2}[5 / 2]^{\circ} 2$ & & & R1c & & \\
\hline
\end{tabular}


Table A1. Cont.

\begin{tabular}{|c|c|c|c|c|c|c|c|c|c|c|c|c|c|c|}
\hline$\lambda_{\text {obs }}{ }^{\text {a }}(\AA)$ & $\sigma_{\mathrm{obs}}{ }^{\mathrm{b}}\left(\mathrm{cm}^{-1}\right)$ & $\lambda_{\text {Ritz }}{ }^{c}(\AA)$ & $\Delta \lambda_{\text {obs-Ritz }}$ & $\begin{array}{l}I_{\text {obs }} \mathrm{d} \\
\text { (arb. u.) }\end{array}$ & Char ${ }^{e}$ & & Lower Level & & Upper Level & $A\left(\mathrm{~s}^{-1}\right)$ & $\operatorname{Acc}^{f}$ & Line Ref. $\mathrm{g}$ & TP Ref. $\mathrm{g}$ & Notes ${ }^{h}$ \\
\hline $2710.2454(16)$ & $36886.093(21)$ & $2710.2434(6)$ & 0.0020 & 2700 & & $\mathrm{~s}^{2}$ & ${ }^{3} \mathrm{P}_{2}$ & $\mathrm{sp}$ & $\left({ }^{3} \mathrm{P}\right)^{3} \mathrm{P}^{\circ}{ }^{5} \mathrm{P}_{2}^{\circ}$ & & & R1c & & \\
\hline $2710.6069(16)$ & $36881.173(21)$ & $2710.6071(7)$ & -0.0001 & 700 & & $4 \mathrm{~d}$ & $(5 / 2)^{2}[1 / 2]_{0}$ & $6 f$ & $(3 / 2)^{2}[3 / 2]^{\circ}{ }_{1}^{2}$ & & & R1c & & \\
\hline $2711.5767(23)$ & $36867.98(3)$ & $2711.5711(13)$ & 0.0057 & 350 & & $s^{2}$ & ${ }^{3} \mathrm{P}_{2}$ & $\mathrm{sp}$ & $\left({ }^{3} \mathrm{P}\right)^{3} \mathrm{P}^{\circ}{ }^{5} \mathrm{P}^{\circ}{ }_{3}$ & & & $\mathrm{R} 2 \mathrm{nc}$ & & \\
\hline $2711.8649(6)$ & $36864.066(8)$ & $2711.8639(3)$ & 0.0010 & 18000 & & $4 \mathrm{~d}$ & $(5 / 2)^{2}[1 / 2]_{1}$ & $6 \mathrm{f}$ & $(5 / 2)^{2}[3 / 2]^{\circ} 2$ & & & R1c & & \\
\hline $2713.5078(5)$ & $36841.748(7)$ & $2713.50740(10)$ & 0.0004 & 400000 & & $4 p$ & ${ }^{1} \mathrm{D}^{\circ}{ }_{2}$ & $5 \mathrm{~s}$ & $(5 / 2)^{2}[5 / 2]_{2}$ & $8.9 e+07$ & $\mathrm{C}+$ & R1c & C94 & \\
\hline $2715.4038(6)$ & $36816.024(8)$ & $2715.4039(6)$ & -0.0000 & 10000 & & $4 \mathrm{~d}$ & $(5 / 2)^{2}[1 / 2]_{1}$ & $6 f$ & $(5 / 2)^{2}[1 / 2]^{\circ} 0$ & & & R1c & & \\
\hline $2718.7770(3)$ & $36770.349(4)$ & $2718.77770(14)$ & -0.0007 & 440000 & & $4 p$ & ${ }^{1} \mathrm{P}_{1}^{\circ}$ & $5 \mathrm{~s}$ & $(3 / 2)^{2}[3 / 2]_{2}$ & $8.0 \mathrm{e}+07$ & $\mathrm{C}+$ & $\mathrm{F}$ & C94 & \\
\hline $2721.6764(2)$ & $36731.179(3)$ & $2721.67628(9)$ & 0.0002 & 300000 & & $4 p$ & ${ }^{3} \mathrm{D}_{2}^{\circ}$ & $5 \mathrm{~s}$ & $(3 / 2)^{2}[3 / 2]_{1}$ & $3.9 \mathrm{e}+07$ & $\mathrm{C}+$ & $\mathrm{F}$ & K82 & $\mathrm{L}$ \\
\hline $2727.6947(6)$ & $36650.142(8)$ & $2727.6948(5)$ & -0.0001 & 620 & & $4 \mathrm{~d}$ & $(5 / 2)^{2}[1 / 2]_{1}$ & $6 f$ & $(5 / 2)^{2}[1 / 2]^{\circ}{ }_{1}$ & & & $\mathrm{R} 2 \mathrm{nc}$ & & \\
\hline $2728.204(7)$ & $36643.30(10)$ & $2728.2019(16)$ & 0.002 & 310 & & $\mathrm{~s}^{2}$ & ${ }^{3} \mathrm{P}_{0}$ & $\mathrm{sp}$ & $\left({ }^{3} \mathrm{P}^{3} \mathrm{P}^{\mathrm{o}} \mathrm{P}^{\mathrm{O}}\right.$ & & & R1c & & \\
\hline 2731.9477(6) & $36593.089(7)$ & $2731.94820(16)$ & -0.0005 & 6300 & & $s^{2}$ & ${ }^{1} \mathrm{D}_{2}$ & $\mathrm{sp}$ & $\left({ }^{3} \mathrm{~F}\right)^{3} \mathrm{P}^{\circ}{ }^{1} \mathrm{D}^{\circ}{ }_{2}$ & & & R1c & & \\
\hline $2737.3415(6)$ & $36520.989(7)$ & 2737.34194(10) & -0.0005 & 48000 & & $4 p$ & ${ }^{1} \mathrm{D}^{\circ}{ }_{2}$ & $5 \mathrm{~s}$ & $(5 / 2)^{2}[5 / 2]_{3}$ & $1.9 e+06$ & $\mathrm{D}$ & $\mathrm{R} 1 \mathrm{c}$ & C94 & \\
\hline $2739.7662(6)$ & $36488.669(8)$ & $2739.76664(14)$ & -0.0004 & 86000 & & $4 p$ & ${ }^{1} \mathrm{P}_{1}^{\circ}$ & $5 s$ & $(3 / 2)^{2}[3 / 2]_{1}$ & $4.0 \mathrm{e}+06$ & $\mathrm{D}$ & R1c & C94 & \\
\hline $2745.2710(6)$ & $36415.506(7)$ & $2745.27056(10)$ & 0.0005 & 140000 & & $4 p$ & ${ }^{3} \mathrm{D}_{3}^{\circ}$ & $5 \mathrm{~s}$ & $(5 / 2)^{2}[5 / 2]_{2}$ & $1.40 \mathrm{e}+07$ & $\mathrm{C}+$ & R1c & C94 & \\
\hline 2757.3283(7) & $36256.276(9)$ & $2757.3290(4)$ & -0.0007 & 770 & & $4 \mathrm{~d}$ & $(3 / 2)^{2}[1 / 2]_{1}$ & $6 f$ & $(3 / 2)^{2}[5 / 2]^{0} 2$ & & & R1c & & \\
\hline $2759.6070(8)$ & $36226.340(11)$ & $2759.6065(7)$ & 0.0005 & 760 & & $4 \mathrm{~d}$ & $(3 / 2)^{2}[1 / 2]_{1}$ & $6 f$ & $(3 / 2)^{2}[3 / 2]^{\circ} 1$ & & & $\mathrm{R} 1 \mathrm{c}$ & & \\
\hline $2762.481(20)$ & $36188.6(3)$ & 2762.4581(4) & 0.023 & 1000 & * & $4 \mathrm{~d}$ & $(3 / 2)^{2}[5 / 2]_{3}$ & $7 \mathrm{f}$ & $(5 / 2)^{2}[7 / 2]^{\circ}{ }_{4}$ & & & R1c & & \\
\hline $2762.481(20)$ & $36188.6(3)$ & $2762.5072(5)$ & -0.026 & 1000 & * & $4 \mathrm{~d}$ & $(3 / 2)^{2}[5 / 2]_{3}$ & $7 \mathrm{f}$ & $(5 / 2)^{2}[5 / 2]^{\circ} 3$ & & & R1c & & \\
\hline $2769.6690(6)$ & $36094.738(7)$ & $2769.66882(9)$ & 0.0002 & 310000 & & $4 p$ & ${ }^{3} \mathrm{D}^{\circ}{ }_{3}$ & $5 \mathrm{~s}$ & $(5 / 2)^{2}[5 / 2]_{3}$ & $6.7 \mathrm{e}+07$ & $\mathrm{C}+$ & R1c & C94 & \\
\hline $2788.2614(7)$ & $35854.067(9)$ & 2788.2619(3) & -0.0005 & 5700 & & $4 \mathrm{~d}$ & $(5 / 2)^{2}[9 / 2]_{5}$ & $6 f$ & $(5 / 2)^{2}[9 / 2]^{\circ}{ }_{5}$ & & & R1c & & \\
\hline $2789.2226(17)$ & $35841.712(21)$ & 2789.2218(12) & 0.0009 & 210 & & $5 p$ & $(5 / 2)^{2}[7 / 2]^{\circ}{ }_{3}$ & $10 \mathrm{~s}$ & $(5 / 2)^{2}[5 / 2]_{2}$ & & & R1c & & \\
\hline $2791.7945(6)$ & $35808.695(8)$ & $2791.7947(4)$ & -0.0002 & 54000 & & $4 \mathrm{~d}$ & $(5 / 2)^{2}[9 / 2]_{5}$ & $6 f$ & $(5 / 2)^{2}[11 / 2]^{\circ} 6$ & & & R1c & & \\
\hline 2792.224(11) & $35803.19(14)$ & $2792.2125(3)$ & 0.012 & 420 & * & $4 \mathrm{~d}$ & $(5 / 2)^{2}[9 / 2]_{5}$ & $6 f$ & $(5 / 2)^{2}[11 / 2]^{\circ}{ }_{5}$ & & & R1c & & \\
\hline 2792.224(11) & $35803.19(14)$ & 2792.2312(4) & -0.007 & 420 & * & $4 \mathrm{~d}$ & $(5 / 2)^{2}[3 / 2]_{2}$ & $6 \mathrm{f}$ & $(5 / 2)^{2}[7 / 2]^{\circ}{ }_{3}$ & & & R1c & & \\
\hline $2793.6079(7)$ & $35785.452(9)$ & 2793.6091(3) & -0.0012 & 620 & & $4 \mathrm{~d}$ & $(5 / 2)^{2}[3 / 2]_{2}$ & $8 p$ & $(5 / 2)^{2}[3 / 2]^{\circ}{ }_{1}$ & & & R1c & & \\
\hline 2795.2979(6) & $35763.818(8)$ & 2795.2980(3) & -0.0001 & 8000 & & $4 \mathrm{~d}$ & $(5 / 2)^{2}[3 / 2]_{2}$ & $6 \mathrm{f}$ & $(5 / 2)^{2}[5 / 2]^{\circ}{ }_{3}$ & & & R1c & & \\
\hline 2795.6571(7) & $35759.223(9)$ & $2795.6567(3)$ & 0.0004 & 4600 & & $4 \mathrm{~d}$ & $(5 / 2)^{2}[9 / 2]_{4}$ & $6 f$ & $(5 / 2)^{2}[9 / 2]^{\circ}{ }_{4}$ & & & R1c & & \\
\hline $2795.8729(11)$ & $35756.463(14)$ & 2795.8732(3) & -0.0003 & 610 & & $4 \mathrm{~d}$ & $(5 / 2)^{2}[9 / 2]_{4}$ & $6 \mathrm{f}$ & $(5 / 2)^{2}[7 / 2]^{7}$ & & & $\mathrm{R} 1 \mathrm{c}$ & & \\
\hline $2796.2625(7)$ & $35751.482(9)$ & $2796.2624(5)$ & 0.0001 & 820 & & $4 \mathrm{~d}$ & $(3 / 2)^{2}[7 / 2]_{3}$ & $6 \mathrm{f}$ & $(3 / 2)^{2}[7 / 2]^{\circ}{ }_{3}$ & & & R1c & & \\
\hline $2797.2549(6)$ & $35738.798(8)$ & 2797.2557(3) & -0.0008 & 7500 & & $4 \mathrm{~d}$ & $(5 / 2)^{2}[3 / 2]_{1}$ & $6 \mathrm{f}$ & $(5 / 2)^{2}[5 / 2]^{\circ}{ }_{2}$ & & & R1c & & \\
\hline 2797.4336(6) & $35736.516(8)$ & 2797.4337(3) & -0.0001 & 8300 & & $4 \mathrm{~d}$ & $(5 / 2)^{2}[3 / 2]_{2}$ & $6 \mathrm{f}$ & $(5 / 2)^{2}[3 / 2]^{\circ}{ }_{2}$ & & & R1c & & \\
\hline 2797.5493(12) & $35735.038(16)$ & 2797.5494(5) & -0.0001 & 410 & & $4 \mathrm{~d}$ & $(5 / 2)^{2}[3 / 2]_{2}$ & $6 \mathrm{f}$ & $(5 / 2)^{2}[3 / 2]^{\circ}{ }_{1}$ & & & R1c & & \\
\hline 2798.8296(17) & $35718.692(21)$ & & & 200 & & $5 p$ & $(5 / 2)^{2}[7 / 2]^{\circ}{ }_{4}$ & $10 \mathrm{~s}$ & $(5 / 2)^{2}[5 / 2]_{3}$ & & & R1c & & \\
\hline $2799.5280(6)$ & $35709.781(8)$ & 2799.5291(3) & -0.0010 & 35000 & & $4 \mathrm{~d}$ & $(5 / 2)^{2}[9 / 2]_{4}$ & $6 \mathrm{f}$ & $(5 / 2)^{2}[11 / 2]^{\circ}{ }_{5}$ & & & R1c & & \\
\hline $2799.6805(6)$ & $35707.837(8)$ & 2799.6812(4) & -0.0007 & 10000 & & $4 \mathrm{~d}$ & $(3 / 2)^{2}[7 / 2]_{3}$ & $6 \mathrm{f}$ & $(3 / 2)^{2}[9 / 2]^{\circ}{ }_{4}$ & & & R1c & & \\
\hline $2801.0500(6)$ & $35690.379(8)$ & $2801.05008(16)$ & -0.0001 & 1000 & & $s^{2}$ & ${ }^{1} \mathrm{D}_{2}$ & $5 p$ & $(5 / 2)^{2}[5 / 2]_{3}{ }_{3}$ & & & $\mathrm{R} 1 \mathrm{c}$ & & \\
\hline $2801.3182(24)$ & $35686.96(3)$ & $2801.3220(17)$ & -0.0038 & 400 & & $4 \mathrm{~d}$ & $(3 / 2)^{2}[3 / 2]_{2}$ & $\mathrm{sp}$ & ${ }^{3} \mathrm{D}_{3}^{\circ}$ & & & $\mathrm{R} 2 \mathrm{nc}$ & & \\
\hline 2803.2711(17) & $35662.102(21)$ & $2803.2706(6)$ & 0.0005 & 390 & & $4 \mathrm{~d}$ & $(5 / 2)^{2}[3 / 2]_{1}$ & $6 \mathrm{f}$ & $(5 / 2)^{2}[1 / 2]^{\circ}{ }_{0}$ & & & R1c & & \\
\hline 2804.1888(17) & $35650.432(21)$ & $2804.1896(12)$ & -0.0008 & 200 & & $5 p$ & $(5 / 2)^{2}[5 / 2]^{0}{ }_{2}$ & $10 \mathrm{~s}$ & $(5 / 2)^{2}[5 / 2]_{2}$ & & & R1c & & \\
\hline $2807.1550(8)$ & $35612.763(10)$ & $2807.1556(5)$ & -0.0006 & 770 & & $4 \mathrm{~d}$ & $(3 / 2)^{2}[7 / 2]_{4}$ & $6 \mathrm{f}$ & $(3 / 2)^{2}[7 / 2]^{\circ}$ & & & R1c & & \\
\hline $2810.3655(17)$ & $35572.082(21)$ & $2810.3657(4)$ & -0.0002 & 190 & & $4 \mathrm{~d}$ & $(3 / 2)^{2}[7 / 2]_{4}$ & $6 \mathrm{f}$ & $(3 / 2)^{2}[9 / 2]^{\circ}$ & & & R1c & & \\
\hline
\end{tabular}


Table A1. Cont.

\begin{tabular}{|c|c|c|c|c|c|c|c|c|c|c|c|c|c|c|}
\hline$\lambda_{\text {obs }}{ }^{a}(\AA)$ & $\sigma_{\mathrm{obs}}{ }^{\mathrm{b}}\left(\mathrm{cm}^{-1}\right)$ & $\lambda_{\text {Ritz }}{ }^{c}(\AA ̊)$ & $\Delta \lambda_{\text {obs-Ritz }}$ & $\begin{array}{c}I_{\text {obs }} \mathrm{d} \\
\text { (arb.u.) }\end{array}$ & Char ${ }^{e}$ & & ower Level & & Jpper Level & $A\left(\mathrm{~s}^{-1}\right)$ & $\operatorname{Acc}^{f}$ & Line Ref. $\mathrm{g}$ & TP Ref. $\mathrm{g}$ & Notes ${ }^{h}$ \\
\hline $2810.8038(6)$ & $35566.536(7)$ & $2810.8039(4)$ & -0.0001 & 14000 & & $4 \mathrm{~d}$ & $(3 / 2)^{2}[7 / 2]_{4}$ & $6 f$ & $(3 / 2)^{2}[9 / 2]^{\circ}{ }_{5}$ & & & R1c & & \\
\hline $2813.6315(7)$ & $35530.793(9)$ & $2813.63100(16)$ & 0.0005 & 2900 & & $\mathrm{~s}^{2}$ & ${ }^{1} \mathrm{D}_{2}$ & $5 p$ & $(5 / 2)^{2}[3 / 2]^{\circ}{ }_{1}$ & & & R1c & & \\
\hline 2816.1978(9) & 35498.417(11) & 2816.1982(5) & -0.0004 & 3700 & & $4 \mathrm{~d}$ & $(3 / 2)^{2}[3 / 2]_{1}$ & $6 \mathrm{f}$ & $(3 / 2)^{2}[3 / 2]^{0}{ }_{2}$ & & & R1c & & \\
\hline $2817.0843(7)$ & $35487.247(9)$ & $2817.08465(18)$ & -0.0004 & 370 & & $\mathrm{~s}^{2}$ & ${ }^{1} \mathrm{D}_{2}$ & $5 p$ & $(5 / 2)^{2}[5 / 2]^{\circ} 2$ & & & R1c & & \\
\hline $2828.6968(7)$ & $35341.570(9)$ & $2828.6970(3)$ & -0.0002 & 3500 & & $4 \mathrm{~d}$ & $(5 / 2)^{2}[5 / 2]_{3}$ & $6 \mathrm{f}$ & $(5 / 2)^{2}[9 / 2]^{\circ} 4$ & & & R1c & & \\
\hline 2828.9184(12) & $35338.802(15)$ & 2828.9187(3) & -0.0003 & 3500 & & $4 \mathrm{~d}$ & $(5 / 2)^{2}[5 / 2]_{3}$ & $6 f$ & $(5 / 2)^{2}[7 / 2]^{\circ}{ }_{4}$ & & & R1c & & \\
\hline $2830.2314(8)$ & $35322.408(10)$ & $2830.2323(3)$ & -0.0008 & 6100 & & $4 \mathrm{~d}$ & $(5 / 2)^{2}[5 / 2]_{3}$ & $6 f$ & $(5 / 2)^{2}[5 / 2]^{\circ} 3$ & & & R1c & & \\
\hline $2832.4214(7)$ & $35295.099(9)$ & $2832.4217(3)$ & -0.0004 & 3400 & & $4 \mathrm{~d}$ & $(5 / 2)^{2}[5 / 2]_{3}$ & $6 f$ & $(5 / 2)^{2}[3 / 2]^{\circ} 2$ & & & R1c & & \\
\hline $2833.053(2)$ & $35287.23(3)$ & 2833.0250(5) & 0.028 & 170 & ? & $\mathrm{sp}$ & $\left({ }^{3} \mathrm{~F}\right)^{3} \mathrm{P}^{0}{ }^{3} \mathrm{D}^{\circ}{ }_{3}$ & $7 \mathrm{~d}$ & $(5 / 2)^{2}[3 / 2]_{2}$ & & & R1c & & $\mathrm{x}$ \\
\hline $2834.9699(17)$ & $35263.372(21)$ & $2834.9704(5)$ & -0.0005 & 510 & & $4 \mathrm{~d}$ & $(3 / 2)^{2}[3 / 2]_{2}$ & $6 f$ & $(3 / 2)^{2}[3 / 2]^{0} 2$ & & & R1c & & \\
\hline $2836.2898(8)$ & $35246.962(10)$ & 2836.2911(5) & -0.0013 & 4600 & & $4 \mathrm{~d}$ & $(3 / 2)^{2}[3 / 2]_{2}$ & $6 f$ & $(3 / 2)^{2}[5 / 2]^{\circ} 3$ & & & R1c & & \\
\hline 2836.6971(17) & $35241.902(21)$ & $2836.7201(6)$ & -0.0230 & 840 & ? & $\mathrm{sp}$ & $\left({ }^{3} \mathrm{~F}\right)^{3} \mathrm{P}^{\circ}{ }^{3} \mathrm{~F}^{\circ}{ }_{2}$ & $9 \mathrm{~s}$ & $(5 / 2)^{2}[5 / 2]_{2}$ & & & R1c & & $\mathrm{x}$ \\
\hline $2837.3682(6)$ & $35233.566(7)$ & $2837.36814(10)$ & 0.0001 & 150000 & & $4 \mathrm{p}$ & ${ }^{3} \mathrm{D}^{\circ}{ }_{1}$ & $5 \mathrm{~s}$ & $(5 / 2)^{2}[5 / 2]_{2}$ & $2.3 e+07$ & $\mathrm{C}+$ & R1c & C94 & \\
\hline $2840.4918(6)$ & $35194.823(8)$ & $2840.49162(17)$ & 0.0002 & 5800 & & $\mathrm{~s}^{2}$ & ${ }^{3} \mathrm{P}_{2}$ & $5 p$ & $(3 / 2)^{2}[3 / 2]^{\circ} 2$ & & & R1c & & \\
\hline $2846.8683(7)$ & $35115.996(8)$ & 2846.8693(5) & -0.0009 & 4300 & & $4 \mathrm{~d}$ & $(5 / 2)^{2}[7 / 2]_{3}$ & $6 \mathrm{f}$ & $(5 / 2)^{2}[7 / 2]^{0}{ }_{3}$ & & & R1c & & \\
\hline $2848.4999(6)$ & $35095.883(8)$ & $2848.5005(3)$ & -0.0005 & 11000 & & $4 \mathrm{~d}$ & $(5 / 2)^{2}[7 / 2]_{3}$ & $6 f$ & $(5 / 2)^{2}[9 / 2]^{\circ} 4$ & & & R1c & & \\
\hline $2848.7252(6)$ & 35093.108(8) & 2848.7252(3) & -0.0000 & 9100 & & $4 \mathrm{~d}$ & $(5 / 2)^{2}[7 / 2]_{3}$ & $6 f$ & $(5 / 2)^{2}[7 / 2]^{\circ} 4$ & & & R1c & & \\
\hline $2849.9526(10)$ & $35077.995(12)$ & $2849.9500(3)$ & 0.0026 & 160 & & $4 \mathrm{~d}$ & $(5 / 2)^{2}[7 / 2]_{3}$ & $6 \mathrm{f}$ & $(5 / 2)^{2}[5 / 2]^{\circ}{ }_{2}$ & & & R1c & & \\
\hline $2851.8949(8)$ & $35054.106(10)$ & $2851.8944(5)$ & 0.0005 & 4700 & & $4 \mathrm{~d}$ & $(5 / 2)^{2}[5 / 2]_{2}$ & $6 \mathrm{f}$ & $(5 / 2)^{2}[7 / 2]^{\circ} 3$ & & & R1c & & \\
\hline $2852.0764(7)$ & $35051.875(8)$ & $2852.0764(3)$ & 0.0001 & 12000 & & $4 \mathrm{~d}$ & $(5 / 2)^{2}[7 / 2]_{4}$ & $6 \mathrm{f}$ & $(5 / 2)^{2}[9 / 2]^{\circ}{ }_{5}$ & & & R1c & & \\
\hline 2852.1785(11) & $35050.621(13)$ & 2852.1793(3) & -0.0008 & 6300 & & $4 \mathrm{~d}$ & $(5 / 2)^{2}[7 / 2]_{4}$ & $6 f$ & $(5 / 2)^{2}[9 / 2]^{\circ}{ }_{4}$ & & & R1c & & \\
\hline $2852.4042(6)$ & $35047.847(8)$ & $2852.4046(3)$ & -0.0004 & 3900 & & $4 \mathrm{~d}$ & $(5 / 2)^{2}[7 / 2]_{4}$ & $6 f$ & $(5 / 2)^{2}[7 / 2]^{\circ} 4$ & & & R1c & & \\
\hline 2853.7403(7) & $35031.439(8)$ & 2853.7401(3) & 0.0002 & 630 & & $4 \mathrm{~d}$ & $(5 / 2)^{2}[7 / 2]_{4}$ & $6 f$ & $(5 / 2)^{2}[5 / 2]^{\circ} 3$ & & & R1c & & \\
\hline \multirow[t]{2}{*}{$2854.9858(7)$} & $35016.157(9)$ & $2854.9860(3)$ & -0.0002 & 2100 & & $4 \mathrm{~d}$ & $(5 / 2)^{2}[5 / 2]_{2}$ & $6 \mathrm{f}$ & $(5 / 2)^{2}[5 / 2]^{\circ} 2$ & & & R1c & & \\
\hline & & $2855.0785(5)$ & & & $\mathrm{m}$ & $4 \mathrm{~d}$ & $(3 / 2)^{2}[5 / 2]_{3}$ & $6 \mathrm{f}$ & $(3 / 2)^{2}[7 / 2]^{\circ} 3$ & & & R1nc & & \\
\hline $2855.0930(17)$ & $35014.842(21)$ & 2855.0937(3) & -0.0007 & 310 & & $4 \mathrm{~d}$ & $(5 / 2)^{2}[5 / 2]_{2}$ & $6 \mathrm{f}$ & $(5 / 2)^{2}[5 / 2]^{\circ} 3$ & & & R1c & & \\
\hline $2855.3206(7)$ & $35012.052(8)$ & 2855.3215(5) & -0.0009 & 4700 & & $4 \mathrm{~d}$ & $(3 / 2)^{2}[5 / 2]_{3}$ & $6 f$ & $(3 / 2)^{2}[7 / 2]^{\circ}{ }_{4}$ & & & R1c & & \\
\hline $2856.2109(7)$ & $35001.138(8)$ & $2856.2100(4)$ & 0.0010 & 310 & & $4 \mathrm{~d}$ & $(5 / 2)^{2}[7 / 2]_{4}$ & $6 f$ & $(5 / 2)^{2}[11 / 2]^{\circ}{ }_{5}$ & & & R1c & & \\
\hline 2857.4418(17) & $34986.062(21)$ & 2857.4425(5) & -0.0007 & 310 & & $4 \mathrm{~d}$ & $(5 / 2)^{2}[5 / 2]_{2}$ & $6 f$ & $(5 / 2)^{2}[3 / 2]^{\circ}{ }_{1}$ & & & R1c & & \\
\hline $2857.7484(6)$ & $34982.309(8)$ & $2857.74808(11)$ & 0.0003 & 19000 & & $4 p$ & ${ }^{3} \mathrm{D}^{\circ}{ }_{2}$ & $5 \mathrm{~s}$ & $(5 / 2)^{2}[5 / 2]_{2}$ & $5 . e+05$ & E & R1c & K82cal & \\
\hline $2859.0051(7)$ & $34966.932(8)$ & 2859.0054(5) & -0.0003 & 5300 & & $4 \mathrm{~d}$ & $(3 / 2)^{2}[5 / 2]_{2}$ & $6 \mathrm{f}$ & $(3 / 2)^{2}[7 / 2]^{0} 3$ & & & R1c & & \\
\hline 2859.9192(7) & $34955.757(9)$ & $2859.9187(4)$ & 0.0005 & 2300 & & $4 \mathrm{~d}$ & $(3 / 2)^{2}[5 / 2]_{2}$ & $6 \mathrm{f}$ & $(3 / 2)^{2}[5 / 2]^{\circ} 2$ & & & R1c & & \\
\hline $2860.2493(9)$ & 34951.723(11) & $2860.24866(19)$ & 0.0006 & 1100 & & $\mathrm{~s}^{2}$ & ${ }^{3} \mathrm{P}_{1}$ & $5 p$ & $(3 / 2)^{2}[3 / 2]^{\circ}{ }_{2}$ & & & R1c & & \\
\hline $2862.3233(7)$ & $34926.399(8)$ & $2862.3233(5)$ & -0.0000 & 3000 & & $4 \mathrm{~d}$ & $(3 / 2)^{2}[5 / 2]_{3}$ & $6 \mathrm{f}$ & $(3 / 2)^{2}[5 / 2]^{\circ} 3$ & & & R1c & & \\
\hline $2866.2706(17)$ & $34878.302(21)$ & 2866.2702(5) & 0.0004 & 740 & & $4 \mathrm{~d}$ & $(3 / 2)^{2}[5 / 2]_{2}$ & $6 f$ & $(3 / 2)^{2}[5 / 2]^{\circ}{ }_{3}$ & & & R1c & & \\
\hline 2868.7914(8) & $34847.656(9)$ & $2868.7906(4)$ & 0.0008 & 440 & & $4 \mathrm{~d}$ & $(5 / 2)^{2}[1 / 2]_{0}$ & $8 p$ & $(5 / 2)^{2}[3 / 2]^{\circ}{ }_{1}$ & & & R1c & & \\
\hline 2872.9461(7) & $34797.263(8)$ & $2872.9460(5)$ & 0.0001 & 580 & & $4 \mathrm{~d}$ & $(5 / 2)^{2}[1 / 2]_{0}$ & $6 \mathrm{f}$ & $(5 / 2)^{2}[3 / 2]^{\circ} 1$ & & & R1c & & \\
\hline 2875.3341(7) & $34768.365(9)$ & 2875.3346(5) & -0.0005 & 570 & & $5 p$ & $(5 / 2)^{2}[3 / 2]^{\circ} 2$ & $8 \mathrm{~d}$ & $(5 / 2)^{2}[5 / 2]_{3}$ & & & R1c & & \\
\hline 2877.6996(6) & $34739.786(7)$ & $2877.69898(16)$ & 0.0007 & 170000 & & $4 p$ & ${ }^{1} \mathrm{P}_{1}^{\circ}$ & $5 s$ & $(5 / 2)^{2}[5 / 2]_{2}$ & $2.3 e+07$ & $\mathrm{C}+$ & R1c & C94 & \\
\hline 2880.7003(7) & $34703.600(9)$ & $2880.69947(17)$ & 0.0009 & 2000 & & $\mathrm{~s}^{\frac{1}{2}}$ & ${ }^{1} \mathrm{D}_{2}$ & $5 p$ & $(5 / 2)^{2}[3 / 2]^{0} 2$ & & & R1c & & \\
\hline 2881.0216(8) & $34699.730(9)$ & $2881.02191(20)$ & -0.0003 & 1000 & & $\mathrm{~s}^{2}$ & ${ }^{3} \mathrm{P}_{1}$ & $5 p$ & $(3 / 2)^{2}[3 / 2]^{\circ}{ }_{1}$ & & & R1c & & \\
\hline 2883.1886(18) & $34673.651(21)$ & & & 2800 & & $5 p$ & $(5 / 2)^{2}[3 / 2]^{\circ} 2$ & $8 \mathrm{~d}$ & $(5 / 2)^{2}[1 / 2]_{1}$ & & & R1c & & \\
\hline
\end{tabular}


Table A1. Cont.

\begin{tabular}{|c|c|c|c|c|c|c|c|c|c|c|c|c|c|c|}
\hline$\lambda_{\text {obs }}{ }^{a}(\AA)$ & $\sigma_{\mathrm{obs}}{ }^{\mathrm{b}}\left(\mathrm{cm}^{-1}\right)$ & $\lambda_{\text {Ritz }}{ }^{\mathrm{c}}(\AA)$ & $\begin{array}{c}\Delta \lambda_{\text {obs-Ritz }} \\
\text { (A) }\end{array}$ & $\begin{array}{l}I_{\text {obs }} \mathrm{d} \\
\text { (arb. u.) }\end{array}$ & Char ${ }^{e}$ & & ower Level & & Upper Level & $A\left(\mathrm{~s}^{-1}\right)$ & $\operatorname{Acc}^{f}$ & Line Ref. $\mathrm{g}$ & TP Ref. $\mathrm{g}$ & Notes ${ }^{h}$ \\
\hline 2884.1954(6) & $34661.548(8)$ & $2884.19598(11)$ & -0.0006 & 41000 & & $4 p$ & ${ }^{3} \mathrm{D}_{2}^{\circ}$ & $5 s$ & $(5 / 2)^{2}[5 / 2]_{3}$ & $1.20 \mathrm{e}+07$ & $\mathrm{C}_{+}$ & R1c & C94 & \\
\hline $2884.7560(7)$ & $34654.812(9)$ & $2884.75571(16)$ & 0.0003 & 280 & & $\mathrm{~s}^{23} \mathrm{P}$ & & $5 p$ & $(3 / 2)^{2}[5 / 2]^{0}$ & & & R1c & & \\
\hline 2897.2206(7) & $34505.726(8)$ & 2897.21944(17) & 0.0011 & 1200 & & $\mathrm{~s}^{2}$ & ${ }^{3} \mathrm{P}_{2}$ & $5 p$ & $(3 / 2)^{2}[1 / 2]^{\circ} 1$ & & & R1c & & \\
\hline 2907.9162(8) & $34378.816(10)$ & 2907.9158(3) & 0.0004 & 1300 & & $s^{2}$ & ${ }^{3} \mathrm{P}_{0}$ & $5 p$ & $(3 / 2)^{2}[3 / 2]^{\circ}{ }_{1}$ & & & R1c & & \\
\hline 2908.863(3) & $34367.63(3)$ & $2908.8746(4)$ & -0.012 & 130 & & $\mathrm{sp}$ & $\left({ }^{3} \mathrm{~F}\right)^{3} \mathrm{P}^{\circ}{ }^{3} \mathrm{D}^{\circ}{ }_{3}$ & $8 \mathrm{~s}$ & $(5 / 2)^{2}[5 / 2]_{3}$ & & & R1c & & $x$ \\
\hline $2912.4526(18)$ & $34325.271(21)$ & & & 250 & & $5 p$ & $(5 / 2)^{2}[3 / 2]^{\circ}{ }_{1}$ & $8 \mathrm{~d}$ & $(5 / 2)^{2}[1 / 2]_{0}$ & & & R1c & & \\
\hline 2917.7762(9) & $34262.646(11)$ & $2917.77629(20)$ & -0.0001 & 620 & & $s^{2}$ & ${ }^{3} \mathrm{P}_{1}$ & $5 p$ & $(3 / 2)^{2}[1 / 2]^{\circ}{ }_{1}$ & & & $\mathrm{R} 1 \mathrm{c}$ & & \\
\hline $2923.2569(18)$ & $34198.411(21)$ & $2923.2586(8)$ & -0.0017 & 240 & & $5 p$ & $(5 / 2)^{2}[7 / 2]^{\circ}{ }_{3}$ & $8 \mathrm{~d}$ & $(5 / 2)^{2}[7 / 2]_{3}$ & & & R1c & & \\
\hline $2926.2499(7)$ & $34163.434(8)$ & 2926.2501(4) & -0.0002 & 240 & & $5 p$ & $(5 / 2)^{2}[3 / 2]^{\circ} 2$ & $9 \mathrm{~s}$ & $(5 / 2)^{2}[5 / 2]_{3}$ & & & $\mathrm{R} 1 \mathrm{c}$ & & \\
\hline 2927.2539(7) & $34151.717(9)$ & 2927.2535(5) & 0.0003 & 2900 & & $5 p$ & $(5 / 2)^{2}[7 / 2]^{\circ}{ }_{3}$ & $8 \mathrm{~d}$ & $(5 / 2)^{2}[9 / 2]_{4}$ & & & R1c & & \\
\hline 2928.1916(18) & $34140.781(21)$ & 2928.18542(19) & 0.0062 & 240 & & $s^{2}$ & ${ }^{3} \mathrm{P}_{1}$ & $5 p$ & $(3 / 2)^{2}[5 / 2]^{\circ}{ }_{2}$ & & & R1c & & \\
\hline 2931.789(3) & $34098.89(4)$ & 2931.7851(4) & 0.004 & 230 & & $\mathrm{sp}$ & $\left({ }^{3} \mathrm{~F}\right)^{3} \mathrm{P}^{\circ}{ }^{3} \mathrm{G}^{\circ}{ }_{3}$ & $8 \mathrm{~s}$ & $(5 / 2)^{2}[5 / 2]_{3}$ & & & R1c & & \\
\hline $2932.2253(7)$ & $34093.818(9)$ & $2932.2254(6)$ & -0.0002 & 1200 & & $5 p$ & $(5 / 2)^{2}[7 / 2]^{\circ} 4$ & $8 \mathrm{~d}$ & $\begin{array}{l}(5 / 2)^{2}[7 / 2]_{4} \\
\end{array}$ & & & R1c & & \\
\hline $2933.565(6)$ & $34078.25(7)$ & 2933.5614(6) & 0.003 & 230 & & $\mathrm{sp}$ & $\left({ }^{3} \mathrm{~F}\right)^{3} \mathrm{P}^{\circ}{ }^{3} \mathrm{~F}^{\circ}{ }_{4}$ & $7 \mathrm{~d}$ & $(5 / 2)^{2}[7 / 2]_{4}$ & & & R1c & & \\
\hline $2936.9553(7)$ & $34038.912(8)$ & $2936.9555(7)$ & -0.0002 & 2900 & & $5 p$ & $(5 / 2)^{2}[7 / 2]^{\circ} 4$ & $8 \mathrm{~d}$ & $(5 / 2)^{2}[9 / 2]_{5}$ & & & $\mathrm{R} 1 \mathrm{c}$ & & \\
\hline 2939.7041(9) & $34007.084(10)$ & $2939.7036(8)$ & 0.0005 & 1100 & & $5 p$ & $(5 / 2)^{2}[5 / 2]^{\circ}{ }_{2}$ & $8 \mathrm{~d}$ & $(5 / 2)^{2}[7 / 2]_{3}$ & & & $\mathrm{R} 1 \mathrm{c}$ & & \\
\hline 2941.2137(18) & $33989.631(21)$ & $2941.2085(6)$ & 0.0052 & 1100 & & $\mathrm{sp}$ & $\left({ }^{3} \mathrm{~F}\right)^{3} \mathrm{P}^{\circ}{ }^{3} \mathrm{~F}^{\circ}{ }_{4}$ & $7 \mathrm{~d}$ & $(5 / 2)^{2}[9 / 2]_{5}$ & & & R1c & & \\
\hline $2942.623(5)$ & $33973.35(6)$ & $2942.6237(18)$ & -0.001 & 230 & & $5 p$ & $(5 / 2)^{2}[3 / 2]^{\circ}{ }_{1}$ & $8 \mathrm{~d}$ & $(5 / 2)^{2}[5 / 2]_{2}$ & & & R1c & & \\
\hline $2945.370(8)$ & $33941.66(9)$ & $2945.3640(3)$ & 0.007 & 6100 & & $s^{2}$ & ${ }^{3} \mathrm{P}_{0}$ & $5 p$ & $(3 / 2)^{2}[1 / 2]^{\circ} 1$ & & & S36c & & \\
\hline 2947.3037(11) & $33919.401(12)$ & 2947.3037(11) & 0.0000 & 670 & & $5 p$ & $(5 / 2)^{2}[3 / 2]^{\circ}{ }_{1}$ & $8 \mathrm{~d}$ & $(5 / 2)^{2}[3 / 2]_{1}$ & & & R1c & & \\
\hline 2949.3463(18) & $33895.911(21)$ & $2949.3453(10)$ & 0.0010 & 550 & & $5 p$ & $(3 / 2)^{2}[5 / 2]^{\circ}{ }_{3}$ & $9 \mathrm{~d}$ & $(5 / 2)^{2}[9 / 2]_{4}$ & & & $\mathrm{R} 1 \mathrm{c}$ & & \\
\hline 2957.3211(19) & $33804.511(21)$ & 2957.3242(6) & -0.0032 & 1100 & & $5 p$ & $(5 / 2)^{2}[5 / 2]^{\circ}{ }_{3}$ & $8 \mathrm{~d}$ & $(5 / 2)^{2}[7 / 2]_{4}$ & & & R1c & & \\
\hline 2959.3285(19) & $33781.581(21)$ & $2959.3276(5)$ & 0.0009 & 740 & & $5 p$ & $(5 / 2)^{2}[5 / 2]^{\circ}{ }_{3}$ & $8 \mathrm{~d}$ & $(5 / 2)^{2}[5 / 2]_{3}$ & & & R1c & & \\
\hline $2968.744(3)$ & $33674.44(3)$ & $2968.7445(4)$ & -0.000 & 200 & & $4 \mathrm{~d}$ & $(3 / 2)^{2}[7 / 2]_{3}$ & $6 f$ & $(5 / 2)^{2}[9 / 2]^{\circ} 4$ & & & R1c & & \\
\hline $2973.6495(7)$ & $33618.897(8)$ & 2973.64712(23) & 0.0024 & 1300 & & $\mathrm{~s}^{2}$ & ${ }^{3} \mathrm{P}_{1}$ & $5 p$ & $(3 / 2)^{2}[1 / 2]^{\circ} 0$ & & & R1c & & \\
\hline $2975.271(5)$ & $33600.57(6)$ & 2975.2681(3) & 0.003 & 300 & & $\mathrm{sp}$ & $\left({ }^{3} \mathrm{~F}\right)^{3} \mathrm{P}^{\circ}{ }^{3} \mathrm{~F}^{\circ}{ }_{3}$ & $7 \mathrm{~d}$ & $(5 / 2)^{2}[7 / 2]_{3}$ & & & R1c & & \\
\hline $2975.6294(8)$ & $33596.529(9)$ & $2975.6286(7)$ & 0.0008 & 1200 & & $5 p$ & $(5 / 2)^{2}[7 / 2]^{\circ}{ }_{3}$ & $9 \mathrm{~s}$ & $(5 / 2)^{2}[5 / 2]_{2}$ & & & $\mathrm{R} 1 \mathrm{c}$ & & \\
\hline 2977.0081(19) & $33580.971(21)$ & $2977.0087(16)$ & -0.0006 & 1100 & & $5 p$ & $(3 / 2)^{2}[5 / 2]^{\circ}{ }_{2}$ & $9 \mathrm{~s}$ & $(3 / 2)^{2}[3 / 2]_{1}$ & & & R1c & & \\
\hline 2981.7867(12) & $33527.156(14)$ & $2981.7860(4)$ & 0.0007 & 1000 & & $5 \mathrm{~s}$ & $(5 / 2)^{2}[5 / 2]_{3}$ & $6 p$ & $(3 / 2)^{2}[3 / 2]^{\circ} 2$ & & & R1c & & \\
\hline 2981.945(3) & $33525.38(3)$ & $2981.9449(5)$ & -0.000 & 1400 & & $\mathrm{sp}$ & $\left({ }^{3} \mathrm{~F}\right)^{3} \mathrm{P}^{\circ}{ }^{3} \mathrm{~F}^{\circ}{ }_{3}$ & $7 \mathrm{~d}$ & $(5 / 2)^{2}[9 / 2]_{4}$ & & & R1c & & \\
\hline $2983.7677(10)$ & $33504.898(11)$ & 2983.7658(3) & 0.0018 & 1100 & & $4 \mathrm{~d}$ & $(5 / 2)^{2}[1 / 2]_{1}$ & $5 f$ & $(3 / 2)^{2}[3 / 2]^{\circ}{ }_{1}$ & & & R1c & & \\
\hline $2986.3345(8)$ & $33476.101(9)$ & 2986.3327(3) & 0.0017 & 5700 & & $4 \mathrm{~d}$ & $(5 / 2)^{2}[1 / 2]_{1}$ & $5 f$ & $(3 / 2)^{2}[3 / 2]^{\circ} 2$ & & & R1c & & \\
\hline 2987.2351(7) & $33466.009(8)$ & 2987.2353(5) & -0.0002 & 1400 & & $5 p$ & $(5 / 2)^{2}[7 / 2]^{\circ} 4$ & $9 \mathrm{~s}$ & $(5 / 2)^{2}[5 / 2]_{3}$ & & & $\mathrm{R} 1 \mathrm{c}$ & & \\
\hline 2992.6695(19) & $33405.241(21)$ & $2992.6698(7)$ & -0.0003 & 460 & & $5 p$ & $(5 / 2)^{2}[5 / 2]^{\circ}{ }_{2}$ & $9 \mathrm{~s}$ & $\begin{array}{l}(5 / 2)^{2}[5 / 2]_{2}\end{array}$ & & & R1c & & \\
\hline 2993.024(5) & $33401.28(5)$ & & & 460 & & $5 p$ & $(3 / 2)^{2}[3 / 2]^{\circ} 2$ & $8 \mathrm{~d}$ & $(3 / 2)^{2}[5 / 2]_{2}$ & & & R1c & & \\
\hline $2993.2667(8)$ & $33398.576(9)$ & $2993.2675(4)$ & -0.0008 & 920 & & $5 s$ & $(5 / 2)^{2}[5 / 2]_{2}$ & $6 p$ & $(3 / 2)^{2}[3 / 2]^{\circ}{ }_{1}$ & & & $\mathrm{R} 1 \mathrm{c}$ & & \\
\hline 2996.8412(19) & $33358.741(21)$ & 2996.8382(6) & 0.0030 & 720 & & $\mathrm{sp}$ & $\left({ }^{3} \mathrm{~F}\right)^{3} \mathrm{P}^{\circ}{ }^{1} \mathrm{~F}^{\circ}{ }_{3}$ & $8 \mathrm{~d}$ & $(5 / 2)^{2}[7 / 2]_{4}$ & & & R1c & & \\
\hline 2998.893(5) & $33335.92(6)$ & $2998.8956(6)$ & -0.003 & 180 & & $\mathrm{sp}$ & $\left({ }^{3} \mathrm{~F}\right)^{3} \mathrm{P}^{\circ}{ }^{1} \mathrm{~F}^{\circ}{ }_{3}$ & $8 \mathrm{~d}$ & $(5 / 2)^{2}[5 / 2]_{3}$ & & & R1c & & \\
\hline 2999.8710(19) & $33325.051(21)$ & 2999.8701(8) & 0.0009 & 530 & & $5 p$ & $(3 / 2)^{2}[5 / 2]^{\circ}{ }_{3}$ & $9 \mathrm{~s}$ & $(3 / 2)^{2}[3 / 2]_{2}$ & & & $\mathrm{R} 1 \mathrm{c}$ & & \\
\hline $3004.058(5)$ & $\begin{array}{l}33278.60(6) \\
\text { (a) }\end{array}$ & $3004.0555(5)$ & 0.003 & 260 & & $4 \mathrm{~d}$ & $(3 / 2)^{2}[3 / 2]_{2}$ & $6 \mathrm{f}$ & $(5 / 2)^{2}[7 / 2]^{\circ}{ }_{3}$ & & & R1c & & \\
\hline $3010.5921(19)$ & $33206.381(21)$ & $3010.5910(4)$ & 0.0011 & 1700 & & $5 \mathrm{~s}$ & $(5 / 2)^{2}[5 / 2]_{2}$ & $6 p$ & $(3 / 2)^{2}[3 / 2]^{\circ} 2$ & & & R1c & & \\
\hline $3012.2768(19)$ & $33187.811(21)$ & $3012.2786(5)$ & -0.0018 & 170 & & $5 \mathrm{~s}$ & $(5 / 2)^{2}[5 / 2]_{3}$ & $6 \mathrm{p}$ & $(3 / 2)^{2}[5 / 2]^{\circ} 3$ & & & R1c & & \\
\hline
\end{tabular}


Table A1. Cont.

\begin{tabular}{|c|c|c|c|c|c|c|c|c|c|c|c|c|c|c|}
\hline$\lambda_{\text {obs }}{ }^{\text {a }}(\AA)$ & $\sigma_{\mathrm{obs}}{ }^{\mathrm{b}}\left(\mathrm{cm}^{-1}\right)$ & $\lambda_{\text {Ritz }}{ }^{c}(\AA)$ & $\begin{array}{c}\Delta \lambda_{\text {obs-Ritz }} \\
\text { (A) }\end{array}$ & $\begin{array}{l}I_{\text {obs }} \mathrm{d} \\
\text { (arb. u.) }\end{array}$ & Char ${ }^{\mathrm{e}}$ & & ower Level & & pper Level & $A\left(\mathrm{~s}^{-1}\right)$ & $\operatorname{Acc}^{f}$ & Line Ref. $\mathrm{g}$ & TP Ref. $\mathrm{g}$ & Notes ${ }^{h}$ \\
\hline $3013.2896(11)$ & $33176.656(12)$ & $3013.2888(5)$ & 0.0008 & 170 & & $5 p$ & $(5 / 2)^{2}[5 / 2]_{3}^{\circ}$ & $9 \mathrm{~s}$ & $(5 / 2)^{2}[5 / 2]_{3}$ & & & R1c & & \\
\hline $3014.5445(8)$ & $33162.846(9)$ & $3014.54413(18)$ & 0.0004 & 6200 & & $\mathrm{~s}^{2}$ & ${ }^{3} \mathrm{P}_{2}$ & $\mathrm{sp}$ & $\left({ }^{3} \mathrm{~F}\right)^{3} \mathrm{P}^{\circ} 1^{1} \mathrm{~F}_{3}^{\circ}$ & & & $\mathrm{R} 1 \mathrm{c}$ & & \\
\hline $3024.1816(19)$ & $33057.171(21)$ & 3024.1822(19) & -0.0005 & 230 & & $5 \mathrm{~s}$ & $(3 / 2)^{2}[3 / 2]_{2}$ & $\mathrm{sp}$ & $\left({ }^{1} G\right)^{3} \mathrm{P}^{\circ} 3^{3} \mathrm{G}^{0}{ }_{3}$ & & & $\mathrm{R} 2 \mathrm{nc}$ & & \\
\hline $3027.388(6)$ & $33022.16(6)$ & $3027.3945(17)$ & -0.006 & 76 & & $5 p$ & $(3 / 2)^{2}[3 / 2]^{\circ}{ }_{1}$ & $9 \mathrm{~s}$ & $(3 / 2)^{2}[3 / 2]_{1}$ & & & R1c & & \\
\hline $3037.6082(20)$ & $32911.061(21)$ & $3037.6081(19)$ & 0.0001 & 140 & & $\mathrm{sp}$ & $\left({ }^{3} \mathrm{~F}\right)^{3} \mathrm{P}^{\circ}{ }^{1} \mathrm{D}_{2}^{\circ}$ & $8 \mathrm{~d}$ & $(5 / 2)^{2}[5 / 2]_{2}$ & & & $\mathrm{R} 1 \mathrm{c}$ & & \\
\hline 3037.801(3) & 32908.97(3) & $3037.8031(6)$ & -0.002 & 710 & & $5 s$ & $(5 / 2)^{2}[5 / 2]_{2}$ & $6 p$ & $(3 / 2)^{2}[5 / 2]^{\circ}{ }_{2}$ & & & R1c & & \\
\hline $3041.6787(8)$ & $32867.019(9)$ & $3041.6786(5)$ & 0.0002 & 4500 & & $5 \mathrm{~s}$ & $(5 / 2)^{2}[5 / 2]_{2}$ & $6 \mathrm{p}$ & $(3 / 2)^{2}[5 / 2]^{\circ} 3$ & & & R1c & & \\
\hline $3042.8556(8)$ & $32854.308(9)$ & $3042.8551(5)$ & 0.0005 & 340 & & $5 p$ & $(5 / 2)^{2}[3 / 2]^{\circ}{ }_{1}$ & $7 \mathrm{~d}$ & $(3 / 2)^{2}[3 / 2]_{2}$ & & & R1c & & \\
\hline $3049.2831(10)$ & $32785.058(11)$ & $3049.2836(8)$ & -0.0005 & 130 & & $5 p$ & $(3 / 2)^{2}[3 / 2]^{\circ} 2$ & $9 \mathrm{~s}$ & $(3 / 2)^{2}[3 / 2]_{2}$ & & & R1c & & \\
\hline $3051.9472(20)$ & $32756.441(21)$ & $3051.9509(7)$ & -0.0038 & 130 & & $\mathrm{sp}$ & $\left({ }^{3} \mathrm{~F}\right)^{3} \mathrm{P}^{\circ}{ }^{1} \mathrm{~F}_{3}^{\circ}$ & $9 \mathrm{~s}$ & $(5 / 2)^{2}[5 / 2]_{2}$ & & & R1c & & \\
\hline $3052.1596(20)$ & $32754.161(21)$ & $3052.15798(24)$ & 0.0016 & 130 & & $\mathrm{~s}^{2}$ & ${ }^{1} \mathrm{D}_{2}$ & $\mathrm{sp}$ & $\left({ }^{3} \mathrm{~F}\right)^{3} \mathrm{P}^{\circ} 3^{3} \mathrm{~F}_{3}^{\circ}$ & & & $\mathrm{R} 1 \mathrm{c}$ & & \\
\hline $3053.5737(15)$ & $32738.993(16)$ & $3053.5735(6)$ & 0.0003 & 190 & & $5 p$ & $(5 / 2)^{2}[3 / 2]^{\circ} 1$ & $7 \mathrm{~d}$ & $(3 / 2)^{2}[1 / 2]_{1}$ & & & R1c & & \\
\hline $3055.6134(8)$ & $32717.140(9)$ & $3055.61251(18)$ & 0.0009 & 500 & & $\mathrm{~s}^{\frac{1}{2}}$ & ${ }^{3} \mathrm{P}_{2}$ & $5 p$ & $(5 / 2)^{2}[5 / 2]^{\circ} 3$ & & & R1c & & \\
\hline 3056.8491(9) & $32703.914(9)$ & $3056.8484(3)$ & 0.0007 & 310 & & $\mathrm{sp}$ & $\left({ }^{3} \mathrm{~F}\right)^{3} \mathrm{P}^{\circ}{ }^{3} \mathrm{~F}^{\circ}{ }_{2}$ & $7 \mathrm{~d}$ & $(5 / 2)^{2}[7 / 2]_{3}$ & & & $\mathrm{R} 1 \mathrm{c}$ & & \\
\hline $3059.8637(9)$ & $32671.695(10)$ & $3059.8632(5)$ & 0.0005 & 420 & & $5 p$ & $(5 / 2)^{2}[5 / 2]^{\circ} 3$ & $7 \mathrm{~d}$ & $(3 / 2)^{2}[7 / 2]_{4}$ & & & R1c & & \\
\hline $3062.2814(8)$ & $32645.902(8)$ & $3062.2814(5)$ & -0.0000 & 590 & & $5 \mathrm{~s}$ & $(5 / 2)^{2}[5 / 2]_{2}$ & $6 p$ & $(3 / 2)^{2}[1 / 2]^{\circ} 1$ & & & R1c & & \\
\hline $3066.6018(12)$ & $32599.910(13)$ & $3066.5985(3)$ & 0.0033 & 400 & & $\mathrm{sp}$ & $\left({ }^{3} \mathrm{~F}\right)^{3} \mathrm{P}^{\circ}{ }^{3} \mathrm{~F}^{\circ}{ }_{3}$ & $8 \mathrm{~s}$ & $(5 / 2)^{2}[5 / 2]_{3}$ & & & R1c & & \\
\hline $3080.3202(20)$ & $32454.730(21)$ & $3080.3284(6)$ & -0.0082 & 400 & & $\mathrm{sp}$ & $\left({ }^{3} \mathrm{~F}\right)^{3} \mathrm{P}^{\circ}{ }^{5} \mathrm{~F}^{\circ}{ }_{2}$ & $7 \mathrm{~s}$ & $(3 / 2)^{2}[3 / 2]_{2}$ & & & R1c & & \\
\hline $3081.4480(14)$ & $32442.852(15)$ & $3081.4527(3)$ & -0.0046 & 150 & & $4 \mathrm{~d}$ & $(5 / 2)^{2}[9 / 2]_{4}$ & $5 f$ & $(3 / 2)^{2}[7 / 2]^{\circ} 4$ & & & R1c & & \\
\hline $3082.935(3)$ & $32427.21(3)$ & $3082.9348(3)$ & -0.000 & 97 & & $4 \mathrm{~d}$ & $(5 / 2)^{2}[3 / 2]_{2}$ & $5 f$ & $(3 / 2)^{2}[5 / 2]^{\circ} 2$ & & & R1c & & \\
\hline 3083.3677(8) & $32422.654(8)$ & $3083.3669(3)$ & 0.0009 & 970 & & $4 \mathrm{~d}$ & $(5 / 2)^{2}[3 / 2]_{2}$ & $5 f$ & $(3 / 2)^{2}[5 / 2]^{0}{ }_{3}$ & & & R1c & & \\
\hline $3085.4342(20)$ & $32400.940(21)$ & $3085.4421(3)$ & -0.0079 & 95 & & $4 \mathrm{~d}$ & $(5 / 2)^{2}[3 / 2]_{1}$ & $5 f$ & $(3 / 2)^{2}[5 / 2]^{\circ} 2$ & & & R1c & & \\
\hline $3085.5818(20)$ & $32399.390(21)$ & $3085.6280(3)$ & -0.0462 & 94 & ? & $4 \mathrm{~d}$ & $(5 / 2)^{2}[9 / 2]_{4}$ & $5 f$ & $(3 / 2)^{2}[5 / 2]^{\circ} 3$ & & & $\mathrm{R} 1 \mathrm{c}$ & & $x$ \\
\hline $3088.7489(7)$ & $32366.170(8)$ & $3088.74858(24)$ & 0.0003 & 550 & & $4 \mathrm{~d}$ & $(5 / 2)^{2}[9 / 2]_{4}$ & $5 f$ & $(3 / 2)^{2}[9 / 2]^{\circ}{ }_{5}$ & & & R1c & & \\
\hline 3097.8651(13) & $32270.929(13)$ & $3097.86615(23)$ & -0.0011 & 410 & & $\mathrm{~s}^{2}$ & ${ }^{3} \mathrm{P}_{1}$ & $5 p$ & $(5 / 2)^{2}[5 / 2]^{\circ} 2$ & & & R1c & & \\
\hline $3110.4745(10)$ & $32140.112(10)$ & $3110.4732(4)$ & 0.0013 & 70 & & $\mathrm{sp}$ & $\left({ }^{3} \mathrm{~F}^{3} \mathrm{P}^{\circ}{ }^{3} \mathrm{D}^{\circ}{ }_{3}\right.$ & $6 \mathrm{~d}$ & $(3 / 2)^{2}[7 / 2]_{4}$ & & & R1c & & \\
\hline $3121.3959(8)$ & $32027.662(8)$ & $3121.3961(5)$ & -0.0002 & 150 & & $4 \mathrm{~d}$ & $(5 / 2)^{2}[9 / 2]_{5}$ & $7 p$ & $(5 / 2)^{2}[7 / 2]^{\circ}{ }_{4}$ & & & R1c & & \\
\hline $3121.6428(7)$ & $32025.129(7)$ & $3121.6415(3)$ & 0.0013 & 240 & & $4 \mathrm{~d}$ & $(5 / 2)^{2}[5 / 2]_{3}$ & $5 f$ & $(3 / 2)^{2}[7 / 2]^{\circ} 4$ & & & R1c & & \\
\hline $3121.8708(21)$ & $32022.790(21)$ & $3121.8736(3)$ & -0.0027 & 90 & & $4 \mathrm{~d}$ & $(5 / 2)^{2}[5 / 2]_{3}$ & $5 f$ & $(3 / 2)^{2}[7 / 2]^{\circ} 3$ & & & R1c & & \\
\hline $3124.7229(8)$ & $31993.563(8)$ & $3124.7222(3)$ & 0.0006 & 440 & & $\mathrm{~s}^{2}$ & ${ }^{3} \mathrm{P}_{0}$ & $5 p$ & $(5 / 2)^{2}[3 / 2]^{\circ} 1$ & & & R1c & & \\
\hline $3139.7885(9)$ & $31840.054(9)$ & $3139.7857(6)$ & 0.0028 & 610 & $\mathrm{bl}$ & $\mathrm{sp}$ & $\left({ }^{3} \mathrm{~F}\right)^{3} \mathrm{P}^{\circ}{ }^{1} \mathrm{D}^{\circ}{ }_{2}$ & $7 \mathrm{~d}$ & $(3 / 2)^{2}[5 / 2]_{2}$ & & & R1c & & \\
\hline $3140.4073(9)$ & 31833.781(9) & $3140.4064(6)$ & 0.0008 & 230 & & $\mathrm{sp}$ & $\left({ }^{3} \mathrm{~F}\right)^{3} \mathrm{P}^{\circ}{ }^{1} \mathrm{D}^{\circ}{ }_{2}$ & $7 \mathrm{~d}$ & $(3 / 2)^{2}[5 / 2]_{3}$ & & & R1c & & \\
\hline $3146.0122(8)$ & $31777.068(8)$ & $3146.0119(3)$ & 0.0003 & 480 & & $4 \mathrm{~d}$ & $(5 / 2)^{2}[7 / 2]_{3}$ & $5 f$ & $(3 / 2)^{2}[7 / 2]^{\circ} 3$ & & & R1c & & \\
\hline $3148.7869(8)$ & $31749.067(8)$ & $3148.7856(5)$ & 0.0013 & 170 & & $\mathrm{sp}$ & $\left({ }^{3} \mathrm{~F}\right)^{3} \mathrm{P}^{\mathrm{o}}{ }^{1} \mathrm{D}^{\circ}{ }_{2}$ & $7 \mathrm{~d}$ & $(3 / 2)^{2}[7 / 2]_{3}$ & & & R1c & & \\
\hline $3149.6815(21)$ & $31740.050(21)$ & $3149.6769(3)$ & 0.0046 & 260 & $\mathrm{bl}$ & $4 \mathrm{~d}$ & $(5 / 2)^{2}[7 / 2]_{3}$ & $5 f$ & $(3 / 2)^{2}[5 / 2]^{\circ} 2$ & & & $\mathrm{R} 1 \mathrm{c}$ & & \\
\hline $3150.2634(9)$ & $31734.187(9)$ & $3150.2636(3)$ & -0.0002 & 88 & & $4 \mathrm{~d}$ & $(5 / 2)^{2}[7 / 2]_{4}$ & $5 f$ & $(3 / 2)^{2}[7 / 2]^{\circ}{ }_{4}$ & & & R1c & & \\
\hline $3150.540(8)$ & $31731.40(8)$ & $3150.4999(3)$ & 0.040 & 4600 & ? & $4 \mathrm{~d}$ & $(5 / 2)^{2}[7 / 2]_{4}$ & $5 f$ & $(3 / 2)^{2}[7 / 2]^{\circ} 3$ & & & S36c & & $x$ \\
\hline $3150.6422(9)$ & $31730.372(9)$ & $3150.64124(19)$ & 0.0010 & 3000 & & $\mathrm{~s}^{2}$ & ${ }^{3} \mathrm{P}_{2}$ & $5 p$ & $(5 / 2)^{2}[3 / 2]^{\circ}{ }_{2}$ & & & R1c & & \\
\hline $3151.0505(7)$ & $31726.261(7)$ & $3151.0506(3)$ & -0.0001 & 10000 & & $5 \mathrm{~s}$ & $(5 / 2)^{2}[5 / 2]_{3}$ & $6 \mathrm{p}$ & $(5 / 2)^{2}[5 / 2]^{0}{ }_{3}$ & & & R1c & & \\
\hline $3152.9000(7)$ & 31707.651(7) & 3152.8992(3) & 0.0008 & 3500 & & $4 \mathrm{~d}$ & $(5 / 2)^{2}[7 / 2]_{3}$ & $5 f$ & $(3 / 2)^{2}[9 / 2]^{\circ} 4$ & & & $\mathrm{R} 1 \mathrm{c}$ & & \\
\hline $3154.0935(8)$ & $31695.653(8)$ & $3154.0934(4)$ & 0.0001 & 550 & & $5 \mathrm{~s}$ & $(5 / 2)^{2}[5 / 2]_{3}$ & $\mathrm{sp}$ & $\left({ }^{3} \mathrm{~F}\right)^{1} \mathrm{P}^{\circ}{ }^{3} \mathrm{~F}_{2}^{\circ}$ & & & R1c & & \\
\hline $3155.3760(9)$ & $31682.771(9)$ & $3155.3765(5)$ & -0.0005 & 380 & & $4 \mathrm{~d}$ & $(5 / 2)^{2}[5 / 2]_{3}$ & $7 p$ & $(5 / 2)^{2}[5 / 2]^{\circ} 3$ & & & R1c & & \\
\hline
\end{tabular}


Table A1. Cont.

\begin{tabular}{|c|c|c|c|c|c|c|c|c|c|c|c|c|c|c|}
\hline$\lambda_{\text {obs }}{ }^{a}(\AA)$ & $\sigma_{\mathrm{obs}}{ }^{\mathrm{b}}\left(\mathrm{cm}^{-1}\right)$ & $\lambda_{\text {Ritz }}{ }^{c}(\AA)$ & $\begin{array}{c}\Delta \lambda_{\text {obs-Ritz }} \\
\text { (A) }\end{array}$ & $\begin{array}{l}I_{\text {obs }} \mathrm{d} \\
\text { (arb. u.) }\end{array}$ & Char ${ }^{\mathrm{e}}$ & & ower Level & & pper Level & $A\left(\mathrm{~s}^{-1}\right)$ & $\operatorname{Acc}^{f}$ & Line Ref. $\mathrm{g}$ & TP Ref. $\mathrm{g}$ & Notes ${ }^{h}$ \\
\hline $3155.8323(21)$ & $31678.190(21)$ & $3155.8290(3)$ & 0.0033 & 190 & & $4 \mathrm{~d}$ & $(5 / 2)^{2}[5 / 2]_{2}$ & $5 f$ & $(3 / 2)^{2}[5 / 2]^{\circ}{ }_{2}$ & & & R1c & & \\
\hline $3156.2820(7)$ & $31673.677(7)$ & $3156.2817(3)$ & 0.0003 & 1900 & & $4 \mathrm{~d}$ & $(5 / 2)^{2}[5 / 2]_{2}$ & $5 f$ & $(3 / 2)^{2}[5 / 2]^{0} 3$ & & & $\mathrm{R} 1 \mathrm{c}$ & & \\
\hline 3157.8901(8) & $31657.548(8)$ & $3157.88937(23)$ & 0.0007 & 1900 & & $4 \mathrm{~d}$ & $(5 / 2)^{2}[7 / 2]_{4}$ & $5 f$ & $(3 / 2)^{2}[9 / 2]^{\circ}{ }_{5}$ & & & R1c & & \\
\hline $3158.6729(8)$ & $31649.703(8)$ & $3158.6734(5)$ & -0.0005 & 3700 & & $5 \mathrm{~s}$ & $(3 / 2)^{2}[3 / 2]_{1}$ & $6 p$ & $(3 / 2)^{2}[3 / 2]^{\circ} 1$ & & & R1c & & \\
\hline $3162.0434(7)$ & $31615.968(7)$ & $3162.0438(4)$ & -0.0004 & 2300 & & $5 p$ & $(5 / 2)^{2}[3 / 2]^{\circ} 2$ & $7 \mathrm{~d}$ & $(5 / 2)^{2}[5 / 2]_{3}$ & & & $\mathrm{R} 1 \mathrm{c}$ & & \\
\hline $3163.6838(11)$ & 31599.575(11) & $3163.6842(4)$ & -0.0003 & 610 & & $4 \mathrm{~d}$ & $(5 / 2)^{2}[5 / 2]_{2}$ & $5 f$ & $(3 / 2)^{2}[3 / 2]^{\circ} 2$ & & & R1c & & \\
\hline $3166.5879(7)$ & $31570.596(7)$ & $3166.5873(5)$ & 0.0006 & 3500 & & $5 p$ & $(5 / 2)^{2}[3 / 2]^{\circ} 2$ & $7 \mathrm{~d}$ & $(5 / 2)^{2}[3 / 2]_{2}$ & & & R1c & & \\
\hline $3170.6936(8)$ & $31529.717(8)$ & $3170.6935(7)$ & 0.0001 & 540 & & $5 p$ & $(3 / 2)^{2}[1 / 2]^{\circ} 0$ & $7 \mathrm{~d}$ & $(3 / 2)^{2}[3 / 2]_{1}$ & & & R1c & & \\
\hline $3173.6092(9)$ & $31500.752(9)$ & $3173.6088(3)$ & 0.0004 & 330 & & $\mathrm{sp}$ & $\left({ }^{3} \mathrm{~F}\right)^{3} \mathrm{P}^{\circ}{ }^{3} \mathrm{D}_{2}^{\circ}$ & $6 \mathrm{~d}$ & $(3 / 2)^{2}[5 / 2]_{3}$ & & & R1c & & \\
\hline $3174.9680(12)$ & 31487.271(11) & $3174.96642(22)$ & 0.0016 & 840 & & $\mathrm{~s}^{\frac{1}{2}}$ & ${ }^{3} \mathrm{P}_{1}$ & $5 p$ & $(5 / 2)^{2}[3 / 2]^{\circ} 2$ & & & R1c & & \\
\hline $3176.3094(16)$ & $31473.974(16)$ & $3176.3108(3)$ & -0.0014 & 230 & & $4 \mathrm{~d}$ & $(5 / 2)^{2}[1 / 2]_{1}$ & $5 f$ & $(5 / 2)^{2}[5 / 2]^{\circ} 2$ & & & $\mathrm{R} 1 \mathrm{c}$ & & \\
\hline 3177.7392(8) & $31459.813(8)$ & $3177.7397(7)$ & -0.0005 & 1700 & & $5 p$ & $(5 / 2)^{2}[3 / 2]^{\circ} 2$ & $7 \mathrm{~d}$ & $(5 / 2)^{2}[1 / 2]_{1}$ & & & R1c & & \\
\hline $3177.9692(8)$ & $31457.536(8)$ & $3177.9705(5)$ & -0.0012 & 2700 & & $5 \mathrm{~s}$ & $(3 / 2)^{2}[3 / 2]_{1}$ & $6 p$ & $(3 / 2)^{2}[3 / 2]^{\circ} 2$ & & & R1c & & \\
\hline $3179.3173(17)$ & $31444.198(17)$ & $3179.3163(4)$ & 0.0010 & 230 & & $4 \mathrm{~d}$ & $(5 / 2)^{2}[1 / 2]_{1}$ & $5 f$ & $(5 / 2)^{2}[3 / 2]^{\circ}{ }_{1}$ & & & $\mathrm{R} 1 \mathrm{c}$ & & \\
\hline 3179.7846(8) & $31439.577(8)$ & $3179.7842(4)$ & 0.0004 & 6400 & & $4 \mathrm{~d}$ & $(5 / 2)^{2}[1 / 2]_{0}$ & $5 f$ & $(3 / 2)^{2}[3 / 2]^{0}{ }_{1}$ & & & R1c & & \\
\hline $3180.2932(9)$ & $31434.550(9)$ & $3180.2935(6)$ & -0.0003 & 470 & & $5 p$ & $(3 / 2)^{2}[1 / 2]_{0}^{\circ} 0$ & $7 \mathrm{~d}$ & $(3 / 2)^{2}[1 / 2]_{1}$ & & & R1c & & \\
\hline $3180.7930(21)$ & $31429.610(21)$ & $3180.7922(4)$ & 0.0008 & 230 & & $\mathrm{sp}$ & $\left({ }^{3} \mathrm{~F}\right)^{3} \mathrm{P}^{\circ}{ }^{3} \mathrm{D}_{2}^{\circ}$ & $6 \mathrm{~d}$ & $(3 / 2)^{2}[3 / 2]_{2}$ & & & R1c & & \\
\hline $3182.1717(7)$ & $31415.994(7)$ & $3182.1718(3)$ & -0.0002 & 11000 & & $4 \mathrm{~d}$ & $(5 / 2)^{2}[1 / 2]_{1}$ & $5 f$ & $(5 / 2)^{2}[3 / 2]^{\circ} 2$ & & & R1c & & \\
\hline 3184.6224(12) & $31391.819(12)$ & $3184.6233(5)$ & -0.0010 & 480 & & $4 \mathrm{~d}$ & $(5 / 2)^{2}[7 / 2]_{4}$ & $7 p$ & $(5 / 2)^{2}[5 / 2]^{\circ} 3$ & & & R1c & & \\
\hline $3184.8404(8)$ & $31389.670(8)$ & $3184.8409(3)$ & -0.0005 & 15000 & & $4 \mathrm{~d}$ & $(5 / 2)^{2}[1 / 2]_{1}$ & $5 f$ & $(5 / 2)^{2}[1 / 2]^{\circ} 1$ & & & $\mathrm{R} 1 \mathrm{c}$ & & \\
\hline $3185.7249(7)$ & $31380.955(7)$ & $3185.7256(4)$ & -0.0006 & 4000 & & $5 \mathrm{~s}$ & $(5 / 2)^{2}[5 / 2]_{3}$ & $6 p$ & $(5 / 2)^{2}[7 / 2]^{0} 4$ & & & R1c & & \\
\hline $3186.0148(7)$ & $31378.100(7)$ & $3186.0153(4)$ & -0.0005 & 6400 & & $4 \mathrm{~d}$ & $(5 / 2)^{2}[1 / 2]_{1}$ & $5 f$ & $(5 / 2)^{2}[1 / 2]_{0}^{\circ}$ & & & R1c & & \\
\hline $3186.3411(8)$ & $31374.887(8)$ & $3186.3415(4)$ & -0.0004 & 3000 & & $5 s$ & $(5 / 2)^{2}[5 / 2]_{2}$ & $\mathrm{sp}$ & $\left({ }^{3} \mathrm{~F}\right)^{1} \mathrm{P}^{\circ}{ }^{3} \mathrm{~F}_{2}^{\circ}{ }_{2}$ & & & R1c & & \\
\hline $3187.0427(13)$ & $31367.980(13)$ & $3187.0386(5)$ & 0.0041 & 1200 & & $5 \mathrm{~s}$ & $(3 / 2)^{2}[3 / 2]_{2}$ & $6 p$ & $(3 / 2)^{2}[3 / 2]^{\circ} 1$ & & & R1c & & \\
\hline 3188.7231(22) & $31351.450(21)$ & $3188.7259(4)$ & -0.0028 & 490 & & $\mathrm{sp}$ & $\left({ }^{3} \mathrm{~F}\right)^{3} \mathrm{P}^{\circ}{ }^{3} \mathrm{D}^{\circ}{ }_{2}$ & $6 \mathrm{~d}$ & $(3 / 2)^{2}[7 / 2]_{3}$ & & & R1c & & \\
\hline $3192.3023(22)$ & $31316.300(21)$ & 3192.3011(3) & 0.0012 & 250 & & $5 s$ & $(5 / 2)^{2}[5 / 2]_{3}$ & $\mathrm{sp}$ & $\left({ }^{1} \mathrm{G}\right)^{3} \mathrm{P}^{\circ}{ }^{3} \mathrm{~F}^{\circ}{ }_{3}$ & & & R1c & & \\
\hline 3198.1052(10) & $31259.480(9)$ & 3198.1052(8) & -0.0001 & 1000 & & $5 p$ & $(5 / 2)^{2}[3 / 2]^{\circ}{ }_{1}$ & $7 \mathrm{~d}$ & $(5 / 2)^{2}[1 / 2]_{0}$ & & & R1c & & \\
\hline $3204.5231(16)$ & $31196.877(16)$ & $3204.5259(6)$ & -0.0029 & 640 & & sp & $\left({ }^{3} \mathrm{~F}\right)^{3} \mathrm{P}^{\circ}{ }^{3} \mathrm{G}_{4}^{\circ}$ & $6 \mathrm{~d}$ & $(5 / 2)^{2}[7 / 2]_{3}$ & & & R1c & & \\
\hline $3206.6848(13)$ & $31175.847(12)$ & $3206.6848(5)$ & -0.0000 & 1800 & & $5 \mathrm{~s}$ & $(3 / 2)^{2}[3 / 2]_{2}$ & $6 p$ & $(3 / 2)^{2}[3 / 2]^{\circ} 2$ & & & R1c & & \\
\hline $3208.3026(16)$ & $31160.127(16)$ & $3208.3076(6)$ & -0.0050 & 1500 & & $5 \mathrm{~s}$ & $(3 / 2)^{2}[3 / 2]_{1}$ & $6 \mathrm{p}$ & $(3 / 2)^{2}[5 / 2]^{0} 2$ & & & R1c & & \\
\hline $3216.9896(14)$ & $31075.987(14)$ & $3216.9895(6)$ & 0.0001 & 520 & & $5 p$ & $(3 / 2)^{2}[5 / 2]^{\circ} 2$ & $7 \mathrm{~d}$ & $(3 / 2)^{2}[5 / 2]_{2}$ & & & R1c & & \\
\hline 3217.3123(22) & $31072.870(21)$ & $3217.3104(4)$ & 0.0019 & 650 & & $5 p$ & $(5 / 2)^{2}[7 / 2]^{\circ}{ }_{3}$ & $7 \mathrm{~d}$ & $(5 / 2)^{2}[5 / 2]_{2}$ & & & R1c & & \\
\hline 3217.6410(17) & $31069.696(17)$ & $3217.6411(6)$ & -0.0001 & 390 & & $5 p$ & $(3 / 2)^{2}[5 / 2]^{\circ} 2$ & $7 \mathrm{~d}$ & $(3 / 2)^{2}[5 / 2]_{3}$ & & & $\mathrm{R} 1 \mathrm{rc}$ & & \\
\hline $3218.2664(15)$ & $31063.658(15)$ & $3218.2653(6)$ & 0.0011 & 260 & & $\mathrm{sp}$ & $\left({ }^{3} \mathrm{~F}\right)^{3} \mathrm{P}^{\circ}{ }^{3} \mathrm{G}_{4}^{\circ}$ & $6 \mathrm{~d}$ & $(5 / 2)^{2}[9 / 2]_{4}$ & & & R1c & & \\
\hline $3218.6278(16)$ & $31060.170(16)$ & $3218.6244(5)$ & 0.0034 & 390 & & $5 p$ & $(5 / 2)^{2}[7 / 2]^{4}{ }_{3}$ & $7 \mathrm{~d}$ & $(5 / 2)^{2}[7 / 2]_{4}$ & & & $\mathrm{R} 1 \mathrm{c}$ & & \\
\hline $3218.7642(8)$ & $31058.854(8)$ & $3218.7651(4)$ & -0.0009 & 2000 & & $5 p$ & $(5 / 2)^{2}[7 / 2]^{\circ} 3$ & $7 \mathrm{~d}$ & $(5 / 2)^{2}[7 / 2]_{3}$ & & & R1c & & \\
\hline $3221.9702(22)$ & $31027.950(21)$ & $3221.9718(6)$ & -0.0016 & 260 & & $5 p$ & $(3 / 2)^{2}[5 / 2]^{\circ} 2$ & $7 \mathrm{~d}$ & $(3 / 2)^{2}[3 / 2]_{2}$ & & & R1c & & \\
\hline $3225.3388(8)$ & $30995.545(8)$ & $3225.3392(3)$ & -0.0004 & 2100 & & $5 s$ & $(5 / 2)^{2}[5 / 2]_{2}$ & $\mathrm{sp}$ & $\left({ }^{1} \mathrm{G}\right)^{3} \mathrm{P}^{\circ}{ }^{3} \mathrm{~F}^{\circ}{ }_{3}$ & & & R1c & & \\
\hline $3226.3290(16)$ & 30986.033(16) & $3226.3301(12)$ & -0.0011 & 260 & & $5 p$ & $(3 / 2)^{2}[1 / 2]^{\circ}{ }_{1}$ & $7 \mathrm{~d}$ & $(3 / 2)^{2}[1 / 2]_{0}$ & & & R1c & & \\
\hline $3226.4395(9)$ & $30984.972(9)$ & $3226.4380(5)$ & 0.0015 & 640 & & $5 p$ & $(3 / 2)^{2}[5 / 2]^{\circ} 2$ & $7 \mathrm{~d}$ & $(3 / 2)^{2}[7 / 2]_{3}$ & & & $\mathrm{R} 1 \mathrm{c}$ & & \\
\hline $3226.5812(10)$ & 30983.611(9) & $3226.5808(5)$ & 0.0004 & 5200 & & $5 p$ & $(5 / 2)^{2}[7 / 2]^{\circ}{ }_{3}$ & $7 \mathrm{~d}$ & $(5 / 2)^{2}[9 / 2]_{4}$ & & & R1c & & \\
\hline $3229.5526(8)$ & $30955.105(8)$ & $3229.5524(5)$ & 0.0003 & 970 & & $5 p$ & $(5 / 2)^{2}[7 / 2]^{\circ} 4$ & $7 \mathrm{~d}$ & $(5 / 2)^{2}[7 / 2]_{4}$ & & & R1c & & \\
\hline
\end{tabular}


Table A1. Cont.

\begin{tabular}{|c|c|c|c|c|c|c|c|c|c|c|c|c|c|c|}
\hline \multirow{2}{*}{$\begin{array}{c}\lambda_{\text {obs }^{\text {a }}}(\AA \AA) \\
3233.3754(13)\end{array}$} & \multirow{2}{*}{$\begin{array}{l}\sigma_{\text {obs }} \mathbf{b}\left(\mathbf{c m}^{-\mathbf{1}}\right) \\
30918.508(12)\end{array}$} & \multirow{2}{*}{$\begin{array}{r}\lambda_{\text {Ritz }}{ }^{\mathrm{c}}(\AA ̊) \\
3233.3722(4)\end{array}$} & \multirow{2}{*}{$\begin{array}{c}\begin{array}{c}\Delta \lambda_{\text {obs-Ritz }} \\
(\AA)\end{array} \\
0.0032\end{array}$} & \multirow{2}{*}{$\begin{array}{c}\begin{array}{c}I_{\text {obs }}{ }^{d} \\
\text { (arb. u.) }\end{array} \\
1000\end{array}$} & \multirow[t]{2}{*}{ Char ${ }^{e}$} & \multicolumn{2}{|c|}{ Lower Level } & \multicolumn{2}{|c|}{ Upper Level } & \multirow[t]{2}{*}{$A\left(\mathrm{~s}^{-1}\right)$} & \multirow[t]{2}{*}{$\operatorname{Acc}^{f}$} & \multirow{2}{*}{$\begin{array}{l}\text { Line Ref. } \mathbf{g} \\
\text { R1c }\end{array}$} & \multirow[t]{2}{*}{ TP Ref. $\mathrm{g}$} & \multirow[t]{2}{*}{ Notes $^{h}$} \\
\hline & & & & & & $5 p$ & $(5 / 2)^{2}[7 / 2]_{4}^{\circ}$ & $7 \mathrm{~d}$ & $(5 / 2)^{2}[5 / 2]_{3}$ & & & & & \\
\hline $3234.6710(14)$ & $30906.125(13)$ & $3234.6691(6)$ & 0.0019 & 510 & & $5 p$ & $(3 / 2)^{2}[1 / 2]^{\circ}{ }_{1}$ & $7 \mathrm{~d}$ & $(3 / 2)^{2}[3 / 2]_{2}$ & & & $\mathrm{R} 1 \mathrm{c}$ & & \\
\hline $3234.7336(10)$ & $30905.527(9)$ & $3234.7341(5)$ & -0.0005 & 930 & & $5 s$ & $(5 / 2)^{2}[5 / 2]_{2}$ & $6 \mathrm{p}$ & $(5 / 2)^{2}[3 / 2]^{\circ} 1$ & & & R1c & & \\
\hline $3237.1430(16)$ & $30882.525(15)$ & $3237.1420(7)$ & 0.0009 & 250 & & $\mathrm{sp}$ & $\left({ }^{3} \mathrm{~F}\right)^{3} \mathrm{P}^{0}{ }^{5} \mathrm{~F}^{\circ}{ }_{3}$ & $7 \mathrm{~s}$ & $(5 / 2)^{2}[5 / 2]_{2}$ & & & R1c & & \\
\hline \multirow[t]{2}{*}{$3237.2417(11)$} & $30881.583(11)$ & $3237.2413(5)$ & 0.0004 & 1100 & & $5 p$ & $(5 / 2)^{2}[5 / 2]^{\circ} 2$ & $7 \mathrm{~d}$ & $(5 / 2)^{2}[5 / 2]_{2}$ & & & R1c & & \\
\hline & & $3237.5629(5)$ & & & $\mathrm{m}$ & $5 p$ & $(5 / 2)^{2}[7 / 2]^{\circ}{ }_{4}$ & $7 \mathrm{~d}$ & $(5 / 2)^{2}[9 / 2]_{4}$ & & & R1nc & & \\
\hline $3237.5736(12)$ & 30878.417(11) & $3237.5752(6)$ & -0.0016 & 1300 & & $5 \mathrm{~s}$ & $(3 / 2)^{2}[3 / 2]_{2}$ & $6 p$ & $(3 / 2)^{2}[5 / 2]^{\circ} 2$ & & & R1c & & \\
\hline $3238.7160(10)$ & $30867.526(10)$ & $3238.7141(4)$ & 0.0019 & 1900 & & $5 p$ & $(5 / 2)^{2}[5 / 2]^{\circ}{ }_{2}$ & $7 \mathrm{~d}$ & $(5 / 2)^{2}[7 / 2]_{3}$ & & & R1c & & \\
\hline $3238.8232(8)$ & $30866.504(7)$ & $3238.8228(6)$ & 0.0005 & 5500 & & $5 p$ & $(5 / 2)^{2}[7 / 2]^{\circ} 4$ & $7 \mathrm{~d}$ & $(5 / 2)^{2}[9 / 2]_{5}$ & & & R1c & & \\
\hline $3239.6499(13)$ & $30858.628(12)$ & $3239.6507(5)$ & -0.0008 & 250 & & $\mathrm{sp}$ & $\left({ }^{3} \mathrm{~F}\right)^{3} \mathrm{P}^{\circ}{ }^{1} \mathrm{D}_{2}^{\circ}$ & $8 \mathrm{~s}$ & $(3 / 2)^{2}[3 / 2]_{2}$ & & & R1c & & \\
\hline $3241.8139(12)$ & $30838.030(11)$ & $3241.8139(5)$ & -0.0000 & 860 & & $5 p$ & $(5 / 2)^{2}[3 / 2]^{\circ}{ }_{1}$ & $7 \mathrm{~d}$ & $(5 / 2)^{2}[5 / 2]_{2}$ & & & $\mathrm{R} 1 \mathrm{c}$ & & \\
\hline $3242.425(10)$ & $30832.21(10)$ & $3242.4129(5)$ & 0.012 & 250 & * & $5 p$ & $(5 / 2)^{2}[5 / 2]^{\circ} 2$ & $7 \mathrm{~d}$ & $(5 / 2)^{2}[5 / 2]_{3}$ & & & R1nc & & \\
\hline $3242.425(10)$ & $30832.21(10)$ & $3242.4292(7)$ & -0.004 & 250 & * & $\mathrm{sp}$ & $\left({ }^{3} \mathrm{~F}\right)^{3} \mathrm{P}^{\circ}{ }^{1} \mathrm{D}_{2}^{\circ}$ & $8 \mathrm{~s}$ & $(3 / 2)^{2}[3 / 2]_{1}$ & & & $\mathrm{R} 1 \mathrm{c}$ & & \\
\hline $3245.9402(14)$ & $30798.829(13)$ & $3245.9405(6)$ & -0.0003 & 1500 & & $5 p$ & $(3 / 2)^{2}[5 / 2]^{\circ}{ }_{3}$ & $7 \mathrm{~d}$ & $(3 / 2)^{2}[5 / 2]_{3}$ & & & $\mathrm{R} 1 \mathrm{c}$ & & \\
\hline $3246.7898(22)$ & $30790.770(21)$ & $3246.7839(7)$ & 0.0059 & 620 & & $5 p$ & $(3 / 2)^{2}[1 / 2]^{\circ}{ }_{1}$ & $7 \mathrm{~d}$ & $(3 / 2)^{2}[1 / 2]_{1}$ & & & R1c & & \\
\hline $3250.4652(8)$ & $30755.955(8)$ & $3250.4650(4)$ & 0.0003 & 11000 & & $4 \mathrm{~d}$ & $(3 / 2)^{2}[1 / 2]_{1}$ & $5 \mathrm{f}$ & $(3 / 2)^{2}[3 / 2]^{\circ} 2$ & & & R1c & & \\
\hline $3250.8739(13)$ & $30752.089(12)$ & $3250.8733(7)$ & 0.0006 & 1200 & & $5 p$ & $(5 / 2)^{2}[3 / 2]^{\circ}{ }_{1}$ & $7 \mathrm{~d}$ & $(5 / 2)^{2}[3 / 2]_{1}$ & & & R1c & & \\
\hline $3251.76(3)$ & $30743.7(3)$ & $3251.7620(6)$ & 0.00 & 240 & * & $\mathrm{sp}$ & $\left({ }^{3} \mathrm{~F}\right)^{3} \mathrm{P}^{\circ}{ }^{3} \mathrm{G}^{\circ}{ }_{4}$ & $5 \mathrm{~g}$ & $(5 / 2)^{2}[11 / 2]_{5}$ & & & R1c & & \\
\hline $3251.76(3)$ & 30743.7(3) & $3251.7913(5)$ & -0.03 & 240 & * & $5 p$ & $(5 / 2)^{2}[3 / 2]^{\circ} 1$ & $7 \mathrm{~d}$ & $(5 / 2)^{2}[3 / 2]_{2}$ & & & R1c & & \\
\hline $3252.7830(8)$ & $30734.041(8)$ & $3252.7838(6)$ & -0.0008 & 2400 & & $5 p$ & $(3 / 2)^{2}[5 / 2]^{\circ}{ }_{3}$ & $7 \mathrm{~d}$ & $(3 / 2)^{2}[7 / 2]_{4}$ & & & R1c & & \\
\hline $3253.1078(12)$ & 30730.972(11) & $3253.1070(5)$ & 0.0008 & 1200 & & $5 s$ & $(5 / 2)^{2}[5 / 2]_{3}$ & $6 \mathrm{p}$ & $(5 / 2)^{2}[3 / 2]^{0} 2$ & & & R1c & & \\
\hline 3257.1234(16) & $30693.086(15)$ & 3257.1222(5) & 0.0012 & 240 & & $5 \mathrm{~s}$ & $(5 / 2)^{2}[5 / 2]_{2}$ & $6 \mathrm{p}$ & $(5 / 2)^{2}[5 / 2]^{\circ} 2$ & & & R1c & & \\
\hline $3260.0249(11)$ & $30665.770(11)$ & $3260.0253(5)$ & -0.0004 & 2400 & & $5 p$ & $(5 / 2)^{2}[5 / 2]^{\circ}{ }_{3}$ & $7 \mathrm{~d}$ & $(5 / 2)^{2}[7 / 2]_{4}$ & & & $\mathrm{R} 1 \mathrm{c}$ & & \\
\hline $3260.1697(17)$ & $30664.408(16)$ & $3260.1697(4)$ & 0.0000 & 240 & & $5 p$ & $(5 / 2)^{2}[5 / 2]^{\circ}{ }_{3}$ & $7 \mathrm{~d}$ & $(5 / 2)^{2}[7 / 2]_{3}$ & & & R1c & & \\
\hline $3261.6469(8)$ & $30650.520(8)$ & $3261.6476(3)$ & -0.0007 & 1800 & & $5 p$ & $(5 / 2)^{2}[3 / 2]^{\circ} 2$ & $8 \mathrm{~s}$ & $(5 / 2)^{2}[5 / 2]_{3}$ & & & R1c & & \\
\hline $3263.5546(14)$ & $30632.604(13)$ & $3263.5530(7)$ & 0.0017 & 350 & & $5 p$ & $(5 / 2)^{2}[3 / 2]^{\circ}{ }_{1}$ & $7 \mathrm{~d}$ & $(5 / 2)^{2}[1 / 2]_{1}$ & & & R1c & & \\
\hline $3263.9177(11)$ & $30629.197(10)$ & $3263.9177(4)$ & 0.0000 & 3500 & & $5 p$ & $(5 / 2)^{2}[5 / 2]^{\circ}{ }_{3}$ & $7 \mathrm{~d}$ & $(5 / 2)^{2}[5 / 2]_{3}$ & & & R1c & & \\
\hline 3265.3948(14) & $30615.342(13)$ & $3265.3933(6)$ & 0.0015 & 1100 & & $5 \mathrm{~s}$ & $(3 / 2)^{2}[3 / 2]_{2}$ & $6 p$ & $(3 / 2)^{2}[1 / 2]^{\circ} 1$ & & & R1c & & \\
\hline $3268.0990(16)$ & $30590.010(15)$ & $3268.0988(7)$ & 0.0002 & 230 & & $5 p$ & $(3 / 2)^{2}[1 / 2]^{\circ}{ }_{0}$ & $8 \mathrm{~s}$ & $(3 / 2)^{2}[3 / 2]_{1}$ & & & $\mathrm{R} 1 \mathrm{c}$ & & \\
\hline $3268.1895(14)$ & 30589.163(13) & $3268.1879(5)$ & 0.0016 & 450 & & $5 p$ & $(5 / 2)^{2}[5 / 2]^{\circ} 3$ & $7 \mathrm{~d}$ & $(5 / 2)^{2}[9 / 2]_{4}$ & & & R1c & & \\
\hline 3268.7591(13) & $30583.833(12)$ & $3268.7589(5)$ & 0.0002 & 1400 & & $5 p$ & $(5 / 2)^{2}[5 / 2]^{\circ}{ }_{3}$ & $7 \mathrm{~d}$ & $(5 / 2)^{2}[3 / 2]_{2}$ & & & R1c & & \\
\hline $3270.1104(16)$ & $30571.195(15)$ & $3270.1113(4)$ & -0.0008 & 1000 & & $\mathrm{sp}$ & $\left({ }^{3} \mathrm{~F}\right)^{3} \mathrm{P}^{\circ}{ }^{3} \mathrm{D}^{\circ}{ }_{1}$ & $6 \mathrm{~d}$ & $(3 / 2)^{2}[5 / 2]_{2}$ & & & $\mathrm{R} 1 \mathrm{c}$ & & \\
\hline $3275.9027(13)$ & 30517.143(12) & $3275.9057(6)$ & -0.0031 & 2200 & & $5 p$ & $(3 / 2)^{2}[3 / 2]^{\circ}{ }_{1}$ & $7 \mathrm{~d}$ & $(3 / 2)^{2}[5 / 2]_{2}$ & & & R1c & & \\
\hline $3278.9653(23)$ & $30488.640(21)$ & $3278.9636(4)$ & 0.0017 & 210 & & $\mathrm{sp}$ & $\left({ }^{3} \mathrm{~F}\right)^{3} \mathrm{P}^{\circ}{ }^{3} \mathrm{D}^{\circ}{ }_{1}$ & $6 \mathrm{~d}$ & $(3 / 2)^{2}[3 / 2]_{2}$ & & & R1c & & \\
\hline $3281.0757(23)$ & $30469.030(21)$ & $3281.0724(6)$ & 0.0034 & 210 & & $5 p$ & $(3 / 2)^{2}[3 / 2]^{\circ}{ }_{1}$ & $7 \mathrm{~d}$ & $(3 / 2)^{2}[3 / 2]_{2}$ & & & $\mathrm{R} 1 \mathrm{c}$ & & \\
\hline $3281.6964(8)$ & $30463.268(8)$ & $3281.69629(23)$ & 0.0001 & 9700 & & $4 d$ & $(5 / 2)^{2}[9 / 2]_{5}$ & $5 f$ & $(5 / 2)^{2}[9 / 2]^{\circ}$ & & & R1c & & \\
\hline $3282.0106(9)$ & $30460.351(8)$ & $3282.01019(24)$ & 0.0005 & 1100 & & $4 \mathrm{~d}$ & $(5 / 2)^{2}[9 / 2]_{5}$ & $5 \mathrm{f}$ & $(5 / 2)^{2}[9 / 2]^{\circ} 4$ & & & R1c & & \\
\hline $3282.6022(11)$ & $30454.862(10)$ & $3282.6016(3)$ & 0.0006 & 530 & & $4 \mathrm{~d}$ & $(5 / 2)^{2}[9 / 2]_{5}$ & $5 \mathrm{f}$ & $(5 / 2)^{2}[7 / 2]^{\circ} 4$ & & & $\mathrm{R} 1 \mathrm{c}$ & & \\
\hline $3283.0958(16)$ & $30450.283(15)$ & $3283.0969(4)$ & -0.0011 & 210 & & $\mathrm{sp}$ & $\left({ }^{3} \mathrm{~F}\right)^{3} \mathrm{P}^{\circ}{ }^{3} \mathrm{D}^{\circ}{ }_{1}$ & $6 \mathrm{~d}$ & $(3 / 2)^{2}[3 / 2]_{1}$ & & & R1c & & \\
\hline 3283.2431(13) & $30448.917(12)$ & $3283.2433(7)$ & -0.0002 & 940 & & $5 p$ & $(3 / 2)^{2}[3 / 2]^{\circ}{ }_{1}$ & $7 \mathrm{~d}$ & $(3 / 2)^{2}[3 / 2]_{1}$ & & & $\mathrm{R} 1 \mathrm{c}$ & & \\
\hline $3290.4174(4)$ & $30382.530(4)$ & $3290.41683(16)$ & 0.0005 & 38000 & & $4 d$ & $(5 / 2)^{2}[9 / 2]_{5}$ & $5 f$ & $(5 / 2)^{2}[11 / 2]^{\circ}{ }_{6}$ & $5.9 \mathrm{e}+07$ & $\mathrm{D}+$ & F_Re & TW & \\
\hline
\end{tabular}


Table A1. Cont.

\begin{tabular}{|c|c|c|c|c|c|c|c|c|c|c|c|c|c|c|}
\hline$\lambda_{\text {obs }}{ }^{\text {a }}(\AA)$ & $\sigma_{\mathrm{obs}}{ }^{\mathrm{b}}\left(\mathrm{cm}^{-1}\right)$ & $\lambda_{\text {Ritz }}{ }^{c}(\AA)$ & $\Delta \lambda_{\text {obs-Ritz }}$ & $\begin{array}{c}I_{\text {obs }} \mathrm{d} \\
\text { (arb. u.) }\end{array}$ & Char ${ }^{e}$ & & ower Level & & Upper Level & $A\left(\mathrm{~s}^{-1}\right)$ & $\operatorname{Acc}^{f}$ & Line Ref. $\mathrm{g}$ & TP Ref. g & Notes ${ }^{h}$ \\
\hline $3291.0597(8)$ & $30376.600(7)$ & $3291.05952(22)$ & 0.0002 & 1100 & & $4 \mathrm{~d}$ & $(5 / 2)^{2}[9 / 2]_{5}$ & $5 f$ & $(5 / 2)^{2}[11 / 2]^{\circ}{ }_{5}$ & & & R1c & & \\
\hline $3291.8090(16)$ & $30369.686(15)$ & 3291.8073(3) & 0.0017 & 2000 & & $4 \mathrm{~d}$ & $(5 / 2)^{2}[9 / 2]_{4}$ & $5 \mathrm{f}$ & $(5 / 2)^{2}[9 / 2]^{\circ}{ }_{5}$ & & & R1c & & \\
\hline $3292.1232(8)$ & $30366.788(7)$ & $3292.1232(3)$ & -0.0000 & 7700 & & $4 \mathrm{~d}$ & $(5 / 2)^{2}[9 / 2]_{4}$ & $5 \mathrm{f}$ & $(5 / 2)^{2}[9 / 2]^{\circ}{ }_{4}$ & & & R1c & & \\
\hline $3292.7189(10)$ & $30361.294(9)$ & 3292.7183(3) & 0.0007 & 3500 & & $4 \mathrm{~d}$ & $(5 / 2)^{2}[9 / 2]_{4}$ & $5 \mathrm{f}$ & $(5 / 2)^{2}[7 / 2]^{\circ}{ }_{4}$ & & & R1c & & \\
\hline $3292.9965(13)$ & $30358.735(12)$ & 3292.9984(3) & -0.0020 & 1500 & & $4 \mathrm{~d}$ & $(5 / 2)^{2}[9 / 2]_{4}$ & $5 \mathrm{f}$ & $(5 / 2)^{2}[7 / 2]^{3}{ }_{3}$ & & & $\mathrm{R} 1 \mathrm{c}$ & & \\
\hline 3293.3326(9) & $30355.637(8)$ & $3293.3327(4)$ & -0.0001 & 2800 & & $4 \mathrm{~d}$ & $(3 / 2)^{2}[7 / 2]_{3}$ & $5 \mathrm{f}$ & $(3 / 2)^{2}[7 / 2]^{\circ}{ }_{3}$ & & & R1c & & \\
\hline $3294.3354(8)$ & $30346.397(7)$ & $3294.3355(3)$ & -0.0002 & 3800 & & $4 \mathrm{~d}$ & $(5 / 2)^{2}[3 / 2]_{2}$ & $5 \mathrm{f}$ & $(5 / 2)^{2}[5 / 2]^{\circ}{ }_{2}$ & & & R1c & & \\
\hline 3295.1019(8) & $30339.338(7)$ & 3295.1018(3) & 0.0001 & 11000 & & $4 \mathrm{~d}$ & $(5 / 2)^{2}[3 / 2]_{2}$ & $5 \mathrm{f}$ & $(5 / 2)^{2}[5 / 2]^{\circ}{ }_{3}$ & & & R1c & & \\
\hline 3297.1983(8) & $30320.048(7)$ & $3297.1986(3)$ & -0.0003 & 9300 & & $4 \mathrm{~d}$ & $(5 / 2)^{2}[3 / 2]_{1}$ & $5 \mathrm{f}$ & $(5 / 2)^{2}[5 / 2]^{\circ} 2$ & & & R1c & & \\
\hline 3297.3461(12) & 30318.689(11) & 3297.3492(4) & -0.0031 & 1200 & & $4 \mathrm{~d}$ & $(3 / 2)^{2}[7 / 2]_{3}$ & $5 \mathrm{f}$ & $(3 / 2)^{2}[5 / 2]^{\circ}$ & & & R1c & & \\
\hline $3297.5688(10)$ & 30316.642(9) & $3297.5686(4)$ & 0.0002 & 1400 & & $4 \mathrm{~d}$ & $(5 / 2)^{2}[3 / 2]_{2}$ & $5 \mathrm{f}$ & $(5 / 2)^{2}[3 / 2]^{\circ}{ }_{1}$ & & & R1c & & \\
\hline $3300.2124(14)$ & $30292.358(13)$ & $3300.2124(4)$ & 0.0000 & 380 & & $\mathrm{sp}$ & $\left({ }^{3} \mathrm{~F}\right)^{3} \mathrm{P}^{0}{ }^{3} \mathrm{G}^{0}{ }_{3}$ & $7 \mathrm{~s}$ & $(3 / 2)^{2}[3 / 2]_{2}$ & & & R1c & & \\
\hline $3300.4370(9)$ & $30290.296(9)$ & $3300.4373(4)$ & -0.0003 & 6100 & & $4 \mathrm{~d}$ & $(5 / 2)^{2}[3 / 2]_{1}$ & $5 \mathrm{f}$ & $(5 / 2)^{2}[3 / 2]^{2}{ }_{1}$ & & & R1c & & \\
\hline $3300.6409(9)$ & $30288.425(9)$ & $3300.6406(3)$ & 0.0003 & 9100 & & $4 \mathrm{~d}$ & $(5 / 2)^{2}[3 / 2]_{2}$ & $5 \mathrm{f}$ & $(5 / 2)^{2}[3 / 2]^{\circ}{ }_{2}$ & & & R1c & & \\
\hline $3300.8815(9)$ & $30286.218(8)$ & $3300.8808(3)$ & 0.0006 & 13000 & & $4 \mathrm{~d}$ & $(3 / 2)^{2}[7 / 2]_{3}$ & $5 \mathrm{f}$ & $(3 / 2)^{2}[9 / 2]^{-}$ & $5.4 \mathrm{e}+07$ & $\mathrm{D}+$ & R1c & TW & \\
\hline $3301.2286(7)$ & $30283.034(6)$ & $3301.22844(23)$ & 0.0001 & 25000 & & $4 \mathrm{~d}$ & $(5 / 2)^{2}[9 / 2]_{4}$ & $5 \mathrm{f}$ & $(5 / 2)^{2}[11 / 2]^{\circ}{ }_{5}$ & $5.6 \mathrm{e}+07$ & $\mathrm{D}+$ & F_Re & TW & \\
\hline 3303.1817(12) & $30265.128(11)$ & $3303.1836(6)$ & -0.0018 & 1400 & & $5 p$ & $(3 / 2)^{2}[3 / 2]^{\circ} 2$ & $7 \mathrm{~d}$ & $(3 / 2)^{2}[5 / 2]_{2}$ & & & R1c & & \\
\hline $3303.5132(21)$ & $30262.091(20)$ & $3303.5122(4)$ & 0.0010 & 5800 & * & $4 \mathrm{~d}$ & $(5 / 2)^{2}[3 / 2]_{2}$ & $5 \mathrm{f}$ & $(5 / 2)^{2}[1 / 2]^{\circ}{ }_{1}$ & & & R1c & & \\
\hline 3303.5132(21) & $30262.091(20)$ & $3303.5147(3)$ & -0.0015 & 5800 & * & $4 \mathrm{~d}$ & $(5 / 2)^{2}[3 / 2]_{1}$ & $5 \mathrm{f}$ & $(5 / 2)^{2}[3 / 2]^{0}{ }_{2}$ & & & R1c & & \\
\hline $3303.8698(10)$ & $30258.825(9)$ & $3303.8706(6)$ & -0.0008 & 2300 & & $5 p$ & $(3 / 2)^{2}[3 / 2]^{\circ} 2$ & $7 \mathrm{~d}$ & $(3 / 2)^{2}[5 / 2]_{3}$ & & & R1c & & \\
\hline $3306.3890(15)$ & 30235.771(14) & $3306.3913(4)$ & -0.0023 & 180 & & $4 d$ & $(5 / 2)^{2}[3 / 2]_{1}$ & $5 \mathrm{f}$ & $(5 / 2)^{2}[1 / 2]^{\circ}{ }_{1}$ & & & R1c & & \\
\hline $3307.6576(11)$ & $30224.175(10)$ & $3307.6570(4)$ & 0.0006 & 1200 & & $4 \mathrm{~d}$ & $(5 / 2)^{2}[3 / 2]_{1}$ & $5 \mathrm{f}$ & $(5 / 2)^{2}[1 / 2]^{\circ}{ }_{0}$ & & & R1c & & \\
\hline 3307.8727(13) & $30222.210(12)$ & $3307.8664(4)$ & 0.0063 & 1200 & & $4 \mathrm{~d}$ & $(3 / 2)^{2}[7 / 2]_{4}$ & $5 \mathrm{f}$ & $(3 / 2)^{2}[7 / 2]^{\circ}{ }_{4}$ & & & R1c & & $x$ \\
\hline $3308.1052(14)$ & $30220.086(13)$ & $3308.1074(5)$ & -0.0023 & 1100 & & $\mathrm{sp}$ & $\left({ }^{3} \mathrm{~F}\right)^{3} \mathrm{P}^{\circ}{ }^{1} \mathrm{~F}^{\circ}{ }_{3}$ & $7 \mathrm{~d}$ & $(5 / 2)^{2}[7 / 2]_{4}$ & & & R1c & & \\
\hline $3308.2526(15)$ & $30218.739(14)$ & $3308.2561(4)$ & -0.0035 & 180 & & $\mathrm{sp}$ & $\left({ }^{3} \mathrm{~F}\right)^{3} \mathrm{P}^{\circ}{ }^{1} \mathrm{~F}_{3}^{\circ}$ & $7 \mathrm{~d}$ & $(5 / 2)^{2}[7 / 2]_{3}$ & & & R1c & & \\
\hline 3308.4337(13) & 30217.085(12) & $3308.4367(6)$ & -0.0029 & 360 & & $5 p$ & $(3 / 2)^{2}[3 / 2]^{\circ} 2$ & $7 \mathrm{~d}$ & $(3 / 2)^{2}[3 / 2]_{2}$ & & & R1c & & \\
\hline $3310.338(3)$ & 30199.70(3) & $3310.3389(4)$ & -0.001 & 89 & & $\mathrm{sp}$ & $\left({ }^{3} \mathrm{~F}\right)^{3} \mathrm{P}^{\circ}{ }^{3} \mathrm{D}^{\circ}{ }_{3}$ & $6 \mathrm{~d}$ & $(5 / 2)^{2}[5 / 2]_{2}$ & & & R1c & & \\
\hline $3312.0278(13)$ & $30184.296(11)$ & $3312.0276(4)$ & 0.0001 & 550 & & $\mathrm{sp}$ & $\left({ }^{3} \mathrm{~F}\right)^{3} \mathrm{P}^{\circ}{ }^{3} \mathrm{D}^{\circ}{ }_{3}$ & $6 \mathrm{~d}$ & $(5 / 2)^{2}[7 / 2]_{4}$ & & & R1c & & \\
\hline 3312.1151(14) & 30183.500(13) & $3312.1155(5)$ & -0.0004 & 970 & & $\mathrm{sp}$ & $\left({ }^{3} \mathrm{~F}\right)^{3} \mathrm{P}^{\circ}{ }^{1} \mathrm{~F}_{3}^{\circ}$ & $7 \mathrm{~d}$ & $(5 / 2)^{2}[5 / 2]_{3}$ & & & R1c & & \\
\hline $3312.6771(10)$ & $30178.380(9)$ & $3312.6783(3)$ & -0.0012 & 610 & & $4 \mathrm{~d}$ & $(3 / 2)^{2}[7 / 2]_{4}$ & $5 \mathrm{f}$ & $(3 / 2)^{2}[5 / 2]^{\circ}{ }_{3}$ & & & R1c & & \\
\hline $3315.7440(8)$ & $30150.467(7)$ & 3315.7431(3) & 0.0010 & 1300 & & $4 \mathrm{~d}$ & $(3 / 2)^{2}[7 / 2]_{4}$ & $5 f$ & $(3 / 2)^{2}[9 / 2]^{\circ}{ }_{4}$ & & & $\mathrm{R} 1 \mathrm{c}$ & & \\
\hline $3316.2756(8)$ & $30145.634(7)$ & $3316.2752(3)$ & 0.0004 & 16000 & & $4 \mathrm{~d}$ & $(3 / 2)^{2}[7 / 2]_{4}$ & $5 \mathrm{f}$ & $(3 / 2)^{2}[9 / 2]^{\circ}$ & $5.6 \mathrm{e}+07$ & $\mathrm{D}+$ & R1c & TW & \\
\hline $3316.5116(16)$ & $30143.489(15)$ & $3316.5129(6)$ & -0.0013 & 260 & & $\mathrm{sp}$ & $\left({ }^{3} \mathrm{~F}\right)^{3} \mathrm{P}^{\circ}{ }^{1} \mathrm{~F}^{\circ}{ }_{3}$ & $7 \mathrm{~d}$ & $(5 / 2)^{2}[9 / 2]_{4}$ & & & R1c & & \\
\hline 3317.1383(8) & $30137.794(7)$ & $3317.1386(4)$ & -0.0003 & 5500 & & $4 \mathrm{~d}$ & $(3 / 2)^{2}[3 / 2]_{1}$ & $5 \mathrm{f}$ & $(3 / 2)^{2}[5 / 2]_{2}$ & & & R1c & & \\
\hline $3318.3147(23)$ & 30127.110(21) & 3318.3232(12) & -0.0085 & 84 & & $\mathrm{sp}$ & $\left({ }^{3} \mathrm{~F}\right)^{3} \mathrm{P}^{\circ}{ }^{5} \mathrm{~F}^{\circ}{ }_{1}$ & $7 \mathrm{~s}$ & $(5 / 2)^{2}[5 / 2]_{2}$ & & & R1c & & \\
\hline 3319.0203(11) & $30120.706(10)$ & $3319.0216(5)$ & -0.0013 & 840 & & $\mathrm{sp}$ & $\left({ }^{3} \mathrm{~F}\right)^{3} \mathrm{P}^{\circ}{ }^{3} \mathrm{D}^{\circ}{ }_{3}$ & $6 \mathrm{~d}$ & $(5 / 2)^{2}[5 / 2]_{3}$ & & & R1c & & \\
\hline 3321.1137(23) & $30101.720(21)$ & 3321.1114(7) & 0.0023 & 500 & & $5 p$ & $(3 / 2)^{2}[3 / 2]_{2}^{\circ}$ & $7 \mathrm{~d}$ & $(3 / 2)^{2}[1 / 2]_{1}$ & & & R1c & & \\
\hline $3321.5526(8)$ & $30097.743(8)$ & $3321.5528(3)$ & -0.0002 & 1400 & & $5 p$ & $(5 / 2)^{2}[7 / 2]^{\circ}{ }_{3}$ & $8 \mathrm{~s}$ & $(5 / 2)^{2}[5 / 2]_{2}$ & & & R1c & & \\
\hline $3321.7168(14)$ & $30096.255(13)$ & $3321.7165(8)$ & 0.0003 & 410 & & $4 \mathrm{~d}$ & $(3 / 2)^{2}[1 / 2]_{1}$ & $7 \mathrm{p}$ & $(5 / 2)^{2}[3 / 2]^{\circ}{ }_{2}$ & & & R1c & & \\
\hline 3322.6363(10) & $30087.927(9)$ & $3322.6351(4)$ & 0.0012 & 2000 & & $4 d$ & $(3 / 2)^{2}[3 / 2]_{1}$ & $5 f$ & $(3 / 2)^{2}[3 / 2]^{\circ}{ }_{1}$ & & & R1c & & \\
\hline $3324.8295(9)$ & $30068.080(8)$ & $3324.8292(7)$ & 0.0003 & 1200 & & $5 p$ & $(3 / 2)^{2}[5 / 2]^{\circ} 2$ & $8 \mathrm{~s}$ & $(3 / 2)^{2}[3 / 2]_{1}$ & & & R1c & & \\
\hline
\end{tabular}


Table A1. Cont.

\begin{tabular}{|c|c|c|c|c|c|c|c|c|c|c|c|c|c|c|}
\hline$\lambda_{\text {obs }}{ }^{a}(\AA)$ & $\sigma_{\mathrm{obs}}{ }^{\mathrm{b}}\left(\mathrm{cm}^{-1}\right)$ & $\lambda_{\text {Ritz }}{ }^{c}(\AA)$ & $\begin{array}{c}\Delta \lambda_{\text {obs-Ritz }} \\
\text { (A) }\end{array}$ & $\begin{array}{l}I_{\text {obs }} \mathrm{d} \\
\text { (arb. u.) }\end{array}$ & Char ${ }^{\mathrm{e}}$ & & ower Level & & pper Level & $A\left(\mathrm{~s}^{-1}\right)$ & $\operatorname{Acc}^{f}$ & Line Ref. $\mathrm{g}$ & TP Ref. $\mathrm{g}$ & Notes ${ }^{h}$ \\
\hline $3325.0235(13)$ & $30066.326(11)$ & $3325.0210(4)$ & 0.0025 & 720 & & $5 s$ & $(5 / 2)^{2}[5 / 2]_{2}$ & $6 p$ & $(5 / 2)^{2}[7 / 2]^{\circ}{ }_{3}$ & & & R1c & & \\
\hline $3325.8187(14)$ & $30059.137(12)$ & $3325.8184(4)$ & 0.0003 & 3000 & & $4 \mathrm{~d}$ & $(3 / 2)^{2}[3 / 2]_{1}$ & $5 f$ & $(3 / 2)^{2}[3 / 2]^{\circ} 2$ & & & $\mathrm{R} 1 \mathrm{c}$ & & \\
\hline $3325.9236(13)$ & $30058.189(11)$ & $3325.9242(4)$ & -0.0006 & 400 & & $5 p$ & $(5 / 2)^{2}[7 / 2]^{\circ}{ }_{3}$ & $8 \mathrm{~s}$ & $(5 / 2)^{2}[5 / 2]_{3}$ & & & R1c & & \\
\hline $3327.9129(16)$ & $30040.222(15)$ & $3327.9161(5)$ & -0.0032 & 160 & & $\mathrm{sp}$ & $\left({ }^{3} \mathrm{~F}\right)^{3} \mathrm{P}^{\circ}{ }^{3} \mathrm{D}_{3}^{\circ}$ & $6 \mathrm{~d}$ & $(5 / 2)^{2}[3 / 2]_{2}$ & & & R1c & & \\
\hline $3335.4075(14)$ & $29972.725(12)$ & $3335.4064(5)$ & 0.0011 & 520 & & $5 p$ & $(3 / 2)^{2}[1 / 2]^{\circ}{ }_{1}$ & $8 \mathrm{~s}$ & $(3 / 2)^{2}[3 / 2]_{2}$ & & & $\mathrm{R} 1 \mathrm{c}$ & & \\
\hline 3337.5951(12) & 29953.080(11) & $3337.5942(4)$ & 0.0008 & 3000 & & $5 p$ & $(5 / 2)^{2}[7 / 2]^{\circ}{ }_{4}$ & $8 \mathrm{~s}$ & $(5 / 2)^{2}[5 / 2]_{3}$ & & & R1c & & \\
\hline $3338.0369(14)$ & 29949.116(12) & $3338.03567(24)$ & 0.0012 & 9200 & & $4 \mathrm{~d}$ & $(5 / 2)^{2}[5 / 2]_{3}$ & $5 f$ & $(5 / 2)^{2}[9 / 2]^{\circ} 4$ & & & R1c & & \\
\hline $3338.6475(9)$ & $29943.638(8)$ & $3338.6475(3)$ & 0.0001 & 9900 & & $4 \mathrm{~d}$ & $(5 / 2)^{2}[5 / 2]_{3}$ & $5 f$ & $(5 / 2)^{2}[7 / 2]^{\circ} 4$ & $2.7 \mathrm{e}+07$ & $\mathrm{D}+$ & R1c & TW & \\
\hline $3338.9360(9)$ & 29941.051(8) & $3338.9355(3)$ & 0.0005 & 3600 & & $4 \mathrm{~d}$ & $(5 / 2)^{2}[5 / 2]_{3}$ & $5 f$ & $(5 / 2)^{2}[7 / 2]^{0} 3$ & & & R1c & & \\
\hline $3339.0850(9)$ & 29939.715(8) & $3339.0845(4)$ & 0.0006 & 3600 & & $4 \mathrm{~d}$ & $(3 / 2)^{2}[3 / 2]_{2}$ & $5 f$ & $(3 / 2)^{2}[7 / 2]^{\circ} 3$ & & & R1c & & \\
\hline $3340.8308(13)$ & $29924.070(11)$ & $3340.8312(8)$ & -0.0003 & 720 & & $5 \mathrm{~s}$ & $(5 / 2)^{2}[5 / 2]_{3}$ & $\mathrm{sp}$ & $\left({ }^{1} \mathrm{G}\right)^{3} \mathrm{P}^{\circ} 3^{3} \mathrm{~F}_{4}^{\circ}$ & & & $\mathrm{R} 1 \mathrm{c}$ & & \\
\hline $3341.7610(13)$ & 29915.741(11) & $3341.7607(5)$ & 0.0003 & 570 & & $\mathrm{sp}$ & $\left({ }^{3} \mathrm{~F}\right)^{3} \mathrm{P}^{0}{ }^{3} \mathrm{G}^{\circ}{ }_{3}$ & $6 \mathrm{~d}$ & $(5 / 2)^{2}[7 / 2]_{4}$ & & & R1c & & \\
\hline $3342.8004(10)$ & $29906.439(9)$ & $3342.8003(4)$ & 0.0001 & 710 & & $5 p$ & $(5 / 2)^{2}[5 / 2]^{\circ} 2$ & $8 \mathrm{~s}$ & $(5 / 2)^{2}[5 / 2]_{2}$ & & & R1c & & \\
\hline $3342.9641(9)$ & $29904.975(8)$ & $3342.9640(3)$ & 0.0001 & 1800 & & $4 \mathrm{~d}$ & $(5 / 2)^{2}[5 / 2]_{3}$ & $5 f$ & $(5 / 2)^{2}[5 / 2]^{\circ} 2$ & & & $\mathrm{R} 1 \mathrm{c}$ & & \\
\hline $3343.2140(24)$ & $29902.740(21)$ & $3343.2134(4)$ & 0.0006 & 280 & & $4 \mathrm{~d}$ & $(3 / 2)^{2}[3 / 2]_{2}$ & $5 f$ & $(3 / 2)^{2}[5 / 2]^{0} 2$ & & & R1c & & \\
\hline $3343.7214(9)$ & $29898.202(8)$ & $3343.7215(3)$ & -0.0001 & 14000 & & $4 \mathrm{~d}$ & $(3 / 2)^{2}[3 / 2]_{2}$ & $5 f$ & $(3 / 2)^{2}[5 / 2]^{\circ} 3$ & & & R1c & & \\
\hline $3343.7515(9)$ & 29897.933(8) & 3343.7531(3) & -0.0016 & 14000 & & $4 \mathrm{~d}$ & $(5 / 2)^{2}[5 / 2]_{3}$ & $5 f$ & $(5 / 2)^{2}[5 / 2]^{\circ}{ }_{3}$ & & & R1c & & \\
\hline $3347.2268(18)$ & 29866.892(16) & $3347.2279(4)$ & -0.0011 & 140 & & $5 p$ & $(5 / 2)^{2}[5 / 2]^{\circ} 2$ & $8 \mathrm{~s}$ & $(5 / 2)^{2}[5 / 2]_{3}$ & & & R1c & & \\
\hline $3347.6754(9)$ & $29862.890(8)$ & $3347.6762(3)$ & -0.0008 & 550 & & $5 p$ & $(5 / 2)^{2}[3 / 2]^{\circ} 1$ & $8 \mathrm{~s}$ & $(5 / 2)^{2}[5 / 2]_{2}$ & & & R1c & & \\
\hline $3348.7955(14)$ & 29852.902(12) & $3348.7967(4)$ & -0.0012 & 540 & & $4 \mathrm{~d}$ & $(3 / 2)^{2}[3 / 2]_{2}$ & $5 f$ & $(3 / 2)^{2}[3 / 2]^{\circ} 1$ & & & R1c & & \\
\hline $3348.8824(14)$ & 29852.127(12) & $3348.8809(5)$ & 0.0015 & 620 & & $\mathrm{sp}$ & $\left({ }^{3} \mathrm{~F}\right)^{3} \mathrm{P}^{0}{ }^{3} \mathrm{G}^{0}{ }_{3}$ & $6 \mathrm{~d}$ & $(5 / 2)^{2}[5 / 2]_{3}$ & & & R1c & & \\
\hline $3349.4567(8)$ & $29847.009(7)$ & $3349.4569(3)$ & -0.0002 & 4200 & & $4 \mathrm{~d}$ & $(5 / 2)^{2}[5 / 2]_{3}$ & $5 f$ & $(5 / 2)^{2}[3 / 2]^{\circ} 2$ & & & R1c & & \\
\hline $3352.0324(12)$ & $29824.075(11)$ & $3352.0304(4)$ & 0.0020 & 6400 & & $4 \mathrm{~d}$ & $(3 / 2)^{2}[3 / 2]_{2}$ & $5 f$ & $(3 / 2)^{2}[3 / 2]^{\circ} 2$ & & & $\mathrm{R} 1 \mathrm{c}$ & & \\
\hline 3352.079 & 29823.66 & $3352.0793(6)$ & & & : & $5 p$ & $(3 / 2)^{2}[5 / 2]^{\circ} 3$ & $8 \mathrm{~s}$ & $(3 / 2)^{2}[3 / 2]_{2}$ & & & & & \\
\hline $3354.0672(12)$ & $29805.983(11)$ & $3354.0677(3)$ & -0.0005 & 330 & & $\mathrm{sp}$ & $\left({ }^{3} \mathrm{~F}\right)^{3} \mathrm{P}^{\circ}{ }^{3} \mathrm{D}_{2}^{\circ}$ & $7 \mathrm{~s}$ & $(3 / 2)^{2}[3 / 2]_{2}$ & & & R1c & & \\
\hline $3355.3107(13)$ & 29794.937(11) & $3355.31179(19)$ & -0.0011 & 130 & & $5 s$ & $(5 / 2)^{2}[5 / 2]_{2}$ & $4 \mathrm{f}$ & $(3 / 2)^{2}[5 / 2]^{\circ} 3$ & & & R1c & & \\
\hline $3356.8877(17)$ & $29780.940(15)$ & $3356.8867(3)$ & 0.0010 & 64 & & $\mathrm{~s}^{2}$ & ${ }^{3} \mathrm{P}_{2}$ & $\mathrm{sp}$ & $\left({ }^{3} \mathrm{~F}\right)^{3} \mathrm{P}^{\circ}{ }^{3} \mathrm{~F}^{\circ}{ }_{3}$ & & & R1c & & \\
\hline $3357.4722(10)$ & $29775.756(9)$ & $3357.4732(5)$ & -0.0010 & 640 & & $\mathrm{sp}$ & $\left({ }^{3} \mathrm{~F}\right)^{3} \mathrm{P}^{\circ}{ }^{1} \mathrm{D}^{\circ}{ }_{2}$ & $7 \mathrm{~d}$ & $(5 / 2)^{2}[5 / 2]_{2}$ & & & R1c & & \\
\hline $3357.9368(14)$ & 29771.636(12) & $3357.9364(5)$ & 0.0004 & 510 & & $\mathrm{sp}$ & $\left({ }^{3} \mathrm{~F}\right)^{3} \mathrm{P}^{\circ}{ }^{3} \mathrm{G}^{\circ}{ }_{3}$ & $6 \mathrm{~d}$ & $(5 / 2)^{2}[3 / 2]_{2}$ & & & $\mathrm{R} 1 \mathrm{c}$ & & \\
\hline $3359.0587(9)$ & $29761.693(8)$ & $3359.0575(4)$ & 0.0012 & 950 & & $\mathrm{sp}$ & $\left({ }^{3} \mathrm{~F}\right)^{3} \mathrm{P}^{\circ}{ }^{1} \mathrm{D}_{2}^{\circ}$ & $7 \mathrm{~d}$ & $(5 / 2)^{2}[7 / 2]_{3}$ & & & R1c & & \\
\hline $3359.7217(9)$ & $29755.820(8)$ & $3359.7209(3)$ & 0.0008 & 670 & & $\mathrm{sp}$ & $\left({ }^{3} \mathrm{~F}\right)^{3} \mathrm{P}^{\circ}{ }^{3} \mathrm{D}^{\circ}{ }_{2}$ & $7 \mathrm{~s}$ & $(3 / 2)^{2}[3 / 2]_{1}$ & & & R1c & & \\
\hline $3363.8287(17)$ & 29719.491(15) & $3363.8300(10)$ & -0.0013 & 310 & & $4 \mathrm{~d}$ & $(3 / 2)^{2}[3 / 2]_{1}$ & $7 p$ & $(5 / 2)^{2}[5 / 2]^{\circ} 2$ & & & R1c & & \\
\hline 3365.4414(17) & $29705.250(15)$ & $3365.4436(7)$ & -0.0021 & 610 & & $\mathrm{sp}$ & $\left({ }^{3} \mathrm{~F}^{3} \mathrm{P}^{\circ}{ }^{5} \mathrm{D}^{\circ}{ }_{3}\right.$ & $5 d$ & $(3 / 2)^{2}[3 / 2]_{2}$ & & & R1c & & \\
\hline $3365.6475(8)$ & $29703.431(7)$ & $3365.6470(3)$ & 0.0005 & 11000 & & $4 \mathrm{~d}$ & $(5 / 2)^{2}[7 / 2]_{3}$ & $5 f$ & $(5 / 2)^{2}[9 / 2]^{\circ} 4$ & $2.9 e+07$ & $\mathrm{D}+$ & R1c & TW & \\
\hline $3366.2696(10)$ & 29697.942(9) & $3366.2690(3)$ & 0.0006 & 10000 & & $4 \mathrm{~d}$ & $(5 / 2)^{2}[7 / 2]_{3}$ & $5 f$ & $(5 / 2)^{2}[7 / 2]^{\circ}{ }_{4}$ & & & R1c & & \\
\hline $3366.5619(8)$ & $29695.364(7)$ & $3366.5618(3)$ & 0.0001 & 6100 & & $4 \mathrm{~d}$ & $(5 / 2)^{2}[7 / 2]_{3}$ & $5 f$ & $(5 / 2)^{2}[7 / 2]^{0} 3$ & & & R1c & & \\
\hline $3366.8560(24)$ & $29692.770(21)$ & $3366.8553(4)$ & 0.0007 & 120 & & $5 \mathrm{~s}$ & $(5 / 2)^{2}[5 / 2]_{2}$ & $4 f$ & $(3 / 2)^{2}[3 / 2]^{\circ} 1$ & & & R1c & & \\
\hline $3370.1508(11)$ & $29663.742(10)$ & 3370.1503(4) & 0.0005 & 610 & & $5 p$ & $(5 / 2)^{2}[5 / 2]^{\circ}{ }_{3}$ & $8 \mathrm{~s}$ & $(5 / 2)^{2}[5 / 2]_{3}$ & & & R1c & & \\
\hline $3370.4529(7)$ & $29661.083(6)$ & $3370.45289(23)$ & -0.0000 & 22000 & & $4 \mathrm{~d}$ & $(5 / 2)^{2}[7 / 2]_{4}$ & $5 f$ & $(5 / 2)^{2}[9 / 2]^{\circ}{ }_{5}$ & $4.3 e+07$ & $\mathrm{D}+$ & F_Re & TW & \\
\hline $3370.6573(11)$ & $29659.285(10)$ & $3370.6573(3)$ & -0.0001 & 1100 & & $4 \mathrm{~d}$ & $(5 / 2)^{2}[7 / 2]_{3}$ & $5 f$ & $(5 / 2)^{2}[5 / 2]^{\circ} 2$ & & & $\mathrm{R} 1 \mathrm{c}$ & & \\
\hline $3370.7846(8)$ & $29658.165(7)$ & $3370.7840(3)$ & 0.0006 & 5900 & & $4 \mathrm{~d}$ & $(5 / 2)^{2}[7 / 2]_{4}$ & $5 f$ & $(5 / 2)^{2}[9 / 2]^{\circ}{ }_{4}$ & & & R1c & & \\
\hline $3371.4075(9)$ & $29652.685(8)$ & $3371.4079(3)$ & -0.0004 & 6200 & & $4 \mathrm{~d}$ & $(5 / 2)^{2}[7 / 2]_{4}$ & $5 f$ & $(5 / 2)^{2}[7 / 2]^{\circ} 4$ & & & R1c & & \\
\hline
\end{tabular}


Table A1. Cont.

\begin{tabular}{|c|c|c|c|c|c|c|c|c|c|c|c|c|c|c|}
\hline$\lambda_{\text {obs }}{ }^{a}(\AA)$ & $\sigma_{\mathrm{obs}}{ }^{\mathrm{b}}\left(\mathrm{cm}^{-1}\right)$ & $\lambda_{\text {Ritz }}{ }^{c}(\AA)$ & $\Delta \lambda_{\text {obs-Ritz }}$ & $\begin{array}{c}I_{\text {obs }} \mathrm{d} \\
\text { (arb. u.) }\end{array}$ & Char ${ }^{e}$ & & ower Level & & Upper Level & $A\left(\mathrm{~s}^{-1}\right)$ & $\operatorname{Acc}^{f}$ & Line Ref. $\mathrm{g}$ & TP Ref. $\mathrm{g}$ & Notes ${ }^{h}$ \\
\hline $3371.7016(9)$ & $29650.099(8)$ & $3371.7016(3)$ & -0.0000 & 870 & & $4 \mathrm{~d}$ & $(5 / 2)^{2}[7 / 2]_{4}$ & $5 f$ & $(5 / 2)^{2}[7 / 2]^{\circ}{ }_{3}$ & & & R1c & & \\
\hline $3373.5914(8)$ & $29633.490(7)$ & $3373.5912(3)$ & 0.0002 & 9700 & & $4 \mathrm{~d}$ & $(5 / 2)^{2}[5 / 2]_{2}$ & $5 f$ & $(5 / 2)^{2}[7 / 2]^{\circ}{ }_{3}$ & & & R1c & & \\
\hline $3374.4423(13)$ & 29626.018(11) & $3374.4427(4)$ & -0.0005 & 170 & & $5 s$ & $(3 / 2)^{2}[3 / 2]_{1}$ & $\mathrm{sp}$ & $\left({ }^{3} \mathrm{~F}\right)^{1} \mathrm{P}^{\circ}{ }^{3} \mathrm{~F}^{\circ}{ }_{2}$ & & & $\mathrm{R} 1 \mathrm{c}$ & & \\
\hline $3374.9515(8)$ & $29621.548(7)$ & $3374.9510(4)$ & 0.0005 & 12000 & & $4 \mathrm{~d}$ & $(3 / 2)^{2}[5 / 2]_{3}$ & $5 f$ & $(3 / 2)^{2}[7 / 2]^{\circ} 4$ & $4.6 \mathrm{e}+07$ & $\mathrm{D}+$ & R1c & TW & \\
\hline $3375.2221(12)$ & 29619.173(11) & $3375.2222(4)$ & -0.0000 & 1400 & & $4 \mathrm{~d}$ & $(3 / 2)^{2}[5 / 2]_{3}$ & $5 f$ & $(3 / 2)^{2}[7 / 2]^{\circ} 3$ & & & $\mathrm{R} 1 \mathrm{c}$ & & \\
\hline $3376.6139(8)$ & $29606.965(7)$ & $3376.6143(3)$ & -0.0004 & 1300 & & $4 \mathrm{~d}$ & $(5 / 2)^{2}[7 / 2]_{4}$ & $5 f$ & $(5 / 2)^{2}[5 / 2]^{\circ}{ }_{3}$ & & & R1c & & \\
\hline 3377.0834(17) & $29602.849(15)$ & $3377.0829(4)$ & 0.0006 & 170 & & $\mathrm{sp}$ & $\left({ }^{3} \mathrm{~F}\right)^{3} \mathrm{P}^{\circ}{ }^{3} \mathrm{~F}_{2}^{\circ}$ & $6 \mathrm{~d}$ & $(3 / 2)^{2}[5 / 2]_{2}$ & & & R1c & & \\
\hline $3377.2601(14)$ & $29601.300(12)$ & $3377.2583(3)$ & 0.0019 & 110 & & $4 \mathrm{~d}$ & $(5 / 2)^{2}[7 / 2]_{3}$ & $5 f$ & $(5 / 2)^{2}[3 / 2]^{\circ}{ }_{2}$ & & & $\mathrm{R} 1 \mathrm{c}$ & & \\
\hline $3377.7037(8)$ & $29597.412(7)$ & $3377.7038(3)$ & -0.0001 & 5300 & & $4 \mathrm{~d}$ & $(5 / 2)^{2}[5 / 2]_{2}$ & $5 f$ & $(5 / 2)^{2}[5 / 2]_{2}^{\circ}$ & & & R1c & & \\
\hline $3378.3846(15)$ & 29591.448(13) & $3378.3831(4)$ & 0.0015 & 170 & & $\mathrm{sp}$ & $\left({ }^{3} \mathrm{~F}\right)^{3} \mathrm{P}^{\circ}{ }^{3} \mathrm{~F}_{2}^{\circ}$ & $6 \mathrm{~d}$ & $(3 / 2)^{2}[5 / 2]_{3}$ & & & R1c & & \\
\hline $3378.5094(8)$ & $29590.354(7)$ & 3378.5094(3) & 0.0001 & 3500 & & $4 \mathrm{~d}$ & $(5 / 2)^{2}[5 / 2]_{2}$ & $5 f$ & $(5 / 2)^{2}[5 / 2]^{\circ}{ }_{3}$ & & & $\mathrm{R} 1 \mathrm{c}$ & & \\
\hline $3379.4421(9)$ & 29582.188(8) & $3379.4410(4)$ & 0.0011 & 500 & & $4 \mathrm{~d}$ & $(3 / 2)^{2}[5 / 2]_{3}$ & $5 f$ & $(3 / 2)^{2}[5 / 2]^{\circ} 2$ & & & R1c & & \\
\hline $3379.9595(9)$ & $29577.660(8)$ & $3379.9602(3)$ & -0.0007 & 3800 & & $4 \mathrm{~d}$ & $(3 / 2)^{2}[5 / 2]_{3}$ & $5 f$ & $(3 / 2)^{2}[5 / 2]^{\circ}$ & & & R1c & & \\
\hline 3380.3311(8) & 29574.409(7) & $3380.33017(22)$ & 0.0009 & 1100 & & $4 \mathrm{~d}$ & $(5 / 2)^{2}[7 / 2]_{4}$ & $5 f$ & $(5 / 2)^{2}[11 / 2]_{5}^{\circ}$ & & & R1c & & \\
\hline $3380.7117(8)$ & 29571.079(7) & $3380.7116(4)$ & 0.0002 & 7600 & & $4 \mathrm{~d}$ & $(3 / 2)^{2}[5 / 2]_{2}$ & $5 f$ & $(3 / 2)^{2}[7 / 2]^{\circ}{ }_{3}$ & $3.4 \mathrm{e}+07$ & $\mathrm{D}+$ & R1c & TW & \\
\hline 3381.1021(11) & 29567.665(10) & $3381.1027(4)$ & -0.0006 & 1400 & & $4 \mathrm{~d}$ & $(5 / 2)^{2}[5 / 2]_{2}$ & $5 f$ & $(5 / 2)^{2}[3 / 2]^{\circ}{ }_{1}$ & & & R1c & & \\
\hline 3384.3322(8) & 29539.446(7) & 3384.3324(3) & -0.0002 & 1300 & & $4 \mathrm{~d}$ & $(5 / 2)^{2}[5 / 2]_{2}$ & $5 \mathrm{f}$ & $(5 / 2)^{2}[3 / 2]^{\circ}{ }_{2}$ & & & R1c & & \\
\hline $3384.7698(14)$ & 29535.627(12) & $3384.7669(6)$ & 0.0029 & 160 & & $5 p$ & $(3 / 2)^{2}[3 / 2]^{\circ} 1$ & $8 \mathrm{~s}$ & $(3 / 2)^{2}[3 / 2]_{2}$ & & & R1c & & \\
\hline $3384.9450(9)$ & 29534.098(8) & $3384.9442(4)$ & 0.0009 & 3600 & & $4 \mathrm{~d}$ & $(3 / 2)^{2}[5 / 2]_{2}$ & $5 f$ & $(3 / 2)^{2}[5 / 2]^{\circ}{ }_{2}$ & & & $\mathrm{R} 1 \mathrm{c}$ & & \\
\hline $3385.4657(9)$ & 29529.556(8) & $3385.4650(3)$ & 0.0006 & 2100 & & $4 \mathrm{~d}$ & $(3 / 2)^{2}[5 / 2]_{2}$ & $5 f$ & $(3 / 2)^{2}[5 / 2]^{\circ}{ }_{3}$ & & & R1c & & \\
\hline 3387.3535(17) & 29513.099(15) & $3387.3515(4)$ & 0.0021 & 100 & & $4 \mathrm{~d}$ & $(5 / 2)^{2}[5 / 2]_{2}$ & $5 f$ & $(5 / 2)^{2}[1 / 2]^{\circ}{ }_{1}$ & & & R1c & & \\
\hline $3387.7986(17)$ & 29509.222(15) & $3387.8000(8)$ & -0.0015 & 210 & & $5 p$ & $(3 / 2)^{2}[3 / 2]^{\circ} 1$ & $8 \mathrm{~s}$ & $(3 / 2)^{2}[3 / 2]_{1}$ & & & R1c & & \\
\hline \multirow[t]{2}{*}{ 3388.4491(14) } & 29503.557(12) & $3388.4504(4)$ & -0.0013 & 420 & & $4 \mathrm{~d}$ & $(3 / 2)^{2}[5 / 2]_{3}$ & $5 f$ & $(3 / 2)^{2}[3 / 2]^{\circ}{ }_{2}$ & & & R1c & & \\
\hline & & $3390.6470(10)$ & & & $\mathrm{m}$ & $4 \mathrm{~d}$ & $(3 / 2)^{2}[3 / 2]_{2}$ & $7 p$ & $(5 / 2)^{2}[5 / 2]_{2}^{\circ}$ & & & R1c & & \\
\hline $3390.664(20)$ & 29484.29(17) & $3390.6678(4)$ & -0.004 & 260 & & $4 \mathrm{~d}$ & $(3 / 2)^{2}[5 / 2]_{2}$ & $5 \mathrm{f}$ & $(3 / 2)^{2}[3 / 2]^{\circ}{ }_{1}$ & & & R1c & & \\
\hline $3390.9325(24)$ & $29481.950(21)$ & $3390.9337(4)$ & -0.0012 & 100 & & $\mathrm{sp}$ & $\left({ }^{3} \mathrm{~F}\right)^{3} \mathrm{P}^{\circ}{ }^{3} \mathrm{~F}_{2}^{\circ}$ & $6 \mathrm{~d}$ & $(3 / 2)^{2}[3 / 2]_{1}$ & & & $\mathrm{R} 1 \mathrm{c}$ & & \\
\hline $3392.7462(24)$ & $29466.190(21)$ & $3392.7444(4)$ & 0.0019 & 130 & & $s^{2}$ & ${ }^{3} \mathrm{P}_{1}$ & $\mathrm{sp}$ & $\left({ }^{3} \mathrm{~F}\right)^{3} \mathrm{P}^{0}{ }^{3} \mathrm{D}^{\circ}{ }_{1}$ & & & R1c & & \\
\hline 3393.9761(18) & $29455.513(16)$ & $3393.9803(13)$ & -0.0042 & 150 & * & $\mathrm{sp}$ & $\left({ }^{3} \mathrm{P}\right)^{3} \mathrm{P}^{\circ}{ }^{5} \mathrm{P}^{\circ}{ }_{2}$ & $7 \mathrm{~g}$ & $(5 / 2)^{2}[7 / 2]_{3}$ & & & R1c & & $\mathrm{x}$ \\
\hline 3393.9761(18) & $29455.513(16)$ & $3393.9829(4)$ & -0.0069 & 150 & * & $4 d$ & $(3 / 2)^{2}[5 / 2]_{2}$ & $5 f$ & $(3 / 2)^{2}[3 / 2]^{\circ}{ }_{2}$ & & & R1c & & $x$ \\
\hline $3393.9909(15)$ & $29455.384(13)$ & $3393.9914(6)$ & -0.0004 & 150 & & $\mathrm{sp}$ & $\left({ }^{3} \mathrm{~F}\right)^{3} \mathrm{P}^{\circ}{ }^{3} \mathrm{G}^{\circ}{ }_{4}$ & $7 \mathrm{~s}$ & $(5 / 2)^{2}[5 / 2]_{3}$ & & & R1c & & \\
\hline $3395.2150(9)$ & $29444.765(8)$ & $3395.2154(4)$ & -0.0004 & 2000 & & $\mathrm{sp}$ & $\left({ }^{3} \mathrm{~F}\right)^{3} \mathrm{P}^{\circ}{ }^{3} \mathrm{D}^{\circ}{ }_{2}$ & $6 \mathrm{~d}$ & $(5 / 2)^{2}[5 / 2]_{2}$ & & & R1c & & \\
\hline $3395.5206(10)$ & 29442.115(9) & 3395.5191(4) & 0.0015 & 450 & & $\mathrm{sp}$ & $\left({ }^{3} \mathrm{~F}\right)^{3} \mathrm{P}^{\circ}{ }^{3} \mathrm{~F}^{\circ}{ }_{2}$ & $6 \mathrm{~d}$ & $(3 / 2)^{2}[7 / 2]_{3}$ & & & R1c & & \\
\hline $3397.3752(9)$ & $29426.043(8)$ & $3397.3754(3)$ & -0.0002 & 740 & & $\mathrm{sp}$ & $\left({ }^{3} \mathrm{~F}\right)^{3} \mathrm{P}^{\circ}{ }^{3} \mathrm{D}^{\circ}{ }_{2}$ & $6 \mathrm{~d}$ & $(5 / 2)^{2}[7 / 2]_{3}$ & & & R1c & & \\
\hline $3400.4535(9)$ & $29399.406(8)$ & $3400.4535(6)$ & 0.0000 & 96 & & $4 d$ & $(3 / 2)^{2}[3 / 2]_{2}$ & $7 p$ & $(5 / 2)^{2}[3 / 2]^{\circ}{ }_{1}$ & & & R1c & & \\
\hline $3402.8301(9)$ & 29378.873(7) & $3402.8303(4)$ & -0.0002 & 1500 & & $4 \mathrm{~d}$ & $(5 / 2)^{2}[1 / 2]_{0}$ & $5 f$ & $(5 / 2)^{2}[3 / 2]^{\circ}{ }_{1}$ & & & R1c & & \\
\hline $3406.835(2)$ & $29344.340(21)$ & $3406.8351(4)$ & -0.000 & 140 & & $5 s$ & $(3 / 2)^{2}[3 / 2]_{2}$ & $\mathrm{sp}$ & $\left({ }^{3} \mathrm{~F}\right)^{1} \mathrm{P}^{\circ}{ }^{3} \mathrm{~F}^{\circ}{ }_{2}$ & & & R1c & & \\
\hline $3409.1599(8)$ & $29324.327(7)$ & 3409.1597(4) & 0.0001 & 1400 & & $4 \mathrm{~d}$ & $(5 / 2)^{2}[1 / 2]_{0}$ & $5 f$ & $(5 / 2)^{2}[1 / 2]^{\circ}{ }_{1}$ & & & R1c & & \\
\hline $3410.4564(17)$ & 29313.180(15) & $3410.4549(5)$ & 0.0015 & 90 & & $5 s$ & $(5 / 2)^{2}[5 / 2]_{2}$ & $\mathrm{sp}$ & $\left({ }^{3} \mathrm{P}\right)^{3} \mathrm{P}^{\circ}{ }^{1} \mathrm{D}^{\circ}{ }_{2}$ & & & R1c & & \\
\hline $3410.6738(13)$ & 29311.311(11) & $3410.6739(9)$ & -0.0000 & 90 & & $5 p$ & $(3 / 2)^{2}[1 / 2]^{\circ}{ }_{1}$ & $7 \mathrm{~d}$ & $(5 / 2)^{2}[1 / 2]_{0}$ & & & R1c & & \\
\hline $3412.2696(10)$ & 29297.604(9) & $3412.2691(4)$ & 0.0005 & 130 & & $\mathrm{sp}$ & $\left({ }^{3} \mathrm{~F}\right)^{3} \mathrm{P}^{\circ}{ }^{3} \mathrm{D}_{2}{ }_{2}$ & $6 \mathrm{~d}$ & $(5 / 2)^{2}[3 / 2]_{1}$ & & & $\mathrm{R} 1 \mathrm{c}$ & & \\
\hline 3413.7074(14) & $29285.265(12)$ & $3413.7080(5)$ & -0.0007 & 180 & & $\mathrm{sp}$ & $\left({ }^{3} \mathrm{~F}\right)^{3} \mathrm{P}^{\circ}{ }^{3} \mathrm{D}^{\circ}{ }_{2}$ & $6 \mathrm{~d}$ & $(5 / 2)^{2}[3 / 2]_{2}$ & & & R1c & & \\
\hline
\end{tabular}


Table A1. Cont.

\begin{tabular}{|c|c|c|c|c|c|c|c|c|c|c|c|c|c|c|}
\hline$\lambda_{\text {obs }}{ }^{\text {a }}(\AA)$ & $\sigma_{\mathrm{obs}} \mathrm{b}^{\mathrm{b}}\left(\mathrm{cm}^{-1}\right)$ & $\lambda_{\text {Ritz }}{ }^{c}(\AA)$ & $\begin{array}{c}\Delta \lambda_{\text {obs-Ritz }} \\
\text { (A) }\end{array}$ & $\begin{array}{l}I_{\text {obs }} \mathrm{d} \\
\text { (arb. u.) }\end{array}$ & Char ${ }^{\mathrm{e}}$ & & ower Level & & pper Level & $A\left(\mathrm{~s}^{-1}\right)$ & $\operatorname{Acc}^{f}$ & Line Ref. $\mathrm{g}$ & TP Ref. $\mathrm{g}$ & Notes ${ }^{h}$ \\
\hline $3413.8952(9)$ & $29283.654(8)$ & $3413.8957(6)$ & -0.0006 & 220 & & $5 p$ & $(3 / 2)^{2}[3 / 2]_{2}^{\circ}$ & $8 \mathrm{~s}$ & $(3 / 2)^{2}[3 / 2]_{2}$ & & & R1c & & \\
\hline 3416.9362(12) & 29257.593(11) & $3416.9349(4)$ & 0.0012 & 170 & & $\mathrm{sp}$ & $\left({ }^{3} \mathrm{~F}\right)^{3} \mathrm{P}^{\circ}{ }^{1} \mathrm{~F}_{3}^{\circ}$ & $8 \mathrm{~s}$ & $(5 / 2)^{2}[5 / 2]_{2}$ & & & $\mathrm{R} 1 \mathrm{c}$ & & \\
\hline $3421.5612(10)$ & $29218.046(8)$ & $3421.5612(4)$ & -0.0001 & 850 & & $\mathrm{sp}$ & $\left({ }^{3} \mathrm{~F}\right)^{3} \mathrm{P}^{\circ} 1^{1} \mathrm{~F}^{\circ}{ }_{3}$ & $8 \mathrm{~s}$ & $\begin{array}{l}(5 / 2)^{2}[5 / 2]_{3} \\
\end{array}$ & & & R1c & & \\
\hline $3428.7658(10)$ & 29156.654(8) & $3428.7659(5)$ & -0.0001 & 330 & & $5 \mathrm{~s}$ & $(3 / 2)^{2}[3 / 2]_{1}$ & $6 p$ & $(5 / 2)^{2}[3 / 2]^{\circ} 1$ & & & R1c & & \\
\hline 3430.1011(12) & $29145.304(10)$ & $3430.1015(4)$ & -0.0004 & 81 & & $\mathrm{~s}^{2}$ & ${ }^{3} \mathrm{P}_{0}$ & $\mathrm{sp}$ & $\left({ }^{3} \mathrm{~F}\right)^{3} \mathrm{P}^{\circ} 3^{3} \mathrm{D}^{\circ}{ }_{1}$ & & & $\mathrm{R} 1 \mathrm{c}$ & & \\
\hline $3433.9380(9)$ & $29112.740(8)$ & $3433.9259(7)$ & 0.0120 & 120 & ? & $4 \mathrm{~d}$ & $(3 / 2)^{2}[5 / 2]_{3}$ & $7 p$ & $(5 / 2)^{2}[7 / 2]^{\circ} 4$ & & & R1c & & $x$ \\
\hline $3437.1829(19)$ & $29085.256(16)$ & $3437.1784(5)$ & 0.0045 & 78 & & $\mathrm{sp}$ & $\left({ }^{3} \mathrm{~F}\right)^{3} \mathrm{P}^{0}{ }^{3} \mathrm{D}^{\circ}{ }_{2}$ & $6 \mathrm{~d}$ & $(5 / 2)^{2}[1 / 2]_{1}$ & & & R1c & & \\
\hline $3444.1360(17)$ & $29026.540(14)$ & $3444.1368(7)$ & -0.0008 & 75 & & $\mathrm{sp}$ & $\left({ }^{3} \mathrm{~F}\right)^{3} \mathrm{P}^{\circ}{ }^{3} \mathrm{D}^{\circ}{ }_{1}$ & $6 \mathrm{~d}$ & $(5 / 2)^{2}[1 / 2]_{0}$ & & & R1c & & \\
\hline $3445.9053(16)$ & 29011.637(13) & $3445.9035(5)$ & 0.0018 & 37 & & $5 p$ & $(3 / 2)^{2}[5 / 2]^{\circ} 2$ & $7 \mathrm{~d}$ & $(5 / 2)^{2}[5 / 2]_{2}$ & & & R1c & & \\
\hline $3451.4534(9)$ & $28965.003(8)$ & $3451.4543(4)$ & -0.0009 & 360 & & $5 \mathrm{~s}$ & $(3 / 2)^{2}[3 / 2]_{2}$ & $\mathrm{sp}$ & $\left({ }^{1} \mathrm{G}\right)^{3} \mathrm{P}^{\circ}{ }^{3} \mathrm{~F}_{3}{ }_{3}$ & & & R1c & & \\
\hline $3453.9306(10)$ & 28944.230(9) & $3453.9308(6)$ & -0.0002 & 540 & & $5 \mathrm{~s}$ & $(3 / 2)^{2}[3 / 2]_{1}$ & $6 p$ & $(5 / 2)^{2}[5 / 2]^{\circ} 2$ & & & $\mathrm{R} 1 \mathrm{c}$ & & \\
\hline $3460.045(3)$ & $28893.080(21)$ & $3460.0456(7)$ & -0.000 & 350 & & $\mathrm{sp}$ & $\left({ }^{3} \mathrm{~F}\right)^{3} \mathrm{P}^{\circ}{ }^{3} \mathrm{~F}^{\circ}{ }_{4}$ & $6 \mathrm{~d}$ & $(5 / 2)^{2}[7 / 2]_{4}$ & & & R1c & & \\
\hline $3460.4304(14)$ & 28889.865(11) & $3460.4309(5)$ & -0.0005 & 69 & & $5 p$ & $(3 / 2)^{2}[1 / 2]^{\circ} 1$ & $7 \mathrm{~d}$ & $(5 / 2)^{2}[5 / 2]_{2}$ & & & R1c & & \\
\hline $3461.9510(15)$ & 28877.176(12) & $3461.9515(5)$ & -0.0005 & 100 & & $5 s$ & $(5 / 2)^{2}[5 / 2]_{2}$ & $\mathrm{sp}$ & $\left({ }^{1} \mathrm{G}\right)^{3} \mathrm{P}^{\circ}{ }^{3} \mathrm{~F}_{2}{ }_{2}$ & & & R1nc & & \\
\hline $3463.4085(10)$ & $28865.024(9)$ & 3463.4094(3) & -0.0009 & 140 & & $\mathrm{sp}$ & $\left({ }^{3} \mathrm{~F}\right)^{3} \mathrm{P}^{0}{ }^{3} \mathrm{D}^{\circ}{ }_{1}$ & $7 \mathrm{~s}$ & $(3 / 2)^{2}[3 / 2]_{2}$ & & & R1c & & \\
\hline $3467.679(3)$ & $28829.480(21)$ & $3467.6793(7)$ & -0.001 & 200 & & sp & $\left({ }^{3} \mathrm{~F}\right)^{3} \mathrm{P}^{\circ}{ }^{3} \mathrm{~F}^{\circ}{ }_{4}$ & $6 \mathrm{~d}$ & $(5 / 2)^{2}[5 / 2]_{3}$ & & & R1c & & \\
\hline $3469.4366(10)$ & $28814.873(9)$ & $3469.4375(4)$ & -0.0009 & 1000 & & $\mathrm{sp}$ & $\left({ }^{3} \mathrm{~F}\right)^{3} \mathrm{P}^{\circ}{ }^{3} \mathrm{D}^{\circ}{ }_{1}$ & $7 \mathrm{~s}$ & $(3 / 2)^{2}[3 / 2]_{1}$ & & & R1c & & \\
\hline $3470.7569(14)$ & 28803.912(11) & $3470.7552(8)$ & 0.0017 & 130 & & $5 p$ & $(3 / 2)^{2}[1 / 2]^{\circ} 1$ & $7 \mathrm{~d}$ & $(5 / 2)^{2}[3 / 2]_{1}$ & & & R1c & & \\
\hline 3471.1558(10) & $28800.602(8)$ & $3471.1560(4)$ & -0.0003 & 260 & & $\mathrm{sp}$ & $\left({ }^{3} \mathrm{~F}\right)^{3} \mathrm{P}^{\circ}{ }^{1} \mathrm{D}_{2}^{\circ}$ & $8 \mathrm{~s}$ & $(5 / 2)^{2}[5 / 2]_{2}$ & & & R1c & & \\
\hline $3472.0275(10)$ & 28793.371(9) & $3472.0278(3)$ & -0.0003 & 130 & & $\mathrm{sp}$ & $\left({ }^{3} \mathrm{~F}\right)^{3} \mathrm{P}^{\circ}{ }^{3} \mathrm{~F}_{3}^{\circ}$ & $7 \mathrm{~s}$ & $(3 / 2)^{2}[3 / 2]_{2}$ & & & R1c & & \\
\hline $3473.0008(10)$ & 28785.302(8) & $3473.0010(4)$ & -0.0002 & 330 & & $5 \mathrm{~s}$ & $(5 / 2)^{2}[5 / 2]_{3}$ & $\mathrm{sp}$ & $\left({ }^{3} \mathrm{~F}\right)^{1} \mathrm{P}^{\circ}{ }^{3} \mathrm{D}^{\circ}{ }_{2}$ & & & R1c & & \\
\hline $3476.4708(17)$ & 28756.571(14) & $3476.4703(7)$ & 0.0005 & 97 & & $\mathrm{sp}$ & $\left({ }^{3} \mathrm{~F}\right)^{3} \mathrm{P}^{\circ}{ }^{3} \mathrm{~F}^{\circ}{ }_{4}$ & $6 \mathrm{~d}$ & $(5 / 2)^{2}[9 / 2]_{4}$ & & & $\mathrm{R} 1 \mathrm{c}$ & & \\
\hline $3478.950(3)$ & $28736.080(21)$ & $3478.9487(7)$ & 0.001 & 640 & & $\mathrm{sp}$ & $\left({ }^{3} \mathrm{~F}\right)^{3} \mathrm{P}^{\circ}{ }^{3} \mathrm{~F}^{\circ}{ }_{4}$ & $6 \mathrm{~d}$ & $(5 / 2)^{2}[9 / 2]_{5}$ & & & $\mathrm{R} 1 \mathrm{c}$ & & \\
\hline $3483.8304(9)$ & $28695.825(8)$ & $3483.8297(4)$ & 0.0006 & 310 & & $4 \mathrm{~d}$ & $(3 / 2)^{2}[1 / 2]_{1}$ & $5 f$ & $(5 / 2)^{2}[3 / 2]^{\circ} 2$ & & & R1c & & \\
\hline 3487.0291(9) & $28669.502(7)$ & $3487.0290(4)$ & 0.0001 & 470 & & $4 \mathrm{~d}$ & $(3 / 2)^{2}[1 / 2]_{1}$ & $5 f$ & $(5 / 2)^{2}[1 / 2]^{\circ} 1$ & & & R1c & & \\
\hline 3488.0212(16) & 28661.348(13) & $3488.0222(6)$ & -0.0010 & 31 & & $5 \mathrm{~s}$ & $(3 / 2)^{2}[3 / 2]_{1}$ & $6 \mathrm{p}$ & $(5 / 2)^{2}[3 / 2]^{\circ}{ }_{2}$ & & & R1c & & \\
\hline 3488.4370(9) & $28657.932(7)$ & $3488.4369(5)$ & 0.0001 & 150 & & $4 \mathrm{~d}$ & $(3 / 2)^{2}[1 / 2]_{1}$ & $5 f$ & $(5 / 2)^{2}[1 / 2]_{0}^{0}$ & & & R1c & & \\
\hline $3501.612(3)$ & 28550.110(21) & $3501.6122(4)$ & -0.000 & 59 & & $5 p$ & $(5 / 2)^{2}[3 / 2]^{\circ} 2$ & $6 \mathrm{~d}$ & $(3 / 2)^{2}[5 / 2]_{2}$ & & & R1c & & \\
\hline $3503.0098(9)$ & $28538.716(8)$ & $3503.0101(3)$ & -0.0003 & 150 & & $5 p$ & $(5 / 2)^{2}[3 / 2]^{\circ} 2$ & $6 \mathrm{~d}$ & $(3 / 2)^{2}[5 / 2]_{3}$ & & & R1c & & \\
\hline $3504.6643(15)$ & 28525.244(12) & $3504.6642(4)$ & 0.0001 & 59 & & $\mathrm{~s}^{\frac{1}{2}}$ & ${ }^{3} \mathrm{P}_{1}$ & $\mathrm{sp}$ & $\left({ }^{3} \mathrm{~F}\right)^{3} \mathrm{P}^{\circ}{ }^{3} \mathrm{D}^{\circ}{ }_{2}$ & & & R1c & & \\
\hline 3506.8362(11) & $28507.578(9)$ & $3506.8359(4)$ & 0.0003 & 330 & & $\mathrm{sp}$ & $\left({ }^{3} \mathrm{~F}\right)^{3} \mathrm{P}^{\circ}{ }^{3} \mathrm{D}^{\circ}{ }_{3}$ & $7 \mathrm{~s}$ & $(5 / 2)^{2}[5 / 2]_{2}$ & & & R1c & & \\
\hline $3507.3014(10)$ & 28503.797(8) & 3507.3011(4) & 0.0003 & 58 & & $\mathrm{sp}$ & $\left({ }^{3} \mathrm{~F}\right)^{3} \mathrm{P}^{\circ}{ }^{3} \mathrm{D}^{\circ}{ }_{1}$ & $6 \mathrm{~d}$ & $(5 / 2)^{2}[5 / 2]_{2}$ & & & $\mathrm{R} 1 \mathrm{c}$ & & \\
\hline 3515.2292(11) & $28439.515(9)$ & $3515.2302(4)$ & -0.0011 & 850 & & $\mathrm{sp}$ & $\left({ }^{3} \mathrm{~F}\right)^{3} \mathrm{P}^{\circ}{ }^{3} \mathrm{D}^{\circ}{ }_{3}$ & $7 \mathrm{~s}$ & $(5 / 2)^{2}[5 / 2]_{3}$ & & & R1c & & \\
\hline 3516.1395(14) & 28432.152(11) & $3516.1396(4)$ & -0.0001 & 110 & & sp & $\left({ }^{3} \mathrm{~F}\right)^{3} \mathrm{P}^{\circ}{ }^{3} \mathrm{~F}^{\circ}{ }_{3}$ & $6 \mathrm{~d}$ & $(5 / 2)^{2}[5 / 2]_{2}$ & & & R1c & & \\
\hline $3516.7786(9)$ & $28426.985(8)$ & $3516.7792(3)$ & -0.0006 & 280 & & $5 \mathrm{~s}$ & $(5 / 2)^{2}[5 / 2]_{3}$ & $\mathrm{sp}$ & $\left({ }^{3} \mathrm{~F}\right)^{1} \mathrm{P}^{\circ} 3^{3} \mathrm{~F}_{3}^{\circ}$ & & & $\mathrm{R} 1 \mathrm{c}$ & & \\
\hline $3518.0440(10)$ & $28416.761(8)$ & $3518.0449(4)$ & -0.0009 & 230 & & $\mathrm{sp}$ & $\left({ }^{3} \mathrm{~F}\right)^{3} \mathrm{P}^{\circ}{ }^{3} \mathrm{~F}^{\circ}{ }_{3}$ & $6 \mathrm{~d}$ & $(5 / 2)^{2}[7 / 2]_{4}$ & & & R1c & & \\
\hline $3518.4565(9)$ & $28413.429(7)$ & $3518.4562(3)$ & 0.0003 & 420 & & sp & $\left({ }^{3} \mathrm{~F}\right)^{3} \mathrm{P}^{\circ}{ }^{3} \mathrm{~F}^{\circ}{ }_{3}$ & $6 \mathrm{~d}$ & $(5 / 2)^{2}[7 / 2]_{3}$ & & & R1c & & \\
\hline $3522.643(3)$ & 28379.660(21) & $3522.6429(6)$ & 0.000 & 84 & & $5 s$ & $(3 / 2)^{2}[3 / 2]_{2}$ & $6 \mathrm{p}$ & $(5 / 2)^{2}[3 / 2]^{\circ} 2$ & & & R1c & & \\
\hline $3525.5026(16)$ & 28356.644(13) & $3525.5023(5)$ & 0.0003 & 55 & & $\mathrm{sp}$ & $\left({ }^{3} \mathrm{~F}\right)^{3} \mathrm{P}^{0}{ }^{3} \mathrm{D}^{\circ}{ }_{1}$ & $6 \mathrm{~d}$ & $(5 / 2)^{2}[3 / 2]_{1}$ & & & R1c & & \\
\hline 3525.9383(15) & 28353.140(12) & $3525.9370(4)$ & 0.0013 & 140 & & sp & $\left({ }^{3} \mathrm{~F}\right)^{3} \mathrm{P}^{\circ}{ }^{3} \mathrm{~F}^{\circ}{ }_{3}$ & $6 \mathrm{~d}$ & $(5 / 2)^{2}[5 / 2]_{3}$ & & & $\mathrm{R} 1 \mathrm{c}$ & & \\
\hline $3534.6784(20)$ & 28283.034(16) & $3534.6781(9)$ & 0.0002 & 27 & & $5 p$ & $(5 / 2)^{2}[3 / 2]^{\circ}{ }_{1}$ & $6 \mathrm{~d}$ & $(3 / 2)^{2}[1 / 2]_{0}$ & & & R1c & & \\
\hline $3534.809(5)$ & 28281.99(4) & $3534.8076(3)$ & 0.002 & 410 & * & $4 \mathrm{~d}$ & $(3 / 2)^{2}[7 / 2]_{3}$ & $5 f$ & $(5 / 2)^{2}[9 / 2]^{\circ} 4$ & & & R1c & & \\
\hline
\end{tabular}


Table A1. Cont.

\begin{tabular}{|c|c|c|c|c|c|c|c|c|c|c|c|c|c|c|}
\hline$\lambda_{\text {obs }}{ }^{a}(\AA)$ & $\sigma_{\mathrm{obs}}^{\mathrm{b}}\left(\mathrm{cm}^{-1}\right)$ & $\lambda_{\text {Ritz }}{ }^{c}(\AA)$ & $\begin{array}{c}\Delta \lambda_{\text {obs-Ritz }} \\
\text { (A) }\end{array}$ & $\begin{array}{c}I_{\text {obs }}{ }^{\mathrm{d}} \\
\text { (arb. u.) }\end{array}$ & Char ${ }^{e}$ & & Lower Level & & Upper Level & $A\left(\mathrm{~s}^{-1}\right)$ & $\operatorname{Acc}^{f}$ & Line Ref. $\mathrm{g}$ & TP Ref. $\mathrm{g}$ & Notes ${ }^{h}$ \\
\hline $3534.809(5)$ & $28281.99(4)$ & $3534.8127(5)$ & -0.004 & 410 & * & $s^{2}$ & ${ }^{3} \mathrm{P}_{2}$ & $\mathrm{sp}$ & $\left({ }^{3} \mathrm{~F}\right)^{3} \mathrm{P}^{\circ}{ }^{3} \mathrm{G}_{3}^{\circ}$ & & & R1c & & \\
\hline $3535.0258(14)$ & $28280.254(11)$ & 3535.0262(4) & -0.0003 & 1100 & & $\mathrm{sp}$ & $\left({ }^{3} \mathrm{~F}\right)^{3} \mathrm{P}^{\circ}{ }^{3} \mathrm{~F}^{\circ}{ }_{3}$ & $6 \mathrm{~d}$ & $(5 / 2)^{2}[9 / 2]_{4}$ & & & R1c & & \\
\hline 3535.4942(10) & $28276.508(8)$ & 3535.4937(3) & 0.0005 & 220 & & $4 \mathrm{~d}$ & $(3 / 2)^{2}[7 / 2]_{3}$ & $5 f$ & $(5 / 2)^{2}[7 / 2]^{0}{ }_{4}$ & & & R1c & & \\
\hline $3535.8163(12)$ & 28273.932(9) & $3535.8167(3)$ & -0.0004 & 190 & & $4 \mathrm{~d}$ & $(3 / 2)^{2}[7 / 2]_{3}$ & $5 \mathrm{f}$ & $(5 / 2)^{2}[7 / 2]^{0}{ }_{3}$ & & & R1c & & \\
\hline $3535.9757(10)$ & 28272.657(8) & 3535.9767(5) & -0.0009 & 140 & & $\mathrm{sp}$ & $\left({ }^{3} \mathrm{~F}\right)^{3} \mathrm{P}^{\circ}{ }^{3} \mathrm{~F}^{\circ}{ }_{3}$ & $6 \mathrm{~d}$ & $(5 / 2)^{2}[3 / 2]_{2}$ & & & R1c & & \\
\hline 3540.1850(15) & 28239.042(12) & 3540.1870(5) & -0.0020 & 110 & & $\mathrm{sp}$ & $\left({ }^{3} \mathrm{~F}\right)^{3} \mathrm{P}^{\circ}{ }^{3} \mathrm{G}^{\circ}{ }_{3}$ & $7 \mathrm{~s}$ & $(5 / 2)^{2}[5 / 2]_{2}$ & & & R1c & & \\
\hline $3540.3356(19)$ & $28237.841(15)$ & $3540.3346(4)$ & 0.0010 & 110 & & $4 d$ & $(3 / 2)^{2}[7 / 2]_{3}$ & $5 f$ & $(5 / 2)^{2}[5 / 2]^{\circ}{ }_{2}$ & & & R1c & & \\
\hline $3541.2200(20)$ & $28230.789(16)$ & 3541.2196(4) & 0.0004 & 27 & & $4 \mathrm{~d}$ & $(3 / 2)^{2}[7 / 2]_{3}$ & $5 f$ & $(5 / 2)^{2}[5 / 2]^{0}{ }_{3}$ & & & R1c & & \\
\hline $3546.7568(21)$ & $28186.719(17)$ & 3546.7551(5) & 0.0017 & 27 & & $5 p$ & $(3 / 2)^{2}[3 / 2]^{\circ} 2$ & $7 \mathrm{~d}$ & $(5 / 2)^{2}[7 / 2]_{3}$ & & & R1c & & \\
\hline $3548.7423(11)$ & 28170.949(9) & 3548.7419(4) & 0.0004 & 1400 & & $\mathrm{sp}$ & $\left({ }^{3} \mathrm{~F}\right)^{3} \mathrm{P}^{\circ}{ }^{3} \mathrm{G}^{\circ}{ }_{3}$ & $7 \mathrm{~s}$ & $(5 / 2)^{2}[5 / 2]_{3}$ & & & R1c & & \\
\hline 3551.4887(13) & 28149.165(10) & 3551.4887(3) & 0.0001 & 530 & & $4 \mathrm{~d}$ & $(3 / 2)^{2}[7 / 2]_{4}$ & $5 f$ & $(5 / 2)^{2}[9 / 2]^{\circ}{ }_{5}$ & & & R1c & & \\
\hline $3551.8563(10)$ & $28146.252(8)$ & 3551.8563(3) & -0.0000 & 210 & & $4 \mathrm{~d}$ & $(3 / 2)^{2}[7 / 2]_{4}$ & $5 \mathrm{f}$ & $(5 / 2)^{2}[9 / 2]^{\circ}{ }_{4}$ & & & R1c & & \\
\hline $3552.5495(12)$ & 28140.760(9) & 3552.5490(3) & 0.0005 & 110 & & $4 \mathrm{~d}$ & $(3 / 2)^{2}[7 / 2]_{4}$ & $5 f$ & $(5 / 2)^{2}[7 / 2]^{\circ}{ }_{4}$ & & & R1c & & \\
\hline $3552.8735(15)$ & 28138.194(12) & 3552.8751(3) & -0.0016 & 53 & & $4 \mathrm{~d}$ & $(3 / 2)^{2}[7 / 2]_{4}$ & $5 f$ & $(5 / 2)^{2}[7 / 2]^{\circ}{ }_{3}$ & & & R1c & & \\
\hline $3555.4287(21)$ & 28117.972(17) & $3555.43262(15)$ & -0.0039 & 53 & & $5 s$ & $(5 / 2)^{2}[5 / 2]_{3}$ & $4 \mathrm{f}$ & $(5 / 2)^{2}[7 / 2]^{\circ}{ }_{4}$ & & & R1c & & \\
\hline $3556.915(5)$ & 28106.22(4) & 3556.9167(3) & -0.001 & 79 & * & $5 s$ & $(5 / 2)^{2}[5 / 2]_{2}$ & sp & $\left({ }^{3} \mathrm{~F}\right)^{1} \mathrm{P}^{\circ}{ }^{3} \mathrm{~F}_{3}^{\circ}{ }_{3}$ & & & R1c & & \\
\hline $3556.915(5)$ & 28106.22(4) & 3556.9231(7) & -0.008 & 79 & * & $5 p$ & $(3 / 2)^{2}[3 / 2]^{\circ}{ }_{2}$ & $7 \mathrm{~d}$ & $(5 / 2)^{2}[3 / 2]_{2}$ & & & R1nc & & \\
\hline $3558.332(4)$ & 28095.03(3) & 3558.3303(4) & 0.002 & 52 & & $4 \mathrm{~d}$ & $(3 / 2)^{2}[7 / 2]_{4}$ & $5 \mathrm{f}$ & $(5 / 2)^{2}[5 / 2]^{\circ}{ }_{3}$ & & & R1c & & \\
\hline 3562.4574(10) & 28062.497(8) & 3562.4572(3) & 0.0002 & 100 & & $4 \mathrm{~d}$ & $(3 / 2)^{2}[7 / 2]_{4}$ & $5 f$ & $(5 / 2)^{2}[11 / 2]^{\circ}{ }_{5}$ & & & R1c & & \\
\hline 3563.1578(10) & 28056.981(8) & $3563.1580(4)$ & -0.0002 & 78 & & $4 \mathrm{~d}$ & $(3 / 2)^{2}[3 / 2]_{1}$ & $5 f$ & $(5 / 2)^{2}[5 / 2]^{\circ}{ }_{2}$ & & & R1c & & \\
\hline $3565.7620(21)$ & 28036.491(17) & 3565.7601(4) & 0.0018 & 52 & & $5 p$ & $(3 / 2)^{2}[5 / 2]^{\circ}{ }_{2}$ & $8 \mathrm{~s}$ & $(5 / 2)^{2}[5 / 2]_{2}$ & & & R1c & & \\
\hline $3565.8472(10)$ & 28035.821(8) & 3565.8496(4) & -0.0024 & 210 & & $5 s$ & $(3 / 2)^{2}[3 / 2]_{2}$ & $6 \mathrm{p}$ & $(5 / 2)^{2}[7 / 2]^{\circ}{ }_{3}$ & & & R1c & & \\
\hline $3566.9405(11)$ & 28027.228(9) & 3566.9405(5) & -0.0000 & 52 & & $4 \mathrm{~d}$ & $(3 / 2)^{2}[3 / 2]_{1}$ & $5 f$ & $(5 / 2)^{2}[3 / 2]^{\circ}{ }_{1}$ & & & R1c & & \\
\hline $3567.801(3)$ & $28020.470(21)$ & $3567.79746(15)$ & 0.003 & 73 & & $5 s$ & $(5 / 2)^{2}[5 / 2]_{3}$ & $4 \mathrm{f}$ & $(5 / 2)^{2}[5 / 2]^{0}{ }_{3}$ & & & R1c & & \\
\hline $3568.7006(18)$ & 28013.405(14) & 3568.7005(4) & 0.0001 & 78 & & $\mathrm{~s}^{2}$ & ${ }^{3} \mathrm{P}_{2}$ & sp & $\left({ }^{3} \mathrm{~F}\right)^{3} \mathrm{P}^{\circ}{ }^{3} \mathrm{D}^{\circ}{ }_{3}$ & & & R1c & & \\
\hline 3573.5908(14) & 27975.072(11) & $3573.58964(18)$ & 0.0011 & 520 & & $5 s$ & $(5 / 2)^{2}[5 / 2]_{3}$ & $4 \mathrm{f}$ & $(5 / 2)^{2}[7 / 2]^{\circ}{ }_{3}$ & & & R1c & & \\
\hline 3575.373(4) & 27961.13(3) & 3575.3666(24) & 0.006 & 26 & * & $\mathrm{sp}$ & $\left({ }^{1} \mathrm{D}\right)^{3} \mathrm{P}^{\circ}{ }^{3} \mathrm{~F}^{\circ}{ }_{2}$ & 9s & $(3 / 2)^{2}[3 / 2]_{1}$ & & & R1nc & & \\
\hline 3575.373(4) & 27961.13(3) & $3575.3746(5)$ & -0.002 & 26 & * & $4 \mathrm{~d}$ & $(3 / 2)^{2}[3 / 2]_{1}$ & $5 f$ & $(5 / 2)^{2}[1 / 2]^{\circ} 0$ & & & R1c & & \\
\hline 3577.5874(19) & 27943.821(15) & 3577.5764(4) & 0.0110 & 51 & ? & $5 s$ & $(3 / 2)^{2}[3 / 2]_{1}$ & $4 \mathrm{f}$ & $(3 / 2)^{2}[3 / 2]^{\circ}{ }_{1}$ & & & R1c & & $\mathrm{x}$ \\
\hline 3578.4051(17) & 27937.436(13) & 3578.4043(6) & 0.0008 & 130 & & $5 s$ & $(5 / 2)^{2}[5 / 2]_{3}$ & $\mathrm{sp}$ & $\left({ }^{3} \mathrm{P}\right)^{3} \mathrm{P}^{\circ}{ }^{5} \mathrm{~S}^{\circ}{ }_{2}$ & & & R1c & & \\
\hline 3581.3166(13) & 27914.724(10) & 3581.3179(4) & -0.0013 & 26 & & $5 p$ & $(3 / 2)^{2}[1 / 2]^{\circ}{ }_{1}$ & $8 \mathrm{~s}$ & $(5 / 2)^{2}[5 / 2]_{2}$ & & & R1c & & \\
\hline 3583.6322(15) & 27896.687(11) & 3583.6328(3) & -0.0005 & 77 & & $\mathrm{sp}$ & $\left({ }^{3} \mathrm{~F}\right)^{3} \mathrm{P}^{\circ}{ }^{3} \mathrm{~F}^{\circ}{ }_{2}$ & $7 \mathrm{~s}$ & $(3 / 2)^{2}[3 / 2]_{2}$ & & & R1c & & \\
\hline $3588.6079(9)$ & $27858.009(7)$ & $3588.6074(3)$ & 0.0005 & 200 & & $4 \mathrm{~d}$ & $(3 / 2)^{2}[3 / 2]_{2}$ & $5 \mathrm{f}$ & $(5 / 2)^{2}[7 / 2]^{\circ}{ }_{3}$ & & & R1c & & \\
\hline $3590.0862(10)$ & 27846.538(8) & 3590.0870(3) & -0.0008 & 650 & & $\mathrm{sp}$ & $\left({ }^{3} \mathrm{~F}\right)^{3} \mathrm{P}^{\circ}{ }^{3} \mathrm{~F}^{\circ}{ }_{2}$ & $7 \mathrm{~s}$ & $(3 / 2)^{2}[3 / 2]_{1}$ & & & R1c & & \\
\hline 3592.1150(19) & 27830.811(15) & 3592.1139(5) & 0.0011 & 51 & & $5 p$ & $(5 / 2)^{2}[7 / 2]^{\circ}{ }_{3}$ & $6 \mathrm{~d}$ & $(3 / 2)^{2}[7 / 2]_{4}$ & & & R1c & & \\
\hline $3592.3528(10)$ & 27828.969(8) & 3592.3522(5) & 0.0005 & 770 & & $5 s$ & $(3 / 2)^{2}[3 / 2]_{1}$ & $\mathrm{sp}$ & $\left({ }^{3} \mathrm{~F}\right)^{1} \mathrm{P}^{\circ}{ }^{3} \mathrm{D}^{\circ}{ }_{1}$ & & & R1c & & \\
\hline $3593.2610(10)$ & 27821.935(8) & $3593.2613(4)$ & -0.0002 & 100 & & $4 \mathrm{~d}$ & $(3 / 2)^{2}[3 / 2]_{2}$ & $5 f$ & $(5 / 2)^{2}[5 / 2]^{\circ}{ }_{2}$ & & & R1c & & \\
\hline 3594.1722(18) & 27814.882(14) & 3594.1729(4) & -0.0008 & 130 & & $4 \mathrm{~d}$ & $(3 / 2)^{2}[3 / 2]_{2}$ & $5 f$ & $(5 / 2)^{2}[5 / 2]^{\circ}{ }_{3}$ & & & R1c & & \\
\hline $3597.100(3)$ & $27792.240(21)$ & $3597.1080(5)$ & -0.008 & 51 & & $4 \mathrm{~d}$ & $(3 / 2)^{2}[3 / 2]_{2}$ & $5 \mathrm{f}$ & $(5 / 2)^{2}[3 / 2]^{\circ}{ }_{1}$ & & & R1c & & \\
\hline $3600.4380(11)$ & 27766.477(8) & 3600.4382(4) & -0.0002 & 76 & & $5 p$ & $(5 / 2)^{2}[5 / 2]^{\circ}{ }_{2}$ & $6 \mathrm{~d}$ & $(3 / 2)^{2}[5 / 2]_{2}$ & & & R1c & & \\
\hline $3600.7635(10)$ & $27763.967(7)$ & $3600.7638(4)$ & -0.0002 & 130 & & $4 \mathrm{~d}$ & $(3 / 2)^{2}[3 / 2]_{2}$ & $5 f$ & $(5 / 2)^{2}[3 / 2]^{\circ}{ }_{2}$ & & & R1c & & \\
\hline 3601.9187(19) & 27755.063(15) & 3601.9162(4) & 0.0025 & 200 & & $5 p$ & $(5 / 2)^{2}[5 / 2]^{\circ}{ }_{2}$ & $6 \mathrm{~d}$ & $(3 / 2)^{2}[5 / 2]_{3}$ & & & R1c & & \\
\hline
\end{tabular}


Table A1. Cont.

\begin{tabular}{|c|c|c|c|c|c|c|c|c|c|c|c|c|c|c|}
\hline$\lambda_{\text {obs }}{ }^{a}(\AA)$ & $\sigma_{\mathrm{obs}}{ }^{\mathrm{b}}\left(\mathrm{cm}^{-1}\right)$ & $\lambda_{\text {Ritz }}{ }^{c}(\AA ̊)$ & $\Delta \lambda_{\text {obs-Ritz }}$ & $\begin{array}{c}I_{\text {obs }} \mathrm{d} \\
\text { (arb.u.) }\end{array}$ & Char ${ }^{e}$ & & Lower Level & & pper Level & $A\left(\mathrm{~s}^{-1}\right)$ & $\operatorname{Acc}^{f}$ & Line Ref. $\mathrm{g}$ & TP Ref. $\mathrm{g}$ & Notes ${ }^{h}$ \\
\hline $3602.2331(11)$ & $27752.641(8)$ & $3602.2324(3)$ & 0.0007 & 1200 & & $\mathrm{sp}$ & $\left({ }^{3} \mathrm{~F}\right)^{3} \mathrm{P}^{\circ}{ }^{3} \mathrm{D}^{\circ}{ }_{2}$ & $7 \mathrm{~s}$ & $(5 / 2)^{2}[5 / 2]_{2}$ & & & R1c & & \\
\hline $3606.6576(11)$ & $27718.596(8)$ & $3606.6576(4)$ & 0.0000 & 1900 & & $5 s$ & $(5 / 2)^{2}[5 / 2]_{3}$ & $\mathrm{sp}$ & $\left({ }^{3} \mathrm{~F}\right)^{1} \mathrm{P}^{\circ}{ }^{3} \mathrm{D}^{\circ}{ }_{3}$ & & & $\mathrm{R} 1 \mathrm{c}$ & & \\
\hline 3611.0901(10) & $27684.573(7)$ & 3611.0902(3) & -0.0001 & 370 & & $\mathrm{sp}$ & $\left({ }^{3} \mathrm{~F}\right)^{3} \mathrm{P}^{\circ}{ }^{3} \mathrm{D}^{\circ}{ }_{2}$ & $7 \mathrm{~s}$ & $(5 / 2)^{2}[5 / 2]_{3}$ & & & R1c & & \\
\hline 3611.172 & 27683.94 & 3611.1721(4) & & & : & $5 p$ & $(5 / 2)^{2}[5 / 2]^{\circ}{ }_{2}$ & $6 \mathrm{~d}$ & $(3 / 2)^{2}[3 / 2]_{2}$ & & & & & \\
\hline $3615.0429(20)$ & $27654.303(16)$ & $3615.04192(19)$ & 0.0010 & 25 & & $5 s$ & $(5 / 2)^{2}[5 / 2]_{2}$ & $4 \mathrm{f}$ & $(5 / 2)^{2}[7 / 2]^{\circ} 3$ & & & R1c & & \\
\hline $3616.8636(10)$ & $27640.382(7)$ & $3616.8630(4)$ & 0.0007 & 890 & & $5 p$ & $(5 / 2)^{2}[3 / 2]^{\circ}{ }_{1}$ & $6 \mathrm{~d}$ & $(3 / 2)^{2}[3 / 2]_{2}$ & & & R1c & & \\
\hline $3621.4008(19)$ & 27605.753(15) & $3621.4013(5)$ & -0.0005 & 26 & & $5 p$ & $(5 / 2)^{2}[5 / 2]^{\circ} 2$ & $6 \mathrm{~d}$ & $(3 / 2)^{2}[7 / 2]_{3}$ & & & R1c & & \\
\hline $3621.8942(11)$ & $27601.992(8)$ & $3621.8927(4)$ & 0.0016 & 77 & & $5 p$ & $(5 / 2)^{2}[3 / 2]^{0}{ }_{1}$ & $6 \mathrm{~d}$ & $(3 / 2)^{2}[3 / 2]_{1}$ & & & R1c & & \\
\hline $3626.350(3)$ & $27568.080(21)$ & $3626.3111(9)$ & 0.039 & 77 & ? & $\mathrm{sp}$ & $\left({ }^{3} \mathrm{~F}\right)^{3} \mathrm{P}^{\circ}{ }^{1} \mathrm{G}^{\circ}{ }_{4}$ & $6 \mathrm{~d}$ & $(5 / 2)^{2}[7 / 2]_{4}$ & & & R1c & & $\mathrm{x}$ \\
\hline 3626.973(3) & $27563.340(21)$ & $3626.9734(4)$ & -0.000 & 51 & & $5 p$ & $(5 / 2)^{2}[5 / 2]^{\circ}{ }_{3}$ & $6 \mathrm{~d}$ & $(3 / 2)^{2}[5 / 2]_{2}$ & & & R1c & & \\
\hline $3628.4741(10)$ & $27551.940(8)$ & $3628.4733(4)$ & 0.0008 & 130 & & $5 p$ & $(5 / 2)^{2}[5 / 2]^{\circ}{ }_{3}$ & $6 \mathrm{~d}$ & $(3 / 2)^{2}[5 / 2]_{3}$ & & & $\mathrm{R} 1 \mathrm{c}$ & & \\
\hline $3629.0907(16)$ & $27547.259(12)$ & $3629.0858(5)$ & 0.0049 & 51 & & $5 s$ & $(3 / 2)^{2}[3 / 2]_{2}$ & $\mathrm{sp}$ & $\left({ }^{3} \mathrm{~F}\right)^{1} \mathrm{P}^{\circ}{ }^{3} \mathrm{D}^{\circ}{ }_{1}$ & & & R1c & & \\
\hline 3629.3186(19) & $27545.529(15)$ & $3629.3176(3)$ & 0.0011 & 26 & & $4 \mathrm{~d}$ & $(3 / 2)^{2}[5 / 2]_{3}$ & $5 \mathrm{f}$ & $(5 / 2)^{2}[9 / 2]^{\circ} 4$ & & & R1c & & \\
\hline $3630.0412(11)$ & $27540.046(8)$ & $3630.0408(3)$ & 0.0004 & 100 & & $4 \mathrm{~d}$ & $(3 / 2)^{2}[5 / 2]_{3}$ & $5 f$ & $(5 / 2)^{2}[7 / 2]^{\circ} 4$ & & & $\mathrm{R} 1 \mathrm{c}$ & & \\
\hline $3630.6454(10)$ & $27535.463(7)$ & $3630.6450(4)$ & 0.0004 & 380 & & $\mathrm{sp}$ & $\left({ }^{3} \mathrm{~F}\right)^{3} \mathrm{P}^{\circ}{ }^{3} \mathrm{~F}^{\circ}{ }_{2}$ & $6 \mathrm{~d}$ & $(5 / 2)^{2}[5 / 2]_{2}$ & & & R1c & & \\
\hline $3631.6198(22)$ & 27528.075(17) & $3631.61939(23)$ & 0.0004 & 51 & & $5 s$ & $(5 / 2)^{2}[5 / 2]_{2}$ & $4 \mathrm{f}$ & $(5 / 2)^{2}[1 / 2]^{\circ} 1$ & & & R1c & & \\
\hline $3633.1159(10)$ & $27516.740(8)$ & $3633.1151(4)$ & 0.0008 & 520 & & $\mathrm{sp}$ & $\left({ }^{3} \mathrm{~F}\right)^{3} \mathrm{P}^{\circ}{ }^{3} \mathrm{~F}^{\circ}{ }_{2}$ & $6 \mathrm{~d}$ & $(5 / 2)^{2}[7 / 2]_{3}$ & & & R1c & & \\
\hline $3635.1554(17)$ & 27501.302(13) & $3635.1443(4)$ & 0.0111 & 210 & $\mathrm{bl}$ & $4 \mathrm{~d}$ & $(3 / 2)^{2}[5 / 2]_{3}$ & $5 f$ & $(5 / 2)^{2}[5 / 2]^{\circ} 2$ & & & R1c & & $\mathrm{x}$ \\
\hline 3636.0783(17) & 27494.322(13) & $3636.0773(4)$ & 0.0009 & 77 & & $4 \mathrm{~d}$ & $(3 / 2)^{2}[5 / 2]_{3}$ & $5 f$ & $(5 / 2)^{2}[5 / 2]^{\circ} 3$ & & & R1c & & \\
\hline $3637.867(3)$ & $27480.800(21)$ & $3637.8663(4)$ & 0.001 & 26 & & $5 p$ & $(5 / 2)^{2}[5 / 2]^{0}{ }_{3}$ & $6 \mathrm{~d}$ & $(3 / 2)^{2}[3 / 2]_{2}$ & & & R1c & & \\
\hline $3641.0912(20)$ & $27456.470(15)$ & $3641.0918(5)$ & -0.0007 & 52 & & $\mathrm{sp}$ & $\left({ }^{3} \mathrm{~F}\right)^{3} \mathrm{P}^{\circ}{ }^{3} \mathrm{~F}_{2}^{\circ}$ & $6 \mathrm{~d}$ & $(5 / 2)^{2}[5 / 2]_{3}$ & & & R1c & & \\
\hline 3641.7928(11) & $27451.180(8)$ & $3641.7903(4)$ & 0.0025 & 1000 & & $\mathrm{~s}^{\frac{1}{2}}$ & ${ }^{1} \mathrm{G}_{4}$ & $5 p$ & $(3 / 2)^{2}[5 / 2]^{\circ} 3$ & & & R1c & & \\
\hline $3642.4505(14)$ & 27446.224(11) & $3642.4488(4)$ & 0.0017 & 77 & & $4 \mathrm{~d}$ & $(3 / 2)^{2}[5 / 2]_{2}$ & $5 f$ & $(5 / 2)^{2}[5 / 2]^{\circ} 3$ & & & $\mathrm{R} 1 \mathrm{c}$ & & \\
\hline $3642.8238(22)$ & 27443.411(17) & $3642.8229(4)$ & 0.0009 & 26 & & $4 \mathrm{~d}$ & $(3 / 2)^{2}[5 / 2]_{3}$ & $5 f$ & $(5 / 2)^{2}[3 / 2]^{\circ} 2$ & & & R1c & & \\
\hline $3643.0146(11)$ & $27441.974(8)$ & $3643.0150(5)$ & -0.0004 & 770 & & $5 p$ & $(5 / 2)^{2}[3 / 2]^{\circ}{ }_{1}$ & $6 \mathrm{~d}$ & $(3 / 2)^{2}[1 / 2]_{1}$ & & & R1c & & \\
\hline $3643.7566(10)$ & $27436.386(7)$ & 3643.7571(5) & -0.0005 & 1300 & & $5 p$ & $(5 / 2)^{2}[5 / 2]^{\circ}{ }_{3}$ & $6 \mathrm{~d}$ & $(3 / 2)^{2}[7 / 2]_{4}$ & & & R1c & & \\
\hline $3647.081(4)$ & 27411.38(3) & $3647.0800(10)$ & 0.001 & 160 & & $\mathrm{sp}$ & $\left({ }^{3} \mathrm{~F}\right)^{3} \mathrm{P}^{\circ}{ }^{1} \mathrm{G}^{\circ}{ }_{4}$ & $6 \mathrm{~d}$ & $(5 / 2)^{2}[9 / 2]_{5}$ & & & R1c & & \\
\hline $3648.2463(20)$ & 27402.622(15) & $3648.2475(5)$ & -0.0011 & 52 & & $5 p$ & $(5 / 2)^{2}[5 / 2]^{\circ}{ }_{3}$ & $6 \mathrm{~d}$ & $(3 / 2)^{2}[7 / 2]_{3}$ & & & R1c & & \\
\hline $3648.8875(15)$ & 27397.807(11) & $3648.8850(4)$ & 0.0025 & 390 & & $5 \mathrm{~s}$ & $(5 / 2)^{2}[5 / 2]_{2}$ & $\mathrm{sp}$ & $\left({ }^{3} \mathrm{~F}\right)^{1} \mathrm{P}^{\circ}{ }^{3} \mathrm{D}^{\circ}{ }_{3}$ & & & R1c & & \\
\hline $3649.2205(22)$ & 27395.307(17) & $3649.2180(4)$ & 0.0025 & 52 & & $4 d$ & $(3 / 2)^{2}[5 / 2]_{2}$ & $5 f$ & $(5 / 2)^{2}[3 / 2]^{\circ} 2$ & & & R1c & & \\
\hline $3651.800(3)$ & $27375.960(21)$ & 3651.7990(5) & 0.001 & 100 & & $\mathrm{sp}$ & $\left({ }^{3} \mathrm{~F}\right)^{3} \mathrm{P}^{\circ}{ }^{3} \mathrm{~F}_{2}^{\circ}$ & $6 \mathrm{~d}$ & $(5 / 2)^{2}[3 / 2]_{2}$ & & & R1c & & \\
\hline $3664.2913(10)$ & $27282.636(8)$ & $3664.2900(5)$ & 0.0014 & 130 & & $5 \mathrm{~s}$ & $(3 / 2)^{2}[3 / 2]_{2}$ & $\mathrm{sp}$ & $\left({ }^{3} \mathrm{P}\right)^{3} \mathrm{P}^{\circ}{ }^{1} \mathrm{D}^{\circ}{ }_{2}$ & & & R1c & & \\
\hline $3678.6688(20)$ & $27176.009(15)$ & $3678.6702(6)$ & -0.0014 & 80 & & $\mathrm{sp}$ & $\left({ }^{3} \mathrm{~F}\right)^{3} \mathrm{P}^{\circ}{ }^{3} \mathrm{~F}^{\circ}{ }_{2}$ & $6 \mathrm{~d}$ & $(5 / 2)^{2}[1 / 2]_{1}$ & & & R1c & & \\
\hline $3682.4238(14)$ & 27148.298(11) & $3682.4254(7)$ & -0.0016 & 3900 & & $\mathrm{sp}$ & $\left({ }^{3} \mathrm{~F}\right)^{3} \mathrm{P}^{\circ}{ }^{3} \mathrm{~F}_{4}^{\circ}$ & $7 \mathrm{~s}$ & $(5 / 2)^{2}[5 / 2]_{3}$ & & & R1c & & \\
\hline $3686.5552(10)$ & $27117.875(8)$ & $3686.5539(4)$ & 0.0013 & 16000 & & $4 p$ & ${ }^{3} \mathrm{~F}_{3}^{\circ}$ & $s^{2}$ & ${ }^{1} \mathrm{G}_{4}$ & $9.7 \mathrm{e}+05$ & $\mathrm{C}+$ & R1c & $\mathrm{O} 07$ & \\
\hline $3693.3279(12)$ & $27068.148(9)$ & 3693.3271(10) & 0.0009 & 81 & & $4 d$ & $(3 / 2)^{2}[1 / 2]_{0}$ & $7 p$ & $(3 / 2)^{2}[1 / 2]^{\circ} 1$ & & & $\mathrm{R} 2 \mathrm{nc}$ & & \\
\hline 3703.9305(11) & $26990.667(8)$ & $3703.9285(5)$ & 0.0020 & 680 & & $\mathrm{sp}$ & $\left({ }^{3} \mathrm{~F}\right)^{3} \mathrm{P}^{0}{ }^{1} \mathrm{~F}^{0} 3$ & $6 \mathrm{~d}$ & $(3 / 2)^{2}[7 / 2]_{4}$ & & & R1c & & \\
\hline 3724.1764(11) & $26843.940(8)$ & $3724.1770(3)$ & -0.0006 & 390 & & $5 p$ & $(5 / 2)^{2}[3 / 2]^{\circ}{ }_{2}$ & $7 \mathrm{~s}$ & $(3 / 2)^{2}[3 / 2]_{2}$ & & & R1c & & \\
\hline $3725.4537(17)$ & 26834.737(12) & $3725.4544(4)$ & -0.0007 & 110 & & $4 p$ & ${ }^{3} \mathrm{~F}_{4}^{\circ}$ & $s^{2}$ & ${ }^{1} \mathrm{G}_{4}$ & & & R1c & & \\
\hline $3728.6574(10)$ & $26811.681(7)$ & $3728.6569(4)$ & 0.0005 & 280 & & $\mathrm{sp}$ & $\left({ }^{3} \mathrm{~F}\right)^{3} \mathrm{P}^{\circ}{ }^{3} \mathrm{D}^{\circ}{ }_{1}$ & $7 \mathrm{~s}$ & $(5 / 2)^{2}[5 / 2]_{2}$ & & & $\mathrm{R} 1 \mathrm{c}$ & & \\
\hline $3730.3433(10)$ & $26799.564(7)$ & $3730.3437(5)$ & -0.0005 & 250 & & $5 s$ & $(5 / 2)^{2}[5 / 2]_{2}$ & $\mathrm{sp}$ & $\left({ }^{3} \mathrm{P}\right)^{3} \mathrm{P}^{\circ}{ }^{3} \mathrm{P}^{\circ}{ }_{1}$ & & & R1c & & \\
\hline 3731.1462(13) & 26793.797(9) & $3731.1479(3)$ & -0.0018 & 170 & & $5 p$ & $(5 / 2)^{2}[3 / 2]^{\circ} 2$ & $7 \mathrm{~s}$ & $(3 / 2)^{2}[3 / 2]_{1}$ & & & R1c & & \\
\hline
\end{tabular}


Table A1. Cont.

\begin{tabular}{|c|c|c|c|c|c|c|c|c|c|c|c|c|c|c|}
\hline$\lambda_{\text {obs }}{ }^{\text {a }}(\AA)$ & $\sigma_{\mathrm{obs}}^{\mathrm{b}}\left(\mathrm{cm}^{-1}\right)$ & $\lambda_{\text {Ritz }}{ }^{c}(\AA ̊)$ & $\begin{array}{c}\Delta \lambda_{\text {obs-Ritz }} \\
\text { (A) }\end{array}$ & $\begin{array}{c}I_{\text {obs }} \mathrm{d} \\
\text { (arb. u.) }\end{array}$ & Char $^{e}$ & & pwer Level & & pper Level & $A\left(\mathrm{~s}^{-1}\right)$ & $\operatorname{Acc}^{f}$ & Line Ref. $\mathrm{g}$ & TP Ref. $\mathrm{g}$ & Notes ${ }^{h}$ \\
\hline $3738.6488(10)$ & $26740.029(7)$ & 3738.6478(3) & 0.0010 & 1700 & & $\mathrm{sp}$ & $\left({ }^{3} \mathrm{~F}\right)^{3} \mathrm{P}^{\circ}{ }^{3} \mathrm{~F}_{3}^{\circ}$ & $7 \mathrm{~s}$ & $(5 / 2)^{2}[5 / 2]_{2}$ & & & R1c & & \\
\hline 3740.3281(11) & $26728.024(8)$ & $3740.3278(4)$ & 0.0003 & 280 & & $5 s$ & $(5 / 2)^{2}[5 / 2]_{3}$ & $\mathrm{sp}$ & $\left({ }^{3} \mathrm{~F}\right)^{1} \mathrm{P}^{\circ}{ }^{3} \mathrm{~F}^{\circ}{ }_{4}$ & & & R1c & & \\
\hline 3742.0597(10) & $26715.656(7)$ & 3742.0592(4) & 0.0006 & 430 & & $5 s$ & $(3 / 2)^{2}[3 / 2]_{1}$ & $\mathrm{sp}$ & $\left({ }^{3} \mathrm{~F}\right)^{1} \mathrm{P}^{\circ}{ }^{3} \mathrm{D}_{2}^{\circ}{ }_{2}$ & & & R1c & & \\
\hline 3748.1909(10) & $26671.956(7)$ & 3748.1901(3) & 0.0008 & 1800 & & $\mathrm{sp}$ & $\left({ }^{3} \mathrm{~F}\right)^{3} \mathrm{P}^{\circ}{ }^{3} \mathrm{~F}^{\circ}{ }_{3}$ & $7 \mathrm{~s}$ & $(5 / 2)^{2}[5 / 2]_{3}$ & & & R1c & & \\
\hline 3749.782(10) & $26660.64(7)$ & 3749.7756(4) & 0.006 & 400 & * & $5 s$ & $(5 / 2)^{2}[5 / 2]_{3}$ & $\mathrm{sp}$ & $\left({ }^{3} \mathrm{P}\right)^{3} \mathrm{P}^{\circ}{ }^{3} \mathrm{D}^{\circ}{ }_{2}$ & & & R1c & & \\
\hline $3749.782(10)$ & $26660.64(7)$ & $3749.7826(5)$ & -0.001 & 400 & * & $\mathrm{sp}$ & $\left({ }^{3} \mathrm{~F}\right)^{3} \mathrm{P}^{\circ}{ }^{1} \mathrm{D}^{\circ}{ }_{2}$ & $6 \mathrm{~d}$ & $(3 / 2)^{2}[5 / 2]_{2}$ & & & R1c & & \\
\hline 3751.3859(10) & 26649.241(7) & 3751.3858(4) & 0.0001 & 310 & & $\mathrm{sp}$ & $\left({ }^{3} \mathrm{~F}\right)^{3} \mathrm{P}^{\circ}{ }^{1} \mathrm{D}^{\circ}{ }_{2}$ & $6 \mathrm{~d}$ & $(3 / 2)^{2}[5 / 2]_{3}$ & & & R1c & & \\
\hline $3761.4270(14)$ & $26578.103(10)$ & $3761.4269(5)$ & 0.0001 & 150 & & $\mathrm{sp}$ & $\left({ }^{3} \mathrm{~F}\right)^{3} \mathrm{P}^{\circ}{ }^{1} \mathrm{D}^{\circ}{ }_{2}$ & $6 \mathrm{~d}$ & $(3 / 2)^{2}[3 / 2]_{2}$ & & & R1c & & \\
\hline 3766.8662(14) & 26539.726(10) & $3766.8669(5)$ & -0.0007 & 230 & & $\mathrm{sp}$ & $\left({ }^{3} \mathrm{~F}\right)^{3} \mathrm{P}^{\circ}{ }^{1} \mathrm{D}^{\circ}{ }_{2}$ & $6 \mathrm{~d}$ & $(3 / 2)^{2}[3 / 2]_{1}$ & & & R1c & & \\
\hline 3772.5256(10) & $26499.913(7)$ & 3772.5262(5) & -0.0006 & 480 & & $\mathrm{sp}$ & $\left({ }^{3} \mathrm{~F}\right)^{3} \mathrm{P}^{\circ}{ }^{1} \mathrm{D}^{\circ}{ }_{2}$ & $6 \mathrm{~d}$ & $(3 / 2)^{2}[7 / 2]_{3}$ & & & R1c & & \\
\hline $3774.9743(20)$ & $26482.724(14)$ & 3774.9751(4) & -0.0008 & 59 & & $5 p$ & $(5 / 2)^{2}[3 / 2]^{\circ} 2$ & $6 \mathrm{~d}$ & $(5 / 2)^{2}[5 / 2]_{2}$ & & & R1c & & \\
\hline 3777.6444(10) & $26464.006(7)$ & 3777.6455(3) & -0.0011 & 150 & & $5 p$ & $(5 / 2)^{2}[3 / 2]^{0}{ }_{2}$ & $6 \mathrm{~d}$ & $(5 / 2)^{2}[7 / 2]_{3}$ & & & R1c & & \\
\hline 3781.9355(12) & 26433.980(9) & 3781.9350(4) & 0.0005 & 290 & & $5 s$ & $(3 / 2)^{2}[3 / 2]_{2}$ & $\mathrm{sp}$ & $\left({ }^{3} \mathrm{~F}\right)^{1} \mathrm{P}^{\circ}{ }^{3} \mathrm{D}^{\circ}{ }_{2}$ & & & R1c & & \\
\hline $3786.2696(10)$ & 26403.721(7) & 3786.2702(5) & -0.0006 & 3200 & & $5 p$ & $(5 / 2)^{2}[3 / 2]^{\circ} 2$ & $6 \mathrm{~d}$ & $(5 / 2)^{2}[5 / 2]_{3}$ & & & R1c & & \\
\hline 3795.4413(11) & 26339.919(8) & 3795.4419(4) & -0.0006 & 450 & & $5 s$ & $(5 / 2)^{2}[5 / 2]_{2}$ & $\mathrm{sp}$ & $\left({ }^{3} \mathrm{P}\right)^{3} \mathrm{P}^{\circ}{ }^{3} \mathrm{D}^{\circ}{ }_{2}$ & & & R1c & & \\
\hline 3796.0675(12) & 26335.574(8) & 3796.0687(4) & -0.0012 & 580 & & $5 p$ & $(5 / 2)^{2}[3 / 2]^{\circ} 2$ & $6 \mathrm{~d}$ & $(5 / 2)^{2}[3 / 2]_{1}$ & & & R1c & & \\
\hline 3796.1694(12) & $26334.867(9)$ & 3796.1694(11) & -0.0000 & 820 & & $5 p$ & $(3 / 2)^{2}[1 / 2]_{1}^{\circ}$ & $6 \mathrm{~d}$ & $(3 / 2)^{2}[1 / 2]_{0}$ & & & R1c & & \\
\hline 3797.8489(10) & 26323.221(7) & $3797.8496(5)$ & -0.0007 & 4200 & & $5 p$ & $(5 / 2)^{2}[3 / 2]^{\circ}{ }_{2}$ & $6 \mathrm{~d}$ & $(5 / 2)^{2}[3 / 2]_{2}$ & & & R1c & & \\
\hline 3801.5563(10) & 26297.551(7) & 3801.5556(5) & 0.0007 & 670 & & $5 p$ & $(3 / 2)^{2}[1 / 2]_{0}^{\circ}$ & $6 \mathrm{~d}$ & $(3 / 2)^{2}[3 / 2]_{1}$ & & & R1c & & \\
\hline $3806.27(5)$ & $26265.0(3)$ & $3806.333(3)$ & -0.06 & 7400 & & $\mathrm{~d}^{10}$ & ${ }^{1} S_{0}$ & $4 \mathrm{~s}$ & ${ }^{1} \mathrm{D}_{2}$ & $1.58 \mathrm{e}+00$ & $\mathrm{C}+$ & T53,M97,P84 & P84 & $\mathrm{X}, \mathrm{E} 2$ \\
\hline 3818.8787(11) & $26178.268(7)$ & 3818.8793(8) & -0.0006 & 2000 & & $5 p$ & $(5 / 2)^{2}[3 / 2]^{\circ}{ }_{1}$ & $6 \mathrm{~d}$ & $(5 / 2)^{2}[1 / 2]_{0}$ & & & R1c & & \\
\hline 3824.8323(11) & 26137.521(8) & 3824.8321(6) & 0.0002 & 1000 & & $5 p$ & $(3 / 2)^{2}[1 / 2]^{\circ} 0$ & $6 \mathrm{~d}$ & $(3 / 2)^{2}[1 / 2]_{1}$ & & & R1c & & \\
\hline $3826.9209(10)$ & $26123.256(7)$ & $3826.9216(6)$ & -0.0007 & 3000 & & $5 p$ & $(5 / 2)^{2}[3 / 2]^{\circ}{ }_{2}$ & $6 \mathrm{~d}$ & $(5 / 2)^{2}[1 / 2]_{1}$ & & & R1c & & \\
\hline $3836.1646(11)$ & $26060.310(7)$ & $3836.1655(3)$ & -0.0010 & 1800 & & $5 p$ & $(5 / 2)^{2}[5 / 2]^{\circ}{ }_{2}$ & $7 \mathrm{~s}$ & $(3 / 2)^{2}[3 / 2]_{2}$ & & & R1c & & \\
\hline 3841.4818(11) & $26024.240(7)$ & 3841.4819(4) & -0.0001 & 590 & & $5 s$ & $(5 / 2)^{2}[5 / 2]_{2}$ & $\mathrm{sp}$ & $\left({ }^{3} \mathrm{P}\right)^{3} \mathrm{P}^{\circ}{ }^{3} \mathrm{D}^{\circ}{ }_{1}$ & & & R1c & & \\
\hline $3842.5889(12)$ & $26016.742(8)$ & 3842.5882(3) & 0.0007 & 2000 & & $5 p$ & $(5 / 2)^{2}[3 / 2]^{\circ}{ }_{1}$ & $7 \mathrm{~s}$ & $(3 / 2)^{2}[3 / 2]_{2}$ & & & R1c & & \\
\hline $3843.5628(13)$ & $26010.150(9)$ & $3843.5624(3)$ & 0.0004 & 150 & & $5 p$ & $(5 / 2)^{2}[5 / 2]^{\circ}{ }_{2}$ & $7 \mathrm{~s}$ & $(3 / 2)^{2}[3 / 2]_{1}$ & & & R1c & & \\
\hline 3849.5824(13) & 25969.479(9) & 3849.5810(5) & 0.0014 & 1700 & & $5 s$ & $(5 / 2)^{2}[5 / 2]_{3}$ & $\mathrm{sp}$ & $\left({ }^{3} \mathrm{P}\right)^{3} \mathrm{P}^{0}{ }^{3} \mathrm{D}^{\circ}{ }_{3}$ & & & R1c & & \\
\hline $3850.0092(15)$ & $25966.600(10)$ & 3850.0099(3) & -0.0007 & 1600 & & $5 p$ & $(5 / 2)^{2}[3 / 2]^{\circ} 1$ & $7 \mathrm{~s}$ & $(3 / 2)^{2}[3 / 2]_{1}$ & & & R1c & & \\
\hline 3851.1050(11) & $25959.212(7)$ & $3851.1020(4)$ & 0.0030 & 890 & & $s^{2}$ & ${ }^{1} \mathrm{G}_{4}$ & $\mathrm{sp}$ & $\left({ }^{3} \mathrm{~F}\right)^{3} \mathrm{P}^{\circ}{ }^{1} \mathrm{~F}_{3}^{\circ}{ }_{3}$ & & & R1c & & \\
\hline $3859.41(6)$ & $25903.4(4)$ & 3859.4330(7) & -0.02 & 180 & & $4 \mathrm{~d}$ & $(5 / 2)^{2}[3 / 2]_{2}$ & $6 \mathrm{p}$ & $(3 / 2)^{2}[3 / 2]_{2}^{\circ}$ & & & S36c & & \\
\hline 3861.3421(11) & $25890.391(8)$ & $3861.3424(4)$ & -0.0004 & 1300 & & $5 p$ & $(5 / 2)^{2}[7 / 2]^{\circ}{ }_{3}$ & $6 \mathrm{~d}$ & $(5 / 2)^{2}[5 / 2]_{2}$ & & & R1c & & \\
\hline 3862.1245(11) & 25885.146(8) & 3862.1243(4) & 0.0002 & 1200 & & $5 p$ & $(3 / 2)^{2}[5 / 2]^{\circ} 2$ & $6 \mathrm{~d}$ & $(3 / 2)^{2}[5 / 2]_{3}$ & & & R1c & & \\
\hline 3863.6403(11) & 25874.991(8) & 3863.6404(4) & -0.0001 & 1200 & & $5 p$ & $(5 / 2)^{2}[7 / 2]^{\circ}{ }_{3}$ & $6 \mathrm{~d}$ & $(5 / 2)^{2}[7 / 2]_{4}$ & & & R1c & & \\
\hline 3864.1370(11) & $25871.665(7)$ & $3864.1365(4)$ & 0.0005 & 3200 & & $5 p$ & $(5 / 2)^{2}[7 / 2]^{\circ}{ }_{3}^{\circ}$ & $6 \mathrm{~d}$ & $(5 / 2)^{2}[7 / 2]_{3}$ & & & R1c & & \\
\hline 3866.3047(11) & 25857.160(7) & 3866.3035(3) & 0.0013 & 1200 & & $5 p$ & $(5 / 2)^{2}[5 / 2]^{\circ}{ }_{3}$ & $7 \mathrm{~s}$ & $(3 / 2)^{2}[3 / 2]_{2}$ & & & R1c & & \\
\hline 3868.3711(11) & $25843.348(7)$ & 3868.3707(3) & 0.0004 & 1100 & & $\mathrm{sp}$ & $\left({ }^{3} \mathrm{~F}\right)^{3} \mathrm{P}^{\mathrm{o}} \mathrm{F}^{3}{ }^{\circ}{ }_{2}$ & $7 \mathrm{~s}$ & $(5 / 2)^{2}[5 / 2]_{2}$ & & & R1c & & \\
\hline $3871.334(3)$ & $25823.570(21)$ & 3871.3321(10) & 0.002 & 210 & & $\mathrm{sp}$ & $\left({ }^{3} \mathrm{~F}\right)^{3} \mathrm{P}^{\circ} 1^{1} \mathrm{G}_{4}^{\circ}$ & $7 \mathrm{~s}$ & $(5 / 2)^{2}[5 / 2]_{3}$ & & & R1c & & \\
\hline $3872.7645(15)$ & $25814.031(10)$ & 3872.7678(5) & -0.0033 & 550 & & $5 p$ & $(3 / 2)^{2}[5 / 2]^{0}{ }_{2}$ & $6 \mathrm{~d}$ & $(3 / 2)^{2}[3 / 2]_{2}$ & & & R1c & & \\
\hline 3873.2075(11) & $25811.078(7)$ & 3873.2058(6) & 0.0017 & 940 & & $5 s$ & $(5 / 2)^{2}[5 / 2]_{3}$ & $\mathrm{sp}$ & $\left({ }^{3} \mathrm{P}\right)^{3} \mathrm{P}^{\circ}{ }^{3} \mathrm{P}^{\circ}{ }_{2}$ & & & R1c & & \\
\hline 3878.5873(12) & $25775.278(8)$ & 3878.5875(3) & -0.0002 & 860 & & $\mathrm{sp}$ & $\left({ }^{3} \mathrm{~F}\right)^{3} \mathrm{P}^{\circ}{ }^{3} \mathrm{~F}_{2}^{\circ}$ & $7 \mathrm{~s}$ & $(5 / 2)^{2}[5 / 2]_{3}$ & & & R1c & & \\
\hline
\end{tabular}


Table A1. Cont.

\begin{tabular}{|c|c|c|c|c|c|c|c|c|c|c|c|c|c|c|}
\hline$\lambda_{\text {obs }}{ }^{\text {a }}(\AA)$ & $\sigma_{\mathrm{obs}}{ }^{\mathrm{b}}\left(\mathrm{cm}^{-1}\right)$ & $\lambda_{\text {Ritz }}{ }^{\mathrm{c}}(\AA)$ & $\begin{array}{c}\Delta \lambda_{\text {obs-Ritz }} \\
\text { (A) }\end{array}$ & $\begin{array}{c}I_{\text {obs }} \mathrm{d} \\
\text { (arb. u.) }\end{array}$ & Char ${ }^{e}$ & & ower Level & & pper Level & $A\left(\mathrm{~s}^{-1}\right)$ & $\operatorname{Acc}^{f}$ & Line Ref. $\mathrm{g}$ & TP Ref. $\mathrm{g}$ & Notes $h$ \\
\hline 3878.667 & 25774.75 & $3878.6670(5)$ & & & : & $5 p$ & $(3 / 2)^{2}[1 / 2]^{\circ}{ }_{1}$ & $6 \mathrm{~d}$ & $(3 / 2)^{2}[5 / 2]_{2}$ & & & & & \\
\hline 3879.3966(11) & $25769.901(7)$ & $3879.3976(4)$ & -0.0010 & 3800 & & $5 p$ & $(5 / 2)^{2}[7 / 2]^{\circ} 4$ & $6 \mathrm{~d}$ & $(5 / 2)^{2}[7 / 2]_{4}$ & & & R1c & & \\
\hline 3879.8973(21) & $25766.576(14)$ & 3879.8977(4) & -0.0005 & 140 & & $5 p$ & $(5 / 2)^{2}[7 / 2]^{\circ} 4$ & $6 \mathrm{~d}$ & $(5 / 2)^{2}[7 / 2]_{3}$ & & & R1c & & \\
\hline $3884.1312(9)$ & $25738.489(6)$ & $3884.1313(5)$ & -0.0001 & 8100 & & $5 p$ & $(5 / 2)^{2}[7 / 2]^{\circ}{ }_{3}$ & $6 \mathrm{~d}$ & $(5 / 2)^{2}[9 / 2]_{4}$ & $3.1 \mathrm{e}+07$ & $\mathrm{D}+$ & F_Re & TW & \\
\hline 3884.5339(11) & $25735.821(7)$ & $3884.5350(5)$ & -0.0010 & 3800 & & $5 p$ & $(3 / 2)^{2}[5 / 2]^{\circ}{ }_{2}$ & $6 \mathrm{~d}$ & $(3 / 2)^{2}[7 / 2]_{3}$ & $3.1 \mathrm{e}+07$ & $\mathrm{D}+$ & R1c & TW & \\
\hline $3885.2865(17)$ & $25730.836(11)$ & $3885.2789(5)$ & 0.0076 & 85 & $\mathrm{bl}$ & $5 p$ & $(5 / 2)^{2}[7 / 2]^{\circ}{ }_{3}$ & $6 \mathrm{~d}$ & $(5 / 2)^{2}[3 / 2]_{2}$ & & & R1c & & $\mathrm{x}$ \\
\hline $3890.0864(14)$ & $25699.088(9)$ & $3890.0865(4)$ & -0.0001 & 2200 & & $5 p$ & $(5 / 2)^{2}[5 / 2]^{\circ}{ }_{2}$ & $6 \mathrm{~d}$ & $\begin{array}{l}(5 / 2)^{2}[5 / 2]_{2} \\
\text {. }\end{array}$ & & & $\mathrm{R} 1 \mathrm{c}$ & & \\
\hline $3891.1277(12)$ & $25692.211(8)$ & $3891.1267(5)$ & 0.0010 & 1500 & & $5 p$ & $(3 / 2)^{2}[1 / 2]^{\circ}{ }_{1}$ & $6 \mathrm{~d}$ & $(3 / 2)^{2}[3 / 2]_{2}$ & & & R1c & & \\
\hline 3892.9237(11) & $25680.358(7)$ & $3892.9223(4)$ & 0.0014 & 3000 & & $5 p$ & $(5 / 2)^{2}[5 / 2]^{\circ} 2$ & $6 \mathrm{~d}$ & $(5 / 2)^{2}[7 / 2]_{3}$ & & & $\mathrm{R} 1 \mathrm{c}$ & & \\
\hline $3894.6198(21)$ & $25669.175(14)$ & $3894.61915(19)$ & 0.0006 & 280 & & $5 s$ & $(3 / 2)^{2}[3 / 2]_{2}$ & $4 \mathrm{f}$ & $(5 / 2)^{2}[5 / 2]^{\circ}{ }^{\circ}$ & & & R1c & & \\
\hline 3896.6915(11) & $25655.527(7)$ & $3896.6912(4)$ & 0.0003 & 1900 & & $5 p$ & $(5 / 2)^{2}[3 / 2]^{\circ}{ }_{1}$ & $6 \mathrm{~d}$ & $(5 / 2)^{2}[5 / 2]_{2}$ & & & R1c & & \\
\hline 3896.9491(20) & 25653.832(13) & $3896.9487(5)$ & 0.0004 & 280 & & $5 p$ & $(3 / 2)^{2}[1 / 2]^{\circ}{ }_{1}$ & $6 \mathrm{~d}$ & $(3 / 2)^{2}[3 / 2]_{1}$ & & & R1c & & \\
\hline $3897.7292(20)$ & $25648.698(13)$ & $3897.7262(5)$ & 0.0030 & 2500 & & $5 s$ & $(5 / 2)^{2}[5 / 2]_{2}$ & $\mathrm{sp}$ & $\left({ }^{3} \mathrm{P}\right)^{3} \mathrm{P}^{\circ}{ }^{3} \mathrm{D}^{\circ}{ }_{3}$ & & & R1c & & \\
\hline $3900.0557(11)$ & $25633.397(7)$ & $3900.0565(5)$ & -0.0008 & 1100 & & $5 p$ & $(5 / 2)^{2}[7 / 2]^{\circ}{ }_{4}$ & $6 \mathrm{~d}$ & $(5 / 2)^{2}[9 / 2]_{4}$ & & & R1c & & \\
\hline 3901.2315(12) & $25625.672(8)$ & $3901.2316(5)$ & -0.0000 & 280 & & $5 p$ & $(3 / 2)^{2}[5 / 2]^{\circ}{ }_{3}$ & $6 \mathrm{~d}$ & $(3 / 2)^{2}[5 / 2]_{2}$ & & & $\mathrm{R} 1 \mathrm{c}$ & & \\
\hline $3902.0837(24)$ & $25620.076(16)$ & $3902.0821(5)$ & 0.0016 & 140 & & $5 p$ & $(5 / 2)^{2}[5 / 2]^{\circ}{ }_{2}$ & $6 \mathrm{~d}$ & $(5 / 2)^{2}[5 / 2]_{3}$ & & & $\mathrm{R} 1 \mathrm{c}$ & & \\
\hline $3902.9667(12)$ & $25614.280(8)$ & $3902.9668(4)$ & -0.0002 & 2500 & & $5 p$ & $(3 / 2)^{2}[5 / 2]^{\circ}{ }_{3}$ & $6 \mathrm{~d}$ & $(3 / 2)^{2}[5 / 2]_{3}$ & & & R1c & & \\
\hline $3903.1761(7)$ & $25612.905(5)$ & $3903.1759(5)$ & 0.0002 & 8200 & & $5 p$ & $(5 / 2)^{2}[7 / 2]^{\circ} 4$ & $6 \mathrm{~d}$ & $(5 / 2)^{2}[9 / 2]_{5}$ & $3.5 \mathrm{e}+07$ & $\mathrm{D}+$ & F_Re & TW & \\
\hline $3905.1121(14)$ & $25600.208(9)$ & 3905.1133(6) & -0.0012 & 830 & & $4 \mathrm{~d}$ & $(3 / 2)^{2}[1 / 2]_{0}$ & $5 f$ & $(3 / 2)^{2}[3 / 2]^{0}{ }_{1}$ & & & $\mathrm{R} 1 \mathrm{c}$ & & \\
\hline $3907.2743(24)$ & $25586.042(16)$ & $3907.2616(8)$ & 0.0127 & 82 & ? & $5 s$ & $(3 / 2)^{2}[3 / 2]_{2}$ & $\mathrm{sp}$ & $\left({ }^{3} \mathrm{P}\right)^{3} \mathrm{P}^{\mathrm{o}}{ }^{5} \mathrm{~S}^{\circ}{ }_{2}$ & & & R1c & & $\mathrm{x}$ \\
\hline 3912.4911(12) & $25551.927(8)$ & $3912.4900(5)$ & 0.0011 & 690 & & $5 p$ & $(5 / 2)^{2}[5 / 2]^{\circ} 2$ & $6 \mathrm{~d}$ & $(5 / 2)^{2}[3 / 2]_{1}$ & & & $\mathrm{R} 1 \mathrm{c}$ & & \\
\hline 3913.8355(14) & $25543.150(9)$ & 3913.8369(5) & -0.0014 & 160 & & $5 p$ & $(3 / 2)^{2}[5 / 2]^{\circ}{ }_{3}$ & $6 \mathrm{~d}$ & $(3 / 2)^{2}[3 / 2]_{2}$ & & & R1c & & \\
\hline 3914.311(3) & $25540.050(21)$ & 3914.3081(9) & 0.002 & 110 & $\mathrm{bl}$ & $4 \mathrm{~d}$ & $(5 / 2)^{2}[9 / 2]_{4}$ & $6 p$ & $(3 / 2)^{2}[5 / 2]^{\circ} 3$ & & & R1c & & \\
\hline $3918.3817(12)$ & $25513.515(8)$ & 3918.3792(4) & 0.0025 & 400 & & $\mathrm{~s}^{2}$ & ${ }^{1} \mathrm{G}_{4}$ & $5 p$ & $(5 / 2)^{2}[5 / 2]^{\circ} 3$ & & & R1c & & \\
\hline 3919.1725(11) & $25508.366(7)$ & 3919.1710(4) & 0.0016 & 1000 & & $5 p$ & $(5 / 2)^{2}[3 / 2]^{\circ}$ & $6 \mathrm{~d}$ & $(5 / 2)^{2}[3 / 2]_{1}$ & & & R1c & & \\
\hline $3920.6546(11)$ & $25498.724(7)$ & $3920.6561(5)$ & -0.0015 & 4000 & & $5 p$ & $(3 / 2)^{2}[5 / 2]^{\circ} 3$ & $6 \mathrm{~d}$ & $(3 / 2)^{2}[7 / 2]_{4}$ & $3.3 e+07$ & D+ & R1c & TW & \\
\hline $3921.071(10)$ & $25496.02(7)$ & $3921.0693(5)$ & 0.002 & 130 & * & $5 p$ & $(5 / 2)^{2}[3 / 2]^{\circ}{ }_{1}$ & $6 \mathrm{~d}$ & $(5 / 2)^{2}[3 / 2]_{2}$ & & & $\mathrm{R} 1 \mathrm{c}$ & & \\
\hline 3921.071(10) & 25496.02(7) & 3921.0811(4) & -0.010 & 130 & * & $5 p$ & $(5 / 2)^{2}[5 / 2]^{\circ}{ }_{3}$ & $6 \mathrm{~d}$ & $(5 / 2)^{2}[5 / 2]_{2}$ & & & R1c & & \\
\hline 3921.4136(15) & $25493.789(10)$ & $3921.4117(6)$ & 0.0019 & 450 & & $5 p$ & $(3 / 2)^{2}[1 / 2]^{\circ} 1$ & $6 \mathrm{~d}$ & $(3 / 2)^{2}[1 / 2]_{1}$ & & & R1c & & \\
\hline 3923.4504(11) & $25480.555(7)$ & $3923.4506(5)$ & -0.0003 & 2100 & & $5 p$ & $(5 / 2)^{2}[5 / 2]^{\circ}{ }_{3}$ & $6 \mathrm{~d}$ & $(5 / 2)^{2}[7 / 2]_{4}$ & & & R1c & & \\
\hline $3923.9630(12)$ & 25477.226(8) & 3923.9622(4) & 0.0008 & 400 & & $5 p$ & $(5 / 2)^{2}[5 / 2]^{\circ}{ }_{3}$ & $6 \mathrm{~d}$ & $\begin{array}{l}(5 / 2)^{2}[7 / 2]_{3} \\
\text {. }\end{array}$ & & & R1c & & \\
\hline 3925.8560(11) & $25464.942(7)$ & $3925.8554(5)$ & 0.0006 & 390 & & $5 p$ & $(3 / 2)^{2}[5 / 2]^{\circ} 3$ & $6 \mathrm{~d}$ & $(3 / 2)^{2}[7 / 2]_{3}$ & & & R1c & & \\
\hline 3933.2684(11) & $25416.953(7)$ & $3933.2688(5)$ & -0.0004 & 2000 & & $5 p$ & $(5 / 2)^{2}[5 / 2]^{\circ}{ }_{3}$ & $6 \mathrm{~d}$ & $(5 / 2)^{2}[5 / 2]_{3}$ & & & $\mathrm{R} 1 \mathrm{c}$ & & \\
\hline 3934.1202(11) & 25411.450(7) & $3934.1175(3)$ & 0.0027 & 470 & & $\mathrm{sp}$ & $\left({ }^{3} \mathrm{~F}\right)^{3} \mathrm{P}^{\circ} 1^{1} \mathrm{~F}^{\circ}{ }_{3}$ & $7 \mathrm{~s}$ & $(3 / 2)^{2}[3 / 2]_{2}$ & & & R1c & & \\
\hline $3935.9626(14)$ & 25399.555(9) & $3935.9560(6)$ & 0.0067 & 380 & $\mathrm{bl}$ & $5 \mathrm{~s}$ & $(3 / 2)^{2}[3 / 2]_{1}$ & $\mathrm{sp}$ & $\left({ }^{3} \mathrm{P}\right)^{3} \mathrm{P}^{\circ}{ }^{3} \mathrm{P}^{\circ}{ }_{0}$ & & & R1c & & $\mathrm{x}$ \\
\hline $3940.9702(12)$ & $25367.282(8)$ & $3940.9707(5)$ & -0.0004 & 300 & & $5 s$ & $(3 / 2)^{2}[3 / 2]_{2}$ & $\mathrm{sp}$ & $\left({ }^{3} \mathrm{~F}\right)^{1} \mathrm{P}^{\circ}{ }^{3} \mathrm{D}^{\circ}{ }_{3}$ & & & R1c & & \\
\hline $3944.5822(12)$ & 25344.054(8) & $3944.5826(5)$ & -0.0004 & 490 & & $5 p$ & $(5 / 2)^{2}[5 / 2]^{\circ}{ }_{3}$ & $6 \mathrm{~d}$ & $(5 / 2)^{2}[9 / 2]_{4}$ & & & R1c & & \\
\hline $3945.5770(12)$ & 25337.664(8) & $3945.5767(5)$ & 0.0004 & 2300 & & $5 p$ & $(3 / 2)^{2}[3 / 2]^{\circ} 1$ & $6 \mathrm{~d}$ & $(3 / 2)^{2}[5 / 2]_{2}$ & & & R1c & & \\
\hline 3945.7664(13) & $25336.448(9)$ & $3945.7662(5)$ & 0.0002 & 1500 & & $5 p$ & $(5 / 2)^{2}[5 / 2]^{\circ}{ }_{3}$ & $6 \mathrm{~d}$ & $(5 / 2)^{2}[3 / 2]_{2}$ & & & $\mathrm{R} 1 \mathrm{c}$ & & \\
\hline 3952.0661(11) & 25296.062(7) & $3952.0660(6)$ & 0.0001 & 980 & & $5 p$ & $(5 / 2)^{2}[3 / 2]^{\circ}{ }_{1}$ & $6 \mathrm{~d}$ & $(5 / 2)^{2}[1 / 2]_{1}$ & & & R1c & & \\
\hline 3958.4734(13) & $25255.118(8)$ & $3958.4707(5)$ & 0.0027 & 840 & & $5 p$ & $(3 / 2)^{2}[3 / 2]^{\circ}{ }_{1}$ & $6 \mathrm{~d}$ & $(3 / 2)^{2}[3 / 2]_{2}$ & & & R1c & & \\
\hline $3974.3504(22)$ & $25154.229(14)$ & $3974.3528(7)$ & -0.0024 & 91 & & $4 \mathrm{~d}$ & $(5 / 2)^{2}[5 / 2]_{2}$ & $6 p$ & $(3 / 2)^{2}[3 / 2]^{\circ} 2$ & & & R1c & & \\
\hline
\end{tabular}


Table A1. Cont.

\begin{tabular}{|c|c|c|c|c|c|c|c|c|c|c|c|c|c|c|}
\hline$\lambda_{\text {obs }}{ }^{a}(\AA)$ & $\sigma_{\mathrm{obs}}{ }^{\mathrm{b}}\left(\mathrm{cm}^{-1}\right)$ & $\lambda_{\text {Ritz }}{ }^{c}(\AA)$ & $\begin{array}{c}\Delta \lambda_{\text {obs-Ritz }} \\
\text { (A) }\end{array}$ & $\begin{array}{l}I_{\text {obs }} \mathrm{d} \\
\text { (arb. u.) }\end{array}$ & Char ${ }^{\mathrm{e}}$ & & ower Level & & pper Level & $A\left(\mathrm{~s}^{-1}\right)$ & $\operatorname{Acc}^{f}$ & Line Ref. $\mathrm{g}$ & TP Ref. $\mathrm{g}$ & Notes ${ }^{h}$ \\
\hline $3975.5387(13)$ & $25146.711(8)$ & $3975.5380(9)$ & 0.0007 & 90 & & $4 \mathrm{~d}$ & $(3 / 2)^{2}[1 / 2]_{0}$ & $7 p$ & $(5 / 2)^{2}[3 / 2]^{\circ}{ }_{1}$ & & & R1c & & \\
\hline $3985.2145(11)$ & $25085.657(7)$ & $3985.2136(5)$ & 0.0009 & 700 & & $5 p$ & $(3 / 2)^{2}[3 / 2]^{\circ} 2$ & $6 \mathrm{~d}$ & $(3 / 2)^{2}[5 / 2]_{2}$ & & & $\mathrm{R} 1 \mathrm{c}$ & & \\
\hline 3987.0237(11) & $25074.274(7)$ & 3987.0244(5) & -0.0007 & 2300 & & $5 p$ & $(3 / 2)^{2}[3 / 2]^{0}$ & $6 \mathrm{~d}$ & $(3 / 2)^{2}[5 / 2]_{3}$ & & & R1c & & \\
\hline $3990.7807(13)$ & $25050.670(8)$ & 3990.7773(5) & 0.0033 & 960 & & $5 s$ & $(3 / 2)^{2}[3 / 2]_{1}$ & $\mathrm{sp}$ & $\left({ }^{3} \mathrm{P}\right)^{3} \mathrm{P}^{\circ}{ }^{3} \mathrm{P}^{\circ}{ }_{1}$ & & & R1c & & \\
\hline 3993.3023(12) & $25034.852(8)$ & 3993.3022(5) & 0.0001 & 2600 & & $\mathrm{sp}$ & $\left({ }^{3} \mathrm{~F}\right)^{3} \mathrm{P}^{\circ}{ }^{1} \mathrm{~F}_{3}^{\circ}$ & $6 \mathrm{~d}$ & $(5 / 2)^{2}[7 / 2]_{4}$ & & & $\mathrm{R} 1 \mathrm{c}$ & & \\
\hline 3998.3675(12) & 25003.138(8) & 3998.3684(5) & -0.0009 & 860 & & $5 p$ & $(3 / 2)^{2}[3 / 2]^{\circ}{ }_{2}$ & $6 \mathrm{~d}$ & $(3 / 2)^{2}[3 / 2]_{2}$ & & & R1c & & \\
\hline 4003.4759(12) & $24971.235(8)$ & $4003.4736(5)$ & 0.0023 & 1600 & & $\mathrm{sp}$ & $\left({ }^{3} \mathrm{~F}\right)^{3} \mathrm{P}^{\circ}{ }^{1} \mathrm{~F}_{3}^{\circ}$ & $6 \mathrm{~d}$ & $(5 / 2)^{2}[5 / 2]_{3}$ & & & R1c & & \\
\hline 4004.5155(14) & 24964.752(9) & $4004.5160(6)$ & -0.0004 & 400 & & $5 p$ & $(3 / 2)^{2}[3 / 2]^{\circ} 2$ & $6 \mathrm{~d}$ & $(3 / 2)^{2}[3 / 2]_{1}$ & & & R1c & & \\
\hline 4006.1651(12) & $24954.473(7)$ & $4006.1666(3)$ & -0.0015 & 1500 & & $\mathrm{sp}$ & $\left({ }^{3} \mathrm{~F}\right)^{3} \mathrm{P}^{\circ}{ }^{1} \mathrm{D}^{\circ}{ }_{2}$ & $7 \mathrm{~s}$ & $(3 / 2)^{2}[3 / 2]_{2}$ & & & R1c & & \\
\hline 4014.2360(12) & 24904.301(7) & $4014.2342(4)$ & 0.0018 & 870 & & $\mathrm{sp}$ & $\left({ }^{3} \mathrm{~F}\right)^{3} \mathrm{P}^{\circ}{ }^{1} \mathrm{D}^{\circ}{ }_{2}$ & $7 \mathrm{~s}$ & $(3 / 2)^{2}[3 / 2]_{1}$ & & & R1c & & \\
\hline 4015.1963(12) & $24898.345(8)$ & 4015.1954(5) & 0.0009 & 860 & & $\mathrm{sp}$ & $\left({ }^{3} \mathrm{~F}\right)^{3} \mathrm{P}^{\circ} 1^{1} \mathrm{~F}_{3}^{\circ}$ & $6 \mathrm{~d}$ & $(5 / 2)^{2}[9 / 2]_{4}$ & & & $\mathrm{R} 1 \mathrm{c}$ & & \\
\hline 4016.4254(14) & $24890.726(9)$ & $4016.4218(6)$ & 0.0036 & 1500 & & $\mathrm{sp}$ & $\left({ }^{3} \mathrm{~F}\right)^{3} \mathrm{P}^{\circ}{ }^{1} \mathrm{~F}_{3}^{\circ}$ & $6 \mathrm{~d}$ & $(5 / 2)^{2}[3 / 2]_{2}$ & & & R1c & & \\
\hline 4030.3508(21) & $24804.727(13)$ & $4030.3525(7)$ & -0.0018 & 330 & & $5 p$ & $(3 / 2)^{2}[3 / 2]^{\circ} 2$ & $6 \mathrm{~d}$ & $(3 / 2)^{2}[1 / 2]_{1}$ & & & R1c & & \\
\hline 4032.6469(12) & $24790.604(7)$ & $4032.6470(3)$ & -0.0001 & 1500 & & $5 p$ & $(5 / 2)^{2}[3 / 2]^{\circ} 2$ & $7 \mathrm{~s}$ & $(5 / 2)^{2}[5 / 2]_{2}$ & & & $\mathrm{R} 1 \mathrm{c}$ & & \\
\hline $4042.549(3)$ & $24729.880(21)$ & $4042.5473(7)$ & 0.002 & 67 & & $4 \mathrm{~d}$ & $(5 / 2)^{2}[1 / 2]_{1}$ & $6 \mathrm{p}$ & $(5 / 2)^{2}[3 / 2]^{\circ}{ }_{1}$ & & & R1c & & \\
\hline $4043.4879(9)$ & $24724.139(6)$ & $4043.4858(5)$ & 0.0021 & 27000 & & $4 p$ & ${ }^{1} \mathrm{~F}_{3}^{\circ}$ & $\mathrm{s}^{\frac{1}{2}}$ & ${ }^{1} \mathrm{G}_{4}$ & $1.14 \mathrm{e}+06$ & $\mathrm{C}+$ & F_Re & $\mathrm{O} 07$ & \\
\hline $4043.7515(4)$ & $24722.527(3)$ & $4043.75122(21)$ & 0.0003 & 17000 & & $5 p$ & $(5 / 2)^{2}[3 / 2]^{\circ} 2$ & $7 \mathrm{~s}$ & $(5 / 2)^{2}[5 / 2]_{3}$ & & & F_Re & & \\
\hline $4053.6529(21)$ & $24662.142(13)$ & 4053.6521(4) & 0.0008 & 3100 & & $5 p$ & $(3 / 2)^{2}[1 / 2]_{0}^{0}$ & $7 \mathrm{~s}$ & $(3 / 2)^{2}[3 / 2]_{1}$ & & & R1c & & \\
\hline 4065.0094(12) & $24593.244(7)$ & $4065.0090(4)$ & 0.0004 & 1600 & & $\mathrm{sp}$ & $\left({ }^{3} \mathrm{~F}\right)^{3} \mathrm{P}^{\circ}{ }^{1} \mathrm{D}_{2}^{\circ}$ & $6 \mathrm{~d}$ & $(5 / 2)^{2}[5 / 2]_{2}$ & & & R1c & & \\
\hline $4065.3723(22)$ & $24591.049(13)$ & 4065.3730(5) & -0.0007 & 230 & & $5 s$ & $(3 / 2)^{2}[3 / 2]_{1}$ & $\mathrm{sp}$ & $\left({ }^{3} \mathrm{P}\right)^{3} \mathrm{P}^{\circ}{ }^{3} \mathrm{D}^{\circ}{ }_{2}$ & & & R1c & & \\
\hline 4068.1058(12) & $24574.526(7)$ & $4068.1056(4)$ & 0.0001 & 2000 & & $\mathrm{sp}$ & $\left({ }^{3} \mathrm{~F}\right)^{3} \mathrm{P}^{\circ}{ }^{1} \mathrm{D}^{\circ}{ }_{2}$ & $6 \mathrm{~d}$ & $(5 / 2)^{2}[7 / 2]_{3}$ & & & R1c & & \\
\hline 4089.4787(13) & 24446.094(8) & $4089.4788(5)$ & -0.0001 & 480 & & $\mathrm{sp}$ & $\left({ }^{3} \mathrm{~F}\right)^{3} \mathrm{P}^{\circ}{ }^{1} \mathrm{D}^{\circ}{ }_{2}$ & $6 \mathrm{~d}$ & $(5 / 2)^{2}[3 / 2]_{1}$ & & & R1c & & \\
\hline 4112.4816(13) & $24309.359(8)$ & $4112.4801(5)$ & 0.0015 & 820 & & $5 s$ & $(3 / 2)^{2}[3 / 2]_{2}$ & $\mathrm{sp}$ & $\left({ }^{3} \mathrm{P}\right)^{3} \mathrm{P}^{\circ}{ }^{3} \mathrm{D}_{2}{ }_{2}$ & & & $\mathrm{R} 1 \mathrm{c}$ & & \\
\hline $4118.2403(24)$ & $24275.367(14)$ & $4118.2398(5)$ & 0.0005 & 57 & & $5 s$ & $(3 / 2)^{2}[3 / 2]_{1}$ & $\mathrm{sp}$ & $\left({ }^{3} \mathrm{P}\right)^{3} \mathrm{P}^{\circ}{ }^{3} \mathrm{D}^{\circ}{ }_{1}$ & & & R1c & & \\
\hline $4125.171(4)$ & $24234.580(21)$ & $4125.1717(8)$ & -0.000 & 11 & & $4 \mathrm{~d}$ & $(5 / 2)^{2}[1 / 2]_{1}$ & $6 p$ & $(5 / 2)^{2}[3 / 2]^{\circ} 2$ & & & R1c & & \\
\hline 4125.9352(17) & $24230.094(10)$ & $4125.9352(9)$ & 0.0001 & 65 & & $5 p$ & $(3 / 2)^{2}[1 / 2]^{\circ} 1$ & $6 \mathrm{~d}$ & $(5 / 2)^{2}[1 / 2]_{0}$ & & & R1c & & \\
\hline $4131.3610(4)$ & $24198.2729(25)$ & $4131.3610(3)$ & 0.0000 & 16000 & & $5 p$ & $(5 / 2)^{2}[7 / 2]^{0}{ }_{3}$ & $7 \mathrm{~s}$ & $(5 / 2)^{2}[5 / 2]_{2}$ & & & F_Re & & \\
\hline 4132.7116(21) & $24190.365(12)$ & $4132.7109(3)$ & 0.0007 & 1000 & & $5 p$ & $(3 / 2)^{2}[5 / 2]^{\circ} 2$ & $7 \mathrm{~s}$ & $(3 / 2)^{2}[3 / 2]_{2}$ & & & $\mathrm{R} 1 \mathrm{c}$ & & \\
\hline $4141.2965(5)$ & $24140.219(3)$ & $4141.2968(4)$ & -0.0003 & 9700 & & $5 p$ & $(3 / 2)^{2}[5 / 2]^{\circ} 2$ & $7 \mathrm{~s}$ & $(3 / 2)^{2}[3 / 2]_{1}$ & & & F_Re & & \\
\hline 4143.0170(13) & $24130.195(7)$ & $4143.01624(20)$ & 0.0007 & 2100 & & $5 p$ & $(5 / 2)^{2}[7 / 2]^{0} 3$ & $7 \mathrm{~s}$ & $(5 / 2)^{2}[5 / 2]_{3}$ & & & R1c & & \\
\hline $4147.8136(20)$ & 24102.291(11) & $4147.8131(6)$ & 0.0004 & 190 & & $4 d$ & $(5 / 2)^{2}[3 / 2]_{2}$ & $6 p$ & $(5 / 2)^{2}[5 / 2]^{\circ} 3$ & & & R1c & & \\
\hline 4151.904(3) & $24078.544(15)$ & $4151.9059(6)$ & -0.002 & 47 & & $4 \mathrm{~d}$ & $\begin{array}{l}(5 / 2)^{2}[9 / 2]_{4} \\
\end{array}$ & $6 \mathrm{p}$ & $(5 / 2)^{2}[5 / 2]^{\circ}{ }^{\circ}$ & & & $\mathrm{R} 1 \mathrm{c}$ & & \\
\hline 4153.6234(13) & $24068.579(7)$ & $4153.6236(3)$ & -0.0003 & 3600 & & $5 p$ & $(3 / 2)^{2}[1 / 2]^{\circ} 1$ & $7 \mathrm{~s}$ & $(3 / 2)^{2}[3 / 2]_{2}$ & & & R1c & & \\
\hline 4154.642(4) & $24062.680(21)$ & $4154.642(4)$ & -0.000 & 9 & & $\mathrm{~s}^{\frac{1}{2}}$ & ${ }^{3} \mathrm{P}_{2}$ & $\mathrm{sp}$ & $\left({ }^{3} \mathrm{~F}\right)^{3} \mathrm{P}^{\circ}{ }^{5} \mathrm{G}^{\circ}{ }_{2}$ & & & R1c & & \\
\hline 4161.13984(15) & $24025.1034(9)$ & $4161.13989(14)$ & -0.00005 & 17000 & & $5 p$ & $(5 / 2)^{2}[7 / 2]^{\circ} 4$ & $7 \mathrm{~s}$ & $(5 / 2)^{2}[5 / 2]_{3}$ & & & F_Re & & \\
\hline $4162.2967(14)$ & $24018.426(8)$ & $4162.2967(4)$ & 0.0000 & 3000 & & $5 p$ & $(3 / 2)^{2}[1 / 2]^{\circ}{ }_{1}$ & $7 \mathrm{~s}$ & $(3 / 2)^{2}[3 / 2]_{1}$ & & & R1c & & \\
\hline $4164.2826(10)$ & $24006.972(6)$ & 4164.2827(3) & -0.0001 & 8700 & & $5 p$ & $(5 / 2)^{2}[5 / 2]^{\circ} 2$ & $7 \mathrm{~s}$ & $(5 / 2)^{2}[5 / 2]_{2}$ & & & F_Re & & \\
\hline $4165.67(15)$ & $23999.0(9)$ & 4165.775(3) & -0.10 & 3 & & $\mathrm{~d}^{10}$ & ${ }^{1} \mathrm{~S}_{0}$ & $4 \mathrm{~s}$ & ${ }^{3} \mathrm{D}_{1}$ & $2.1 \mathrm{e}-12$ & $\mathrm{C}+$ & M97 & TW & $\mathrm{X}, \mathrm{M} 1$ \\
\hline $4166.5884(23)$ & 23993.687(13) & $4166.5874(5)$ & 0.0010 & 350 & & $5 s$ & $(3 / 2)^{2}[3 / 2]_{2}$ & $\mathrm{sp}$ & $\left({ }^{3} \mathrm{P}\right)^{3} \mathrm{P}^{\circ}{ }^{3} \mathrm{D}^{\circ}{ }_{1}$ & & & R1c & & \\
\hline $4171.8513(5)$ & 23963.419(3) & $4171.8521(3)$ & -0.0007 & 10000 & & $5 p$ & $(5 / 2)^{2}[3 / 2]^{\circ} 1$ & $7 \mathrm{~s}$ & $(5 / 2)^{2}[5 / 2]_{2}$ & & & F_Re & & \\
\hline $4176.1248(12)$ & $23938.897(7)$ & $4176.1247(3)$ & 0.0001 & 1400 & & $5 p$ & $(5 / 2)^{2}[5 / 2]^{\circ}{ }_{2}$ & $7 \mathrm{~s}$ & $(5 / 2)^{2}[5 / 2]_{3}$ & & & $\mathrm{R} 1 \mathrm{c}$ & & \\
\hline
\end{tabular}


Table A1. Cont.

\begin{tabular}{|c|c|c|c|c|c|c|c|c|c|c|c|c|c|c|}
\hline$\lambda_{\text {obs }}{ }^{\text {a }}(\AA)$ & $\sigma_{\mathrm{obs}}{ }^{\mathrm{b}}\left(\mathrm{cm}^{-1}\right)$ & $\lambda_{\text {Ritz }}{ }^{c}(\AA)$ & $\begin{array}{c}\Delta \lambda_{\text {obs-Ritz }} \\
\text { (A) }\end{array}$ & $\begin{array}{l}I_{\text {obs }} \mathrm{d} \\
\text { (arb. u.) }\end{array}$ & Char ${ }^{\mathrm{e}}$ & & ower Level & & pper Level & $A\left(\mathrm{~s}^{-1}\right)$ & $\operatorname{Acc}^{f}$ & Line Ref. $\mathrm{g}$ & TP Ref. $\mathrm{g}$ & Notes ${ }^{h}$ \\
\hline $4179.5117(4)$ & $23919.4987(21)$ & $4179.5113(3)$ & 0.0003 & 12000 & & $5 p$ & $(3 / 2)^{2}[5 / 2]_{3}^{\circ}$ & $7 \mathrm{~s}$ & $(3 / 2)^{2}[3 / 2]_{2}$ & & & F_Re & & \\
\hline $4185.149(4)$ & $23887.280(21)$ & $4185.1555(11)$ & -0.006 & 8 & & $\mathrm{~s}^{2}$ & ${ }^{1} \mathrm{D}_{2}$ & $\mathrm{sp}$ & $\left({ }^{3} \mathrm{~F}\right)^{3} \mathrm{P}^{\circ}{ }^{5} \mathrm{D}^{\circ}{ }_{3}$ & & & $\mathrm{R} 1 \mathrm{c}$ & & \\
\hline 4195.3590(16) & $23829.148(9)$ & $4195.3583(5)$ & 0.0007 & 210 & & $5 p$ & $(3 / 2)^{2}[5 / 2]_{2}^{\circ}$ & $6 \mathrm{~d}$ & $(5 / 2)^{2}[5 / 2]_{2}$ & & & R1c & & \\
\hline 4195.7735(13) & 23826.794(8) & $4195.7740(7)$ & -0.0005 & 340 & & $4 \mathrm{~d}$ & $(5 / 2)^{2}[9 / 2]_{5}$ & $6 p$ & $(5 / 2)^{2}[7 / 2]^{\circ} 4$ & & & R1c & & \\
\hline 4198.6561(13) & $23810.436(8)$ & $4198.6568(4)$ & -0.0007 & 240 & & $5 p$ & $(3 / 2)^{2}[5 / 2]^{\circ}{ }_{2}$ & $6 \mathrm{~d}$ & $(5 / 2)^{2}[7 / 2]_{3}$ & & & $\mathrm{R} 1 \mathrm{c}$ & & \\
\hline 4199.435(4) & $23806.020(21)$ & 4199.4464(9) & -0.011 & 110 & & $4 \mathrm{~d}$ & $(3 / 2)^{2}[3 / 2]_{1}$ & $6 p$ & $(3 / 2)^{2}[3 / 2]^{\circ}{ }_{1}$ & & & R1c & & \\
\hline $4201.735(3)$ & $23792.990(17)$ & $4201.7309(10)$ & 0.004 & 15 & & $5 p$ & $(3 / 2)^{2}[3 / 2]^{\circ}{ }_{1}$ & $6 \mathrm{~d}$ & $(5 / 2)^{2}[1 / 2]_{0}$ & & & R1c & & \\
\hline $4201.888(4)$ & $23792.120(21)$ & $4201.8877(16)$ & 0.001 & 22 & & $\mathrm{sp}$ & $\left({ }^{3} \mathrm{P}\right)^{3} \mathrm{P}^{\circ}{ }^{5} \mathrm{D}_{3}^{\circ}$ & $8 \mathrm{~d}$ & $(5 / 2)^{2}[9 / 2]_{4}$ & & & R1c & & \\
\hline $4207.672(3)$ & 23759.417(16) & $4207.5870(9)$ & 0.085 & 15 & ? & $\mathrm{sp}$ & $\left({ }^{3} \mathrm{~F}\right)^{3} \mathrm{P}^{\circ}{ }^{3} \mathrm{G}_{4}^{\circ}$ & $5 \mathrm{~d}$ & $(3 / 2)^{2}[5 / 2]_{3}$ & & & R1c & & $\mathrm{x}$ \\
\hline 4209.321(4) & $23750.110(21)$ & $4209.3213(10)$ & -0.000 & 29 & & $4 \mathrm{~d}$ & $(3 / 2)^{2}[1 / 2]_{1}$ & $6 p$ & $(3 / 2)^{2}[1 / 2]^{\circ} 1$ & & & R1c & & \\
\hline $4211.8649(8)$ & $23735.766(5)$ & $4211.86561(23)$ & -0.0007 & 11000 & & $5 p$ & $(5 / 2)^{2}[5 / 2]^{\circ}{ }_{3}$ & $7 \mathrm{~s}$ & $(5 / 2)^{2}[5 / 2]_{3}$ & & & F_Re & & \\
\hline $4212.315(3)$ & 23733.232(16) & $4212.3160(7)$ & -0.001 & 14 & & $4 \mathrm{~d}$ & $(5 / 2)^{2}[9 / 2]_{4}$ & $6 p$ & $(5 / 2)^{2}[7 / 2]^{\circ} 4$ & & & R1c & & \\
\hline $4216.9124(13)$ & $23707.356(7)$ & $4216.9115(5)$ & 0.0008 & 640 & & $5 p$ & $(3 / 2)^{2}[1 / 2]^{\circ} 1$ & $6 \mathrm{~d}$ & $(5 / 2)^{2}[5 / 2]_{2}$ & & & R1c & & \\
\hline $4219.38(20)$ & $23693.5(11)$ & $4219.5840(6)$ & -0.20 & 340 & & $4 \mathrm{~d}$ & $(5 / 2)^{2}[3 / 2]_{2}$ & $\mathrm{sp}$ & $\left({ }^{1} \mathrm{G}\right)^{3} \mathrm{P}^{\circ}{ }^{3} \mathrm{~F}_{3}{ }_{3}$ & & & S36c & & \\
\hline $4221.427(2)$ & $23682.000(14)$ & $4221.4276(5)$ & -0.000 & 69 & & $5 p$ & $(3 / 2)^{2}[5 / 2]^{\circ} 2$ & $6 \mathrm{~d}$ & $(5 / 2)^{2}[3 / 2]_{1}$ & & & R1c & & \\
\hline 4223.8195(14) & $23668.588(8)$ & $4223.8197(6)$ & -0.0002 & 100 & & $4 \mathrm{~d}$ & $(5 / 2)^{2}[9 / 2]_{4}$ & $\mathrm{sp}$ & $\left({ }^{1} \mathrm{G}\right)^{3} \mathrm{P}^{\circ}{ }^{3} \mathrm{~F}^{\circ}{ }_{3}$ & & & R1c & & \\
\hline $4224.344(4)$ & $23665.650(21)$ & $4224.3455(3)$ & -0.002 & 14 & & $4 \mathrm{~d}$ & $(5 / 2)^{2}[1 / 2]_{1}$ & $4 f$ & $(3 / 2)^{2}[5 / 2]^{\circ} 2$ & & & R1c & & \\
\hline $4225.1956(14)$ & $23660.880(8)$ & $4225.1967(6)$ & -0.0012 & 140 & & $4 \mathrm{~d}$ & $(5 / 2)^{2}[5 / 2]_{3}$ & $6 p$ & $(5 / 2)^{2}[5 / 2]^{0} 3$ & & & R1c & & \\
\hline 4227.9422(14) & $23645.509(8)$ & $4227.9397(5)$ & 0.0025 & 7900 & & $4 p$ & ${ }^{3} \mathrm{D}^{\circ}{ }_{3}$ & $s^{\frac{1}{2}}$ & ${ }^{1} \mathrm{G}_{4}$ & $5.1 \mathrm{e}+05$ & B & R1c & $\mathrm{O} 07$ & \\
\hline $4230.4486(14)$ & $23631.500(8)$ & $4230.4495(4)$ & -0.0009 & 4000 & & $5 p$ & $(3 / 2)^{2}[3 / 2]^{\circ}{ }_{1}^{\circ}$ & $7 \mathrm{~s}$ & $(3 / 2)^{2}[3 / 2]_{2}$ & & & R1c & & \\
\hline $4232.8346(15)$ & $23618.180(8)$ & $4232.8362(6)$ & -0.0016 & 130 & & $5 s$ & $(3 / 2)^{2}[3 / 2]_{2}$ & $\mathrm{sp}$ & $\left({ }^{3} \mathrm{P}\right)^{3} \mathrm{P}^{\circ}{ }^{3} \mathrm{D}^{\circ}{ }_{3}$ & & & R1c & & \\
\hline 4239.4445(20) & 23581.356(11) & $4239.4468(4)$ & -0.0023 & 7000 & & $5 p$ & $(3 / 2)^{2}[3 / 2]^{\circ}{ }_{1}$ & $7 \mathrm{~s}$ & $(3 / 2)^{2}[3 / 2]_{1}$ & & & F_Re & & \\
\hline $4240.410(3)$ & $23575.985(14)$ & $4240.4120(8)$ & -0.002 & 64 & & $4 \mathrm{~d}$ & $(5 / 2)^{2}[3 / 2]_{1}$ & $6 p$ & $(5 / 2)^{2}[3 / 2]^{0}{ }_{1}$ & & & $\mathrm{R} 1 \mathrm{c}$ & & \\
\hline $4241.31(4)$ & $23570.97(22)$ & $4241.271(3)$ & 0.04 & 48 & * & $\mathrm{sp}$ & $\left({ }^{3} \mathrm{P}\right)^{3} \mathrm{P}^{0}{ }^{5} \mathrm{D}^{\circ}{ }_{4}$ & $8 \mathrm{~d}$ & $(5 / 2)^{2}[7 / 2]_{4}$ & & & R1nc & & \\
\hline $4241.31(4)$ & $23570.97(22)$ & $4241.3236(9)$ & -0.01 & 48 & * & $4 \mathrm{~d}$ & $(3 / 2)^{2}[3 / 2]_{2}$ & $6 p$ & $(3 / 2)^{2}[3 / 2]^{0} 1$ & & & $\mathrm{R} 1 \mathrm{c}$ & & \\
\hline $4243.2516(15)$ & $23560.199(8)$ & $4243.2501(5)$ & 0.0015 & 320 & & $5 p$ & $(3 / 2)^{2}[1 / 2]^{\circ}{ }_{1}$ & $6 \mathrm{~d}$ & $(5 / 2)^{2}[3 / 2]_{1}$ & & & R1c & & \\
\hline $4246.9860(20)$ & 23539.483(11) & $4246.9715(4)$ & 0.0145 & 390 & * & $5 p$ & $(3 / 2)^{2}[5 / 2]^{\circ}{ }_{3}$ & $6 \mathrm{~d}$ & $(5 / 2)^{2}[7 / 2]_{3}$ & & & R1nc & & $\mathrm{x}$ \\
\hline $4246.9860(20)$ & 23539.483(11) & $4246.9847(7)$ & 0.0013 & 390 & * & $4 \mathrm{~d}$ & $(3 / 2)^{2}[1 / 2]_{0}$ & $5 f$ & $(5 / 2)^{2}[3 / 2]^{\circ} 1$ & & & R1c & & \\
\hline 4251.0194(15) & $23517.149(8)$ & $4251.0200(6)$ & -0.0006 & 300 & & $4 \mathrm{~d}$ & $(5 / 2)^{2}[1 / 2]_{1}$ & $4 \mathrm{f}$ & $(3 / 2)^{2}[3 / 2]^{\circ} 1$ & & & R1c & & \\
\hline $4254.630(4)$ & 23497.190(21) & $4254.6275(11)$ & 0.003 & 12 & & $4 \mathrm{~d}$ & $(3 / 2)^{2}[7 / 2]_{3}$ & $6 p$ & $(3 / 2)^{2}[5 / 2]^{0} 2$ & & & $\mathrm{R} 2 \mathrm{nc}$ & & \\
\hline $4255.6348(14)$ & $23491.644(8)$ & $4255.6343(4)$ & 0.0006 & 1100 & & $4 \mathrm{~d}$ & $(5 / 2)^{2}[1 / 2]_{1}$ & $4 \mathrm{f}$ & $(3 / 2)^{2}[3 / 2]^{0} 2$ & & & $\mathrm{R} 1 \mathrm{c}$ & & \\
\hline $4256.846(4)$ & $23484.960(21)$ & $4256.8484(7)$ & -0.002 & 30 & & $4 \mathrm{~d}$ & $(3 / 2)^{2}[1 / 2]_{0}$ & $5 f$ & $(5 / 2)^{2}[1 / 2]^{\circ}{ }_{1}$ & & & R1c & & \\
\hline $4267.611(4)$ & $23425.720(21)$ & $4267.6128(13)$ & -0.002 & 120 & & $\mathrm{~s}^{2}$ & ${ }^{1} \mathrm{G}_{4}$ & $\mathrm{sp}$ & $\left({ }^{3} \mathrm{~F}\right)^{3} \mathrm{P}^{\circ}{ }^{1} \mathrm{G}^{\circ}{ }_{4}$ & & & R1c & & \\
\hline 4268.103(4) & $23423.020(21)$ & $4268.1012(12)$ & 0.002 & 18 & & $\mathrm{sp}$ & $\left({ }^{3} \mathrm{~F}\right)^{3} \mathrm{P}^{\circ}{ }^{5} \mathrm{~F}_{4}^{\circ}$ & $5 \mathrm{~d}$ & $(5 / 2)^{2}[9 / 2]_{5}$ & & & R1c & & \\
\hline $4276.0484(9)$ & $23379.499(5)$ & $4276.0496(4)$ & -0.0012 & 8700 & & $5 p$ & $(3 / 2)^{2}[3 / 2]_{2}^{\circ}$ & $7 \mathrm{~s}$ & $(3 / 2)^{2}[3 / 2]_{2}$ & & & F_Re & & \\
\hline $4277.805(4)$ & 23369.900(21) & $4277.8016(6)$ & 0.003 & 150 & & $4 \mathrm{~d}$ & $(5 / 2)^{2}[7 / 2]_{4}$ & $6 p$ & $(5 / 2)^{2}[5 / 2]^{\circ} 3$ & & & R1c & & \\
\hline $4279.9621(15)$ & $23358.120(8)$ & 4279.9594(3) & 0.0028 & 5100 & & $\mathrm{sp}$ & $\left({ }^{3} \mathrm{~F}\right)^{3} \mathrm{P}^{0}{ }^{1} \mathrm{~F}_{3}$ & $7 \mathrm{~s}$ & $(5 / 2)^{2}[5 / 2]_{2}$ & & & R1c & & \\
\hline $4280.845(4)$ & $23353.300(21)$ & $4280.8437(6)$ & 0.002 & 6 & & $4 \mathrm{~d}$ & $(5 / 2)^{2}[5 / 2]_{2}$ & $6 \mathrm{p}$ & $(5 / 2)^{2}[5 / 2]^{\circ}{ }_{3}$ & & & R1c & & \\
\hline $4281.838(3)$ & $23347.889(15)$ & $4281.8368(8)$ & 0.001 & 29 & & $5 p$ & $(3 / 2)^{2}[1 / 2]^{\circ}{ }_{1}$ & $6 \mathrm{~d}$ & $(5 / 2)^{2}[1 / 2]_{1}$ & & & R1c & & \\
\hline $4285.2433(13)$ & $23329.334(7)$ & $4285.2420(4)$ & 0.0012 & 2000 & & $5 p$ & $(3 / 2)^{2}[3 / 2]^{\circ} 2$ & $7 \mathrm{~s}$ & $(3 / 2)^{2}[3 / 2]_{1}$ & & & $\mathrm{R} 1 \mathrm{c}$ & & \\
\hline $4286.465(3)$ & 23322.683(16) & $4286.4615(7)$ & 0.004 & 11 & & $4 \mathrm{~d}$ & $(5 / 2)^{2}[5 / 2]_{2}$ & $\mathrm{sp}$ & $\left({ }^{3} \mathrm{~F}\right)^{1} \mathrm{P}^{\circ}{ }^{3} \mathrm{~F}_{2}^{\circ}{ }_{2}$ & & & R1c & & \\
\hline $4287.13(6)$ & 23319.1(3) & $4287.0450(10)$ & 0.08 & 180 & & $4 \mathrm{~d}$ & $(3 / 2)^{2}[7 / 2]_{4}$ & $6 p$ & $(3 / 2)^{2}[5 / 2]^{0} 3$ & & & S36c & & \\
\hline
\end{tabular}


Table A1. Cont.

\begin{tabular}{|c|c|c|c|c|c|c|c|c|c|c|c|c|c|c|}
\hline$\lambda_{\text {obs }}{ }^{a}(\AA)$ & $\sigma_{\mathrm{obs}}{ }^{\mathrm{b}}\left(\mathrm{cm}^{-1}\right)$ & $\lambda_{\text {Ritz }}{ }^{c}(\AA)$ & $\begin{array}{c}\Delta \lambda_{\text {obs-Ritz }} \\
\text { (A) }\end{array}$ & $\begin{array}{l}I_{\text {obs }} \mathrm{d} \\
\text { (arb. u.) }\end{array}$ & Char ${ }^{\mathrm{e}}$ & & pwer Level & & pper Level & $A\left(\mathrm{~s}^{-1}\right)$ & $\operatorname{Acc}^{f}$ & Line Ref. $\mathrm{g}$ & TP Ref. $\mathrm{g}$ & Notes ${ }^{h}$ \\
\hline $4287.779(3)$ & 23315.537(17) & $4287.7744(7)$ & 0.005 & 11 & & $4 \mathrm{~d}$ & $(5 / 2)^{2}[5 / 2]_{3}$ & $6 p$ & $(5 / 2)^{2}[7 / 2]^{\circ}{ }_{4}$ & & & R1c & & \\
\hline $4291.084(3)$ & $23297.580(16)$ & $4291.0824(24)$ & 0.002 & 640 & & $5 s$ & $(5 / 2)^{2}[5 / 2]_{3}$ & $\mathrm{sp}$ & $\left({ }^{3} \mathrm{P}\right)^{3} \mathrm{P}^{\circ}{ }^{5} \mathrm{D}^{\circ}$ & & & $\mathrm{R} 1 \mathrm{c}$ & & \\
\hline $4292.4705(14)$ & $23290.055(8)$ & 4292.4694(3) & 0.0011 & 9000 & & $\mathrm{sp}$ & $\left({ }^{3} \mathrm{~F}\right)^{3} \mathrm{P}^{\circ} \mathrm{F}^{\circ} \mathrm{F}_{3}$ & $7 \mathrm{~s}$ & $(5 / 2)^{2}[5 / 2]_{3}$ & & & R1c & & \\
\hline $4296.123(3)$ & $23270.256(14)$ & $4296.1187(5)$ & 0.004 & 17 & & $5 p$ & $(3 / 2)^{2}[3 / 2]^{\circ}{ }_{1}$ & $6 \mathrm{~d}$ & $(5 / 2)^{2}[5 / 2]_{2}$ & & & R1c & & \\
\hline $4299.696(3)$ & $23250.920(16)$ & $4299.6946(6)$ & 0.001 & 11 & & $4 d$ & $(5 / 2)^{2}[5 / 2]_{3}$ & $\mathrm{sp}$ & $\left({ }^{1} \mathrm{G}\right)^{3} \mathrm{P}^{\circ}{ }^{3} \mathrm{~F}^{0}{ }_{3}$ & & & $\mathrm{R} 1 \mathrm{c}$ & & \\
\hline $4308.710(3)$ & $23202.274(16)$ & $4308.7114(9)$ & -0.001 & 28 & & $4 \mathrm{~d}$ & $(3 / 2)^{2}[5 / 2]_{2}$ & $6 \mathrm{p}$ & $(3 / 2)^{2}[3 / 2]^{\circ}{ }_{1}$ & & & R1c & & \\
\hline 4320.7611(19) & 23137.564(10) & $4320.7616(7)$ & -0.0005 & 270 & & $4 \mathrm{~d}$ & $(5 / 2)^{2}[1 / 2]_{1}$ & $\mathrm{sp}$ & $\left({ }^{3} \mathrm{P}\right)^{3} \mathrm{P}^{\circ}{ }^{1} \mathrm{D}^{\circ}{ }_{2}$ & & & $\mathrm{R} 1 \mathrm{c}$ & & \\
\hline $4335.639(3)$ & $23058.167(16)$ & 4335.6353(9) & 0.004 & 17 & & $4 \mathrm{~d}$ & $(3 / 2)^{2}[5 / 2]_{3}$ & $6 p$ & $(3 / 2)^{2}[3 / 2]^{\circ} 2$ & & & $\mathrm{R} 1 \mathrm{c}$ & & \\
\hline $4341.039(3)$ & $23029.484(15)$ & $4341.0414(14)$ & -0.002 & 17 & & $5 s$ & $(5 / 2)^{2}[5 / 2]_{3}$ & $\mathrm{sp}$ & $\left({ }^{3} \mathrm{P}\right)^{3} \mathrm{P}^{\circ}{ }^{5} \mathrm{D}^{\circ}{ }_{3}$ & & & R1c & & \\
\hline 4343.152(4) & $23018.280(21)$ & $4343.1532(5)$ & -0.001 & 11 & & $5 p$ & $(3 / 2)^{2}[3 / 2]^{\circ} 2$ & $6 \mathrm{~d}$ & $(5 / 2)^{2}[5 / 2]_{2}$ & & & R1c & & \\
\hline $4344.700(4)$ & $23010.080(21)$ & $4344.6972(9)$ & 0.003 & 6 & & $4 \mathrm{~d}$ & $(3 / 2)^{2}[5 / 2]_{2}$ & $6 p$ & $(3 / 2)^{2}[3 / 2]^{\circ} 2$ & & & R1c & & \\
\hline $4346.687(4)$ & 22999.560(21) & $4346.6883(5)$ & -0.001 & 240 & & $5 p$ & $(3 / 2)^{2}[3 / 2]^{\circ}{ }_{2}$ & $6 \mathrm{~d}$ & $(5 / 2)^{2}[7 / 2]_{3}$ & & & R1c & & \\
\hline 4357.339(4) & $22943.340(21)$ & $4357.3345(6)$ & 0.004 & 6 & & $4 d$ & $(5 / 2)^{2}[5 / 2]_{2}$ & $\mathrm{sp}$ & $\left({ }^{1} \mathrm{G}\right)^{3} \mathrm{P}^{\circ}{ }^{3} \mathrm{~F}^{\circ}{ }_{3}$ & & & $\mathrm{R} 1 \mathrm{c}$ & & \\
\hline $4360.102(3)$ & 22928.797(14) & $4360.0973(17)$ & 0.005 & 47 & & $5 s$ & $(5 / 2)^{2}[5 / 2]_{3}$ & $\mathrm{sp}$ & $\left({ }^{3} \mathrm{P}\right)^{3} \mathrm{P}^{\circ}{ }^{5} \mathrm{D}_{2}^{\circ}$ & & & $\mathrm{R} 1 \mathrm{c}$ & & \\
\hline $4365.3705(14)$ & $22901.127(7)$ & $4365.3697(3)$ & 0.0008 & 7300 & & $\mathrm{sp}$ & $\left({ }^{3} \mathrm{~F}\right)^{3} \mathrm{P}^{\circ}{ }^{1} \mathrm{D}^{\circ}{ }_{2}$ & $7 \mathrm{~s}$ & $(5 / 2)^{2}[5 / 2]_{2}$ & & & R1c & & \\
\hline $4373.460(3)$ & 22858.770(15) & $4373.4591(7)$ & 0.001 & 18 & & $5 p$ & $(3 / 2)^{2}[3 / 2]^{\circ}{ }_{2}$ & $6 \mathrm{~d}$ & $(5 / 2)^{2}[3 / 2]_{2}$ & & & R1c & & \\
\hline $4375.8(5)$ & $22847(3)$ & $4375.690(3)$ & 0.1 & 2000 & & $\mathrm{~d}^{10}$ & ${ }^{1} \mathrm{~S}_{0}$ & $4 \mathrm{~s}$ & ${ }^{3} \mathrm{D}_{2}$ & $9 . e-02$ & $\mathrm{D}+$ & A73,P84 & A08adj & $\mathrm{X}, \mathrm{E} 2$ \\
\hline $4378.3840(14)$ & 22833.061(7) & $4378.3848(3)$ & -0.0007 & 1500 & & $\mathrm{sp}$ & $\left({ }^{3} \mathrm{~F}\right)^{3} \mathrm{P}^{\circ}{ }^{1} \mathrm{D}^{\circ}{ }_{2}$ & $7 \mathrm{~s}$ & $(5 / 2)^{2}[5 / 2]_{3}$ & & & R1c & & \\
\hline $4396.423(3)$ & $22739.375(15)$ & $4396.4196(6)$ & 0.004 & 13 & & $4 d$ & $(5 / 2)^{2}[9 / 2]_{4}$ & $6 p$ & $(5 / 2)^{2}[7 / 2]^{\circ} 3$ & & & R1c & & \\
\hline $4402.363(3)$ & $22708.694(16)$ & $4402.3616(15)$ & 0.002 & 33 & & $5 s$ & $(5 / 2)^{2}[5 / 2]_{2}$ & $\mathrm{sp}$ & $\left({ }^{3} \mathrm{P}\right)^{3} \mathrm{P}^{\circ}{ }^{5} \mathrm{D}_{3}^{\circ}$ & & & R1c & & \\
\hline $4410.941(3)$ & 22664.535(17) & $4410.9371(9)$ & 0.004 & 7 & & $4 \mathrm{~d}$ & $(5 / 2)^{2}[1 / 2]_{0}$ & $6 \mathrm{p}$ & $(5 / 2)^{2}[3 / 2]^{\circ}{ }_{1}$ & & & R1c & & \\
\hline $4419.088(4)$ & $22622.750(21)$ & $4419.0825(3)$ & 0.006 & 7 & & $4 \mathrm{~d}$ & $(5 / 2)^{2}[3 / 2]_{2}$ & $4 \mathrm{f}$ & $(3 / 2)^{2}[7 / 2]^{\circ} 3$ & & & R1c & & \\
\hline $4422.583(3)$ & $22604.871(16)$ & $4422.5816(15)$ & 0.002 & 15 & & $5 s$ & $(5 / 2)^{2}[5 / 2]_{2}$ & $\mathrm{sp}$ & $\left({ }^{3} \mathrm{P}\right)^{3} \mathrm{P}^{\circ}{ }^{5} \mathrm{D}^{\circ}$ & & & $\mathrm{R} 1 \mathrm{c}$ & & \\
\hline $4435.693(3)$ & $22538.066(15)$ & $4435.6906(4)$ & 0.002 & 80 & & $4 \mathrm{~d}$ & $(5 / 2)^{2}[3 / 2]_{2}$ & $4 \mathrm{f}$ & $(3 / 2)^{2}[5 / 2]^{\circ}{ }_{2}$ & & & R1c & & \\
\hline $4440.8836(15)$ & 22511.721(8) & $4440.8827(4)$ & 0.0009 & 520 & & $4 \mathrm{~d}$ & $(5 / 2)^{2}[3 / 2]_{1}$ & $4 \mathrm{f}$ & $(3 / 2)^{2}[5 / 2]^{\circ} 2$ & & & $\mathrm{R} 1 \mathrm{c}$ & & \\
\hline $4441.709(14)$ & $22507.54(7)$ & $4441.7193(7)$ & -0.011 & 180 & & $\mathrm{sp}$ & $\left({ }^{3} \mathrm{~F}\right)^{3} \mathrm{P}^{\circ}{ }^{3} \mathrm{D}_{3}^{\circ}$ & $5 \mathrm{~d}$ & $(3 / 2)^{2}[7 / 2]_{4}$ & & & $\mathrm{R} 1 \mathrm{c}$ & & \\
\hline $4444.8314(14)$ & $22491.727(7)$ & $4444.8307(3)$ & 0.0007 & 1200 & & $4 d$ & $(5 / 2)^{2}[3 / 2]_{2}$ & $4 \mathrm{f}$ & $(3 / 2)^{2}[5 / 2]^{\circ}{ }_{3}$ & & & R1c & & \\
\hline $4462.6902(16)$ & $22401.721(8)$ & $4462.6875(5)$ & 0.0027 & 1600 & & $4 \mathrm{~d}$ & $(5 / 2)^{2}[9 / 2]_{4}$ & $4 \mathrm{f}$ & $(3 / 2)^{2}[9 / 2]^{\circ}{ }_{5}$ & & & $\mathrm{R} 1 \mathrm{c}$ & & \\
\hline 4485.280 & 22288.90 & $4485.2800(6)$ & & & : & $4 \mathrm{~d}$ & $(5 / 2)^{2}[1 / 2]_{1}$ & $\mathrm{sp}$ & $\left({ }^{3} \mathrm{~F}\right)^{1} \mathrm{P}^{\circ}{ }^{3} \mathrm{D}_{2}^{\circ}$ & & & W93 & & \\
\hline $4495.356(3)$ & $22238.942(15)$ & 4495.3577(8) & -0.002 & 38 & & $\mathrm{sp}$ & $\left({ }^{3} \mathrm{~F}\right)^{3} \mathrm{P}^{\circ}{ }^{3} \mathrm{G}^{\circ}{ }_{3}$ & $5 d$ & $(3 / 2)^{2}[7 / 2]_{4}$ & & & R1c & & \\
\hline $4505.9982(8)$ & $22186.418(4)$ & $4505.9996(4)$ & -0.0013 & 11000 & & $4 p$ & ${ }^{3} \mathrm{P}_{2}^{\circ}{ }_{2}$ & $\mathrm{~s}^{2}$ & ${ }^{3} \mathrm{P}_{1}$ & $6.3 e+05$ & C & F_Re & H71,N88 & $\mathrm{L}$ \\
\hline 4515.5194(15) & $22139.637(7)$ & 4515.5195(3) & -0.0001 & 960 & & $4 \mathrm{~d}$ & $(5 / 2)^{2}[5 / 2]_{3}$ & $4 \mathrm{f}$ & $(3 / 2)^{2}[7 / 2]^{\circ} 4$ & & & $\mathrm{R} 1 \mathrm{c}$ & & \\
\hline 4516.0492(15) & $22137.040(7)$ & $4516.0499(4)$ & -0.0007 & 2700 & & $5 p$ & $(3 / 2)^{2}[5 / 2]^{\circ} 2$ & $7 \mathrm{~s}$ & $(5 / 2)^{2}[5 / 2]_{2}$ & & & R1c & & \\
\hline $4529.983(3)$ & 22068.951(15) & $4529.9803(3)$ & 0.002 & 70 & & $5 p$ & $(3 / 2)^{2}[5 / 2]_{2}^{\circ}$ & $7 \mathrm{~s}$ & $(5 / 2)^{2}[5 / 2]_{3}$ & & & R1c & & \\
\hline $4533.814(4)$ & $22050.300(21)$ & $4533.8122(3)$ & 0.002 & 54 & & $4 \mathrm{~d}$ & $(5 / 2)^{2}[5 / 2]_{3}$ & $4 \mathrm{f}$ & $(3 / 2)^{2}[5 / 2]^{\circ} 3$ & & & $\mathrm{R} 1 \mathrm{c}$ & & \\
\hline $4541.0325(15)$ & 22015.251(7) & $4541.0337(4)$ & -0.0013 & 6900 & & $5 p$ & $(3 / 2)^{2}[1 / 2]^{\circ}{ }_{1}$ & $7 \mathrm{~s}$ & $(5 / 2)^{2}[5 / 2]_{2}$ & & & R1c & & \\
\hline $4542.15(4)$ & 22009.82(19) & 4542.1167(8) & 0.04 & 390 & * & $4 d$ & $(5 / 2)^{2}[3 / 2]_{2}$ & $\mathrm{sp}$ & $\left({ }^{3} \mathrm{P}\right)^{3} \mathrm{P}^{\circ}{ }^{1} \mathrm{D}_{2}{ }_{2}$ & & & $\mathrm{R} 1 \mathrm{c}$ & & \\
\hline $4542.15(4)$ & $22009.82(19)$ & $4542.1719(10)$ & -0.02 & 390 & * & $4 \mathrm{~d}$ & $(3 / 2)^{2}[1 / 2]_{1}$ & $6 p$ & $(5 / 2)^{2}[3 / 2]^{\circ} 1$ & & & R1c & & \\
\hline $4546.433(3)$ & 21989.099(12) & $4546.4353(6)$ & -0.002 & 410 & & $\mathrm{sp}$ & $\left({ }^{3} \mathrm{~F}\right)^{3} \mathrm{P}^{\circ}{ }^{3} \mathrm{D}^{\circ}{ }_{2}$ & $5 d$ & $(3 / 2)^{2}[5 / 2]_{3}$ & & & R1c & & \\
\hline 4551.807(3) & 21963.141(13) & $4551.8069(8)$ & -0.000 & 260 & & $4 d$ & $(3 / 2)^{2}[7 / 2]_{3}$ & $\mathrm{sp}$ & $\left({ }^{3} \mathrm{~F}\right)^{1} \mathrm{P}^{\circ}{ }^{3} \mathrm{~F}_{2}^{\circ}$ & & & $\mathrm{R} 1 \mathrm{c}$ & & \\
\hline 4555.9211(5) & $21943.307(3)$ & 4555.9193(4) & 0.0018 & 18000 & & $4 p$ & ${ }^{3} \mathrm{P}_{2}^{\circ}$ & $s^{2}$ & ${ }^{3} \mathrm{P}_{2}$ & $7.1 \mathrm{e}+05$ & C & F_Re & H71,N88 & $\mathrm{L}$ \\
\hline
\end{tabular}


Table A1. Cont.

\begin{tabular}{|c|c|c|c|c|c|c|c|c|c|c|c|c|c|c|}
\hline$\lambda_{\text {obs }}{ }^{\text {a }}(\AA)$ & $\sigma_{\mathrm{obs}}{ }^{\mathrm{b}}\left(\mathrm{cm}^{-1}\right)$ & $\lambda_{\text {Ritz }}{ }^{c}(\AA ̊)$ & $\begin{array}{c}\Delta \lambda_{\text {obs-Ritz }} \\
(\mathrm{A})\end{array}$ & $\begin{array}{l}I_{\text {obs }} \mathrm{d} \\
\text { (arb. u.) }\end{array}$ & Char ${ }^{e}$ & & ower Level & & pper Level & $A\left(\mathrm{~s}^{-1}\right)$ & $\operatorname{Acc}^{f}$ & Line Ref. $\mathrm{g}$ & TP Ref. $\mathrm{g}$ & Notes ${ }^{h}$ \\
\hline 4557.5077(15) & $21935.668(7)$ & $4557.5079(4)$ & -0.0002 & 1100 & & $4 \mathrm{~d}$ & $(5 / 2)^{2}[7 / 2]_{3}$ & $4 \mathrm{f}$ & $(3 / 2)^{2}[7 / 2]^{\circ}{ }_{3}$ & & & R1c & & \\
\hline $4558.7(5)$ & $21929.9(24)$ & $4558.949(4)$ & -0.2 & 3000 & & $\mathrm{~d}^{10}$ & ${ }^{1} \mathrm{~S}_{0}$ & $4 \mathrm{~s}$ & ${ }^{3} \mathrm{D}_{3}$ & & & A73 & & $\mathrm{X}, \mathrm{HF}$ \\
\hline \multirow[t]{2}{*}{$4575.180(7)$} & 21850.94(3) & $4575.1748(4)$ & 0.005 & 550 & * & $4 \mathrm{~d}$ & $(5 / 2)^{2}[7 / 2]_{3}$ & $4 f$ & $(3 / 2)^{2}[5 / 2]^{\circ} 2$ & & & R1c & & \\
\hline & & $4575.185(3)$ & & & $\mathrm{m}$ & $\mathrm{sp}$ & $\left({ }^{3} \mathrm{P}\right)^{3} \mathrm{P}^{\circ}{ }^{3} \mathrm{D}^{\circ}{ }_{2}$ & $10 \mathrm{~s}$ & $(5 / 2)^{2}[5 / 2]_{2}$ & & & R1c & & \\
\hline $4575.180(7)$ & 21850.94(3) & $4575.1871(7)$ & -0.007 & 550 & * & $\mathrm{sp}$ & $\left({ }^{3} \mathrm{~F}\right)^{3} \mathrm{P}^{\circ}{ }^{3} \mathrm{D}^{\circ}{ }_{2}$ & $5 \mathrm{~d}$ & $(3 / 2)^{2}[3 / 2]_{2}$ & & & $\mathrm{R} 1 \mathrm{c}$ & & \\
\hline $4575.6529(15)$ & $21848.681(7)$ & $4575.6532(3)$ & -0.0003 & 850 & & $4 d$ & $(5 / 2)^{2}[7 / 2]_{4}$ & $4 \mathrm{f}$ & $(3 / 2)^{2}[7 / 2]^{\circ} 4$ & & & R1c & & \\
\hline $4584.899(3)$ & $21804.623(12)$ & 4584.8994(3) & -0.001 & 60 & & $4 \mathrm{~d}$ & $(5 / 2)^{2}[7 / 2]_{3}$ & $4 \mathrm{f}$ & $(3 / 2)^{2}[5 / 2]^{0}{ }^{0}$ & & & $\mathrm{R} 1 \mathrm{c}$ & & \\
\hline $4586.274(3)$ & 21798.084(13) & $4586.2719(3)$ & 0.002 & 300 & & $5 p$ & $(3 / 2)^{2}[5 / 2]^{\circ} 3$ & $7 \mathrm{~s}$ & $(5 / 2)^{2}[5 / 2]_{3}$ & & & R1c & & \\
\hline 4588.1661(15) & $21789.095(7)$ & $4588.1667(4)$ & -0.0006 & 620 & & $4 d$ & $(5 / 2)^{2}[5 / 2]_{2}$ & $4 \mathrm{f}$ & $(3 / 2)^{2}[5 / 2]^{\circ} 2$ & & & $\mathrm{R} 1 \mathrm{c}$ & & \\
\hline 4589.601(3) & $21782.285(12)$ & $4589.6035(8)$ & -0.003 & 220 & & $4 \mathrm{~d}$ & $(3 / 2)^{2}[3 / 2]_{1}$ & $\mathrm{sp}$ & $\left({ }^{3} \mathrm{~F}\right)^{1} \mathrm{P}^{\circ}{ }^{3} \mathrm{~F}_{2}^{\circ}$ & & & R1c & & \\
\hline 4594.330(3) & $21759.863(13)$ & $4594.3638(14)$ & -0.034 & 200 & ? & $\mathrm{sp}$ & $\left({ }^{3} \mathrm{~F}\right)^{3} \mathrm{P}^{\circ} 5^{\circ} \mathrm{F}_{3}$ & $6 \mathrm{~s}$ & $(3 / 2)^{2}[3 / 2]_{2}$ & & & R1c & & $\mathrm{x}$ \\
\hline $4594.436(3)$ & $21759.358(15)$ & 4594.4374(3) & -0.001 & 130 & & $4 \mathrm{~d}$ & $(5 / 2)^{2}[7 / 2]_{4}$ & $4 \mathrm{f}$ & $(3 / 2)^{2}[5 / 2]^{\circ} 3$ & & & R1c & & \\
\hline $4596.9056(15)$ & $21747.671(7)$ & $4596.9063(5)$ & -0.0007 & 4800 & & $4 \mathrm{~d}$ & $(5 / 2)^{2}[7 / 2]_{3}$ & $4 \mathrm{f}$ & $(3 / 2)^{2}[9 / 2]^{\circ} 4$ & & & R1c & & \\
\hline $4597.9473(15)$ & $21742.744(7)$ & $4597.9466(3)$ & 0.0007 & 3200 & & $4 \mathrm{~d}$ & $(5 / 2)^{2}[5 / 2]_{2}$ & $4 \mathrm{f}$ & $(3 / 2)^{2}[5 / 2]^{0} 3$ & & & R1c & & \\
\hline $4606.5041(21)$ & $21702.356(10)$ & $4606.4944(5)$ & 0.0097 & 730 & $\mathrm{bl}$ & $4 \mathrm{~d}$ & $(5 / 2)^{2}[7 / 2]_{4}$ & $4 \mathrm{f}$ & $(3 / 2)^{2}[9 / 2]^{\circ} 4$ & & & $\mathrm{R} 1 \mathrm{c}$ & & $\mathrm{x}$ \\
\hline $4608.4661(16)$ & 21693.117(7) & $4608.4662(5)$ & -0.0001 & 3100 & & $4 \mathrm{~d}$ & $(5 / 2)^{2}[7 / 2]_{4}$ & $4 \mathrm{f}$ & $(3 / 2)^{2}[9 / 2]^{\circ} 5$ & & & $\mathrm{R} 1 \mathrm{c}$ & & \\
\hline $4609.416(3)$ & $21688.646(14)$ & $4609.3996(6)$ & 0.016 & 560 & & $\mathrm{sp}$ & $\left({ }^{3} \mathrm{~F}\right)^{3} \mathrm{P}^{\circ} \mathrm{D}^{\circ}{ }_{2}$ & $5 \mathrm{~d}$ & $(3 / 2)^{2}[7 / 2]_{3}$ & & & R1c & & $\mathrm{x}$ \\
\hline $4619.659(3)$ & $21640.559(15)$ & $4619.6507(6)$ & 0.008 & 82 & & $4 \mathrm{~d}$ & $(5 / 2)^{2}[5 / 2]_{2}$ & $4 \mathrm{f}$ & $(3 / 2)^{2}[3 / 2]^{\circ}{ }_{1}$ & & & R1c & & \\
\hline $4625.097(3)$ & $21615.114(13)$ & $4625.1004(5)$ & -0.003 & 2100 & & $4 \mathrm{~d}$ & $(5 / 2)^{2}[5 / 2]_{2}$ & $4 \mathrm{f}$ & $(3 / 2)^{2}[3 / 2]^{0}{ }^{1}$ & & & R1c & & \\
\hline $4631.801(3)$ & $21583.829(16)$ & $4631.8076(7)$ & -0.007 & 90 & & $4 \mathrm{~d}$ & $(3 / 2)^{2}[7 / 2]_{3}$ & $\mathrm{sp}$ & $\left({ }^{1} \mathrm{G}\right)^{3} \mathrm{P}^{\circ}{ }^{3} \mathrm{~F}_{3}^{\circ}$ & & & R1c & & \\
\hline $4635.260(3)$ & 21567.722(12) & $4635.2599(16)$ & 0.000 & 230 & & $4 d$ & $(5 / 2)^{2}[7 / 2]_{4}$ & $\mathrm{sp}$ & $\left({ }^{1} \mathrm{G}\right)^{3} \mathrm{P}^{\circ}{ }^{3} \mathrm{~F}_{4}^{\circ}$ & & & $\mathrm{R} 1 \mathrm{c}$ & & \\
\hline $4639.5854(16)$ & $21547.616(8)$ & $4639.5835(9)$ & 0.0019 & 1100 & & $4 \mathrm{~d}$ & $(5 / 2)^{2}[3 / 2]_{1}$ & $\mathrm{sp}$ & $\left({ }^{1} \mathrm{G}\right)^{3} \mathrm{P}^{\circ} 3^{3} \mathrm{~F}_{2}^{\circ}$ & & & R1nc & & \\
\hline $4647.012(5)$ & $21513.180(21)$ & $4647.0121(12)$ & -0.000 & 950 & & $4 \mathrm{~d}$ & $(5 / 2)^{2}[9 / 2]_{5}$ & $\mathrm{sp}$ & $\left({ }^{1} \mathrm{G}\right){ }^{3} \mathrm{P}^{\circ}{ }^{3} \mathrm{H}^{\circ}{ }_{5}$ & & & R1c & & \\
\hline $4649.2705(16)$ & $21502.730(7)$ & $4649.27084(23)$ & -0.0003 & 6700 & & $4 \mathrm{~d}$ & $(5 / 2)^{2}[1 / 2]_{1}$ & $4 \mathrm{f}$ & $(5 / 2)^{2}[5 / 2]^{\circ} 2$ & & & R1c & & \\
\hline $4660.3044(17)$ & $21451.820(8)$ & $4660.3070(7)$ & -0.0026 & 5600 & & $4 \mathrm{~d}$ & $(5 / 2)^{2}[1 / 2]_{0}$ & $4 \mathrm{f}$ & $(3 / 2)^{2}[3 / 2]^{0}{ }_{1}$ & & & R1c & & \\
\hline $4661.3627(16)$ & $21446.950(7)$ & $4661.3620(3)$ & 0.0007 & 7100 & & $4 \mathrm{~d}$ & $(5 / 2)^{2}[1 / 2]_{1}$ & $4 \mathrm{f}$ & $(5 / 2)^{2}[3 / 2]^{\circ} 1$ & & & R1c & & \\
\hline 4662.654(3) & $21441.010(12)$ & $4662.6475(11)$ & 0.007 & 13000 & & $4 \mathrm{~d}$ & $(5 / 2)^{2}[1 / 2]_{1}$ & $\mathrm{sp}$ & $\left({ }^{3} \mathrm{P}\right)^{3} \mathrm{P}^{0}{ }^{5} \mathrm{~S}^{\circ}{ }_{2}$ & & & $\mathrm{R} 1 \mathrm{c}$ & & \\
\hline $4667.315(2)$ & 21419.599(11) & $4667.3119(12)$ & 0.003 & 11000 & & $4 \mathrm{~d}$ & $(5 / 2)^{2}[9 / 2]_{4}$ & $\mathrm{sp}$ & $\left({ }^{1} \mathrm{G}\right)^{3} \mathrm{P}^{\circ}{ }^{3} \mathrm{H}^{\circ}{ }_{5}$ & & & R1c & & \\
\hline $4671.7016(2)$ & 21399.4867(11) & $4671.70176(20)$ & -0.0002 & 29000 & & $4 \mathrm{~d}$ & $(5 / 2)^{2}[1 / 2]_{1}$ & $4 f$ & $(5 / 2)^{2}[3 / 2]^{\circ}{ }_{2}$ & $5.6 \mathrm{e}+07$ & $\mathrm{D}+$ & F_Re & TW & \\
\hline $4673.5772(5)$ & $21390.8989(25)$ & 4673.5774(5) & -0.0002 & 20000 & & $4 \mathrm{~d}$ & $(5 / 2)^{2}[1 / 2]_{1}$ & $4 \mathrm{f}$ & $(5 / 2)^{2}[1 / 2]^{\circ} 0$ & $1.6 \mathrm{e}+08$ & $\mathrm{D}+$ & F_Re & TW & $\mathrm{L}$ \\
\hline $4681.9938(5)$ & $21352.4459(22)$ & 4681.9935(3) & 0.0003 & 36000 & & $4 \mathrm{~d}$ & $(5 / 2)^{2}[1 / 2]_{1}$ & $4 \mathrm{f}$ & $(5 / 2)^{2}[1 / 2]^{0}{ }_{1}$ & $1.1 \mathrm{e}+08$ & $\mathrm{D}+$ & F_Re & TW & $\mathrm{L}$ \\
\hline $4687.7662(18)$ & 21326.153(8) & $4687.7648(4)$ & 0.0014 & 3000 & & $5 p$ & $(3 / 2)^{2}[3 / 2]^{\circ} 2$ & $7 \mathrm{~s}$ & $(5 / 2)^{2}[5 / 2]_{2}$ & & & $\mathrm{R} 1 \mathrm{c}$ & & \\
\hline $4702.125(3)$ & 21261.032(13) & 4702.1291(9) & -0.004 & 550 & & $4 d$ & $(5 / 2)^{2}[5 / 2]_{2}$ & $\mathrm{sp}$ & $\left({ }^{3} \mathrm{P}\right)^{3} \mathrm{P}^{\circ}{ }^{1} \mathrm{D}_{2}{ }_{2}$ & & & $\mathrm{R} 1 \mathrm{c}$ & & \\
\hline 4737.909(4) & $21100.456(16)$ & $4737.9023(11)$ & 0.006 & 170 & & $4 \mathrm{~d}$ & $(3 / 2)^{2}[3 / 2]_{1}$ & $6 p$ & $(5 / 2)^{2}[5 / 2]^{\circ} 2$ & & & R1c & & \\
\hline $4744.588(3)$ & 21070.751(11) & $4744.5892(8)$ & -0.001 & 520 & & $\mathrm{sp}$ & $\left({ }^{3} \mathrm{~F}\right)^{3} \mathrm{P}^{0}{ }^{3} \mathrm{D}^{\circ}{ }_{1}$ & $5 \mathrm{~d}$ & $(3 / 2)^{2}[5 / 2]_{2}$ & & & R1c & & \\
\hline $4753.4684(16)$ & $21031.389(7)$ & 4753.4691(7) & -0.0007 & 2100 & & $4 \mathrm{~d}$ & $(5 / 2)^{2}[9 / 2]_{4}$ & $\mathrm{sp}$ & $\left({ }^{1} \mathrm{G}\right)^{3} \mathrm{P}^{0}{ }^{3} \mathrm{H}^{\circ}{ }_{4}$ & & & $\mathrm{R} 1 \mathrm{c}$ & & \\
\hline $4758.4334(9)$ & $21009.445(4)$ & 4758.4334(7) & 0.0000 & 15000 & & $4 p$ & ${ }^{3} \mathrm{P}^{\circ}$ & $s^{2}$ & ${ }^{3} \mathrm{P}_{0}$ & $1.3 e+06$ & $\mathrm{C}$ & F_Re & H71,N88 & \\
\hline $4765.913(3)$ & 20976.472(14) & $4765.9115(6)$ & 0.002 & 62 & & $\mathrm{sp}$ & $\left({ }^{3} \mathrm{~F}\right)^{3} \mathrm{P}^{\circ}{ }^{3} \mathrm{~F}_{3}^{\circ}$ & $5 \mathrm{~d}$ & $(3 / 2)^{2}[5 / 2]_{3}$ & & & $\mathrm{R} 1 \mathrm{c}$ & & \\
\hline $4766.7392(16)$ & $20972.837(7)$ & $4766.7394(9)$ & -0.0002 & 4500 & & $4 d$ & $(5 / 2)^{2}[1 / 2]_{1}$ & $\mathrm{sp}$ & $\left({ }^{3} \mathrm{P}\right)^{3} \mathrm{P}^{\circ}{ }^{3} \mathrm{P}^{\circ}{ }_{0}$ & & & $\mathrm{R} 1 \mathrm{c}$ & & \\
\hline 4772.9651(17) & 20945.481(7) & $4772.9656(5)$ & -0.0005 & 590 & & $4 \mathrm{~d}$ & $(3 / 2)^{2}[1 / 2]_{1}$ & $4 f$ & $(3 / 2)^{2}[5 / 2]^{0} 2$ & & & R1c & & \\
\hline 4781.0855(17) & $20909.907(8)$ & $4781.0769(8)$ & 0.0086 & 670 & & $\mathrm{sp}$ & $\left({ }^{3} \mathrm{~F}\right)^{3} \mathrm{P}^{0}{ }^{3} \mathrm{D}^{\circ}{ }_{1}$ & $5 \mathrm{~d}$ & $(3 / 2)^{2}[3 / 2]_{2}$ & & & R1c & & $\mathrm{x}$ \\
\hline $4792.709(3)$ & 20859.194(14) & $4792.7117(21)$ & -0.002 & 430 & & $5 s$ & $(3 / 2)^{2}[3 / 2]_{1}$ & $\mathrm{sp}$ & $\left({ }^{3} \mathrm{P}\right)^{3} \mathrm{P}^{\circ}{ }^{\circ} \mathrm{D}^{\circ}{ }_{2}$ & & & R1c & & \\
\hline
\end{tabular}


Table A1. Cont.

\begin{tabular}{|c|c|c|c|c|c|c|c|c|c|c|c|c|c|c|}
\hline$\lambda_{\text {obs }}{ }^{\text {a }(\AA)}$ & $\sigma_{\mathrm{obs}}{ }^{\mathrm{b}}\left(\mathrm{cm}^{-1}\right)$ & $\lambda_{\text {Ritz }}{ }^{c}(\AA)$ & $\begin{array}{c}\Delta \lambda_{\text {obs-Ritz }} \\
\text { (A) }\end{array}$ & $\begin{array}{c}I_{\text {obs }} \mathrm{d} \\
\text { (arb. u.) }\end{array}$ & Char ${ }^{e}$ & & Lower Level & & Upper Level & $A\left(\mathrm{~s}^{-1}\right)$ & $\operatorname{Acc}^{f}$ & Line Ref. $\mathrm{g}$ & TP Ref. $\mathrm{g}$ & Notes ${ }^{h}$ \\
\hline $4797.306(3)$ & 20839.208(14) & 4797.3080(18) & -0.002 & 150 & & $5 s$ & $(5 / 2)^{2}[5 / 2]_{3}$ & $\mathrm{sp}$ & $\left({ }^{1} \mathrm{D}\right)^{3} \mathrm{P}^{\circ}{ }^{3} \mathrm{D}^{\circ}{ }_{2}$ & & & R1c & & \\
\hline $4800.112(3)$ & 20827.027(11) & $4800.1106(8)$ & 0.001 & 590 & & $\mathrm{sp}$ & $\left({ }^{3} \mathrm{~F}\right)^{3} \mathrm{P}^{\circ}{ }^{3} \mathrm{D}^{\circ}{ }_{1}$ & $5 \mathrm{~d}$ & $(3 / 2)^{2}[3 / 2]$ & & & R1c & & \\
\hline $4800.584(3)$ & $20824.980(14)$ & $4800.5808(9)$ & 0.003 & 370 & & $4 \mathrm{~d}$ & $(5 / 2)^{2}[5 / 2]_{2}$ & $\mathrm{sp}$ & $\left({ }^{1} \mathrm{G}\right)^{3} \mathrm{P}^{\circ}{ }^{3} \mathrm{~F}_{2}^{\circ}$ & & & R1nc & & \\
\hline $4805.6557(19)$ & 20803.001(8) & $4805.6527(6)$ & 0.0030 & 3600 & & $4 \mathrm{~d}$ & $(5 / 2)^{2}[3 / 2]_{2}$ & $\mathrm{sp}$ & $\left({ }^{3} \mathrm{~F}\right)^{1} \mathrm{P}^{\circ}{ }^{3} \mathrm{~F}_{3}^{\circ}$ & & & R1c & & \\
\hline $4807.0463(17)$ & 20796.983(7) & $4807.0463(8)$ & 0.0000 & 7000 & & $4 \mathrm{~d}$ & $(3 / 2)^{2}[1 / 2]_{1}$ & $4 f$ & $(3 / 2)^{2}[3 / 2]^{\circ}$ & & & R1c & & \\
\hline 4811.1487(21) & 20779.250(9) & $4811.1475(6)$ & 0.0012 & 1000 & & $4 \mathrm{~d}$ & $(5 / 2)^{2}[9 / 2]_{4}$ & $\mathrm{sp}$ & $\left({ }^{3} \mathrm{~F}\right)^{1} \mathrm{P}^{\circ} 3^{3} \mathrm{~F}_{3}^{\circ}$ & & & R1c & & \\
\hline $4812.9476(5)$ & 20771.4831(22) & $4812.9474(4)$ & 0.0003 & 36000 & & $4 \mathrm{~d}$ & $(3 / 2)^{2}[1 / 2]_{1}$ & $4 f$ & $(3 / 2)^{2}[3 / 2]^{\circ} 2$ & $1.3 e+08$ & $\mathrm{D}+$ & F_Re & TW & \\
\hline $4814.868(4)$ & 20763.199(16) & $4814.868(4)$ & 0.000 & 310 & & $5 \mathrm{~s}$ & $\begin{array}{l}(5 / 2)^{2}[5 / 2]_{3} \\
\end{array}$ & $\mathrm{sp}$ & $\left.\left({ }^{1} \mathrm{D}\right)\right)^{3} \mathrm{P}^{\circ}{ }^{3} \mathrm{~F}_{4}^{\circ}$ & & & $\mathrm{R} 1 \mathrm{c}$ & & \\
\hline $4820.269(3)$ & 20739.934(15) & $4820.2672(6)$ & 0.002 & 160 & & $\mathrm{sp}$ & $\left({ }^{3} \mathrm{~F}\right)^{3} \mathrm{P}^{\circ}{ }^{3} \mathrm{~F}^{\circ}{ }_{3}$ & $5 \mathrm{~d}$ & $(3 / 2)^{2}[7 / 2]_{4}$ & & & $\mathrm{R} 1 \mathrm{c}$ & & \\
\hline $4829.3349(17)$ & 20701.001(7) & $4829.3340(3)$ & 0.0009 & 1100 & & $4 \mathrm{~d}$ & $(5 / 2)^{2}[9 / 2]_{5}$ & $4 \mathrm{f}$ & $(5 / 2)^{2}[9 / 2]^{\circ} 4$ & & & R1c & & \\
\hline 4832.2454(17) & 20688.533(7) & $4832.2439(5)$ & 0.0014 & 14000 & & $4 \mathrm{p}$ & ${ }^{3} \mathrm{P}^{\circ}$ & $s^{2}$ & ${ }^{3} \mathrm{P}_{1}$ & $2.5 \mathrm{e}+05$ & $\mathrm{C}$ & R1c & H71,N88 & \\
\hline 4833.761(3) & $20682.046(12)$ & $4833.7603(9)$ & 0.001 & 1000 & & $4 \mathrm{~d}$ & $(3 / 2)^{2}[1 / 2]_{1}$ & $\mathrm{sp}$ & $\left({ }^{3} \mathrm{~F}\right)^{1} \mathrm{P}^{\circ}{ }^{3} \mathrm{D}^{\circ}{ }_{1}$ & & & R1c & & \\
\hline 4834.673(3) & 20678.146(12) & $4834.6703(18)$ & 0.002 & 440 & & $5 \mathrm{~s}$ & $(3 / 2)^{2}[3 / 2]_{2}$ & $\mathrm{sp}$ & $\left({ }^{3} \mathrm{P}\right)^{3} \mathrm{P}^{\circ}{ }^{5} \mathrm{D}^{\circ}{ }_{3}$ & & & R1c & & \\
\hline 4836.799(2) & 20669.054(11) & 4836.7982(8) & 0.001 & 790 & & sp & $\left({ }^{3} \mathrm{~F}\right)^{3} \mathrm{P}^{\circ}{ }^{\circ} \mathrm{D}^{\circ}{ }_{3}$ & $5 \mathrm{~d}$ & $(5 / 2)^{2}[7 / 2]_{4}$ & & & R1c & & \\
\hline 4837.434(3) & $20666.341(15)$ & $4837.4350(20)$ & -0.001 & 360 & & $4 \mathrm{~d}$ & $(5 / 2)^{2}[1 / 2]_{0}$ & $\mathrm{sp}$ & $\left({ }^{3} \mathrm{P}\right)^{3} \mathrm{P}^{\circ}{ }^{1} \mathrm{P}^{\circ}{ }_{1}$ & & & $\mathrm{R} 2 \mathrm{nc}$ & & \\
\hline $4840.1833(17)$ & 20654.604(7) & $4840.1829(8)$ & 0.0005 & 1100 & & $4 \mathrm{~d}$ & $(3 / 2)^{2}[7 / 2]_{3}$ & $6 p$ & $(5 / 2)^{2}[7 / 2]^{0} 3$ & & & $\mathrm{R} 1 \mathrm{c}$ & & \\
\hline $4841.832(3)$ & 20647.573(15) & $4841.8320(24)$ & -0.000 & 91 & ? & $4 \mathrm{f}$ & $(5 / 2)^{2}[9 / 2]^{\circ}$ & $8 \mathrm{~g}$ & $(5 / 2)^{2}[11 / 2]_{6}$ & & & $\mathrm{R} 2 \mathrm{nc}$ & & \\
\hline $4847.3822(17)$ & $20623.930(7)$ & $4847.3823(8)$ & -0.0000 & 1500 & & $4 \mathrm{~d}$ & $(5 / 2)^{2}[1 / 2]_{1}$ & $\mathrm{sp}$ & $\left({ }^{3} \mathrm{P}\right)^{3} \mathrm{P}^{\circ}{ }^{3} \mathrm{P}^{\circ}{ }_{1}$ & & & R1c & & \\
\hline $4851.2625(11)$ & 20607.434(5) & 4851.2617(4) & 0.0008 & 12000 & & $4 \mathrm{~d}$ & $(5 / 2)^{2}[9 / 2]_{4}$ & $4 f$ & $(5 / 2)^{2}[9 / 2]^{\circ} 4$ & $2.9 \mathrm{e}+07$ & $\mathrm{D}+$ & F_Re & TW & \\
\hline $4854.98733(18)$ & 20591.6237(7) & $4854.98743(17)$ & -0.00011 & 34000 & & $4 \mathrm{~d}$ & $(5 / 2)^{2}[9 / 2]_{5}$ & $4 \mathrm{f}$ & $(5 / 2)^{2}[9 / 2]^{\circ}$ & $3.5 \mathrm{e}+07$ & $\mathrm{D}+$ & F_Re & TW & $\mathrm{L}$ \\
\hline $4859.068(7)$ & 20574.33(3) & 4859.0673(19) & 0.001 & 97 & & $5 \mathrm{~s}$ & $(3 / 2)^{2}[3 / 2]_{2}$ & $\mathrm{sp}$ & $\left({ }^{3} \mathrm{P}\right)^{3} \mathrm{P}^{\circ}{ }^{5} \mathrm{D}^{\circ}{ }_{1}$ & & & $\mathrm{R} 1 \mathrm{c}$ & & \\
\hline $4861.5612(18)$ & $20563.780(8)$ & $4861.56049(20)$ & 0.0008 & 3600 & & $4 \mathrm{~d}$ & $(5 / 2)^{2}[9 / 2]_{5}$ & $4 f$ & $(5 / 2)^{2}[7 / 2]^{\circ} 4$ & & & R1c & & \\
\hline $4866.254(3)$ & 20543.951(12) & $4866.2550(8)$ & -0.001 & 700 & & $\mathrm{sp}$ & $\left({ }^{3} \mathrm{~F}\right)^{3} \mathrm{P}^{\circ}{ }^{3} \mathrm{D}^{\circ}{ }_{3}$ & $5 \mathrm{~d}$ & $(5 / 2)^{2}[5 / 2]_{3}$ & & & R1c & & \\
\hline 4873.3056(17) & $20514.223(7)$ & $4873.3035(5)$ & 0.0021 & 9900 & & $4 \mathrm{~d}$ & $(3 / 2)^{2}[7 / 2]_{3}$ & $4 f$ & $(3 / 2)^{2}[7 / 2]^{0} 3$ & $2.4 \mathrm{e}+07$ & $\mathrm{D}+$ & F_Re & TW & \\
\hline 4877.151(3) & 20498.050(13) & 4877.1492(3) & 0.002 & 100 & & $4 \mathrm{~d}$ & $(5 / 2)^{2}[9 / 2]_{4}$ & $4 f$ & $(5 / 2)^{2}[9 / 2]^{\circ}$ & & & R1c & & \\
\hline $4883.2352(17)$ & $20472.510(7)$ & $4883.2351(4)$ & 0.0001 & 2900 & & $4 \mathrm{~d}$ & $(3 / 2)^{2}[7 / 2]_{3}$ & $4 \mathrm{f}$ & $(3 / 2)^{2}[7 / 2]^{\circ}{ }_{4}$ & & & R1c & & \\
\hline 4883.7832(17) & $20470.213(7)$ & $4883.7825(3)$ & 0.0007 & 2900 & & $4 \mathrm{~d}$ & $(5 / 2)^{2}[9 / 2]_{4}$ & $4 f$ & $(5 / 2)^{2}[7 / 2]^{\circ} 4$ & & & $\mathrm{R} 1 \mathrm{c}$ & & \\
\hline $4889.7005(17)$ & 20445.441(7) & 4889.6998(5) & 0.0008 & 12000 & & $4 p$ & ${ }^{3} \mathrm{P}^{\circ}{ }_{1}$ & $s^{2}$ & ${ }^{3} \mathrm{P}_{2}$ & $1.9 \mathrm{e}+05$ & $\mathrm{C}$ & R1c & H71,N88 & \\
\hline $4893.506(3)$ & 20429.543(11) & 4893.5089(5) & -0.003 & 770 & & $4 \mathrm{~d}$ & $(3 / 2)^{2}[7 / 2]_{3}$ & $4 f$ & $(3 / 2)^{2}[5 / 2]^{\circ}{ }_{2}$ & & & R1c & & \\
\hline 4896.414(3) & 20417.408(14) & $4896.4163(10)$ & -0.002 & 1900 & & $4 \mathrm{~d}$ & $(3 / 2)^{2}[1 / 2]_{1}$ & $\mathrm{sp}$ & $\left({ }^{3} \mathrm{P}\right)^{3} \mathrm{P}^{\circ} 1^{\circ} \mathrm{D}_{2}$ & & & R1c & & \\
\hline $4900.472(3)$ & 20400.501(11) & 4900.4712(9) & 0.001 & 920 & & $\mathrm{sp}$ & $\left({ }^{3} \mathrm{~F}\right)^{3} \mathrm{P}^{\circ}{ }^{3} \mathrm{G}^{\circ}{ }_{3}$ & $5 \mathrm{~d}$ & $(5 / 2)^{2}[7 / 2]_{4}$ & & & R1c & & \\
\hline $4901.42635(21)$ & 20396.5291(9) & 4901.42634(15) & 0.00002 & 26000 & & $4 \mathrm{~d}$ & $(5 / 2)^{2}[3 / 2]_{2}$ & $4 \mathrm{f}$ & $(5 / 2)^{2}[5 / 2]^{\circ} 3$ & $6.2 \mathrm{e}+07$ & D+ & F_Re & TW & \\
\hline 4904.534(4) & 20383.605(16) & $4904.5146(21)$ & 0.020 & 110 & ? & $4 \mathrm{f}$ & $(5 / 2)^{2}[3 / 2]^{\circ} 1$ & $9 \mathrm{~s}$ & $(3 / 2)^{2}[3 / 2]_{2}$ & & & $\mathrm{R} 1 \mathrm{c}$ & & $\mathrm{x}$ \\
\hline $4905.768(3)$ & 20378.478(11) & 4905.7667(5) & 0.001 & 580 & & $4 \mathrm{~d}$ & $(3 / 2)^{2}[7 / 2]_{4}$ & $4 \mathrm{f}$ & $(3 / 2)^{2}[7 / 2]^{\circ}{ }_{3}$ & & & R1c & & \\
\hline $4906.5663(3)$ & 20375.1629(11) & $4906.56612(16)$ & 0.0001 & 21000 & & $4 \mathrm{~d}$ & $(5 / 2)^{2}[3 / 2]_{2}$ & $4 \mathrm{f}$ & $(5 / 2)^{2}[5 / 2]^{\circ} 2$ & $4.4 \mathrm{e}+07$ & $\mathrm{D}+$ & F_Re & TW & \\
\hline 4907.1427(24) & $20372.770(10)$ & 4907.1424(4) & 0.0002 & 1000 & & $4 \mathrm{~d}$ & $(5 / 2)^{2}[9 / 2]_{4}$ & $4 f$ & $(5 / 2)^{2}[5 / 2]^{\circ}{ }_{3}$ & & & $\mathrm{R} 1 \mathrm{c}$ & & \\
\hline $4908.2819(24)$ & 20368.041(10) & 4908.2838(8) & -0.0019 & 970 & & $4 \mathrm{~d}$ & $\begin{array}{l}(5 / 2)^{2}[7 / 2]_{3} \\
\end{array}$ & $\mathrm{sp}$ & $\left({ }^{1} \mathrm{G}\right)^{3} \mathrm{P}^{\circ}{ }^{3} \mathrm{H}^{\circ}{ }_{4}^{\circ}$ & & & F_Re & & \\
\hline $4909.0397(13)$ & 20364.897(5) & $4909.0390(5)$ & 0.0007 & 6100 & & $4 \mathrm{~d}$ & $(5 / 2)^{2}[9 / 2]_{5}$ & $4 f$ & $(5 / 2)^{2}[11 / 2]^{\circ}{ }_{5}$ & & & $\mathrm{~F} R \mathrm{Re}$ & & \\
\hline 4909.73351(2) & $20362.01913(10)$ & $4909.733510(20)$ & 0.00000 & 160000 & & $4 \mathrm{~d}$ & $(5 / 2)^{2}[9 / 2]_{5}$ & $4 f$ & $(5 / 2)^{2}[11 / 2]_{6}^{\circ}$ & $2.04 \mathrm{e}+08$ & $\mathrm{~B}+$ & F_Re & C84 & $\mathrm{L}$ \\
\hline $4912.3645(5)$ & 20351.1138(21) & 4912.3644(3) & 0.0001 & 17000 & & $4 \mathrm{~d}$ & $(5 / 2)^{2}[3 / 2]_{2}$ & $4 \mathrm{f}$ & $(5 / 2)^{2}[7 / 2]^{\circ}{ }_{3}^{\circ}$ & & & F_Re & & \\
\hline 4912.91989(16) & $20348.8131(7)$ & 4912.91987(14) & 0.00002 & 29000 & & $4 \mathrm{~d}$ & $(5 / 2)^{2}[3 / 2]_{1}$ & $4 \mathrm{f}$ & $(5 / 2)^{2}[5 / 2]^{\circ} 2$ & $6.0 \mathrm{e}+07$ & $\mathrm{D}+$ & F_Re & TW & \\
\hline 4915.8321(17) & $20336.758(7)$ & 4915.8312(4) & 0.0009 & 11000 & & $4 \mathrm{~d}$ & $(3 / 2)^{2}[7 / 2]_{4}$ & $4 f$ & $(3 / 2)^{2}[7 / 2]^{\circ}{ }_{4}$ & $2.5 \mathrm{e}+07$ & $\mathrm{D}+$ & F_Re & TW & \\
\hline 4918.1079(21) & 20327.348(9) & 4918.1061(4) & 0.0019 & 2400 & & $4 \mathrm{~d}$ & $\begin{array}{l}(5 / 2)^{2}[9 / 2]_{4} \\
\end{array}$ & $4 f$ & $(5 / 2)^{2}[7 / 2]^{\circ}{ }^{\circ}$ & & & R1c & & \\
\hline
\end{tabular}


Table A1. Cont.

\begin{tabular}{|c|c|c|c|c|c|c|c|c|c|c|c|c|c|c|}
\hline$\lambda_{\text {obs }}{ }^{\text {a }}(\AA)$ & $\sigma_{\mathrm{obs}}{ }^{\mathrm{b}}\left(\mathrm{cm}^{-1}\right)$ & $\lambda_{\text {Ritz }}{ }^{c}(\AA ̊)$ & $\begin{array}{c}\Delta \lambda_{\text {obs-Ritz }} \\
(\mathrm{A})\end{array}$ & $\begin{array}{l}I_{\text {obs }} \mathrm{d} \\
\text { (arb. u.) }\end{array}$ & Char ${ }^{e}$ & & wer Level & & Upper Level & $A\left(\mathrm{~s}^{-1}\right)$ & $\operatorname{Acc}^{f}$ & Line Ref. $\mathrm{g}$ & TP Ref. $\mathrm{g}$ & Notes ${ }^{h}$ \\
\hline $4918.3779(5)$ & $20326.2320(20)$ & $4918.3778(4)$ & 0.0000 & 54000 & & $4 \mathrm{~d}$ & $(3 / 2)^{2}[7 / 2]_{3}$ & $4 f$ & $(3 / 2)^{2}[9 / 2]^{\circ}{ }_{4}$ & $2.9 \mathrm{e}+08$ & C & F_Re & H71 & \\
\hline $4920.0348(12)$ & 20319.387(5) & $4920.0345(4)$ & 0.0003 & 6800 & & $4 \mathrm{~d}$ & $(5 / 2)^{2}[3 / 2]_{2}$ & $4 f$ & $(5 / 2)^{2}[3 / 2]^{\circ} 1$ & & & F_Re & & \\
\hline $4921.4627(21)$ & 20313.492(9) & $4921.4666(12)$ & -0.0039 & 5900 & & $4 \mathrm{~d}$ & $(5 / 2)^{2}[3 / 2]_{2}$ & $\mathrm{sp}$ & $\left({ }^{3} \mathrm{P}\right)^{3} \mathrm{P}^{0}{ }^{5} \mathrm{~S}^{\circ}{ }_{2}$ & & & F_Re & & \\
\hline $4926.4228(5)$ & $20293.0397(22)$ & 4926.4232(3) & -0.0004 & 26000 & & $4 \mathrm{~d}$ & $(5 / 2)^{2}[3 / 2]_{1}$ & $4 f$ & $(5 / 2)^{2}[3 / 2]^{\circ} 1$ & $9 . e+07$ & $\mathrm{D}+$ & F_Re & TW & \\
\hline 4926.811(4) & 20291.440(17) & $4926.8186(15)$ & -0.007 & 740 & & $\mathrm{sp}$ & $\left({ }^{3} \mathrm{~F}\right)^{3} \mathrm{P}^{\circ}{ }^{5} \mathrm{~F}_{4}^{\circ}$ & $6 \mathrm{~s}$ & $(5 / 2)^{2}[5 / 2]_{3}$ & & & R1c & & \\
\hline 4927.860(3) & 20287.121(14) & $4927.8590(12)$ & 0.001 & 830 & & $4 \mathrm{~d}$ & $(5 / 2)^{2}[3 / 2]_{1}$ & $\mathrm{sp}$ & $\left({ }^{3} \mathrm{P}\right)^{3} \mathrm{P}^{\circ}{ }^{5} \mathrm{~S}^{\circ}{ }_{2}$ & & & R1c & & \\
\hline 4930.713(3) & $20275.383(11)$ & $4930.7110(8)$ & 0.002 & 620 & & $\mathrm{sp}$ & $\left({ }^{3} \mathrm{~F}\right)^{3} \mathrm{P}^{\circ}{ }^{3} \mathrm{G}_{3}^{\circ}$ & $5 \mathrm{~d}$ & $(5 / 2)^{2}[5 / 2]_{3}$ & & & $\mathrm{R} 1 \mathrm{c}$ & & \\
\hline $4931.55496(20)$ & $20271.9213(8)$ & $4931.55505(16)$ & -0.00009 & 28000 & & $4 \mathrm{~d}$ & $(5 / 2)^{2}[3 / 2]_{2}$ & $4 \mathrm{f}$ & $(5 / 2)^{2}[3 / 2]^{\circ} 2$ & $7.1 \mathrm{e}+07$ & $\mathrm{D}+$ & F_Re & TW & \\
\hline 4931.6982(5) & $20271.3325(20)$ & 4931.6981(4) & 0.0001 & 140000 & & $4 \mathrm{~d}$ & $(5 / 2)^{2}[9 / 2]_{4}$ & $4 f$ & $(5 / 2)^{2}[11 / 2]_{5}^{\circ}$ & $1.9 e+08$ & $\mathrm{C}$ & F_Re & H71 & $\mathrm{L}$ \\
\hline $4933.0777(24)$ & $20265.664(10)$ & 4933.0745(5) & 0.0032 & 1100 & & $4 \mathrm{~d}$ & $(5 / 2)^{2}[9 / 2]_{5}$ & $\mathrm{sp}$ & $\left({ }^{3} \mathrm{~F}\right)^{1} \mathrm{P}^{\circ}{ }^{3} \mathrm{G}^{\circ}{ }_{4}$ & & & $\mathrm{R} 1 \mathrm{c}$ & & \\
\hline $4937.22029(23)$ & $20248.6601(9)$ & $4937.22031(22)$ & -0.00002 & 26000 & & $4 \mathrm{~d}$ & $(3 / 2)^{2}[3 / 2]_{1}$ & $4 f$ & $(3 / 2)^{2}[5 / 2]^{\circ} 2$ & $1.1 \mathrm{e}+08$ & $\mathrm{D}+$ & F_Re & TW & \\
\hline 4937.516(3) & 20247.449(11) & 4937.5188(4) & -0.003 & 1100 & & $4 \mathrm{~d}$ & $(3 / 2)^{2}[7 / 2]_{4}$ & $4 \mathrm{f}$ & $(3 / 2)^{2}[5 / 2]^{\circ} 3$ & & & $\mathrm{R} 1 \mathrm{c}$ & & \\
\hline 4937.9740(4) & $20245.5693(16)$ & 4937.97372(21) & 0.0003 & 16000 & & $4 \mathrm{~d}$ & $(5 / 2)^{2}[3 / 2]_{1}$ & $4 \mathrm{f}$ & $(5 / 2)^{2}[3 / 2]^{\circ}{ }_{2}$ & $2.8 \mathrm{e}+07$ & $\mathrm{D}+$ & F_Re & TW & \\
\hline $4939.648(3)$ & 20238.709(11) & $4939.6526(8)$ & -0.005 & 1200 & & $4 \mathrm{~d}$ & $(3 / 2)^{2}[3 / 2]_{2}$ & $6 p$ & $(5 / 2)^{2}[7 / 2]^{\circ} 3$ & & & $\mathrm{R} 1 \mathrm{c}$ & & \\
\hline $4940.0695(17)$ & $20236.982(7)$ & $4940.0693(6)$ & 0.0002 & 4700 & & $4 \mathrm{~d}$ & $(5 / 2)^{2}[3 / 2]_{1}$ & $4 f$ & $(5 / 2)^{2}[1 / 2]^{\circ} 0$ & & & $\mathrm{R} 1 \mathrm{c}$ & & \\
\hline $4940.912(8)$ & 20233.53(3) & $4940.9093(20)$ & 0.003 & 130 & & $5 s$ & $(5 / 2)^{2}[5 / 2]_{2}$ & $\mathrm{sp}$ & $\left({ }^{1} \mathrm{D}\right)^{3} \mathrm{P}^{\circ}{ }^{3} \mathrm{D}^{\circ}{ }_{1}$ & & & $\mathrm{R} 1 \mathrm{c}$ & & \\
\hline $4943.0240(7)$ & $20224.886(3)$ & $4943.0250(4)$ & -0.0010 & 20000 & & $4 \mathrm{~d}$ & $(5 / 2)^{2}[3 / 2]_{2}$ & $4 \mathrm{f}$ & $(5 / 2)^{2}[1 / 2]^{\circ} 1$ & $4.0 \mathrm{e}+07$ & $\mathrm{D}+$ & F_Re & TW & \\
\hline $4949.4750(18)$ & 20198.526(7) & 4949.4735(4) & 0.0015 & 3700 & & $4 \mathrm{~d}$ & $(5 / 2)^{2}[3 / 2]_{1}$ & $4 \mathrm{f}$ & $(5 / 2)^{2}[1 / 2]^{\circ} 1$ & & & $\mathrm{R} 1 \mathrm{c}$ & & \\
\hline 4951.4463(18) & 20190.484(7) & 4951.4464(6) & -0.0000 & 4200 & & $4 \mathrm{~d}$ & $(3 / 2)^{2}[7 / 2]_{4}$ & $4 f$ & $(3 / 2)^{2}[9 / 2]^{\circ} 4$ & & & F_Re & & \\
\hline 4951.6184(14) & 20189.783(6) & 4951.6201(3) & -0.0016 & 12000 & & $4 \mathrm{~d}$ & $(5 / 2)^{2}[5 / 2]_{3}$ & $4 \mathrm{f}$ & $(5 / 2)^{2}[9 / 2]^{\circ}{ }_{4}$ & & & F_Re & & \\
\hline $4953.7244(5)$ & 20181.1995(20) & 4953.7246(4) & -0.0002 & 82000 & & $4 \mathrm{~d}$ & $(3 / 2)^{2}[7 / 2]_{4}$ & $4 f$ & $(3 / 2)^{2}[9 / 2]^{\circ} 5$ & $3.1 \mathrm{e}+08$ & C & F_Re & H71 & \\
\hline $4955.9564(18)$ & $20172.111(7)$ & $4955.9566(5)$ & -0.0002 & 6300 & & $4 \mathrm{~d}$ & $(5 / 2)^{2}[9 / 2]_{4}$ & $\mathrm{sp}$ & $\left({ }^{3} \mathrm{~F}\right)^{1} \mathrm{P}^{\circ}{ }^{3} \mathrm{G}_{4}^{\circ}$ & & & $\mathrm{R} 1 \mathrm{c}$ & & \\
\hline $4969.8062(18)$ & $20115.896(7)$ & $4969.8046(6)$ & 0.0016 & 4400 & & $4 \mathrm{~d}$ & $(5 / 2)^{2}[7 / 2]_{3}$ & $\mathrm{sp}$ & $\left({ }^{3} \mathrm{~F}\right){ }^{1} \mathrm{P}^{\circ}{ }^{3} \mathrm{~F}^{\circ}{ }_{3}$ & $1.1 \mathrm{e}+07$ & $\mathrm{D}+$ & R1c & TW & \\
\hline 4971.222(4) & $20110.167(15)$ & 4971.2261(9) & -0.004 & 280 & & $\mathrm{sp}$ & $\left({ }^{3} \mathrm{~F}\right)^{3} \mathrm{P}^{\circ}{ }^{3} \mathrm{G}_{3}^{\circ}$ & $5 \mathrm{~d}$ & $(5 / 2)^{2}[3 / 2]_{2}$ & & & R1c & & \\
\hline 4973.136(3) & $20102.429(12)$ & 4973.1394(7) & -0.004 & 280 & & $\mathrm{sp}$ & $\left({ }^{3} \mathrm{~F}\right)^{3} \mathrm{P}^{\circ} 3^{3} \mathrm{~F}^{\circ}{ }_{2}$ & $5 \mathrm{~d}$ & $(3 / 2)^{2}[5 / 2]_{2}$ & & & R1c & & \\
\hline 4973.6975(19) & $20100.158(8)$ & 4973.6959(8) & 0.0016 & 9000 & & $4 \mathrm{~d}$ & $(3 / 2)^{2}[3 / 2]_{1}$ & $4 \mathrm{f}$ & $(3 / 2)^{2}[3 / 2]^{\circ} 1$ & $3.6 \mathrm{e}+07$ & D+ & F_Re & TW & \\
\hline 4974.1542(15) & $20098.313(6)$ & 4974.1533(5) & 0.0008 & 9700 & & $4 \mathrm{~d}$ & $(3 / 2)^{2}[3 / 2]_{2}$ & $4 f$ & $(3 / 2)^{2}[7 / 2]^{\circ}{ }_{3}$ & $3.0 \mathrm{e}+07$ & $\mathrm{D}+$ & F_Re & TW & \\
\hline 4975.064(3) & 20094.637(12) & $4975.0659(8)$ & -0.002 & 560 & & $4 \mathrm{~d}$ & $(5 / 2)^{2}[3 / 2]_{2}$ & $\mathrm{sp}$ & $\left({ }^{3} \mathrm{~F}\right)^{1} \mathrm{P}^{\circ}{ }^{3} \mathrm{D}_{3}^{\circ}$ & & & $\mathrm{R} 1 \mathrm{c}$ & & \\
\hline $4980.0153(19)$ & $20074.659(8)$ & $4980.0135(6)$ & 0.0018 & 11000 & & $4 \mathrm{~d}$ & $(3 / 2)^{2}[3 / 2]_{1}$ & $4 f$ & $(3 / 2)^{2}[3 / 2]^{\circ}{ }_{2}$ & & & R1c & & \\
\hline 4981.0125(23) & $20070.640(9)$ & $4981.0133(6)$ & -0.0008 & 670 & & $4 \mathrm{~d}$ & $(5 / 2)^{2}[7 / 2]_{4}$ & $\mathrm{sp}$ & $\left({ }^{3} \mathrm{~F}\right)^{1} \mathrm{P}^{\mathrm{o} 3} \mathrm{~F}_{3}^{\circ}$ & & & R1c & & \\
\hline $4985.1421(23)$ & 20054.014(9) & 4985.1381(6) & 0.0040 & 5100 & & $4 \mathrm{~d}$ & $(5 / 2)^{2}[5 / 2]_{2}$ & $\mathrm{sp}$ & $\left({ }^{3} \mathrm{~F}\right)^{1} \mathrm{P}^{\circ} 3^{3} \mathrm{~F}_{3}^{\circ}$ & $2.1 \mathrm{e}+07$ & $\mathrm{D}+$ & R1c & TW & \\
\hline $4985.50499(7)$ & $20052.5541(3)$ & $4985.50498(7)$ & 0.00001 & 70000 & & $4 \mathrm{~d}$ & $(5 / 2)^{2}[5 / 2]_{3}$ & $4 \mathrm{f}$ & $(5 / 2)^{2}[7 / 2]^{\circ}{ }_{4}$ & $9.7 \mathrm{e}+07$ & $\mathrm{C}+$ & F_Re & TW & \\
\hline 4988.327(4) & $20041.212(15)$ & $4988.326(4)$ & 0.000 & 430 & & $5 \mathrm{~d}$ & $(5 / 2)^{2}[9 / 2]_{5}$ & $8 \mathrm{f}$ & $(5 / 2)^{2}[11 / 2]^{\circ} 6$ & $5.7 \mathrm{e}+06$ & $\mathrm{D}+$ & $\mathrm{R} 1 \mathrm{c}$ & TW & \\
\hline $4995.2054(22)$ & 20013.614(9) & 4995.2055(5) & -0.0001 & 800 & & $4 \mathrm{~d}$ & $(3 / 2)^{2}[3 / 2]_{2}$ & $4 f$ & $(3 / 2)^{2}[5 / 2]^{\circ} 2_{2}$ & & & R1c & & \\
\hline 4999.584(3) & 19996.088(12) & $4999.5826(20)$ & 0.001 & 440 & & $5 \mathrm{~d}$ & $(5 / 2)^{2}[9 / 2]_{4}$ & $8 \mathrm{f}$ & $(5 / 2)^{2}[11 / 2]_{5}^{\circ}$ & & & R1c & & \\
\hline $5002.294(3)$ & $19985.255(12)$ & $5002.2998(10)$ & -0.006 & 440 & & $4 \mathrm{~d}$ & $(3 / 2)^{2}[3 / 2]_{1}$ & $\mathrm{sp}$ & $\left({ }^{3} \mathrm{~F}\right)^{1} \mathrm{P}^{\circ}{ }^{3} \mathrm{D}^{\circ}{ }_{1}$ & & & $\mathrm{R} 1 \mathrm{c}$ & & \\
\hline $5006.79983(15)$ & $19967.2680(6)$ & $5006.79978(15)$ & 0.00005 & 46000 & & $4 \mathrm{~d}$ & $(3 / 2)^{2}[3 / 2]_{2}$ & $4 f$ & $(3 / 2)^{2}[5 / 2]^{0} 3$ & $1.20 \mathrm{e}+08$ & $\mathrm{C}_{+}$ & F_Re & TW & \\
\hline $5009.8505(3)$ & 19955.1095(10) & $5009.85058(17)$ & -0.0001 & 35000 & & $4 \mathrm{~d}$ & $(5 / 2)^{2}[5 / 2]_{3}$ & $4 \mathrm{f}$ & $(5 / 2)^{2}[5 / 2]^{\circ} 3$ & $5.4 \mathrm{e}+07$ & D+ & F_Re & TW & \\
\hline $5012.6197(3)$ & $19944.0855(12)$ & 5012.6199(3) & -0.0002 & 37000 & & $4 \mathrm{~d}$ & $(5 / 2)^{2}[7 / 2]_{3}$ & $4 f$ & $(5 / 2)^{2}[9 / 2]^{\circ} 4$ & $9.6 \mathrm{e}+07$ & $\mathrm{C}+$ & F_Re & TW & $\mathrm{L}$ \\
\hline $5015.2190(8)$ & 19933.749(3) & $5015.22038(21)$ & -0.0014 & 11000 & & $4 \mathrm{~d}$ & $(5 / 2)^{2}[5 / 2]_{3}$ & $4 \mathrm{f}$ & $(5 / 2)^{2}[5 / 2]^{\circ}{ }_{2}$ & & & F_Re & & \\
\hline $5020.126(3)$ & 19914.266(10) & $5020.1256(5)$ & 0.000 & 2000 & & $4 p$ & ${ }^{3} \mathrm{~F}_{3}^{\circ}$ & $\mathrm{s}^{2}$ & & & & $\mathrm{R} 1 \mathrm{c}$ & & \\
\hline $5021.2785(4)$ & $19909.6939(15)$ & $5021.27849(23)$ & 0.0000 & 32000 & & $4 d$ & $(5 / 2)^{2}[5 / 2]_{3}$ & $4 f$ & $(5 / 2)^{2}[7 / 2]^{\circ}{ }_{3}$ & $3.2 \mathrm{e}+07$ & E & F_Re & TW & $\mathrm{L}$ \\
\hline
\end{tabular}


Table A1. Cont.

\begin{tabular}{|c|c|c|c|c|c|c|c|c|c|c|c|c|c|c|}
\hline$\lambda_{\text {obs }}{ }^{\text {a }(\AA)}$ & $\sigma_{\mathrm{obs}}{ }^{\mathrm{b}}\left(\mathrm{cm}^{-1}\right)$ & $\lambda_{\text {Ritz }}{ }^{\mathrm{c}}(\AA)$ & $\begin{array}{c}\Delta \lambda_{\text {obs-Ritz }} \\
\text { (A) }\end{array}$ & $\begin{array}{c}I_{\text {obs }} \mathrm{d} \\
\text { (arb. u.) }\end{array}$ & Char ${ }^{e}$ & & ower Level & & Jpper Level & $A\left(\mathrm{~s}^{-1}\right)$ & $\operatorname{Acc}^{f}$ & Line Ref. $\mathrm{g}$ & TP Ref. $\mathrm{g}$ & Notes ${ }^{h}$ \\
\hline $5022.599(3)$ & 19904.459(11) & $5022.6044(7)$ & -0.005 & 760 & & $\mathrm{sp}$ & $\left({ }^{3} \mathrm{~F}\right)^{3} \mathrm{P}^{\circ}{ }^{3} \mathrm{D}^{\circ}{ }_{2}$ & $5 \mathrm{~d}$ & $(5 / 2)^{2}[7 / 2]_{3}$ & & & R1c & & \\
\hline $5024.0251(16)$ & $19898.810(6)$ & $5024.0227(3)$ & 0.0024 & 8700 & & $4 \mathrm{~d}$ & $(5 / 2)^{2}[7 / 2]_{4}$ & $4 \mathrm{f}$ & $(5 / 2)^{2}[9 / 2]^{\circ}{ }_{4}$ & & & $\mathrm{~F}$ Re & & \\
\hline $5030.789(3)$ & 19872.057(11) & $5030.7892(12)$ & -0.000 & 1400 & & $4 \mathrm{~d}$ & $(5 / 2)^{2}[5 / 2]_{3}$ & $\mathrm{sp}$ & $\left({ }^{3} \mathrm{P}\right)^{3} \mathrm{P}^{0}{ }^{5} \mathrm{~S}_{2}{ }_{2}$ & & & $\mathrm{R} 1 \mathrm{c}$ & & \\
\hline $5031.299(3)$ & $19870.040(10)$ & $5031.2968(8)$ & 0.003 & 1400 & & $4 \mathrm{~d}$ & $(3 / 2)^{2}[5 / 2]_{2}$ & $6 p$ & $(5 / 2)^{2}[7 / 2]^{\circ} 3$ & & & R1c & & \\
\hline $5032.544(3)$ & 19865.125(11) & $5032.5461(8)$ & -0.002 & 1100 & & $4 \mathrm{~d}$ & $\begin{array}{l}(3 / 2)^{2}[3 / 2]_{2} \\
\text { nat }\end{array}$ & $4 \mathrm{f}$ & $(3 / 2)^{2}[3 / 2]^{\circ}{ }_{1}^{\circ}$ & & & R1c & & \\
\hline 5034.171(3) & 19858.705(13) & $5034.1729(8)$ & -0.002 & 460 & & $\mathrm{sp}$ & $\left({ }^{3} \mathrm{~F}\right)^{3} \mathrm{P}^{\circ}{ }^{3} \mathrm{~F}^{\mathrm{O}}{ }_{2}$ & $5 \mathrm{~d}$ & $(3 / 2)^{2}[3 / 2]_{1}$ & & & R1c & & \\
\hline $5036.7302(21)$ & $19848.616(8)$ & $5036.7320(7)$ & -0.0019 & 540 & & $4 \mathrm{~d}$ & $(5 / 2)^{2}[1 / 2]_{1}$ & $\mathrm{sp}$ & $\left({ }^{3} \mathrm{P}\right)^{3} \mathrm{P}^{\circ}{ }^{3} \mathrm{D}^{\circ}{ }_{1}$ & & & $\mathrm{R} 1 \mathrm{c}$ & & \\
\hline $5039.0109(13)$ & 19839.632(5) & $5039.0141(6)$ & -0.0032 & 17000 & & $4 \mathrm{~d}$ & $(3 / 2)^{2}[3 / 2]_{2}$ & $4 \mathrm{f}$ & $(3 / 2)^{2}[3 / 2]^{1}{ }_{2}$ & $3.9 \mathrm{e}+07$ & $\mathrm{D}+$ & F_Re & TW & \\
\hline $5041.3310(9)$ & $19830.502(4)$ & $5041.33123(23)$ & -0.0003 & 14000 & & $4 \mathrm{~d}$ & $(5 / 2)^{2}[5 / 2]_{3}$ & $4 \mathrm{f}$ & $(5 / 2)^{2}[3 / 2]^{\circ} 2$ & $1.6 \mathrm{e}+07$ & $\mathrm{D}+$ & F_Re & TW & \\
\hline 5042.093(3) & 19827.505(11) & $5042.0348(18)$ & 0.058 & 310 & ? & $\mathrm{sp}$ & $\left({ }^{3} \mathrm{~F}\right)^{3} \mathrm{P}^{\circ}{ }^{1} \mathrm{G}^{\circ}{ }_{4}$ & $5 \mathrm{~d}$ & $(3 / 2)^{2}[7 / 2]_{3}$ & & & $\mathrm{R} 1 \mathrm{c}$ & & $\mathrm{x}$ \\
\hline 5044.2776(21) & $19818.918(8)$ & $5044.2768(10)$ & 0.0008 & 940 & & $4 \mathrm{~d}$ & $(5 / 2)^{2}[3 / 2]_{1}$ & $\mathrm{sp}$ & $\left({ }^{3} \mathrm{P}\right)^{3} \mathrm{P}^{\circ}{ }^{3} \mathrm{P}^{\circ} 0$ & & & R1c & & \\
\hline $5047.34772(23)$ & $19806.8629(9)$ & $5047.34770(18)$ & 0.00002 & 27000 & & $4 \mathrm{~d}$ & $(5 / 2)^{2}[7 / 2]_{3}$ & $4 \mathrm{f}$ & $(5 / 2)^{2}[7 / 2]^{\circ} 4$ & & & F_Re & & \\
\hline $5051.79210(4)$ & $19789.43784(14)$ & $5051.79209(4)$ & 0.00000 & 120000 & & $4 \mathrm{~d}$ & $(5 / 2)^{2}[7 / 2]_{4}$ & $4 \mathrm{f}$ & $(5 / 2)^{2}[9 / 2]^{\circ}$ & $1.55 \mathrm{e}+08$ & $\mathrm{C}+$ & F_Re & TW & $\mathrm{L}$ \\
\hline $5054.344(3)$ & $\begin{array}{l}19779.447(10) \\
\text { a }\end{array}$ & $5054.3487(6)$ & -0.005 & 950 & & sp & $\left({ }^{3} \mathrm{~F}\right)^{3} \mathrm{P}^{\circ} 3^{3} \mathrm{~F}^{\circ}{ }_{2}$ & $5 \mathrm{~d}$ & $(3 / 2)^{2}[7 / 2]_{3}$ & & & $\mathrm{R} 1 \mathrm{c}$ & & \\
\hline $5054.7758(20)$ & $19777.757(8)$ & $5054.7728(5)$ & 0.0030 & 1300 & & $4 \mathrm{~d}$ & $(3 / 2)^{2}[5 / 2]_{3}$ & $4 \mathrm{f}$ & $(3 / 2)^{2}[7 / 2]^{\circ} 3$ & & & $\mathrm{R} 1 \mathrm{c}$ & & \\
\hline $5058.90906(16)$ & 19761.5981(6) & $5058.90923(14)$ & -0.00017 & 48000 & & $4 \mathrm{~d}$ & $(5 / 2)^{2}[7 / 2]_{4}$ & $4 \mathrm{f}$ & $(5 / 2)^{2}[7 / 2]^{\circ} 4$ & $4.8 \mathrm{e}+07$ & $\mathrm{D}+$ & F_Re & TW & \\
\hline $5059.418(5)$ & 19759.611(21) & 5059.4131(13) & 0.005 & 160 & & $6 \mathrm{~s}$ & $(5 / 2)^{2}[5 / 2]_{2}$ & $6 \mathrm{f}$ & $(3 / 2)^{2}[5 / 2]_{2}{ }_{2}$ & & & R1c & & \\
\hline $5060.6437(9)$ & 19754.825(4) & $5060.6415(5)$ & 0.0022 & 16000 & & $4 p$ & ${ }^{3} \mathrm{P}_{0}^{\circ}$ & $\mathrm{s}^{2}$ & ${ }^{3} \mathrm{P}_{1}$ & $4.1 \mathrm{e}+05$ & $\mathrm{C}$ & F_Re & H71 & $\mathrm{L}$ \\
\hline $5065.45858(8)$ & $19736.0471(3)$ & $5065.45861(8)$ & -0.00002 & 70000 & & $4 \mathrm{~d}$ & $(3 / 2)^{2}[5 / 2]_{3}$ & $4 \mathrm{f}$ & $(3 / 2)^{2}[7 / 2]^{\circ} 4$ & $1.61 \mathrm{e}+08$ & $\mathrm{C}_{+}$ & F_Re & TW & \\
\hline $5067.09416(17)$ & 19729.6767(7) & $5067.09423(17)$ & -0.00008 & 46000 & & $4 \mathrm{~d}$ & $(3 / 2)^{2}[5 / 2]_{2}$ & $4 \mathrm{f}$ & $(3 / 2)^{2}[7 / 2]^{\circ} 3$ & $1.23 \mathrm{e}+08$ & $\mathrm{C}+$ & F_Re & TW & \\
\hline 5069.431(3) & 19720.584(11) & $5069.4315(10)$ & -0.001 & 1600 & & $4 \mathrm{~d}$ & $(3 / 2)^{2}[3 / 2]_{1}$ & $\mathrm{sp}$ & $\left({ }^{3} \mathrm{P}\right)^{3} \mathrm{P}^{\circ}{ }^{1} \mathrm{D}_{2}^{\circ}$ & & & $\mathrm{R} 1 \mathrm{c}$ & & \\
\hline $5072.3034(6)$ & $19709.4147(23)$ & $5072.30253(22)$ & 0.0008 & 32000 & & $4 \mathrm{~d}$ & $(5 / 2)^{2}[7 / 2]_{3}$ & $4 \mathrm{f}$ & $(5 / 2)^{2}[5 / 2]^{\circ} 3$ & & & F_Re & & \\
\hline $5076.5136(20)$ & 19693.069(8) & $5076.5143(5)$ & -0.0007 & 830 & & $4 \mathrm{~d}$ & $(3 / 2)^{2}[5 / 2]_{3}$ & $4 \mathrm{f}$ & $(3 / 2)^{2}[5 / 2]^{\circ}{ }_{2}$ & & & $\mathrm{R} 1 \mathrm{c}$ & & \\
\hline $5077.8070(18)$ & 19688.053(7) & $5077.8071(3)$ & -0.0001 & 6300 & & $4 \mathrm{~d}$ & $(5 / 2)^{2}[7 / 2]_{3}$ & $4 \mathrm{f}$ & $(5 / 2)^{2}[5 / 2]^{\circ} 2$ & & & R1c & & \\
\hline $5081.099(4)$ & $19675.296(14)$ & $5081.1053(16)$ & -0.006 & 330 & & $5 \mathrm{~s}$ & $(3 / 2)^{2}[3 / 2]_{1}$ & sp & $\left({ }^{1} \mathrm{D}\right)^{3} \mathrm{P}^{\circ} \mathrm{P}^{\circ}{ }_{1}^{\circ}$ & & & R1c & & \\
\hline $5082.525(4)$ & $19669.778(14)$ & $5082.5262(15)$ & -0.001 & 160 & & $5 p$ & $(5 / 2)^{2}[3 / 2]^{\circ}{ }_{1}$ & $5 d$ & $(3 / 2)^{2}[1 / 2]_{0}$ & & & R1c & & \\
\hline 5083.9795(12) & 19664.150(5) & $5083.97879(22)$ & 0.0007 & 21000 & & $4 \mathrm{~d}$ & $(5 / 2)^{2}[7 / 2]_{4}$ & $4 \mathrm{f}$ & $(5 / 2)^{2}[5 / 2]^{\circ}{ }_{3}$ & & & F_Re & & \\
\hline $5084.0173(5)$ & $19664.0034(18)$ & $5084.0175(3)$ & -0.0002 & 14000 & & $4 \mathrm{~d}$ & $(5 / 2)^{2}[7 / 2]_{3}$ & $4 \mathrm{f}$ & $(5 / 2)^{2}[7 / 2]^{\circ} 3$ & & & F_Re,R1r & & \\
\hline $5088.27603(10)$ & $19647.5455(4)$ & $5088.27603(9)$ & 0.00000 & 57000 & & $4 \mathrm{~d}$ & $(5 / 2)^{2}[5 / 2]_{2}$ & $4 \mathrm{f}$ & $(5 / 2)^{2}[5 / 2]^{\circ} 3$ & & & F_Re & & \\
\hline $5088.4882(8)$ & 19646.726(3) & $5088.4896(4)$ & -0.0013 & 25000 & & $4 \mathrm{~d}$ & $(3 / 2)^{2}[5 / 2]_{3}$ & $4 \mathrm{f}$ & $(3 / 2)^{2}[5 / 2]^{\circ}{ }_{3}$ & $4.8 \mathrm{e}+07$ & $\mathrm{D}+$ & F_Re & TW & \\
\hline $5088.9426(11)$ & $19644.972(4)$ & $5088.9421(5)$ & 0.0005 & 19000 & & $4 \mathrm{~d}$ & $(3 / 2)^{2}[5 / 2]_{2}$ & $4 \mathrm{f}$ & $(3 / 2)^{2}[5 / 2]^{\circ} 2_{2}$ & $5.2 \mathrm{e}+07$ & $\mathrm{D}+$ & F_Re & TW & \\
\hline $5089.492(5)$ & $19642.851(21)$ & $5089.4972(7)$ & -0.005 & 330 & & $\mathrm{sp}$ & $\left({ }^{3} \mathrm{~F}\right)^{3} \mathrm{P}^{\circ}{ }^{3} \mathrm{D}^{\circ}{ }_{2}$ & $5 \mathrm{~d}$ & $(5 / 2)^{2}[3 / 2]_{1}$ & & & R1c & & \\
\hline $5093.81535(17)$ & $19626.1799(7)$ & $5093.81536(14)$ & -0.00001 & 41000 & & $4 \mathrm{~d}$ & $(5 / 2)^{2}[5 / 2]_{2}$ & $4 \mathrm{f}$ & $(5 / 2)^{2}[5 / 2]^{\circ} 2$ & $4.9 e+07$ & $\mathrm{D}+$ & F_Re & TW & \\
\hline 5094.442(3) & 19623.766(11) & $5094.4408(7)$ & 0.001 & 330 & & $\mathrm{sp}$ & $\left({ }^{3} \mathrm{~F}\right)^{3} \mathrm{P}^{\circ}{ }^{3} \mathrm{D}^{\circ}{ }_{2}$ & $5 \mathrm{~d}$ & $(5 / 2)^{2}[3 / 2]_{2}$ & & & $\mathrm{R} 1 \mathrm{c}$ & & \\
\hline 5095.7489(19) & $19618.733(7)$ & $5095.7478(3)$ & 0.0011 & 3100 & & $4 \mathrm{~d}$ & $(5 / 2)^{2}[7 / 2]_{4}$ & $4 \mathrm{f}$ & $(5 / 2)^{2}[7 / 2]^{\circ}{ }_{3}$ & & & R1c & & \\
\hline $5100.0629(10)$ & $19602.138(4)$ & $5100.0650(3)$ & -0.0021 & 12000 & & $4 \mathrm{~d}$ & $(5 / 2)^{2}[5 / 2]_{2}$ & $4 \mathrm{f}$ & $(5 / 2)^{2}[7 / 2]^{\circ}{ }_{3}$ & & & F_Re & & \\
\hline $5100.9790(21)$ & 19598.618(8) & $5100.9762(5)$ & 0.0028 & 9900 & & $4 \mathrm{~d}$ & $(3 / 2)^{2}[5 / 2]_{2}$ & $4 \mathrm{f}$ & $(3 / 2)^{2}[5 / 2]^{\circ} 3$ & $1.6 \mathrm{e}+07$ & $\mathrm{D}+$ & R1c & TW & \\
\hline $5103.283(3)$ & $19589.770(13)$ & $5103.2831(6)$ & -0.000 & 830 & & $4 \mathrm{~d}$ & $(3 / 2)^{2}[5 / 2]_{3}$ & $4 \mathrm{f}$ & $(3 / 2)^{2}[9 / 2]^{\circ} 4$ & & & R1c & & \\
\hline 5104.573(3) & $19584.818(11)$ & $5104.5755(3)$ & -0.002 & 830 & & $4 \mathrm{~d}$ & $(5 / 2)^{2}[7 / 2]_{3}$ & $4 \mathrm{f}$ & $(5 / 2)^{2}[3 / 2]^{\circ} 2$ & & & $\mathrm{R} 1 \mathrm{c}$ & & \\
\hline $5108.3335(19)$ & $\begin{array}{l}19570.402(7) \\
\text { (1) }\end{array}$ & $5108.3328(4)$ & 0.0007 & 7300 & & $4 \mathrm{~d}$ & $(5 / 2)^{2}[5 / 2]_{2}$ & $4 \mathrm{f}$ & $(5 / 2)^{2}[3 / 2]^{\circ}{ }_{1}$ & & & R1c & & \\
\hline $5109.825(3)$ & 19564.691(13) & $5109.8270(21)$ & -0.002 & 500 & & sp & $\left({ }^{1} \mathrm{D}\right)^{3} \mathrm{P}^{\circ}{ }^{\circ} \mathrm{D}^{\circ}{ }_{1}$ & $5 \mathrm{~g}$ & $(3 / 2)^{2}[5 / 2]_{2}$ & & & R1c & & \\
\hline $5109.880(6)$ & 19564.481(21) & $5109.8767(13)$ & 0.003 & 330 & & $4 \mathrm{~d}$ & $(5 / 2)^{2}[5 / 2]_{2}$ & $\mathrm{sp}$ & $\left({ }^{3} \mathrm{P}\right)^{3} \mathrm{P}^{\circ}{ }^{5} \mathrm{~S}^{\circ}{ }_{2}$ & & & R1c & & \\
\hline $5110.3417(19)$ & $\begin{array}{l}19562.712(7) \\
\text { (1) }\end{array}$ & $5110.3409(5)$ & 0.0008 & 1700 & & $4 \mathrm{~d}$ & $(5 / 2)^{2}[7 / 2]_{4}$ & $4 f$ & $(5 / 2)^{2}[11 / 2]^{\circ}{ }_{5}$ & & & R1c & & \\
\hline
\end{tabular}


Table A1. Cont.

\begin{tabular}{|c|c|c|c|c|c|c|c|c|c|c|c|c|c|c|}
\hline$\lambda_{\text {obs }}{ }^{\text {a }(\AA)}$ & $\sigma_{\mathrm{obs}}{ }^{\mathrm{b}}\left(\mathrm{cm}^{-1}\right)$ & $\lambda_{\text {Ritz }}{ }^{\mathrm{c}}(\AA)$ & $\begin{array}{c}\Delta \lambda_{\text {obs-Ritz }} \\
\text { (A) }\end{array}$ & $\begin{array}{c}I_{\text {obs }} \mathrm{d} \\
\text { (arb. u.) }\end{array}$ & Char ${ }^{e}$ & & ower Level & & pper Level & $A\left(\mathrm{~s}^{-1}\right)$ & $\operatorname{Acc}^{f}$ & Line Ref. $\mathrm{g}$ & TP Ref. $\mathrm{g}$ & Notes ${ }^{h}$ \\
\hline $5115.535(3)$ & $19542.852(13)$ & $5115.5386(11)$ & -0.004 & 170 & & $\mathrm{sp}$ & $\left({ }^{3} \mathrm{~F}\right)^{3} \mathrm{P}^{\circ}{ }^{3} \mathrm{D}^{\circ}{ }_{1}$ & $5 \mathrm{~d}$ & $(5 / 2)^{2}[1 / 2]_{0}$ & & & R1c & & \\
\hline $5120.7535(4)$ & $19522.9360(15)$ & $5120.75319(19)$ & 0.0003 & 20000 & & $4 \mathrm{~d}$ & $(5 / 2)^{2}[5 / 2]_{2}$ & $4 \mathrm{f}$ & $(5 / 2)^{2}[3 / 2]^{0} 2$ & $2.0 \mathrm{e}+07$ & $\mathrm{D}+$ & F_Re & TW & \\
\hline $5121.765(3)$ & $19519.080(13)$ & $5121.7672(7)$ & -0.002 & 1000 & & $4 \mathrm{~d}$ & $(3 / 2)^{2}[5 / 2]_{3}$ & $4 \mathrm{f}$ & $(3 / 2)^{2}[3 / 2]^{0} 2$ & & & $\mathrm{R} 1 \mathrm{c}$ & & \\
\hline $5124.4745(7)$ & $19508.760(3)$ & $5124.4753(5)$ & -0.0007 & 30000 & & $4 \mathrm{~d}$ & $(5 / 2)^{2}[7 / 2]_{3}$ & $\mathrm{sp}$ & $\left({ }^{3} \mathrm{~F}\right)^{1} \mathrm{P}^{\circ}{ }^{3} \mathrm{G}^{\circ}{ }_{4}$ & & & F_Re & & \\
\hline $5127.682(3)$ & $19496.556(13)$ & $5127.7027(9)$ & -0.020 & 570 & & $4 \mathrm{~d}$ & $\begin{array}{l}(3 / 2)^{2}[5 / 2]_{2} \\
\text { nats }\end{array}$ & $4 f$ & $(3 / 2)^{2}[3 / 2]^{0}{ }_{1}$ & & & $\mathrm{R} 1 \mathrm{c}$ & & $x$ \\
\hline $5127.7428(21)$ & $19496.326(8)$ & $5127.7327(8)$ & 0.0101 & 630 & ? & $4 \mathrm{~d}$ & $(5 / 2)^{2}[3 / 2]_{2}$ & $\mathrm{sp}$ & $\left({ }^{3} \mathrm{P}\right)^{3} \mathrm{P}^{\circ} \mathrm{P}^{\circ}{ }_{1}$ & & & R1c & & $\mathrm{x}$ \\
\hline $5130.583(3)$ & $19485.533(12)$ & $5130.5829(10)$ & 0.000 & 920 & & $4 \mathrm{~d}$ & $(3 / 2)^{2}[3 / 2]_{2}$ & $\mathrm{sp}$ & $\left({ }^{3} \mathrm{P}\right)^{3} \mathrm{P}^{\circ}{ }^{1} \mathrm{D}_{2}^{\circ}$ & & & $\mathrm{R} 1 \mathrm{c}$ & & \\
\hline $5133.123(3)$ & $19475.890(11)$ & $5133.1211(4)$ & 0.002 & 500 & & $4 \mathrm{~d}$ & $\begin{array}{l}(5 / 2)^{2}[5 / 2]_{2} \\
\text { nat }\end{array}$ & $4 f$ & $(5 / 2)^{2}[1 / 2]^{\circ}{ }_{1}$ & & & R1c & & \\
\hline $5134.6720(24)$ & $19470.016(9)$ & $5134.6725(9)$ & -0.0005 & 170 & & $4 \mathrm{~d}$ & $(5 / 2)^{2}[3 / 2]_{1}$ & $\mathrm{sp}$ & $\left({ }^{3} \mathrm{P}\right)^{3} \mathrm{P}^{\circ}{ }^{3} \mathrm{P}^{\circ} 1$ & & & $\mathrm{R} 1 \mathrm{c}$ & & \\
\hline 5136.3939(19) & 19463.489(7) & $5136.3932(5)$ & 0.0007 & 1300 & & $4 \mathrm{~d}$ & $(5 / 2)^{2}[7 / 2]_{4}$ & $\mathrm{sp}$ & $\left({ }^{3} \mathrm{~F}\right)^{1} \mathrm{P}^{\circ}{ }^{3} \mathrm{G}^{\circ}{ }_{4}$ & & & R1c & & \\
\hline $5138.613(4)$ & $19455.085(17)$ & $5138.6113(20)$ & 0.001 & 340 & & $4 \mathrm{~d}$ & $(3 / 2)^{2}[5 / 2]_{3}$ & $\mathrm{sp}$ & $\left({ }^{1} \mathrm{G}\right)^{3} \mathrm{P}^{\circ} 3^{3} \mathrm{~F}^{\circ}{ }_{4}$ & & & R1c & & \\
\hline $5151.206(2)$ & 19407.522(9) & $5151.2073(8)$ & -0.001 & 2500 & & $4 \mathrm{~d}$ & $(5 / 2)^{2}[7 / 2]_{3}$ & $\mathrm{sp}$ & $\left({ }^{3} \mathrm{~F}\right)^{1} \mathrm{P}^{\circ}{ }^{3} \mathrm{D}_{3}^{\circ}$ & & & R1c & & \\
\hline $5157.255(4)$ & $19384.761(14)$ & $5157.2580(9)$ & -0.003 & 340 & & $\mathrm{sp}$ & $\left({ }^{3} \mathrm{~F}\right)^{3} \mathrm{P}^{\circ}{ }^{3} \mathrm{D}^{\circ}{ }_{3}$ & $6 \mathrm{~s}$ & $(3 / 2)^{2}[3 / 2]_{2}$ & & & R1c & & \\
\hline $5158.0914(6)$ & 19381.6168(21) & $5158.0916(4)$ & -0.0002 & 13000 & & $4 \mathrm{~d}$ & $(5 / 2)^{2}[1 / 2]_{0}$ & $4 \mathrm{f}$ & $(5 / 2)^{2}[3 / 2]^{0}$ & $2.4 \mathrm{e}+07$ & $\mathrm{D}+$ & $\mathrm{F}$ Re & TW & \\
\hline 5159.103(4) & 19377.817(17) & $5159.0971(13)$ & 0.006 & 2300 & & $\mathrm{sp}$ & $\left({ }^{3} \mathrm{~F}\right)^{3} \mathrm{P}^{\circ}{ }^{3} \mathrm{~F}^{\circ}{ }_{4}$ & $5 \mathrm{~d}$ & $(5 / 2)^{2}[7 / 2]_{4}$ & & & $\mathrm{R} 1 \mathrm{c}$ & & \\
\hline $5163.252(3)$ & $19362.244(11)$ & $5163.2501(8)$ & 0.002 & 330 & & $4 \mathrm{~d}$ & $(5 / 2)^{2}[7 / 2]_{4}$ & $\mathrm{sp}$ & $\left({ }^{3} \mathrm{~F}\right)^{1} \mathrm{P}^{\circ}{ }^{3} \mathrm{D}_{3}{ }_{3}$ & & & $\mathrm{R} 1 \mathrm{c}$ & & \\
\hline $5167.6923(22)$ & $19345.609(8)$ & $5167.6825(8)$ & 0.0098 & 11000 & ? & $4 \mathrm{~d}$ & $(5 / 2)^{2}[5 / 2]_{2}$ & $\mathrm{sp}$ & $\left({ }^{3} \mathrm{~F}\right)^{1} \mathrm{P}^{\circ}{ }^{3} \mathrm{D}^{\circ}{ }_{3}^{\circ}$ & & & R1c & & $x$ \\
\hline $5171.644(3)$ & $19330.828(12)$ & $5171.6406(13)$ & 0.003 & 570 & & $4 \mathrm{~d}$ & $(3 / 2)^{2}[7 / 2]_{3}$ & $\mathrm{sp}$ & $\left({ }^{3} \mathrm{~F}\right)^{1} \mathrm{P}^{\circ}{ }^{3} \mathrm{G}_{3}^{\circ}{ }_{3}$ & & & R1c & & \\
\hline 5175.9651(23) & $\begin{array}{l}19314.689(9) \\
\text { ( }\end{array}$ & $5175.9637(23)$ & 0.0014 & 1400 & * & $4 \mathrm{~d}$ & $(3 / 2)^{2}[3 / 2]_{1}$ & $\mathrm{sp}$ & $\left({ }^{3} \mathrm{P}\right)^{3} \mathrm{P}^{\circ}{ }^{1} \mathrm{P}^{\circ}$ & & & R1nc & & \\
\hline 5175.9651(23) & $19314.689(9)$ & $5175.9653(10)$ & -0.0002 & 1400 & * & $4 \mathrm{~d}$ & $(5 / 2)^{2}[1 / 2]_{1}$ & $\mathrm{sp}$ & $\left({ }^{3} \mathrm{P}\right){ }^{3} \mathrm{P}^{\circ}{ }^{3} \mathrm{P}_{2}^{\circ}$ & & & R1c & & \\
\hline $5183.3664(7)$ & 19287.110(3) & $5183.3664(5)$ & -0.0000 & 19000 & & $4 \mathrm{~d}$ & $(5 / 2)^{2}[1 / 2]_{0}$ & $4 f$ & $(5 / 2)^{2}[1 / 2]^{\circ}{ }_{1}$ & $2.4 \mathrm{e}+07$ & $\mathrm{D}+$ & F_Re & TW & \\
\hline $5184.052(3)$ & 19284.561(11) & $5184.0520(11)$ & -0.000 & 340 & & $4 \mathrm{~d}$ & $(3 / 2)^{2}[3 / 2]_{1}$ & $\mathrm{sp}$ & $\left({ }^{1} \mathrm{G}\right)^{3} \mathrm{P}^{\circ}{ }^{3} \mathrm{~F}_{2}{ }_{2}$ & & & R1nc & & \\
\hline $5192.638(6)$ & $19252.671(21)$ & $5192.6239(13)$ & 0.015 & 240 & & $\mathrm{sp}$ & $\left({ }^{3} \mathrm{~F}\right)^{3} \mathrm{P}^{\circ} 3^{\circ} \mathrm{F}_{4}^{\circ}$ & $5 \mathrm{~d}$ & $(5 / 2)^{2}[5 / 2]_{3}$ & & & R1c & & \\
\hline $5194.349(4)$ & $19246.331(16)$ & $5194.3399(17)$ & 0.009 & 170 & & $\mathrm{sp}$ & $\left({ }^{3} \mathrm{~F}\right)^{3} \mathrm{P}^{\circ}{ }^{5} \mathrm{~F}^{\circ}{ }_{2}$ & $6 \mathrm{~s}$ & $(5 / 2)^{2}[5 / 2]_{2}$ & & & R1c & & \\
\hline $5205.112(4)$ & $19206.536(17)$ & $5205.1059(8)$ & 0.006 & 500 & & $\mathrm{sp}$ & $\left({ }^{3} \mathrm{~F}\right)^{3} \mathrm{P}^{\circ}{ }^{3} \mathrm{D}^{\circ}{ }_{2}$ & $5 \mathrm{~d}$ & $(5 / 2)^{2}[1 / 2]_{1}$ & & & R1c & & \\
\hline $5207.1357(22)$ & 19199.070(8) & $5207.1340(15)$ & 0.0018 & 29000 & & $4 \mathrm{~d}$ & $(3 / 2)^{2}[7 / 2]_{4}$ & $\mathrm{sp}$ & $\left({ }^{1} \mathrm{G}\right){ }^{3} \mathrm{P}^{\circ}{ }^{3} \mathrm{H}^{\circ}{ }_{5}$ & & & R1c & & \\
\hline $5229.518(3)$ & $19116.900(10)$ & $5229.5194(11)$ & -0.002 & 3200 & & $4 \mathrm{~d}$ & $(3 / 2)^{2}[5 / 2]_{2}$ & $\mathrm{sp}$ & $\left({ }^{3} \mathrm{P}\right)^{3} \mathrm{P}^{\circ}{ }^{1} \mathrm{D}^{\circ}{ }_{2}$ & & & $\mathrm{R} 1 \mathrm{c}$ & & \\
\hline $5229.709(6)$ & $19116.201(21)$ & $5229.7107(10)$ & -0.002 & 170 & & $\mathrm{sp}$ & $\left({ }^{3} \mathrm{~F}\right)^{3} \mathrm{P}^{\circ}{ }^{3} \mathrm{G}_{3}^{\circ}$ & $6 \mathrm{~s}$ & $(3 / 2)^{2}[3 / 2]_{2}$ & & & R1c & & \\
\hline $5234.471(23)$ & $\begin{array}{l}19098.81(8) \\
\text { (n) }\end{array}$ & $5234.429(3)$ & 0.042 & 170 & * & $\mathrm{sp}$ & $\left({ }^{3} \mathrm{P}\right)^{3} \mathrm{P}^{\circ} 1^{1} \mathrm{P}^{\circ}$ & $9 \mathrm{~s}$ & $(3 / 2)^{2}[3 / 2]$ & & & R1nc & & \\
\hline $5234.471(23)$ & 19098.81(8) & $5234.4943(14)$ & -0.023 & 170 & * & $\mathrm{sp}$ & $\left({ }^{3} \mathrm{~F}\right)^{3} \mathrm{P}^{\circ}{ }^{3} \mathrm{~F}^{\circ}{ }_{4}$ & $5 \mathrm{~d}$ & $(5 / 2)^{2}[9 / 2]_{4}$ & & & R1c & & \\
\hline $5239.5473(24)$ & $19080.307(9)$ & $5239.5490(8)$ & -0.0017 & 330 & & $4 \mathrm{~d}$ & $(5 / 2)^{2}[9 / 2]_{4}$ & $\mathrm{sp}$ & $\left({ }^{3} \mathrm{~F}\right)^{1} \mathrm{P}^{\circ} 3^{3} \mathrm{~F}_{4}^{\circ}$ & & & R1c & & \\
\hline $5245.340(3)$ & $19059.237(12)$ & $5245.3423(13)$ & -0.003 & 19000 & & sp & $\left({ }^{3} \mathrm{~F}\right)^{3} \mathrm{P}^{\circ}{ }^{3} \mathrm{~F}^{\circ}{ }_{4}$ & $5 \mathrm{~d}$ & $(5 / 2)^{2}[9 / 2]_{5}$ & & & R1c & & \\
\hline 5247.109(4) & $19052.811(16)$ & $5247.1155(9)$ & -0.007 & 160 & & $4 \mathrm{~d}$ & $(3 / 2)^{2}[7 / 2]_{3}$ & $\mathrm{sp}$ & $\left({ }^{3} \mathrm{~F}\right)^{1} \mathrm{P}^{\circ}{ }^{3} \mathrm{D}^{\circ}{ }_{2}$ & & & $\mathrm{R} 1 \mathrm{c}$ & & \\
\hline $5247.976(4)$ & 19049.661(16) & $5247.9750(7)$ & 0.001 & 160 & & $5 p$ & $(5 / 2)^{2}[3 / 2]^{\circ}$ & $5 \mathrm{~d}$ & $(3 / 2)^{2}[5 / 2]_{2}$ & & & R1c & & \\
\hline $5251.545(3)$ & $19036.718(9)$ & $5251.5445(8)$ & 0.000 & 330 & & $4 \mathrm{~d}$ & $(5 / 2)^{2}[3 / 2]_{2}$ & $\mathrm{sp}$ & $\left({ }^{3} \mathrm{P}\right)^{3} \mathrm{P}^{\circ}{ }^{3} \mathrm{D}^{\circ}$ & & & R1c & & \\
\hline $5254.2141(20)$ & 19027.046(7) & $5254.2139(6)$ & 0.0002 & 350 & & $5 p$ & $(5 / 2)^{2}[3 / 2]_{2}^{\circ}$ & $5 \mathrm{~d}$ & $(3 / 2)^{2}[5 / 2]_{3}$ & & & $\mathrm{R} 1 \mathrm{c}$ & & \\
\hline $5258.8249(21)$ & $19010.364(8)$ & $5258.8237(8)$ & 0.0011 & 580 & & $4 \mathrm{~d}$ & $(5 / 2)^{2}[3 / 2]_{1}$ & $\mathrm{sp}$ & $\left({ }^{3} \mathrm{P}\right)^{3} \mathrm{P}^{\circ}{ }^{3} \mathrm{D}^{\circ}$ & & & R1c & & \\
\hline $5261.0461(22)$ & $19002.338(8)$ & $5261.0443(8)$ & 0.0018 & 330 & & sp & $\left({ }^{3} \mathrm{~F}\right)^{3} \mathrm{P}^{\circ}{ }^{3} \mathrm{D}^{\circ}$ & $5 \mathrm{~d}$ & $(5 / 2)^{2}[5 / 2]_{2}$ & & & R1c & & \\
\hline $5269.9892(14)$ & $18970.091(5)$ & $5269.9904(6)$ & -0.0012 & 23000 & & $4 p$ & ${ }^{3} \mathrm{P}_{2}^{\circ}$ & $\mathrm{s}^{2}$ & ${ }^{1} \mathrm{D}_{2}$ & & & F_Re & & \\
\hline $5276.5244(15)$ & $18946.597(5)$ & $5276.5241(9)$ & 0.0003 & 16000 & & $4 \mathrm{~d}$ & $(3 / 2)^{2}[7 / 2]_{3}$ & $\mathrm{sp}$ & $\left({ }^{1} \mathrm{G}\right)^{3} \mathrm{P}^{\circ}{ }^{3} \mathrm{H}^{\circ}{ }_{4}$ & & & F_Re & & \\
\hline $5280.9533(23)$ & $18930.707(8)$ & $5280.9563(7)$ & -0.0030 & 480 & & sp & $\left({ }^{3} \mathrm{~F}\right)^{3} \mathrm{P}^{\circ}{ }^{3} \mathrm{~F}^{\circ}{ }_{3}$ & $5 \mathrm{~d}$ & $(5 / 2)^{2}[5 / 2]$ & & & R1c & & \\
\hline $5285.360(4)$ & $18914.923(13)$ & $5285.3600(13)$ & 0.000 & 480 & & $4 \mathrm{~d}$ & $(3 / 2)^{2}[3 / 2]_{2}$ & $\mathrm{sp}$ & $\left({ }^{3} \mathrm{~F}\right)^{1} \mathrm{P}^{\circ}{ }^{3} \mathrm{G}_{3}{ }_{3}$ & & & R1c & & \\
\hline $5289.1103(21)$ & 18901.512(8) & $5289.1096(6)$ & 0.0007 & 800 & & $\mathrm{sp}$ & $\left({ }^{3} \mathrm{~F}\right)^{3} \mathrm{P}^{\circ} 3^{\circ} \mathrm{F}_{3}$ & $5 \mathrm{~d}$ & $(5 / 2)^{2}[7 / 2]_{4}$ & & & R1c & & \\
\hline
\end{tabular}


Table A1. Cont.

\begin{tabular}{|c|c|c|c|c|c|c|c|c|c|c|c|c|c|c|}
\hline$\lambda_{\text {obs }}{ }^{a}(\AA)$ & $\sigma_{\mathrm{obs}}^{\mathrm{b}}\left(\mathrm{cm}^{-1}\right)$ & $\lambda_{\text {Ritz }}{ }^{c}(\AA)$ & $\begin{array}{c}\Delta \lambda_{\text {obs-Ritz }} \\
\text { (A) }\end{array}$ & $\begin{array}{c}I_{\text {obs }}{ }^{\mathrm{d}} \\
\text { (arb. u.) }\end{array}$ & Char ${ }^{e}$ & & ower Level & & Upper Level & $A\left(\mathrm{~s}^{-1}\right)$ & $\operatorname{Acc}^{f}$ & Line Ref. $\mathrm{g}$ & TP Ref. $\mathrm{g}$ & Notes ${ }^{h}$ \\
\hline $5291.8196(20)$ & $18891.835(7)$ & $5291.8216(7)$ & -0.0020 & 1600 & & $\mathrm{sp}$ & $\left({ }^{3} \mathrm{~F}\right)^{3} \mathrm{P}^{\circ}{ }^{3} \mathrm{~F}_{3}^{\circ}$ & $5 \mathrm{~d}$ & $(5 / 2)^{2}[7 / 2]_{3}$ & & & R1c & & \\
\hline $5297.4020(23)$ & 18871.927(8) & $5297.4047(9)$ & -0.0028 & 480 & & $4 \mathrm{~d}$ & $(3 / 2)^{2}[3 / 2]_{1}$ & sp & $\left({ }^{3} \mathrm{~F}\right)^{1} \mathrm{P}^{\circ}{ }^{3} \mathrm{D}^{\circ}{ }_{2}$ & & & R1c & & \\
\hline $5310.771(3)$ & 18824.421(11) & $5310.7694(24)$ & 0.001 & 310 & & $4 \mathrm{f}$ & $(5 / 2)^{2}[1 / 2]^{\circ}{ }_{1}$ & $7 \mathrm{~g}$ & $(5 / 2)^{2}[3 / 2]_{2}$ & & & R1c & & \\
\hline $5314.6020(22)$ & $18810.851(8)$ & $5314.6025(9)$ & -0.0005 & 480 & & $4 d$ & $(3 / 2)^{2}[7 / 2]_{4}$ & $\mathrm{sp}$ & $\left({ }^{1} \mathrm{G}\right)^{3} \mathrm{P}^{\circ}{ }^{3} \mathrm{H}^{\circ}{ }_{4}$ & & & R1c & & \\
\hline $5315.989(4)$ & 18805.942(15) & $5315.9868(7)$ & 0.003 & 160 & & $5 p$ & $(5 / 2)^{2}[3 / 2]^{\circ} 2$ & $5 \mathrm{~d}$ & $(3 / 2)^{2}[3 / 2]_{1}$ & & & R1c & & \\
\hline $5321.666(3)$ & $18785.883(12)$ & $5321.666(3)$ & 0.000 & 310 & & $4 \mathrm{f}$ & $(5 / 2)^{2}[1 / 2]^{\circ}{ }_{0}$ & $7 \mathrm{~g}$ & $(5 / 2)^{2}[3 / 2]_{1}$ & & & R1c & & \\
\hline $5321.840(3)$ & $18785.267(11)$ & $5321.8352(21)$ & 0.005 & 310 & * & $4 \mathrm{f}$ & $(5 / 2)^{2}[3 / 2]^{0}{ }_{2}$ & $7 \mathrm{~g}$ & $(5 / 2)^{2}[5 / 2]_{2}$ & & & R1nc & & \\
\hline $5321.840(3)$ & 18785.267(11) & $5321.8386(23)$ & 0.002 & 310 & * & $4 \mathrm{f}$ & $(5 / 2)^{2}[3 / 2]^{\circ}{ }_{2}$ & $7 \mathrm{~g}$ & $(5 / 2)^{2}[5 / 2]_{3}$ & & & R1c & & \\
\hline $5324.070(4)$ & 18777.398(13) & $5324.0734(24)$ & -0.003 & 310 & & $4 \mathrm{f}$ & $(5 / 2)^{2}[3 / 2]^{\circ} 2$ & $7 \mathrm{~g}$ & $(5 / 2)^{2}[3 / 2]_{2}$ & & & R1c & & \\
\hline $5324.3542(21)$ & $18776.397(7)$ & $5324.3531(6)$ & 0.0011 & 1500 & & $\mathrm{sp}$ & $\left({ }^{3} \mathrm{~F}\right)^{3} \mathrm{P}^{\circ}{ }^{3} \mathrm{~F}_{3}^{\circ}$ & $5 \mathrm{~d}$ & $(5 / 2)^{2}[5 / 2]_{3}$ & & & R1c & & \\
\hline $5325.319(3)$ & 18772.994(12) & $5325.3132(19)$ & 0.006 & 310 & & $4 \mathrm{f}$ & $(5 / 2)^{2}[11 / 2]^{\circ}{ }_{5}$ & $7 \mathrm{~g}$ & $(5 / 2)^{2}[11 / 2]_{5}$ & & & R1c & & \\
\hline $5327.9841(23)$ & $18763.605(8)$ & & & 920 & & $4 \mathrm{f}$ & $(5 / 2)^{2}[11 / 2]^{\circ}{ }_{6}$ & $7 \mathrm{~g}$ & $(5 / 2)^{2}[13 / 2]_{7}$ & $1.1 \mathrm{e}+07$ & $\mathrm{D}+$ & R1c & TW & \\
\hline $5328.8109(22)$ & 18760.694(8) & & & 1600 & & $4 \mathrm{f}$ & $(5 / 2)^{2}[11 / 2]^{\circ}{ }_{5}$ & $7 \mathrm{~g}$ & $(5 / 2)^{2}[13 / 2]_{6}$ & $1.1 \mathrm{e}+07$ & $\mathrm{D}+$ & R1c & TW & \\
\hline $5328.963(4)$ & $18760.157(13)$ & & & 460 & & $4 \mathrm{f}$ & $(3 / 2)^{2}[3 / 2]^{\circ}{ }_{2}$ & $7 \mathrm{~g}$ & $(3 / 2)^{2}[5 / 2]_{3}$ & $9 . e+06$ & D+ & R1c & TW & \\
\hline $5331.63(4)$ & $18750.78(14)$ & $5331.6093(19)$ & 0.02 & 150 & * & $4 \mathrm{f}$ & $(5 / 2)^{2}[7 / 2]^{\circ}{ }_{4}$ & $8 \mathrm{~d}$ & $(5 / 2)^{2}[7 / 2]_{4}$ & & & R1nc & & $\mathrm{x}$ \\
\hline 5331.63(4) & 18750.78(14) & $5331.674(3)$ & -0.05 & 150 & * & $5 s$ & $(3 / 2)^{2}[3 / 2]_{2}$ & sp & $\left({ }^{1} \mathrm{D}\right)^{3} \mathrm{P}^{\circ}{ }^{3} \mathrm{D}^{\circ}{ }_{3}$ & & & R1c & & $\mathrm{X}$ \\
\hline $5335.317(3)$ & 18737.818(12) & $5335.3169(21)$ & -0.000 & 300 & & $4 \mathrm{f}$ & $(5 / 2)^{2}[3 / 2]^{\circ}{ }_{1}$ & $7 \mathrm{~g}$ & $(5 / 2)^{2}[5 / 2]_{2}$ & & & R2nc & & \\
\hline $5335.518(3)$ & 18737.112(12) & $5335.5179(6)$ & -0.000 & 300 & & $4 p$ & ${ }^{3} \mathrm{~F}_{2}^{\circ}$ & $\mathrm{s}^{2}$ & ${ }^{3} \mathrm{P}_{1}$ & & & R1c & & \\
\hline $5336.203(3)$ & 18734.707(12) & & & 150 & & $4 \mathrm{f}$ & $(3 / 2)^{2}[3 / 2]^{\circ}{ }_{1}$ & $7 \mathrm{~g}$ & $(3 / 2)^{2}[5 / 2]_{2}$ & & & R1c & & \\
\hline 5337.580(13) & $18729.87(5)$ & $5337.5664(20)$ & 0.013 & 150 & * & $4 \mathrm{f}$ & $(5 / 2)^{2}[3 / 2]^{\circ}{ }_{1}$ & $7 \mathrm{~g}$ & $(5 / 2)^{2}[3 / 2]_{2}$ & & & R1c & & \\
\hline $5337.580(13)$ & $18729.87(5)$ & $5337.593(3)$ & -0.013 & 150 & * & $4 \mathrm{f}$ & $(5 / 2)^{2}[3 / 2]^{\circ}{ }_{1}$ & $7 \mathrm{~g}$ & $(5 / 2)^{2}[3 / 2]_{1}$ & & & R1c & & \\
\hline $5338.460(3)$ & 18726.784(10) & $5338.4584(6)$ & 0.002 & 300 & & $4 \mathrm{~d}$ & $(3 / 2)^{2}[1 / 2]_{1}$ & $4 \mathrm{f}$ & $(5 / 2)^{2}[3 / 2]^{\circ}{ }_{1}$ & & & R1c & & \\
\hline $5340.098(3)$ & 18721.041(11) & $5340.0973(8)$ & 0.001 & 1300 & & $4 \mathrm{~d}$ & $(5 / 2)^{2}[3 / 2]_{2}$ & sp & $\left({ }^{3} \mathrm{P}\right)^{3} \mathrm{P}^{\circ}{ }^{3} \mathrm{D}^{\circ}{ }_{1}$ & & & R1c & & \\
\hline $5340.878(3)$ & $18718.305(12)$ & $5340.8750(20)$ & 0.003 & 300 & & $4 \mathrm{f}$ & $(5 / 2)^{2}[7 / 2]_{3}{ }_{3}$ & $7 \mathrm{~g}$ & $(5 / 2)^{2}[9 / 2]_{4}$ & & & R1c & & \\
\hline \multirow[t]{2}{*}{ 5342.197(3) } & 18713.684(12) & $5342.1987(24)$ & -0.001 & 150 & & $4 \mathrm{f}$ & $(5 / 2)^{2}[7 / 2]^{\circ}{ }_{3}$ & $7 \mathrm{~g}$ & $(5 / 2)^{2}[7 / 2]_{3}$ & & & R1c & & \\
\hline & & $5344.316(4)$ & & & $\mathrm{m}$ & $4 \mathrm{f}$ & $(3 / 2)^{2}[9 / 2]^{\circ}{ }_{4}$ & $7 \mathrm{~g}$ & $(3 / 2)^{2}[9 / 2]_{4}$ & & & R1nc & & \\
\hline $5344.366(4)$ & 18706.091(16) & $5344.3657(21)$ & 0.000 & 150 & * & $4 \mathrm{f}$ & $(5 / 2)^{2}[7 / 2]^{\circ}{ }_{3}$ & $7 \mathrm{~g}$ & $(5 / 2)^{2}[5 / 2]_{2}$ & & & R1nc & & \\
\hline $5344.366(4)$ & 18706.091(16) & $5344.3692(24)$ & -0.003 & 150 & * & $4 \mathrm{f}$ & $(5 / 2)^{2}[7 / 2]^{\circ}{ }_{3}$ & $7 \mathrm{~g}$ & $(5 / 2)^{2}[5 / 2]_{3}$ & & & R1c & & \\
\hline $5345.160(3)$ & 18703.312(10) & $5345.154(3)$ & 0.006 & 420 & * & $4 \mathrm{f}$ & $(3 / 2)^{2}[9 / 2]^{\circ}$ & $7 \mathrm{~g}$ & $(3 / 2)^{2}[11 / 2]_{5}$ & & & R1c & & \\
\hline $5345.160(3)$ & $18703.312(10)$ & & & 420 & * & $4 \mathrm{f}$ & $(3 / 2)^{2}[9 / 2]^{\circ}{ }_{5}$ & $7 \mathrm{~g}$ & $(3 / 2)^{2}[11 / 2]_{6}$ & $1.1 \mathrm{e}+07$ & $\mathrm{D}+$ & R1nc & TW & \\
\hline $5345.576(2)$ & 18701.857(9) & $5345.5723(9)$ & 0.003 & 300 & & $\mathrm{sp}$ & $\left({ }^{3} \mathrm{~F}\right)^{3} \mathrm{P}^{\circ}{ }^{3} \mathrm{D}^{\circ}{ }_{1}$ & $5 \mathrm{~d}$ & $(5 / 2)^{2}[3 / 2]_{1}$ & & & R1c & & \\
\hline 5347.6243(22) & 18694.693(8) & $5347.6243(8)$ & -0.0000 & 1500 & & $4 \mathrm{~d}$ & $(5 / 2)^{2}[3 / 2]_{1}$ & sp & $\left({ }^{3} \mathrm{P}\right)^{3} \mathrm{P}^{\circ}{ }^{3} \mathrm{D}^{\circ}{ }_{1}$ & & & R1c & & \\
\hline $5347.684(3)$ & 18694.484(11) & $5347.6891(8)$ & -0.005 & 750 & & $4 \mathrm{~d}$ & $(3 / 2)^{2}[7 / 2]_{3}$ & $\mathrm{sp}$ & $\left({ }^{3} \mathrm{~F}\right)^{1} \mathrm{P}^{\circ}{ }^{3} \mathrm{~F}_{3}^{\circ}$ & & & R1c & & \\
\hline $5347.809(3)$ & $18694.047(12)$ & $5347.809(3)$ & 0.000 & 420 & & $4 \mathrm{f}$ & $(3 / 2)^{2}[9 / 2]^{\circ}{ }_{4}$ & $7 \mathrm{~g}$ & $(3 / 2)^{2}[11 / 2]_{5}$ & $1.1 \mathrm{e}+07$ & $\mathrm{D}+$ & R1c & TW & \\
\hline $5349.076(4)$ & $18689.619(13)$ & $5349.0730(24)$ & 0.003 & 150 & & $4 \mathrm{f}$ & $(5 / 2)^{2}[5 / 2]^{\circ} 2$ & $7 \mathrm{~g}$ & $(5 / 2)^{2}[7 / 2]_{3}$ & & & R1c & & \\
\hline $5351.245(4)$ & $18682.043(14)$ & $5351.2456(21)$ & -0.000 & 150 & * & $4 \mathrm{f}$ & $(5 / 2)^{2}[5 / 2]^{\circ}{ }_{2}$ & $7 \mathrm{~g}$ & $(5 / 2)^{2}[5 / 2]_{2}$ & & & R1nc & & \\
\hline $5351.245(4)$ & $18682.043(14)$ & $5351.2491(24)$ & -0.004 & 150 & * & $4 \mathrm{f}$ & $(5 / 2)^{2}[5 / 2]^{\circ}{ }_{2}$ & $7 \mathrm{~g}$ & $(5 / 2)^{2}[5 / 2]_{3}$ & & & R1c & & \\
\hline $5351.579(4)$ & $18680.878(12)$ & $5351.5804(12)$ & -0.001 & 300 & & $4 \mathrm{~d}$ & $(3 / 2)^{2}[5 / 2]_{2}$ & sp & $\left({ }^{1} \mathrm{G}\right)^{3} \mathrm{P}^{\circ}{ }^{3} \mathrm{~F}^{\circ}{ }_{2}$ & & & R1nc & & \\
\hline $5352.0268(21)$ & $18679.315(7)$ & $5352.0245(5)$ & 0.0023 & 6000 & & $4 \mathrm{~d}$ & $(3 / 2)^{2}[1 / 2]_{1}$ & $4 \mathrm{f}$ & $(5 / 2)^{2}[3 / 2]^{\circ}{ }_{2}$ & & & $\mathrm{R} 1 \mathrm{c}$ & & \\
\hline $5353.863(4)$ & $18672.908(12)$ & $5353.8649(20)$ & -0.002 & 300 & & $4 \mathrm{f}$ & $(5 / 2)^{2}[5 / 2]^{\circ}{ }_{3}$ & $7 \mathrm{~g}$ & $(5 / 2)^{2}[9 / 2]_{4}$ & & & R1c & & \\
\hline $5354.4850(20)$ & $18670.740(7)$ & $5354.4863(8)$ & -0.0013 & 4500 & & $4 \mathrm{~d}$ & $(3 / 2)^{2}[1 / 2]_{1}$ & $4 \mathrm{f}$ & $(5 / 2)^{2}[1 / 2]_{0}$ & & & R1c & & \\
\hline $5355.186(3)$ & $18668.294(11)$ & $5355.1870(24)$ & -0.001 & 410 & & $4 \mathrm{f}$ & $(5 / 2)^{2}[5 / 2]_{3}{ }_{3}$ & $7 \mathrm{~g}$ & $(5 / 2)^{2}[7 / 2]_{4}$ & $7.4 \mathrm{e}+06$ & $\mathrm{D}+$ & R1c & TW & \\
\hline $5356.8109(21)$ & $18662.633(7)$ & $5356.8091(8)$ & 0.0018 & 2100 & & $4 \mathrm{~d}$ & $(5 / 2)^{2}[5 / 2]_{3}$ & sp & $\left({ }^{3} \mathrm{~F}\right)^{1} \mathrm{P}^{\circ}{ }^{3} \mathrm{~F}^{\circ}{ }_{4}$ & & & R1c & & \\
\hline
\end{tabular}


Table A1. Cont.

\begin{tabular}{|c|c|c|c|c|c|c|c|c|c|c|c|c|c|c|}
\hline$\lambda_{\text {obs }}{ }^{\text {a }}(\AA)$ & $\sigma_{\mathrm{obs}}{ }^{\mathrm{b}}\left(\mathrm{cm}^{-1}\right)$ & $\lambda_{\text {Ritz }}{ }^{c}(\AA)$ & $\begin{array}{c}\Delta \lambda_{\text {obs-Ritz }} \\
\text { (A) }\end{array}$ & $\begin{array}{c}I_{\text {obs }} \mathrm{d} \\
\text { (arb. u.) }\end{array}$ & Char ${ }^{e}$ & & ower Level & & Jpper Level & $A\left(\mathrm{~s}^{-1}\right)$ & $\operatorname{Acc}^{f}$ & Line Ref. $\mathrm{g}$ & TP Ref. $\mathrm{g}$ & Notes $h$ \\
\hline $5357.374(4)$ & $18660.670(13)$ & $5357.3727(21)$ & 0.002 & 300 & $*$ & $4 \mathrm{f}$ & $(5 / 2)^{2}[5 / 2]_{3}^{\circ}$ & $7 \mathrm{~g}$ & $(5 / 2)^{2}[5 / 2]_{2}$ & & & R1nc & & \\
\hline $5357.374(4)$ & $18660.670(13)$ & $5357.3761(24)$ & -0.002 & 300 & * & $4 f$ & $(5 / 2)^{2}[5 / 2]^{\circ}{ }_{3}$ & $7 \mathrm{~g}$ & $\begin{array}{l}(5 / 2)^{2}[5 / 2]_{3} \\
\end{array}$ & & & R1c & & \\
\hline $5361.907(4)$ & 18644.894(12) & $5361.914(4)$ & -0.007 & 150 & * & $4 \mathrm{f}$ & $(3 / 2)^{2}[5 / 2]^{\circ}{ }_{3}$ & $7 \mathrm{~g}$ & $(3 / 2)^{2}[7 / 2]_{3}$ & & & R1c & & \\
\hline $5361.907(4)$ & $18644.894(12)$ & & & 150 & * & $4 f$ & $(3 / 2)^{2}[5 / 2]^{\circ}{ }_{3}$ & $7 \mathrm{~g}$ & $(3 / 2)^{2}[7 / 2]_{4}$ & $9 . e+06$ & $\mathrm{D}+$ & R1nc & TW & \\
\hline $5365.5363(20)$ & 18632.284(7) & $5365.5363(6)$ & -0.0000 & 5500 & & $4 \mathrm{~d}$ & $(3 / 2)^{2}[1 / 2]_{1}$ & $4 \mathrm{f}$ & $(5 / 2)^{2}[1 / 2]^{\circ}$ & & & R1c & & \\
\hline $5366.243(3)$ & 18629.831(11) & $5366.2476(7)$ & -0.005 & 440 & & $\mathrm{sp}$ & $\left({ }^{3} \mathrm{~F}\right)^{3} \mathrm{P}^{\circ}{ }^{3} \mathrm{D}^{\circ}{ }_{2}$ & $6 \mathrm{~s}$ & $(3 / 2)^{2}[3 / 2]_{2}$ & & & R1c & & \\
\hline $5368.3825(21)$ & $18622.406(7)$ & $5368.3839(7)$ & -0.0013 & 7400 & & $\mathrm{sp}$ & $\left({ }^{3} \mathrm{~F}\right)^{3} \mathrm{P}^{\circ}{ }^{3} \mathrm{~F}^{\circ}{ }_{3}$ & $5 \mathrm{~d}$ & $(5 / 2)^{2}[9 / 2]_{4}$ & & & $\mathrm{R} 1 \mathrm{c}$ & & \\
\hline $5371.6264(21)$ & 18611.160(7) & $5371.6264(7)$ & 0.0001 & 880 & & $\mathrm{sp}$ & $\left({ }^{3} \mathrm{~F}\right)^{3} \mathrm{P}^{\circ} 3^{3} \mathrm{~F}^{\mathrm{o}}{ }_{3}$ & $5 \mathrm{~d}$ & $(5 / 2)^{2}[3 / 2]_{2}$ & & & R1c & & \\
\hline $5375.276(4)$ & $18598.524(12)$ & $5375.276(4)$ & 0.000 & 290 & & $4 \mathrm{f}$ & $(3 / 2)^{2}[5 / 2]_{2}^{\circ}$ & $7 \mathrm{~g}$ & $(3 / 2)^{2}[7 / 2]_{3}$ & $8.1 \mathrm{e}+06$ & $\mathrm{D}+$ & R1c & TW & \\
\hline $5381.9479(23)$ & $18575.468(8)$ & $5381.9511(21)$ & -0.0032 & 430 & * & $4 f$ & $(5 / 2)^{2}[7 / 2]^{\circ}{ }_{4}$ & $7 \mathrm{~g}$ & $(5 / 2)^{2}[9 / 2]_{4}$ & & & R1c & & \\
\hline $5381.9479(23)$ & $18575.468(8)$ & $5381.9485(20)$ & -0.0006 & 430 & * & $4 \mathrm{f}$ & $(5 / 2)^{2}[7 / 2]^{\circ}{ }_{4}$ & $7 \mathrm{~g}$ & $(5 / 2)^{2}[9 / 2]_{5}$ & $8.2 e+06$ & D+ & R1nc & TW & \\
\hline $5382.329(4)$ & 18574.152(15) & $5382.3347(19)$ & -0.006 & 140 & & $4 f$ & $(5 / 2)^{2}[7 / 2]^{\circ}{ }_{4}$ & $7 \mathrm{~g}$ & $(5 / 2)^{2}[11 / 2]_{5}$ & & & R1c & & \\
\hline $5383.289(4)$ & $18570.842(12)$ & $5383.2871(24)$ & 0.001 & 140 & & $4 \mathrm{f}$ & $(5 / 2)^{2}[7 / 2]^{\circ}{ }_{4}$ & $7 \mathrm{~g}$ & $(5 / 2)^{2}[7 / 2]_{4}$ & & & R1c & & \\
\hline $5384.818(3)$ & $18565.569(11)$ & $5384.8222(16)$ & -0.005 & 430 & & $4 \mathrm{~d}$ & $(3 / 2)^{2}[1 / 2]_{0}$ & $6 \mathrm{p}$ & $(3 / 2)^{2}[1 / 2]^{\circ}$ & & & R1c & & \\
\hline $5386.435(4)$ & $18559.995(14)$ & $5386.437(4)$ & -0.003 & 420 & * & $4 \mathrm{f}$ & $(3 / 2)^{2}[7 / 2]^{\circ}{ }_{4}$ & $7 \mathrm{~g}$ & $(3 / 2)^{2}[9 / 2]_{4}$ & & & $\mathrm{R} 1 \mathrm{c}$ & & \\
\hline $5386.435(4)$ & $18559.995(14)$ & & & 420 & * & $4 f$ & $(3 / 2)^{2}[7 / 2]^{\circ}{ }_{4}$ & $7 \mathrm{~g}$ & $(3 / 2)^{2}[9 / 2]_{5}$ & $1.0 \mathrm{e}+07$ & $\mathrm{D}+$ & R1nc & TW & \\
\hline $5386.8405(22)$ & $18558.597(8)$ & $5386.8419(10)$ & -0.0014 & 720 & & $4 \mathrm{~d}$ & $(5 / 2)^{2}[1 / 2]_{0}$ & $\mathrm{sp}$ & $\left({ }^{3} \mathrm{P}\right)^{3} \mathrm{P}^{\circ}{ }^{3} \mathrm{P}^{\circ}{ }_{1}$ & & & R1c & & \\
\hline $5390.029(4)$ & 18547.619(13) & $5390.0296(21)$ & -0.001 & 140 & * & $4 f$ & $(5 / 2)^{2}[9 / 2]^{\circ}{ }_{5}$ & $7 \mathrm{~g}$ & $(5 / 2)^{2}[9 / 2]_{4}$ & & & R1c & & \\
\hline $5390.029(4)$ & $18547.619(13)$ & $5390.0270(20)$ & 0.002 & 140 & * & $4 f$ & $(5 / 2)^{2}[9 / 2]^{\circ}$ & $7 \mathrm{~g}$ & $\begin{array}{l}(5 / 2)^{2}[9 / 2]_{5} \\
\text {. }\end{array}$ & & & R1nc & & \\
\hline $5390.4163(22)$ & $18546.286(8)$ & $5390.4169(13)$ & -0.0006 & 4800 & & $4 \mathrm{~d}$ & $(3 / 2)^{2}[5 / 2]_{2}$ & $\mathrm{sp}$ & $\left({ }^{3} \mathrm{~F}\right)^{1} \mathrm{P}^{\circ}{ }^{3} \mathrm{G}^{\circ}{ }_{3}$ & & & R1c & & \\
\hline $5391.6966(22)$ & $18541.882(8)$ & $5391.6987(10)$ & -0.0021 & 1700 & & $4 \mathrm{~d}$ & $(5 / 2)^{2}[9 / 2]_{5}$ & $\mathrm{sp}$ & $\left({ }^{3} \mathrm{~F}\right)^{1} \mathrm{P}^{\circ}{ }^{3} \mathrm{G}^{\circ}{ }_{5}$ & & & $\mathrm{R} 1 \mathrm{c}$ & & \\
\hline 5393.9372(21) & $18534.180(7)$ & $5393.9372(7)$ & 0.0001 & 2600 & & $\mathrm{sp}$ & $\left({ }^{3} \mathrm{~F}\right)^{3} \mathrm{P}^{\circ}{ }^{3} \mathrm{D}^{\circ}{ }_{2}$ & $6 \mathrm{~s}$ & $(3 / 2)^{2}[3 / 2]_{1}$ & & & R1c & & \\
\hline $5397.2943(21)$ & $18522.652(7)$ & $5397.2952(5)$ & -0.0008 & 580 & & $4 \mathrm{~d}$ & $(3 / 2)^{2}[7 / 2]_{3}$ & $4 f$ & $(5 / 2)^{2}[9 / 2]^{\circ}$ & & & R1c & & \\
\hline 5397.37 & 18522.38 & $5397.3730(17)$ & & & : & $\mathrm{sp}$ & $\left({ }^{3} \mathrm{~F}\right)^{1} \mathrm{P}^{\circ}{ }^{3} \mathrm{D}^{\circ}{ }_{3}$ & $9 \mathrm{~s}$ & $(5 / 2)^{2}[5 / 2]_{3}$ & & & & & \\
\hline $5398.573(4)$ & $18518.266(12)$ & $5398.573(4)$ & -0.000 & 140 & & $4 f$ & $(3 / 2)^{2}[7 / 2]^{\circ}$ & $7 \mathrm{~g}$ & $(3 / 2)^{2}[9 / 2]_{4}$ & $9 . e+06$ & $\mathrm{D}+$ & R1c & TW & \\
\hline $5403.714(4)$ & $18500.647(13)$ & $5403.7093(9)$ & 0.005 & 140 & & $5 p$ & $(5 / 2)^{2}[3 / 2]^{\circ} 2$ & $5 \mathrm{~d}$ & $(3 / 2)^{2}[1 / 2]_{1}$ & & & R1c & & \\
\hline 5405.652(3) & $18494.013(11)$ & $5405.6515(6)$ & 0.001 & 280 & & $4 p$ & ${ }^{3} \mathrm{~F}_{2}^{\circ}$ & $\mathrm{s}^{2}$ & ${ }^{3} \mathrm{P}_{2}$ & & & $\mathrm{R} 1 \mathrm{c}$ & & \\
\hline $5407.447(3)$ & $18487.877(11)$ & $5407.4452(23)$ & 0.001 & 280 & & $5 s$ & $(3 / 2)^{2}[3 / 2]_{2}$ & $\mathrm{sp}$ & $\left({ }^{1} \mathrm{D}\right)^{3} \mathrm{P}^{\circ}{ }^{3} \mathrm{D}_{2}^{\circ}$ & & & R1c & & \\
\hline $5408.383(4)$ & $18484.675(12)$ & $5408.3840(24)$ & -0.001 & 280 & & $5 s$ & $(3 / 2)^{2}[3 / 2]_{1}$ & $\mathrm{sp}$ & $\left({ }^{1} \mathrm{D}\right)^{3} \mathrm{P}^{\circ}{ }^{3} \mathrm{D}^{\circ}{ }_{1}$ & & & R1c & & \\
\hline $5422.002(4)$ & $18438.246(12)$ & $5422.0053(21)$ & -0.003 & 140 & & $4 \mathrm{f}$ & $(5 / 2)^{2}[9 / 2]^{\circ}{ }_{4}$ & $7 g$ & $(5 / 2)^{2}[9 / 2]_{4}$ & & & R1c & & \\
\hline $5422.392(3)$ & $18436.920(11)$ & $5422.3947(19)$ & -0.003 & 270 & & $4 f$ & $(5 / 2)^{2}[9 / 2]^{\circ}{ }_{4}$ & $7 \mathrm{~g}$ & $(5 / 2)^{2}[11 / 2]_{5}$ & $7.2 \mathrm{e}+06$ & $\mathrm{D}+$ & R1c & TW & \\
\hline $5428.274(3)$ & 18416.944(11) & $5428.2724(9)$ & 0.001 & 140 & & $4 \mathrm{~d}$ & $(5 / 2)^{2}[7 / 2]_{3}$ & $\mathrm{sp}$ & $\left({ }^{3} \mathrm{~F}\right)^{1} \mathrm{P}^{\circ}{ }^{3} \mathrm{~F}_{4}^{\circ}$ & & & R1c & & \\
\hline $5437.1438(21)$ & $18386.899(7)$ & 5437.1431(5) & 0.0006 & 2100 & & $4 \mathrm{~d}$ & $(3 / 2)^{2}[7 / 2]_{4}$ & $4 \mathrm{f}$ & $(5 / 2)^{2}[9 / 2]^{\circ} 4$ & & & $\mathrm{R} 1 \mathrm{c}$ & & \\
\hline $5437.5787(23)$ & $18385.428(8)$ & $5437.5790(4)$ & -0.0002 & 6700 & & $4 \mathrm{~d}$ & $(3 / 2)^{2}[7 / 2]_{3}$ & $4 \mathrm{f}$ & $(5 / 2)^{2}[7 / 2]^{\circ}{ }_{4}$ & & & R1c & & \\
\hline 5441.6461(22) & $18371.686(7)$ & $5441.6471(9)$ & -0.0011 & 2100 & & $4 \mathrm{~d}$ & $(5 / 2)^{2}[7 / 2]_{4}$ & $\mathrm{sp}$ & $\left({ }^{3} \mathrm{~F}\right)^{1} \mathrm{P}^{\circ}{ }^{3} \mathrm{~F}_{4}^{\circ}$ & & & R1c & & \\
\hline 5448.1914(23) & $18349.615(8)$ & $5448.1938(9)$ & -0.0024 & 260 & & $4 \mathrm{~d}$ & $(5 / 2)^{2}[7 / 2]_{3}$ & $\mathrm{sp}$ & $\left({ }^{3} \mathrm{P}\right)^{3} \mathrm{P}^{\circ}{ }^{3} \mathrm{D}^{\circ}{ }_{2}$ & & & $\mathrm{R} 1 \mathrm{c}$ & & \\
\hline $5449.409(4)$ & $18345.516(12)$ & $5449.4072(10)$ & 0.002 & 2700 & & $4 \mathrm{~d}$ & $(5 / 2)^{2}[3 / 2]_{2}$ & $\mathrm{sp}$ & $\left({ }^{3} \mathrm{P}\right)^{3} \mathrm{P}^{\circ}{ }^{3} \mathrm{D}_{3}^{\circ}$ & & & R1c & & \\
\hline $5458.0942(22)$ & $18316.323(7)$ & $5458.0941(9)$ & 0.0001 & 390 & & $4 \mathrm{~d}$ & $(3 / 2)^{2}[5 / 2]_{3}$ & $\mathrm{sp}$ & $\left({ }^{3} \mathrm{~F}\right)^{1} \mathrm{P}^{\circ}{ }^{3} \mathrm{D}^{\circ}{ }_{2}$ & & & R1c & & \\
\hline $5466.57(6)$ & $18287.94(20)$ & $5466.5527(5)$ & 0.01 & 1600 & * & $4 \mathrm{~d}$ & $(3 / 2)^{2}[7 / 2]_{3}$ & $4 \mathrm{f}$ & $(5 / 2)^{2}[5 / 2]^{\circ} 3$ & & & $\mathrm{R} 1 \mathrm{c}$ & & \\
\hline $5466.57(6)$ & $18287.94(20)$ & $5466.6267(9)$ & -0.06 & 1600 & * & $4 \mathrm{~d}$ & $(5 / 2)^{2}[5 / 2]_{2}$ & $\mathrm{sp}$ & $\left({ }^{3} \mathrm{P}\right)^{3} \mathrm{P}^{\circ}{ }^{3} \mathrm{D}^{\circ}$ & & & R1c & & \\
\hline $5468.563(3)$ & $18281.260(9)$ & $5468.5633(19)$ & -0.000 & 510 & & $5 \mathrm{~s}$ & $(3 / 2)^{2}[3 / 2]_{1}$ & $\mathrm{sp}$ & $\left({ }^{1} \mathrm{D}\right)^{3} \mathrm{P}^{\circ}{ }^{3} \mathrm{~F}_{2}^{\circ}$ & & & R1c & & \\
\hline $5469.376(3)$ & $18278.541(10)$ & $5469.3734(8)$ & 0.003 & 1300 & & $4 \mathrm{~d}$ & $(3 / 2)^{2}[3 / 2]_{2}$ & $\mathrm{sp}$ & $\left({ }^{3} \mathrm{~F}\right)^{1} \mathrm{P}^{\circ} 3^{3} \mathrm{~F}_{3}^{\circ}$ & & & R1c & & \\
\hline
\end{tabular}


Table A1. Cont.

\begin{tabular}{|c|c|c|c|c|c|c|c|c|c|c|c|c|c|c|}
\hline$\lambda_{\text {obs }}{ }^{\text {a }}(\AA)$ & $\sigma_{\mathrm{obs}}{ }^{\mathrm{b}}\left(\mathrm{cm}^{-1}\right)$ & $\lambda_{\text {Ritz }}{ }^{\mathrm{c}}(\AA)$ & $\begin{array}{c}\Delta \lambda_{\text {obs-Ritz }} \\
\text { (A) }\end{array}$ & $\begin{array}{l}I_{\text {obs }} \mathrm{d} \\
\text { (arb. u.) }\end{array}$ & Char ${ }^{\mathrm{e}}$ & & ower Level & & Upper Level & $A\left(\mathrm{~s}^{-1}\right)$ & $\operatorname{Acc}^{f}$ & Line Ref. $\mathrm{g}$ & TP Ref. $\mathrm{g}$ & Notes $h$ \\
\hline $5469.6826(21)$ & $18277.518(7)$ & $5469.6819(4)$ & 0.0006 & 4400 & & $4 \mathrm{~d}$ & $(3 / 2)^{2}[7 / 2]_{4}$ & $4 f$ & $(5 / 2)^{2}[9 / 2]^{\circ}{ }_{5}$ & & & R1c & & \\
\hline $5472.465(3)$ & $18268.225(11)$ & $5472.4631(10)$ & 0.002 & 130 & & $4 \mathrm{~d}$ & $(3 / 2)^{2}[5 / 2]_{2}$ & $\mathrm{sp}$ & $\left({ }^{3} \mathrm{~F}\right)^{1} \mathrm{P}^{\circ}{ }^{3} \mathrm{D}^{\circ}{ }_{2}$ & & & R1c & & \\
\hline $5472.9475(24)$ & $18266.614(8)$ & $5472.9467(5)$ & 0.0007 & 740 & & $4 \mathrm{~d}$ & $(3 / 2)^{2}[7 / 2]_{3}$ & $4 \mathrm{f}$ & $(5 / 2)^{2}[5 / 2]^{\circ}{ }_{2}$ & & & R1c & & \\
\hline 5473.01 & 18266.42 & $5473.006(2)$ & & & : & $\mathrm{sp}$ & $\left({ }^{3} \mathrm{~F}\right)^{1} \mathrm{P}^{\circ}{ }^{3} \mathrm{~F}^{\circ}{ }_{3}$ & $7 \mathrm{~g}$ & $(5 / 2)^{2}[9 / 2]_{4}$ & & & & & \\
\hline \multirow{2}{*}{$5473.119(4)$} & $18266.040(12)$ & $5473.1211(8)$ & -0.002 & 920 & & $5 p$ & $(5 / 2)^{2}[5 / 2]^{\circ}{ }_{2}$ & $5 \mathrm{~d}$ & $(3 / 2)^{2}[5 / 2]_{2}$ & & & R1c & & \\
\hline & & $5473.1623(16)$ & & & $\mathrm{m}$ & $4 \mathrm{f}$ & $(5 / 2)^{2}[7 / 2]^{\circ}{ }_{3}$ & 9s & $(5 / 2)^{2}[5 / 2]_{3}$ & & & R1nc & & \\
\hline $5477.127(3)$ & $18252.676(11)$ & 5477.1275(13) & -0.001 & 130 & & $4 \mathrm{~d}$ & $(3 / 2)^{2}[1 / 2]_{1}$ & $\mathrm{sp}$ & $\left({ }^{3} \mathrm{P}\right)^{3} \mathrm{P}^{\circ}{ }^{3} \mathrm{P}_{0}^{\circ}$ & & & R1c & & \\
\hline $5478.0268(23)$ & $18249.677(8)$ & $5478.0262(4)$ & 0.0006 & 3700 & & $4 \mathrm{~d}$ & $(3 / 2)^{2}[7 / 2]_{4}$ & $4 \mathrm{f}$ & $(5 / 2)^{2}[7 / 2]^{\circ} 4$ & & & R1c & & \\
\hline $5479.9089(22)$ & $18243.409(7)$ & 5479.9071(7) & 0.0018 & 3700 & & $5 p$ & $(5 / 2)^{2}[5 / 2]^{\circ} 2$ & $5 \mathrm{~d}$ & $(3 / 2)^{2}[5 / 2]_{3}$ & & & $\mathrm{R} 1 \mathrm{c}$ & & \\
\hline $5480.1613(24)$ & $18242.569(8)$ & $5480.1619(5)$ & -0.0006 & 1900 & & $4 \mathrm{~d}$ & $(3 / 2)^{2}[7 / 2]_{3}$ & $4 \mathrm{f}$ & $(5 / 2)^{2}[7 / 2]^{0}{ }_{3}$ & & & R1c & & \\
\hline $5482.524(3)$ & $18234.708(10)$ & $5482.5262(15)$ & -0.002 & 790 & & $\mathrm{sp}$ & $\left({ }^{3} \mathrm{~F}\right)^{3} \mathrm{P}^{0} \mathrm{~B}^{\circ}{ }_{4}$ & $6 \mathrm{~s}$ & $(5 / 2)^{2}[5 / 2]_{3}$ & & & R1c & & \\
\hline $5486.205(3)$ & $18222.473(11)$ & $5486.2037(8)$ & 0.001 & 120 & & $5 p$ & $(5 / 2)^{2}[3 / 2]^{\circ}{ }_{1}$ & $5 \mathrm{~d}$ & $(3 / 2)^{2}[5 / 2]_{2}$ & & & R1c & & \\
\hline $5493.5268(22)$ & $18198.186(7)$ & $5493.5289(6)$ & -0.0021 & 240 & & $5 p$ & $(5 / 2)^{2}[7 / 2]^{\circ}{ }_{3}$ & $5 \mathrm{~d}$ & $(3 / 2)^{2}[7 / 2]_{4}$ & & & R1c & & \\
\hline $5495.0454(23)$ & 18193.157(8) & $5495.0446(23)$ & 0.0008 & 430 & & $5 s$ & $(3 / 2)^{2}[3 / 2]_{2}$ & $\mathrm{sp}$ & $\left({ }^{1} \mathrm{D}\right)^{3} \mathrm{P}^{\circ}{ }^{3} \mathrm{~F}^{\circ}{ }_{3}$ & & & R1c & & \\
\hline $5496.868(3)$ & 18187.126(11) & $5496.8685(11)$ & -0.001 & 510 & & $4 \mathrm{~d}$ & $(5 / 2)^{2}[3 / 2]_{2}$ & $\mathrm{sp}$ & $\left({ }^{3} \mathrm{P}\right)^{3} \mathrm{P}^{\circ}{ }^{3} \mathrm{P}^{\circ}{ }_{2}$ & & & $\mathrm{R} 1 \mathrm{c}$ & & \\
\hline $5504.021(4)$ & $18163.488(12)$ & $5504.0559(5)$ & -0.035 & 120 & ? & $4 \mathrm{~d}$ & $(3 / 2)^{2}[7 / 2]_{3}$ & $4 f$ & $(5 / 2)^{2}[3 / 2]^{\circ}{ }_{2}$ & & & $\mathrm{R} 1 \mathrm{c}$ & & $\mathrm{x}$ \\
\hline $5504.844(3)$ & 18160.774(11) & $5504.8442(11)$ & -0.000 & 240 & & $4 \mathrm{~d}$ & $(5 / 2)^{2}[3 / 2]_{1}$ & $\mathrm{sp}$ & $\left({ }^{3} \mathrm{P}\right)^{3} \mathrm{P}^{\circ}{ }^{3} \mathrm{P}^{\circ}{ }_{2}$ & & & R1c & & \\
\hline $5507.428(4)$ & 18152.254(12) & $5507.4337(4)$ & -0.006 & 240 & & $4 \mathrm{~d}$ & $(3 / 2)^{2}[7 / 2]_{4}$ & $4 f$ & $(5 / 2)^{2}[5 / 2]^{\circ}{ }_{3}$ & & & R1c & & \\
\hline $5512.8649(22)$ & $18134.351(7)$ & $5512.8650(6)$ & -0.0001 & 240 & & $5 p$ & $(5 / 2)^{2}[7 / 2]^{\circ}{ }_{3}$ & $5 \mathrm{~d}$ & $(3 / 2)^{2}[7 / 2]_{3}$ & & & R1c & & \\
\hline $5521.249(3)$ & $18106.815(9)$ & $5521.2475(5)$ & 0.001 & 230 & & $4 \mathrm{~d}$ & $(3 / 2)^{2}[7 / 2]_{4}$ & $4 \mathrm{f}$ & $(5 / 2)^{2}[7 / 2]^{\circ}{ }_{3}$ & & & R1c & & \\
\hline $5521.729(3)$ & 18105.239(9) & 5521.7315(8) & -0.002 & 1100 & & $5 p$ & $(5 / 2)^{2}[5 / 2]^{\circ} 2$ & $5 \mathrm{~d}$ & $(3 / 2)^{2}[3 / 2]_{2}$ & & & $\mathrm{R} 1 \mathrm{c}$ & & \\
\hline $5525.438(3)$ & 18093.088(9) & $5525.4391(6)$ & -0.001 & 230 & & $5 p$ & $(5 / 2)^{2}[7 / 2]^{\circ}{ }_{4}$ & $5 \mathrm{~d}$ & $(3 / 2)^{2}[7 / 2]_{4}$ & & & R1c & & \\
\hline 5525.53 & 18092.78 & $5525.532(6)$ & & & : & $\mathrm{sp}$ & $\left({ }^{3} \mathrm{~F}\right)^{1} \mathrm{P}^{\circ}{ }^{3} \mathrm{D}^{\circ}{ }_{2}$ & $8 \mathrm{~d}$ & $(5 / 2)^{2}[5 / 2]_{2}$ & & & & & \\
\hline $5527.1968(22)$ & $18087.330(7)$ & $5527.1993(7)$ & -0.0025 & 2200 & & $4 \mathrm{~d}$ & $(3 / 2)^{2}[7 / 2]_{3}$ & $\mathrm{sp}$ & $\left({ }^{3} \mathrm{~F}\right)^{1} \mathrm{P}^{\circ}{ }^{3} \mathrm{G}_{4}^{\circ}$ & & & R1c & & \\
\hline $5527.6813(22)$ & $18085.744(7)$ & $5527.6804(5)$ & 0.0009 & 690 & & $4 \mathrm{~d}$ & $(3 / 2)^{2}[3 / 2]_{1}$ & $4 f$ & $(5 / 2)^{2}[5 / 2]^{0}{ }_{2}$ & & & R1c & & \\
\hline $5534.668(3)$ & $18062.913(10)$ & $5534.6726(8)$ & -0.004 & 230 & & $5 p$ & $(5 / 2)^{2}[5 / 2]^{\circ} 3$ & $5 \mathrm{~d}$ & $(3 / 2)^{2}[5 / 2]_{2}$ & & & R1c & & \\
\hline $5535.0494(22)$ & $18061.669(7)$ & $5535.0478(8)$ & 0.0016 & 4100 & & $5 p$ & $(5 / 2)^{2}[3 / 2]^{\circ}{ }_{1}$ & $5 \mathrm{~d}$ & $(3 / 2)^{2}[3 / 2]_{2}$ & & & $\mathrm{R} 1 \mathrm{c}$ & & \\
\hline $5537.670(20)$ & 18053.12(7) & $5537.6662(21)$ & 0.004 & 340 & & $\mathrm{sp}$ & $\left({ }^{3} \mathrm{~F}\right)^{3} \mathrm{P}^{\circ} 1^{1} \mathrm{G}_{4}^{\circ}$ & $5 \mathrm{~d}$ & $(5 / 2)^{2}[7 / 2]_{4}$ & & & R1c & & \\
\hline $5538.3836(22)$ & $18050.796(7)$ & $5538.3835(6)$ & 0.0001 & 880 & & $4 d$ & $(3 / 2)^{2}[7 / 2]_{4}$ & $4 \mathrm{f}$ & $(5 / 2)^{2}[11 / 2]^{\circ}{ }_{5}$ & & & R1c & & \\
\hline $5541.6120(22)$ & $18040.280(7)$ & $5541.6123(6)$ & -0.0003 & 1300 & & $5 p$ & $(5 / 2)^{2}[5 / 2]^{\circ}{ }_{3}$ & $5 \mathrm{~d}$ & $(3 / 2)^{2}[5 / 2]_{3}$ & & & R1c & & \\
\hline $5543.5368(22)$ & $18034.016(7)$ & $5543.5385(7)$ & -0.0016 & 1900 & & $\mathrm{sp}$ & $\left({ }^{3} \mathrm{~F}^{3} \mathrm{P}^{\circ}{ }^{3} \mathrm{~F}^{\circ}{ }_{2}\right.$ & $5 \mathrm{~d}$ & $(5 / 2)^{2}[5 / 2]_{2}$ & & & R1c & & \\
\hline $5544.7815(22)$ & $18029.968(7)$ & $5544.7803(6)$ & 0.0012 & 220 & & $4 \mathrm{~d}$ & $(3 / 2)^{2}[3 / 2]_{1}$ & $4 \mathrm{f}$ & $(5 / 2)^{2}[3 / 2]^{\circ}{ }_{1}$ & & & R1c & & \\
\hline $5555.5122(22)$ & 17995.143(7) & $5555.5123(7)$ & -0.0001 & 5500 & & $\mathrm{sp}$ & $\left({ }^{3} \mathrm{~F}\right)^{3} \mathrm{P}^{\circ}{ }^{3} \mathrm{~F}^{\circ}{ }_{2}$ & $5 \mathrm{~d}$ & $(5 / 2)^{2}[7 / 2]_{3}$ & & & $\mathrm{R} 1 \mathrm{c}$ & & \\
\hline $5558.314(3)$ & $17986.072(11)$ & $5558.3108(10)$ & 0.003 & 110 & & $4 \mathrm{~d}$ & $(3 / 2)^{2}[7 / 2]_{3}$ & $\mathrm{sp}$ & $\left({ }^{3} \mathrm{~F}\right)^{1} \mathrm{P}^{\circ}{ }^{3} \mathrm{D}^{\circ}{ }_{3}$ & & & R1c & & \\
\hline $5559.417(4)$ & 17982.504(11) & $5559.4167(5)$ & 0.000 & 110 & & $4 \mathrm{~d}$ & $(3 / 2)^{2}[3 / 2]_{1}$ & $4 \mathrm{f}$ & $(5 / 2)^{2}[3 / 2]^{\circ}{ }_{2}$ & & & R1c & & \\
\hline $5560.5761(24)$ & $17978.755(8)$ & $5560.5739(8)$ & 0.0022 & 610 & & $5 p$ & $(5 / 2)^{2}[3 / 2]^{\circ}{ }_{1}$ & $5 \mathrm{~d}$ & $(3 / 2)^{2}[3 / 2]_{1}$ & & & $\mathrm{R} 1 \mathrm{c}$ & & \\
\hline $5562.074(3)$ & $17973.913(11)$ & $5562.0730(8)$ & 0.001 & 110 & & $4 \mathrm{~d}$ & $(3 / 2)^{2}[3 / 2]_{1}$ & $4 \mathrm{f}$ & $(5 / 2)^{2}[1 / 2]^{0}$ & & & R1c & & \\
\hline $5562.6460(23)$ & $17972.065(7)$ & $5562.6478(9)$ & -0.0018 & 770 & & $4 \mathrm{~d}$ & $(5 / 2)^{2}[5 / 2]_{2}$ & $\mathrm{sp}$ & $\left({ }^{3} \mathrm{P}\right)^{3} \mathrm{P}^{\circ}{ }^{3} \mathrm{D}^{\circ}{ }_{1}$ & & & R1c & & \\
\hline $5567.002(3)$ & $17958.004(9)$ & $5567.0018(8)$ & -0.000 & 210 & & $4 \mathrm{~d}$ & $(3 / 2)^{2}[5 / 2]_{3}$ & $\mathrm{sp}$ & $\left({ }^{3} \mathrm{~F}\right)^{1} \mathrm{P}^{\circ}{ }^{3} \mathrm{~F}_{3}^{\circ}$ & & & $\mathrm{R} 1 \mathrm{c}$ & & \\
\hline $5569.670(3)$ & $17949.401(11)$ & $5569.6723(16)$ & -0.002 & 210 & & $5 \mathrm{~d}$ & $(5 / 2)^{2}[9 / 2]_{5}$ & $7 f$ & $(5 / 2)^{2}[9 / 2]^{\circ}$ & & & R1c & & \\
\hline $5571.6406(24)$ & $17943.052(8)$ & $5571.6415(7)$ & -0.0009 & 210 & & $5 p$ & $(5 / 2)^{2}[5 / 2]^{\circ} 2$ & $5 \mathrm{~d}$ & $(3 / 2)^{2}[7 / 2]_{3}$ & & & R1c & & \\
\hline $5578.4356(23)$ & 17921.196(7) & 5578.4337(17) & 0.0019 & 520 & & $5 \mathrm{~d}$ & $(5 / 2)^{2}[9 / 2]_{5}$ & $7 f$ & $(5 / 2)^{2}[11 / 2]_{6}^{\circ}$ & $1.0 \mathrm{e}+07$ & $\mathrm{D}+$ & R1c & TW & \\
\hline
\end{tabular}


Table A1. Cont.

\begin{tabular}{|c|c|c|c|c|c|c|c|c|c|c|c|c|c|c|}
\hline$\lambda_{\text {obs }}{ }^{\text {a }}(\AA)$ & $\sigma_{\mathrm{obs}}{ }^{\mathrm{b}}\left(\mathrm{cm}^{-1}\right)$ & $\lambda_{\text {Ritz }}{ }^{c}(\AA)$ & $\begin{array}{c}\Delta \lambda_{\text {obs-Ritz }} \\
(\mathrm{A})\end{array}$ & $\begin{array}{l}I_{\text {obs }} \mathrm{d} \\
\text { (arb. u.) }\end{array}$ & Char ${ }^{e}$ & & Lower Level & & Upper Level & $A\left(\mathrm{~s}^{-1}\right)$ & $\operatorname{Acc}^{f}$ & Line Ref. $\mathrm{g}$ & TP Ref. $\mathrm{g}$ & Notes ${ }^{h}$ \\
\hline $5579.431(4)$ & 17917.999(12) & $5579.4269(20)$ & 0.004 & 210 & & $5 \mathrm{~d}$ & $(5 / 2)^{2}[3 / 2]_{2}$ & $7 \mathrm{f}$ & $(5 / 2)^{2}[5 / 2]^{\circ}{ }_{3}$ & & & R1c & & \\
\hline $5581.9475(24)$ & $17909.921(8)$ & $5581.9507(9)$ & -0.0032 & 310 & & $4 \mathrm{~d}$ & $(3 / 2)^{2}[5 / 2]_{2}$ & sp & $\left({ }^{3} \mathrm{~F}\right)^{1} \mathrm{P}^{0}{ }^{3} \mathrm{~F}^{\circ}$ & & & R1c & & \\
\hline $5582.173(4)$ & 17909.196(11) & $5582.1725(18)$ & 0.001 & 100 & & $5 \mathrm{~d}$ & $(5 / 2)^{2}[9 / 2]_{4}$ & $7 \mathrm{f}$ & $(5 / 2)^{2}[9 / 2]^{\circ}{ }_{4}$ & & & R1c & & \\
\hline $5583.864(3)$ & $17903.773(11)$ & $5583.8665(11)$ & -0.002 & 210 & & $4 \mathrm{~d}$ & $(3 / 2)^{2}[1 / 2]_{1}$ & $\mathrm{sp}$ & $\left({ }^{3} \mathrm{P}\right)^{3} \mathrm{P}^{\circ} \mathrm{P}^{\circ} \mathrm{P}_{1}^{\circ}$ & & & R1c & & \\
\hline $5584.346(3)$ & $17902.230(11)$ & $5584.3413(22)$ & 0.004 & 480 & & $5 \mathrm{~d}$ & $(3 / 2)^{2}[7 / 2]_{3}$ & $7 \mathrm{f}$ & $(3 / 2)^{2}[9 / 2]^{\circ}$ & $9 . e+06$ & $\mathrm{D}+$ & R1c & TW & \\
\hline 5591.382(3) & $17879.700(9)$ & $5591.3777(6)$ & 0.005 & 210 & & $\mathrm{sp}$ & $\left({ }^{3} \mathrm{~F}\right)^{3} \mathrm{P}^{\circ}{ }^{3} \mathrm{~F}^{\circ}{ }_{2}$ & $5 \mathrm{~d}$ & $(5 / 2)^{2}[5 / 2]_{3}$ & & & R1c & & \\
\hline $5592.5298(24)$ & $17876.032(8)$ & $5592.5272(19)$ & 0.0026 & 510 & & $5 \mathrm{~d}$ & $(5 / 2)^{2}[9 / 2]_{4}$ & $7 \mathrm{f}$ & $(5 / 2)^{2}[11 / 2]^{\circ}{ }_{5}$ & $9 . e+06$ & $\mathrm{D}+$ & $\mathrm{R} 1 \mathrm{c}$ & TW & \\
\hline $5593.7697(13)$ & $17872.070(4)$ & $5593.7708(4)$ & -0.0011 & 5400 & & $4 \mathrm{~d}$ & $(3 / 2)^{2}[3 / 2]_{2}$ & $4 \mathrm{f}$ & $(5 / 2)^{2}[5 / 2]_{3}^{\circ}$ & & & F_Re & & \\
\hline $5600.4662(23)$ & 17850.701(7) & $5600.4661(5)$ & 0.0001 & 500 & & $4 \mathrm{~d}$ & $(3 / 2)^{2}[3 / 2]_{2}$ & $4 \mathrm{f}$ & $(5 / 2)^{2}[5 / 2]^{\circ} 2$ & & & $\mathrm{R} 1 \mathrm{c}$ & & \\
\hline $5600.581(4)$ & $17850.334(11)$ & $5600.5810(10)$ & 0.000 & 300 & & $4 \mathrm{~d}$ & $(3 / 2)^{2}[7 / 2]_{4}$ & $\mathrm{sp}$ & $\left({ }^{3} \mathrm{~F}\right)^{1} \mathrm{P}^{\circ}{ }^{3} \mathrm{D}^{\circ}{ }_{3}$ & & & R1c & & \\
\hline $5607.282(3)$ & 17829.001(9) & $5607.2823(22)$ & 0.000 & 300 & & $5 \mathrm{~d}$ & $(3 / 2)^{2}[7 / 2]_{4}$ & $7 \mathrm{f}$ & $(3 / 2)^{2}[9 / 2]^{\circ}{ }_{5}$ & 8.e+06 & D+ & R1c & TW & \\
\hline $5608.027(3)$ & $17826.635(8)$ & $5608.0216(5)$ & 0.005 & 200 & & $4 \mathrm{~d}$ & $(3 / 2)^{2}[3 / 2]_{2}$ & $4 \mathrm{f}$ & $(5 / 2)^{2}[7 / 2]^{\circ} 3$ & & & R1c & & \\
\hline $5615.2350(23)$ & $17803.751(7)$ & $5615.2379(6)$ & -0.0030 & 5500 & & $5 p$ & $(5 / 2)^{2}[5 / 2]^{\circ} 3$ & $5 \mathrm{~d}$ & $(3 / 2)^{2}[7 / 2]_{4}$ & & & R1c & & \\
\hline $5618.016(3)$ & 17794.938(11) & $5618.0200(6)$ & -0.004 & 98 & & $4 \mathrm{~d}$ & $(3 / 2)^{2}[3 / 2]_{2}$ & $4 \mathrm{f}$ & $(5 / 2)^{2}[3 / 2]^{\circ} 1$ & & & R1c & & \\
\hline $5619.885(7)$ & 17789.021(21) & $5619.8874(16)$ & -0.003 & 190 & & $4 \mathrm{~d}$ & $(3 / 2)^{2}[3 / 2]_{2}$ & $\mathrm{sp}$ & $\left({ }^{3} \mathrm{P}\right)^{3} \mathrm{P}^{\mathrm{o}} \mathrm{S}^{5}{ }_{2}$ & & & $\mathrm{R} 1 \mathrm{c}$ & & \\
\hline $5620.7814(23)$ & $17786.183(7)$ & $5620.7805(5)$ & 0.0008 & 850 & & $4 \mathrm{~d}$ & $(3 / 2)^{2}[5 / 2]_{3}$ & $4 f$ & $(5 / 2)^{2}[9 / 2]^{\circ} 4$ & & & $\mathrm{R} 1 \mathrm{c}$ & & \\
\hline $5621.7027(24)$ & $17783.268(8)$ & $5621.7019(10)$ & 0.0009 & 530 & & $4 \mathrm{~d}$ & $(5 / 2)^{2}[1 / 2]_{0}$ & $\mathrm{sp}$ & $\left({ }^{3} \mathrm{P}\right)^{3} \mathrm{P}^{\circ}{ }^{3} \mathrm{D}^{\circ}{ }_{1}$ & & & R1c & & \\
\hline $5629.240(3)$ & 17759.456(11) & $5629.218(3)$ & 0.022 & 190 & ? & $5 \mathrm{~d}$ & $(5 / 2)^{2}[5 / 2]_{3}$ & $7 \mathrm{f}$ & $(5 / 2)^{2}[7 / 2]^{\circ} 3$ & & & R1c & & $x$ \\
\hline $5630.589(4)$ & $17755.202(11)$ & $5630.5900(18)$ & -0.001 & 95 & & $5 \mathrm{~d}$ & 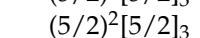 & $7 f$ & $(5 / 2)^{2}[9 / 2]^{\circ} 4$ & & & R1c & & \\
\hline 5631.153(3) & $17753.425(11)$ & $5631.1561(18)$ & -0.003 & 190 & & $5 \mathrm{~d}$ & $(5 / 2)^{2}[5 / 2]_{3}$ & $7 \mathrm{f}$ & $(5 / 2)^{2}[7 / 2]^{\circ}$ & & & R1c & & \\
\hline $5631.358(3)$ & 17752.777(11) & $5631.3600(20)$ & -0.002 & 95 & & $5 \mathrm{~d}$ & $(5 / 2)^{2}[5 / 2]_{3}$ & $7 \mathrm{f}$ & $(5 / 2)^{2}[5 / 2]_{3}{ }_{3}$ & & & $\mathrm{R} 1 \mathrm{c}$ & & \\
\hline $5633.0442(23)$ & $17747.464(7)$ & $5633.0461(5)$ & -0.0020 & 3400 & & $4 \mathrm{~d}$ & $(3 / 2)^{2}[3 / 2]_{2}$ & $4 f$ & $(5 / 2)^{2}[3 / 2]^{\circ}{ }_{2}$ & & & R1c & & \\
\hline $5633.602(3)$ & $17745.707(10)$ & $5633.6032(12)$ & -0.001 & 950 & & $4 \mathrm{~d}$ & $(5 / 2)^{2}[5 / 2]_{3}$ & $\mathrm{sp}$ & $\left({ }^{3} \mathrm{P}\right)^{3} \mathrm{P}^{\circ}{ }^{3} \mathrm{P}^{\circ}{ }_{2}$ & & & R1c & & \\
\hline $5635.5118(23)$ & $17739.693(7)$ & $5635.5130(11)$ & -0.0012 & 3000 & & $4 \mathrm{~d}$ & $(5 / 2)^{2}[7 / 2]_{4}$ & $\mathrm{sp}$ & $\left({ }^{3} \mathrm{~F}\right)^{1} \mathrm{P}^{\circ}{ }^{3} \mathrm{G}_{5}^{\circ}$ & & & R1c & & \\
\hline $5637.162(4)$ & $17734.501(13)$ & $5637.1546(21)$ & 0.007 & 190 & & $\mathrm{sp}$ & $\left({ }^{3} \mathrm{~F}\right)^{3} \mathrm{P}^{\circ} 1^{\circ} \mathrm{G}_{4}^{\circ}$ & $5 d$ & $(5 / 2)^{2}[9 / 2]_{5}$ & & & R1c & & \\
\hline $5637.468(3)$ & $17733.536(8)$ & $5637.4686(8)$ & -0.000 & 1100 & & $\mathrm{sp}$ & $\left({ }^{3} \mathrm{~F}\right)^{3} \mathrm{P}^{\circ}{ }^{3} \mathrm{~F}^{\circ}{ }_{2}$ & $5 d$ & $(5 / 2)^{2}[3 / 2]_{1}$ & & & R1c & & \\
\hline $5641.2645(23)$ & $17721.603(7)$ & $5641.2645(18)$ & -0.0000 & 2300 & & $5 p$ & $(3 / 2)^{2}[1 / 2]^{\circ} 1$ & $5 \mathrm{~d}$ & $(3 / 2)^{2}[1 / 2]_{0}$ & & & $\mathrm{R} 1 \mathrm{c}$ & & \\
\hline $5643.5338(24)$ & $17714.477(8)$ & $5643.5346(7)$ & -0.0008 & 280 & & $\mathrm{sp}$ & $\left({ }^{3} \mathrm{~F}\right)^{3} \mathrm{P}^{\circ} 3^{\circ} \mathrm{F}_{2}$ & $5 \mathrm{~d}$ & $(5 / 2)^{2}[3 / 2]_{2}$ & & & R1c & & \\
\hline $5648.0170(24)$ & $17700.416(8)$ & $5648.0161(6)$ & 0.0009 & 430 & & $4 \mathrm{~d}$ & $(3 / 2)^{2}[3 / 2]_{2}$ & $4 \mathrm{f}$ & $(5 / 2)^{2}[1 / 2]^{\circ}{ }_{1}$ & & & R1c & & \\
\hline $5651.708(4)$ & $17688.857(11)$ & $5651.7093(9)$ & -0.001 & 370 & & $\mathrm{sp}$ & $\left({ }^{3} \mathrm{~F}\right)^{3} \mathrm{P}^{\circ}{ }^{3} \mathrm{D}^{\circ}{ }_{1}$ & $6 \mathrm{~s}$ & $(3 / 2)^{2}[3 / 2]_{2}$ & & & R1c & & \\
\hline $5656.632(3)$ & $17673.459(10)$ & $5656.6271(10)$ & 0.005 & 7300 & & $5 p$ & $(5 / 2)^{2}[3 / 2]^{0} 1$ & $5 \mathrm{~d}$ & $(3 / 2)^{2}[1 / 2]_{1}$ & & & R1c & & \\
\hline $5664.4833(23)$ & $17648.963(7)$ & $5664.4829(5)$ & 0.0004 & 2900 & & $4 \mathrm{~d}$ & $(3 / 2)^{2}[5 / 2]_{3}$ & $4 \mathrm{f}$ & $(5 / 2)^{2}[7 / 2]^{\circ}$ & & & R1c & & \\
\hline 5667.434(3) & $17639.775(11)$ & $5667.4344(19)$ & -0.001 & 180 & & $5 \mathrm{~d}$ & $(5 / 2)^{2}[7 / 2]_{3}$ & $7 \mathrm{f}$ & $(5 / 2)^{2}[9 / 2]^{2}$ & $5.8 \mathrm{e}+06$ & $\mathrm{D}+$ & $\mathrm{R} 1 \mathrm{c}$ & TW & \\
\hline $5668.007(3)$ & $17637.991(10)$ & $5668.0080(18)$ & -0.001 & 180 & & $5 \mathrm{~d}$ & $(5 / 2)^{2}[7 / 2]_{3}$ & $7 \mathrm{f}$ & $(5 / 2)^{2}[7 / 2]^{4}{ }_{4}$ & & & R1c & & \\
\hline $5670.329(3)$ & $17630.769(10)$ & $5670.3245(16)$ & 0.004 & 350 & & $5 \mathrm{~d}$ & $(5 / 2)^{2}[7 / 2]_{4}$ & $7 \mathrm{f}$ & $(5 / 2)^{2}[9 / 2]^{\circ}$ & $7.4 \mathrm{e}+06$ & $\mathrm{D}+$ & R1c & TW & \\
\hline $5674.694(4)$ & $17617.207(12)$ & $5674.6947(7)$ & -0.001 & 180 & * & $\mathrm{sp}$ & $\left({ }^{3} \mathrm{~F}\right)^{3} \mathrm{P}^{\circ}{ }^{3} \mathrm{~F}_{3}^{\circ}$ & $6 \mathrm{~s}$ & $(3 / 2)^{2}[3 / 2]_{2}$ & & & $\mathrm{R} 1 \mathrm{c}$ & & \\
\hline 5674.694(4) & $17617.207(12)$ & $5674.6968(9)$ & -0.003 & 180 & * & $\mathrm{sp}$ & $\left({ }^{3} \mathrm{~F}\right)^{3} \mathrm{P}^{\circ} 1^{1} \mathrm{~F}_{3}^{\circ}$ & $5 \mathrm{~d}$ & $(3 / 2)^{2}[5 / 2]_{2}$ & & & R1nc & & \\
\hline $5676.005(3)$ & 17613.137(10) & $5676.0028(11)$ & 0.002 & 1300 & & $4 \mathrm{~d}$ & $(5 / 2)^{2}[7 / 2]_{4}$ & $\mathrm{sp}$ & $\left({ }^{3} \mathrm{P}\right)^{3} \mathrm{P}^{\circ}{ }^{3} \mathrm{D}^{\circ}{ }_{3}$ & & & R1c & & \\
\hline $5678.556(3)$ & $17605.224(11)$ & 5678.554(3) & 0.002 & 87 & & $5 \mathrm{~d}$ & $(5 / 2)^{2}[5 / 2]_{2}$ & $7 \mathrm{f}$ & $(5 / 2)^{2}[7 / 2]^{\circ}{ }_{3}$ & & & $\mathrm{R} 1 \mathrm{c}$ & & \\
\hline $5681.353(3)$ & $17596.559(11)$ & $5681.3597(11)$ & -0.007 & 87 & & $4 \mathrm{~d}$ & $\begin{array}{l}(5 / 2)^{2}[5 / 2]_{2} \\
\text { nat }\end{array}$ & sp & $\left({ }^{3} \mathrm{P}\right)^{3} \mathrm{P}^{\circ}{ }^{3} \mathrm{D}_{3}$ & & & R1c & & \\
\hline $5681.998(3)$ & $17594.559(9)$ & $5681.9923(7)$ & 0.006 & 430 & & $\mathrm{sp}$ & $\left({ }^{3} \mathrm{~F}\right)^{3} \mathrm{P}^{\circ}{ }^{1} \mathrm{~F}^{\circ}{ }_{3}$ & $5 \mathrm{~d}$ & $(3 / 2)^{2}[5 / 2]_{3}$ & & & R1c & & \\
\hline $5682.433(3)$ & 17593.214(9) & $5682.4316(9)$ & 0.001 & 2100 & & $\mathrm{sp}$ & $\left({ }^{3} \mathrm{~F}\right)^{3} \mathrm{P}^{\circ}{ }^{3} \mathrm{D}^{\circ}{ }_{1}$ & $6 \mathrm{~s}$ & $(3 / 2)^{2}[3 / 2]_{1}$ & & & R1c & & \\
\hline
\end{tabular}


Table A1. Cont.

\begin{tabular}{|c|c|c|c|c|c|c|c|c|c|c|c|c|c|c|}
\hline$\lambda_{\text {obs }}{ }^{\text {a }}(\AA)$ & $\sigma_{\mathrm{obs}}{ }^{\mathrm{b}}\left(\mathrm{cm}^{-1}\right)$ & $\lambda_{\text {Ritz }}{ }^{c}(\AA)$ & $\begin{array}{c}\Delta \lambda_{\text {obs-Ritz }} \\
\text { (A) }\end{array}$ & $\begin{array}{l}I_{\text {obs }} \mathrm{d} \\
\text { (arb. u.) }\end{array}$ & Char ${ }^{e}$ & & ower Level & & pper Level & $A\left(\mathrm{~s}^{-1}\right)$ & $\operatorname{Acc}^{f}$ & Line Ref. $\mathrm{g}$ & TP Ref. $\mathrm{g}$ & Notes ${ }^{h}$ \\
\hline $5689.83(4)$ & $17570.34(12)$ & $5689.8869(11)$ & -0.06 & 170 & & $4 \mathrm{~d}$ & $(3 / 2)^{2}[3 / 2]_{2}$ & $\mathrm{sp}$ & $\left({ }^{3} \mathrm{~F}\right)^{1} \mathrm{P}^{\circ}{ }^{3} \mathrm{D}^{\circ}{ }_{3}$ & & & S36c & & \\
\hline $5694.526(3)$ & $17555.852(10)$ & 5694.5253(14) & 0.001 & 170 & & $4 \mathrm{~d}$ & $(3 / 2)^{2}[3 / 2]_{1}$ & $\mathrm{sp}$ & $\left({ }^{3} \mathrm{P}\right)^{3} \mathrm{P}^{\circ}{ }^{3} \mathrm{P}^{\circ} 0$ & & & R1c & & \\
\hline 5695.931(3) & $17551.521(10)$ & $5695.9321(5)$ & -0.001 & 340 & & $4 \mathrm{~d}$ & $(3 / 2)^{2}[5 / 2]_{3}$ & $4 \mathrm{f}$ & $(5 / 2)^{2}[5 / 2]^{\circ} 3$ & & & R1c & & \\
\hline $5706.012(4)$ & $17520.514(12)$ & $5706.0055(7)$ & 0.006 & 450 & & $4 p$ & ${ }^{1} \mathrm{~F}^{\circ}{ }_{3}$ & $s^{2}$ & ${ }^{3} \mathrm{P}_{2}$ & & & R1c & & \\
\hline $5710.7111(24)$ & $17506.097(7)$ & $5710.7089(5)$ & 0.0022 & 250 & & $4 \mathrm{~d}$ & $(3 / 2)^{2}[5 / 2]_{3}$ & $4 \mathrm{f}$ & $(5 / 2)^{2}[7 / 2]^{\circ}{ }_{3}$ & & & R1c & & \\
\hline $5711.586(4)$ & 17503.416(11) & $5711.5823(5)$ & 0.003 & 250 & & $4 \mathrm{~d}$ & $(3 / 2)^{2}[5 / 2]_{2}$ & $4 \mathrm{f}$ & $(5 / 2)^{2}[5 / 2]^{\circ} 3$ & & & R1c & & \\
\hline $5721.7840(24)$ & $\begin{array}{l}17472.220(7) \\
\text { (1) }\end{array}$ & $5721.7856(7)$ & -0.0015 & 4100 & & $4 p$ & ${ }^{3} \mathrm{P}^{\circ}$ & $\mathrm{s}^{2}$ & ${ }^{1} \mathrm{D}_{2}$ & & & $\mathrm{R} 1 \mathrm{c}$ & & \\
\hline $5726.442(3)$ & $17458.009(11)$ & $5726.4406(6)$ & 0.001 & 160 & & $4 \mathrm{~d}$ & $(3 / 2)^{2}[5 / 2]_{2}$ & $4 \mathrm{f}$ & $(5 / 2)^{2}[7 / 2]^{\circ}{ }_{3}$ & & & R1c & & \\
\hline $5731.004(4)$ & $17444.110(12)$ & $5731.0007(11)$ & 0.004 & 160 & & $4 \mathrm{~d}$ & $(3 / 2)^{2}[1 / 2]_{1}$ & $\mathrm{sp}$ & $\left({ }^{3} \mathrm{P}\right)^{3} \mathrm{P}^{\circ}{ }^{\circ} \mathrm{D}^{\circ}{ }_{2}$ & & & R1c & & \\
\hline $5736.659(4)$ & $17426.915(11)$ & $5736.6603(5)$ & -0.001 & 160 & & $4 \mathrm{~d}$ & $(3 / 2)^{2}[5 / 2]_{3}$ & $4 f$ & $(5 / 2)^{2}[3 / 2]^{\circ} 2$ & & & R1c & & \\
\hline $5752.536(4)$ & $17378.819(11)$ & $5752.5355(6)$ & 0.000 & 580 & & $4 \mathrm{~d}$ & $(3 / 2)^{2}[5 / 2]_{2}$ & $4 f$ & $(5 / 2)^{2}[3 / 2]^{\circ} 2$ & & & $\mathrm{R} 1 \mathrm{c}$ & & \\
\hline $5759.015(4)$ & $17359.266(11)$ & $5759.0170(20)$ & -0.002 & 150 & & $\mathrm{sp}$ & $\left({ }^{3} \mathrm{~F}\right)^{1} \mathrm{P}^{\circ}{ }^{3} \mathrm{G}_{5}^{\circ}$ & $6 \mathrm{~g}$ & $(5 / 2)^{2}[11 / 2]_{6}$ & & & R1c & & \\
\hline $5759.4219(24)$ & $\begin{array}{l}17358.040(7) \\
\text { (1) }\end{array}$ & $5759.4213(7)$ & 0.0005 & 6300 & & $\mathrm{sp}$ & $\left({ }^{3} \mathrm{~F}\right)^{3} \mathrm{P}^{\circ} 1^{1} \mathrm{~F}_{3}^{\circ}$ & $5 \mathrm{~d}$ & $(3 / 2)^{2}[7 / 2]_{4}$ & & & R1c & & \\
\hline $5761.220(3)$ & $17352.622(8)$ & $5761.2165(10)$ & 0.004 & 2000 & & $\mathrm{sp}$ & $\left({ }^{3} \mathrm{~F}\right)^{3} \mathrm{P}^{\circ}{ }^{3} \mathrm{D}^{\circ}{ }_{3}$ & $6 \mathrm{~s}$ & $\begin{array}{l}(5 / 2)^{2}[5 / 2]_{2} \\
\text { nats }\end{array}$ & & & R1c & & \\
\hline $5761.8052(24)$ & $17350.860(7)$ & $5761.8056(7)$ & -0.0003 & 2200 & & $4 \mathrm{~d}$ & $(3 / 2)^{2}[5 / 2]_{3}$ & $\mathrm{sp}$ & $\left({ }^{3} \mathrm{~F}\right)^{1} \mathrm{P}^{\circ}{ }^{3} \mathrm{G}^{\circ}{ }_{4}$ & & & $\mathrm{R} 1 \mathrm{c}$ & & \\
\hline $5768.143(4)$ & $17331.796(11)$ & $5768.1482(7)$ & -0.005 & 150 & & $4 \mathrm{~d}$ & $(3 / 2)^{2}[5 / 2]_{2}$ & $4 f$ & $(5 / 2)^{2}[1 / 2]^{\circ}{ }_{1}$ & & & $\mathrm{R} 1 \mathrm{c}$ & & \\
\hline $5779.656(4)$ & $17297.270(12)$ & $5779.6592(8)$ & -0.003 & 360 & & $\mathrm{sp}$ & $\left({ }^{3} \mathrm{~F}\right)^{3} \mathrm{P}^{\circ} 3^{\circ} \mathrm{F}_{2}^{\circ}$ & $5 \mathrm{~d}$ & $(5 / 2)^{2}[1 / 2]$ & & & R1c & & \\
\hline $5783.920(4)$ & $17284.519(11)$ & 5783.9194(20) & 0.001 & 6400 & & $5 p$ & $(3 / 2)^{2}[3 / 2]^{\circ}{ }_{1}^{2}$ & $5 \mathrm{~d}$ & $(3 / 2)^{2}[1 / 2]_{0}$ & & & R1c & & \\
\hline $5801.130(7)$ & $17233.242(21)$ & $5801.116(3)$ & 0.014 & 140 & & $5 s$ & $(5 / 2)^{2}[5 / 2]_{3}$ & $\mathrm{sp}$ & $\left({ }^{3} \mathrm{P}\right)^{3} \mathrm{P}^{\circ}{ }^{5} \mathrm{P}_{2}^{\circ}$ & & & R1c & & \\
\hline $5805.989(3)$ & $17218.822(8)$ & $5805.9869(11)$ & 0.002 & 4700 & & $\mathrm{sp}$ & $\left({ }^{3} \mathrm{~F}\right)^{3} \mathrm{P}^{\circ}{ }^{3} \mathrm{D}^{\circ}{ }_{3}$ & $6 \mathrm{~s}$ & $(5 / 2)^{2}[5 / 2]_{3}$ & & & R1c & & \\
\hline $5825.823(2)$ & $17160.200(7)$ & $5825.8247(9)$ & -0.002 & 9100 & & $\mathrm{sp}$ & $\left({ }^{3} \mathrm{~F}\right)^{3} \mathrm{P}^{\circ}{ }^{1} \mathrm{D}^{\circ}{ }_{2}$ & $5 \mathrm{~d}$ & $(3 / 2)^{2}[5 / 2]_{2}$ & $1.1 \mathrm{e}+07$ & $\mathrm{D}+$ & $\mathrm{R} 1 \mathrm{c}$ & TW & \\
\hline $5833.5146(24)$ & $17137.573(7)$ & $5833.5141(7)$ & 0.0005 & 6400 & & $\mathrm{sp}$ & $\left({ }^{3} \mathrm{~F}\right)^{3} \mathrm{P}^{\circ}{ }^{1} \mathrm{D}^{\circ}{ }_{2}$ & $5 \mathrm{~d}$ & $(3 / 2)^{2}[5 / 2]_{3}$ & $7.9 \mathrm{e}+06$ & $\mathrm{D}+$ & R1c & TW & \\
\hline $5836.619(4)$ & $17128.458(13)$ & 5836.6234(11) & -0.004 & 130 & & $4 \mathrm{~d}$ & $(3 / 2)^{2}[1 / 2]_{1}$ & $\mathrm{sp}$ & $\left({ }^{3} \mathrm{P}\right)^{3} \mathrm{P}^{\circ}{ }^{3} \mathrm{D}^{\circ}{ }_{1}$ & & & R1c & & \\
\hline $5842.495(3)$ & 17111.233(8) & $5842.4918(7)$ & 0.003 & 1900 & & $4 p$ & ${ }^{1} \mathrm{D}^{\circ}{ }_{2}$ & $\mathrm{~s}^{\frac{1}{2}}$ & ${ }^{3} \mathrm{P}_{1}$ & & & R1c & & \\
\hline $5851.787(4)$ & $17084.062(11)$ & 5851.7811(12) & 0.006 & 930 & & $\mathrm{sp}$ & $\left({ }^{3} \mathrm{~F}\right)^{3} \mathrm{P}^{\circ}{ }^{3} \mathrm{G}_{3}^{\circ}$ & $6 \mathrm{~s}$ & $(5 / 2)^{2}[5 / 2]_{2}$ & & & R1c & & \\
\hline $5858.54(10)$ & $17064.4(3)$ & $5858.353(5)$ & 0.19 & 9100 & & $\mathrm{sp}$ & $\left({ }^{3} \mathrm{~F}\right)^{1} \mathrm{P}^{\circ}{ }^{3} \mathrm{~F}^{\circ}{ }_{2}$ & $7 \mathrm{~g}$ & $\begin{array}{l}(3 / 2)^{2}[7 / 2]_{3} \\
\end{array}$ & & & S36cn & & $\mathrm{x}$ \\
\hline $5890.443(4)$ & $16971.949(11)$ & $5890.4592(12)$ & -0.016 & 120 & & $4 \mathrm{~d}$ & $(3 / 2)^{2}[3 / 2]_{2}$ & $\mathrm{sp}$ & $\left({ }^{3} \mathrm{P}\right)^{3} \mathrm{P}^{\circ}{ }^{3} \mathrm{P}^{\circ}{ }_{1}$ & & & R1c & & $\mathrm{x}$ \\
\hline $5897.971(3)$ & $\begin{array}{l}16950.287(7) \\
\text { (n) }\end{array}$ & $5897.9758(12)$ & -0.005 & 23000 & & $\mathrm{sp}$ & $\left({ }^{3} \mathrm{~F}\right)^{3} \mathrm{P}^{\circ}{ }^{3} \mathrm{G}_{3}^{\circ}$ & $6 \mathrm{~s}$ & $(5 / 2)^{2}[5 / 2]_{3}$ & & & R1c & & \\
\hline $5901.188(3)$ & $16941.047(10)$ & $5901.1910(8)$ & -0.003 & 1700 & & $4 \mathrm{p}$ & ${ }^{3} \mathrm{~F}^{\circ}{ }_{3}^{\circ}$ & $s^{2}$ & ${ }^{1} \mathrm{D}_{2}$ & & & R1c & & \\
\hline $5909.753(3)$ & 16916.494(9) & 5909.7579(9) & -0.005 & 1500 & & sp & $\left({ }^{3} \mathrm{~F}\right)^{3} \mathrm{P}^{\circ}{ }^{1} \mathrm{D}^{\circ}{ }_{2}$ & $5 \mathrm{~d}$ & $(3 / 2)^{2}[3 / 2]_{1}$ & & & R1c & & \\
\hline $5910.813(4)$ & $16913.460(11)$ & $5910.8150(20)$ & -0.002 & 57 & & $\mathrm{sp}$ & $\left({ }^{3} \mathrm{~F}\right)^{1} \mathrm{P}^{\circ}{ }^{3} \mathrm{~F}_{4}^{\circ}$ & $7 \mathrm{~d}$ & $\begin{array}{l}(5 / 2)^{2}[9 / 2]_{5} \\
\text {. }\end{array}$ & & & R1c & & \\
\hline $5926.691(3)$ & $16868.147(8)$ & $5926.6916(6)$ & -0.000 & 1300 & & $4 \mathrm{p}$ & ${ }^{1} \mathrm{D}^{\circ}{ }_{2}$ & $s^{2}$ & $\begin{array}{l}3 \mathrm{P}_{2} \\
{ }_{3}\end{array}$ & & & R1c & & \\
\hline 5929.634(3) & $16859.777(10)$ & $5929.6380(11)$ & -0.004 & 110 & & $4 \mathrm{~d}$ & $(3 / 2)^{2}[7 / 2]_{4}$ & $\mathrm{sp}$ & $\left({ }^{3} \mathrm{~F}\right)^{1} \mathrm{P}^{\circ}{ }^{3} \mathrm{~F}_{4}^{\circ}$ & & & $\mathrm{R} 1 \mathrm{c}$ & & \\
\hline $5937.576(2)$ & 16837.224(7) & $5937.5815(7)$ & -0.005 & 2000 & & $\mathrm{sp}$ & $\left({ }^{3} \mathrm{~F}\right)^{3} \mathrm{P}^{\circ}{ }^{1} \mathrm{D}^{\circ}{ }_{2}$ & $5 \mathrm{~d}$ & $(3 / 2)^{2}[7 / 2]_{3}$ & $1.0 \mathrm{e}+07$ & $\mathrm{D}+$ & R1c & TW & \\
\hline $5939.775(7)$ & 16830.992(21) & $5939.7773(20)$ & -0.002 & 54 & & $\mathrm{sp}$ & $\left({ }^{1} \mathrm{G}\right)^{3} \mathrm{P}^{\circ}{ }^{3} \mathrm{H}^{\circ}{ }_{4}$ & $6 \mathrm{~g}$ & $(3 / 2)^{2}[11 / 2]_{5}$ & & & R1c & & \\
\hline 5941.1951(4) & $16826.9685(10)$ & 5941.1951(3) & -0.0001 & 31000 & & $5 p$ & $(5 / 2)^{2}[3 / 2]^{\circ}{ }_{2}$ & $5 \mathrm{~d}$ & $(5 / 2)^{2}[5 / 2]_{3}$ & $3.6 \mathrm{e}+07$ & $\mathrm{D}+$ & F_Re & TW & \\
\hline $5941.826(4)$ & $16825.183(11)$ & 5941.8291(17) & -0.003 & 1600 & & $4 \mathrm{~d}$ & $(3 / 2)^{2}[1 / 2]_{0}$ & $6 p$ & $(5 / 2)^{2}[3 / 2]^{0}{ }_{1}$ & & & $\mathrm{R} 1 \mathrm{c}$ & & \\
\hline $5979.015(3)$ & $16720.532(8)$ & $5979.0193(8)$ & -0.004 & 2800 & & $\mathrm{sp}$ & $\left({ }^{3} \mathrm{~F}\right)^{3} \mathrm{P}^{\circ}{ }^{3} \mathrm{~F}^{\circ}{ }_{2}$ & $6 \mathrm{~s}$ & $(3 / 2)^{2}[3 / 2]$ & & & R1c & & \\
\hline 5988.3141(17) & $16694.567(5)$ & $5988.3135(14)$ & 0.0006 & 7600 & & $5 p$ & $(5 / 2)^{2}[3 / 2]^{\circ}{ }_{1}$ & $5 \mathrm{~d}$ & $(5 / 2)^{2}[1 / 2]_{0}$ & 9.e+07 & $\mathrm{D}+$ & F_Re & TW & \\
\hline $5993.259(3)$ & $16680.793(7)$ & 5993.2605(8) & -0.001 & 3200 & & $5 p$ & $(5 / 2)^{2}[3 / 2]_{2}^{\circ}$ & $5 \mathrm{~d}$ & $(5 / 2)^{2}[3 / 2]_{1}$ & & & R1c & & \\
\hline $5995.5902(19)$ & $16674.307(5)$ & $5995.5876(10)$ & 0.0026 & 4600 & & $5 p$ & $(3 / 2)^{2}[1 / 2]_{0}^{\circ}$ & $5 \mathrm{~d}$ & $(3 / 2)^{2}[3 / 2]_{1}$ & $3.0 \mathrm{e}+07$ & $\mathrm{D}+$ & F_Re & TW & \\
\hline $6000.1169(8)$ & $16661.7272(22)$ & $6000.1168(6)$ & 0.0001 & 38000 & & $5 p$ & $(5 / 2)^{2}[3 / 2]_{2}^{\circ}$ & $5 \mathrm{~d}$ & $(5 / 2)^{2}[3 / 2]_{2}$ & $7.5 \mathrm{e}+07$ & $\mathrm{D}+$ & F_Re & TW & \\
\hline $6002.304(4)$ & $16655.656(10)$ & $6002.305(3)$ & -0.000 & 98 & & $6 \mathrm{~s}$ & $(5 / 2)^{2}[5 / 2]_{3}$ & $\mathrm{sp}$ & $\left({ }^{1} \mathrm{D}\right)^{1} \mathrm{P}^{\circ}{ }^{1} \mathrm{D}^{\circ}$ & & & R1c & & \\
\hline
\end{tabular}


Table A1. Cont.

\begin{tabular}{|c|c|c|c|c|c|c|c|c|c|c|c|c|c|c|}
\hline$\lambda_{\text {obs }}{ }^{\text {a }(\AA)}$ & $\sigma_{\mathrm{obs}}{ }^{\mathrm{b}}\left(\mathrm{cm}^{-1}\right)$ & $\lambda_{\text {Ritz }}{ }^{c}(\AA ̊)$ & $\begin{array}{c}\Delta \lambda_{\text {obs-Ritz }} \\
\text { (A) }\end{array}$ & $\begin{array}{c}I_{\text {obs }} \mathrm{d} \\
\text { (arb. u.) }\end{array}$ & Char ${ }^{e}$ & & Lower Level & & pper Level & $A\left(\mathrm{~s}^{-1}\right)$ & $\operatorname{Acc}^{f}$ & Line Ref. $\mathrm{g}$ & TP Ref. $\mathrm{g}$ & Notes ${ }^{h}$ \\
\hline $6013.411(3)$ & 16624.893(7) & $6013.4138(8)$ & -0.003 & 3200 & & $\mathrm{sp}$ & $\left({ }^{3} \mathrm{~F}\right)^{3} \mathrm{P}^{\circ}{ }^{3} \mathrm{~F}_{2}^{\circ}$ & $6 \mathrm{~s}$ & $(3 / 2)^{2}[3 / 2]_{1}$ & & & R1c & & \\
\hline $6018.369(5)$ & $16611.196(13)$ & 6018.3711(12) & -0.002 & 480 & & $\mathrm{sp}$ & $\left({ }^{3} \mathrm{~F}\right)^{3} \mathrm{P}^{\circ}{ }^{1} \mathrm{D}_{2}^{\circ}$ & $5 \mathrm{~d}$ & $(3 / 2)^{2}[1 / 2]_{1}$ & & & R1c & & \\
\hline 6023.263(3) & $16597.700(7)$ & $6023.2636(9)$ & -0.000 & 3900 & & $\mathrm{sp}$ & $\left({ }^{3} \mathrm{~F}\right)^{3} \mathrm{P}^{\circ}{ }^{3} \mathrm{D}^{\circ}{ }_{2}$ & $6 \mathrm{~s}$ & $(5 / 2)^{2}[5 / 2]_{2}$ & & & R1c & & \\
\hline $6050.917(4)$ & $16521.845(11)$ & $6050.916(3)$ & 0.001 & 91 & & $6 \mathrm{~s}$ & $(5 / 2)^{2}[5 / 2]_{2}$ & $\mathrm{sp}$ & $\left({ }^{1} \mathrm{D}\right)^{1} \mathrm{P}^{\circ}{ }^{1} \mathrm{D}_{2}^{\circ}$ & & & R1c & & \\
\hline 6071.492(4) & $16465.858(10)$ & $6071.4896(22)$ & 0.002 & 130 & & $\mathrm{sp}$ & $\left({ }^{3} \mathrm{~F}\right)^{1} \mathrm{P}^{\circ}{ }^{3} \mathrm{G}_{3}^{\circ}$ & $6 \mathrm{~g}$ & $(3 / 2)^{2}[9 / 2]_{4}$ & & & R1c & & \\
\hline 6072.217(3) & $16463.891(7)$ & $6072.2167(9)$ & 0.001 & 4200 & & $\mathrm{sp}$ & $\left({ }^{3} \mathrm{~F}\right)^{3} \mathrm{P}^{\circ}{ }^{3} \mathrm{D}^{\circ}{ }_{2}$ & $6 \mathrm{~s}$ & $(5 / 2)^{2}[5 / 2]_{3}$ & & & R1c & & \\
\hline 6080.3334(19) & $16441.915(5)$ & $6080.3370(7)$ & -0.0036 & 11000 & & $4 p$ & ${ }^{3} \mathrm{D}_{3}^{\circ}$ & $s^{2}$ & ${ }^{3} \mathrm{P}_{2}$ & & & F_Re & & \\
\hline 6083.086(4) & $16434.475(10)$ & $6083.0848(22)$ & 0.001 & 130 & & $6 \mathrm{p}$ & $(5 / 2)^{2}[7 / 2]^{0}{ }_{3}^{3}$ & $8 \mathrm{~d}$ & $(5 / 2)^{2}[9 / 2]_{4}$ & $6.9 e+06$ & $\mathrm{D}+$ & $\mathrm{R} 1 \mathrm{c}$ & TW & \\
\hline 6085.021(4) & $16429.250(11)$ & 6085.021(3) & -0.000 & 87 & & $4 \mathrm{~d}$ & $(5 / 2)^{2}[1 / 2]_{1}$ & $\mathrm{sp}$ & $\left({ }^{3} \mathrm{P}\right)^{3} \mathrm{P}^{\circ}{ }^{5} \mathrm{D}^{\circ}{ }_{1}$ & & & $\mathrm{R} 1 \mathrm{c}$ & & \\
\hline 6097.328(3) & $16396.088(8)$ & $6097.3235(10)$ & 0.004 & 4800 & & $5 p$ & $(3 / 2)^{2}[5 / 2]^{\circ}{ }^{\circ}$ & $5 \mathrm{~d}$ & $(3 / 2)^{2}[5 / 2]_{2}$ & $1.9 \mathrm{e}+07$ & $\mathrm{D}+$ & R1c & TW & \\
\hline 6099.989(3) & 16388.936(7) & 6099.9896(7) & -0.001 & 3700 & & $5 p$ & $(5 / 2)^{2}[7 / 2]^{\circ}{ }_{3}$ & $5 \mathrm{~d}$ & $(5 / 2)^{2}[5 / 2]_{2}$ & & & $\mathrm{R} 1 \mathrm{c}$ & & \\
\hline $6105.747(3)$ & $16373.480(7)$ & 6105.7469(8) & 0.000 & 3600 & & $5 p$ & $(3 / 2)^{2}[5 / 2]^{\circ} 2$ & $5 \mathrm{~d}$ & $(3 / 2)^{2}[5 / 2]_{3}$ & $9 . e+06$ & $\mathrm{D}+$ & R1c & TW & \\
\hline $6107.4055(21)$ & $16369.034(6)$ & 6107.4083(12) & -0.0028 & 5100 & & $5 p$ & $(3 / 2)^{2}[1 / 2]_{0}^{\circ}$ & $5 \mathrm{~d}$ & $(3 / 2)^{2}[1 / 2]_{1}$ & $3.3 e+07$ & $\mathrm{D}+$ & F_Re & TW & \\
\hline $6110.872(3)$ & $16359.748(7)$ & $6110.8706(7)$ & 0.002 & 3700 & & $5 p$ & $(5 / 2)^{2}[7 / 2]^{0}{ }_{3}$ & $5 \mathrm{~d}$ & $(5 / 2)^{2}[7 / 2]_{4}$ & & & $\mathrm{R} 1 \mathrm{c}$ & & \\
\hline $6114.4926(14)$ & $16350.061(4)$ & 6114.4911(7) & 0.0016 & 15000 & & $5 p$ & $(5 / 2)^{2}[7 / 2]^{\circ} 3$ & $5 \mathrm{~d}$ & $(5 / 2)^{2}[7 / 2]_{3}$ & $3.4 \mathrm{e}+07$ & $\mathrm{D}+$ & F_Re & TW & \\
\hline 6150.3818(9) & $16254.6548(23)$ & $6150.3813(6)$ & 0.0005 & 16000 & & $5 p$ & $(5 / 2)^{2}[7 / 2]^{\circ}$ & $5 \mathrm{~d}$ & $(5 / 2)^{2}[7 / 2]_{4}$ & $3.6 \mathrm{e}+07$ & $\mathrm{D}+$ & F_Re & TW & \\
\hline 6154.2215(8) & $16244.5134(21)$ & 6154.2211(7) & 0.0004 & 25000 & & $5 p$ & $(5 / 2)^{2}[3 / 2]_{2}{ }_{2}$ & $5 \mathrm{~d}$ & $(5 / 2)^{2}[1 / 2]_{1}$ & $1.0 \mathrm{e}+08$ & $\mathrm{D}+$ & F_Re & TW & \\
\hline $6157.716(4)$ & $16235.296(10)$ & 6157.7151(10) & 0.000 & 4700 & & $5 p$ & $(3 / 2)^{2}[5 / 2]^{\circ} 2$ & $5 \mathrm{~d}$ & $(3 / 2)^{2}[3 / 2]_{2}$ & & & $\mathrm{R} 1 \mathrm{c}$ & & \\
\hline 6157.956(8) & 16234.662(21) & 6157.9650(6) & -0.009 & 530 & & $5 p$ & $(5 / 2)^{2}[7 / 2]^{\circ}{ }_{3}$ & $5 \mathrm{~d}$ & $\begin{array}{l}(5 / 2)^{2}[5 / 2]_{3} \\
\end{array}$ & & & R1c & & \\
\hline $6160.573(4)$ & $16227.765(11)$ & 6160.5707(14) & 0.003 & 320 & & $4 \mathrm{~d}$ & $(3 / 2)^{2}[7 / 2]_{4}$ & $\mathrm{sp}$ & $\left({ }^{3} \mathrm{~F}\right)^{1} \mathrm{P}^{\circ} 3^{3} \mathrm{G}^{\circ}$ & & & R1c & & \\
\hline 6172.033(3) & $16197.634(7)$ & $6172.0339(8)$ & -0.000 & 16000 & & $5 p$ & $(5 / 2)^{2}[5 / 2]^{2} 2$ & $5 \mathrm{~d}$ & $(5 / 2)^{2}[5 / 2]_{2}$ & $3.4 \mathrm{e}+07$ & $\mathrm{D}+$ & F_Re & TW & \\
\hline $6174.296(5)$ & $16191.698(12)$ & $6174.2920(12)$ & 0.004 & 190 & & $4 \mathrm{~d}$ & $(3 / 2)^{2}[5 / 2]_{3}$ & $\mathrm{sp}$ & $\left({ }^{3} \mathrm{P}\right)^{3} \mathrm{P}^{\circ}{ }^{3} \mathrm{D}^{\circ}$ & & & R1c & & \\
\hline $6186.8770(14)$ & $16158.772(4)$ & $6186.8803(8)$ & -0.0033 & 18000 & & $5 p$ & $\begin{array}{l}(5 / 2)^{2}[5 / 2]^{\circ}, 2 \\
2\end{array}$ & $5 \mathrm{~d}$ & $(5 / 2)^{2}[7 / 2]_{3}$ & $3.6 \mathrm{e}+07$ & $\mathrm{D}+$ & $\mathrm{F}$ Re & TW & \\
\hline 6188.6763(11) & $16154.075(3)$ & $6188.6761(7)$ & 0.0002 & 12000 & & $5 p$ & $(5 / 2)^{2}[3 / 2]^{\circ} 1$ & $5 \mathrm{~d}$ & $(5 / 2)^{2}[5 / 2]_{2}$ & $3.1 \mathrm{e}+07$ & $\mathrm{D}+$ & F_Re & TW & \\
\hline $6189.317(4)$ & 16152.402(11) & 6189.3237(10) & -0.007 & 760 & & $5 p$ & $(3 / 2)^{2}[5 / 2]^{\circ}{ }_{2}$ & $5 \mathrm{~d}$ & $(3 / 2)^{2}[3 / 2]_{1}$ & & & R1c & & \\
\hline 6192.684(4) & $16143.620(11)$ & 6192.6855(12) & -0.001 & 76 & & $4 \mathrm{~d}$ & $(3 / 2)^{2}[5 / 2]_{2}$ & $\mathrm{sp}$ & $\left({ }^{3} \mathrm{P}\right)^{3} \mathrm{P}^{\circ}{ }^{3} \mathrm{D}^{\circ}{ }_{2}$ & & & R1c & & \\
\hline 6198.091(3) & $16129.537(7)$ & $6198.0890(5)$ & 0.002 & 5000 & & $5 p$ & $(5 / 2)^{2}[7 / 2]^{\circ} 4$ & $5 \mathrm{~d}$ & $(5 / 2)^{2}[5 / 2]_{3}$ & $9 . e+06$ & D+ & $\mathrm{R} 1 \mathrm{c}$ & TW & \\
\hline 6199.751(3) & $16125.219(8)$ & $6199.7460(10)$ & 0.005 & 1700 & & $5 p$ & $(3 / 2)^{2}[5 / 2]^{\circ}{ }^{\circ}$ & $5 \mathrm{~d}$ & $(3 / 2)^{2}[5 / 2]_{2}$ & & & R1c & & \\
\hline $6203.715(4)$ & $16114.915(11)$ & 6203.712(3) & 0.004 & 37 & & $6 p$ & $(5 / 2)^{2}[3 / 2]^{\circ}{ }_{2}$ & $8 \mathrm{~d}$ & $(5 / 2)^{2}[5 / 2]_{3}$ & & & $\mathrm{R} 1 \mathrm{c}$ & & \\
\hline $6204.2569(19)$ & $16113.507(5)$ & $6204.2577(10)$ & -0.0008 & 11000 & & $5 p$ & $(3 / 2)^{2}[1 / 2]^{\circ}$ & $5 \mathrm{~d}$ & $(3 / 2)^{2}[3 / 2]_{2}$ & $4.9 \mathrm{e}+07$ & $\mathrm{D}+$ & F_Re & TW & \\
\hline 6208.4527(19) & $16102.618(5)$ & 6208.4549(8) & -0.0022 & 9900 & & $5 p$ & $(3 / 2)^{2}[5 / 2]_{3}^{\circ}$ & $5 \mathrm{~d}$ & $(3 / 2)^{2}[5 / 2]_{3}$ & $2.9 \mathrm{e}+07$ & $\mathrm{D}+$ & F_Re & TW & \\
\hline $6208.988(4)$ & $16101.230(10)$ & 6208.9891(14) & -0.001 & 75 & & $4 \mathrm{~d}$ & $(3 / 2)^{2}[7 / 2]_{4}$ & $\mathrm{sp}$ & $\left({ }^{3} \mathrm{P}\right)^{3} \mathrm{P}^{\circ}{ }^{3} \mathrm{D}^{\circ}{ }_{3}$ & & & $\mathrm{R} 1 \mathrm{c}$ & & \\
\hline 6214.542(4) & $16086.839(11)$ & & & 150 & & $6 \mathrm{p}$ & $(5 / 2)^{2}[3 / 2]^{\circ} 2$ & $8 \mathrm{~d}$ & $(5 / 2)^{2}[3 / 2]_{2}$ & & & $\mathrm{R} 1 \mathrm{c}$ & & \\
\hline 6216.9386(8) & $16080.6383(21)$ & $6216.9385(6)$ & 0.0001 & 39000 & & $5 p$ & $(5 / 2)^{2}[7 / 2]^{\circ}{ }_{3}$ & $5 \mathrm{~d}$ & $\begin{array}{l}(5 / 2)^{2}[9 / 2]_{4} \\
\text {. }\end{array}$ & $9.4 \mathrm{e}+07$ & $\mathrm{C}+$ & F_Re & TW & \\
\hline 6219.8492(9) & $16073.1134(22)$ & 6219.8488(7) & 0.0005 & 24000 & & $5 p$ & $(3 / 2)^{2}[5 / 2]^{\circ} 2$ & $5 \mathrm{~d}$ & $(3 / 2)^{2}[7 / 2]_{3}$ & $9.4 \mathrm{e}+07$ & $\mathrm{C}+$ & F_Re & TW & \\
\hline $6221.288(4)$ & $16069.396(11)$ & 6221.2875(7) & 0.001 & 740 & & $5 p$ & $(5 / 2)^{2}[7 / 2]^{2}{ }_{3}$ & $5 \mathrm{~d}$ & $(5 / 2)^{2}[3 / 2]_{2}$ & & & $\mathrm{R} 1 \mathrm{c}$ & & \\
\hline $6231.396(3)$ & 16043.331(8) & 6231.3934(6) & 0.002 & 290 & & $5 p$ & $(5 / 2)^{2}[5 / 2]^{\circ}{ }_{2}$ & $5 \mathrm{~d}$ & $\begin{array}{l}(5 / 2)^{2}[5 / 2]_{3} \\
\end{array}$ & & & R1c & & \\
\hline $6236.344(3)$ & $16030.601(7)$ & 6236.3471(10) & -0.003 & 1200 & & $5 p$ & $(3 / 2)^{2}[1 / 2]^{\circ}$ & $5 \mathrm{~d}$ & $(3 / 2)^{2}[3 / 2]_{1}$ & & & R1c & & \\
\hline $6250.417(3)$ & $15994.508(8)$ & $6250.4216(8)$ & -0.005 & 720 & & $5 p$ & $(5 / 2)^{2}[5 / 2]^{0}{ }_{3}$ & $5 \mathrm{~d}$ & $(5 / 2)^{2}[5 / 2]_{2}$ & & & $\mathrm{R} 1 \mathrm{c}$ & & \\
\hline 6257.838(3) & $15975.542(7)$ & 6257.8373(7) & 0.000 & 3400 & & $5 p$ & $(5 / 2)^{2}[7 / 2]^{\circ} 4$ & $5 \mathrm{~d}$ & $\begin{array}{l}(5 / 2)^{2}[9 / 2]_{4} \\
\text {. }\end{array}$ & $4.1 \mathrm{e}+06$ & $\mathrm{D}+$ & F_Re & TW & \\
\hline 6261.8477(13) & 15965.311(3) & $6261.8464(6)$ & 0.0013 & 19000 & & $5 p$ & $(5 / 2)^{2}[5 / 2]^{\circ}{ }_{3}$ & $5 \mathrm{~d}$ & $(5 / 2)^{2}[7 / 2]_{4}$ & $3.7 \mathrm{e}+07$ & $\mathrm{D}+$ & F_Re & TW & \\
\hline $6265.650(3)$ & $15955.623(7)$ & $6265.6480(8)$ & 0.002 & 2800 & & $5 p$ & $(5 / 2)^{2}[5 / 2]^{\circ}{ }_{3}$ & $5 \mathrm{~d}$ & $(5 / 2)^{2}[7 / 2]_{3}$ & & & $\mathrm{R} 1 \mathrm{c}$ & & \\
\hline 6273.34762(8) & $15936.04484(21)$ & 6273.34763(8) & -0.00000 & 47000 & & $5 p$ & $(5 / 2)^{2}[7 / 2]^{\circ}{ }_{4}^{\circ}$ & $5 \mathrm{~d}$ & $\begin{array}{l}(5 / 2)^{2}[9 / 2]_{5} \\
\text {. }\end{array}$ & $1.06 \mathrm{e}+08$ & $\mathrm{C}_{+}$ & $\mathrm{F} \_\mathrm{Re}$ & TW & \\
\hline
\end{tabular}


Table A1. Cont.

\begin{tabular}{|c|c|c|c|c|c|c|c|c|c|c|c|c|c|c|}
\hline$\lambda_{\text {obs }}{ }^{\text {a }}(\AA)$ & $\sigma_{\mathrm{obs}}{ }^{\mathrm{b}}\left(\mathrm{cm}^{-1}\right)$ & $\lambda_{\text {Ritz }}{ }^{c}(\AA ̊)$ & $\begin{array}{c}\Delta \lambda_{\text {obs-Ritz }} \\
\text { (A) }\end{array}$ & $\begin{array}{c}I_{\text {obs }} \mathrm{d} \\
\text { (arb. u.) }\end{array}$ & Char ${ }^{e}$ & & Lower Level & & Jpper Level & $A\left(\mathrm{~s}^{-1}\right)$ & $\operatorname{Acc}^{f}$ & Line Ref. $\mathrm{g}$ & TP Ref. $\mathrm{g}$ & Notes ${ }^{h}$ \\
\hline $6276.660(5)$ & $15927.636(14)$ & 6276.6713(19) & -0.012 & 14000 & & $\mathrm{sp}$ & $\left({ }^{3} \mathrm{~F}\right)^{3} \mathrm{P}^{\circ}{ }^{3} \mathrm{~F}_{4}^{\circ}$ & $6 \mathrm{~s}$ & $(5 / 2)^{2}[5 / 2]_{3}$ & & & R1c & & \\
\hline $6288.695(3)$ & $15897.154(7)$ & $6288.6936(9)$ & 0.001 & 6600 & & $5 p$ & $(5 / 2)^{2}[5 / 2]^{4}{ }^{4}$ & $5 \mathrm{~d}$ & $(5 / 2)^{2}[3 / 2]_{1}$ & $1.7 \mathrm{e}+07$ & $\mathrm{D}+$ & R1c & TW & \\
\hline 6296.239(4) & 15878.107(11) & 6296.2430(8) & -0.004 & 34 & & $5 p$ & $(5 / 2)^{2}[5 / 2]^{2} 2$ & $5 \mathrm{~d}$ & $(5 / 2)^{2}[3 / 2]_{2}$ & & & R1c & & \\
\hline $6299.864(5)$ & $15868.969(11)$ & $6299.810(3)$ & 0.054 & 34 & ? & $\mathrm{sp}$ & $\left({ }^{3} \mathrm{P}\right)^{3} \mathrm{P}^{\circ} 1^{\circ} \mathrm{D}_{2}$ & $6 \mathrm{~g}$ & $(3 / 2)^{2}[5 / 2]_{3}$ & & & R1c & & $\mathrm{x}$ \\
\hline $6301.0135(8)$ & $15866.0749(21)$ & 6301.0137(7) & -0.0001 & 27000 & & $5 p$ & $(3 / 2)^{2}[5 / 2]^{2}{ }_{3}$ & $5 \mathrm{~d}$ & $\begin{array}{l}(3 / 2)^{2}[7 / 2]_{4} \\
\end{array}$ & $1.0 \mathrm{e}+08$ & $\mathrm{C}+$ & $\mathrm{F}$ Re & TW & \\
\hline 6305.9712(10) & 15853.601(3) & 6305.9718(8) & -0.0006 & 12000 & & $5 p$ & $(5 / 2)^{2}[3 / 2]^{\circ} 1$ & $5 \mathrm{~d}$ & $(5 / 2)^{2}[3 / 2]_{1}$ & $5.7 \mathrm{e}+07$ & $\mathrm{D}+$ & F_Re & TW & \\
\hline $6311.3006(21)$ & $15840.214(5)$ & 6311.3059(5) & -0.0053 & 18000 & & $5 p$ & $(5 / 2)^{2}[5 / 2]^{\circ}{ }_{3}$ & $5 \mathrm{~d}$ & $(5 / 2)^{2}[5 / 2]_{3}$ & $3.5 \mathrm{e}+07$ & $\mathrm{D}+$ & F_Re & TW & \\
\hline 6312.491(3) & $15837.227(7)$ & 6312.4915(11) & -0.000 & 14000 & & $5 p$ & $(3 / 2)^{2}[3 / 2]^{\circ}{ }_{1}$ & $5 \mathrm{~d}$ & $(3 / 2)^{2}[5 / 2]_{2}$ & $5.8 \mathrm{e}+07$ & $\mathrm{D}+$ & $\mathrm{R} 1 \mathrm{c}$ & TW & \\
\hline 6313.564(3) & $15834.535(7)$ & $6313.5627(7)$ & 0.001 & 1000 & & $5 p$ & $(5 / 2)^{2}[3 / 2]^{\circ} 1$ & $5 \mathrm{~d}$ & $(5 / 2)^{2}[3 / 2]_{2}$ & & & $\mathrm{R} 1 \mathrm{c}$ & & \\
\hline 6317.788(3) & $15823.950(7)$ & 6317.7831(12) & 0.004 & 1300 & & $4 p$ & ${ }^{3} \mathrm{D}^{\circ}{ }_{1}$ & $s^{2}$ & ${ }^{3} \mathrm{P}_{0}$ & & & R1c & & \\
\hline 6318.944(5) & $15821.053(13)$ & 6318.9422(15) & 0.002 & 33 & & $4 \mathrm{~d}$ & $(3 / 2)^{2}[3 / 2]_{2}$ & $\mathrm{sp}$ & $\left({ }^{3} \mathrm{P}\right)^{3} \mathrm{P}^{\circ}{ }^{3} \mathrm{D}^{\circ}{ }_{3}$ & & & R1c & & \\
\hline $6326.465(3)$ & $15802.245(7)$ & $6326.4649(8)$ & 0.000 & 2500 & & $5 p$ & $(3 / 2)^{2}[5 / 2]^{\circ}{ }_{3}$ & $5 \mathrm{~d}$ & $(3 / 2)^{2}[7 / 2]_{3}$ & $5.0 \mathrm{e}+06$ & $\mathrm{D}+$ & R1c & TW & \\
\hline 6357.414(3) & $15725.318(7)$ & 6357.4193(13) & -0.005 & 16000 & & $5 p$ & $(3 / 2)^{2}[1 / 2]^{\circ}$ & $5 \mathrm{~d}$ & $(3 / 2)^{2}[1 / 2]_{1}$ & $5.5 \mathrm{e}+07$ & $\mathrm{D}+$ & F_Re & TW & \\
\hline $6363.566(5)$ & $15710.115(11)$ & $6363.568(4)$ & -0.001 & 32 & & $6 \mathrm{p}$ & $(3 / 2)^{2}[5 / 2]^{\circ}{ }_{3}$ & $9 \mathrm{~d}$ & $(5 / 2)^{2}[9 / 2]_{4}$ & & & $\mathrm{R} 1 \mathrm{c}$ & & \\
\hline 6373.267(3) & $15686.203(8)$ & 6373.2678(7) & -0.001 & 6900 & & $5 p$ & $(5 / 2)^{2}[5 / 2]^{\circ} 3$ & $5 \mathrm{~d}$ & $(5 / 2)^{2}[9 / 2]_{4}$ & & & $\mathrm{R} 1 \mathrm{c}$ & & \\
\hline 6377.248(7) & $15676.410(17)$ & 6377.2432(12) & 0.005 & 13000 & & $5 p$ & $(3 / 2)^{2}[3 / 2]^{\circ} 1$ & $5 \mathrm{~d}$ & $(3 / 2)^{2}[3 / 2]_{2}$ & & & $\mathrm{R} 1 \mathrm{c}$ & & \\
\hline 6377.842(3) & 15674.951(7) & 6377.8383(7) & 0.004 & 19000 & & $5 p$ & $(5 / 2)^{2}[5 / 2]_{3}{ }_{3}$ & $5 \mathrm{~d}$ & $\begin{array}{l}(5 / 2)^{2}[3 / 2]_{2}\end{array}$ & $1.9 \mathrm{e}+07$ & $\mathrm{D}+$ & F_Re & TW & \\
\hline $6380.758(4)$ & 15667.788(9) & 6380.7645(7) & -0.007 & 1300 & & $5 p$ & $(5 / 2)^{2}[3 / 2]^{\circ} 2$ & $6 \mathrm{~s}$ & $(3 / 2)^{2}[3 / 2]_{2}$ & & & $\mathrm{R} 1 \mathrm{c}$ & & \\
\hline 6385.263(3) & 15656.734(7) & $6385.2618(10)$ & 0.001 & 1200 & & $\mathrm{sp}$ & $\left({ }^{3} \mathrm{~F}\right)^{3} \mathrm{P}^{\circ}{ }^{3} \mathrm{D}^{\circ}$ & $6 \mathrm{~s}$ & $(5 / 2)^{2}[5 / 2]_{2}$ & & & R1c & & \\
\hline 6393.074(6) & $15637.604(15)$ & 6393.0792(20) & -0.005 & 130 & * & sp & $\left({ }^{3} \mathrm{~F}\right)^{1} \mathrm{P}^{\circ}{ }^{3} \mathrm{G}_{4}^{\circ}$ & $6 \mathrm{~g}$ & $\begin{array}{l}(5 / 2)^{2}[9 / 2]_{4} \\
\text {. }\end{array}$ & & & R1c & & \\
\hline 6393.074(6) & $15637.604(15)$ & $6393.0735(21)$ & 0.001 & 130 & * & $\mathrm{sp}$ & $\left({ }^{3} \mathrm{~F}\right)^{1} \mathrm{P}^{\circ}{ }^{3} \mathrm{G}_{4}^{\circ}$ & $6 \mathrm{~g}$ & $(5 / 2)^{2}[9 / 2]_{5}$ & & & R1nc & & \\
\hline 6393.957(3) & $15635.446(8)$ & 6393.9587(13) & -0.002 & 2100 & & $\mathrm{sp}$ & $\left({ }^{3} \mathrm{~F}\right)^{1} \mathrm{P}^{\circ}{ }^{3} \mathrm{G}_{4}^{\circ}$ & $6 \mathrm{~g}$ & $(5 / 2)^{2}[11 / 2]_{5}$ & $3.4 \mathrm{e}+06$ & $\mathrm{D}+$ & R1c & TW & \\
\hline $6403.384(3)$ & $15612.428(8)$ & $6403.3840(15)$ & -0.000 & 9600 & & $4 \mathrm{~d}$ & $(3 / 2)^{2}[1 / 2]$ & $4 \mathrm{f}$ & $(3 / 2)^{2}[3 / 2]^{0}$ & $2.7 \mathrm{e}+07$ & $\mathrm{D}+$ & R1c & TW & \\
\hline 6411.142(4) & $15593.535(10)$ & $6411.1518(12)$ & -0.010 & 10000 & & $5 p$ & $(3 / 2)^{2}[3 / 2]^{\circ} 1$ & $5 \mathrm{~d}$ & $(3 / 2)^{2}[3 / 2]_{1}$ & $5.8 \mathrm{e}+07$ & $\mathrm{D}+$ & F_Re & TW & \\
\hline 6414.569(3) & $15585.204(7)$ & 6414.5612(11) & 0.008 & 12000 & & $5 p$ & $(3 / 2)^{2}[3 / 2]^{\circ}{ }_{2}$ & $5 \mathrm{~d}$ & $(3 / 2)^{2}[5 / 2]_{2}$ & $1.4 \mathrm{e}+07$ & $\mathrm{D}+$ & $\mathrm{R} 1 \mathrm{c}$ & TW & \\
\hline $6414.625(3)$ & $15585.069(7)$ & $6414.6165(8)$ & 0.008 & 16000 & & $\mathrm{sp}$ & $\left({ }^{3} \mathrm{~F}\right)^{3} \mathrm{P}^{\circ}{ }^{3} \mathrm{~F}_{3}^{\circ}{ }_{3}$ & $6 \mathrm{~s}$ & $(5 / 2)^{2}[5 / 2]_{2}$ & & & R1c & & \\
\hline \multirow[t]{2}{*}{$6418.160(4)$} & $15576.483(10)$ & $6418.1566(24)$ & 0.004 & 2100 & & $4 \mathrm{f}$ & $(5 / 2)^{2}[1 / 2]^{\circ} 1$ & $6 \mathrm{~g}$ & $(5 / 2)^{2}[3 / 2]_{2}$ & $1.5 \mathrm{e}+07$ & D+ & $\mathrm{R} 1 \mathrm{c}$ & TW & \\
\hline & & 6418.192(3) & & & $\mathrm{m}$ & $4 \mathrm{f}$ & $(5 / 2)^{2}[1 / 2]^{\circ}$ & $6 \mathrm{~g}$ & $(5 / 2)^{2}[3 / 2]_{1}$ & & & R1nc & & \\
\hline 6419.941(9) & $15572.162(21)$ & $6419.9515(7)$ & -0.010 & 62 & & $5 p$ & $(5 / 2)^{2}[3 / 2]^{\circ} 2$ & $6 \mathrm{~s}$ & $(3 / 2)^{2}[3 / 2]_{1}$ & & & R1c & & \\
\hline 6423.8842(11) & 15562.604(3) & 6423.8846(8) & -0.0003 & 19000 & & $5 p$ & $(3 / 2)^{2}[3 / 2]^{2}$ & $5 \mathrm{~d}$ & $(3 / 2)^{2}[5 / 2]_{3}$ & $5.6 \mathrm{e}+07$ & $\mathrm{C}+$ & F_Re & TW & \\
\hline $6427.416(9)$ & $15554.052(21)$ & 6427.411(3) & 0.005 & 31 & & $4 f$ & $(5 / 2)^{2}[3 / 2]_{2}^{\circ}$ & $6 \mathrm{~g}$ & $\begin{array}{l}(5 / 2)^{2}[7 / 2]_{3} \\
\end{array}$ & & & R1c & & \\
\hline $6432.416(3)$ & $15541.962(7)$ & 6432.415(3) & 0.001 & 5600 & & $4 \mathrm{f}$ & $(5 / 2)^{2}[3 / 2]^{\circ} 2$ & $6 \mathrm{~g}$ & $(5 / 2)^{2}[5 / 2]_{3}$ & $1.5 \mathrm{e}+07$ & D+ & R1c & TW & \\
\hline $6433.597(4)$ & $15539.109(10)$ & 6433.5944(22) & 0.003 & 920 & & $4 \mathrm{f}$ & $(5 / 2)^{2}[11 / 2]^{\circ}{ }_{6}$ & $6 \mathrm{~g}$ & $(5 / 2)^{2}[11 / 2]_{6}$ & $2.7 \mathrm{e}+06$ & $\mathrm{D}+$ & $\mathrm{R} 1 \mathrm{c}$ & TW & \\
\hline 6434.081(5) & $15537.941(12)$ & 6434.075(3) & 0.006 & 610 & & $4 \mathrm{f}$ & $(5 / 2)^{2}[1 / 2]^{\circ} 0$ & $6 \mathrm{~g}$ & $(5 / 2)^{2}[3 / 2]_{1}$ & & & R1c & & \\
\hline 6434.799(3) & 15536.208(8) & 6434.7944(13) & 0.004 & 610 & & $4 \mathrm{f}$ & $(5 / 2)^{2}[11 / 2]^{\circ}{ }_{5}$ & $6 \mathrm{~g}$ & $\begin{array}{l}(5 / 2)^{2}[11 / 2]_{5} \\
5\end{array}$ & & & R1c & & \\
\hline $6437.596(3)$ & $15529.458(8)$ & 6437.5974(24) & -0.002 & 460 & & $4 \mathrm{f}$ & $(5 / 2)^{2}[3 / 2]^{\circ} 2$ & $6 \mathrm{~g}$ & $(5 / 2)^{2}[3 / 2]_{2}$ & $6.7 \mathrm{e}+06$ & $\mathrm{D}+$ & $\mathrm{R} 1 \mathrm{c}$ & TW & \\
\hline $6441.202(5)$ & $15520.764(12)$ & 6441.1920(9) & 0.010 & 1200 & & $4 p$ & ${ }^{3} \mathrm{~F}_{2}^{\mathrm{O}}$ & $s^{2}$ & ${ }^{1} \mathrm{D}_{2}$ & & & R1c & & \\
\hline 6441.677(3) & $15519.618(8)$ & $6441.6790(8)$ & -0.002 & 13000 & & sp & $\left({ }^{3} \mathrm{~F}\right)^{3} \mathrm{P}^{\circ} 1^{1} \mathrm{~F}^{\circ}{ }_{3}$ & $5 \mathrm{~d}$ & $(5 / 2)^{2}[7 / 2]_{4}$ & & & R1c & & \\
\hline 6441.7371(11) & $15519.474(3)$ & & & 10000 & & $4 f$ & $(5 / 2)^{2}[11 / 2]^{\circ}{ }_{6}$ & $6 \mathrm{~g}$ & $\begin{array}{l}(5 / 2)^{2}[13 / 2]_{7} \\
\text { (5) }\end{array}$ & $2.4 \mathrm{e}+07$ & D+ & F_Re & TW & \\
\hline $6442.964(3)$ & 15516.519(7) & $6442.964(3)$ & -0.000 & 7600 & & $4 f$ & $(5 / 2)^{2}[11 / 2]^{\circ}{ }_{5}$ & $6 \mathrm{~g}$ & $(5 / 2)^{2}[13 / 2]_{6}$ & $2.3 e+07$ & $\mathrm{D}+$ & R1c & TW & \\
\hline 6443.587(3) & $15515.019(8)$ & 6443.587(3) & 0.000 & 4900 & & $4 \mathrm{f}$ & $(3 / 2)^{2}[3 / 2]^{\circ} 2$ & $6 \mathrm{~g}$ & $(3 / 2)^{2}[5 / 2]_{3}$ & $2.0 \mathrm{e}+07$ & $\mathrm{D}+$ & R1c & TW & \\
\hline $6445.692(20)$ & 15509.95(5) & 6445.673(3) & 0.020 & 1400 & * & $6 p$ & $(5 / 2)^{2}[3 / 2]_{2}{ }_{2}$ & $9 \mathrm{~s}$ & $(5 / 2)^{2}[5 / 2]_{3}$ & & & R1nc & & \\
\hline 6445.692(20) & 15509.95(5) & $6445.7022(9)$ & -0.010 & 1400 & * & $\mathrm{sp}$ & $\left({ }^{3} \mathrm{~F}\right)^{3} \mathrm{P}^{\circ} 1^{1} \mathrm{~F}_{3}^{\circ}$ & $5 \mathrm{~d}$ & $\begin{array}{l}(5 / 2)^{2}[7 / 2]_{3} \\
\end{array}$ & & & R1c & & \\
\hline
\end{tabular}


Table A1. Cont.

\begin{tabular}{|c|c|c|c|c|c|c|c|c|c|c|c|c|c|c|}
\hline$\lambda_{\text {obs }}{ }^{a}(\AA)$ & $\sigma_{\mathrm{obs}}{ }^{\mathrm{b}}\left(\mathrm{cm}^{-1}\right)$ & $\lambda_{\text {Ritz }}{ }^{c}(\AA)$ & $\begin{array}{c}\Delta \lambda_{\text {obs-Ritz }} \\
\text { (A) }\end{array}$ & $\begin{array}{l}I_{\text {obs }} \mathrm{d} \\
\text { (arb. u.) }\end{array}$ & Char ${ }^{e}$ & & ower Level & & pper Level & $A\left(\mathrm{~s}^{-1}\right)$ & $\operatorname{Acc}^{f}$ & Line Ref. $\mathrm{g}$ & TP Ref. $\mathrm{g}$ & Notes $h$ \\
\hline $6447.627(5)$ & 15505.296(13) & 6447.632(3) & -0.005 & 61 & & $\mathrm{sp}$ & $\left({ }^{1} \mathrm{G}\right)^{3} \mathrm{P}^{\circ}{ }^{3} \mathrm{~F}_{3}^{\circ}$ & $8 \mathrm{~d}$ & $(5 / 2)^{2}[9 / 2]_{4}$ & & & R1c & & \\
\hline $6448.558(3)$ & $15503.059(8)$ & 6448.5593(8) & -0.002 & 20000 & & $4 p$ & ${ }^{3} \mathrm{D}^{\circ}{ }_{1}^{3}$ & $s^{2}$ & ${ }^{3} \mathrm{P}_{1}$ & & & R1c & & \\
\hline 6449.63(3) & $15500.49(7)$ & 6449.6175(15) & 0.01 & 30 & * & $4 \mathrm{~d}$ & $(3 / 2)^{2}[5 / 2]_{3}$ & $\mathrm{sp}$ & $\left({ }^{3} \mathrm{P}\right)^{3} \mathrm{P}^{\circ}{ }^{3} \mathrm{D}^{\circ}{ }_{3}$ & & & R1c & & \\
\hline 6449.63(3) & $15500.49(7)$ & $6449.655(3)$ & -0.03 & 30 & * & $\mathrm{sp}$ & $\left({ }^{3} \mathrm{P}\right)^{3} \mathrm{P}^{\circ}{ }^{5} \mathrm{~S}^{\circ}{ }_{2}$ & $6 \mathrm{~g}$ & $(5 / 2)^{2}[5 / 2]_{2}$ & & & R1nc & & \\
\hline 6449.63(3) & $15500.49(7)$ & $6449.659(4)$ & -0.03 & 30 & * & $\mathrm{sp}$ & $\left({ }^{3} \mathrm{P}\right)^{3} \mathrm{P}^{\circ}{ }^{5} \mathrm{~S}^{\circ}{ }_{2}$ & $6 \mathrm{~g}$ & $(5 / 2)^{2}[5 / 2]_{3}$ & & & R1nc & & \\
\hline $6450.874(3)$ & 15497.493(8) & 6450.8738(17) & -0.000 & 610 & & $4 \mathrm{~d}$ & $(3 / 2)^{2}[1 / 2]_{0}$ & $\mathrm{sp}$ & $\left({ }^{3} \mathrm{~F}\right)^{1} \mathrm{P}^{\circ}{ }^{\circ} \mathrm{D}^{\circ}{ }_{1}$ & & & R1c & & \\
\hline $6452.116(3)$ & $15494.508(7)$ & 6452.1161(24) & 0.000 & 1300 & & $4 \mathrm{f}$ & $(5 / 2)^{2}[3 / 2]^{\circ}{ }_{1}$ & $6 \mathrm{~g}$ & $(5 / 2)^{2}[5 / 2]_{2}$ & $1.4 \mathrm{e}+07$ & $\mathrm{D}+$ & $\mathrm{R} 1 \mathrm{c}$ & TW & \\
\hline 6454.161(3) & $15489.601(8)$ & & & 910 & & $4 \mathrm{f}$ & $(3 / 2)^{2}[3 / 2]^{\circ}{ }_{1}$ & $6 \mathrm{~g}$ & $(3 / 2)^{2}[5 / 2]_{2}$ & & & R1c & & \\
\hline 6454.894(9) & $15487.842(21)$ & 6454.895(3) & -0.001 & 30 & & $6 p$ & $(5 / 2)^{2}[7 / 2]^{\circ}{ }_{4}$ & $8 \mathrm{~d}$ & $(5 / 2)^{2}[7 / 2]_{4}$ & & & $\mathrm{R} 1 \mathrm{rc}$ & & \\
\hline 6456.119(13) & $15484.90(3)$ & 6456.140(9) & -0.021 & 30 & & $5 s$ & $(3 / 2)^{2}[3 / 2]_{1}$ & $\mathrm{sp}$ & $\left({ }^{3} \mathrm{P}\right)^{3} \mathrm{P}^{\circ} \mathrm{P}_{1}^{\circ}$ & & & R1c & & \\
\hline $6457.187(3)$ & $15482.342(7)$ & 6457.1848(19) & 0.002 & 2600 & & $4 \mathrm{f}$ & $(5 / 2)^{2}[7 / 2]^{\circ}{ }_{3}$ & $6 \mathrm{~g}$ & $(5 / 2)^{2}[9 / 2]_{4}$ & & & R1c & & \\
\hline $6457.368(4)$ & $15481.908(10)$ & $6457.371(3)$ & -0.004 & 1600 & & $4 \mathrm{f}$ & $(5 / 2)^{2}[3 / 2]^{\circ}{ }_{1}$ & $6 \mathrm{~g}$ & $(5 / 2)^{2}[3 / 2]_{1}$ & & & R1c & & \\
\hline 6458.263(9) & $15479.762(21)$ & 6458.259(3) & 0.004 & 61 & & $4 \mathrm{f}$ & $(3 / 2)^{2}[9 / 2]^{\circ}{ }_{5}$ & $6 \mathrm{~g}$ & $(3 / 2)^{2}[9 / 2]_{5}$ & & & R1c & & \\
\hline $6460.304(3)$ & $15474.870(8)$ & 6460.304(3) & 0.001 & 1000 & & $4 \mathrm{f}$ & $(5 / 2)^{2}[7 / 2]^{\circ}{ }_{3}$ & $6 \mathrm{~g}$ & $(5 / 2)^{2}[7 / 2]_{3}$ & & & R1c & & \\
\hline $6462.140(3)$ & $15470.475(8)$ & $6462.1413(20)$ & -0.002 & 60 & & $4 \mathrm{f}$ & $(3 / 2)^{2}[9 / 2]^{\circ}{ }_{4}$ & $6 \mathrm{~g}$ & $(3 / 2)^{2}[9 / 2]_{4}$ & & & $\mathrm{R} 1 \mathrm{c}$ & & \\
\hline $6462.70(10)$ & $15469.13(24)$ & 6462.6086(21) & 0.09 & 1000 & & $4 \mathrm{f}$ & $(3 / 2)^{2}[7 / 2]^{\circ}{ }_{3}$ & $7 \mathrm{~d}$ & $(3 / 2)^{2}[7 / 2]_{3}$ & & & S36c & & \\
\hline 6465.342(15) & $15462.81(4)$ & $6465.3543(24)$ & -0.012 & 30 & * & $4 \mathrm{f}$ & $(5 / 2)^{2}[7 / 2]^{\circ}{ }_{3}$ & $6 \mathrm{~g}$ & $(5 / 2)^{2}[5 / 2]_{2}$ & & & R1c & & \\
\hline $6465.342(15)$ & 15462.81(4) & $6465.359(3)$ & -0.017 & 30 & * & $4 \mathrm{f}$ & $(5 / 2)^{2}[7 / 2]^{\circ}{ }_{3}$ & $6 \mathrm{~g}$ & $(5 / 2)^{2}[5 / 2]_{3}$ & & & R1c & & \\
\hline $6466.245(3)$ & $15460.653(7)$ & & & 5600 & & $4 f$ & $(3 / 2)^{2}[9 / 2]^{\circ}{ }_{5}$ & $6 \mathrm{~g}$ & $(3 / 2)^{2}[11 / 2]_{6}$ & $2.3 e+07$ & $\mathrm{D}+$ & R1c & TW & \\
\hline 6470.1386(19) & $15451.350(5)$ & $6470.1383(19)$ & 0.0003 & 8600 & & $4 \mathrm{f}$ & $(3 / 2)^{2}[9 / 2]^{\circ} 4$ & $6 \mathrm{~g}$ & $(3 / 2)^{2}[11 / 2]_{5}$ & $2.3 e+07$ & D+ & F_Re & TW & \\
\hline $6470.160(3)$ & $15451.298(7)$ & $6470.1668(8)$ & -0.006 & 15000 & & $\mathrm{sp}$ & $\left({ }^{3} \mathrm{~F}\right)^{3} \mathrm{P}^{\circ}{ }^{3} \mathrm{~F}_{3}^{\circ}$ & $6 \mathrm{~s}$ & $(5 / 2)^{2}[5 / 2]_{3}$ & & & F_Re & & \\
\hline $6475.426(4)$ & 15438.734(9) & $6475.4257(24)$ & -0.000 & 1000 & & $4 \mathrm{f}$ & $(5 / 2)^{2}[5 / 2]^{\circ}{ }_{2}$ & $6 \mathrm{~g}$ & $(5 / 2)^{2}[5 / 2]_{2}$ & $7.5 \mathrm{e}+06$ & $\mathrm{D}+$ & $\mathrm{R} 1 \mathrm{c}$ & TW & \\
\hline 6476.184(4) & $15436.925(9)$ & 6476.1819(19) & 0.003 & 1000 & & $4 \mathrm{f}$ & $(5 / 2)^{2}[5 / 2]^{\circ}{ }_{3}$ & $6 \mathrm{~g}$ & $(5 / 2)^{2}[9 / 2]_{4}$ & & & R1c & & \\
\hline 6477.868(9) & $15432.912(21)$ & $6477.860(3)$ & 0.008 & 120 & & $6 p$ & $(5 / 2)^{2}[7 / 2]^{\circ}{ }_{4}$ & $8 \mathrm{~d}$ & $(5 / 2)^{2}[9 / 2]_{5}$ & & & R1c & & \\
\hline $6479.316(3)$ & $15429.463(7)$ & 6479.3164(21) & -0.000 & 4100 & & $4 f$ & $(5 / 2)^{2}[5 / 2]^{\circ}{ }_{3}$ & $6 \mathrm{~g}$ & $(5 / 2)^{2}[7 / 2]_{4}$ & $1.5 \mathrm{e}+07$ & $\mathrm{D}+$ & R1c & TW & \\
\hline \multirow[t]{2}{*}{$6481.436(3)$} & $15424.418(7)$ & $6481.4350(12)$ & 0.001 & 15000 & & $5 p$ & $(3 / 2)^{2}[3 / 2]^{\circ} 2$ & $5 \mathrm{~d}$ & $(3 / 2)^{2}[3 / 2]_{2}$ & $3.4 \mathrm{e}+07$ & D+ & R1c & TW & \\
\hline & & 6484.404(3) & & & $\mathrm{m}$ & $4 \mathrm{f}$ & $(5 / 2)^{2}[5 / 2]_{3}^{\circ}$ & $6 \mathrm{~g}$ & $\begin{array}{l}(5 / 2)^{2}[5 / 2]_{3} \\
\end{array}$ & $6.5 \mathrm{e}+06$ & $\mathrm{D}+$ & $\mathrm{R} 1 \mathrm{nc}$ & TW & \\
\hline $6484.417(3)$ & $15417.326(8)$ & 6484.4174(9) & -0.000 & 7800 & & $5 p$ & $(5 / 2)^{2}[3 / 2]^{\circ}{ }_{1}$ & $5 \mathrm{~d}$ & $(5 / 2)^{2}[1 / 2]_{1}$ & $1.4 \mathrm{e}+07$ & $\mathrm{D}+$ & F_Re & TW & \\
\hline $6488.816(3)$ & $15406.875(7)$ & $6488.814(3)$ & 0.002 & 2700 & & $4 \mathrm{f}$ & $(3 / 2)^{2}[5 / 2]^{\circ}{ }_{3}$ & $6 \mathrm{~g}$ & $(3 / 2)^{2}[7 / 2]_{4}$ & $1.9 \mathrm{e}+07$ & $\mathrm{D}+$ & $\mathrm{R} 1 \mathrm{c}$ & TW & \\
\hline $6494.029(3)$ & $15394.507(6)$ & $6494.0320(6)$ & -0.003 & 18000 & & $\mathrm{sp}$ & $\left({ }^{3} \mathrm{~F}\right)^{3} \mathrm{P}^{\circ} 1^{\circ}{ }_{3}^{\circ}$ & $5 \mathrm{~d}$ & $(5 / 2)^{2}[5 / 2]_{3}$ & & & F_Re & & \\
\hline 6497.040(9) & $15387.372(21)$ & 6497.041(3) & -0.001 & 89 & & $4 f$ & $(3 / 2)^{2}[5 / 2]^{\circ}$ & $6 \mathrm{~g}$ & $(3 / 2)^{2}[5 / 2]_{3}$ & & & $\mathrm{R} 1 \mathrm{c}$ & & \\
\hline 6508.401(3) & $15360.514(7)$ & $6508.400(3)$ & 0.001 & 2700 & & $4 \mathrm{f}$ & $(3 / 2)^{2}[5 / 2]^{\circ} 2$ & $6 \mathrm{~g}$ & $(3 / 2)^{2}[7 / 2]_{3}$ & $1.7 \mathrm{e}+07$ & D+ & R1c & TW & \\
\hline $6516.458(3)$ & $15341.522(8)$ & 6516.4637(12) & -0.006 & 6600 & & $5 p$ & $(3 / 2)^{2}[3 / 2]^{\circ}{ }_{2}$ & $5 \mathrm{~d}$ & $(3 / 2)^{2}[3 / 2]_{1}$ & & & $\mathrm{R} 1 \mathrm{c}$ & & \\
\hline 6517.316(3) & 15339.501(7) & 6517.3166(21) & -0.000 & 6600 & & $4 \mathrm{f}$ & $(5 / 2)^{2}[7 / 2]^{\circ}{ }_{4}$ & $6 \mathrm{~g}$ & $\begin{array}{l}(5 / 2)^{2}[9 / 2]_{5} \\
\text { nat }\end{array}$ & $1.7 \mathrm{e}+07$ & $\mathrm{D}+$ & R1c & TW & \\
\hline 6518.236(4) & 15337.337(9) & $6518.2366(12)$ & -0.001 & 150 & & $4 \mathrm{f}$ & $(5 / 2)^{2}[7 / 2]^{\circ}{ }_{4}$ & $6 \mathrm{~g}$ & $(5 / 2)^{2}[11 / 2]_{5}$ & & & R1c & & \\
\hline $6520.497(3)$ & $15332.019(8)$ & $6520.4970(21)$ & -0.000 & 1000 & & $4 \mathrm{f}$ & $(5 / 2)^{2}[7 / 2]^{\circ}{ }_{4}$ & $6 \mathrm{~g}$ & $(5 / 2)^{2}[7 / 2]_{4}$ & $5.7 \mathrm{e}+06$ & $\mathrm{D}+$ & $\mathrm{R} 1 \mathrm{c}$ & TW & \\
\hline $6521.270(6)$ & $15330.200(13)$ & 6521.2756(14) & -0.005 & 480 & & $4 p$ & ${ }^{1} \mathrm{P}_{1}^{\circ}$ & $s^{2}$ & ${ }^{3} \mathrm{P}_{0}$ & & & R1c & & \\
\hline 6523.820(3) & $15324.209(7)$ & $6523.820(3)$ & -0.000 & 4500 & & $4 \mathrm{f}$ & $(3 / 2)^{2}[7 / 2]^{\circ}{ }_{4}$ & $6 \mathrm{~g}$ & $(3 / 2)^{2}[9 / 2]_{5}$ & $2.1 \mathrm{e}+07$ & D+ & R1c & TW & \\
\hline 6525.641(9) & $15319.932(21)$ & $6525.650(3)$ & -0.008 & 29 & & $4 \mathrm{f}$ & $(5 / 2)^{2}[7 / 2]^{\circ}{ }_{4}$ & $6 \mathrm{~g}$ & $(5 / 2)^{2}[5 / 2]_{3}$ & & & $\mathrm{R} 1 \mathrm{c}$ & & \\
\hline $6526.646(6)$ & $15317.574(13)$ & 6526.655(3) & -0.009 & 88 & & $4 f$ & $(3 / 2)^{2}[7 / 2]^{\circ}{ }_{4}$ & $6 \mathrm{~g}$ & $(3 / 2)^{2}[7 / 2]_{4}$ & & & R1c & & \\
\hline 6529.167(3) & $15311.658(8)$ & $6529.1668(21)$ & 0.001 & 1000 & & $4 \mathrm{f}$ & $(5 / 2)^{2}[9 / 2]^{\circ}{ }_{5}$ & $6 \mathrm{~g}$ & $(5 / 2)^{2}[9 / 2]_{5}$ & $4.6 \mathrm{e}+06$ & $\mathrm{D}+$ & R1c & TW & \\
\hline $6530.082(3)$ & $15309.514(7)$ & 6530.0829(22) & -0.001 & 13000 & & $4 \mathrm{f}$ & $(5 / 2)^{2}[9 / 2]^{\circ}{ }_{5}$ & $6 \mathrm{~g}$ & $(5 / 2)^{2}[11 / 2]_{6}$ & $2.0 \mathrm{e}+07$ & $\mathrm{D}+$ & R1c & TW & \\
\hline
\end{tabular}


Table A1. Cont.

\begin{tabular}{|c|c|c|c|c|c|c|c|c|c|c|c|c|c|c|}
\hline$\lambda_{\text {obs }}{ }^{\text {a }}(\AA)$ & $\sigma_{\mathrm{obs}}{ }^{\mathrm{b}}\left(\mathrm{cm}^{-1}\right)$ & $\lambda_{\text {Ritz }}{ }^{\mathrm{c}}(\AA)$ & $\begin{array}{c}\Delta \lambda_{\text {obs-Ritz }} \\
\text { (A) }\end{array}$ & $\begin{array}{c}I_{\text {obs }} \mathrm{d} \\
\text { (arb. u.) }\end{array}$ & Char ${ }^{e}$ & & ower Level & & pper Level & $A\left(\mathrm{~s}^{-1}\right)$ & $\operatorname{Acc}^{f}$ & Line Ref. $\mathrm{g}$ & TP Ref. $\mathrm{g}$ & Notes ${ }^{h}$ \\
\hline $6536.679(9)$ & 15294.062(21) & $6536.6969(17)$ & -0.017 & 29 & & $4 \mathrm{~d}$ & $(3 / 2)^{2}[5 / 2]_{2}$ & $\mathrm{sp}$ & $\left({ }^{3} \mathrm{P}\right)^{3} \mathrm{P}^{\circ}{ }^{3} \mathrm{P}^{\circ}{ }_{2}$ & & & R1c & & \\
\hline 6541.637(3) & $15282.471(7)$ & 6541.6369(20) & 0.000 & 3400 & & $4 \mathrm{f}$ & $(3 / 2)^{2}[7 / 2]_{3}^{\circ}$ & $6 \mathrm{~g}$ & $(3 / 2)^{2}[9 / 2]_{4}$ & $1.9 \mathrm{e}+07$ & $\mathrm{D}+$ & R1c & TW & \\
\hline $6544.485(6)$ & $15275.822(14)$ & $6544.480(3)$ & 0.004 & 58 & * & $4 \mathrm{f}$ & $(3 / 2)^{2}[7 / 2]^{\circ}{ }_{3}$ & $6 \mathrm{~g}$ & $(3 / 2)^{2}[7 / 2]_{4}$ & & & R1nc & & \\
\hline $6544.485(6)$ & $15275.822(14)$ & 6544.489(3) & -0.004 & 58 & * & $4 \mathrm{f}$ & $(3 / 2)^{2}[7 / 2]^{\circ}{ }_{3}$ & $6 \mathrm{~g}$ & $(3 / 2)^{2}[7 / 2]_{3}$ & & & R1c & & \\
\hline $6550.304(5)$ & $15262.251(12)$ & 6550.3097(9) & -0.006 & 150 & & $5 p$ & $(3 / 2)^{2}[3 / 2]^{\circ}$ & $5 \mathrm{~d}$ & $(3 / 2)^{2}[7 / 2]_{3}$ & & & R1c & & \\
\hline 6551.286(3) & $15259.964(8)$ & 6551.2873(8) & -0.002 & 1900 & & $4 p$ & ${ }^{3} \mathrm{D}^{\circ}{ }_{1}$ & $\mathrm{~s}^{2}$ & ${ }^{3} \mathrm{P}_{2}$ & & & R1c & & \\
\hline 6554.791(6) & $15251.804(13)$ & 6554.7923(9) & -0.002 & 5100 & & $4 p$ & ${ }^{3} \mathrm{D}_{2}{ }_{2}$ & $s^{2}$ & ${ }^{3} \mathrm{P}_{1}$ & & & $\mathrm{R} 1 \mathrm{c}$ & & \\
\hline 6556.193(6) & $15248.542(14)$ & $6556.206(3)$ & -0.013 & 430 & & $4 \mathrm{~d}$ & $(5 / 2)^{2}[1 / 2]_{1}$ & $\mathrm{sp}$ & $\left({ }^{1} \mathrm{D}\right)^{3} \mathrm{P}^{\circ}{ }^{3} \mathrm{P}^{\circ}{ }_{1}$ & & & R1c & & \\
\hline $6559.657(4)$ & $15240.489(9)$ & $6559.6523(8)$ & 0.005 & 13000 & & $\mathrm{sp}$ & $\left({ }^{3} \mathrm{~F}\right)^{3} \mathrm{P}^{\circ}{ }^{\circ} \mathrm{F}^{\circ}{ }_{3}$ & $5 d$ & $(5 / 2)^{2}[9 / 2]_{4}$ & $4.9 \mathrm{e}+06$ & $\mathrm{D}+$ & R1c & TW & \\
\hline 6564.492(3) & 15229.264(8) & 6564.4941(8) & -0.002 & 10000 & & $\mathrm{sp}$ & $\left({ }^{3} \mathrm{~F}\right)^{3} \mathrm{P}^{\circ} 1^{1} \mathrm{~F}_{3}^{\circ}$ & $5 \mathrm{~d}$ & $(5 / 2)^{2}[3 / 2]_{2}$ & & & R1c & & \\
\hline 6567.949(6) & $15221.249(13)$ & 6567.9621(7) & -0.013 & 290 & & $5 s$ & $(5 / 2)^{2}[5 / 2]_{2}$ & $5 p$ & $(3 / 2)^{2}[3 / 2]^{\circ}$ & $5.1 \mathrm{e}+05$ & $\mathrm{C}$ & R1c & B00 & \\
\hline 6576.147(6) & $15202.273(13)$ & 6576.1510(20) & -0.004 & 1000 & & $4 \mathrm{f}$ & $(5 / 2)^{2}[9 / 2]^{\circ}{ }_{4}$ & $6 \mathrm{~g}$ & $(5 / 2)^{2}[9 / 2]_{4}$ & $4.3 e+06$ & $\mathrm{D}+$ & R1c & TW & \\
\hline $6577.0812(15)$ & $15200.114(3)$ & 6577.0816(12) & -0.0003 & 5700 & & $4 \mathrm{f}$ & $(5 / 2)^{2}[9 / 2]^{\circ}{ }_{4}$ & $6 \mathrm{~g}$ & $(5 / 2)^{2}[11 / 2]_{5}$ & $1.5 \mathrm{e}+07$ & $\mathrm{D}+$ & F_Re & TW & \\
\hline $6579.376(9)$ & $15194.812(21)$ & $6579.386(3)$ & -0.010 & 57 & & $4 \mathrm{f}$ & $(5 / 2)^{2}[9 / 2]^{\circ} 4$ & $6 \mathrm{~g}$ & $(5 / 2)^{2}[7 / 2]_{3}$ & & & $\mathrm{R} 1 \mathrm{c}$ & & \\
\hline 6592.896(9) & $15163.652(21)$ & 6592.900(3) & -0.004 & 120 & & $5 s$ & $(3 / 2)^{2}[3 / 2]_{1}$ & $\mathrm{sp}$ & $\left({ }^{3} \mathrm{P}\right)^{3} \mathrm{P}^{\circ}{ }^{5} \mathrm{P}_{2}^{\circ}$ & & & $\mathrm{R} 1 \mathrm{c}$ & & \\
\hline $6602.096(9)$ & $15142.522(21)$ & 6602.094(3) & 0.003 & 57 & & $6 p$ & $(5 / 2)^{2}[5 / 2]^{\circ}{ }_{3}$ & $8 \mathrm{~d}$ & $(5 / 2)^{2}[7 / 2]_{4}$ & & & $\mathrm{R} 1 \mathrm{c}$ & & \\
\hline 6603.527(9) & $15139.242(21)$ & $6603.523(4)$ & 0.003 & 29 & & $6 p$ & $(3 / 2)^{2}[5 / 2]^{\circ}{ }_{3}$ & $9 \mathrm{~s}$ & $\begin{array}{l}(3 / 2)^{2}[3 / 2]_{2}\end{array}$ & & & R1c & & \\
\hline 6612.087(9) & $15119.642(21)$ & 6612.086(3) & 0.001 & 57 & & $6 p$ & $(5 / 2)^{2}[5 / 2]^{\circ}{ }_{3}$ & $8 \mathrm{~d}$ & $(5 / 2)^{2}[5 / 2]_{3}$ & & & R1c & & \\
\hline 6624.291(3) & 15091.787(7) & $6624.2890(9)$ & 0.002 & 8200 & & $\mathrm{sp}$ & $\left({ }^{3} \mathrm{~F}\right)^{3} \mathrm{P}^{\circ} 1^{1} \mathrm{D}_{2}$ & $5 \mathrm{~d}$ & $\begin{array}{l}(5 / 2)^{2}[5 / 2]_{2} \\
\text {. }\end{array}$ & $2.2 \mathrm{e}+07$ & $\mathrm{D}+$ & R1c & TW & \\
\hline $6626.243(3)$ & $15087.341(8)$ & 6626.2420(20) & 0.001 & 710 & & $5 d$ & $(5 / 2)^{2}[1 / 2]_{1}$ & $8 p$ & $(5 / 2)^{2}[3 / 2]^{\circ}{ }_{1}$ & & & R1c & & \\
\hline 6631.476(3) & $15075.436(7)$ & $6631.4733(7)$ & 0.003 & 3300 & & $5 p$ & $(5 / 2)^{2}[7 / 2]^{\circ}{ }_{3}$ & $6 s$ & $(3 / 2)^{2}[3 / 2]_{2}$ & & & $\mathrm{R} 1 \mathrm{c}$ & & \\
\hline 6635.173(7) & 15067.037(17) & 6635.1692(18) & 0.003 & 85 & & $5 d$ & $(5 / 2)^{2}[1 / 2]_{1}$ & $6 \mathrm{f}$ & $(5 / 2)^{2}[5 / 2]^{\circ} 2$ & & & R1c & & \\
\hline 6641.395(3) & $15052.920(7)$ & $6641.3938(9)$ & 0.002 & 8000 & & $\mathrm{sp}$ & $\left({ }^{3} \mathrm{~F}\right)^{3} \mathrm{P}^{\circ}{ }^{1} \mathrm{D}_{2}^{\circ}{ }_{2}$ & $5 \mathrm{~d}$ & $(5 / 2)^{2}[7 / 2]_{3}$ & $2.6 \mathrm{e}+07$ & $\mathrm{D}+$ & $\mathrm{R} 1 \mathrm{c}$ & TW & \\
\hline 6647.796(4) & $15038.427(10)$ & 6647.7986(18) & -0.003 & 850 & & $5 \mathrm{~d}$ & $(5 / 2)^{2}[1 / 2]_{1}$ & $6 \mathrm{f}$ & $(5 / 2)^{2}[3 / 2]^{\circ} 2$ & & & R1c & & \\
\hline 6648.764(5) & $15036.237(11)$ & 6648.7717(14) & -0.008 & 7300 & & $5 p$ & $(3 / 2)^{2}[3 / 2]^{\circ}{ }_{2}$ & $5 d$ & $(3 / 2)^{2}[1 / 2]_{1}$ & $1.1 \mathrm{e}+07$ & $\mathrm{D}+$ & R1c & TW & \\
\hline 6651.323(4) & $15030.453(10)$ & $6651.3254(21)$ & -0.003 & 700 & & $\mathrm{sp}$ & $\left({ }^{3} \mathrm{~F}\right)^{1} \mathrm{P}^{\circ}{ }^{3} \mathrm{~F}^{\circ}{ }_{3}$ & $6 \mathrm{~g}$ & $(5 / 2)^{2}[9 / 2]_{4}$ & $3.8 \mathrm{e}+06$ & D+ & R1c & TW & \\
\hline 6654.635(9) & $15022.972(21)$ & $6654.635(3)$ & -0.000 & 56 & & $\mathrm{sp}$ & $\left({ }^{3} \mathrm{~F}\right)^{1} \mathrm{P}^{\circ}{ }^{3} \mathrm{~F}^{\circ}{ }_{3}$ & $6 \mathrm{~g}$ & $(5 / 2)^{2}[7 / 2]_{3}$ & & & $\mathrm{R} 1 \mathrm{c}$ & & \\
\hline $6660.961(3)$ & $15008.705(7)$ & $6660.9606(8)$ & 0.000 & 9200 & & $4 p$ & ${ }^{3} \mathrm{D}_{2}^{\circ}$ & $s^{2}$ & ${ }^{3} \mathrm{P}_{2}$ & & & R1c & & \\
\hline $6663.950(9)$ & $15001.972(21)$ & $6663.9466(6)$ & 0.004 & 560 & & $5 \mathrm{~s}$ & $(5 / 2)^{2}[5 / 2]_{3}$ & $5 p$ & $(3 / 2)^{2}[5 / 2]^{0}{ }_{3}$ & & & R1c & & \\
\hline $6692.710(6)$ & $14937.507(14)$ & 6692.7144(7) & -0.005 & 700 & & $\mathrm{sp}$ & $\left({ }^{3} \mathrm{~F}\right)^{3} \mathrm{P}^{\circ}{ }^{1} \mathrm{D}^{\circ}{ }_{2}$ & $5 \mathrm{~d}$ & $(5 / 2)^{2}[5 / 2]_{3}$ & & & R1c & & \\
\hline 6711.983(6) & $14894.615(12)$ & $6711.980(3)$ & 0.003 & 56 & & $5 d$ & $(3 / 2)^{2}[1 / 2]_{1}$ & $6 \mathrm{f}$ & $(3 / 2)^{2}[5 / 2]^{0} 2$ & & & R1c & & \\
\hline $6716.700(3)$ & 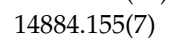 & $6716.7062(8)$ & -0.007 & 1300 & & $5 p$ & $(5 / 2)^{2}[5 / 2]^{\circ}{ }_{2}$ & $6 \mathrm{~s}$ & $(3 / 2)^{2}[3 / 2]_{2}$ & & & R1c & & \\
\hline $6717.687(6)$ & $14881.968(12)$ & $6717.687(4)$ & -0.001 & 280 & & $5 s$ & $(3 / 2)^{2}[3 / 2]_{2}$ & $\mathrm{sp}$ & $\left({ }^{3} \mathrm{P}\right)^{3} \mathrm{P}^{\circ}{ }^{5} \mathrm{P}_{2}{ }_{2}$ & & & $\mathrm{R} 1 \mathrm{c}$ & & \\
\hline 6725.481(10) & $14864.722(21)$ & 6725.490(4) & -0.010 & 28 & & $5 \mathrm{~d}$ & $(3 / 2)^{2}[1 / 2]_{1}$ & $6 \mathrm{f}$ & $(3 / 2)^{2}[3 / 2]^{\circ}{ }_{1}$ & & & $\mathrm{R} 2 \mathrm{c}$ & & \\
\hline $6725.834(10)$ & $14863.942(21)$ & $6725.850(8)$ & -0.016 & 28 & & $5 s$ & $(3 / 2)^{2}[3 / 2]_{2}$ & sp & $\left({ }^{3} \mathrm{P}\right)^{3} \mathrm{P}^{\circ}{ }^{5} \mathrm{P}_{3}^{\mathrm{o}}{ }_{3}$ & & & R1nc & & \\
\hline $6736.397(5)$ & $14840.633(10)$ & 6736.4201(7) & -0.023 & 6300 & & $5 p$ & $(5 / 2)^{2}[3 / 2]^{\circ}{ }_{1}$ & $6 \mathrm{~s}$ & $(3 / 2)^{2}[3 / 2]_{2}$ & & & R1c & & $\mathrm{x}$ \\
\hline 6739.599(3) & $\begin{array}{l}14833.582(7) \\
\text { (1) }\end{array}$ & $6739.598(3)$ & 0.002 & 140 & & $5 d$ & $(3 / 2)^{2}[1 / 2]_{1}$ & $6 \mathrm{f}$ & $(3 / 2)^{2}[3 / 2]^{\circ} 2$ & $7.4 \mathrm{e}+06$ & $\mathrm{D}+$ & R1c & TW & \\
\hline 6743.740(7) & $14824.474(16)$ & 6743.737(3) & 0.003 & 56 & & $5 \mathrm{~d}$ & $(5 / 2)^{2}[1 / 2]_{1}$ & $6 \mathrm{f}$ & $(5 / 2)^{2}[1 / 2]^{\circ}{ }_{1}$ & & & $\mathrm{R} 2 \mathrm{nc}$ & & \\
\hline 6758.855(4) & $14791.323(8)$ & $6758.8577(10)$ & -0.003 & 6300 & & $\mathrm{sp}$ & $\left({ }^{3} \mathrm{~F}\right)^{3} \mathrm{P}^{\circ}{ }^{1} \mathrm{D}^{\circ}{ }_{2}$ & $5 \mathrm{~d}$ & $(5 / 2)^{2}[3 / 2]_{1}$ & $1.0 \mathrm{e}+07$ & $\mathrm{D}+$ & R1c & TW & \\
\hline $6760.142(5)$ & $14788.506(11)$ & $6760.1422(8)$ & 0.000 & 56 & & $5 p$ & $(5 / 2)^{2}[5 / 2]^{\circ}$ & $6 \mathrm{~s}$ & $(3 / 2)^{2}[3 / 2]_{1}$ & & & R1c & & \\
\hline 6765.794(10) & $14776.152(21)$ & 6765.7884(19) & 0.006 & 28 & & $\mathrm{sp}$ & $\left({ }^{1} \mathrm{G}\right)^{3} \mathrm{P}^{\circ} 3^{3} \mathrm{H}^{\circ}{ }_{4}$ & $6 \mathrm{~g}$ & $(5 / 2)^{2}[11 / 2]_{5}$ & & & R1c & & \\
\hline $6767.561(7)$ & $14772.294(16)$ & $6767.5789(9)$ & -0.018 & 28 & & $\mathrm{sp}$ & $\left({ }^{3} \mathrm{~F}\right)^{3} \mathrm{P}^{\circ}{ }^{1} \mathrm{D}^{\circ}{ }_{2}$ & $5 \mathrm{~d}$ & $(5 / 2)^{2}[3 / 2]_{2}$ & & & R1c & & \\
\hline 6770.361(3) & 14766.186(7) & $6770.3604(10)$ & 0.000 & 3100 & & $4 p$ & ${ }^{1} \mathrm{P}^{\circ}{ }_{1}$ & $\mathrm{~s}^{2}$ & ${ }^{3} \mathrm{P}_{2}$ & & & R1c & & \\
\hline
\end{tabular}


Table A1. Cont.

\begin{tabular}{|c|c|c|c|c|c|c|c|c|c|c|c|c|c|c|}
\hline$\lambda_{\text {obs }}{ }^{\text {a }}(\AA)$ & $\sigma_{\mathrm{obs}}{ }^{\mathrm{b}}\left(\mathrm{cm}^{-1}\right)$ & $\lambda_{\text {Ritz }}{ }^{c}(\AA)$ & $\begin{array}{c}\Delta \lambda_{\text {obs-Ritz }} \\
\text { (A) }\end{array}$ & $\begin{array}{c}I_{\text {obs }} \mathrm{d} \\
\text { (arb. u.) }\end{array}$ & Char ${ }^{e}$ & & ower Level & & Jpper Level & $A\left(\mathrm{~s}^{-1}\right)$ & $\operatorname{Acc}^{f}$ & Line Ref. $\mathrm{g}$ & TP Ref. $\mathrm{g}$ & Notes ${ }^{h}$ \\
\hline 6779.440(7) & 14746.410(15) & 6779.4453(18) & -0.005 & 28 & & $5 p$ & $(3 / 2)^{2}[1 / 2]^{\circ}{ }_{1}$ & $5 d$ & $(5 / 2)^{2}[1 / 2]_{0}$ & & & R1c & & \\
\hline $6780.114(4)$ & $14744.945(9)$ & $6780.1122(7)$ & 0.002 & 4900 & & $5 p$ & $(5 / 2)^{2}[3 / 2]^{0}{ }_{1}$ & $6 \mathrm{~s}$ & $(3 / 2)^{2}[3 / 2]_{1}$ & & & R1c & & \\
\hline 6786.482(5) & 14731.110(11) & 6786.4808(7) & 0.001 & 84 & & $5 s$ & $(5 / 2)^{2}[5 / 2]_{3}$ & $5 p$ & $(3 / 2)^{2}[5 / 2]^{0} 2$ & & & R1c & & \\
\hline 6802.138(3) & $\begin{array}{l}14697.204(7) \\
\text { (n) }\end{array}$ & 6802.1387(19) & -0.001 & 220 & & $5 \mathrm{~d}$ & $(5 / 2)^{2}[9 / 2]_{5}$ & $6 f$ & $(5 / 2)^{2}[9 / 2]^{\circ}{ }_{5}$ & $3.5 e+06$ & $\mathrm{D}+$ & R1c & TW & \\
\hline $6806.215(3)$ & 14688.401(7) & $6806.2136(9)$ & 0.001 & 4100 & & $\mathrm{sp}$ & $\left({ }^{3} \mathrm{~F}\right)^{3} \mathrm{P}^{\circ}{ }^{3} \mathrm{~F}^{\circ}{ }_{2}$ & $6 \mathrm{~s}$ & $(5 / 2)^{2}[5 / 2]_{2}$ & & & R1c & & \\
\hline 6809.6453(23) & 14681.001(5) & 6809.6436(8) & 0.0016 & 5200 & & $5 p$ & $(5 / 2)^{2}[5 / 2]^{\circ}{ }_{3}$ & $6 \mathrm{~s}$ & $(3 / 2)^{2}[3 / 2]_{2}$ & & & F_Re & & \\
\hline $6814.690(14)$ & $14670.13(3)$ & 6814.6913(22) & -0.001 & 28 & & $5 \mathrm{~d}$ & $(5 / 2)^{2}[3 / 2]_{2}$ & $8 p$ & $(5 / 2)^{2}[3 / 2]^{\circ}{ }_{1}$ & & & R1c & & \\
\hline $6821.060(5)$ & 14656.434(11) & 6821.0571(19) & 0.002 & 140 & & $5 d$ & $(5 / 2)^{2}[9 / 2]_{4}$ & $6 f$ & $(5 / 2)^{2}[9 / 2]^{\circ}{ }_{4}$ & $3.2 \mathrm{e}+06$ & $\mathrm{D}+$ & R1c & TW & \\
\hline 6822.342(8) & $14653.679(17)$ & $6822.3460(19)$ & -0.004 & 28 & & $5 \mathrm{~d}$ & $(5 / 2)^{2}[9 / 2]_{4}$ & $6 f$ & $(5 / 2)^{2}[7 / 2]^{\circ} 4$ & & & R1c & & \\
\hline 6823.201(3) & $14651.833(7)$ & 6823.2010(23) & 0.000 & 6400 & & $5 \mathrm{~d}$ & $(5 / 2)^{2}[9 / 2]_{5}$ & $6 f$ & $(5 / 2)^{2}[11 / 2]^{\circ}{ }_{6}$ & $1.8 \mathrm{e}+07$ & $\mathrm{D}+$ & R1c & TW & \\
\hline $6824.750(4)$ & $14648.508(8)$ & 6824.7493(19) & 0.001 & 700 & & $5 \mathrm{~d}$ & $(5 / 2)^{2}[3 / 2]_{2}$ & $6 f$ & $(5 / 2)^{2}[5 / 2]^{\circ}{ }_{3}$ & $7.2 \mathrm{e}+06$ & $\mathrm{D}+$ & R1c & TW & \\
\hline 6830.531(3) & 14636.112(7) & $6830.5296(22)$ & 0.001 & 1300 & & $5 \mathrm{~d}$ & $(3 / 2)^{2}[7 / 2]_{3}$ & $6 f$ & $(3 / 2)^{2}[9 / 2]^{\circ} 4$ & $1.8 \mathrm{e}+07$ & $\mathrm{D}+$ & R1c & TW & \\
\hline 6833.026(4) & $14630.766(9)$ & 6833.0244(19) & 0.002 & 560 & & $5 \mathrm{~d}$ & $(5 / 2)^{2}[3 / 2]_{1}$ & $6 \mathrm{f}$ & $(5 / 2)^{2}[5 / 2]^{\circ}{ }_{2}$ & $8.1 \mathrm{e}+06$ & $\mathrm{D}+$ & R1c & TW & \\
\hline 6837.491(4) & $14621.212(8)$ & $6837.4936(18)$ & -0.002 & 720 & & $5 \mathrm{~d}$ & $(5 / 2)^{2}[3 / 2]_{2}$ & $6 f$ & $(5 / 2)^{2}[3 / 2]^{\circ}{ }_{2}$ & $9 . e+06$ & $\mathrm{D}+$ & R1c & TW & \\
\hline 6844.156(3) & $14606.974(7)$ & $6844.1542(20)$ & 0.002 & 3200 & & $5 \mathrm{~d}$ & $(5 / 2)^{2}[9 / 2]_{4}$ & $6 f$ & $(5 / 2)^{2}[11 / 2]^{\circ}{ }_{5}$ & $1.8 \mathrm{e}+07$ & $\mathrm{D}+$ & R1c & TW & \\
\hline 6846.104(20) & $14602.82(4)$ & $6846.065(3)$ & 0.039 & 880 & & $\mathrm{sp}$ & $\left({ }^{3} \mathrm{~F}\right)^{3} \mathrm{P}^{\circ}{ }^{1} \mathrm{G}^{\circ}{ }_{4}$ & $6 \mathrm{~s}$ & $(5 / 2)^{2}[5 / 2]_{3}$ & & & R1c & & \\
\hline 6847.112(7) & $14600.669(14)$ & 6847.112(3) & -0.000 & 170 & & $5 \mathrm{~d}$ & $(5 / 2)^{2}[3 / 2]_{1}$ & $6 \mathrm{f}$ & $(5 / 2)^{2}[3 / 2]^{\circ}{ }_{1}$ & & & R1c & & \\
\hline 6849.991(6) & $14594.532(13)$ & 6849.988(4) & 0.003 & 56 & & $4 \mathrm{~d}$ & $(5 / 2)^{2}[1 / 2]_{1}$ & $\mathrm{sp}$ & $\left({ }^{1} \mathrm{D}\right)^{3} \mathrm{P}^{\circ}{ }^{3} \mathrm{P}^{\circ}{ }_{0}^{\circ}$ & & & R1c & & \\
\hline 6852.428(7) & 14589.342(14) & 6852.432(3) & -0.004 & 84 & & $5 d$ & $(3 / 2)^{2}[3 / 2]_{1}$ & $6 f$ & $(3 / 2)^{2}[5 / 2]^{0} 2$ & & & R1c & & \\
\hline 6863.061(3) & $14566.739(7)$ & $6863.0599(23)$ & 0.001 & 2100 & & $5 \mathrm{~d}$ & $(3 / 2)^{2}[7 / 2]_{4}$ & $6 \mathrm{f}$ & $(3 / 2)^{2}[9 / 2]^{\circ}$ & $1.8 \mathrm{e}+07$ & D+ & R1c & TW & \\
\hline 6867.88(20) & $14556.5(4)$ & 6868.184(4) & -0.30 & 470 & & $4 \mathrm{~d}$ & $(5 / 2)^{2}[5 / 2]_{2}$ & $\mathrm{sp}$ & $\left({ }^{3} \mathrm{P}\right)^{3} \mathrm{P}^{\circ}{ }^{5} \mathrm{D}_{2}^{\circ}$ & & & S36c & & \\
\hline $6868.790(3)$ & $14554.589(7)$ & 6868.7864(9) & 0.004 & 2900 & & $\mathrm{sp}$ & $\left({ }^{3} \mathrm{~F}\right)^{3} \mathrm{P}^{\circ}{ }^{3} \mathrm{~F}^{\circ}{ }_{2}$ & $6 \mathrm{~s}$ & $(5 / 2)^{2}[5 / 2]_{3}$ & & & R1c & & \\
\hline $6872.230(3)$ & $14547.303(7)$ & 6872.2293(10) & 0.001 & 3200 & & $4 p$ & $1^{1} \mathrm{~F}_{3}^{2}$ & $s^{2}$ & ${ }^{1} \mathrm{D}_{2}$ & & & R1c & & \\
\hline $6879.403(3)$ & $14532.136(7)$ & $6879.4043(7)$ & -0.001 & 2500 & & $5 s$ & $(5 / 2)^{2}[5 / 2]_{2}$ & $5 p$ & $(3 / 2)^{2}[1 / 2]^{\circ}{ }_{1}$ & $5.5 e+06$ & C & R1c & B00 & \\
\hline $6893.496(6)$ & $14502.427(12)$ & 6893.4896(18) & 0.006 & 220 & & $5 \mathrm{~d}$ & $(5 / 2)^{2}[5 / 2]_{3}$ & $6 f$ & $(5 / 2)^{2}[9 / 2]^{\circ}$ & & & R1c & & \\
\hline 6894.810(4) & $14499.663(9)$ & 6894.8061(18) & 0.004 & 700 & & $5 \mathrm{~d}$ & $(5 / 2)^{2}[5 / 2]_{3}$ & $6 \mathrm{f}$ & $(5 / 2)^{2}[7 / 2]^{\circ} 4$ & $1.0 \mathrm{e}+07$ & $\mathrm{D}+$ & R1c & TW & \\
\hline $6902.617(4)$ & $14483.263(9)$ & 6902.6139(19) & 0.003 & 560 & & $5 \mathrm{~d}$ & $(5 / 2)^{2}[5 / 2]_{3}$ & $6 \mathrm{f}$ & $(5 / 2)^{2}[5 / 2]^{\circ} 3$ & $8.0 \mathrm{e}+06$ & $\mathrm{D}+$ & R1c & TW & \\
\hline 6915.653(10) & $14455.962(21)$ & 6915.6509(18) & 0.002 & 56 & & $5 \mathrm{~d}$ & $(5 / 2)^{2}[5 / 2]_{3}$ & $6 f$ & $(5 / 2)^{2}[3 / 2]^{\circ}{ }_{2}$ & & & R1c & & \\
\hline $6928.602(6)$ & $14428.946(12)$ & 6928.592(3) & 0.009 & 340 & & $5 \mathrm{~d}$ & $(3 / 2)^{2}[3 / 2]_{2}$ & $6 f$ & $(3 / 2)^{2}[5 / 2]^{\circ} 3$ & $1.1 \mathrm{e}+07$ & $\mathrm{D}+$ & R1c & TW & \\
\hline 6937.552(4) & $14410.331(8)$ & 6937.5472(7) & 0.005 & 2100 & & $5 s$ & $(5 / 2)^{2}[5 / 2]_{2}$ & $5 p$ & $(3 / 2)^{2}[5 / 2]^{\circ} 2$ & $3.9 \mathrm{e}+06$ & $\mathrm{C}$ & R1c & B00 & \\
\hline 6939.099(7) & $14407.119(14)$ & 6939.098(3) & 0.001 & 280 & & $5 d$ & $(5 / 2)^{2}[7 / 2]_{3}$ & $6 f$ & $(5 / 2)^{2}[7 / 2]^{\circ} 3$ & $4.8 \mathrm{e}+06$ & $\mathrm{D}+$ & R1c & TW & \\
\hline 6948.795(7) & 14387.015(15) & 6948.7964(20) & -0.001 & 2000 & & $5 \mathrm{~d}$ & $(5 / 2)^{2}[7 / 2]_{3}$ & $6 f$ & $(5 / 2)^{2}[9 / 2]^{\circ}{ }_{4}$ & $1.1 \mathrm{e}+07$ & $\mathrm{D}+$ & R1c & TW & \\
\hline $6950.139(6)$ & $14384.234(13)$ & 6950.1341(20) & 0.005 & 1700 & & $5 \mathrm{~d}$ & $(5 / 2)^{2}[7 / 2]_{3}$ & $6 \mathrm{f}$ & $(5 / 2)^{2}[7 / 2]^{\circ} 4$ & $3.1 \mathrm{e}+06$ & $\mathrm{D}+$ & R1c & TW & \\
\hline 6952.870(4) & $\begin{array}{l}14378.583(7) \\
\text { (1) }\end{array}$ & 6952.8664(20) & 0.004 & 4200 & & $5 \mathrm{~d}$ & $(5 / 2)^{2}[7 / 2]_{4}$ & $6 \mathrm{f}$ & $(5 / 2)^{2}[9 / 2]^{\circ}{ }_{5}$ & $1.4 \mathrm{e}+07$ & $\mathrm{D}+$ & R1c & TW & \\
\hline $6953.480(10)$ & $14377.322(21)$ & 6953.4782(20) & 0.002 & 110 & & $5 \mathrm{~d}$ & $(5 / 2)^{2}[7 / 2]_{4}$ & $6 \mathrm{f}$ & $(5 / 2)^{2}[9 / 2]^{\circ}{ }_{4}$ & & & R1c & & \\
\hline $6953.930(6)$ & 14376.391(13) & 6953.914(3) & 0.016 & 2100 & & $5 \mathrm{~d}$ & $(3 / 2)^{2}[5 / 2]_{3}$ & $6 f$ & $(3 / 2)^{2}[7 / 2]^{\circ} 4$ & $1.5 \mathrm{e}+07$ & $\mathrm{D}+$ & R1c & TW & \\
\hline 6954.820(10) & 14374.552(21) & 6954.8176(19) & 0.002 & 230 & & $5 \mathrm{~d}$ & $(5 / 2)^{2}[7 / 2]_{4}$ & $6 f$ & $(5 / 2)^{2}[7 / 2]^{\circ} 4$ & $4.4 \mathrm{e}+06$ & $\mathrm{D}+$ & R1c & TW & \\
\hline 6957.884(10) & $14368.222(20)$ & 6957.869(3) & 0.015 & 1800 & * & $5 \mathrm{~d}$ & $\begin{array}{l}(5 / 2)^{2}[5 / 2]_{2} \\
\text { nat }\end{array}$ & $6 \mathrm{f}$ & $(5 / 2)^{2}[7 / 2]^{\circ}{ }_{3}$ & $1.1 \mathrm{e}+07$ & $\mathrm{D}+$ & R1c & TW & \\
\hline 6957.884(10) & $14368.222(20)$ & 6957.876(4) & 0.008 & 1800 & * & $\mathrm{sp}$ & $\left({ }^{1} \mathrm{G}\right)^{3} \mathrm{P}^{\circ}{ }^{3} \mathrm{H}^{\circ}{ }_{5}$ & $6 \mathrm{~g}$ & $(5 / 2)^{2}[13 / 2]_{6}$ & & & R1c & & \\
\hline 6957.884(10) & $14368.222(20)$ & 6957.876(3) & 0.008 & 1800 & * & $5 d$ & $(3 / 2)^{2}[5 / 2]_{3}$ & $6 \mathrm{f}$ & $(3 / 2)^{2}[5 / 2]^{\circ}{ }_{2}$ & & & R1c & & \\
\hline $6963.433(10)$ & $14356.772(21)$ & 6963.427(3) & 0.006 & 230 & & $5 \mathrm{~d}$ & $(3 / 2)^{2}[5 / 2]_{2}$ & $6 f$ & $(3 / 2)^{2}[7 / 2]^{\circ} 3$ & $1.3 \mathrm{e}+07$ & $\mathrm{D}+$ & R1c & TW & \\
\hline $6968.846(10)$ & $14345.622(21)$ & $6968.847(3)$ & -0.002 & 57 & & $5 d$ & $(3 / 2)^{2}[5 / 2]_{2}$ & $6 f$ & $(3 / 2)^{2}[5 / 2]^{\circ} 2$ & & & R1c & & \\
\hline 6976.305(10) & $14330.282(21)$ & 6976.2990(20) & 0.006 & 230 & & $5 \mathrm{~d}$ & $(5 / 2)^{2}[5 / 2]_{2}$ & $6 f$ & $(5 / 2)^{2}[5 / 2]^{\circ}{ }_{2}$ & $5.6 \mathrm{e}+06$ & $\mathrm{D}+$ & R1c & TW & \\
\hline
\end{tabular}


Table A1. Cont.

\begin{tabular}{|c|c|c|c|c|c|c|c|c|c|c|c|c|c|c|}
\hline$\lambda_{\text {obs }}{ }^{a}(\AA)$ & $\sigma_{\mathrm{obs}}{ }^{\mathrm{b}}\left(\mathrm{cm}^{-1}\right)$ & $\lambda_{\text {Ritz }}{ }^{c}(\AA)$ & $\begin{array}{c}\Delta \lambda_{\text {obs-Ritz }} \\
\text { (A) }\end{array}$ & $\begin{array}{l}I_{\text {obs }} \mathrm{d} \\
\text { (arb. u.) }\end{array}$ & Char ${ }^{e}$ & & ower Level & & pper Level & $A\left(\mathrm{~s}^{-1}\right)$ & $\operatorname{Acc}^{f}$ & Line Ref. $\mathrm{g}$ & TP Ref. $\mathrm{g}$ & Notes ${ }^{h}$ \\
\hline $6977.571(4)$ & $14327.683(8)$ & 6977.5641(10) & 0.007 & 4300 & & $5 p$ & $(3 / 2)^{2}[5 / 2]_{2}^{\circ}$ & $5 \mathrm{~d}$ & $(5 / 2)^{2}[5 / 2]_{2}$ & & & R1c & & \\
\hline 6986.548(8) & 14309.273(17) & $6986.5269(20)$ & 0.021 & 2100 & & $5 p$ & $(3 / 2)^{2}[3 / 2]^{\circ}{ }_{1}$ & $5 \mathrm{~d}$ & $(5 / 2)^{2}[1 / 2]_{0}$ & & & R1c & & \\
\hline 6996.556(5) & $14288.805(9)$ & 6996.5446(10) & 0.012 & 1400 & & $5 p$ & $(3 / 2)^{2}[5 / 2]^{\circ}{ }_{2}$ & $5 \mathrm{~d}$ & $(5 / 2)^{2}[7 / 2]_{3}$ & & & R1c & & \\
\hline $7022.859(4)$ & $14235.289(7)$ & $7022.8519(9)$ & 0.007 & 2800 & & $\mathrm{sp}$ & $\left({ }^{3} \mathrm{~F}\right)^{3} \mathrm{P}^{\circ} 1^{1} \mathrm{~F}_{3}^{\circ}$ & $6 \mathrm{~s}$ & $(3 / 2)^{2}[3 / 2]_{2}$ & & & R1c & & \\
\hline 7037.388(4) & $14205.899(8)$ & 7037.3853(10) & 0.003 & 2900 & & $5 p$ & $(3 / 2)^{2}[1 / 2]^{\circ}{ }_{1}$ & $5 \mathrm{~d}$ & $(5 / 2)^{2}[5 / 2]_{2}$ & & & R1c & & \\
\hline 7083.772(6) & $14112.882(12)$ & 7083.7743(13) & -0.003 & 730 & & $5 p$ & $(3 / 2)^{2}[1 / 2]_{0}^{\circ}$ & $5 \mathrm{~d}$ & $(5 / 2)^{2}[1 / 2]_{1}$ & & & R1c & & \\
\hline 7123.112(11) & $14034.939(21)$ & $7123.106(3)$ & 0.006 & 59 & * & $6 \mathrm{~s}$ & $(5 / 2)^{2}[5 / 2]_{2}$ & $7 p$ & $(5 / 2)^{2}[5 / 2]^{\circ}{ }^{\circ}$ & & & $\mathrm{R} 1 \mathrm{c}$ & & \\
\hline 7123.112(11) & $14034.939(21)$ & 7123.1061(21) & 0.006 & 59 & * & $\mathrm{sp}$ & $\left({ }^{3} \mathrm{~F}\right)^{1} \mathrm{P}^{\circ}{ }^{\circ} \mathrm{G}^{\circ}{ }_{5}$ & $5 g$ & $(3 / 2)^{2}[11 / 2]_{6}$ & & & R1c & & \\
\hline 7123.112(11) & $14034.939(21)$ & 7123.1212(21) & -0.010 & 59 & * & $\mathrm{sp}$ & $\left({ }^{3} \mathrm{~F}\right)^{1} \mathrm{P}^{\circ}{ }^{3} \mathrm{G}_{5}^{\circ}$ & $5 \mathrm{~g}$ & $(3 / 2)^{2}[11 / 2]_{5}$ & & & $\mathrm{R} 1 \mathrm{c}$ & & \\
\hline $7127.032(8)$ & $14027.218(15)$ & 7127.0305(11) & 0.002 & 150 & & $5 p$ & $(3 / 2)^{2}[5 / 2]^{\circ}$ & $5 \mathrm{~d}$ & $(5 / 2)^{2}[3 / 2]_{1}$ & & & R1c & & \\
\hline $7139.649(5)$ & $14002.431(10)$ & 7139.653(3) & -0.004 & 1500 & & $6 \mathrm{~s}$ & $(5 / 2)^{2}[5 / 2]_{3}$ & $7 p$ & $(5 / 2)^{2}[7 / 2]^{\circ} 4$ & & & R1c & & \\
\hline $7157.757(5)$ & 13967.006(10) & 7157.7517(8) & 0.006 & 1500 & & $5 s$ & $(5 / 2)^{2}[5 / 2]_{3}$ & $\mathrm{sp}$ & $\left({ }^{3} \mathrm{~F}\right)^{3} \mathrm{P}^{\circ 1} \mathrm{D}^{\circ}{ }_{2}$ & $5.2 \mathrm{e}+05$ & $\mathrm{C}$ & R1c & B00 & \\
\hline 7182.106(7) & $13919.655(14)$ & $7182.104(5)$ & 0.002 & 60 & & $6 \mathrm{~s}$ & $(5 / 2)^{2}[5 / 2]_{2}$ & $7 p$ & $(5 / 2)^{2}[5 / 2]^{\circ} 2$ & & & R1c & & \\
\hline $7189.458(4)$ & $13905.422(7)$ & 7189.4534(12) & 0.004 & 1300 & & $5 p$ & $(3 / 2)^{2}[1 / 2]^{\circ}{ }_{1}$ & $5 \mathrm{~d}$ & $(5 / 2)^{2}[3 / 2]_{1}$ & & & R1c & & \\
\hline $7194.900(3)$ & $13894.903(6)$ & 7194.8933(10) & 0.007 & 6800 & & $4 p$ & ${ }^{1} \mathrm{D}^{\circ}{ }_{2}$ & $s^{2}$ & ${ }^{1} \mathrm{D}_{2}$ & & & F_Re & & \\
\hline $7255.7937(20)$ & $13778.293(4)$ & $7255.7899(9)$ & 0.0038 & 6400 & & $\mathrm{sp}$ & $\left({ }^{3} \mathrm{~F}\right)^{3} \mathrm{P}^{\circ}{ }^{1} \mathrm{D}_{2}^{\circ}$ & $6 \mathrm{~s}$ & $(3 / 2)^{2}[3 / 2]_{2}$ & $6.5 \mathrm{e}+06$ & $\mathrm{D}+$ & F_Re & TW & \\
\hline $7271.507(6)$ & 13748.519(11) & 7271.4991(10) & 0.008 & 460 & & $5 p$ & $(3 / 2)^{2}[5 / 2]^{\circ}{ }_{3}$ & $5 \mathrm{~d}$ & $(5 / 2)^{2}[9 / 2]_{4}$ & & & R1c & & \\
\hline 7306.511(4) & $13682.653(7)$ & $7306.5042(9)$ & 0.007 & 3700 & & $\mathrm{sp}$ & $\left({ }^{3} \mathrm{~F}\right)^{3} \mathrm{P}^{\circ}{ }^{1} \mathrm{D}^{\circ}{ }_{2}$ & $6 \mathrm{~s}$ & $(3 / 2)^{2}[3 / 2]_{1}$ & & & R1c & & \\
\hline 7326.007(4) & 13646.242(7) & 7326.0039(8) & 0.003 & 6900 & & $5 s$ & $(5 / 2)^{2}[5 / 2]_{2}$ & $\mathrm{sp}$ & $\left({ }^{3} \mathrm{~F}\right)^{3} \mathrm{P}^{\circ} 1^{1} \mathrm{D}_{2}^{\circ}$ & $1.80 \mathrm{e}+07$ & B & R1c & B00 & \\
\hline 7331.6940(19) & $13635.656(4)$ & 7331.6941(7) & -0.0001 & 5100 & & $5 p$ & $(5 / 2)^{2}[3 / 2]^{\circ}{ }_{2}$ & $6 \mathrm{~s}$ & $(5 / 2)^{2}[5 / 2]_{2}$ & & & F_Re & & \\
\hline $7382.275(3)$ & $13542.229(6)$ & $7382.2720(14)$ & 0.003 & 3500 & & $4 \mathrm{~d}$ & $(3 / 2)^{2}[1 / 2]_{0}$ & $4 \mathrm{f}$ & $(5 / 2)^{2}[3 / 2]^{\circ} 1$ & $1.6 \mathrm{e}+07$ & $\mathrm{D}+$ & F_Re & TW & \\
\hline $7396.156(6)$ & $13516.815(11)$ & $7396.1523(12)$ & 0.003 & 1900 & & $5 p$ & $(3 / 2)^{2}[3 / 2]^{\circ}{ }_{2}$ & $5 d$ & $(5 / 2)^{2}[5 / 2]_{2}$ & & & $\mathrm{R} 1 \mathrm{c}$ & & \\
\hline $7399.8836(16)$ & $13510.005(3)$ & 7399.8787(7) & 0.0049 & 14000 & & $5 s$ & $(5 / 2)^{2}[5 / 2]_{3}$ & $\mathrm{sp}$ & $\left({ }^{3} \mathrm{~F}\right)^{3} \mathrm{P}^{\circ}{ }^{1} \mathrm{~F}^{\circ}{ }_{3}$ & $2.57 \mathrm{e}+07$ & B & F_Re & B00 & \\
\hline 7404.3561(11) & $13501.8442(20)$ & $7404.3532(6)$ & 0.0029 & 55000 & & $5 p$ & $(5 / 2)^{2}[3 / 2]_{2}^{\circ}$ & $6 \mathrm{~s}$ & $(5 / 2)^{2}[5 / 2]_{3}$ & $2.0 \mathrm{e}+07$ & $\mathrm{D}+$ & F_Re & TW & $\mathrm{L}$ \\
\hline 7417.483(5) & $13477.950(9)$ & 7417.4819(12) & 0.001 & 2400 & & $5 p$ & $(3 / 2)^{2}[3 / 2]_{2}^{\circ}$ & $5 \mathrm{~d}$ & $\begin{array}{l}(5 / 2)^{2}[7 / 2]_{3} \\
\end{array}$ & & & $\mathrm{R} 1 \mathrm{c}$ & & \\
\hline $7420.556(4)$ & $13472.368(7)$ & $7420.5668(9)$ & -0.010 & 4100 & & $5 s$ & $(3 / 2)^{2}[3 / 2]_{1}$ & $5 p$ & $(3 / 2)^{2}[3 / 2]^{\circ} 2$ & $3.1 \mathrm{e}+06$ & C & R1c & B00 & \\
\hline $7422.602(5)$ & $13468.656(9)$ & 7422.5904(11) & 0.011 & 790 & & $4 p$ & ${ }^{3} \mathrm{D}^{\circ}{ }_{3}$ & $\mathrm{~s}^{2}$ & ${ }^{1} \mathrm{D}_{2}$ & & & $\mathrm{R} 1 \mathrm{c}$ & & \\
\hline 7434.155(4) & $13447.725(7)$ & 7434.1525(15) & 0.002 & 3700 & & $4 \mathrm{~d}$ & $(3 / 2)^{2}[1 / 2]_{0}$ & $4 \mathrm{f}$ & $(5 / 2)^{2}[1 / 2]^{\circ}{ }_{1}$ & $1.3 \mathrm{e}+07$ & $\mathrm{D}+$ & R1c & TW & \\
\hline 7438.1504(13) & $13440.5007(24)$ & $7438.1505(10)$ & -0.0001 & 11000 & & $5 p$ & $(3 / 2)^{2}[1 / 2]^{\circ}{ }_{0}$ & $6 \mathrm{~s}$ & $(3 / 2)^{2}[3 / 2]_{1}$ & $1.0 \mathrm{e}+07$ & $\mathrm{D}+$ & F_Re & TW & \\
\hline $7481.554(8)$ & $13362.528(15)$ & 7481.5552(10) & -0.001 & 190 & & $5 p$ & $(3 / 2)^{2}[3 / 2]_{2}^{\circ}$ & $5 \mathrm{~d}$ & $(5 / 2)^{2}[5 / 2]_{3}$ & & & $\mathrm{R} 1 \mathrm{c}$ & & \\
\hline 7493.289(8) & $13341.602(14)$ & 7493.2883(20) & 0.000 & 130 & & $6 p$ & $(5 / 2)^{2}[7 / 2]_{3}^{\circ}$ & $7 \mathrm{~d}$ & $\begin{array}{l}(5 / 2)^{2}[7 / 2]_{3} \\
\end{array}$ & $3.0 \mathrm{e}+06$ & $\mathrm{D}+$ & R1c & TW & \\
\hline 7562.0141(17) & $13220.350(3)$ & $7562.0167(9)$ & -0.0026 & 16000 & & $5 s$ & $(3 / 2)^{2}[3 / 2]_{1}$ & $5 p$ & $(3 / 2)^{2}[3 / 2]^{\circ} 1$ & $3.8 \mathrm{e}+07$ & B & F_Re & B00 & \\
\hline $7564.324(8)$ & $13216.314(14)$ & 7564.3057(14) & 0.018 & 64 & & $5 p$ & $(3 / 2)^{2}[3 / 2]^{\circ}{ }_{2}$ & $5 \mathrm{~d}$ & $(5 / 2)^{2}[3 / 2]_{1}$ & & & $\mathrm{R} 1 \mathrm{c}$ & & \\
\hline 7575.242(7) & $13197.266(12)$ & 7575.2309(12) & 0.011 & 220 & & $5 p$ & $(3 / 2)^{2}[3 / 2]^{\circ}$ & $5 \mathrm{~d}$ & $(5 / 2)^{2}[3 / 2]_{2}$ & & & R1c & & \\
\hline 7579.023(3) & $13190.681(5)$ & $7579.0283(10)$ & -0.005 & 20000 & & $5 s$ & $(3 / 2)^{2}[3 / 2]_{2}$ & $5 p$ & $(3 / 2)^{2}[3 / 2]^{0} 2$ & $4.1 \mathrm{e}+07$ & B & F_Re & B00 & \\
\hline $7579.850(5)$ & 13189.242(9) & 7579.8494(9) & 0.001 & 8400 & & $5 s$ & $(5 / 2)^{2}[5 / 2]_{2}$ & $\mathrm{sp}$ & $\left({ }^{3} \mathrm{~F}\right)^{3} \mathrm{P}^{\circ} \mathrm{F}^{\circ}{ }_{3}$ & $1.15 \mathrm{e}+07$ & B & F_Re & B00 & \\
\hline 7583.273(8) & $13183.289(13)$ & 7583.339(4) & -0.066 & 64 & ? & $4 \mathrm{~d}$ & $(5 / 2)^{2}[1 / 2]_{0}$ & $\mathrm{sp}$ & $\left({ }^{1} \mathrm{D}\right)^{3} \mathrm{P}^{\circ}{ }^{3} \mathrm{P}^{\mathrm{o}}$ & & & $\mathrm{R} 1 \mathrm{c}$ & & $x$ \\
\hline $7652.3326(7)$ & $13064.3147(12)$ & $7652.3337(5)$ & -0.0011 & 26000 & & $5 s$ & $(5 / 2)^{2}[5 / 2]_{3}$ & $5 p$ & $(5 / 2)^{2}[5 / 2]^{\circ}{ }_{3}$ & $2.35 \mathrm{e}+07$ & B & F_Re & B00 & \\
\hline 7664.6451(12) & $13043.3283(20)$ & $7664.6465(7)$ & -0.0014 & 52000 & & $5 p$ & $(5 / 2)^{2}[7 / 2]^{\circ}{ }_{3}$ & $6 \mathrm{~s}$ & $(5 / 2)^{2}[5 / 2]_{2}$ & $3.5 \mathrm{e}+07$ & $\mathrm{D}+$ & F_Re & TW & $\mathrm{L}$ \\
\hline $7681.787(4)$ & $\begin{array}{l}13014.223(7) \\
\text { (1) }\end{array}$ & $7681.7968(10)$ & -0.010 & 570 & & $5 p$ & $(3 / 2)^{2}[5 / 2]_{2}^{\circ}$ & $6 \mathrm{~s}$ & $(3 / 2)^{2}[3 / 2]_{2}$ & & & $\mathrm{R} 1 \mathrm{c}$ & & \\
\hline $7712.418(6)$ & $12962.535(9)$ & $7712.421(3)$ & -0.003 & 640 & & $6 \mathrm{p}$ & $(5 / 2)^{2}[3 / 2]^{\circ}{ }_{2}$ & $7 \mathrm{~d}$ & $(5 / 2)^{2}[5 / 2]_{3}$ & $3.7 \mathrm{e}+06$ & $\mathrm{D}+$ & R1c & TW & \\
\hline $7726.637(4)$ & 12938.681(6) & $7726.6439(10)$ & -0.007 & 9000 & & $5 s$ & $(3 / 2)^{2}[3 / 2]_{2}$ & $5 p$ & $(3 / 2)^{2}[3 / 2]^{\circ}{ }_{1}$ & $1.70 \mathrm{e}+07$ & B & F_Re & B00 & \\
\hline $7738.6656(12)$ & $12918.5693(21)$ & $7738.6644(9)$ & 0.0012 & 30000 & & $5 p$ & $(3 / 2)^{2}[5 / 2]_{2}^{\circ}$ & $6 \mathrm{~s}$ & $(3 / 2)^{2}[3 / 2]_{1}$ & $4.5 \mathrm{e}+07$ & $\mathrm{D}+$ & F_Re & TW & $\mathrm{L}$ \\
\hline
\end{tabular}


Table A1. Cont.

\begin{tabular}{|c|c|c|c|c|c|c|c|c|c|c|c|c|c|c|}
\hline$\lambda_{\text {obs }}{ }^{a}(\AA)$ & $\sigma_{\mathrm{obs}}{ }^{\mathrm{b}}\left(\mathrm{cm}^{-1}\right)$ & $\lambda_{\text {Ritz }}{ }^{c}(\AA)$ & $\begin{array}{c}\Delta \lambda_{\text {obs-Ritz }} \\
\text { (A) }\end{array}$ & $\begin{array}{l}I_{\text {obs }} \mathrm{d} \\
\text { (arb. u.) }\end{array}$ & Char ${ }^{e}$ & & ower Level & & pper Level & $A\left(\mathrm{~s}^{-1}\right)$ & $\operatorname{Acc}^{f}$ & Line Ref. $\mathrm{g}$ & TP Ref. $\mathrm{g}$ & Notes ${ }^{h}$ \\
\hline $7739.499(8)$ & 12917.179(14) & $7739.505(4)$ & -0.006 & 1600 & & $6 p$ & $(5 / 2)^{2}[3 / 2]_{2}^{\circ}$ & $7 \mathrm{~d}$ & $(5 / 2)^{2}[3 / 2]_{2}$ & $7.1 \mathrm{e}+06$ & $\mathrm{D}+$ & R1c & TW & \\
\hline $7744.0889(18)$ & $12909.522(3)$ & 7744.0905(8) & -0.0016 & 7800 & & $5 p$ & $(5 / 2)^{2}[7 / 2]^{\circ}{ }_{3}$ & $6 \mathrm{~s}$ & $(5 / 2)^{2}[5 / 2]_{3}$ & & & F_Re & & \\
\hline $7754.3688(20)$ & $12892.408(3)$ & $7754.3653(10)$ & 0.0035 & 11000 & & $5 p$ & $(3 / 2)^{2}[1 / 2]^{\circ}{ }_{1}$ & $6 \mathrm{~s}$ & $(3 / 2)^{2}[3 / 2]_{2}$ & $9 . e+06$ & $\mathrm{D}+$ & F_Re & TW & \\
\hline $7766.516(9)$ & $12872.244(16)$ & $7766.511(7)$ & 0.006 & 32 & & $6 p$ & $(3 / 2)^{2}[1 / 2]^{\circ}$ & $7 \mathrm{~d}$ & $(3 / 2)^{2}[1 / 2]_{0}$ & & & R1c & & \\
\hline 7773.196(7) & 12861.182(12) & 7773.1992(9) & -0.003 & 3200 & & $5 s$ & $(5 / 2)^{2}[5 / 2]_{3}$ & $5 p$ & $(5 / 2)^{2}[5 / 2]^{\circ}{ }_{2}$ & $1.08 \mathrm{e}+06$ & $\mathrm{C}$ & R1c & B00 & \\
\hline 7778.7353(13) & $12852.0236(22)$ & 7778.7347(8) & 0.0006 & 25000 & & $5 p$ & $(5 / 2)^{2}[5 / 2]^{\circ} 2$ & $6 s$ & $(5 / 2)^{2}[5 / 2]_{2}$ & $1.3 e+07$ & $\mathrm{D}+$ & F_Re & TW & $\mathrm{L}$ \\
\hline 7805.1886(13) & $12808.4659(21)$ & 7805.1878(7) & 0.0008 & 26000 & & $5 p$ & $(5 / 2)^{2}[3 / 2]^{\circ}{ }_{1}$ & $6 \mathrm{~s}$ & $(5 / 2)^{2}[5 / 2]_{2}$ & $1.6 \mathrm{e}+07$ & $\mathrm{D}+$ & F_Re & TW & $\mathrm{L}$ \\
\hline 7807.6534(12) & $12804.4224(20)$ & $7807.6526(7)$ & 0.0007 & 82000 & & $5 p$ & $(5 / 2)^{2}[7 / 2]^{\circ} 4$ & $6 \mathrm{~s}$ & $(5 / 2)^{2}[5 / 2]_{3}$ & $3.6 \mathrm{e}+07$ & $\mathrm{C}+$ & F_Re & TW & $\mathrm{L}$ \\
\hline 7812.3182(19) & 12796.777(3) & $7812.3164(10)$ & 0.0018 & 10000 & & $5 p$ & $(3 / 2)^{2}[1 / 2]^{\circ}{ }_{1}$ & $6 \mathrm{~s}$ & $(3 / 2)^{2}[3 / 2]_{1}$ & $1.2 \mathrm{e}+07$ & $\mathrm{D}+$ & F_Re & TW & \\
\hline $7820.577(4)$ & $12783.264(6)$ & $7820.5768(10)$ & -0.000 & 7300 & & $5 s$ & $(3 / 2)^{2}[3 / 2]_{1}$ & $5 p$ & $(3 / 2)^{2}[1 / 2]^{\circ}{ }_{1}$ & $1.51 \mathrm{e}+07$ & B & F_Re & B00 & \\
\hline $7825.6528(12)$ & $12774.9718(20)$ & $7825.6530(7)$ & -0.0002 & 59000 & & $5 s$ & $(5 / 2)^{2}[5 / 2]_{3}$ & $5 \mathrm{p}$ & $(5 / 2)^{2}[7 / 2]^{\circ} 4$ & $5.2 \mathrm{e}+07$ & B & F_Re & B00 & $\mathrm{L}$ \\
\hline 7845.0792(12) & $12743.3378(20)$ & 7845.0795(9) & -0.0003 & 24000 & & $5 p$ & $(3 / 2)^{2}[5 / 2]^{\circ}{ }_{3}$ & $6 \mathrm{~s}$ & $(3 / 2)^{2}[3 / 2]_{2}$ & $3.8 \mathrm{e}+07$ & $\mathrm{D}+$ & F_Re & TW & $\mathrm{L}$ \\
\hline 7853.984(9) & $12728.890(15)$ & $7853.980(4)$ & 0.004 & 190 & & $6 p$ & $(5 / 2)^{2}[5 / 2]^{\circ}{ }_{2}$ & $7 \mathrm{~d}$ & $\begin{array}{l}(5 / 2)^{2}[5 / 2]_{2}\end{array}$ & & & $\mathrm{R} 1 \mathrm{c}$ & & \\
\hline $7860.576(5)$ & $12718.216(7)$ & $7860.5740(10)$ & 0.002 & 5700 & & $5 p$ & $(5 / 2)^{2}[5 / 2]^{\circ} 2$ & $6 \mathrm{~s}$ & $(5 / 2)^{2}[5 / 2]_{3}$ & & & R1c & & \\
\hline 7862.652(9) & $12714.857(14)$ & 7862.654(3) & -0.002 & 250 & & $6 p$ & $(5 / 2)^{2}[5 / 2]^{\circ} 2$ & $7 \mathrm{~d}$ & $(5 / 2)^{2}[7 / 2]_{3}$ & $2.9 e+06$ & $\mathrm{D}+$ & $\mathrm{R} 1 \mathrm{c}$ & TW & \\
\hline $7886.076(9)$ & $12677.091(15)$ & 7886.097(5) & -0.021 & 61 & & $6 p$ & $(3 / 2)^{2}[1 / 2]^{\circ}{ }_{1}$ & $7 \mathrm{~d}$ & $(3 / 2)^{2}[1 / 2]_{1}$ & & & $\mathrm{R} 1 \mathrm{c}$ & & \\
\hline $7890.568(4)$ & $12669.874(6)$ & $7890.5666(8)$ & 0.001 & 3500 & & $5 s$ & $(5 / 2)^{2}[5 / 2]_{3}$ & $5 p$ & $(5 / 2)^{2}[7 / 2]^{\circ} 3$ & $5.8 \mathrm{e}+06$ & B & F_Re & B00 & \\
\hline $7895.8050(13)$ & $12661.4700(22)$ & 7895.8039(8) & 0.0011 & 21000 & & $5 s$ & $(3 / 2)^{2}[3 / 2]_{1}$ & $5 p$ & $(3 / 2)^{2}[5 / 2]^{\circ} 2$ & $4.3 e+07$ & B & F_Re & B00 & $\mathrm{L}$ \\
\hline $7902.5482(13)$ & $12650.6660(21)$ & 7902.5499(8) & -0.0017 & 25000 & & $5 s$ & $(3 / 2)^{2}[3 / 2]_{2}$ & $5 p$ & $(3 / 2)^{2}[5 / 2]^{\circ}{ }^{\circ}$ & $4.9 \mathrm{e}+07$ & B & F_Re & B00 & $\mathrm{L}$ \\
\hline 7907.351(10) & $12642.983(16)$ & 7907.364(5) & -0.014 & 61 & & $6 p$ & $(5 / 2)^{2}[5 / 2]^{\circ} 2$ & $7 \mathrm{~d}$ & $(5 / 2)^{2}[3 / 2]_{1}$ & & & $\mathrm{R} 1 \mathrm{c}$ & & \\
\hline $7944.4368(6)$ & $12583.9633(10)$ & 7944.4365(5) & 0.0003 & 17000 & & $5 s$ & $(5 / 2)^{2}[5 / 2]_{2}$ & $5 p$ & $(5 / 2)^{2}[3 / 2]^{\circ} 1$ & $4.7 \mathrm{e}+07$ & B & F_Re & B00 & $\mathrm{L}$ \\
\hline 7967.067(5) & $12548.220(8)$ & $7967.065(4)$ & 0.002 & 2100 & & $6 p$ & $(3 / 2)^{2}[5 / 2]^{\circ}{ }_{3}$ & $7 \mathrm{~d}$ & $(3 / 2)^{2}[7 / 2]_{4}$ & $1.0 \mathrm{e}+07$ & $\mathrm{D}+$ & $\mathrm{R} 1 \mathrm{c}$ & TW & \\
\hline 7972.031(5) & $12540.406(7)$ & $7972.0306(10)$ & 0.000 & 7800 & & $5 s$ & $(5 / 2)^{2}[5 / 2]_{2}$ & $5 p$ & $(5 / 2)^{2}[5 / 2]^{\circ} 2$ & $2.9 \mathrm{e}+07$ & B & R1c & B00 & \\
\hline 7981.574(9) & $12525.413(15)$ & 7981.4451(23) & 0.128 & 59 & ? & $4 \mathrm{f}$ & $(5 / 2)^{2}[7 / 2]^{\circ}{ }_{3}$ & $6 \mathrm{~d}$ & $(3 / 2)^{2}[7 / 2]_{4}$ & & & R1c & & $x$ \\
\hline 7988.1598(13) & $12515.0857(20)$ & $7988.1620(7)$ & -0.0022 & 47000 & & $5 p$ & $(5 / 2)^{2}[5 / 2]^{\circ}{ }_{3}$ & $6 \mathrm{~s}$ & $(5 / 2)^{2}[5 / 2]_{3}$ & $1.7 \mathrm{e}+07$ & $\mathrm{D}+$ & F_Re & TW & L \\
\hline $7996.793(3)$ & $12501.575(5)$ & 7996.7854(10) & 0.007 & 9000 & & $5 s$ & $(3 / 2)^{2}[3 / 2]_{2}$ & $5 p$ & $(3 / 2)^{2}[1 / 2]^{\circ} 1$ & $3.2 \mathrm{e}+07$ & B & F_Re & B00 & \\
\hline $8006.515(5)$ & $12486.394(8)$ & $8006.525(4)$ & -0.009 & 440 & & $6 p$ & $(3 / 2)^{2}[5 / 2]^{\circ}{ }_{2}$ & $7 \mathrm{~d}$ & $(3 / 2)^{2}[7 / 2]_{3}$ & $5.9 e+06$ & $\mathrm{D}+$ & $\mathrm{R} 2 \mathrm{nc}$ & TW & \\
\hline $8026.479(3)$ & $12455.338(5)$ & $8026.4828(12)$ & -0.004 & 7100 & & $5 p$ & $(3 / 2)^{2}[3 / 2]^{\circ}{ }_{1}$ & $6 \mathrm{~s}$ & $(3 / 2)^{2}[3 / 2]_{2}$ & $8.2 \mathrm{e}+06$ & $\mathrm{D}+$ & F_Re & TW & \\
\hline \multirow[t]{2}{*}{$8042.489(7)$} & $12430.544(11)$ & $8042.494(4)$ & -0.005 & 230 & & $6 \mathrm{p}$ & $(5 / 2)^{2}[3 / 2]^{\circ}{ }_{1}$ & $7 \mathrm{~d}$ & $(5 / 2)^{2}[3 / 2]_{1}$ & & & $\mathrm{R} 1 \mathrm{c}$ & & \\
\hline & & $8053.200(6)$ & & & $\mathrm{m}$ & $6 p$ & $(3 / 2)^{2}[5 / 2]^{\circ} 2$ & $7 \mathrm{~d}$ & $(3 / 2)^{2}[1 / 2]_{1}$ & & & R1c & & \\
\hline $8053.339(16)$ & $12413.796(24)$ & $8053.358(4)$ & -0.019 & 230 & & $\mathrm{sp}$ & $\left({ }^{1} \mathrm{G}\right)^{3} \mathrm{P}^{\circ}{ }^{3} \mathrm{~F}^{\circ}{ }_{3}$ & $7 d$ & $(5 / 2)^{2}[7 / 2]_{4}$ & $2.3 e+06$ & $\mathrm{D}+$ & R1c & TW & \\
\hline $8054.227(7)$ & $12412.428(11)$ & $8054.239(3)$ & -0.012 & 110 & & $\mathrm{sp}$ & $\left({ }^{1} \mathrm{G}\right)^{3} \mathrm{P}^{\circ}{ }^{3} \mathrm{~F}^{\circ}{ }_{3}$ & $7 \mathrm{~d}$ & $(5 / 2)^{2}[7 / 2]_{3}$ & & & R1c & & \\
\hline $8074.999(7)$ & $12380.498(11)$ & $8074.999(3)$ & 0.000 & 420 & & $6 p$ & $(5 / 2)^{2}[7 / 2]^{\circ}{ }_{3}$ & $8 \mathrm{~s}$ & $(5 / 2)^{2}[5 / 2]_{2}$ & $3.5 e+06$ & $\mathrm{D}+$ & $\mathrm{R} 1 \mathrm{c}$ & TW & \\
\hline $8075.456(7)$ & 12379.797(11) & $8075.4576(10)$ & -0.001 & 2300 & & $5 s$ & $(3 / 2)^{2}[3 / 2]_{2}$ & $5 p$ & $(3 / 2)^{2}[5 / 2]^{\circ}{ }^{\circ}$ & $2.8 \mathrm{e}+05$ & $\mathrm{C}$ & R1c & B00 & \\
\hline $8088.5846(15)$ & $12359.7038(23)$ & $8088.5888(11)$ & -0.0042 & 13000 & & $5 p$ & $(3 / 2)^{2}[3 / 2]^{\circ}{ }_{1}$ & $6 \mathrm{~s}$ & $(3 / 2)^{2}[3 / 2]_{1}$ & $1.6 \mathrm{e}+07$ & $\mathrm{D}+$ & F_Re & TW & \\
\hline $8095.5267(14)$ & $12349.1051(21)$ & $8095.5268(8)$ & -0.0001 & 26000 & & $5 s$ & $(5 / 2)^{2}[5 / 2]_{2}$ & $5 p$ & $(5 / 2)^{2}[7 / 2]^{\circ} 3$ & $3.7 \mathrm{e}+07$ & B & F_Re & B00 & \\
\hline $8098.519(7)$ & 12344.542(11) & $8098.5396(14)$ & -0.020 & 550 & & $\mathrm{sp}$ & $\left({ }^{3} \mathrm{~F}\right)^{1} \mathrm{P}^{\circ}{ }^{3} \mathrm{G}^{\circ}$ & $5 g$ & $(3 / 2)^{2}[9 / 2]_{5}$ & & & $\mathrm{R} 1 \mathrm{c}$ & & \\
\hline $8100.868(7)$ & $12340.963(11)$ & $8100.883(3)$ & -0.015 & 83 & & $6 p$ & $(5 / 2)^{2}[7 / 2]^{\circ} 3$ & $8 \mathrm{~s}$ & $(5 / 2)^{2}[5 / 2]_{3}$ & & & R1c & & \\
\hline $8103.352(7)$ & $12337.180(11)$ & $8103.353(4)$ & -0.001 & 550 & & $\mathrm{sp}$ & $\left({ }^{1} \mathrm{G}\right)^{3} \mathrm{P}^{\circ}{ }^{3} \mathrm{~F}^{\circ}{ }_{3}$ & $7 \mathrm{~d}$ & $(5 / 2)^{2}[9 / 2]_{4}$ & $2.7 \mathrm{e}+06$ & $\mathrm{D}+$ & $\mathrm{R} 1 \mathrm{c}$ & TW & \\
\hline $8154.003(6)$ & $12260.545(9)$ & $8154.012(4)$ & -0.009 & 2000 & & $6 p$ & $(5 / 2)^{2}[7 / 2]^{\circ}{ }_{4}^{\circ}$ & $7 \mathrm{~d}$ & $\begin{array}{l}(5 / 2)^{2}[9 / 2]_{5} \\
\text {. }\end{array}$ & $5.4 \mathrm{e}+06$ & $\mathrm{D}+$ & R1c & TW & \\
\hline $8192.221(6)$ & $12203.347(9)$ & $8192.2333(13)$ & -0.012 & 10700 & & $5 p$ & $(3 / 2)^{2}[3 / 2]^{\circ} 2$ & $6 \mathrm{~s}$ & $(3 / 2)^{2}[3 / 2]_{2}$ & $2.0 \mathrm{e}+07$ & $\mathrm{D}+$ & F_Re & TW & \\
\hline $8192.330(3)$ & $12203.185(4)$ & $8192.3279(10)$ & 0.002 & 3300 & & $\mathrm{sp}$ & $\left({ }^{3} \mathrm{~F}\right)^{3} \mathrm{P}^{\circ}{ }^{1} \mathrm{~F}^{\circ}{ }_{3}$ & $6 \mathrm{~s}$ & $(5 / 2)^{2}[5 / 2]_{2}$ & $5.9 \mathrm{e}+06$ & $\mathrm{D}+$ & F_Re & TW & \\
\hline
\end{tabular}


Table A1. Cont.

\begin{tabular}{|c|c|c|c|c|c|c|c|c|c|c|c|c|c|c|}
\hline$\lambda_{\text {obs }}{ }^{\text {a }(\AA)}$ & $\sigma_{\mathrm{obs}}{ }^{\mathrm{b}}\left(\mathrm{cm}^{-1}\right)$ & $\lambda_{\text {Ritz }}{ }^{c}(\AA ̊)$ & $\begin{array}{c}\Delta \lambda_{\text {obs-Ritz }} \\
\text { (A) }\end{array}$ & $\begin{array}{c}I_{\text {obs }} \mathrm{d} \\
\text { (arb. u.) }\end{array}$ & Char ${ }^{e}$ & & ower Level & & Jpper Level & $A\left(\mathrm{~s}^{-1}\right)$ & $\operatorname{Acc}^{f}$ & Line Ref. $\mathrm{g}$ & TP Ref. $\mathrm{g}$ & Notes $h$ \\
\hline $8201.898(7)$ & $12188.949(11)$ & $8201.908(4)$ & -0.010 & 52 & & $6 p$ & $(3 / 2)^{2}[3 / 2]_{2}^{\circ}$ & $7 \mathrm{~d}$ & $(3 / 2)^{2}[7 / 2]_{3}$ & & & R1c & & \\
\hline $8235.272(4)$ & 12139.553(6) & $8235.2788(14)$ & -0.007 & 5600 & & $5 s$ & $(3 / 2)^{2}[3 / 2]_{1}$ & $5 p$ & $(3 / 2)^{2}[1 / 2]_{0}^{\circ}$ & $4.6 \mathrm{e}+07$ & B & F_Re & B00 & \\
\hline $8256.944(6)$ & 12107.691(9) & $8256.9412(13)$ & 0.002 & 3000 & & $5 p$ & $(3 / 2)^{2}[3 / 2]^{\circ}{ }_{2}$ & $6 \mathrm{~s}$ & $(3 / 2)^{2}[3 / 2]_{1}$ & & & F_Re & & \\
\hline $8277.5525(14)$ & $12077.5460(21)$ & $8277.5527(8)$ & -0.0003 & 20000 & & $5 s$ & $(5 / 2)^{2}[5 / 2]_{3}$ & $5 p$ & $(5 / 2)^{2}[3 / 2]^{\circ} 2$ & $4.3 e+07$ & B & F_Re & B00 & \\
\hline $8283.1521(14)$ & $12069.3813(21)$ & $8283.1520(9)$ & 0.0000 & 23000 & & $\mathrm{sp}$ & $\left({ }^{3} \mathrm{~F}\right)^{3} \mathrm{P}^{\circ}{ }^{1} \mathrm{~F}_{3}^{\circ}$ & $6 \mathrm{~s}$ & $(5 / 2)^{2}[5 / 2]_{3}$ & & & $\mathrm{~F} \operatorname{Re}$ & & \\
\hline $8298.461(7)$ & 12047.116(11) & $8298.465(3)$ & -0.004 & 72 & & $\mathrm{sp}$ & $\left({ }^{3} \mathrm{~F}\right)^{1} \mathrm{P}^{\circ}{ }^{3} \mathrm{~F}^{\circ}{ }_{2}$ & $7 \mathrm{~d}$ & $(5 / 2)^{2}[5 / 2]_{2}$ & & & $\mathrm{R} 1 \mathrm{c}$ & & \\
\hline $8306.475(5)$ & $12035.493(7)$ & $8306.4866(14)$ & -0.011 & 890 & & $4 p$ & ${ }^{3} \mathrm{D}_{2}^{\circ}$ & $\mathrm{s}^{2}$ & ${ }^{1} \mathrm{D}_{2}$ & & & $\mathrm{R} 1 \mathrm{c}$ & & \\
\hline $8308.148(7)$ & $12033.070(11)$ & $8308.150(3)$ & -0.002 & 120 & & sp & $\left({ }^{3} \mathrm{~F}\right)^{1} \mathrm{P}^{\circ}{ }^{3} \mathrm{~F}^{\circ}{ }_{2}$ & $7 \mathrm{~d}$ & $(5 / 2)^{2}[7 / 2]_{3}$ & & & R1c & & \\
\hline $8328.395(6)$ & $12003.817(8)$ & $8328.393(3)$ & 0.001 & 470 & & $6 p$ & $(5 / 2)^{2}[5 / 2]_{3}^{\circ}$ & $7 \mathrm{~d}$ & $(5 / 2)^{2}[7 / 2]_{4}$ & $3.1 \mathrm{e}+06$ & $\mathrm{D}+$ & R1c & TW & \\
\hline $8333.074(7)$ & $11997.077(10)$ & 8333.072(4) & 0.001 & 470 & & $6 p$ & $(5 / 2)^{2}[3 / 2]^{\circ}$ & $8 \mathrm{~s}$ & $(5 / 2)^{2}[5 / 2]_{3}$ & $2.3 e+06$ & $\mathrm{D}+$ & R1c & TW & \\
\hline $8333.617(8)$ & 11996.294(12) & $8333.6418(20)$ & -0.024 & 580 & & $\mathrm{sp}$ & $\left({ }^{3} \mathrm{~F}\right)^{1} \mathrm{P}^{\circ}{ }^{3} \mathrm{G}^{\circ}{ }_{5}$ & $5 g$ & $\begin{array}{l}(5 / 2)^{2}[9 / 2]_{5} \\
\end{array}$ & & & R1c & & \\
\hline $8336.333(5)$ & $11992.386(8)$ & $8336.3323(20)$ & 0.001 & 3500 & & $\mathrm{sp}$ & $\left({ }^{3} \mathrm{~F}\right)^{1} \mathrm{P}^{\circ}{ }^{3} \mathrm{G}_{5}^{\circ}$ & $5 g$ & $(5 / 2)^{2}[11 / 2]_{6}$ & $1.4 \mathrm{e}+06$ & $\mathrm{D}+$ & R1c & TW & \\
\hline 8353.837(7) & $11967.259(10)$ & 8353.843(3) & -0.006 & 230 & & $6 p$ & $(5 / 2)^{2}[5 / 2]^{\circ}{ }_{3}$ & $7 \mathrm{~d}$ & $(5 / 2)^{2}[5 / 2]_{3}$ & $3.1 \mathrm{e}+06$ & $\mathrm{D}+$ & R1c & TW & \\
\hline $8381.868(7)$ & $11927.237(11)$ & 8381.872(4) & -0.004 & 45 & & $6 p$ & $(5 / 2)^{2}[5 / 2]^{\circ}{ }_{3}$ & $7 \mathrm{~d}$ & $(5 / 2)^{2}[9 / 2]_{4}$ & & & $\mathrm{R} 1 \mathrm{c}$ & & \\
\hline $8385.624(7)$ & $11921.895(11)$ & $8385.629(4)$ & -0.005 & 67 & & $6 p$ & $(5 / 2)^{2}[5 / 2]^{\circ}{ }_{3}$ & $7 \mathrm{~d}$ & $(5 / 2)^{2}[3 / 2]_{2}$ & & & $\mathrm{R} 1 \mathrm{c}$ & & \\
\hline 8394.566(8) & 11909.195(11) & $8394.5745(12)$ & -0.008 & 110 & & $4 \mathrm{f}$ & $(5 / 2)^{2}[9 / 2]^{\circ}{ }_{4}$ & $5 g$ & $(3 / 2)^{2}[9 / 2]_{5}$ & & & $\mathrm{R} 1 \mathrm{c}$ & & \\
\hline $8402.897(7)$ & $11897.388(9)$ & $8402.9047(11)$ & -0.007 & 1600 & & $5 s$ & $(3 / 2)^{2}[3 / 2]_{1}$ & $\mathrm{sp}$ & $\left({ }^{3} \mathrm{~F}\right)^{3} \mathrm{P}^{\circ}{ }^{1} \mathrm{D}^{\circ}{ }_{2}$ & $3.9 \mathrm{e}+06$ & C & R1c & B00 & \\
\hline $8450.062(8)$ & $11830.982(11)$ & $8450.078(3)$ & -0.016 & 63 & & $\mathrm{sp}$ & $\left({ }^{3} \mathrm{~F}\right)^{1} \mathrm{P}^{\circ}{ }^{3} \mathrm{D}^{\circ}{ }_{2}$ & $6 \mathrm{~d}$ & $\begin{array}{r}(3 / 2)^{2}[5 / 2]_{3} \\
{ }^{1} \mathrm{D}\end{array}$ & & & R1c & & \\
\hline $8477.298(5)$ & $11792.971(7)$ & $8477.3077(17)$ & -0.009 & 3900 & & $4 p$ & ${ }^{1} \mathrm{P}^{\circ}{ }_{1}$ & $s^{2}$ & textsubscript2 & & & R1c & & \\
\hline 8503.394(5) & $11756.780(7)$ & $8503.3976(9)$ & -0.004 & 3600 & & $5 \mathrm{~s}$ & $(5 / 2)^{2}[5 / 2]_{2}$ & $5 p$ & $(5 / 2)^{2}[3 / 2]^{\circ}{ }_{2}$ & $1.6 \mathrm{e}+06$ & C & R1c & B00 & \\
\hline $8511.0626(20)$ & $11746.187(3)$ & $8511.0647(10)$ & -0.0021 & 11000 & & $\mathrm{sp}$ & $\left({ }^{3} \mathrm{~F}\right)^{3} \mathrm{P}^{\circ}{ }^{1} \mathrm{D}_{2}{ }_{2}$ & $6 \mathrm{~s}$ & $(5 / 2)^{2}[5 / 2]_{2}$ & $1.0 \mathrm{e}+07$ & $\mathrm{D}+$ & F_Re & TW & \\
\hline $8535.196(11)$ & $11712.975(15)$ & $8535.2020(23)$ & -0.006 & 77 & & $\mathrm{sp}$ & $\left({ }^{3} \mathrm{P}\right)^{3} \mathrm{P}^{\circ}{ }^{3} \mathrm{D}^{\circ}{ }_{1}$ & $5 g$ & $\begin{array}{l}(5 / 2)^{2}[5 / 2]_{2} \\
\end{array}$ & & & $\mathrm{R} 1 \mathrm{c}$ & & \\
\hline 8574.093(11) & $11659.838(15)$ & $8574.083(3)$ & 0.010 & 110 & & $\mathrm{sp}$ & $\left({ }^{3} \mathrm{~F}\right)^{1} \mathrm{P}^{\circ}{ }^{3} \mathrm{~F}_{4}^{\circ}$ & $6 \mathrm{~d}$ & $(5 / 2)^{2}[9 / 2]_{5}$ & & & R1c & & \\
\hline $8606.681(10)$ & $11615.690(13)$ & $8606.6732(12)$ & 0.008 & 690 & & $5 s$ & $(3 / 2)^{2}[3 / 2]_{2}$ & $\mathrm{sp}$ & $\left({ }^{3} \mathrm{~F}\right)^{3} \mathrm{P}^{\circ}{ }^{1} \mathrm{D}_{2}{ }_{2}$ & $7.9 \mathrm{e}+06$ & B & $\mathrm{R} 1 \mathrm{c}$ & B00 & \\
\hline 8609.132(5) & $11612.384(7)$ & $8609.1359(12)$ & -0.004 & 1100 & & $\mathrm{sp}$ & $\left({ }^{3} \mathrm{~F}\right)^{3} \mathrm{P}^{\circ}{ }^{1} \mathrm{D}^{\circ}{ }_{2}$ & $6 \mathrm{~s}$ & $(5 / 2)^{2}[5 / 2]_{3}$ & & & R1c & & \\
\hline $8729.820(9)$ & $11451.845(12)$ & $8729.822(3)$ & -0.002 & 160 & & $\mathrm{sp}$ & $\left({ }^{1} \mathrm{G}\right)^{3} \mathrm{P}^{\circ}{ }^{3} \mathrm{H}^{\circ}{ }_{4}$ & $5 \mathrm{~g}$ & $(3 / 2)^{2}[11 / 2]_{5}$ & & & R1c & & \\
\hline $8730.233(7)$ & $11451.303(9)$ & $8730.232(3)$ & 0.002 & 110 & & $\mathrm{sp}$ & $\left({ }^{1} \mathrm{G}\right)^{3} \mathrm{P}^{\circ} 3^{3} \mathrm{~F}^{\circ}{ }_{3}$ & $8 \mathrm{~s}$ & $(5 / 2)^{2}[5 / 2]_{2}$ & & & R1c & & \\
\hline $8797.094(7)$ & $11364.269(9)$ & $8797.0968(22)$ & -0.002 & 380 & & $\mathrm{sp}$ & $\left({ }^{3} \mathrm{~F}\right)^{1} \mathrm{P}^{\circ} 3^{3} \mathrm{~F}_{4}^{\circ}$ & $5 g$ & $(5 / 2)^{2}[9 / 2]_{5}$ & $1.6 \mathrm{e}+06$ & $\mathrm{D}+$ & R1c & TW & \\
\hline $8800.120(8)$ & $11360.362(10)$ & $8800.122(3)$ & -0.002 & 74 & & $\mathrm{sp}$ & $\left({ }^{3} \mathrm{~F}\right)^{1} \mathrm{P}^{\circ}{ }^{3} \mathrm{~F}_{4}^{\circ}$ & $5 \mathrm{~g}$ & $(5 / 2)^{2}[11 / 2]_{5}$ & & & R1c & & \\
\hline $8810.397(8)$ & $11347.110(10)$ & $8810.397(4)$ & 0.000 & 290 & & $6 p$ & $(5 / 2)^{2}[7 / 2]^{\circ}{ }_{4}$ & $8 \mathrm{~s}$ & $(5 / 2)^{2}[5 / 2]_{3}$ & $2.4 \mathrm{e}+06$ & $\mathrm{D}+$ & R1c & TW & \\
\hline $8937.241(8)$ & $11186.064(10)$ & 8937.281(3) & -0.039 & 13 & & $5 \mathrm{~d}$ & $(5 / 2)^{2}[5 / 2]_{3}$ & $5 \mathrm{f}$ & $(3 / 2)^{2}[7 / 2]^{\circ} 4$ & & & R1c & & $\mathrm{x}$ \\
\hline $8959.159(9)$ & $11158.698(11)$ & $8959.1604(12)$ & -0.001 & 1300 & & $5 s$ & $(3 / 2)^{2}[3 / 2]_{2}$ & $\mathrm{sp}$ & $\left({ }^{3} \mathrm{~F}\right)^{3} \mathrm{P}^{\circ} 1 \mathrm{~F}_{3}^{\circ}$ & $1.6 \mathrm{e}+05$ & C & R1c & B00 & \\
\hline $8967.150(9)$ & $11148.754(11)$ & $8967.143(4)$ & 0.007 & 250 & & $\mathrm{sp}$ & $\left({ }^{1} \mathrm{G}\right)^{3} \mathrm{P}^{\circ} 3^{3} \mathrm{~F}_{2}{ }_{2}$ & $6 \mathrm{~d}$ & $(3 / 2)^{2}[1 / 2]_{1}$ & & & $\mathrm{R} 1 \mathrm{rc}$ & & \\
\hline $8991.446(9)$ & $11118.629(11)$ & 8991.447(7) & -0.001 & 12 & & $\mathrm{sp}$ & $\left({ }^{3} \mathrm{P}\right)^{3} \mathrm{P}^{\circ} 1^{1} \mathrm{P}_{1}^{\circ}$ & $6 \mathrm{~d}$ & $(3 / 2)^{2}[1 / 2]_{1}$ & & & $\mathrm{R} 2 \mathrm{nc}$ & & \\
\hline 9001.419(8) & $11106.311(9)$ & $9001.428(5)$ & -0.009 & 25 & & $6 p$ & $(3 / 2)^{2}[3 / 2]^{\circ}{ }_{1}$ & $8 \mathrm{~s}$ & $(3 / 2)^{2}[3 / 2]_{2}$ & & & R1c & & \\
\hline $9005.757(8)$ & $11100.961(9)$ & $9005.754(4)$ & 0.003 & 4300 & & $\mathrm{sp}$ & $\left({ }^{3} \mathrm{~F}\right)^{1} \mathrm{P}^{\circ}{ }^{3} \mathrm{G}_{3}^{\circ}$ & $5 g$ & $(3 / 2)^{2}[9 / 2]_{4}$ & $2.5 \mathrm{e}+06$ & $\mathrm{D}+$ & $\mathrm{R} 1 \mathrm{c}$ & TW & \\
\hline 9015.187(9) & 11089.349(11) & 9015.144(4) & 0.043 & 310 & & $\mathrm{sp}$ & $\left({ }^{3} \mathrm{~F}\right)^{1} \mathrm{P}^{\circ}{ }^{3} \mathrm{G}_{3}^{\circ}$ & $5 \mathrm{~g}$ & $(3 / 2)^{2}[7 / 2]_{4}$ & & & R1rc & & $\mathrm{x}$ \\
\hline $9029.347(8)$ & $11071.959(9)$ & $9029.343(4)$ & 0.003 & 300 & & $\mathrm{sp}$ & $\left({ }^{3} \mathrm{~F}\right)^{1} \mathrm{P}^{\circ}{ }^{3} \mathrm{~F}^{\circ}{ }_{2}$ & $8 \mathrm{~s}$ & $(5 / 2)^{2}[5 / 2]_{2}$ & & & R1c & & \\
\hline 9036.147(8) & $11063.627(9)$ & 9036.132(4) & 0.015 & 600 & & $\mathrm{sp}$ & $\left({ }^{1} \mathrm{G}\right)^{3} \mathrm{P}^{\circ}{ }^{3} \mathrm{H}^{\circ}{ }_{5}$ & $5 \mathrm{~g}$ & $(3 / 2)^{2}[11 / 2]_{6}$ & $9 . e+05$ & $\mathrm{D}+$ & $\mathrm{R} 1 \mathrm{rc}$ & TW & \\
\hline 9067.974(8) & $11024.796(10)$ & $9067.9717(16)$ & 0.002 & 180 & & $5 s$ & $(5 / 2)^{2}[5 / 2]_{3}$ & $\mathrm{sp}$ & $\left({ }^{3} \mathrm{~F}\right)^{3} \mathrm{P}^{\circ} 3^{3} \mathrm{~F}^{\mathrm{o}}{ }_{2}$ & $3.9 \mathrm{e}+05$ & C & R1c & B00 & \\
\hline 9086.933(9) & $11001.793(11)$ & 9086.928(4) & 0.005 & 1200 & & $6 p$ & $(5 / 2)^{2}[5 / 2]^{\circ}{ }_{3}$ & $8 \mathrm{~s}$ & $(5 / 2)^{2}[5 / 2]_{3}$ & $1.7 \mathrm{e}+06$ & $\mathrm{D}+$ & $\mathrm{R} 1 \mathrm{c}$ & TW & \\
\hline
\end{tabular}


Table A1. Cont.

\begin{tabular}{|c|c|c|c|c|c|c|c|c|c|c|c|c|c|c|}
\hline$\lambda_{\text {obs }}{ }^{\text {a }}(\AA)$ & $\sigma_{\mathrm{obs}}{ }^{\mathrm{b}}\left(\mathrm{cm}^{-1}\right)$ & $\lambda_{\text {Ritz }}{ }^{c}(\AA)$ & $\begin{array}{c}\Delta \lambda_{\text {obs-Ritz }} \\
\text { (A) }\end{array}$ & $\begin{array}{c}I_{\text {obs }} \mathrm{d} \\
\text { (arb. u.) }\end{array}$ & Char ${ }^{\mathrm{e}}$ & & ower Level & & Upper Level & $A\left(\mathrm{~s}^{-1}\right)$ & $\operatorname{Acc}^{f}$ & Line Ref. $\mathrm{g}$ & TP Ref. $\mathrm{g}$ & Notes $h$ \\
\hline 9089.413(9) & 10998.792(11) & 9089.4094(24) & 0.003 & 46 & & $5 d$ & $(5 / 2)^{2}[7 / 2]_{3}$ & $5 f$ & $(3 / 2)^{2}[9 / 2]^{\circ}{ }_{4}$ & & & R1c & & \\
\hline $9093.704(9)$ & 10993.602(11) & $9093.632(4)$ & 0.072 & 12 & & $\mathrm{sp}$ & $\left({ }^{3} \mathrm{P}\right)^{3} \mathrm{P}^{\circ}{ }^{1} \mathrm{D}^{\circ}$ & $6 \mathrm{~d}$ & $(3 / 2)^{2}[5 / 2]_{2}$ & & & R1c & & $\mathrm{x}$ \\
\hline 9096.064(9) & $10990.749(11)$ & $9096.054(6)$ & 0.011 & 81 & & $5 \mathrm{~d}$ & $(5 / 2)^{2}[1 / 2]_{1}$ & $7 p$ & $(5 / 2)^{2}[3 / 2]^{0}{ }_{2}$ & & & R1c & & \\
\hline 9097.529(9) & $10988.980(11)$ & 9097.525(3) & 0.004 & 460 & & $\mathrm{sp}$ & $\left({ }^{3} \mathrm{P}\right)^{3} \mathrm{P}^{\circ}{ }^{3} \mathrm{P}_{2}^{\circ}$ & $7 \mathrm{~s}$ & $(5 / 2)^{2}[5 / 2]_{3}$ & & & R1c & & \\
\hline 9098.493(9) & $10987.815(11)$ & 9098.489(3) & 0.005 & 69 & & $5 \mathrm{~d}$ & $(5 / 2)^{2}[5 / 2]_{2}$ & $5 \mathrm{f}$ & $(3 / 2)^{2}[5 / 2]_{3}^{0}$ & & & R1c & & \\
\hline 9101.459(12) & 10984.235(15) & $9101.4285(22)$ & 0.030 & 23 & & $5 \mathrm{~d}$ & $(5 / 2)^{2}[7 / 2]_{4}$ & $5 f$ & $(3 / 2)^{2}[9 / 2]^{\circ}{ }_{5}$ & & & R1c & & \\
\hline 9103.234(6) & 10982.093(8) & $9103.2366(12)$ & -0.003 & 2600 & & $5 p$ & $(3 / 2)^{2}[5 / 2]^{\circ}{ }_{2}$ & $6 \mathrm{~s}$ & $(5 / 2)^{2}[5 / 2]_{2}$ & & & R1c & & \\
\hline $9107.895(9)$ & $10976.473(11)$ & $9107.878(6)$ & 0.016 & 230 & & $5 s$ & $(5 / 2)^{2}[5 / 2]_{3}$ & $\mathrm{sp}$ & $\left({ }^{3} \mathrm{~F}\right)^{3} \mathrm{P}^{\circ}{ }^{1} \mathrm{G}_{4}^{\circ}$ & $9.1 \mathrm{e}+04$ & $\mathrm{C}$ & R1c & B00 & \\
\hline 9115.700(9) & 10967.075(11) & $9115.643(10)$ & 0.057 & 46 & ? & $5 f$ & $(5 / 2)^{2}[11 / 2]^{\circ}{ }_{5}$ & $9 \mathrm{~d}$ & $(5 / 2)^{2}[9 / 2]_{4}$ & & & R1c & & $\mathrm{x}$ \\
\hline 9125.941(9) & $10954.767(11)$ & 9125.932(4) & 0.009 & 340 & & $\mathrm{sp}$ & $\left({ }^{1} \mathrm{G}\right)^{3} \mathrm{P}^{\circ}{ }^{3} \mathrm{~F}_{2}^{\circ}$ & $5 \mathrm{~g}$ & $(3 / 2)^{2}[7 / 2]_{3}$ & & & R2nc & & \\
\hline 9154.181(18) & $10920.973(21)$ & 9154.160(4) & 0.021 & 170 & & $\mathrm{sp}$ & $\left({ }^{1} \mathrm{G}\right)^{3} \mathrm{P}^{\circ} 3^{3} \mathrm{~F}^{\circ}{ }_{2}$ & $5 \mathrm{~g}$ & $(3 / 2)^{2}[5 / 2]_{3}$ & & & $\mathrm{R} 2 \mathrm{c}$ & & \\
\hline $9158.409(9)$ & 10915.931(11) & 9158.393(4) & 0.016 & 450 & & $\mathrm{sp}$ & $\left({ }^{3} \mathrm{P}\right)^{3} \mathrm{P}^{\circ}{ }^{3} \mathrm{P}^{\circ}{ }_{1}$ & $5 \mathrm{~g}$ & $(5 / 2)^{2}[3 / 2]_{1}$ & & & R1c & & \\
\hline $9179.306(12)$ & 10891.081(14) & 9179.319(7) & -0.014 & 610 & & $5 \mathrm{~d}$ & $(3 / 2)^{2}[1 / 2]_{1}$ & $7 \mathrm{p}$ & $(3 / 2)^{2}[1 / 2]^{0}{ }_{1}$ & & & $\mathrm{R} 2 \mathrm{nc}$ & & \\
\hline 9205.3214(22) & $10860.301(3)$ & 9205.3242(12) & -0.0028 & 5800 & & $5 p$ & $(3 / 2)^{2}[1 / 2]^{\circ}{ }_{1}$ & $6 \mathrm{~s}$ & $(5 / 2)^{2}[5 / 2]_{2}$ & & & F_Re & & \\
\hline $9219.491(10)$ & $10843.610(12)$ & $9219.466(5)$ & 0.025 & 32 & & $5 \mathrm{~d}$ & $(5 / 2)^{2}[5 / 2]_{3}$ & $7 p$ & $(5 / 2)^{2}[5 / 2]{ }_{3}$ & & & $\mathrm{R} 1 \mathrm{c}$ & & \\
\hline $9226.742(10)$ & $10835.088(12)$ & $9226.7371(12)$ & 0.005 & 730 & & $5 \mathrm{~s}$ & $(3 / 2)^{2}[3 / 2]_{1}$ & $5 p$ & $(5 / 2)^{2}[3 / 2]^{\circ}{ }_{1}$ & $1.3 e+06$ & C & R1c & B00 & \\
\hline $9230.567(11)$ & $10830.599(13)$ & $9230.577(3)$ & -0.010 & 370 & & $\mathrm{sp}$ & $\left({ }^{3} \mathrm{P}^{3} \mathrm{P}^{\circ}{ }^{3} \mathrm{D}^{\circ}\right.$ & $7 \mathrm{~s}$ & $(5 / 2)^{2}[5 / 2]_{3}$ & & & R1c & & \\
\hline $9234.272(8)$ & $10826.253(10)$ & $9234.258(3)$ & 0.014 & 1300 & & $\mathrm{sp}$ & $\left({ }^{3} \mathrm{~F}\right)^{1} \mathrm{P}^{\circ}{ }^{3} \mathrm{D}^{\circ}{ }_{3}$ & $6 \mathrm{~d}$ & $(5 / 2)^{2}[7 / 2]_{4}$ & & & R1c & & \\
\hline 9277.399(12) & $10775.926(14)$ & $9277.400(9)$ & -0.000 & 11 & ? & $5 f$ & $(5 / 2)^{2}[9 / 2]_{5}^{\circ}$ & $8 \mathrm{~g}$ & $(5 / 2)^{2}[11 / 2]_{6}$ & $4.6 \mathrm{e}+06$ & $\mathrm{D}+$ & R1nc & TW & \\
\hline 9296.634(12) & 10753.631(14) & $9296.580(6)$ & 0.054 & 21 & & $\mathrm{sp}$ & $\left({ }^{1} \mathrm{D}\right)^{3} \mathrm{P}^{\circ} 3^{3} \mathrm{~F}_{2}^{\circ}$ & $5 \mathrm{~d}$ & $(3 / 2)^{2}[5 / 2]_{3}$ & & & R1c & & $\mathrm{x}$ \\
\hline 9312.387(13) & $10735.440(15)$ & $9312.450(9)$ & -0.064 & 21 & & $5 f$ & $(5 / 2)^{2}[9 / 2]^{\circ}{ }_{5}$ & $7 \mathrm{~g}$ & $(3 / 2)^{2}[11 / 2]_{5}$ & & & R1c & & $\mathrm{x}$ \\
\hline 9317.944(9) & $10729.037(10)$ & 9317.943(4) & 0.002 & 260 & & $\mathrm{sp}$ & $\left({ }^{3} \mathrm{~F}\right)^{1} \mathrm{P}^{\circ}{ }^{3} \mathrm{D}^{\circ}{ }_{1}$ & $6 \mathrm{~d}$ & $(3 / 2)^{2}[5 / 2]_{2}$ & & & R1c & & \\
\hline 9324.302(9) & $10721.722(10)$ & $9324.3128(20)$ & -0.011 & 21 & & $\mathrm{sp}$ & $\left({ }^{3} \mathrm{~F}\right)^{1} \mathrm{P}^{\circ}{ }^{3} \mathrm{G}^{\circ}{ }_{4}$ & $6 \mathrm{~d}$ & 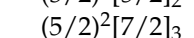 & & & R1c & & \\
\hline $9331.893(6)$ & $10713.000(7)$ & $9331.8965(12)$ & -0.004 & 2400 & & $5 \mathrm{~s}$ & $(3 / 2)^{2}[3 / 2]_{2}$ & $5 p$ & $(5 / 2)^{2}[5 / 2]_{3}{ }_{3}$ & $1.2 \mathrm{e}+06$ & $\mathrm{C}$ & R1c & B00 & \\
\hline 9339.711(8) & 10704.032(9) & 9339.7151(17) & -0.004 & 360 & & $5 s$ & $(5 / 2)^{2}[5 / 2]_{2}$ & $\mathrm{sp}$ & $\left({ }^{3} \mathrm{~F}\right)^{3} \mathrm{P}^{\circ} 3^{3} \mathrm{~F}_{2}{ }_{2}$ & $6.8 \mathrm{e}+05$ & C & R1c & B00 & \\
\hline 9364.118(14) & $10676.133(16)$ & $9364.046(7)$ & 0.072 & 10 & & $4 \mathrm{~d}$ & $(3 / 2)^{2}[7 / 2]_{4}$ & $\mathrm{sp}$ & $\left({ }^{1} \mathrm{D}\right)^{3} \mathrm{P}^{\circ}{ }^{3} \mathrm{~F}^{\circ}{ }_{3}$ & & & R1c & & $\mathrm{x}$ \\
\hline $9374.435(19)$ & $10664.383(21)$ & 9374.4412(23) & -0.006 & 10 & & $4 \mathrm{f}$ & $(5 / 2)^{2}[3 / 2]^{\circ} 2$ & $6 \mathrm{~d}$ & $(5 / 2)^{2}[5 / 2]_{2}$ & & & R1c & & \\
\hline 9391.049(12) & $10645.517(14)$ & $9390.9259(21)$ & 0.123 & 21 & ? & $4 f$ & $(5 / 2)^{2}[3 / 2]_{2}^{\circ}$ & $6 \mathrm{~d}$ & $(5 / 2)^{2}[7 / 2]_{3}$ & & & R1c & & $\mathrm{x}$ \\
\hline $9415.060(9)$ & $10618.368(10)$ & $9415.044(6)$ & 0.016 & 21 & & $4 \mathrm{~d}$ & $(3 / 2)^{2}[7 / 2]_{3}$ & $\mathrm{sp}$ & $\left({ }^{1} \mathrm{D}\right)^{3} \mathrm{P}^{\circ}{ }^{3} \mathrm{~F}^{\circ}{ }_{2}$ & & & R1c & & \\
\hline $9424.164(12)$ & $10608.110(14)$ & $9424.151(4)$ & 0.013 & 100 & & $\mathrm{sp}$ & $\left({ }^{3} \mathrm{~F}\right)^{1} \mathrm{P}^{\circ}{ }^{3} \mathrm{D}^{\circ}{ }_{1}$ & $6 \mathrm{~d}$ & $(3 / 2)^{2}[3 / 2]_{1}$ & & & R1c & & \\
\hline $9444.862(10)$ & $10584.863(11)$ & $9444.858(10)$ & 0.005 & 10 & & $4 \mathrm{~d}$ & $(3 / 2)^{2}[5 / 2]_{2}$ & $\mathrm{sp}$ & $\left({ }^{1} \mathrm{D}\right)^{3} \mathrm{P}^{\circ}{ }^{3} \mathrm{D}^{\circ}{ }_{3}$ & & & R1c & & \\
\hline $9451.495(11)$ & $10577.435(12)$ & $9451.5130(13)$ & -0.018 & 1400 & & $5 p$ & $(3 / 2)^{2}[5 / 2]^{\circ}$ & $6 \mathrm{~s}$ & $(5 / 2)^{2}[5 / 2]_{3}$ & & & R1c & & \\
\hline $9458.336(9)$ & $10569.785(10)$ & $9458.335(3)$ & 0.000 & 72 & & $4 \mathrm{f}$ & $(5 / 2)^{2}[7 / 2]^{\circ}{ }_{3}$ & $6 \mathrm{~d}$ & $(5 / 2)^{2}[7 / 2]_{4}$ & & & R1c & & \\
\hline $9459.889(10)$ & 10568.049(11) & 9459.895(4) & -0.005 & 31 & & $\mathrm{sp}$ & $\left({ }^{3} \mathrm{~F}\right)^{1} \mathrm{P}^{\circ}{ }^{3} \mathrm{G}^{\circ}{ }_{4}$ & $6 \mathrm{~d}$ & $\begin{array}{l}(5 / 2)^{2}[9 / 2]_{5} \\
\end{array}$ & & & R1c & & \\
\hline $9460.791(9)$ & $10567.042(10)$ & $9460.790(5)$ & 0.001 & 1000 & & sp & $\left({ }^{3} \mathrm{P}\right)^{3} \mathrm{P}^{\circ}{ }^{3} \mathrm{P}^{\circ} \mathrm{O}^{4}$ & $5 \mathrm{~g}$ & $(5 / 2)^{2}[3 / 2]_{1}$ & & & R1c & & \\
\hline $9461.294(10)$ & $10566.480(11)$ & $9461.3089(22)$ & -0.015 & 10 & & $4 \mathrm{f}$ & $(5 / 2)^{2}[7 / 2]^{\circ}{ }_{3}$ & $6 \mathrm{~d}$ & $(5 / 2)^{2}[7 / 2]_{3}$ & & & R1c & & \\
\hline $9473.011(12)$ & 10553.411(13) & $9473.0050(12)$ & 0.006 & 1300 & & $5 s$ & $(3 / 2)^{2}[3 / 2]_{2}$ & $5 p$ & $(5 / 2)^{2}[3 / 2]^{0}$ & $1.13 e+06$ & C & R1c & B00 & \\
\hline $9474.119(10)$ & 10552.176(11) & $9474.106(5)$ & 0.013 & 10 & & $5 \mathrm{~d}$ & $(5 / 2)^{2}[7 / 2]_{4}$ & $7 p$ & $(5 / 2)^{2}[7 / 2]^{1}{ }_{4}$ & & & R1c & & \\
\hline $9485.256(10)$ & 10539.787(11) & $9485.2726(23)$ & -0.017 & 21 & & $4 \mathrm{f}$ & $(5 / 2)^{2}[5 / 2]^{0} 3$ & $6 \mathrm{~d}$ & $(5 / 2)^{2}[5 / 2]_{2}$ & & & R1c & & \\
\hline $9492.663(19)$ & $10531.563(21)$ & $9492.653(4)$ & 0.010 & 10 & & $4 \mathrm{f}$ & $(3 / 2)^{2}[3 / 2]^{\circ}{ }_{1}$ & $6 \mathrm{~d}$ & $(3 / 2)^{2}[3 / 2]_{2}$ & & & R1c & & \\
\hline $9493.808(21)$ & $10530.293(23)$ & $9493.987(16)$ & -0.179 & 10 & $?$ & $\mathrm{sp}$ & $\left({ }^{3} \mathrm{P}\right)^{3} \mathrm{P}^{\circ}{ }^{5} \mathrm{P}^{\circ}$ & $6 \mathrm{~s}$ & $(3 / 2)^{2}[3 / 2]$ & & & $\mathrm{R} 2 \mathrm{c}$ & & $\mathrm{x}$ \\
\hline $9500.248(10)$ & $10523.154(11)$ & $9500.262(3)$ & -0.014 & 52 & & $\mathrm{sp}$ & $\left({ }^{3} \mathrm{P}\right)^{3} \mathrm{P}^{\circ}{ }^{3} \mathrm{D}^{\circ}{ }_{1}$ & $7 \mathrm{~s}$ & $(5 / 2)^{2}[5 / 2]_{2}$ & & & R1c & & \\
\hline $9502.093(10)$ & 10521.111(11) & $9502.1498(21)$ & -0.057 & 10 & ? & $4 f$ & $(5 / 2)^{2}[5 / 2]^{0}{ }_{3}^{1}$ & $6 \mathrm{~d}$ & $(5 / 2)^{2}[7 / 2]_{3}$ & & & R1c & & $\mathrm{x}$ \\
\hline
\end{tabular}


Table A1. Cont.

\begin{tabular}{|c|c|c|c|c|c|c|c|c|c|c|c|c|c|c|}
\hline$\lambda_{\text {obs }}{ }^{a}(\AA)$ & $\sigma_{\mathrm{obs}}{ }^{\mathrm{b}}\left(\mathrm{cm}^{-1}\right)$ & $\lambda_{\text {Ritz }}{ }^{c}(\AA)$ & $\begin{array}{c}\Delta \lambda_{\text {obs-Ritz }} \\
\text { (A) }\end{array}$ & $\begin{array}{l}I_{\text {obs }} \mathrm{d} \\
\text { (arb. u.) }\end{array}$ & Char ${ }^{e}$ & & Lower Level & & Jpper Level & $A\left(\mathrm{~s}^{-1}\right)$ & $\operatorname{Acc}^{f}$ & Line Ref. $\mathrm{g}$ & TP Ref. $\mathrm{g}$ & Notes ${ }^{h}$ \\
\hline 9504.184(9) & $10518.796(10)$ & $9504.220(4)$ & -0.035 & 360 & & $\mathrm{sp}$ & $\left({ }^{3} \mathrm{P}\right)^{3} \mathrm{P}^{\circ}{ }^{1} \mathrm{D}_{2}^{\circ}$ & $5 g$ & $(3 / 2)^{2}[7 / 2]_{3}$ & & & R1c & & $x$ \\
\hline $9505.598(10)$ & 10517.232(11) & 9505.605(3) & -0.008 & 10 & & $4 \mathrm{f}$ & $(5 / 2)^{2}[3 / 2]^{\circ}{ }_{2}$ & $6 \mathrm{~d}$ & $(5 / 2)^{2}[3 / 2]_{1}$ & & & R1c & & \\
\hline $9510.299(19)$ & $10512.033(21)$ & $9510.298(7)$ & 0.001 & 42 & & $\mathrm{sp}$ & $\left({ }^{3} \mathrm{P}\right)^{3} \mathrm{P}^{\circ}{ }^{5} \mathrm{P}_{2}$ & $6 \mathrm{~s}$ & $(3 / 2)^{2}[3 / 2]_{2}$ & & & R1c & & \\
\hline $9512.262(8)$ & $10509.864(9)$ & $9512.2653(15)$ & -0.004 & 2900 & & $5 s$ & $(3 / 2)^{2}[3 / 2]_{2}$ & $5 p$ & $(5 / 2)^{2}[5 / 2]^{\circ} 2$ & $1.3 e+06$ & C & R1c & B00 & \\
\hline 9515.588(11) & 10506.190(12) & 9515.595(3) & -0.007 & 210 & & $4 \mathrm{f}$ & $\begin{array}{l}(5 / 2)^{2}[7 / 2]^{\circ}{ }_{3} \\
\end{array}$ & $6 \mathrm{~d}$ & $(5 / 2)^{2}[5 / 2]_{3}$ & & & R1c & & \\
\hline 9527.379(10) & 10493.188(11) & 9527.376(4) & 0.003 & 31 & & $4 \mathrm{f}$ & $(3 / 2)^{2}[3 / 2]^{\circ} 1$ & $6 \mathrm{~d}$ & $(3 / 2)^{2}[3 / 2]_{1}$ & & & R1c & & \\
\hline $9530.897(10)$ & $10489.315(11)$ & $9530.905(3)$ & -0.008 & 160 & & $4 \mathrm{f}$ & $(5 / 2)^{2}[11 / 2]^{\circ}{ }_{5}$ & $6 \mathrm{~d}$ & $\begin{array}{l}(5 / 2)^{2}[9 / 2]_{4} \\
\end{array}$ & & & R1c & & \\
\hline $9546.928(10)$ & 10471.701(11) & 9546.928(3) & -0.000 & 210 & & $4 \mathrm{f}$ & $(5 / 2)^{2}[11 / 2]^{\circ}{ }_{6}$ & $6 \mathrm{~d}$ & $(5 / 2)^{2}[9 / 2]_{5}$ & & & R1c & & \\
\hline 9548.691(10) & 10469.768(11) & 9548.702(3) & -0.011 & 21 & & $4 \mathrm{f}$ & $(5 / 2)^{2}[3 / 2]^{\circ} 1$ & $6 \mathrm{~d}$ & $(5 / 2)^{2}[3 / 2]_{1}$ & & & R1c & & \\
\hline $9565.600(10)$ & 10451.261(11) & 9565.623(4) & -0.024 & 53 & & $4 f$ & $(3 / 2)^{2}[9 / 2]^{\circ}$ & $6 \mathrm{~d}$ & $(3 / 2)^{2}[7 / 2]_{4}$ & & & R1c & & \\
\hline $9585.611(10)$ & 10429.442(11) & 9585.628(3) & -0.017 & 32 & & $4 f$ & $(3 / 2)^{2}[5 / 2]^{\circ}{ }_{3}$ & $6 \mathrm{~d}$ & $(3 / 2)^{2}[3 / 2]_{2}$ & & & R1c & & \\
\hline $9597.666(20)$ & $10416.343(21)$ & 9597.614(7) & 0.052 & 11 & & $\mathrm{sp}$ & $\left({ }^{3} \mathrm{P}\right)^{3} \mathrm{P}^{\circ}{ }^{5} \mathrm{P}_{2}^{\circ}$ & $6 \mathrm{~s}$ & $(3 / 2)^{2}[3 / 2]_{1}$ & & & R1c & & \\
\hline $9599.817(10)$ & $10414.009(11)$ & $9599.843(3)$ & -0.026 & 11 & & $4 \mathrm{f}$ & $(5 / 2)^{2}[5 / 2]^{\circ}{ }_{2}$ & $6 \mathrm{~d}$ & $(5 / 2)^{2}[3 / 2]_{1}$ & & & R1c & & \\
\hline $9602.377(10)$ & 10411.232(11) & 9602.374(3) & 0.003 & 22 & & $4 \mathrm{f}$ & $(3 / 2)^{2}[7 / 2]^{\circ}{ }_{4}$ & $6 \mathrm{~d}$ & $(3 / 2)^{2}[5 / 2]_{3}$ & & & R1c & & \\
\hline $9605.15(9)$ & $10408.22(10)$ & $9605.075(6)$ & 0.08 & 54 & * & $5 \mathrm{~d}$ & $(5 / 2)^{2}[5 / 2]_{3}$ & $7 p$ & $(5 / 2)^{2}[3 / 2]^{\circ} 2$ & & & R1c & & \\
\hline $9605.15(9)$ & $10408.22(10)$ & 9605.193(4) & -0.04 & 54 & * & $4 \mathrm{f}$ & $(3 / 2)^{2}[9 / 2]^{\circ} 4$ & $6 \mathrm{~d}$ & $(3 / 2)^{2}[7 / 2]_{3}$ & & & R1c & & \\
\hline $9610.896(10)$ & $10402.004(11)$ & $9610.884(5)$ & 0.012 & 430 & & $5 \mathrm{~d}$ & $(5 / 2)^{2}[1 / 2]_{0}$ & $5 f$ & $(3 / 2)^{2}[3 / 2]^{0}$ & $5.9 e+06$ & $\mathrm{D}+$ & R1c & TW & \\
\hline $9613.596(20)$ & 10399.083(21) & 9613.594(3) & 0.002 & 65 & & $4 f$ & $(5 / 2)^{2}[9 / 2]^{\circ}{ }_{5}$ & $6 \mathrm{~d}$ & $(5 / 2)^{2}[7 / 2]_{4}$ & & & R1c & & \\
\hline $9630.420(10)$ & $10380.916(11)$ & $9630.428(3)$ & -0.009 & 11 & & $4 f$ & $(3 / 2)^{2}[7 / 2]^{\circ}{ }_{3}$ & $6 \mathrm{~d}$ & $(3 / 2)^{2}[5 / 2]_{2}$ & & & R1c & & \\
\hline $9630.969(11)$ & $10380.324(11)$ & 9631.023(3) & -0.054 & 11 & & $4 \mathrm{f}$ & $(5 / 2)^{2}[5 / 2]^{3}{ }_{3}$ & $6 \mathrm{~d}$ & $(5 / 2)^{2}[3 / 2]_{2}$ & & & R1c & & $\mathrm{x}$ \\
\hline 9637.149(11) & 10373.668(12) & 9637.148(3) & 0.000 & 1100 & & $\mathrm{sp}$ & $\left({ }^{3} \mathrm{~F}\right)^{1} \mathrm{P}^{\circ}{ }^{3} \mathrm{D}^{\circ}{ }_{3}$ & $5 \mathrm{~g}$ & $(5 / 2)^{2}[9 / 2]_{4}$ & & & R1c & & \\
\hline $9646.751(10)$ & $10363.342(11)$ & $9646.770(3)$ & -0.019 & 22 & & $4 f$ & $(5 / 2)^{2}[7 / 2]^{\circ} 4$ & $6 \mathrm{~d}$ & $(5 / 2)^{2}[5 / 2]_{3}$ & & & R1c & & \\
\hline $9649.361(10)$ & $10360.539(11)$ & $9649.378(3)$ & -0.017 & 110 & * & $\mathrm{sp}$ & $\left({ }^{3} \mathrm{~F}\right)^{1} \mathrm{P}^{\circ}{ }^{3} \mathrm{D}_{3}{ }_{3}$ & $5 \mathrm{~g}$ & $(5 / 2)^{2}[7 / 2]_{4}$ & & & R1c & & $\mathrm{x}$ \\
\hline 9649.361(10) & $10360.539(11)$ & 9649.399(3) & -0.038 & 110 & * & $\mathrm{sp}$ & $\left({ }^{3} \mathrm{~F}\right)^{1} \mathrm{P}^{\circ}{ }^{3} \mathrm{D}^{\circ}{ }_{3}$ & $5 \mathrm{~g}$ & $(5 / 2)^{2}[7 / 2]_{3}$ & & & R1nc & & $\mathrm{x}$ \\
\hline $9651.107(10)$ & $10358.665(11)$ & 9651.115(4) & -0.008 & 22 & & $4 f$ & $(3 / 2)^{2}[3 / 2]^{\circ}{ }_{2}^{\circ}$ & $6 \mathrm{~d}$ & $(3 / 2)^{2}[1 / 2]_{1}$ & & & R1c & & \\
\hline 9657.363(11) & 10351.954(11) & $9657.369(4)$ & -0.005 & 22 & & $4 \mathrm{f}$ & $(5 / 2)^{2}[1 / 2]^{\circ}{ }_{1}$ & $6 \mathrm{~d}$ & $(5 / 2)^{2}[1 / 2]_{1}$ & & & R1c & & \\
\hline $9664.119(10)$ & $10344.717(11)$ & $9664.140(3)$ & -0.020 & 11 & & $4 \mathrm{f}$ & $(3 / 2)^{2}[5 / 2]^{\circ} 2$ & $6 \mathrm{~d}$ & $(3 / 2)^{2}[3 / 2]_{1}$ & & & R1c & & \\
\hline $9670.167(10)$ & $10338.248(11)$ & $9670.172(8)$ & -0.006 & 11 & & $6 \mathrm{~d}$ & $(5 / 2)^{2}[9 / 2]_{4}$ & $8 \mathrm{f}$ & $(5 / 2)^{2}[11 / 2]^{\circ}{ }_{5}$ & $3.5 \mathrm{e}+06$ & $\mathrm{D}+$ & R1c & TW & \\
\hline $9688.602(8)$ & 10318.577(8) & $9688.6189(13)$ & -0.017 & 1600 & & $5 s$ & $(3 / 2)^{2}[3 / 2]_{2}$ & $5 p$ & $(5 / 2)^{2}[7 / 2]_{3}^{\circ}$ & $2.5 \mathrm{e}+05$ & $\mathrm{C}$ & R1c & B00 & \\
\hline $9715.855(20)$ & $10289.633(21)$ & 9715.789(3) & 0.066 & 12 & & $4 \mathrm{f}$ & $(5 / 2)^{2}[9 / 2]^{\circ} 4$ & $6 \mathrm{~d}$ & $(5 / 2)^{2}[7 / 2]_{4}$ & & & R1c & & $\mathrm{x}$ \\
\hline $9718.927(10)$ & $10286.381(11)$ & $9718.9263(24)$ & 0.000 & 23 & & $4 f$ & $(5 / 2)^{2}[9 / 2]^{\circ}$ & $6 \mathrm{~d}$ & $\begin{array}{l}(5 / 2)^{2}[7 / 2]_{3} \\
\text {. }\end{array}$ & & & R1c & & \\
\hline $9732.121(21)$ & $10272.435(22)$ & $9732.1007(17)$ & 0.021 & 1100 & * & $\mathrm{sp}$ & $\left({ }^{3} \mathrm{~F}\right)^{1} \mathrm{P}^{\circ}{ }^{3} \mathrm{G}_{4}^{\circ}$ & $5 \mathrm{~g}$ & $(5 / 2)^{2}[9 / 2]_{5}$ & & & R1nc & & \\
\hline $9732.148(13)$ & $10272.407(13)$ & $9732.1264(21)$ & 0.022 & 1100 & * & $\mathrm{sp}$ & $\left({ }^{3} \mathrm{~F}\right)^{1} \mathrm{P}^{\circ}{ }^{3} \mathrm{G}_{4}^{\circ}$ & $5 \mathrm{~g}$ & $(5 / 2)^{2}[9 / 2]_{4}$ & $2.1 \mathrm{e}+06$ & $\mathrm{D}+$ & F_Re & TW & \\
\hline $9734.473(10)$ & $10269.953(11)$ & $9734.488(3)$ & -0.015 & 24 & & $4 \mathrm{f}$ & $(5 / 2)^{2}[7 / 2]^{4} 4$ & $6 \mathrm{~d}$ & $\begin{array}{l}(5 / 2)^{2}[9 / 2]_{5} \\
\text { nat }\end{array}$ & & & R1c & & \\
\hline \multirow[t]{2}{*}{ 9735.802(4) } & 10268.551(4) & $9735.8031(20)$ & -0.001 & 6700 & & $\mathrm{sp}$ & $\left({ }^{3} \mathrm{~F}\right)^{1} \mathrm{P}^{\circ}{ }^{3} \mathrm{G}^{\circ}{ }_{4}$ & $5 \mathrm{~g}$ & $\begin{array}{l}(5 / 2)^{2}[11 / 2]_{5} \\
5\end{array}$ & $9 . e+06$ & $\mathrm{D}+$ & F_Re & TW & \\
\hline & & $9781.688(5)$ & & & $\mathrm{m}$ & $\mathrm{sp}$ & $\left({ }^{3} \mathrm{~F}\right)^{1} \mathrm{P}^{\circ}{ }^{3} \mathrm{D}^{\circ}{ }_{1}$ & $5 \mathrm{~g}$ & $(3 / 2)^{2}[5 / 2]_{2}$ & & & $\mathrm{R} 1 \mathrm{c}$ & & \\
\hline $9781.844(13)$ & $10220.219(13)$ & $9781.880(4)$ & -0.037 & 120 & & $4 f$ & $(3 / 2)^{2}[7 / 2]^{0}{ }_{3}^{1}$ & $6 \mathrm{~d}$ & $(3 / 2)^{2}[7 / 2]_{3}$ & & & R1c & & $x$ \\
\hline $9792.465(10)$ & 10209.134(11) & $9792.4709(19)$ & -0.006 & 50 & & $4 f$ & $(5 / 2)^{2}[1 / 2]^{\circ}$ & $5 \mathrm{~g}$ & $(5 / 2)^{2}[5 / 2]_{2}$ & & & R1c & & \\
\hline $9794.085(10)$ & $10207.445(10)$ & $9794.067(3)$ & 0.018 & 38 & & $\mathrm{sp}$ & $\left({ }^{3} \mathrm{P}\right)^{3} \mathrm{P}^{\circ}{ }^{3} \mathrm{D}^{\circ}{ }_{2}$ & $7 \mathrm{~s}$ & $(5 / 2)^{2}[5 / 2]_{2}$ & & & R1c & & \\
\hline $9813.2038(22)$ & 10187.5583(23) & $9813.2047(20)$ & -0.0009 & 8600 & & $4 \mathrm{f}$ & $(5 / 2)^{2}[1 / 2]^{\circ} 1$ & $5 \mathrm{~g}$ & $\begin{array}{l}(5 / 2)^{2}[3 / 2]_{2} \\
\text {. }\end{array}$ & $4.1 \mathrm{e}+07$ & $\mathrm{C}+$ & F_Re & TW & \\
\hline 9813.334(8) & $10187.423(8)$ & 9813.325(4) & 0.009 & 2600 & & $4 \mathrm{f}$ & $(5 / 2)^{2}[1 / 2]^{\circ}{ }_{1}$ & $5 g$ & $(5 / 2)^{2}[3 / 2]_{1}$ & $1.7 \mathrm{e}+07$ & $\mathrm{D}+$ & R1c & TW & \\
\hline 9817.398(9) & 10183.206(9) & 9817.3974(12) & 0.001 & 240 & & $4 f$ & $(5 / 2)^{2}[3 / 2]_{2}{ }_{2}$ & $5 \mathrm{~g}$ & $(5 / 2)^{2}[7 / 2]_{3}$ & $1.8 \mathrm{e}+06$ & $\mathrm{D}+$ & R1c & TW & \\
\hline $9824.237(10)$ & $10176.117(11)$ & 9824.2394(9) & -0.002 & 100 & & $4 f$ & $(5 / 2)^{2}[11 / 2]_{6}^{\circ}$ & $5 g$ & $\begin{array}{l}(5 / 2)^{2}[9 / 2]_{5} \\
\text {. }\end{array}$ & & & $\mathrm{R} 1 \mathrm{c}$ & & \\
\hline
\end{tabular}


Table A1. Cont.

\begin{tabular}{|c|c|c|c|c|c|c|c|c|c|c|c|c|c|c|}
\hline$\lambda_{\text {obs }}{ }^{\text {a }}(\AA)$ & $\sigma_{\mathrm{obs}}{ }^{\mathrm{b}}\left(\mathrm{cm}^{-1}\right)$ & $\lambda_{\text {Ritz }}{ }^{c}(\AA)$ & $\begin{array}{c}\Delta \lambda_{\text {obs-Ritz }} \\
(\mathrm{A})\end{array}$ & $\begin{array}{l}I_{\text {obs }} \mathrm{d} \\
\text { (arb. u.) }\end{array}$ & Char ${ }^{e}$ & & ower Level & & Upper Level & $A\left(\mathrm{~s}^{-1}\right)$ & $\operatorname{Acc}^{f}$ & Line Ref. $\mathrm{g}$ & TP Ref. $\mathrm{g}$ & Notes $h$ \\
\hline $9827.041(12)$ & 10173.213(12) & $9827.0477(21)$ & -0.006 & 78 & & $4 \mathrm{f}$ & $(5 / 2)^{2}[11 / 2]^{\circ}{ }_{5}$ & $5 g$ & $(5 / 2)^{2}[9 / 2]_{4}$ & & & R1c & & \\
\hline $\begin{array}{l}9827.982(4) \\
\text { (n) }\end{array}$ & $10172.240(4)$ & $9827.9787(7)$ & 0.003 & 3200 & & $4 f$ & $(5 / 2)^{2}[11 / 2]^{\circ}{ }_{6}$ & $5 g$ & $\begin{array}{l}(5 / 2)^{2}[11 / 2]_{6} \\
\text { (n) }\end{array}$ & $7.5 \mathrm{e}+06$ & $\mathrm{D}+$ & F_Re & TW & \\
\hline $9828.973(10)$ & 10171.214(10) & $9828.9743(16)$ & -0.001 & 710 & & $5 p$ & $(3 / 2)^{2}[3 / 2]^{\circ}{ }_{2}$ & $6 \mathrm{~s}$ & $(5 / 2)^{2}[5 / 2]_{2}$ & & & $\mathrm{R} 1 \mathrm{c}$ & & \\
\hline $9830.795(4)$ & $10169.329(4)$ & $9830.7965(20)$ & -0.001 & 2700 & & $4 f$ & $(5 / 2)^{2}[11 / 2]^{\circ}{ }_{5}$ & $5 \mathrm{~g}$ & $(5 / 2)^{2}[11 / 2]_{5}$ & $7.7 \mathrm{e}+06$ & $\mathrm{D}+$ & F_Re & TW & \\
\hline 9835.299(10) & 10164.672(11) & $9835.299(3)$ & -0.001 & 100 & & $4 \mathrm{f}$ & $(3 / 2)^{2}[3 / 2]^{\circ}{ }_{2}$ & $5 g$ & $(3 / 2)^{2}[7 / 2]_{3}$ & & & $\mathrm{R} 1 \mathrm{c}$ & & \\
\hline 9837.8361(21) & $10162.0505(22)$ & 9837.8372(17) & -0.0011 & 10000 & & $4 \mathrm{f}$ & $(5 / 2)^{2}[3 / 2]^{\circ}{ }_{2}$ & $5 g$ & $(5 / 2)^{2}[5 / 2]_{3}$ & $4.4 \mathrm{e}+07$ & $\mathrm{C}+$ & F_Re & TW & \\
\hline $9850.495(5)$ & 10148.991(6) & 9850.504(4) & -0.010 & 2400 & & $4 \mathrm{f}$ & $(5 / 2)^{2}[1 / 2]_{0}^{\circ}$ & $5 g$ & $(5 / 2)^{2}[3 / 2]_{1}$ & $2.8 \mathrm{e}+07$ & $\mathrm{D}+$ & F_Re & TW & \\
\hline $9858.723(7)$ & $10140.521(8)$ & $9858.7254(24)$ & -0.002 & 2500 & & $4 \mathrm{f}$ & $(5 / 2)^{2}[3 / 2]^{\circ}{ }_{2}$ & $5 \mathrm{~g}$ & $(5 / 2)^{2}[3 / 2]_{2}$ & $1.9 \mathrm{e}+07$ & $\mathrm{D}+$ & R1c & TW & \\
\hline $9861.28027(17)$ & $10137.89135(18)$ & & & 21000 & & $4 \mathrm{f}$ & $(5 / 2)^{2}[11 / 2]_{6}^{\circ}$ & $5 \mathrm{~g}$ & $(5 / 2)^{2}[13 / 2]_{7}$ & $6.7 \mathrm{e}+07$ & B & F_Re & TW & \\
\hline $9864.13643(18)$ & 10134.95593(19) & & & 19000 & & $4 f$ & $(5 / 2)^{2}[11 / 2]^{\circ}{ }_{5}$ & $5 g$ & $(5 / 2)^{2}[13 / 2]_{6}$ & $6.6 \mathrm{e}+07$ & B & F_Re & TW & \\
\hline $9868.0934(21)$ & $10130.8920(22)$ & $9868.0940(21)$ & -0.0006 & 9100 & & $4 \mathrm{f}$ & $(3 / 2)^{2}[3 / 2]_{2}^{\circ}$ & $5 g$ & $(3 / 2)^{2}[5 / 2]_{3}$ & $5.6 \mathrm{e}+07$ & $\mathrm{C}_{+}$ & F_Re & TW & \\
\hline $9870.816(16)$ & 10128.098(17) & $9870.8012(20)$ & 0.014 & 97 & & $5 \mathrm{~s}$ & $(5 / 2)^{2}[5 / 2]_{3}$ & $\mathrm{sp}$ & $\left({ }^{3} \mathrm{~F}\right)^{3} \mathrm{P}^{\circ}{ }^{3} \mathrm{~F}_{3}^{\circ}$ & $5.4 \mathrm{e}+05$ & $\mathrm{C}$ & $\mathrm{R} 1 \mathrm{c}$ & B00 & \\
\hline 9878.465(8) & $10120.255(9)$ & 9878.193(5) & 0.273 & 280 & ? & $\mathrm{sp}$ & $\left({ }^{3} \mathrm{P}\right)^{3} \mathrm{P}^{\circ} 5 \mathrm{~S}^{\circ}{ }_{2}$ & $5 g$ & $(5 / 2)^{2}[5 / 2]_{2}$ & & & R1c & & $\mathrm{x}$ \\
\hline 9881.464(3) & 10117.184(3) & $9881.4639(15)$ & 0.000 & 8400 & & $4 \mathrm{f}$ & $(5 / 2)^{2}[7 / 2]^{\circ}{ }_{3}$ & $5 \mathrm{~g}$ & $(5 / 2)^{2}[9 / 2]_{4}$ & $2.1 \mathrm{e}+07$ & $\mathrm{D}$ & F_Re & TW & \\
\hline 9883.730(9) & 10114.864(9) & $9883.7091(24)$ & 0.021 & 560 & & $4 \mathrm{f}$ & $(3 / 2)^{2}[9 / 2]^{\circ}{ }_{5}$ & $5 \mathrm{~g}$ & $(3 / 2)^{2}[9 / 2]_{5}$ & $5.4 \mathrm{e}+06$ & $\mathrm{D}+$ & $\mathrm{R} 1 \mathrm{c}$ & TW & \\
\hline $9883.9672(10)$ & $10114.6216(10)$ & $9883.9674(10)$ & -0.0001 & 5100 & & $4 f$ & $(5 / 2)^{2}[3 / 2]^{\circ} 1$ & $5 \mathrm{~g}$ & $(5 / 2)^{2}[5 / 2]_{2}$ & $3.9 \mathrm{e}+07$ & $\mathrm{C}+$ & F_Re & TW & \\
\hline 9884.996(11) & $10113.569(11)$ & 9884.989(4) & 0.007 & 28 & & $6 p$ & $(5 / 2)^{2}[7 / 2]^{\circ}{ }_{3}$ & $6 \mathrm{~d}$ & $(3 / 2)^{2}[7 / 2]_{4}$ & & & R1c & & \\
\hline 9892.939(4) & $10105.448(4)$ & $9892.940(4)$ & -0.000 & 4800 & & $4 f$ & $(3 / 2)^{2}[3 / 2]^{\circ} 1$ & $5 \mathrm{~g}$ & $(3 / 2)^{2}[5 / 2]_{2}$ & $3.1 \mathrm{e}+07$ & $\mathrm{D}$ & F_Re & TW & \\
\hline 9894.337(7) & 10104.021(7) & $9894.3443(14)$ & -0.007 & 5300 & & $4 f$ & $(5 / 2)^{2}[7 / 2]^{\circ}{ }_{3}$ & $5 g$ & $\begin{array}{l}(5 / 2)^{2}[7 / 2]_{3} \\
\end{array}$ & $1.0 \mathrm{e}+07$ & $\mathrm{D}+$ & $\mathrm{R} 1 \mathrm{c}$ & TW & \\
\hline 9899.567(11) & 10098.683(11) & 9899.414(6) & 0.153 & 57 & ? & $\mathrm{sp}$ & $\left({ }^{3} \mathrm{P}\right)^{3} \mathrm{P}^{\circ}{ }^{5} \mathrm{~S}^{\circ}{ }_{2}$ & $5 \mathrm{~g}$ & $(5 / 2)^{2}[3 / 2]_{1}$ & & & R1c & & $\mathrm{x}$ \\
\hline $9905.136(23)$ & $10093.005(24)$ & 9905.091(3) & 0.045 & 1800 & * & $4 \mathrm{f}$ & $(5 / 2)^{2}[3 / 2]^{\circ} 1$ & $5 \mathrm{~g}$ & $(5 / 2)^{2}[3 / 2]_{2}$ & $3.7 \mathrm{e}+06$ & $\mathrm{D}+$ & F_Re & TW & \\
\hline $9905.18(8)$ & $10092.97(8)$ & $9905.214(4)$ & -0.04 & 1800 & * & $4 f$ & $(5 / 2)^{2}[3 / 2]^{\circ}$ & $5 g$ & $(5 / 2)^{2}[3 / 2]_{1}$ & $1.5 \mathrm{e}+07$ & $\mathrm{D}+$ & $\mathrm{R} 1 \mathrm{c}$ & TW & \\
\hline $9911.988(12)$ & $10086.028(12)$ & 9911.986(3) & 0.002 & 58 & & $\mathrm{sp}$ & $\left({ }^{3} \mathrm{~F}\right)^{1} \mathrm{P}^{\circ}{ }^{3} \mathrm{D}^{\circ}{ }_{2}$ & $7 \mathrm{~s}$ & $(3 / 2)^{2}[3 / 2]_{1}$ & & & R1c & & \\
\hline $9915.090(21)$ & $10082.873(22)$ & $9915.0672(18)$ & 0.022 & 630 & * & $4 \mathrm{f}$ & $(5 / 2)^{2}[7 / 2]^{\circ}{ }_{3}$ & $5 \mathrm{~g}$ & $(5 / 2)^{2}[5 / 2]_{2}$ & & & R1c & & \\
\hline $9915.090(21)$ & $10082.873(22)$ & $9915.1061(20)$ & -0.017 & 630 & * & $4 f$ & $(5 / 2)^{2}[7 / 2]^{\circ}{ }_{3}^{\circ}$ & $5 g$ & $(5 / 2)^{2}[5 / 2]_{3}$ & & & R1c & & \\
\hline 9916.4171(3) & $10081.5233(3)$ & $9916.4172(3)$ & -0.0001 & 15000 & & $4 \mathrm{f}$ & $(3 / 2)^{2}[9 / 2]^{\circ}$ & $5 \mathrm{~g}$ & $(3 / 2)^{2}[11 / 2]_{6}$ & $6.6 \mathrm{e}+07$ & B & F_Re & TW & \\
\hline $9917.9509(10)$ & $10079.9642(10)$ & $9917.9510(10)$ & -0.0001 & 9800 & & $4 \mathrm{f}$ & $(5 / 2)^{2}[5 / 2]^{\circ} 2$ & $5 \mathrm{~g}$ & $(5 / 2)^{2}[7 / 2]_{3}$ & $3.8 \mathrm{e}+07$ & $\mathrm{C}+$ & F_Re & TW & \\
\hline 9925.5883(4) & $10072.2080(4)$ & $9925.5883(4)$ & -0.0000 & 13000 & & $4 f$ & $(3 / 2)^{2}[9 / 2]_{4}^{\circ}$ & $5 g$ & $(3 / 2)^{2}[11 / 2]_{5}$ & $6.5 \mathrm{e}+07$ & B & F_Re & TW & \\
\hline $9926.0209(15)$ & $10071.7690(16)$ & $9926.0211(13)$ & -0.0002 & 4900 & & $4 \mathrm{f}$ & $(5 / 2)^{2}[5 / 2]^{\circ}{ }_{3}$ & $5 g$ & $(5 / 2)^{2}[9 / 2]_{4}$ & & & F_Re & & \\
\hline $9938.775(4)$ & $10058.844(4)$ & $9938.7730(16)$ & 0.002 & 5300 & & $4 f$ & $(5 / 2)^{2}[5 / 2]^{\circ} 2$ & $5 \mathrm{~g}$ & $(5 / 2)^{2}[5 / 2]_{2}$ & $2.1 \mathrm{e}+07$ & $\mathrm{C}+$ & F_Re & TW & \\
\hline $9938.9960(6)$ & $10058.6207(6)$ & $9938.9960(6)$ & 0.0000 & 11000 & & $4 f$ & $(5 / 2)^{2}[5 / 2]_{3}^{\circ}$ & $5 g$ & $\begin{array}{l}(5 / 2)^{2}[7 / 2]_{4} \\
\text {. }\end{array}$ & $4.4 \mathrm{e}+07$ & $\mathrm{C}+$ & F_Re & TW & \\
\hline 9948.895(11) & 10048.612(11) & $9948.8880(21)$ & 0.007 & 61 & & $4 \mathrm{f}$ & $(3 / 2)^{2}[5 / 2]^{\circ}{ }_{3}$ & $5 \mathrm{~g}$ & $(3 / 2)^{2}[9 / 2]_{4}$ & & & $\mathrm{R} 1 \mathrm{c}$ & & \\
\hline $9959.970(3)$ & $10037.439(3)$ & $9959.9679(18)$ & 0.002 & 5800 & & $4 \mathrm{f}$ & $(5 / 2)^{2}[5 / 2]^{\circ}{ }_{3}$ & $5 g$ & $(5 / 2)^{2}[5 / 2]_{3}$ & $1.9 \mathrm{e}+07$ & $\mathrm{C}+$ & F_Re & TW & \\
\hline $9960.3485(8)$ & $10037.0575(8)$ & $9960.3485(8)$ & -0.0000 & 11000 & & $4 \mathrm{f}$ & $(3 / 2)^{2}[5 / 2]^{\circ}{ }_{3}^{\circ}$ & $5 \mathrm{~g}$ & $(3 / 2)^{2}[7 / 2]_{4}$ & $5.6 \mathrm{e}+07$ & B & F_Re & TW & \\
\hline 9981.399(12) & 10015.890(12) & $9981.379(3)$ & 0.020 & 130 & & $4 \mathrm{f}$ & $(5 / 2)^{2}[5 / 2]^{\circ}{ }_{3}$ & $5 g$ & $(5 / 2)^{2}[3 / 2]_{2}$ & $1.9 \mathrm{e}+06$ & $\mathrm{D}+$ & $\mathrm{R} 1 \mathrm{c}$ & TW & \\
\hline 9994.027(11) & $10003.234(11)$ & 9994.019(3) & 0.008 & 900 & & $4 f$ & $(3 / 2)^{2}[5 / 2]^{\circ}{ }_{3}$ & $5 \mathrm{~g}$ & $(3 / 2)^{2}[5 / 2]_{3}$ & $9 . e+06$ & $\mathrm{D}+$ & $\mathrm{R} 1 \mathrm{c}$ & TW & \\
\hline $10006.588(3)$ & $9990.678(3)$ & $10006.5881(24)$ & -0.001 & 8400 & & $4 f$ & $(3 / 2)^{2}[5 / 2]^{\circ}{ }_{2}$ & $5 g$ & $(3 / 2)^{2}[7 / 2]_{3}$ & $4.9 \mathrm{e}+07$ & $\mathrm{C}+$ & F_Re & TW & \\
\hline $10022.9672(3)$ & $9974.3510(3)$ & $10022.9672(3)$ & -0.0000 & 20000 & & $4 \mathrm{f}$ & $(5 / 2)^{2}[7 / 2]^{\circ}{ }_{4}$ & $5 \mathrm{~g}$ & $(5 / 2)^{2}[9 / 2]_{5}$ & $4.9 e+07$ & B & F_Re & TW & \\
\hline $10026.894(4)$ & $9970.445(4)$ & $10026.8943(17)$ & -0.001 & 1500 & & $4 \mathrm{f}$ & $(5 / 2)^{2}[7 / 2]^{\circ} 4$ & $5 \mathrm{~g}$ & $(5 / 2)^{2}[11 / 2]_{5}$ & & & F_Re & & \\
\hline $10036.224(3)$ & $9961.176(3)$ & 10036.2243(9) & -0.000 & 5700 & & $4 \mathrm{f}$ & $(5 / 2)^{2}[7 / 2]^{\circ} 4$ & $5 g$ & $(5 / 2)^{2}[7 / 2]_{4}$ & $1.64 \mathrm{e}+07$ & $\mathrm{C}+$ & F_Re & TW & \\
\hline $10038.0920(5)$ & $9959.3223(5)$ & $10038.0919(5)$ & 0.0001 & 14000 & & $4 \mathrm{f}$ & $(3 / 2)^{2}[7 / 2]^{\circ}{ }_{4}$ & $5 g$ & $(3 / 2)^{2}[9 / 2]_{5}$ & $6.0 \mathrm{e}+07$ & B & F_Re & TW & \\
\hline 10040.499(13) & 9956.935(13) & $10040.481(5)$ & 0.017 & 560 & & $4 f$ & $(3 / 2)^{2}[5 / 2]^{2} 2$ & $5 \mathrm{~g}$ & $(3 / 2)^{2}[5 / 2]_{2}$ & $9 . e+06$ & $\mathrm{D}+$ & R1c & TW & \\
\hline
\end{tabular}


Table A1. Cont.

\begin{tabular}{|c|c|c|c|c|c|c|c|c|c|c|c|c|c|c|}
\hline$\lambda_{\text {obs }}{ }^{a}(\AA)$ & $\sigma_{\mathrm{obs}}{ }^{\mathrm{b}}\left(\mathrm{cm}^{-1}\right)$ & $\lambda_{\text {Ritz }}{ }^{c}(\AA)$ & $\begin{array}{c}\Delta \lambda_{\text {obs-Ritz }} \\
\text { (A) }\end{array}$ & $\begin{array}{l}I_{\text {obs }} \mathrm{d} \\
\text { (arb. u.) }\end{array}$ & Char ${ }^{e}$ & & ower Level & & Upper Level & $A\left(\mathrm{~s}^{-1}\right)$ & $\operatorname{Acc}^{f}$ & Line Ref. $\mathrm{g}$ & TP Ref. $\mathrm{g}$ & Notes $h$ \\
\hline 10049.773(12) & $9947.746(12)$ & 10049.7881(19) & -0.015 & 1200 & & $4 \mathrm{f}$ & $(3 / 2)^{2}[7 / 2]^{\circ}{ }_{4}$ & $5 \mathrm{~g}$ & $(3 / 2)^{2}[7 / 2]_{4}$ & $9 . e+06$ & $\mathrm{D}+$ & R1c & TW & \\
\hline 10051.0231(20) & $9946.5092(20)$ & $10051.0218(6)$ & 0.0014 & 5100 & & $4 f$ & $(5 / 2)^{2}[9 / 2]^{\circ}{ }_{5}$ & $5 g$ & $(5 / 2)^{2}[9 / 2]_{5}$ & $1.32 \mathrm{e}+07$ & $\mathrm{C}+$ & F_Re & TW & \\
\hline $10054.93572(22)$ & $9942.63880(21)$ & $10054.93573(22)$ & -0.00001 & 24000 & & $4 \mathrm{f}$ & $(5 / 2)^{2}[9 / 2]^{\circ}{ }_{5}$ & $5 \mathrm{~g}$ & $(5 / 2)^{2}[11 / 2]_{6}$ & $5.8 \mathrm{e}+07$ & B & F_Re & TW & \\
\hline 10057.609(11) & 9939.996(11) & $10057.6090(20)$ & -0.000 & 180 & & $4 f$ & $(5 / 2)^{2}[7 / 2]^{\circ}{ }_{4}$ & $5 \mathrm{~g}$ & $(5 / 2)^{2}[5 / 2]_{3}$ & $1.7 \mathrm{e}+06$ & $\mathrm{D}+$ & R1c & TW & \\
\hline 10080.3499(10) & $9917.5719(10)$ & $10080.3499(10)$ & -0.0000 & 11000 & & $4 f$ & $(3 / 2)^{2}[7 / 2]^{\circ}{ }_{3}$ & $5 g$ & $(3 / 2)^{2}[9 / 2]_{4}$ & $5.5 \mathrm{e}+07$ & B & F_Re & TW & \\
\hline $10092.14(3)$ & 9905.99(3) & 10092.1155(21) & 0.02 & 990 & * & $4 \mathrm{f}$ & $(3 / 2)^{2}[7 / 2]^{\circ}{ }_{3}$ & $5 \mathrm{~g}$ & $(3 / 2)^{2}[7 / 2]_{4}$ & $1.4 \mathrm{e}+06$ & $\mathrm{D}+$ & Rinc & TW & \\
\hline 10092.14(3) & 9905.99(3) & 10092.151(3) & -0.01 & 990 & * & $4 \mathrm{f}$ & $(3 / 2)^{2}[7 / 2]^{\circ}{ }_{3}$ & $5 g$ & $(3 / 2)^{2}[7 / 2]_{3}$ & $7.3 e+06$ & $\mathrm{D}+$ & R1c & TW & \\
\hline $10162.808(8)$ & 9837.103(8) & 10162.8099(18) & -0.001 & 3800 & & $4 \mathrm{f}$ & $(5 / 2)^{2}[9 / 2]^{\circ}{ }_{4}$ & $5 \mathrm{~g}$ & $(5 / 2)^{2}[9 / 2]_{4}$ & $1.25 \mathrm{e}+07$ & $\mathrm{C}+$ & F_Re & TW & \\
\hline 10166.8202(21) & $9833.2217(20)$ & 10166.8192(16) & 0.0010 & 18000 & & $4 \mathrm{f}$ & $(5 / 2)^{2}[9 / 2]^{\circ}{ }_{4}$ & $5 \mathrm{~g}$ & $(5 / 2)^{2}[11 / 2]_{5}$ & $4.4 \mathrm{e}+07$ & B & F_Re & TW & \\
\hline $10176.433(12)$ & 9823.933(11) & $10176.4346(17)$ & -0.002 & 1700 & & $4 f$ & $(5 / 2)^{2}[9 / 2]^{\circ}{ }_{4}$ & $5 \mathrm{~g}$ & $(5 / 2)^{2}[7 / 2]_{3}$ & $1.4 \mathrm{e}+06$ & $\mathrm{D}+$ & $\mathrm{R} 1 \mathrm{c}$ & TW & \\
\hline 10193.660(10) & 9807.331(10) & 10193.6484(21) & 0.012 & 1100 & & $5 s$ & $(5 / 2)^{2}[5 / 2]_{2}$ & $\mathrm{sp}$ & $\left({ }^{3} \mathrm{~F}^{3} \mathrm{P}^{\circ}{ }^{3} \mathrm{~F}^{\mathrm{o}}{ }_{3}\right.$ & & & R1c & & \\
\hline $10225.073(10)$ & 9777.201(10) & $10225.077(4)$ & -0.003 & 1700 & & $6 p$ & $(5 / 2)^{2}[7 / 2]^{\circ}{ }_{3}$ & $5 g$ & $(3 / 2)^{2}[9 / 2]_{4}$ & & & R1c & & \\
\hline $10237.22(3)$ & $9765.60(3)$ & 10237.183(4) & 0.03 & 120 & * & $6 p$ & $(5 / 2)^{2}[7 / 2]^{\circ}{ }_{3}$ & $5 g$ & $(3 / 2)^{2}[7 / 2]_{4}$ & & & R1nc & & \\
\hline 10237.22(3) & $9765.60(3)$ & $10237.219(4)$ & -0.00 & 120 & * & $6 \mathrm{p}$ & $(5 / 2)^{2}[7 / 2]^{\circ}{ }_{3}$ & $5 \mathrm{~g}$ & $(3 / 2)^{2}[7 / 2]_{3}$ & & & R1c & & \\
\hline $10247.043(10)$ & $9756.239(9)$ & $10247.038(4)$ & 0.005 & 1300 & & $\mathrm{sp}$ & $\left({ }^{3} \mathrm{~F}\right)^{1} \mathrm{P}^{\circ}{ }^{3} \mathrm{D}^{\circ}{ }_{2}$ & $6 \mathrm{~d}$ & $(5 / 2)^{2}[7 / 2]_{3}$ & $1.9 e+06$ & $\mathrm{D}+$ & $\mathrm{R} 1 \mathrm{c}$ & TW & \\
\hline $10278.686(16)$ & $9726.204(15)$ & $10278.6604(14)$ & 0.026 & 540 & & $5 s$ & $(3 / 2)^{2}[3 / 2]_{2}$ & $5 p$ & $(5 / 2)^{2}[3 / 2]^{\circ} 2$ & & & $\mathrm{R} 1 \mathrm{c}$ & & \\
\hline 10297.40(3) & $9708.53(3)$ & 10297.147(5) & 0.25 & 56 & ? & $\mathrm{sp}$ & $\left({ }^{1} \mathrm{G}\right)^{3} \mathrm{P}^{\circ}{ }^{3} \mathrm{H}^{\circ}{ }_{4}$ & $6 \mathrm{~d}$ & $(5 / 2)^{2}[9 / 2]_{5}$ & & & R1c & & $x$ \\
\hline $10343.469(10)$ & 9665.287(9) & 10343.472(3) & -0.003 & 6100 & & $\mathrm{sp}$ & $\left({ }^{3} \mathrm{~F}\right)^{1} \mathrm{P}^{\circ}{ }^{3} \mathrm{~F}_{3}^{\circ}$ & $5 \mathrm{~g}$ & $(5 / 2)^{2}[9 / 2]_{4}$ & $1.17 \mathrm{e}+07$ & $\mathrm{C}+$ & R1c & TW & \\
\hline $10357.603(10)$ & $9652.098(9)$ & $10357.586(3)$ & 0.017 & 780 & & $\mathrm{sp}$ & $\left({ }^{3} \mathrm{~F}\right)^{1} \mathrm{P}^{\circ}{ }^{3} \mathrm{~F}^{\circ}{ }_{3}$ & $5 g$ & $\begin{array}{l}(5 / 2)^{2}[7 / 2]_{3} \\
\end{array}$ & $4.8 \mathrm{e}+06$ & $\mathrm{D}+$ & R1c & TW & \\
\hline $10380.323(21)$ & $9630.972(20)$ & $10380.297(3)$ & 0.026 & 130 & * & $\mathrm{sp}$ & $\left({ }^{3} \mathrm{~F}\right)^{1} \mathrm{P}^{\circ}{ }^{3} \mathrm{~F}^{\circ}{ }_{3}$ & $5 g$ & $\begin{array}{l}(5 / 2)^{2}[5 / 2]_{2} \\
\text { ton }\end{array}$ & & & R1nc & & \\
\hline $10380.323(21)$ & $9630.972(20)$ & $10380.340(3)$ & -0.016 & 130 & * & $\mathrm{sp}$ & $\left({ }^{3} \mathrm{~F}\right)^{1} \mathrm{P}^{\circ}{ }^{3} \mathrm{~F}^{\circ}{ }_{3}$ & $5 \mathrm{~g}$ & $(5 / 2)^{2}[5 / 2]_{3}$ & & & R1c & & \\
\hline 10424.302(8) & $9590.341(7)$ & $10424.309(3)$ & -0.007 & 3700 & & $5 \mathrm{~d}$ & $(5 / 2)^{2}[1 / 2]_{1}$ & $5 \mathrm{f}$ & $(5 / 2)^{2}[3 / 2]^{\circ} 2$ & $1.2 \mathrm{e}+07$ & $\mathrm{D}+$ & F_Re & TW & \\
\hline 10453.012(7) & $9564.000(7)$ & $10453.004(4)$ & 0.008 & 4800 & & $5 d$ & $(5 / 2)^{2}[1 / 2]_{1}$ & $5 \mathrm{f}$ & $(5 / 2)^{2}[1 / 2]^{\circ} 1$ & $2.7 \mathrm{e}+07$ & $\mathrm{D}+$ & F_Re & TW & \\
\hline $10465.664(10)$ & $9552.438(10)$ & $10465.665(4)$ & -0.002 & 3800 & & $5 \mathrm{~d}$ & $(5 / 2)^{2}[1 / 2]_{1}$ & $5 \mathrm{f}$ & $(5 / 2)^{2}[1 / 2]^{\circ} 0$ & $3.6 \mathrm{e}+07$ & $\mathrm{D}+$ & F_Re & TW & \\
\hline $10575.703(11)$ & $9453.046(10)$ & $10575.690(6)$ & 0.013 & 180 & & $6 p$ & $(5 / 2)^{2}[5 / 2]^{\circ}{ }_{2}$ & $6 \mathrm{~d}$ & $(3 / 2)^{2}[7 / 2]_{3}$ & $3.0 \mathrm{e}+06$ & $\mathrm{D}+$ & $\mathrm{R} 1 \mathrm{c}$ & TW & \\
\hline 10609.294(11) & $9423.116(10)$ & $10609.300(5)$ & -0.006 & 240 & & $5 d$ & $(3 / 2)^{2}[1 / 2]_{1}$ & $5 \mathrm{f}$ & $(3 / 2)^{2}[3 / 2]^{\circ} 1$ & $1.0 \mathrm{e}+07$ & D+ & R1c & TW & \\
\hline $10624.895(12)$ & $9409.280(11)$ & 10624.901(4) & -0.007 & 96 & & $\mathrm{sp}$ & $\left({ }^{1} \mathrm{G}\right)^{3} \mathrm{P}^{\circ}{ }^{3} \mathrm{H}^{\circ}{ }_{4}$ & $5 \mathrm{~g}$ & $\begin{array}{l}(5 / 2)^{2}[11 / 2]_{5} \\
\text { nat }\end{array}$ & & & R1c & & \\
\hline 10641.797(11) & 9394.335(9) & $10641.822(5)$ & -0.025 & 3600 & & $5 \mathrm{~d}$ & $(3 / 2)^{2}[1 / 2]_{1}$ & $5 \mathrm{f}$ & $(3 / 2)^{2}[3 / 2]^{\circ} 2$ & $3.0 \mathrm{e}+07$ & $\mathrm{D}+$ & F_Re & TW & \\
\hline 10742.332(8) & 9306.416(7) & 10742.3392(23) & -0.007 & 4800 & & $5 d$ & $(5 / 2)^{2}[9 / 2]_{5}$ & $5 \mathrm{f}$ & $(5 / 2)^{2}[9 / 2]^{\circ}{ }_{5}$ & $7.7 \mathrm{e}+06$ & $\mathrm{D}+$ & F_Re & TW & \\
\hline 10745.703(12) & $9303.497(11)$ & $10745.703(3)$ & -0.001 & 110 & & $5 \mathrm{~d}$ & $(5 / 2)^{2}[9 / 2]_{5}$ & $5 f$ & $(5 / 2)^{2}[9 / 2]^{\circ}{ }_{4}$ & & & $\mathrm{R} 1 \mathrm{c}$ & & \\
\hline 10752.044(14) & $9298.010(12)$ & $10752.046(3)$ & -0.002 & 53 & & $5 \mathrm{~d}$ & $(5 / 2)^{2}[9 / 2]_{5}$ & $5 \mathrm{f}$ & $(5 / 2)^{2}[7 / 2]^{\circ} 4$ & & & R1c & & \\
\hline $10768.359(14)$ & 9283.923(12) & $10768.360(4)$ & -0.001 & 210 & & $5 d$ & $(3 / 2)^{2}[7 / 2]_{3}$ & $5 f$ & $(3 / 2)^{2}[7 / 2]^{\circ} 3$ & $5.7 \mathrm{e}+06$ & D+ & R1c & TW & \\
\hline $10787.828(10)$ & $9267.168(9)$ & $10787.827(3)$ & 0.001 & 210 & & $5 \mathrm{~d}$ & $(5 / 2)^{2}[3 / 2]_{2}$ & $5 f$ & $(5 / 2)^{2}[7 / 2]^{\circ}{ }_{3}$ & $1.9 \mathrm{e}+06$ & $\mathrm{D}+$ & $\mathrm{R} 1 \mathrm{c}$ & TW & \\
\hline $10791.529(10)$ & $9263.990(9)$ & $10791.519(3)$ & 0.010 & 1400 & & $5 \mathrm{~d}$ & $(5 / 2)^{2}[9 / 2]_{4}$ & $5 \mathrm{f}$ & $(5 / 2)^{2}[9 / 2]^{\circ}{ }_{4}^{\circ}$ & 7.e+06 & $\mathrm{D}+$ & R1c & TW & \\
\hline 10797.923(12) & 9258.504(11) & 10797.915(3) & 0.008 & 160 & & $5 d$ & $(5 / 2)^{2}[9 / 2]_{4}$ & $5 \mathrm{f}$ & $(5 / 2)^{2}[7 / 2]^{\circ}{ }_{4}$ & & & R1c & & \\
\hline $10800.96(3)$ & $9255.90(3)$ & $10800.929(3)$ & 0.03 & 110 & * & $5 \mathrm{~d}$ & $(5 / 2)^{2}[9 / 2]_{4}$ & $5 f$ & $(5 / 2)^{2}[7 / 2]^{\circ} 3$ & & & $\mathrm{R} 1 \mathrm{c}$ & & \\
\hline $10800.985(12)$ & $9255.879(10)$ & $10800.972(7)$ & 0.014 & 110 & * & $\mathrm{sp}$ & $\left({ }^{3} \mathrm{~F}\right)^{3} \mathrm{P}^{\circ}{ }^{5} \mathrm{D}_{3}$ & $4 \mathrm{~d}$ & $(3 / 2)^{2}[5 / 2]_{2}$ & & & F_Re & & \\
\hline $10836.3452(6)$ & $9225.6765(5)$ & $10836.3453(6)$ & -0.0001 & 8300 & & $5 d$ & $(5 / 2)^{2}[9 / 2]_{5}$ & $5 \mathrm{f}$ & $(5 / 2)^{2}[11 / 2]^{\circ} 6$ & $4.2 \mathrm{e}+07$ & D+ & F_Re & TW & \\
\hline 10849.473(3) & 9214.514(3) & $10849.477(3)$ & -0.004 & 6300 & & $5 \mathrm{~d}$ & $(3 / 2)^{2}[7 / 2]_{3}$ & $5 \mathrm{f}$ & $(3 / 2)^{2}[9 / 2]^{\circ} 4$ & $4.1 \mathrm{e}+07$ & $\mathrm{D}+$ & F_Re & TW & \\
\hline $10852.407(10)$ & 9212.022(9) & $10852.401(4)$ & 0.007 & 2100 & & $5 \mathrm{~d}$ & $(5 / 2)^{2}[3 / 2]_{1}$ & $5 f$ & $(5 / 2)^{2}[5 / 2]^{\circ} 2$ & $1.8 \mathrm{e}+07$ & $\mathrm{D}+$ & $\mathrm{R} 1 \mathrm{c}$ & TW & \\
\hline 10865.050(17) & 9201.303(15) & $10865.010(5)$ & 0.039 & 100 & & $5 d$ & $(5 / 2)^{2}[3 / 2]_{2}$ & $5 \mathrm{f}$ & $(5 / 2)^{2}[3 / 2]^{\circ} 1$ & & & $\mathrm{R} 1 \mathrm{c}$ & & \\
\hline $10885.074(16)$ & $9184.376(14)$ & $10885.066(5)$ & 0.008 & 200 & * & $\mathrm{sp}$ & $\left({ }^{1} \mathrm{G}\right)^{3} \mathrm{P}^{\circ}{ }^{3} \mathrm{~F}^{\circ}{ }_{3}$ & $6 \mathrm{~d}$ & $(3 / 2)^{2}[7 / 2]_{4}$ & $2.1 \mathrm{e}+06$ & $\mathrm{D}+$ & $\mathrm{R} 1 \mathrm{c}$ & TW & \\
\hline
\end{tabular}


Table A1. Cont.

\begin{tabular}{|c|c|c|c|c|c|c|c|c|c|c|c|c|c|c|}
\hline$\lambda_{\text {obs }}{ }^{\text {a }}(\AA)$ & $\sigma_{\mathrm{obs}}{ }^{\mathrm{b}}\left(\mathrm{cm}^{-1}\right)$ & $\lambda_{\text {Ritz }}{ }^{c}(\AA ̊)$ & $\begin{array}{c}\Delta \lambda_{\text {obs-Ritz }} \\
\text { (A) }\end{array}$ & $\begin{array}{c}I_{\text {obs }} \mathrm{d} \\
\text { (arb. u.) }\end{array}$ & Char ${ }^{e}$ & & wer Level & & Jpper Level & $A\left(\mathrm{~s}^{-1}\right)$ & $\operatorname{Acc}^{f}$ & Line Ref. $\mathrm{g}$ & TP Ref. $\mathrm{g}$ & Notes ${ }^{h}$ \\
\hline $10885.074(16)$ & 9184.376(14) & 10885.064(8) & 0.010 & 200 & $*$ & $\mathrm{sp}$ & $\left({ }^{3} \mathrm{~F}\right)^{1} \mathrm{P}^{\circ}{ }^{3} \mathrm{D}^{\circ}{ }_{1}$ & $6 \mathrm{~d}$ & $(5 / 2)^{2}[1 / 2]_{0}$ & & & R1c & & \\
\hline $10889.9718(12)$ & $9180.2457(11)$ & $10889.9720(12)$ & -0.0003 & 6800 & & $5 \mathrm{~d}$ & $(5 / 2)^{2}[9 / 2]_{4}$ & $5 \mathrm{f}$ & $(5 / 2)^{2}[11 / 2]^{\circ}$ & $4.2 \mathrm{e}+07$ & $\mathrm{D}+$ & $\mathrm{F} R \mathrm{Re}$ & TW & \\
\hline 10904.886(9) & 9167.691(7) & $10904.902(5)$ & -0.016 & 2600 & & $5 d$ & $(3 / 2)^{2}[3 / 2]_{1}$ & $5 \mathrm{f}$ & $(3 / 2)^{2}[5 / 2]^{\circ}{ }_{2}$ & $2.8 \mathrm{e}+07$ & $\mathrm{D}+$ & F_Re & TW & \\
\hline $10925.167(13)$ & $9150.672(11)$ & $10925.155(4)$ & 0.012 & 97 & & $5 \mathrm{~d}$ & $(3 / 2)^{2}[7 / 2]_{4}$ & $5 \mathrm{f}$ & $(3 / 2)^{2}[9 / 2]^{\circ} 4$ & $1.3 e+06$ & $\mathrm{D}+$ & R1c & TW & \\
\hline $10929.791(10)$ & $9146.800(9)$ & $10929.799(4)$ & -0.008 & 480 & & $5 \mathrm{~d}$ & $(5 / 2)^{2}[3 / 2]_{2}$ & $5 \mathrm{f}$ & $(5 / 2)^{2}[1 / 2]^{\circ} 1$ & $1.2 \mathrm{e}+07$ & $\mathrm{D}+$ & R1c & TW & \\
\hline $10930.9327(21)$ & $9145.8452(17)$ & $10930.9342(19)$ & -0.0015 & 5300 & & $5 \mathrm{~d}$ & $(3 / 2)^{2}[7 / 2]_{4}$ & $5 \mathrm{f}$ & $(3 / 2)^{2}[9 / 2]^{\circ}{ }_{5}$ & $4.2 \mathrm{e}+07$ & $\mathrm{D}+$ & F_Re & TW & \\
\hline $10959.809(11)$ & $9121.748(9)$ & $10959.819(5)$ & -0.010 & 4600 & & $5 \mathrm{~d}$ & $(3 / 2)^{2}[3 / 2]_{2}$ & $5 \mathrm{f}$ & $(3 / 2)^{2}[7 / 2]^{\circ}{ }^{\circ}$ & $3.0 \mathrm{e}+06$ & $\mathrm{D}+$ & $\mathrm{R} 1 \mathrm{c}$ & TW & \\
\hline 10964.502(11) & $9117.844(9)$ & $10964.527(5)$ & -0.025 & 3200 & & $5 d$ & $(3 / 2)^{2}[3 / 2]_{1}$ & $5 \mathrm{f}$ & $(3 / 2)^{2}[3 / 2]^{\circ}{ }_{1}$ & $1.0 \mathrm{e}+07$ & $\mathrm{D}+$ & R1c & TW & \\
\hline 10966.532(11) & 9116.156(9) & $10966.524(5)$ & 0.008 & 900 & & $5 \mathrm{~d}$ & $(5 / 2)^{2}[3 / 2]_{1}$ & $5 \mathrm{f}$ & $(5 / 2)^{2}[1 / 2]^{\circ} 0$ & 7.e+06 & $\mathrm{D}+$ & R1c & TW & \\
\hline $10973.938(5)$ & $9110.004(4)$ & 10973.944(3) & -0.006 & 3100 & & $5 \mathrm{~d}$ & $(5 / 2)^{2}[5 / 2]_{3}$ & $5 \mathrm{f}$ & $(5 / 2)^{2}[9 / 2]^{\circ} 4$ & $5.5 \mathrm{e}+06$ & $\mathrm{D}+$ & F_Re & TW & \\
\hline 10980.553(5) & $9104.516(4)$ & $10980.559(3)$ & -0.006 & 4700 & & $5 d$ & $(5 / 2)^{2}[5 / 2]_{3}$ & $5 \mathrm{f}$ & $(5 / 2)^{2}[7 / 2]^{\circ}{ }_{4}$ & $2.0 \mathrm{e}+07$ & D+ & F_Re & TW & \\
\hline $10983.677(10)$ & 9101.926(8) & 10983.675(3) & 0.002 & 1600 & & $5 \mathrm{~d}$ & $(5 / 2)^{2}[5 / 2]_{3}$ & $5 \mathrm{f}$ & $(5 / 2)^{2}[7 / 2]^{\circ} 3$ & $3.9 \mathrm{e}+06$ & $\mathrm{D}+$ & R1c & TW & \\
\hline $11008.410(12)$ & 9081.477(10) & $11008.408(4)$ & 0.002 & 3300 & & $\mathrm{sp}$ & $\left({ }^{3} \mathrm{~F}\right)^{1} \mathrm{P}^{\circ}{ }^{3} \mathrm{D}^{\circ}{ }_{3}$ & $7 \mathrm{~s}$ & $(5 / 2)^{2}[5 / 2]_{3}$ & & & R1c & & \\
\hline $11009.932(6)$ & $9080.221(5)$ & $11009.932(4)$ & -0.000 & 3200 & & $5 d$ & $(3 / 2)^{2}[3 / 2]_{2}$ & $5 \mathrm{f}$ & $(3 / 2)^{2}[5 / 2]^{\circ} 3$ & $2.9 \mathrm{e}+07$ & $\mathrm{D}+$ & F_Re & TW & \\
\hline $11027.380(13)$ & 9065.854(11) & $11027.388(3)$ & -0.008 & 190 & & $5 \mathrm{~d}$ & $(5 / 2)^{2}[5 / 2]_{3}$ & $5 \mathrm{f}$ & $(5 / 2)^{2}[5 / 2]^{\circ} 2$ & $1.6 \mathrm{e}+06$ & $\mathrm{D}+$ & Ric & TW & \\
\hline $11035.977(10)$ & $9058.792(8)$ & $11035.978(4)$ & -0.002 & 2700 & & $5 \mathrm{~d}$ & $(5 / 2)^{2}[5 / 2]_{3}$ & $5 \mathrm{f}$ & $(5 / 2)^{2}[5 / 2]^{\circ} 3$ & $1.6 \mathrm{e}+07$ & $\mathrm{D}+$ & R1c & TW & \\
\hline $11098.345(11)$ & $9007.885(9)$ & $11098.352(3)$ & -0.006 & 150 & & $5 d$ & $(5 / 2)^{2}[5 / 2]_{3}$ & $5 \mathrm{f}$ & $(5 / 2)^{2}[3 / 2]^{\circ} 2$ & $4.5 \mathrm{e}+06$ & $\mathrm{D}+$ & R1c & TW & \\
\hline $11100.523(11)$ & 9006.118(9) & $11100.529(5)$ & -0.006 & 150 & & $5 \mathrm{~d}$ & $(3 / 2)^{2}[3 / 2]_{2}$ & $5 \mathrm{f}$ & $(3 / 2)^{2}[3 / 2]^{\circ} 2$ & $1.1 \mathrm{e}+07$ & $\mathrm{D}+$ & R1c & TW & \\
\hline $11114.771(5)$ & 8994.573(4) & $11114.773(3)$ & -0.001 & 2500 & & $5 \mathrm{~d}$ & $(5 / 2)^{2}[7 / 2]_{3}$ & $5 \mathrm{f}$ & $(5 / 2)^{2}[9 / 2]^{\circ} 4$ & $2.4 \mathrm{e}+07$ & $\mathrm{D}+$ & F_Re & TW & \\
\hline $11121.559(10)$ & 8989.083(8) & $11121.558(3)$ & 0.001 & 3300 & & $5 d$ & $(5 / 2)^{2}[7 / 2]_{3}$ & $5 \mathrm{f}$ & $(5 / 2)^{2}[7 / 2]^{\circ}{ }_{4}$ & $9 . e+06$ & $\mathrm{E}$ & R1c & TW & \\
\hline $11123.1488(20)$ & $8987.7987(16)$ & $11123.1487(18)$ & 0.0001 & 6400 & & $5 \mathrm{~d}$ & $(5 / 2)^{2}[7 / 2]_{4}$ & $5 \mathrm{f}$ & $(5 / 2)^{2}[9 / 2]^{\circ} 5$ & $3.3 e+07$ & $\mathrm{D}+$ & F_Re & TW & \\
\hline $11124.761(11)$ & $8986.496(9)$ & $11124.755(4)$ & 0.006 & 2400 & & $5 \mathrm{~d}$ & $(5 / 2)^{2}[7 / 2]_{3}$ & $5 \mathrm{f}$ & $(5 / 2)^{2}[7 / 2]^{\circ}{ }^{\circ}$ & $1.2 \mathrm{e}+07$ & $\mathrm{D}+$ & $\mathrm{R} 1 \mathrm{c}$ & TW & \\
\hline 11125.451(5) & $8985.939(4)$ & $11125.454(4)$ & -0.003 & 3400 & & $5 d$ & $(3 / 2)^{2}[5 / 2]_{3}$ & $5 \mathrm{f}$ & $(3 / 2)^{2}[7 / 2]^{\circ}{ }_{4}$ & $3.5 \mathrm{e}+07$ & $\mathrm{D}+$ & F_Re & TW & \\
\hline $11126.754(11)$ & $8984.886(9)$ & $11126.756(3)$ & -0.001 & 2100 & & $5 \mathrm{~d}$ & $(5 / 2)^{2}[7 / 2]_{4}$ & $5 \mathrm{f}$ & $(5 / 2)^{2}[9 / 2]^{\circ} 4$ & $4.5 \mathrm{e}+06$ & $\mathrm{D}+$ & Ric & TW & \\
\hline $11133.556(12)$ & $8979.397(9)$ & $11133.556(3)$ & 0.000 & 2300 & & $5 \mathrm{~d}$ & $(5 / 2)^{2}[7 / 2]_{4}$ & $5 \mathrm{f}$ & $(5 / 2)^{2}[7 / 2]^{\circ} 4$ & $9 . \mathrm{e}+06$ & $\mathrm{D}+$ & R1c & TW & \\
\hline $11141.872(21)$ & $8972.695(17)$ & $11141.845(5)$ & 0.027 & 490 & & $\mathrm{sp}$ & $\left({ }^{3} \mathrm{~F}\right)^{1} \mathrm{P}^{\circ}{ }^{3} \mathrm{D}^{\circ}{ }_{1}$ & $7 \mathrm{~s}$ & $(3 / 2)^{2}[3 / 2]_{1}$ & & & R1c & & \\
\hline $11156.494(10)$ & $8960.935(8)$ & $11156.492(5)$ & 0.002 & 3400 & & $5 \mathrm{~d}$ & $(3 / 2)^{2}[5 / 2]_{2}$ & $5 \mathrm{f}$ & $(3 / 2)^{2}[7 / 2]^{\circ} 3$ & $3.2 \mathrm{e}+07$ & D+ & R1c & TW & \\
\hline $11173.078(11)$ & 8947.635(8) & $11173.081(4)$ & -0.003 & 2200 & & $5 \mathrm{~d}$ & $(5 / 2)^{2}[5 / 2]_{2}$ & $5 \mathrm{f}$ & $(5 / 2)^{2}[7 / 2]^{\circ} 3$ & $2.2 \mathrm{e}+07$ & $\mathrm{D}+$ & F_Re & TW & \\
\hline $11180.070(13)$ & $8942.039(11)$ & $11180.072(4)$ & -0.002 & 800 & & $5 d$ & $(3 / 2)^{2}[5 / 2]_{3}$ & $5 \mathrm{f}$ & $(3 / 2)^{2}[5 / 2]^{\circ} 3$ & $1.0 \mathrm{e}+07$ & $\mathrm{D}+$ & R1c & TW & \\
\hline $11190.552(13)$ & $8933.663(11)$ & $11190.534(4)$ & 0.018 & 94 & & $5 \mathrm{~d}$ & $(5 / 2)^{2}[7 / 2]_{4}$ & $5 \mathrm{f}$ & $(5 / 2)^{2}[5 / 2]^{\circ}{ }_{3}$ & $2.0 \mathrm{e}+06$ & $\mathrm{D}+$ & R1c & TW & \\
\hline $11202.751(12)$ & 8923.935(9) & $11202.717(5)$ & 0.034 & 350 & & $5 \mathrm{~d}$ & $(3 / 2)^{2}[5 / 2]_{2}$ & $5 \mathrm{f}$ & $(3 / 2)^{2}[5 / 2]^{\circ} 2_{2}$ & $1.1 \mathrm{e}+07$ & $\mathrm{D}+$ & R1c & TW & \\
\hline $11208.490(13)$ & 8919.366(11) & $11208.425(5)$ & 0.065 & 84 & & $5 d$ & $(3 / 2)^{2}[5 / 2]_{2}$ & $5 \mathrm{f}$ & $(3 / 2)^{2}[5 / 2]^{\circ} 3$ & $1.2 \mathrm{e}+06$ & D+ & R1c & TW & $\mathrm{x}$ \\
\hline $11218.324(11)$ & $8911.547(9)$ & $11218.317(4)$ & 0.007 & 1200 & & $5 \mathrm{~d}$ & $(5 / 2)^{2}[5 / 2]_{2}$ & $5 \mathrm{f}$ & $(5 / 2)^{2}[5 / 2]^{\circ} 2$ & $1.6 \mathrm{e}+07$ & $\mathrm{D}+$ & R1c & TW & \\
\hline 11227.209(11) & $8904.495(9)$ & $11227.208(4)$ & 0.000 & 1100 & & $5 \mathrm{~d}$ & $(5 / 2)^{2}[5 / 2]_{2}$ & $5 \mathrm{f}$ & $(5 / 2)^{2}[5 / 2]^{\circ}{ }_{3}$ & $5.4 \mathrm{e}+06$ & $\mathrm{D}+$ & R1c & TW & \\
\hline 23063.7 & 4335.82 & 23063.701(9) & & & : & $4 \mathrm{~s}$ & ${ }^{3} \mathrm{D}_{3}$ & $4 \mathrm{~s}$ & ${ }^{1} \mathrm{D}_{2}$ & $2.0 \mathrm{e}-01$ & $\mathrm{C}+$ & & TW,G64 & M1 \\
\hline 29261.7 & 3417.43 & $29261.733(10)$ & & & $:$ & $4 \mathrm{~s}$ & ${ }^{3} \mathrm{D}_{2}$ & $4 \mathrm{~s}$ & ${ }^{1} \mathrm{D}_{2}$ & $1.55 \mathrm{e}-02$ & $\mathrm{C}+$ & & TW,G64 & M1 \\
\hline 44127.2 & 2266.18 & $44127.15(3)$ & & & : & $4 \mathrm{~s}$ & ${ }^{3} \mathrm{D}_{1}$ & $4 \mathrm{~s}$ & ${ }^{1} \mathrm{D}_{2}$ & $2.7 \mathrm{e}-02$ & $\mathrm{C}+$ & & TW,G64 & M1 \\
\hline
\end{tabular}


Table A1. Cont.

\begin{tabular}{|c|c|c|c|c|c|c|c|c|c|c|c|c|c|c|}
\hline$\lambda_{\mathrm{obs}}{ }^{\mathrm{a}}(\AA)$ & $\sigma_{\mathrm{obs}}^{\mathrm{b}}\left(\mathrm{cm}^{-1}\right)$ & $\lambda_{\text {Ritz }}{ }^{c}(\AA)$ & $\begin{array}{c}\Delta \lambda_{\text {obs-Ritz }} \\
\text { (A) }\end{array}$ & $\begin{array}{c}I_{\text {obs }} \mathrm{d} \\
\text { (arb. u.) }\end{array}$ & Char ${ }^{\mathrm{e}}$ & & Lower Level & & Upper Level & $A\left(\mathrm{~s}^{-1}\right)$ & $\operatorname{Acc}^{f}$ & Line Ref. $\mathrm{g}$ & TP Ref. $\mathrm{g}$ & Notes $h$ \\
\hline 48317.6 & 2069.639 & $48317.60(4)$ & & & : & $4 \mathrm{~s}$ & ${ }^{3} \mathrm{D}_{3}$ & $4 \mathrm{~s}$ & ${ }^{3} \mathrm{D}_{1}$ & $9 . e-08$ & E & & TW & E2 \\
\hline 86861.8 & 1151.254 & $86861.79(7)$ & & & & $4 \mathrm{~s}$ & ${ }^{3} \mathrm{D}_{2}$ & $4 \mathrm{~s}$ & ${ }^{3} \mathrm{D}_{1}$ & $5.6 \mathrm{e}-02$ & $\mathrm{C}+$ & & TW,G64 & M1 \\
\hline 108887 & 918.385 & 108886.80(16) & & & : & $4 \mathrm{~s}$ & ${ }^{3} \mathrm{D}_{3}$ & $4 \mathrm{~s}$ & ${ }^{3} \mathrm{D}_{2}$ & $1.8 \mathrm{e}-02$ & $C+$ & & TW,G64 & M1 \\
\hline
\end{tabular}

a Observed wavelength between $2000 \AA$ and $20000 \AA$ is given in standard air; outside of this region, it is in vacuum. The standard uncertainty in the last decimal place is given in parentheses after the value. Conversion from air to vacuum was made using the five-parameter formula from Peck and Reeder [38]

b Observed wavenumber in vacuum;

c Ritz wavelength and its uncertainty were obtained in the least-squares level optimization procedure using the LOPT code [39]. For lines that alone determine one of the energy levels of the transition, this column is blank;

d Observed intensities from different experiments have been normalized to a uniform scale (see text). They are proportional to the energy flux under the line profile and have uncertainties of a factor of three on average. Intensities of parity-forbidden transitions are given on a different scale, since most of them were observed only in nebulas;

e Line character code: bl—blended line; $\mathrm{p}$-perturbed by a close line; * - the given intensity value is shared by two or more transitions; $\mathrm{m}$-masked by another strong line (no wavelength measurement available); - - the value given in the observed wavelength column is a rounded Ritz wavelength (no wavelength measurement available); ?-questionable identification; f Transition probability accuracy code: A+—transition probability uncertainty is likely $\leq 2 \%$; $\mathrm{B}+-\leq 7 \%$; $\mathrm{B}-\leq 7 \%$; $\mathrm{C}+-\leq 18 \%$; $\mathrm{C}-\leq 25 \%$; $\mathrm{D}-\leq 50 \%$; E- $>50 \%$;

g Key to observed wavelength and transition probability references: A73-Aller et al. [4]; A08—Andersson et al. [40]; B00-Biémont et al. [41]; B09—Brown et al. [42]; C84-Cederquist et al. [43]; C94—Crespo López-Urrutia et al. [44]; D05—Dong and Fritzsche [45]; G64—Garstang [46]; H71—Hefferlin et al. [47]; K66—Kaufman and Ward [15]; K82—Kono and Hattori [48]; M97-McKenna et al. [5]; N88—Neger and Jäger [49]; O07—Ortiz et al. [50]; P84—Prior [37]; P97—Pinnington et al. [51]; R1—Ross [2]; R2—Ross [16]; S36—Shenstone [1]; T53-Thackeray [3]; W93-Wagatsuma and Hirokawa [36]; F-this work, FTS measurements with Cu/Ge/Pt/Ar hollow cathode; F Re-this work, measurements with Cu/Re/Ar

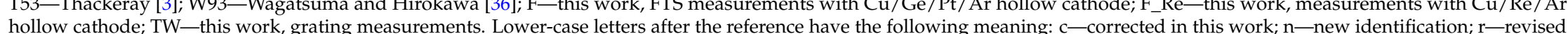
hollow cathode; TW-this work, grating measurements. Lower-case letters after the reference have the following meaning: c-corimed
identification; cal-calculated $A$-value; se- $A$-value was semiempirically adjusted by ratio of observed and calculated lifetime;

${ }^{\mathrm{h}}$ Notes: X—excluded from level-optimization procedure; L-lasing line; M1—magnetic-dipole transition; E2—electric-quadrupole transition; HF-hyperfine-induced transition. 
Table A2. Energy levels of Cu II.

\begin{tabular}{|c|c|c|c|c|c|c|c|c|c|c|}
\hline & Label $^{\text {a }}$ & Configuration & Term & $J$ & Level $^{\mathrm{b}}, \mathrm{cm}^{-1}$ & Unc. ${ }^{\mathrm{c}}, \mathrm{cm}^{-1}$ & Landé $g^{\mathrm{d}}$ & Leading Percentages ${ }^{\mathrm{e}}$ & Note $^{f}$ & $N_{\text {lines }} \mathrm{g}$ \\
\hline $\mathrm{d}^{10}$ & ${ }^{1} \mathrm{~S}$ & $3 d^{10}$ & ${ }^{1} \mathrm{~S}$ & 0 & 0.000 & 0.017 & & $97 \%+2 \% 4 d^{1} S$ & & 15 \\
\hline $4 \mathrm{~s}$ & ${ }^{3} \mathrm{D}$ & $3 d^{9} 4 s$ & ${ }^{3} \mathrm{D}$ & 3 & 21928.7326 & 0.0014 & 1.32 & $98 \%$ & & 59 \\
\hline $4 \mathrm{~s}$ & ${ }^{3} \mathrm{D}$ & $3 d^{9} 4 s$ & ${ }^{3} \mathrm{D}$ & 2 & 22847.1176 & - & 1.16 & $89 \%+9 \% 4 s^{1} \mathrm{D}$ & & 77 \\
\hline $4 \mathrm{~s}$ & ${ }^{3} \mathrm{D}$ & $3 d^{9} 4 s$ & ${ }^{3} \mathrm{D}$ & 1 & 23998.3718 & 0.0009 & 0.48 & $98 \%$ & & 48 \\
\hline $4 \mathrm{~s}$ & ${ }^{1} \mathrm{D}$ & $3 d^{9} 4 s$ & ${ }^{1} \mathrm{D}$ & 2 & 26264.5502 & 0.0012 & 1.00 & $89 \%+9 \% 4 s^{3} D$ & & 64 \\
\hline $4 p$ & ${ }^{3} \mathrm{P}^{\circ}$ & $3 d^{9} 4 p$ & ${ }^{3} \mathrm{P}^{\circ}$ & 2 & 66418.6849 & 0.0014 & 1.49 & $96 \%+2 \% 4 p^{3} D^{\circ}$ & & 31 \\
\hline $4 \mathrm{p}$ & ${ }^{3} \mathrm{P}^{\circ}$ & $3 d^{9} 4 p$ & ${ }^{3} \mathrm{P}^{\circ}$ & 1 & 67916.5572 & 0.0011 & 1.49 & $95 \%+2 \% 4 p^{1} \mathrm{P}^{\circ}$ & & 42 \\
\hline $4 \mathrm{p}$ & ${ }^{3} \mathrm{~F}^{\circ}$ & $3 d^{9} 4 p$ & ${ }^{3} \mathrm{~F}^{\circ}$ & 3 & 68447.7349 & 0.0013 & 1.06 & $62 \%+34 \% 4 p^{1} \mathrm{~F}^{\circ}$ & & 32 \\
\hline $4 \mathrm{p}$ & ${ }^{3} \mathrm{~F}^{\circ}$ & $3 d^{9} 4 p$ & ${ }^{3} \mathrm{~F}^{\circ}$ & 4 & 68730.8876 & 0.0017 & 1.23 & $98 \%$ & & 23 \\
\hline $4 \mathrm{p}$ & ${ }^{3} \mathrm{P}^{\circ}$ & $3 d^{9} 4 p$ & ${ }^{3} \mathrm{P}^{\circ}$ & 0 & 68850.2628 & 0.0014 & & $98 \%$ & & 12 \\
\hline $\mathrm{s}^{2}$ & ${ }^{3} \mathrm{~F}$ & $3 d^{8} 4 s^{2}$ & ${ }^{3} \mathrm{~F}$ & 4 & 69704.7015 & 0.0019 & & $95 \%+4 \% \mathrm{p}^{2}\left({ }^{3} \mathrm{~F}\right)^{1} \mathrm{~S}^{3} \mathrm{~F}$ & & 32 \\
\hline $4 p$ & ${ }^{3} \mathrm{~F}^{\circ}$ & $3 d^{9} 4 p$ & ${ }^{3} \mathrm{~F}^{\circ}$ & 2 & 69867.9849 & 0.0011 & 0.67 & $88 \%+6 \% 4 p^{3} \mathrm{D}^{\circ}$ & & 37 \\
\hline $4 \mathrm{p}$ & ${ }^{1} \mathrm{~F}^{\circ}$ & $3 d^{9} 4 p$ & ${ }^{1} \mathrm{~F}^{\circ}$ & 3 & 70841.4669 & 0.0014 & & $49 \%+31 \% 4 p^{3} D^{\circ}+17 \% 4 p^{3} F^{\circ}$ & & 40 \\
\hline $4 \mathrm{p}$ & ${ }^{1} \mathrm{D}^{\circ}$ & $3 d^{9} 4 p$ & ${ }^{1} \mathrm{D}^{\circ}$ & 2 & 71493.8548 & 0.0007 & 1.08 & $54 \%+35 \% 4 p^{3} D^{\circ}+9 \% 4 p^{3} \mathrm{~F}^{\circ}$ & & 36 \\
\hline $\mathrm{s}^{2}$ & ${ }^{3} \mathrm{~F}$ & $3 d^{8} 4 s^{2}$ & ${ }^{3} \mathrm{~F}$ & 3 & 71531.542 & 0.003 & & $95 \%+4 \% \mathrm{p}^{2}\left({ }^{3} \mathrm{~F}\right)^{1} \mathrm{~S}^{3} \mathrm{~F}$ & & 57 \\
\hline $4 p$ & ${ }^{3} \mathrm{D}^{\circ}$ & $3 d^{9} 4 p$ & ${ }^{3} \mathrm{D}^{\circ}$ & 3 & 71920.0961 & 0.0008 & & $64 \%+19 \% 4 p^{3} \mathrm{~F}^{\circ}+15 \% 4 \mathrm{p}^{1} \mathrm{~F}^{\circ}$ & & 36 \\
\hline$s^{2}$ & ${ }^{3} \mathrm{~F}$ & $3 d^{8} 4 s^{2}$ & ${ }^{3} \mathrm{~F}$ & 2 & 72723.817 & 0.004 & & $94 \%+4 \% \mathrm{p}^{2}\left({ }^{3} \mathrm{~F}\right)^{1} \mathrm{~S}^{3} \mathrm{~F}$ & & 52 \\
\hline $4 \mathrm{p}$ & ${ }^{3} \mathrm{D}^{\circ}$ & $3 d^{9} 4 p$ & ${ }^{3} \mathrm{D}^{\circ}$ & 1 & 73102.0408 & 0.0007 & 0.47 & $92 \%+3 \% 4 p^{1} \mathrm{P}^{\circ}$ & & 31 \\
\hline $4 \mathrm{p}$ & ${ }^{3} \mathrm{D}^{\circ}$ & $3 d^{9} 4 p$ & ${ }^{3} \mathrm{D}^{\circ}$ & 2 & 73353.2957 & 0.0008 & 0.99 & $54 \%+41 \% 4 p^{1} D^{\circ}$ & & 39 \\
\hline $4 \mathrm{p}$ & ${ }^{1} \mathrm{P}^{\circ}$ & $3 d^{9} 4 p$ & ${ }^{1} \mathrm{P}^{\circ}$ & 1 & 73595.8143 & 0.0018 & 1.04 & $93 \%+4 \% 4 p^{3} D^{\circ}$ & & 36 \\
\hline$s^{2}$ & ${ }^{1} \mathrm{D}$ & $3 d^{8} 4 s^{2}$ & ${ }^{1} \mathrm{D}$ & 2 & 85388.772 & 0.003 & & $73 \%+21 \% \mathrm{~s}^{23} \mathrm{P}$ & & 49 \\
\hline$s^{2}$ & ${ }^{3} \mathrm{P}$ & $3 d^{8} 4 s^{2}$ & ${ }^{3} \mathrm{P}$ & 2 & 88362.001 & 0.003 & & $74 \%+21 \% \mathrm{~s}^{21} \mathrm{D}$ & & 44 \\
\hline$s^{2}$ & ${ }^{3} \mathrm{P}$ & $3 d^{8} 4 s^{2}$ & ${ }^{3} \mathrm{P}$ & 1 & 88605.096 & 0.004 & & $95 \%+4 \% \mathrm{p}^{2}\left({ }^{3} \mathrm{P}\right){ }^{1} \mathrm{~S}^{3} \mathrm{P}$ & & 38 \\
\hline$s^{2}$ & ${ }^{3} \mathrm{P}$ & $3 d^{8} 4 s^{2}$ & ${ }^{3} \mathrm{P}$ & 0 & 88926.002 & 0.003 & & $95 \%+4 \% \mathrm{p}^{2}\left({ }^{3} \mathrm{P}\right)^{1} \mathrm{~S}^{3} \mathrm{P}$ & & 17 \\
\hline$s^{2}$ & ${ }^{1} \mathrm{G}$ & $3 d^{8} 4 s^{2}$ & ${ }^{1} \mathrm{G}$ & 4 & 95565.619 & 0.003 & 0.98 & $95 \%+4 \% \mathrm{p}^{2}\left({ }^{1} \mathrm{G}\right)^{1} \mathrm{~S}^{1} \mathrm{G}$ & & 18 \\
\hline $\mathrm{sp}$ & $\left({ }^{3} \mathrm{~F}\right)^{3} \mathrm{P}^{\circ}{ }^{5} \mathrm{D}^{\circ}$ & $3 d^{8}\left({ }^{3} \mathrm{~F}\right) 4 \mathrm{~s} 4 \mathrm{p}\left({ }^{3} \mathrm{P}^{\circ}\right)$ & ${ }^{5} \mathrm{D}^{\circ}$ & 4 & 107942.795 & 0.010 & & $93 \%+4 \% \operatorname{sp}\left({ }^{3} \mathrm{P}\right)^{3} \mathrm{P}^{\circ}{ }^{5} \mathrm{D}^{\circ}$ & & 2 \\
\hline $5 s$ & $(5 / 2)^{2}[5 / 2]$ & $3 \mathrm{~d}^{9}\left({ }^{2} \mathrm{D}_{5 / 2}\right) 5 \mathrm{~s}$ & ${ }^{2}[5 / 2]$ & 3 & 108014.8372 & 0.0012 & & $100 \%$ & & 48 \\
\hline $5 s$ & $(5 / 2)^{2}[5 / 2]$ & $3 d^{9}\left({ }^{2} D_{5 / 2}\right) 5 s$ & $2[5 / 2]$ & 2 & 108335.6078 & 0.0012 & & $98 \%+2 \% 5 s(3 / 2)^{2}[3 / 2]$ & & 48 \\
\hline $\mathrm{sp}$ & $\left({ }^{3} \mathrm{~F}\right)^{3} \mathrm{P}^{\circ}{ }^{5} \mathrm{D}^{\circ}$ & $3 d^{8}\left({ }^{3} \mathrm{~F}\right) 4 s 4 p\left({ }^{3} \mathrm{P}^{\circ}\right)$ & ${ }^{5} \mathrm{D}^{\circ}$ & 3 & 109276.015 & 0.006 & & $91 \%+4 \% \operatorname{sp}\left({ }^{3} \mathrm{P}\right)^{3} \mathrm{P}^{\circ}{ }^{5} \mathrm{D}^{\circ}$ & & 8 \\
\hline $5 \mathrm{~s}$ & $(3 / 2)^{2}[3 / 2]$ & $3 \mathrm{~d}^{9}\left({ }^{2} \mathrm{D}_{3 / 2}\right) 5 \mathrm{~s}$ & ${ }^{2}[3 / 2]$ & 1 & 110084.4773 & 0.0011 & & $100 \%$ & & 34 \\
\hline $\mathrm{sp}$ & $\left({ }^{3} \mathrm{~F}\right)^{3} \mathrm{P}^{\circ}{ }^{5} \mathrm{D}^{\circ}$ & $3 \mathrm{~d}^{8}\left({ }^{3} \mathrm{~F}\right) 4 \mathrm{~s} 4 \mathrm{p}\left({ }^{3} \mathrm{P}^{\circ}\right)$ & ${ }^{5} \mathrm{D}^{\circ}$ & 2 & 110363.725 & 0.008 & & $91 \%+5 \%$ sp $\left({ }^{3} \mathrm{P}\right)^{3} \mathrm{P}^{\circ}{ }^{5} \mathrm{D}^{\circ}$ & & 4 \\
\hline $5 s$ & $(3 / 2)^{2}[3 / 2]$ & $3 d^{9}\left({ }^{2} D_{3 / 2}\right) 5 s$ & ${ }^{2}[3 / 2]$ & 2 & 110366.1542 & 0.0011 & & $98 \%+2 \% 5 s(5 / 2)^{2}[5 / 2]$ & & 47 \\
\hline $\mathrm{sp}$ & $\left({ }^{3} \mathrm{~F}\right)^{3} \mathrm{P}^{\circ 5} \mathrm{G}^{\circ}$ & $3 \mathrm{~d}^{8}\left({ }^{3} \mathrm{~F}\right) 4 \mathrm{~s} 4 \mathrm{p}\left({ }^{3} \mathrm{P}^{\circ}\right)$ & ${ }^{5} \mathrm{G}^{\circ}$ & 5 & 110631.196 & 0.009 & & $82 \%+13 \%$ sp $\left({ }^{3} \mathrm{~F}\right)^{3} \mathrm{P}^{\circ}{ }^{5} \mathrm{~F}^{\circ}$ & & 1 \\
\hline $\mathrm{sp}$ & $\left({ }^{3} \mathrm{~F}\right)^{3} \mathrm{P}^{\circ}{ }^{5} \mathrm{D}^{\circ}$ & $3 d^{8}\left({ }^{3} \mathrm{~F}\right) 4 \mathrm{~s} 4 \mathrm{p}\left({ }^{3} \mathrm{p}^{\circ}\right)$ & ${ }^{5} \mathrm{D}^{\circ}$ & 1 & 111124.39 & 0.14 & & $93 \%+5 \% \operatorname{sp}\left({ }^{3} \mathrm{P}\right)^{3} \mathrm{P}^{\circ}{ }^{5} \mathrm{D}^{\circ}$ & & 1 \\
\hline $\mathrm{sp}$ & $\left({ }^{3} \mathrm{~F}\right)^{3} \mathrm{P}^{\circ} \mathrm{G}^{\circ}$ & $3 \mathrm{~d}^{8}\left({ }^{3} \mathrm{~F}\right) 4 \mathrm{~s} 4 \mathrm{p}\left({ }^{3} \mathrm{P}^{\circ}\right)$ & ${ }^{5} \mathrm{G}^{\circ}$ & 4 & 111218.705 & 0.008 & & $83 \%+10 \%$ sp $\left({ }^{3} \mathrm{~F}\right)^{3} \mathrm{P}^{\circ}{ }^{5} \mathrm{~F}^{\circ}+5 \%$ sp $\left({ }^{3} \mathrm{~F}\right)^{3} \mathrm{P}^{\circ}{ }^{3} \mathrm{G}^{\circ}$ & & 2 \\
\hline $\mathrm{sp}$ & $\left({ }^{3} \mathrm{~F}\right)^{3} \mathrm{P}^{\circ} \mathrm{G}^{\circ}$ & $3 \mathrm{~d}^{8}\left({ }^{3} \mathrm{~F}\right) 4 \mathrm{~s} 4 \mathrm{p}\left({ }^{3} \mathrm{P}^{\circ}\right)$ & ${ }^{5} \mathrm{G}^{\circ}$ & 3 & 111876.412 & 0.008 & & $89 \%+7 \%$ sp $\left({ }^{3} \mathrm{~F}\right)^{3} \mathrm{P}^{\circ}{ }^{5} \mathrm{~F}^{\circ}$ & & 3 \\
\hline sp & $\left({ }^{3} \mathrm{~F}\right)^{3} \mathrm{P}^{\circ}{ }^{5} \mathrm{~F}^{\circ}$ & $3 \mathrm{~d}^{8}\left({ }^{3} \mathrm{~F}\right) 4 \mathrm{~s} 4 \mathrm{p}\left({ }^{3} \mathrm{P}^{\circ}\right)$ & ${ }^{5} \mathrm{~F}^{\circ}$ & 5 & 112401.632 & 0.022 & & $86 \%+12 \% \operatorname{sp}\left({ }^{3} \mathrm{~F}\right)^{3} \mathrm{P}^{\circ}{ }^{5} \mathrm{G}^{\circ}$ & & 1 \\
\hline sp & $\left({ }^{3}\right)^{3} \mathrm{P}^{\circ} 5^{5} \mathrm{G}^{\circ}$ & $3 d^{8}\left({ }^{3} \mathrm{~F}\right) 4 \mathrm{~s} 4 \mathrm{p}\left({ }^{3} \mathrm{P}^{\circ}\right)$ & ${ }^{5} \mathrm{G}^{\circ}$ & 2 & 112424.679 & 0.023 & & $95 \%+3 \%$ sp $\left({ }^{3} \mathrm{~F}\right)^{3} \mathrm{P}^{\circ}{ }^{5} \mathrm{~F}^{\circ}$ & & 2 \\
\hline $\mathrm{sp}$ & $\left({ }^{3} \mathrm{~F}\right)^{3} \mathrm{P}^{\circ} 5^{5} \mathrm{~F}^{\circ}$ & $3 d^{8}\left({ }^{3} \mathrm{~F}\right) 4 s 4 p\left({ }^{3} \mathrm{p}^{\circ}\right)$ & ${ }^{5} \mathrm{~F}^{\circ}$ & 4 & 113302.823 & 0.006 & & $84 \%+9 \%$ sp $\left({ }^{3} \mathrm{~F}\right)^{3} \mathrm{P}^{\circ 5} \mathrm{G}^{\circ}$ & & 5 \\
\hline $\mathrm{sp}$ & $\left({ }^{3} \mathrm{~F}\right)^{3} \mathrm{P}^{\circ} \mathrm{F}^{\circ}$ & $3 \mathrm{~d}^{8}\left({ }^{3} \mathrm{~F}\right) 4 \mathrm{~s} 4 \mathrm{p}\left({ }^{3} \mathrm{P}^{\circ}\right)$ & ${ }^{5} \mathrm{~F}^{\circ}$ & 3 & 114000.452 & 0.007 & & $86 \%+7 \%$ sp $\left({ }^{3} \mathrm{~F}\right)^{3} \mathrm{P}^{\circ 5} \mathrm{G}^{\circ}$ & & 5 \\
\hline
\end{tabular}


Table A2. Cont.

\begin{tabular}{|c|c|c|c|c|c|c|c|c|c|c|}
\hline & Label $^{\text {a }}$ & Configuration & Term & $J$ & Level $^{\mathrm{b}}, \mathrm{cm}^{-1}$ & Unc. ${ }^{\mathrm{c}}, \mathrm{cm}^{-1}$ & Landé $g^{\mathrm{d}}$ & Leading Percentages ${ }^{\mathrm{e}}$ & Note ${ }^{\mathrm{f}}$ & $N_{\text {lines }}{ }^{8}$ \\
\hline $\mathrm{sp}$ & $\left({ }^{3} \mathrm{~F}\right)^{3} \mathrm{P}^{\circ}{ }^{5} \mathrm{~F}^{\circ}$ & $3 d^{8}\left({ }^{3} F\right) 4 s 4 p\left({ }^{3} P^{\circ}\right)$ & ${ }^{5} \mathrm{~F}^{\circ}$ & 2 & 114481.674 & 0.006 & & $92 \%+3 \%$ sp $\left({ }^{3} \mathrm{~F}\right)^{3} \mathrm{P}^{\circ}{ }^{5} \mathrm{G}^{\circ}$ & & 6 \\
\hline $4 \mathrm{~d}$ & $(5 / 2)^{2}[1 / 2]$ & $3 \mathrm{~d}^{9}\left({ }^{2} \mathrm{D}_{5 / 2}\right) 4 \mathrm{~d}$ & ${ }^{2}[1 / 2]$ & 1 & 114511.2386 & 0.0012 & & $91 \%+7 \% 4 \mathrm{~d}(3 / 2)^{2}[1 / 2]+1 \% 4 \mathrm{~d} 2$ & & 39 \\
\hline $\mathrm{sp}$ & $\left({ }^{3} \mathrm{~F}\right)^{3} \mathrm{P}^{0}{ }^{5} \mathrm{~F}^{0}$ & $3 d^{8}\left({ }^{3} F\right) 4 s 4 p\left({ }^{3} P^{\circ}\right)$ & ${ }^{5} \mathrm{~F}^{\circ}$ & 1 & 114755.953 & 0.011 & & $97 \%$ & & 3 \\
\hline $\mathrm{sp}$ & $\left({ }^{3} \mathrm{~F}\right)^{3} \mathrm{P}^{\circ}{ }^{3} \mathrm{G}^{\circ}$ & $3 \mathrm{~d}^{8}\left({ }^{3} \mathrm{~F}\right) 4 \mathrm{~s} 4 \mathrm{p}\left({ }^{3} \mathrm{P}^{\circ}\right)$ & ${ }^{3} G^{\circ}$ & 4 & 115359.532 & 0.005 & & $71 \%+20 \% \operatorname{sp}\left({ }^{3} \mathrm{~F}\right)^{3} \mathrm{P}^{\circ}{ }^{1} \mathrm{G}^{\circ}+6 \% \operatorname{sp}\left({ }^{3} \mathrm{~F}\right)^{3} \mathrm{P}^{\circ}{ }^{5} \mathrm{G}^{\circ}$ & & 7 \\
\hline $\mathrm{sp}$ & $\left({ }^{3} \mathrm{~F}\right)^{3} \mathrm{P}^{\circ}{ }^{3} \mathrm{G}^{\circ}$ & $3 d^{8}\left({ }^{3} F\right) 4 s 4 p\left({ }^{3} P^{\circ}\right)$ & ${ }^{3} \mathrm{G}^{\circ}$ & 5 & 115546.114 & 0.011 & & $93 \%+6 \% \operatorname{sp}\left({ }^{3} \mathrm{~F}\right)^{3} \mathrm{P}^{\circ}{ }^{5} \mathrm{G}^{\circ}$ & & 1 \\
\hline $4 \mathrm{~d}$ & $(5 / 2)^{2}[9 / 2]$ & $3 \mathrm{~d}^{9}\left({ }^{2} \mathrm{D}_{5 / 2}\right) 4 \mathrm{~d}$ & ${ }^{2}[9 / 2]$ & 5 & 115568.99497 & 0.0012 & & $99 \%$ & & 22 \\
\hline $4 \mathrm{~d}$ & $(5 / 2)^{2}[3 / 2]$ & $3 d^{9}\left({ }^{2} D_{5 / 2}\right) 4 d$ & ${ }^{2}[3 / 2]$ & 2 & 115638.8036 & 0.0011 & & $93 \%+4 \% 4 d(5 / 2)^{2}[5 / 2]+2 \% 4 d(3 / 2)^{2}[3 / 2]$ & & 42 \\
\hline $4 d$ & $(5 / 2)^{2}[9 / 2]$ & $3 d^{9}\left({ }^{2} D_{5 / 2}\right) 4 d$ & ${ }^{2}[9 / 2]$ & 4 & 115662.5622 & 0.0014 & & $97 \%+1 \% 4 d(5 / 2)^{2}[7 / 2]$ & & 32 \\
\hline $4 \mathrm{~d}$ & $(5 / 2)^{2}[3 / 2]$ & $3 d^{9}\left({ }^{2} D_{5 / 2}\right) 4 d$ & ${ }^{2}[3 / 2]$ & 1 & 115665.1539 & 0.0011 & & $97 \%+1 \% 4 \mathrm{~d}(3 / 2)^{2}[1 / 2]+1 \% 4 \mathrm{~d}(3 / 2)^{2}[3 / 2]$ & & 30 \\
\hline $4 \mathrm{~d}$ & $(5 / 2)^{2}[5 / 2]$ & $3 d^{9}\left({ }^{2} D_{5 / 2}\right) 4 d$ & ${ }^{2}[5 / 2]$ & 3 & 116080.2237 & 0.0010 & & $97 \%+1 \% 4 d(5 / 2)^{2}[7 / 2]+1 \% 4 d(3 / 2)^{2}[5 / 2]$ & & 35 \\
\hline $4 \mathrm{~d}$ & $(5 / 2)^{2}[7 / 2]$ & $3 d^{9}\left({ }^{2} D_{5 / 2}\right) 4 d$ & ${ }^{2}[7 / 2]$ & 3 & 116325.9148 & 0.0011 & & $93 \%+4 \% 4 d(3 / 2)^{2}[7 / 2]+1 \% 4 d(5 / 2)^{2}[5 / 2]$ & & 37 \\
\hline $4 \mathrm{~d}$ & $(5 / 2)^{2}[7 / 2]$ & $3 d^{9}\left({ }^{2} D_{5 / 2}\right) 4 d$ & ${ }^{2}[7 / 2]$ & 4 & 116371.18040 & 0.0011 & & $95 \%+2 \% 4 d(3 / 2)^{2}[7 / 2]+1 \% 4 d(5 / 2)^{2}[9 / 2]$ & & 35 \\
\hline $\mathrm{sp}$ & $\left({ }^{3} \mathrm{~F}\right)^{3} \mathrm{P}^{\circ}{ }^{3} \mathrm{D}^{\circ}$ & $3 d^{8}\left({ }^{3} F\right) 4 s 4 p\left({ }^{3} P^{\circ}\right)$ & ${ }^{3} \mathrm{D}^{\circ}$ & 3 & 116375.406 & 0.003 & & $44 \%+35 \%$ sp $\left({ }^{3} \mathrm{~F}\right)^{3} \mathrm{P}^{\circ}{ }^{3} \mathrm{G}^{\circ}+13 \%$ sp $\left({ }^{3} \mathrm{~F}\right)^{3} \mathrm{P}^{\circ}{ }^{3} \mathrm{~F}^{\circ}$ & & 17 \\
\hline $4 \mathrm{~d}$ & $(5 / 2)^{2}[5 / 2]$ & $3 \mathrm{~d}^{9}\left({ }^{2} \mathrm{D}_{5 / 2}\right) 4 \mathrm{~d}$ & ${ }^{2}[5 / 2]$ & 2 & 116387.7873 & 0.0010 & & $92 \%+3 \% 4 \mathrm{~d}(3 / 2)^{2}[3 / 2]+3 \% 4 \mathrm{~d}(5 / 2)^{2}[3 / 2]$ & & 43 \\
\hline $4 \mathrm{~d}$ & $(5 / 2)^{2}[1 / 2]$ & $3 d^{9}\left({ }^{2} D_{5 / 2}\right) 4 d$ & ${ }^{2}[1 / 2]$ & 0 & 116576.5758 & 0.0018 & & $53 \%+45 \% 4 \mathrm{~d}(3 / 2)^{2}[1 / 2]$ & & 16 \\
\hline $\mathrm{sp}$ & $\left({ }^{3} \mathrm{~F}\right)^{3} \mathrm{P}^{\circ}{ }^{3} \mathrm{G}^{\circ}$ & $3 \mathrm{~d}^{8}\left({ }^{3} \mathrm{~F}\right) 4 \mathrm{~s} 4 \mathrm{p}\left({ }^{3} \mathrm{P}^{\circ}\right)$ & ${ }^{3} \mathrm{G}^{\circ}$ & 3 & 116643.960 & 0.003 & & $57 \%+36 \%$ sp $\left({ }^{3} \mathrm{~F}\right)^{3} \mathrm{P}^{\circ}{ }^{3} \mathrm{D}^{\circ}$ & & 17 \\
\hline $\mathrm{sp}$ & $\left({ }^{3} \mathrm{~F}\right)^{3} \mathrm{P}^{\circ}{ }^{3} \mathrm{D}^{\circ}$ & $3 \mathrm{~d}^{8}\left({ }^{3} \mathrm{~F}\right) 4 \mathrm{~s} 4 \mathrm{p}\left({ }^{3} \mathrm{P}^{\circ}\right)$ & ${ }^{3} \mathrm{D}^{\circ}$ & 2 & 117130.340 & 0.003 & & $75 \%+10 \% \operatorname{sp}\left({ }^{3} \mathrm{~F}\right)^{3} \mathrm{P}^{\circ}{ }^{3} \mathrm{~F}^{\circ}$ & & 27 \\
\hline $4 \mathrm{~d}$ & $(3 / 2)^{2}[1 / 2]$ & $3 d^{9}\left({ }^{2} D_{3 / 2}\right) 4 d$ & ${ }^{2}[1 / 2]$ & 1 & 117231.4014 & 0.0019 & & $91 \%+7 \% 4 \mathrm{~d}(5 / 2)^{2}[1 / 2]$ & & 28 \\
\hline $\mathrm{sp}$ & $\left({ }^{3} \mathrm{~F}\right)^{3} \mathrm{P}^{\circ}{ }^{3} \mathrm{~F}^{\circ}$ & $3 d^{8}\left({ }^{3} F\right) 4 s 4 p\left({ }^{3} P^{\circ}\right)$ & ${ }^{3} \mathrm{~F}^{\circ}$ & 4 & 117666.626 & 0.005 & & $88 \%+3 \%$ sp $\left({ }^{3} \mathrm{~F}\right)^{3} \mathrm{P}^{\circ}{ }^{5} \mathrm{~F}^{\circ}$ & & 15 \\
\hline $4 \mathrm{~d}$ & $(3 / 2)^{2}[7 / 2]$ & $3 \mathrm{~d}^{9}\left({ }^{2} \mathrm{D}_{3 / 2}\right) 4 \mathrm{~d}$ & ${ }^{2}[7 / 2]$ & 3 & 117747.3504 & 0.0015 & & $95 \%+4 \% 4 d(5 / 2)^{2}[7 / 2]$ & & 37 \\
\hline $4 \mathrm{~d}$ & $(3 / 2)^{2}[7 / 2]$ & $3 d^{9}\left({ }^{2} D_{3 / 2}\right) 4 d$ & ${ }^{2}[7 / 2]$ & 4 & 117883.0985 & 0.0013 & & $96 \%+2 \% 4 d(5 / 2)^{2}[7 / 2]$ & & 35 \\
\hline $4 \mathrm{~d}$ & $(3 / 2)^{2}[3 / 2]$ & $3 d^{9}\left({ }^{2} D_{3 / 2}\right) 4 d$ & ${ }^{2}[3 / 2]$ & 1 & 117928.2197 & 0.0018 & & $98 \%$ & & 31 \\
\hline $\mathrm{sp}$ & $\left({ }^{3} \mathrm{~F}\right)^{3} \mathrm{P}^{\circ}{ }^{3} \mathrm{D}^{\circ}$ & $3 d^{8}\left({ }^{3} F\right) 4 s 4 p\left({ }^{3} P^{\circ}\right)$ & ${ }^{3} \mathrm{D}^{\circ}$ & 1 & 118071.302 & 0.003 & & $87 \%+6 \% \operatorname{sp}\left({ }^{1} \mathrm{D}\right)^{3} \mathrm{P}^{\circ}{ }^{3} \mathrm{D}^{\circ}$ & & 22 \\
\hline $\mathrm{sp}$ & $\left({ }^{3} \mathrm{~F}\right)^{3} \mathrm{P}^{\circ}{ }^{3} \mathrm{~F}^{\circ}$ & $3 \mathrm{~d}^{8}\left({ }^{3} \mathrm{~F}\right) 4 \mathrm{~s} 4 \mathrm{p}\left({ }^{3} \mathrm{P}^{\circ}\right)$ & ${ }^{3} \mathrm{~F}^{\circ}$ & 3 & 118142.950 & 0.003 & & $63 \%+14 \% \operatorname{sp}\left({ }^{3} \mathrm{~F}\right)^{3} \mathrm{P}^{\circ}{ }^{1} \mathrm{~F}^{\circ}+10 \% \mathrm{sp}\left({ }^{3} \mathrm{~F}\right)^{3} \mathrm{P}^{\circ}{ }^{3} \mathrm{D}^{\circ}$ & & 32 \\
\hline $4 \mathrm{~d}$ & $(3 / 2)^{2}[3 / 2]$ & $3 \mathrm{~d}^{9}\left({ }^{2} \mathrm{D}_{3 / 2}\right) 4 \mathrm{~d}$ & ${ }^{2}[3 / 2]$ & 2 & 118163.2663 & 0.0015 & & $82 \%+12 \% 4 \mathrm{~d}(3 / 2)^{2}[5 / 2]+2 \% 4 \mathrm{~d}(5 / 2)^{2}[5 / 2]$ & & 42 \\
\hline $4 \mathrm{~d}$ & $(3 / 2)^{2}[5 / 2]$ & $3 \mathrm{~d}^{9}\left({ }^{2} \mathrm{D}_{3 / 2}\right) 4 \mathrm{~d}$ & ${ }^{2}[5 / 2]$ & 3 & 118483.8135 & 0.0015 & & $98 \%+1 \% 4 \mathrm{~d}(5 / 2)^{2}[5 / 2]$ & & 37 \\
\hline $4 \mathrm{~d}$ & $(3 / 2)^{2}[5 / 2]$ & $3 d^{9}\left({ }^{2} D_{3 / 2}\right) 4 d$ & ${ }^{2}[5 / 2]$ & 2 & 118531.9058 & 0.0016 & & $86 \%+12 \% 4 \mathrm{~d}(3 / 2)^{2}[3 / 2]+1 \% 4 \mathrm{~d}(5 / 2)^{2}[3 / 2]$ & & 35 \\
\hline $\mathrm{sp}$ & $\left({ }^{3} \mathrm{~F}\right)^{3} \mathrm{P}^{\mathrm{o}} \mathrm{G}^{\circ}$ & $3 d^{8}\left({ }^{3} F\right) 4 s 4 p\left({ }^{3} P^{\circ}\right)$ & ${ }^{1} \mathrm{G}^{\circ}$ & 4 & 118991.330 & 0.007 & & $75 \%+20 \% \operatorname{sp}\left({ }^{3} \mathrm{~F}\right)^{3} \mathrm{P}^{\circ}{ }^{3} \mathrm{G}^{\circ}$ & & 9 \\
\hline $\mathrm{sp}$ & $\left({ }^{3} \mathrm{~F}\right)^{3} \mathrm{P}^{\circ}{ }^{3} \mathrm{~F}^{\circ}$ & $3 \mathrm{~d}^{8}\left({ }^{3} \mathrm{~F}\right) 4 \mathrm{~s} 4 \mathrm{p}\left({ }^{3} \mathrm{P}^{\circ}\right)$ & ${ }^{3} \mathrm{~F}^{\circ}$ & 2 & 119039.6355 & 0.0019 & & $82 \%+8 \% \operatorname{sp}\left({ }^{3} \mathrm{~F}\right)^{3} \mathrm{P}^{\circ}{ }^{3} \mathrm{D}^{\circ}$ & & 34 \\
\hline $5 p$ & $(5 / 2)^{2}[3 / 2]^{\circ}$ & $3 \mathrm{~d}^{9}\left({ }^{2} \mathrm{D}_{5 / 2}\right) 5 \mathrm{p}$ & ${ }^{2}[3 / 2]^{\circ}$ & 2 & 120092.3828 & 0.0012 & & $96 \%+2 \% s p+1 \% 5 p(3 / 2)^{2}[3 / 2]^{\circ}$ & & 40 \\
\hline $5 p$ & $(5 / 2)^{2}[7 / 2]^{\circ}$ & $3 d^{9}\left({ }^{2} D_{5 / 2}\right) 5 p$ & ${ }^{2}[7 / 2]^{\circ}$ & 3 & 120684.7128 & 0.0013 & & $86 \%+13 \% \mathrm{sp}$ & & 33 \\
\hline $5 p$ & $(5 / 2)^{2}[7 / 2]^{\circ}$ & $3 d^{9}\left({ }^{2} D_{5 / 2}\right) 5 p$ & ${ }^{2}[7 / 2]^{\circ}$ & 4 & 120789.80865 & 0.0013 & & $95 \%+4 \% \mathrm{sp}$ & & 20 \\
\hline $5 p$ & $(5 / 2)^{2}[5 / 2]^{\circ}$ & $3 d^{9}\left({ }^{2} D_{5 / 2}\right) 5 p$ & ${ }^{2}[5 / 2]^{\circ}$ & 2 & 120876.0141 & 0.0015 & & $51 \%+41 \% s p+3 \% 5 p(3 / 2)^{2}[3 / 2]^{\circ}$ & & 41 \\
\hline $5 \mathrm{p}$ & $(5 / 2)^{2}[3 / 2]^{\circ}$ & $3 d^{9}\left({ }^{2} D_{5 / 2}\right) 5 p$ & ${ }^{2}[3 / 2]^{\circ}$ & 1 & 120919.5715 & 0.0012 & & $83 \%+15 \% 5 p(3 / 2)^{2}[1 / 2]^{\circ}+1 \% s p$ & & 43 \\
\hline $5 p$ & $(5 / 2)^{2}[5 / 2]^{\circ}$ & $3 d^{9}\left({ }^{2} D_{5 / 2}\right) 5 p$ & ${ }^{2}[5 / 2]^{\circ}$ & 3 & 121079.1501 & 0.0012 & & $54 \%+35 \% \mathrm{sp}+3 \% 5 p(3 / 2)^{2}[5 / 2]^{\circ}$ & & 45 \\
\hline sp & $\left({ }^{3} \mathrm{~F}\right)^{3} \mathrm{P}^{\circ}{ }^{1} \mathrm{~F}^{\circ}$ & $3 d^{8}\left({ }^{3} \mathrm{~F}\right) 4 \mathrm{~s} 4 \mathrm{p}\left({ }^{3} \mathrm{P}^{\circ}\right)$ & ${ }^{1} \mathrm{~F}^{\circ}$ & 3 & 121524.8509 & 0.0014 & & $39 \%+43 \% 5 \mathrm{p}^{3} \mathrm{D}^{\circ}+6 \% \mathrm{sp}\left({ }^{3} \mathrm{~F}\right)^{3} \mathrm{P}^{\circ}{ }^{3} \mathrm{~F}^{\circ}$ & $\mathrm{cd}$ & 37 \\
\hline $\mathrm{sp}$ & $\left({ }^{3} \mathrm{~F}\right)^{3} \mathrm{P}^{\circ}{ }^{1} \mathrm{D}^{\circ}$ & $3 \mathrm{~d}^{8}\left({ }^{3} \mathrm{~F}\right) 4 \mathrm{~s} 4 \mathrm{p}\left({ }^{3} \mathrm{P}^{\circ}\right)$ & ${ }^{1} \mathrm{D}^{\circ}$ & 2 & 121981.8546 & 0.0015 & & $36 \%+32 \% 5 p^{3} \mathrm{D}^{\circ}+22 \% 5 \mathrm{p}^{3} \mathrm{~F}^{\circ}$ & & 44 \\
\hline $5 p$ & $(3 / 2)^{2}[1 / 2]^{\circ}$ & $3 d^{9}\left({ }^{2} D_{3 / 2}\right) 5 p$ & ${ }^{2}[1 / 2]^{\circ}$ & 0 & 122224.0199 & 0.0020 & & $99 \%$ & & 13 \\
\hline $4 \mathrm{~d}$ & $(3 / 2)^{2}[1 / 2]$ & $3 d^{9}\left({ }^{2} D_{3 / 2}\right) 4 d$ & ${ }^{2}[1 / 2]$ & 0 & 122415.957 & 0.003 & & $45 \%+34 \% 4 d(5 / 2)^{2}[1 / 2]+10 \% 5 d(5 / 2)^{2}[1 / 2]$ & & 12 \\
\hline $5 p$ & $(3 / 2)^{2}[5 / 2]^{\circ}$ & $3 d^{9}\left({ }^{2} D_{3 / 2}\right) 5 p$ & ${ }^{2}[5 / 2]^{\circ}$ & 2 & 122745.9491 & 0.0013 & & $85 \%+4 \% 5 p(3 / 2)^{2}[3 / 2]^{\circ}+4 \% 5 p(5 / 2)^{2}[5 / 2]^{\circ}$ & & 39 \\
\hline
\end{tabular}


Table A2. Cont.

\begin{tabular}{|c|c|c|c|c|c|c|c|c|c|c|}
\hline & Label $^{\text {a }}$ & Configuration & Term & $\bar{J}$ & Level $^{\mathrm{b}}, \mathrm{cm}^{-1}$ & Unc. ${ }^{\mathrm{c}}, \mathrm{cm}^{-1}$ & Landé $g^{\mathrm{d}}$ & Leading Percentages $\mathrm{e}$ & Note $^{f}$ & $N_{\text {lines }} \mathrm{g}$ \\
\hline $5 p$ & $(3 / 2)^{2}[1 / 2]^{\circ}$ & $3 d^{9}\left({ }^{2} D_{3 / 2}\right) 5 p$ & ${ }^{2}[1 / 2]^{\circ}$ & 1 & 122867.7407 & 0.0015 & & $75 \%+14 \% 5 p(5 / 2)^{2}[3 / 2]^{\circ}+9 \% 5 p(3 / 2)^{2}[3 / 2]^{\circ}$ & & 39 \\
\hline $5 \mathrm{p}$ & $(3 / 2)^{2}[5 / 2]^{\circ}$ & $3 d^{9}\left({ }^{2} D_{3 / 2}\right) 5 p$ & ${ }^{2}[5 / 2]^{\circ}$ & 3 & 123016.8175 & 0.0014 & & $91 \%+7 \% \mathrm{sp}+1 \% 5 \mathrm{p}(5 / 2)^{2}[7 / 2]^{\circ}$ & & 27 \\
\hline $5 p$ & $(3 / 2)^{2}[3 / 2]^{\circ}$ & $3 d^{9}\left({ }^{2} D_{3 / 2}\right) 5 p$ & ${ }^{2}[3 / 2]^{\circ}$ & 1 & 123304.823 & 0.003 & & $86 \%+9 \% 5 p(3 / 2)^{2}[1 / 2]^{\circ}+4 \% s p$ & & 27 \\
\hline $5 \mathrm{p}$ & $(3 / 2)^{2}[3 / 2]^{\circ}$ & $3 d^{9}\left({ }^{2} D_{3 / 2}\right) 5 p$ & ${ }^{2}[3 / 2]^{\circ}$ & 2 & 123556.8261 & 0.0016 & & $76 \%+13 \% s p+3 \% 5 p(5 / 2)^{2}[5 / 2]^{\circ}$ & & 43 \\
\hline $\mathrm{sp}$ & $\left({ }^{3} \mathrm{P}\right)^{3} \mathrm{P}^{\circ}{ }^{5} \mathrm{P}^{\circ}$ & $3 \mathrm{~d}^{8}\left({ }^{3} \mathrm{P}\right) 4 \mathrm{~s} 4 \mathrm{p}\left({ }^{3} \mathrm{P}^{\circ}\right)$ & ${ }^{5} \mathrm{P}^{\circ}$ & 3 & 125230.061 & 0.017 & & $88 \%+8 \% \operatorname{sp}\left({ }^{1} \mathrm{D}\right){ }^{3} \mathrm{P}^{\circ}{ }^{3} \mathrm{D}^{\circ}$ & & 6 \\
\hline $\mathrm{sp}$ & $\left({ }^{3} \mathrm{P}\right)^{3} \mathrm{P}^{\circ}{ }^{5} \mathrm{P}^{\circ}$ & $3 d^{8}\left({ }^{3} \mathrm{P}\right) 4 \mathrm{~s} 4 \mathrm{p}\left({ }^{3} \mathrm{P}^{\circ}\right)$ & ${ }^{5} \mathrm{P}^{\circ}$ & 2 & 125248.121 & 0.008 & & $88 \%+3 \% \mathrm{sp}\left({ }^{1} \mathrm{D}\right)^{3} \mathrm{P}^{\circ}{ }^{3} \mathrm{P}^{\circ}$ & & 11 \\
\hline $\mathrm{sp}$ & $\left({ }^{3} \mathrm{P}\right)^{3} \mathrm{P}^{\circ}{ }^{5} \mathrm{P}^{\circ}$ & $3 d^{8}\left({ }^{3} \mathrm{P}\right) 4 \mathrm{~s} 4 \mathrm{p}\left({ }^{3} \mathrm{P}^{\circ}\right)$ & ${ }^{5} \mathrm{P}^{\circ}$ & 1 & 125569.33 & 0.04 & & $94 \%+2 \% \mathrm{sp}\left({ }^{1} \mathrm{D}\right)^{3} \mathrm{P}^{\circ}{ }^{3} \mathrm{P}^{\circ}$ & & 5 \\
\hline $\mathrm{sp}$ & $\left({ }^{1} \mathrm{D}\right)^{3} \mathrm{P}^{\circ}{ }^{3} \mathrm{~F}^{\circ}$ & $3 d^{8}\left({ }^{1} D\right) 4 s 4 p\left({ }^{3} P^{\circ}\right)$ & ${ }^{3} \mathrm{~F}^{\circ}$ & 2 & 128365.736 & 0.006 & & $69 \%+15 \% \operatorname{sp}\left({ }^{1} \mathrm{D}\right)^{3} \mathrm{P}^{\circ}{ }^{3} \mathrm{D}^{\circ}$ & & 10 \\
\hline $\mathrm{sp}$ & $\left({ }^{1} \mathrm{D}\right)^{3} \mathrm{P}^{\circ}{ }^{3} \mathrm{~F}^{\circ}$ & $3 d^{8}\left({ }^{1} \mathrm{D}\right) 4 \mathrm{~s} 4 \mathrm{p}\left({ }^{3} \mathrm{P}^{\circ}\right)$ & ${ }^{3} \mathrm{~F}^{\circ}$ & 3 & 128559.314 & 0.008 & & $68 \%+11 \% \operatorname{sp}\left({ }^{3} \mathrm{P}\right){ }^{3} \mathrm{P}^{\circ}{ }^{5} \mathrm{D}^{\circ}+9 \% \mathrm{sp}\left({ }^{1} \mathrm{D}\right){ }^{3} \mathrm{P}^{\circ}{ }^{3} \mathrm{D}^{\circ}$ & & 6 \\
\hline $\mathrm{sp}$ & $\left({ }^{1} \mathrm{D}\right)^{3} \mathrm{P}^{\circ}{ }^{3} \mathrm{D}^{\circ}$ & $3 d^{8}\left({ }^{1} D\right) 4 s 4 p\left({ }^{3} \mathrm{P}^{\circ}\right)$ & ${ }^{3} \mathrm{D}^{\circ}$ & 1 & 128569.150 & 0.008 & & $66 \%+9 \% \operatorname{sp}\left({ }^{1} \mathrm{D}\right)^{3} \mathrm{P}^{\circ}{ }^{3} \mathrm{P}^{\circ}+9 \% \operatorname{sp}\left({ }^{3} \mathrm{P}\right)^{3} \mathrm{P}^{\circ}{ }^{3} \mathrm{P}^{\circ}$ & & 7 \\
\hline $\mathrm{sp}$ & $\left({ }^{1} \mathrm{D}\right)^{3} \mathrm{P}^{\circ}{ }^{3} \mathrm{~F}^{\circ}$ & $3 \mathrm{~d}^{8}\left({ }^{1} \mathrm{D}\right) 4 \mathrm{~s} 4 \mathrm{p}\left({ }^{3} \mathrm{P}^{\circ}\right)$ & ${ }^{3} \mathrm{~F}^{\circ}$ & 4 & 128778.037 & 0.017 & & $64 \%+28 \% \operatorname{sp}\left({ }^{3} \mathrm{P}\right){ }^{3} \mathrm{P}^{\circ}{ }^{5} \mathrm{D}^{\circ}$ & & 2 \\
\hline $\mathrm{sp}$ & $\left({ }^{1} \mathrm{D}\right)^{3} \mathrm{P}^{\circ}{ }^{3} \mathrm{D}^{\circ}$ & $3 d^{8}\left({ }^{1} \mathrm{D}\right) 4 \mathrm{~s} 4 \mathrm{p}\left({ }^{3} \mathrm{P}^{\circ}\right)$ & ${ }^{3} \mathrm{D}^{\circ}$ & 2 & 128854.036 & 0.008 & & $59 \%+19 \% \mathrm{sp}\left({ }^{1} \mathrm{D}\right){ }^{3} \mathrm{P}^{\circ}{ }^{3} \mathrm{~F}^{\circ}$ & & 8 \\
\hline$s^{2}$ & $1_{S}$ & $3 d^{8} 4 s^{2}$ & ${ }^{1} \mathrm{~S}$ & 0 & 128910.03 & 0.06 & & $94 \%+5 \% \mathrm{p}^{2}\left({ }^{1} \mathrm{~S}\right)^{1} \mathrm{~S}^{1} \mathrm{~S}$ & $\mathrm{~N}$ & 1 \\
\hline $\mathrm{sp}$ & $\left({ }^{1} \mathrm{D}\right)^{3} \mathrm{P}^{\circ}{ }^{3} \mathrm{P}^{\circ}$ & $3 d^{8}\left({ }^{1} D\right) 4 s 4 p\left({ }^{3} P^{\circ}\right)$ & ${ }^{3} \mathrm{P}^{\circ}$ & 0 & 129105.778 & 0.009 & & $63 \%+32 \% \operatorname{sp}\left({ }^{3} \mathrm{P}\right)^{3} \mathrm{P}^{\circ}{ }^{3} \mathrm{P}^{\circ}$ & & 3 \\
\hline $\mathrm{sp}$ & $\left({ }^{1} \mathrm{D}\right)^{3} \mathrm{P}^{\circ}{ }^{3} \mathrm{D}^{\circ}$ & $3 d^{8}\left({ }^{1} D\right) 4 s 4 p\left({ }^{3} \mathrm{P}^{\circ}\right)$ & ${ }^{3} \mathrm{D}^{\circ}$ & 3 & 129116.774 & 0.011 & & $69 \%+10 \%$ sp $\left({ }^{1} \mathrm{D}\right){ }^{3} \mathrm{P}^{\circ}{ }^{3} \mathrm{~F}^{\circ}+7 \% \mathrm{sp}\left({ }^{3} \mathrm{P}\right)^{3} \mathrm{P}^{\circ}{ }^{5} \mathrm{P}^{\circ}$ & & 6 \\
\hline $\mathrm{sp}$ & $\left({ }^{1} \mathrm{D}\right)^{3} \mathrm{P}^{\circ}{ }^{3} \mathrm{P}^{\circ}$ & $3 \mathrm{~d}^{8}\left({ }^{1} \mathrm{D}\right) 4 \mathrm{~s} 4 \mathrm{p}\left({ }^{3} \mathrm{P}^{\circ}\right)$ & ${ }^{3} \mathrm{P}^{\circ}$ & 1 & 129759.750 & 0.006 & & $56 \%+19 \% \operatorname{sp}\left({ }^{1} \mathrm{D}\right){ }^{3} \mathrm{P}^{\circ}{ }^{3} \mathrm{D}^{\circ}+18 \% \operatorname{sp}\left({ }^{3} \mathrm{P}\right){ }^{3} \mathrm{P}^{\circ}{ }^{3} \mathrm{P}^{\circ}$ & & 9 \\
\hline $\mathrm{sp}$ & $\left({ }^{1} \mathrm{D}\right)^{3} \mathrm{P}^{\circ}{ }^{3} \mathrm{P}^{\circ}$ & $3 d^{8}\left({ }^{1} \mathrm{D}\right) 4 \mathrm{~s} 4 \mathrm{p}\left({ }^{3} \mathrm{P}^{\circ}\right)$ & ${ }^{3} \mathrm{P}^{\circ}$ & 2 & 130386.404 & 0.016 & & $74 \%+12 \% \mathrm{sp}\left({ }^{3} \mathrm{P}\right)^{3} \mathrm{P}^{\circ}{ }^{3} \mathrm{P}^{\circ}+8 \% \mathrm{sp}\left({ }^{1} \mathrm{D}\right)^{3} \mathrm{P}^{\circ}{ }^{3} \mathrm{D}^{\circ}$ & & 8 \\
\hline $\mathrm{sp}$ & $\left({ }^{3} \mathrm{P}\right)^{3} \mathrm{P}^{\circ}{ }^{5} \mathrm{D}^{\circ}$ & $3 \mathrm{~d}^{8}\left({ }^{3} \mathrm{P}\right) 4 \mathrm{~s} 4 \mathrm{p}\left({ }^{3} \mathrm{P}^{\circ}\right)$ & ${ }^{5} \mathrm{D}^{\circ}$ & 1 & 130940.488 & 0.008 & & $91 \%+5 \%$ sp $\left({ }^{3} \mathrm{~F}\right)^{3} \mathrm{P}^{\circ}{ }^{5} \mathrm{D}^{\circ}$ & & 8 \\
\hline $\mathrm{sp}$ & $\left({ }^{3} \mathrm{P}^{3} \mathrm{P}^{\circ}{ }^{5} \mathrm{D}^{\circ}\right.$ & $3 d^{8}\left({ }^{3} \mathrm{P}\right) 4 \mathrm{~s} 4 \mathrm{p}\left({ }^{3} \mathrm{P}^{\circ}\right)$ & ${ }^{5} \mathrm{D}^{\circ}$ & 2 & 130943.661 & 0.009 & & $88 \%+5 \%$ sp $\left({ }^{3} \mathrm{~F}\right)^{3} \mathrm{P}^{\circ}{ }^{5} \mathrm{D}^{\circ}$ & & 9 \\
\hline $\mathrm{sp}$ & $\left({ }^{3} \mathrm{P}\right)^{3} \mathrm{P}^{\circ}{ }^{5} \mathrm{D}^{\circ}$ & $3 \mathrm{~d}^{8}\left({ }^{3} \mathrm{P}\right) 4 \mathrm{~s} 4 \mathrm{p}\left({ }^{3} \mathrm{P}^{\circ}\right)$ & ${ }^{5} \mathrm{D}^{\circ}$ & 0 & 130953.558 & 0.016 & & $91 \%+6 \%$ sp $\left({ }^{3} \mathrm{~F}\right)^{3} \mathrm{P}^{\circ}{ }^{5} \mathrm{D}^{\circ}$ & $\mathrm{N}$ & 2 \\
\hline $\mathrm{sp}$ & $\left({ }^{3} \mathrm{P}\right)^{3} \mathrm{P}^{\circ}{ }^{5} \mathrm{D}^{\circ}$ & $3 \mathrm{~d}^{8}\left({ }^{3} \mathrm{P}\right) 4 \mathrm{~s} 4 \mathrm{p}\left({ }^{3} \mathrm{P}^{\circ}\right)$ & ${ }^{5} \mathrm{D}^{\circ}$ & 3 & 131044.310 & 0.008 & & $81 \%+11 \% \operatorname{sp}\left({ }^{1} \mathrm{D}\right)^{3} \mathrm{P}^{\circ}{ }^{3} \mathrm{~F}^{\circ}$ & & 10 \\
\hline $\mathrm{sp}$ & $\left({ }^{3} \mathrm{P}^{3} \mathrm{P}^{\circ}{ }^{5} \mathrm{D}^{\circ}\right.$ & $3 d^{8}\left({ }^{3} \mathrm{P}\right) 4 \mathrm{~s} 4 \mathrm{p}\left({ }^{3} \mathrm{P}^{\circ}\right)$ & ${ }^{5} \mathrm{D}^{\circ}$ & 4 & 131312.426 & 0.013 & & $66 \%+25 \%$ sp $\left({ }^{1} \mathrm{D}\right){ }^{3} \mathrm{P}^{\circ}{ }^{3} \mathrm{~F}^{\circ}+5 \% \mathrm{sp}\left({ }^{1} \mathrm{G}\right)^{3} \mathrm{P}^{\circ}{ }^{3} \mathrm{~F}^{\circ}$ & & 5 \\
\hline $6 \mathrm{~s}$ & $(5 / 2)^{2}[5 / 2]$ & $3 d^{9}\left({ }^{2} D_{5 / 2}\right) 6 s$ & ${ }^{2}[5 / 2]$ & 3 & 133594.2323 & 0.0013 & & $100 \%$ & & 27 \\
\hline $6 s$ & $(5 / 2)^{2}[5 / 2]$ & $3 d^{9}\left({ }^{2} D_{5 / 2}\right) 6 s$ & $2[5 / 2]$ & 2 & 133728.0387 & 0.0013 & & $100 \%$ & & 29 \\
\hline $\mathrm{sp}$ & $\left({ }^{3} \mathrm{P}\right)^{3} \mathrm{P}^{\circ}{ }^{3} \mathrm{P}^{\circ}$ & $3 \mathrm{~d}^{8}\left({ }^{3} \mathrm{P}\right) 4 \mathrm{~s} 4 \mathrm{p}\left({ }^{3} \mathrm{P}^{\circ}\right)$ & ${ }^{3} \mathrm{P}^{\circ}$ & 2 & 133825.927 & 0.004 & & $55 \%+22 \%$ sp $\left({ }^{3} \mathrm{P}\right){ }^{3} \mathrm{P}^{\circ}{ }^{3} \mathrm{D}^{\circ}+9 \% \mathrm{sp}\left({ }^{1} \mathrm{D}\right){ }^{3} \mathrm{P}^{\circ}{ }^{3} \mathrm{P}^{\circ}$ & & 13 \\
\hline $\mathrm{sp}$ & $\left({ }^{3} \mathrm{P}\right)^{3} \mathrm{P}^{\circ}{ }^{3} \mathrm{D}^{\circ}$ & $3 \mathrm{~d}^{8}\left({ }^{3} \mathrm{P}\right) 4 \mathrm{~s} 4 \mathrm{p}\left({ }^{3} \mathrm{P}^{\circ}\right)$ & ${ }^{3} \mathrm{D}^{\circ}$ & 3 & 133984.325 & 0.004 & & $47 \%+31 \% \mathrm{sp}\left({ }^{3} \mathrm{~F}\right){ }^{1} \mathrm{P}^{\circ}{ }^{3} \mathrm{D}^{\circ}+6 \% \mathrm{sp}\left({ }^{1} \mathrm{D}\right){ }^{3} \mathrm{P}^{\circ}{ }^{3} \mathrm{D}^{\circ}$ & & 14 \\
\hline $\mathrm{sp}$ & $\left({ }^{3} \mathrm{~F}\right)^{1} \mathrm{P}^{\circ}{ }^{3} \mathrm{G}^{\circ}$ & $3 d^{8}\left({ }^{3} F\right) 4 s 4 p\left({ }^{1} P^{\circ}\right)$ & ${ }^{3} \mathrm{G}^{\circ}$ & 5 & 134110.870 & 0.004 & & $93 \%+2 \% 4 \mathrm{f}^{3} \mathrm{G}^{\circ}$ & & 9 \\
\hline $\mathrm{sp}$ & $\left({ }^{3} \mathrm{P}\right)^{3} \mathrm{P}^{\circ}{ }^{3} \mathrm{D}^{\circ}$ & $3 \mathrm{~d}^{8}\left({ }^{3} \mathrm{P}\right) 4 \mathrm{~s} 4 \mathrm{p}\left({ }^{3} \mathrm{P}^{\circ}\right)$ & ${ }^{3} \mathrm{D}^{\circ}$ & 1 & 134359.847 & 0.003 & & $52 \%+24 \% \operatorname{sp}\left({ }^{3} \mathrm{P}\right){ }^{3} \mathrm{P}^{\circ}{ }^{3} \mathrm{P}^{\circ}+10 \% \operatorname{sp}\left({ }^{1} \mathrm{D}\right)^{3} \mathrm{P}^{\circ}{ }^{3} \mathrm{P}^{\circ}$ & & 16 \\
\hline $\mathrm{sp}$ & $\left({ }^{3} \mathrm{P}^{3} \mathrm{P}^{\circ}{ }^{3} \mathrm{D}^{\circ}\right.$ & $3 d^{8}\left({ }^{3} \mathrm{P}\right) 4 \mathrm{~s} 4 \mathrm{p}\left({ }^{3} \mathrm{P}^{\circ}\right)$ & ${ }^{3} \mathrm{D}^{\circ}$ & 2 & 134675.522 & 0.003 & & $50 \%+22 \% s p\left({ }^{3} \mathrm{P}\right)^{3} \mathrm{P}^{\circ}{ }^{3} \mathrm{P}^{\circ}+7 \% \mathrm{sp}\left({ }^{3} \mathrm{~F}\right)^{1} \mathrm{P}^{\circ}{ }^{3} \mathrm{D}^{\circ}$ & & 18 \\
\hline $\mathrm{sp}$ & $\left({ }^{3} \mathrm{~F}\right)^{1} \mathrm{P}^{\circ}{ }^{3} \mathrm{~F}^{\circ}$ & $3 d^{8}\left({ }^{3} \mathrm{~F}\right) 4 \mathrm{~s} 4 \mathrm{p}\left({ }^{1} \mathrm{P}^{\circ}\right)$ & ${ }^{3} \mathrm{~F}^{\circ}$ & 4 & 134742.863 & 0.003 & & $50 \%+31 \%$ sp $\left({ }^{3} \mathrm{~F}\right)^{1} \mathrm{P}^{\circ}{ }^{3} \mathrm{G}^{\circ}+7 \% \mathrm{sp}\left({ }^{1} \mathrm{G}\right)^{3} \mathrm{P}^{\circ}{ }^{3} \mathrm{~F}^{\circ}$ & $\mathrm{cd}$ & 13 \\
\hline sp & $\left({ }^{3} \mathrm{P}\right)^{3} \mathrm{P}^{\circ}{ }^{3} \mathrm{P}^{\circ}$ & $3 \mathrm{~d}^{8}\left({ }^{3} \mathrm{P}\right) 4 \mathrm{~s} 4 \mathrm{p}\left({ }^{3} \mathrm{P}^{\circ}\right)$ & ${ }^{3} \mathrm{P}^{\circ}$ & 1 & 135135.168 & 0.003 & & $38 \%+32 \% \operatorname{sp}\left({ }^{3} \mathrm{P}\right){ }^{3} \mathrm{P}^{\circ}{ }^{3} \mathrm{D}^{\circ}+14 \% \mathrm{sp}\left({ }^{1} \mathrm{D}\right){ }^{3} \mathrm{P}^{\circ}{ }^{3} \mathrm{P}^{\circ}$ & & 10 \\
\hline $\mathrm{sp}$ & $\left({ }^{3} \mathrm{P}\right)^{3} \mathrm{P}^{\circ}{ }^{3} \mathrm{P}^{\circ}$ & $3 d^{8}\left({ }^{3} \mathrm{P}\right) 4 \mathrm{~s} 4 \mathrm{p}\left({ }^{3} \mathrm{P}^{\circ}\right)$ & ${ }^{3} \mathrm{P}^{\circ}$ & 0 & 135484.075 & 0.004 & & $60 \%+31 \% \operatorname{sp}\left({ }^{1} \mathrm{D}\right)^{3} \mathrm{P}^{\circ}{ }^{3} \mathrm{P}^{\circ}+5 \% 4 \mathrm{f}^{3} \mathrm{P}^{\circ}$ & & 6 \\
\hline $6 \mathrm{~s}$ & $(3 / 2)^{2}[3 / 2]$ & $3 d^{9}\left({ }^{2} D_{3 / 2}\right) 6 s$ & ${ }^{2}[3 / 2]$ & 1 & 135664.5204 & 0.0015 & & $100 \%$ & & 21 \\
\hline & & 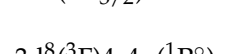 & & & & & & $26 \% \operatorname{sp}\left({ }^{3} \mathrm{P}\right)^{3} \mathrm{P}^{\circ}{ }^{3} \mathrm{D}^{\circ}+14 \% \operatorname{sp}\left({ }^{3} \mathrm{~F}\right)^{1} \mathrm{P}^{\circ}{ }^{3} \mathrm{~F}^{\circ}+13 \% \mathrm{sp}\left({ }^{3} \mathrm{~F}\right)^{1} \mathrm{P}^{\circ}$ & & \\
\hline $\mathrm{sp}$ & $\left({ }^{3} \mathrm{~F}\right)^{1} \mathrm{P}^{\circ}{ }^{3} \mathrm{D}^{\circ}$ & $3 \mathrm{~d}^{8}\left({ }^{3} \mathrm{~F}\right) 4 \mathrm{~s} 4 \mathrm{p}\left({ }^{1} \mathrm{P}^{\circ}\right)$ & ${ }^{3} \mathrm{D}^{\circ}$ & 3 & 135733.433 & 0.003 & & ${ }^{3} \mathrm{D}^{\circ}$ & & 20 \\
\hline $6 s$ & $(3 / 2)^{2}[3 / 2]$ & $3 d^{9}\left({ }^{2} D_{3 / 2}\right) 6 s$ & ${ }^{2}[3 / 2]$ & 2 & 135760.1548 & 0.0016 & & $99 \%$ & & 27 \\
\hline $\mathrm{sp}$ & $\left({ }^{3} \mathrm{~F}\right)^{1} \mathrm{P}^{\circ}{ }^{3} \mathrm{G}^{\circ}$ & $3 d^{8}\left({ }^{3} F\right) 4 s 4 p\left({ }^{1} \mathrm{P}^{\circ}\right)$ & ${ }^{3} \mathrm{G}^{\circ}$ & 4 & 135834.6720 & 0.0019 & & $49 \%+22 \% \operatorname{sp}\left({ }^{3} \mathrm{~F}\right)^{1} \mathrm{P}^{\circ}{ }^{3} \mathrm{~F}^{\circ}+9 \% 4 \mathrm{f}^{3} \mathrm{G}^{\circ}$ & & 17 \\
\hline $4 \mathrm{f}$ & $(5 / 2)^{2}[1 / 2]^{\circ}$ & $3 d^{9}\left({ }^{2} D_{5 / 2}\right) 4 f$ & ${ }^{2}[1 / 2]^{\circ}$ & 1 & 135863.6857 & 0.0016 & & $97 \%+2 \% s p+1 \% 4 f(5 / 2)^{2}[3 / 2]^{\circ}$ & & 19 \\
\hline $4 f$ & $(5 / 2)^{2}[1 / 2]^{\circ}$ & $3 d^{9}\left({ }^{2} D_{5 / 2}\right) 4 f$ & ${ }^{2}[1 / 2]^{\circ}$ & 0 & 135902.1365 & 0.0023 & & $94 \%+4 \%$ sp & & 8 \\
\hline $4 f$ & $(5 / 2)^{2}[3 / 2]^{\circ}$ & $3 d^{9}\left({ }^{2} D_{5 / 2}\right) 4 f$ & ${ }^{2}[3 / 2]^{\circ}$ & 2 & 135910.7245 & 0.0011 & & $94 \%+4 \% 4 f(5 / 2)^{2}[5 / 2]^{\circ}+2 \% s p$ & & 23 \\
\hline
\end{tabular}


Table A2. Cont.

\begin{tabular}{|c|c|c|c|c|c|c|c|c|c|c|}
\hline & Label $^{\text {a }}$ & Configuration & Term & $J$ & Level $^{\mathrm{b}}, \mathrm{cm}^{-1}$ & Unc. ${ }^{c}, \mathrm{~cm}^{-1}$ & Landé $g^{\mathrm{d}}$ & Leading Percentages ${ }^{\mathrm{e}}$ & Note $^{\mathrm{f}}$ & $N_{\text {lines }} \mathrm{g}$ \\
\hline $4 \mathrm{f}$ & $(5 / 2)^{2}[11 / 2]^{\circ}$ & $3 d^{9}\left({ }^{2} D_{5 / 2}\right) 4 f$ & ${ }^{2}[11 / 2]^{\circ}$ & 6 & 135931.01412 & 0.0012 & & $99 \%+1 \% \mathrm{sp}$ & & 5 \\
\hline $4 \mathrm{f}$ & $(5 / 2)^{2}[11 / 2]^{\circ}$ & $3 d^{9}\left({ }^{2} D_{5 / 2}\right) 4 f$ & ${ }^{2}[11 / 2]^{\circ}$ & 5 & 135933.89499 & 0.0019 & & $98 \%+1 \% \mathrm{sp}$ & & 11 \\
\hline $\mathrm{sp}$ & $\left({ }^{3} \mathrm{P}\right)^{3} \mathrm{P}^{\circ}{ }^{5} \mathrm{~S}^{\circ}$ & $3 d^{8}\left({ }^{3} \mathrm{P}\right) 4 \mathrm{~s} 4 \mathrm{p}\left({ }^{3} \mathrm{P}^{\circ}\right)$ & ${ }^{5} \mathrm{~S}^{\circ}$ & 2 & 135952.279 & 0.005 & & $83 \%+4 \% 4 \mathrm{f}^{1} \mathrm{D}^{\circ}$ & & 12 \\
\hline $4 \mathrm{f}$ & $(5 / 2)^{2}[3 / 2]^{\circ}$ & $3 d^{9}\left({ }^{2} D_{5 / 2}\right) 4 f$ & ${ }^{2}[3 / 2]^{\circ}$ & 1 & 135958.1919 & 0.0016 & & $97 \%+2 \% s p+1 \% 4 f(5 / 2)^{2}[1 / 2]^{\circ}$ & & 20 \\
\hline $4 \mathrm{f}$ & $(5 / 2)^{2}[7 / 2]^{\circ}$ & $3 d^{9}\left({ }^{2} D_{5 / 2}\right) 4 f$ & ${ }^{2}[7 / 2]^{\circ}$ & 3 & 135989.9176 & 0.0012 & & $41 \%+37 \% s p+11 \% 4 f(5 / 2)^{2}[5 / 2]^{\circ}$ & & 33 \\
\hline $4 \mathrm{f}$ & $(5 / 2)^{2}[5 / 2]^{\circ}$ & $3 d^{9}\left({ }^{2} D_{5 / 2}\right) 4 f$ & ${ }^{2}[5 / 2]^{\circ}$ & 2 & 136013.9671 & 0.0011 & & $84 \%+9 \% s p+5 \% 4 f(5 / 2)^{2}[3 / 2]^{\circ}$ & & 17 \\
\hline $4 \mathrm{f}$ & $(5 / 2)^{2}[5 / 2]^{\circ}$ & $3 d^{9}\left({ }^{2} D_{5 / 2}\right) 4 f$ & ${ }^{2}[5 / 2]^{\circ}$ & 3 & 136035.3328 & 0.0010 & & $86 \%+11 \%$ 4f $(5 / 2)^{2}[7 / 2]^{\circ}+3 \% s p$ & & 28 \\
\hline $4 \mathrm{f}$ & $(5 / 2)^{2}[7 / 2]^{\circ}$ & $3 d^{9}\left({ }^{2} D_{5 / 2}\right) 4 f$ & ${ }^{2}[7 / 2]^{\circ}$ & 4 & 136132.77781 & 0.0010 & & $88 \%+8 \% 4 f(5 / 2)^{2}[9 / 2]^{\circ}+3 \% \mathrm{sp}$ & & 26 \\
\hline $4 \mathrm{f}$ & $(5 / 2)^{2}[9 / 2]^{\circ}$ & $3 d^{9}\left({ }^{2} D_{5 / 2}\right) 4 f$ & ${ }^{2}[9 / 2]^{\circ}$ & 5 & 136160.61825 & 0.0011 & & $98 \%+2 \%$ sp & & 14 \\
\hline $4 \mathrm{f}$ & $(5 / 2)^{2}[9 / 2]^{\circ}$ & $3 d^{9}\left({ }^{2} D_{5 / 2}\right) 4 f$ & ${ }^{2}[9 / 2]^{\circ}$ & 4 & 136269.9996 & 0.0014 & & $75 \%+14 \% \mathrm{sp}+9 \%$ ff $(5 / 2)^{2}[7 / 2]^{\circ}$ & & 20 \\
\hline $5 \mathrm{~d}$ & $(5 / 2)^{2}[1 / 2]$ & $3 d^{9}\left({ }^{2} D_{5 / 2}\right) 5 d$ & ${ }^{2}[1 / 2]$ & 1 & 136336.8971 & 0.0020 & & $98 \%+1 \% 5 \mathrm{~d}(3 / 2)^{2}[1 / 2]$ & & 17 \\
\hline $\mathrm{sp}$ & $\left({ }^{3} \mathrm{~F}\right)^{1} \mathrm{P}^{\circ}{ }^{3} \mathrm{~F}^{\circ}$ & $3 d^{8}\left({ }^{3} \mathrm{~F}\right) 4 \mathrm{~s} 4 \mathrm{p}\left({ }^{1} \mathrm{P}^{\circ}\right)$ & ${ }^{3} \mathrm{~F}^{\circ}$ & 3 & 136441.817 & 0.003 & & $25 \%+25 \%$ sp $\left({ }^{3} \mathrm{~F}\right){ }^{1} \mathrm{P}^{\circ}{ }^{3} \mathrm{G}^{\circ}+11 \% \mathrm{sp}\left({ }^{1} \mathrm{G}\right){ }^{3} \mathrm{P}^{\circ}{ }^{3} \mathrm{~F}^{\circ}$ & & 21 \\
\hline sp & $\left({ }^{1} \mathrm{G}\right)^{3} \mathrm{P}^{\circ}{ }^{3} \mathrm{H}^{\circ}$ & $3 d^{8}\left({ }^{1} G\right) 4 s 4 p\left({ }^{3} P^{\circ}\right)$ & ${ }^{3} \mathrm{H}^{\circ}$ & 4 & 136693.948 & 0.003 & & $97 \%$ & & 10 \\
\hline $5 \mathrm{~d}$ & $(5 / 2)^{2}[9 / 2]$ & $3 d^{9}\left({ }^{2} D_{5 / 2}\right) 5 d$ & ${ }^{2}[9 / 2]$ & 5 & 136725.85349 & 0.0013 & & $100 \%$ & & 14 \\
\hline $5 \mathrm{~d}$ & $(5 / 2)^{2}[3 / 2]$ & $3 d^{9}\left({ }^{2} D_{5 / 2}\right) 5 d$ & ${ }^{2}[3 / 2]$ & 2 & 136754.1104 & 0.0018 & & $97 \%+2 \% 5 d(5 / 2)^{2}[5 / 2]$ & & 25 \\
\hline $5 \mathrm{~d}$ & $(5 / 2)^{2}[9 / 2]$ & $3 d^{9}\left({ }^{2} D_{5 / 2}\right) 5 d$ & ${ }^{2}[9 / 2]$ & 4 & 136765.3514 & 0.0018 & & $99 \%$ & & 20 \\
\hline $5 d$ & $(5 / 2)^{2}[3 / 2]$ & $3 d^{9}\left({ }^{2} D_{5 / 2}\right) 5 d$ & ${ }^{2}[3 / 2]$ & 1 & 136773.1713 & 0.0021 & & $99 \%$ & & 19 \\
\hline $\mathrm{sp}$ & $\left({ }^{3} \mathrm{~F}\right)^{1} \mathrm{P}^{\circ}{ }^{3} \mathrm{D}^{\circ}$ & $3 d^{8}\left({ }^{3} \mathrm{~F}\right) 4 \mathrm{~s} 4 \mathrm{p}\left({ }^{1} \mathrm{P}^{\circ}\right)$ & ${ }^{3} \mathrm{D}^{\circ}$ & 2 & 136800.137 & 0.003 & & $41 \%+18 \% \operatorname{sp}\left({ }^{3} \mathrm{P}\right)^{3} \mathrm{P}^{\circ}{ }^{1} \mathrm{D}^{\circ}+14 \% 6 \mathrm{p}$ & & 14 \\
\hline $5 \mathrm{~d}$ & $(5 / 2)^{2}[5 / 2]$ & $3 d^{9}\left({ }^{2} D_{5 / 2}\right) 5 d$ & ${ }^{2}[5 / 2]$ & 3 & 136919.3511 & 0.0014 & & $99 \%+1 \% 5 \mathrm{~d}(5 / 2)^{2}[7 / 2]$ & & 36 \\
\hline $5 \mathrm{~d}$ & $(5 / 2)^{2}[7 / 2]$ & $3 \mathrm{~d}^{9}\left({ }^{2} \mathrm{D}_{5 / 2}\right) 5 \mathrm{~d}$ & ${ }^{2}[7 / 2]$ & 3 & 137034.778 & 0.003 & & $99 \%+1 \% 5 d(5 / 2)^{2}[5 / 2]+1 \% 5 d(3 / 2)^{2}[7 / 2]$ & & 23 \\
\hline $5 \mathrm{~d}$ & $(5 / 2)^{2}[7 / 2]$ & $3 \mathrm{~d}^{9}\left({ }^{2} \mathrm{D}_{5 / 2}\right) 5 \mathrm{~d}$ & ${ }^{2}[7 / 2]$ & 4 & 137044.4647 & 0.0017 & & $99 \%$ & & 22 \\
\hline $5 d$ & $(5 / 2)^{2}[5 / 2]$ & $3 d^{9}\left({ }^{2} D_{5 / 2}\right) 5 d$ & ${ }^{2}[5 / 2]$ & 2 & 137073.6465 & 0.0019 & & $97 \%+2 \% 5 d(5 / 2)^{2}[3 / 2]$ & & 24 \\
\hline $\mathrm{sp}$ & $\left({ }^{3} \mathrm{~F}\right)^{1} \mathrm{P}^{\circ}{ }^{3} \mathrm{G}^{\circ}$ & $3 d^{8}\left({ }^{3} \mathrm{~F}\right) 4 \mathrm{~s} 4 \mathrm{p}\left({ }^{1} \mathrm{P}^{\circ}\right)$ & ${ }^{3} \mathrm{G}^{\circ}$ & 3 & 137078.190 & 0.005 & & $52 \%+19 \% \operatorname{sp}\left({ }^{3} \mathrm{~F}\right)^{1} \mathrm{P}^{\circ}{ }^{3} \mathrm{~F}^{\circ}+14 \% \mathrm{sp}\left({ }^{1} \mathrm{G}\right)^{3} \mathrm{P}^{\circ}{ }^{3} \mathrm{~F}^{\circ}$ & & 7 \\
\hline $\mathrm{sp}$ & $\left({ }^{1} \mathrm{G}\right)^{3} \mathrm{P}^{\circ}{ }^{3} \mathrm{H}^{\circ}$ & $3 d^{8}\left({ }^{1} G\right) 4 s 4 p\left({ }^{3} P^{\circ}\right)$ & ${ }^{3} \mathrm{H}^{\circ}$ & 5 & 137082.175 & 0.005 & & $96 \%+2 \% 4 \mathrm{f} 1 \mathrm{H}^{\circ}$ & & 6 \\
\hline sp & $\left({ }^{1} \mathrm{G}\right)^{3} \mathrm{P}^{\circ}{ }^{3} \mathrm{~F}^{\circ}$ & $3 \mathrm{~d}^{8}\left({ }^{1} \mathrm{G}\right) 4 \mathrm{~s} 4 \mathrm{p}\left({ }^{3} \mathrm{P}^{\circ}\right)$ & ${ }^{3} \mathrm{~F}^{\circ}$ & 2 & 137212.779 & 0.004 & & $36 \%+36 \% \operatorname{sp}\left({ }^{3} \mathrm{~F}\right)^{1} \mathrm{P}^{\circ}{ }^{3} \mathrm{~F}^{\circ}+14 \% \mathrm{sp}\left({ }^{3} \mathrm{P}\right)^{3} \mathrm{P}^{\circ}{ }^{1} \mathrm{D}^{\circ}$ & RJ & 12 \\
\hline $\mathrm{sp}$ & $\left({ }^{3} \mathrm{P}\right)^{3} \mathrm{P}^{\circ}{ }^{1} \mathrm{P}^{\circ}$ & $3 d^{8}\left({ }^{3} \mathrm{P}\right) 4 \mathrm{~s} 4 \mathrm{p}\left({ }^{3} \mathrm{P}^{\circ}\right)$ & ${ }^{1} \mathrm{P}^{\circ}$ & 1 & 137242.914 & 0.008 & & $82 \%+5 \% \operatorname{sp}\left({ }^{3} \mathrm{P}\right)^{3} \mathrm{P}^{\circ}{ }^{3} \mathrm{P}^{\circ}$ & $\mathrm{N}$ & 6 \\
\hline $5 d$ & $(5 / 2)^{2}[1 / 2]$ & $3 \mathrm{~d}^{9}\left({ }^{2} \mathrm{D}_{5 / 2}\right) 5 \mathrm{~d}$ & ${ }^{2}[1 / 2]$ & 0 & 137614.140 & 0.004 & & $60 \%+37 \% 5 \mathrm{~d}(3 / 2)^{2}[1 / 2]+1 \% 6 \mathrm{~d}(5 / 2)^{2}[1 / 2]$ & & 7 \\
\hline $\mathrm{sp}$ & $\left({ }^{3} \mathrm{P}\right)^{3} \mathrm{P}^{\circ}{ }^{1} \mathrm{D}^{\circ}$ & $3 d^{8}\left({ }^{3} \mathrm{P}\right) 4 \mathrm{~s} 4 \mathrm{p}\left({ }^{3} \mathrm{P}^{\circ}\right)$ & ${ }^{1} \mathrm{D}^{\circ}$ & 2 & 137648.800 & 0.004 & & $50 \%+9 \% \operatorname{sp}\left({ }^{3} \mathrm{P}\right)^{3} \mathrm{P}^{\circ}{ }^{3} \mathrm{D}^{\circ}+9 \% \operatorname{sp}\left({ }^{3} \mathrm{~F}\right)^{1} \mathrm{P}^{\circ}{ }^{3} \mathrm{D}^{\circ}$ & & 15 \\
\hline $\mathrm{sp}$ & $\left({ }^{3} \mathrm{~F}\right)^{1} \mathrm{P}^{\circ}{ }^{3} \mathrm{D}^{\circ}$ & $3 d^{8}\left({ }^{3} F\right) 4 s 4 p\left({ }^{1} \mathrm{P}^{\circ}\right)$ & ${ }^{3} \mathrm{D}^{\circ}$ & 1 & 137913.450 & 0.004 & & $28 \%+16 \% 4 \mathrm{f}^{3} \mathrm{D}^{\circ}+16 \% 4 \mathrm{f}$ & & 13 \\
\hline $\mathrm{sp}$ & $\left({ }^{1} \mathrm{G}\right)^{3} \mathrm{P}^{\circ}{ }^{3} \mathrm{~F}^{\circ}$ & $3 d^{8}\left({ }^{1} G\right) 4 s 4 p\left({ }^{3} \mathrm{P}^{\circ}\right)$ & ${ }^{3} \mathrm{~F}^{\circ}$ & 4 & 137938.904 & 0.007 & & $49 \%+39 \% 6 \mathrm{p}^{3} \mathrm{~F}^{\circ}+5 \% \mathrm{sp}\left({ }^{3} \mathrm{~F}\right)^{1} \mathrm{P}^{\circ}{ }^{3} \mathrm{~F}^{\circ}$ & $\mathrm{cd}$ & 6 \\
\hline $4 \mathrm{f}$ & $(3 / 2)^{2}[3 / 2]^{\circ}$ & $3 \mathrm{~d}^{9}\left({ }^{2} \mathrm{D}_{3 / 2}\right) 4 \mathrm{f}$ & ${ }^{2}[3 / 2]^{\circ}$ & 2 & 138002.8856 & 0.0023 & & $94 \%+3 \% s p+2 \% 4 f(3 / 2)^{2}[5 / 2]^{\circ}$ & & 10 \\
\hline $4 \mathrm{f}$ & $(3 / 2)^{2}[3 / 2]^{\circ}$ & $3 d^{9}\left({ }^{2} D_{3 / 2}\right) 4 f$ & ${ }^{2}[3 / 2]^{\circ}$ & 1 & 138028.384 & 0.003 & & $59 \%+26 \% s p+10 \% 6 p$ & & 14 \\
\hline $4 \mathrm{f}$ & $(3 / 2)^{2}[9 / 2]^{\circ}$ & $3 d^{9}\left({ }^{2} D_{3 / 2}\right) 4 f$ & ${ }^{2}[9 / 2]^{\circ}$ & 5 & 138064.2971 & 0.0021 & & $99 \%+1 \% \mathrm{sp}$ & & 10 \\
\hline $4 \mathrm{f}$ & $(3 / 2)^{2}[9 / 2]^{\circ}$ & $3 d^{9}\left({ }^{2} D_{3 / 2}\right) 4 f$ & ${ }^{2}[9 / 2]^{\circ}$ & 4 & 138073.5826 & 0.0022 & & $98 \%+1 \% \mathrm{sp}$ & & 10 \\
\hline $4 \mathrm{f}$ & $(3 / 2)^{2}[5 / 2]^{\circ}$ & $3 d^{9}\left({ }^{2} D_{3 / 2}\right) 4 f$ & ${ }^{2}[5 / 2]^{\circ}$ & 3 & 138130.5345 & 0.0015 & & $97 \%+1 \% 4 f(3 / 2)^{2}[7 / 2]^{\circ}$ & & 17 \\
\hline $4 \mathrm{f}$ & $(3 / 2)^{2}[5 / 2]^{\circ}$ & $3 d^{9}\left({ }^{2} D_{3 / 2}\right) 4 f$ & ${ }^{2}[5 / 2]^{\circ}$ & 2 & 138176.8797 & 0.0018 & & $89 \%+7 \% \mathrm{sp}+1 \% 4 \mathrm{f}(3 / 2)^{2}[3 / 2]^{\circ}$ & & 17 \\
\hline $4 \mathrm{f}$ & $(3 / 2)^{2}[7 / 2]^{\circ}$ & $3 d^{9}\left({ }^{2} D_{3 / 2}\right) 4 f$ & ${ }^{2}[7 / 2]^{\circ}$ & 4 & 138219.8605 & 0.0015 & & $98 \%$ & & 12 \\
\hline $4 \mathrm{f}$ & $(3 / 2)^{2}[7 / 2]^{\circ}$ & $3 d^{9}\left({ }^{2} D_{3 / 2}\right) 4 f$ & ${ }^{2}[7 / 2]^{\circ}$ & 3 & 138261.5822 & 0.0017 & & $93 \%+1 \% \mathrm{sp}+1 \% 4 \mathrm{f}(3 / 2)^{2}[5 / 2]^{\circ}$ & & 19 \\
\hline $6 \mathrm{p}$ & $(5 / 2)^{2}[7 / 2]^{\circ}$ & $3 d^{9}\left({ }^{2} D_{5 / 2}\right) 6 p$ & ${ }^{2}[7 / 2]^{\circ}$ & 3 & 138401.956 & 0.003 & & $62 \%+29 \% s p+2 \% 6 p(5 / 2)^{2}[5 / 2]^{\circ}$ & & 21 \\
\hline $\mathrm{sp}$ & $\left({ }^{3} \mathrm{P}\right)^{3} \mathrm{P}^{0}{ }^{3} \mathrm{~S}^{\circ}$ & $3 d^{8}\left({ }^{3} \mathrm{P}\right) 4 \mathrm{~s} 4 \mathrm{p}\left({ }^{3} \mathrm{P}^{\circ}\right)$ & ${ }^{3} S^{\circ}$ & 1 & 138516.49 & 0.03 & & $97 \%$ & $\mathrm{~N}$ & 5 \\
\hline
\end{tabular}


Table A2. Cont.

\begin{tabular}{|c|c|c|c|c|c|c|c|c|c|c|}
\hline & Label $^{\text {a }}$ & Configuration & Term & $J$ & Level $^{\mathrm{b}}, \mathrm{cm}^{-1}$ & Unc. ${ }^{\mathrm{c}}, \mathrm{cm}^{-1}$ & Landé $g^{\mathrm{d}}$ & Leading Percentages ${ }^{\mathrm{e}}$ & Note $^{f}$ & $N_{\text {lines }} \mathrm{g}$ \\
\hline $5 d$ & $(3 / 2)^{2}[1 / 2]$ & $3 d^{9}\left({ }^{2} D_{3 / 2}\right) 5 d$ & ${ }^{2}[1 / 2]$ & 1 & 138593.046 & 0.003 & & $98 \%+1 \% 5 d(5 / 2)^{2}[1 / 2]$ & & 17 \\
\hline $6 \mathrm{p}$ & $(5 / 2)^{2}[3 / 2]^{\circ}$ & $3 d^{9}\left({ }^{2} D_{5 / 2}\right) 6 p$ & ${ }^{2}[3 / 2]^{\circ}$ & 2 & 138745.817 & 0.005 & & $91 \%+4 \% s p+3 \% 6 p(5 / 2)^{2}[5 / 2]^{\circ}$ & & 14 \\
\hline $5 \mathrm{~d}$ & $(3 / 2)^{2}[7 / 2]$ & $3 d^{9}\left({ }^{2} D_{3 / 2}\right) 5 d$ & ${ }^{2}[7 / 2]$ & 3 & 138819.0637 & 0.0019 & & $99 \%+1 \% 5 d(5 / 2)^{2}[7 / 2]$ & & 15 \\
\hline $5 d$ & $(3 / 2)^{2}[7 / 2]$ & $3 d^{9}\left({ }^{2} D_{3 / 2}\right) 5 d$ & ${ }^{2}[7 / 2]$ & 4 & 138882.8921 & 0.0019 & & $99 \%$ & & 15 \\
\hline $5 \mathrm{~d}$ & $(3 / 2)^{2}[3 / 2]$ & $3 d^{9}\left({ }^{2} D_{3 / 2}\right) 5 d$ & $2[3 / 2]$ & 1 & 138898.334 & 0.003 & & $99 \%$ & & 18 \\
\hline $5 \mathrm{~d}$ & $(3 / 2)^{2}[3 / 2]$ & $3 d^{9}\left({ }^{2} D_{3 / 2}\right) 5 d$ & $2[3 / 2]$ & 2 & 138981.246 & 0.003 & & $97 \%+2 \% 5 d(3 / 2)^{2}[5 / 2]+1 \% 5 d(5 / 2)^{2}[3 / 2]$ & & 19 \\
\hline $6 \mathrm{p}$ & $(5 / 2)^{2}[5 / 2]^{\circ}$ & $3 d^{9}\left({ }^{2} D_{5 / 2}\right) 6 p$ & ${ }^{2}[5 / 2]^{\circ}$ & 2 & 139028.705 & 0.005 & & $41 \%+40 \% \mathrm{sp}+11 \% 6 \mathrm{p}(3 / 2)^{2}[5 / 2]^{\circ}$ & & 11 \\
\hline $5 \mathrm{~d}$ & $(3 / 2)^{2}[5 / 2]$ & $3 d^{9}\left({ }^{2} D_{3 / 2}\right) 5 d$ & ${ }^{2}[5 / 2]$ & 3 & 139119.4295 & 0.0020 & & $100 \%$ & & 18 \\
\hline $5 d$ & $(3 / 2)^{2}[5 / 2]$ & $3 d^{9}\left({ }^{2} D_{3 / 2}\right) 5 d$ & ${ }^{2}[5 / 2]$ & 2 & 139142.049 & 0.003 & & $97 \%+2 \% 5 d(3 / 2)^{2}[3 / 2]$ & & 24 \\
\hline $6 \mathrm{p}$ & $(5 / 2)^{2}[3 / 2]^{\circ}$ & $3 d^{9}\left({ }^{2} D_{5 / 2}\right) 6 p$ & $2[3 / 2]^{\circ}$ & 1 & 139241.130 & 0.005 & & $73 \%+7 \% 6 p(3 / 2)^{2}[3 / 2]^{\circ}+4 \% 6 p(3 / 2)^{2}[1 / 2]^{\circ}$ & & 13 \\
\hline $\mathrm{sp}$ & $\left({ }^{1} \mathrm{G}\right)^{3} \mathrm{P}^{\circ}{ }^{3} \mathrm{~F}^{\circ}$ & $3 d^{8}\left({ }^{1} G\right) 4 s 4 p\left({ }^{3} P^{\circ}\right)$ & ${ }^{3} \mathrm{~F}^{0}$ & 3 & 139331.149 & 0.003 & & $21 \%+46 \% 6 p^{1} \mathrm{~F}^{\circ}+15 \% 6 \mathrm{p}^{3} \mathrm{D}^{\circ}$ & $\mathrm{cd}$ & 21 \\
\hline $6 \mathrm{p}$ & $(5 / 2)^{2}[7 / 2]^{\circ}$ & $3 d^{9}\left({ }^{2} D_{5 / 2}\right) 6 p$ & ${ }^{2}[7 / 2]^{\circ}$ & 4 & 139395.786 & 0.004 & & $59 \%+37 \% \mathrm{sp}$ & & 11 \\
\hline $\mathrm{sp}$ & $\left({ }^{3} \mathrm{~F}\right)^{1} \mathrm{P}^{\circ}{ }^{3} \mathrm{~F}^{\circ}$ & $3 d^{8}\left({ }^{3} F\right) 4 s 4 p\left({ }^{1} P^{\circ}\right)$ & ${ }^{3} \mathrm{~F}^{\circ}$ & 2 & 139710.491 & 0.004 & & $19 \%+42 \% 6 \mathrm{p}^{1} \mathrm{D}^{\circ}+15 \% \mathrm{sp}\left({ }^{1} \mathrm{G}\right)^{3} \mathrm{P}^{\circ}{ }^{3} \mathrm{~F}^{\circ}$ & & 15 \\
\hline $6 \mathrm{p}$ & $(5 / 2)^{2}[5 / 2]^{\circ}$ & $3 d^{9}\left({ }^{2} D_{5 / 2}\right) 6 p$ & ${ }^{2}[5 / 2]^{\circ}$ & 3 & 139741.097 & 0.003 & & $59 \%+31 \% s p+4 \% 6 p(5 / 2)^{2}[7 / 2]^{\circ}$ & & 18 \\
\hline $5 d$ & $(3 / 2)^{2}[1 / 2]$ & $3 \mathrm{~d}^{9}\left({ }^{2} \mathrm{D}_{3 / 2}\right) 5 \mathrm{~d}$ & ${ }^{2}[1 / 2]$ & 0 & 140589.344 & 0.006 & & $47 \%+20 \% 6 d(5 / 2)^{2}[1 / 2]+17 \% 5 d(5 / 2)^{2}[1 / 2]$ & & 4 \\
\hline $6 \mathrm{p}$ & $(3 / 2)^{2}[1 / 2]^{\circ}$ & $3 d^{9}\left({ }^{2} D_{3 / 2}\right) 6 p$ & ${ }^{2}[1 / 2]^{\circ}$ & 1 & 140981.510 & 0.005 & & $94 \%+3 \% 6 p(5 / 2)^{2}[3 / 2]^{\circ}+2 \% s p$ & & 8 \\
\hline $6 \mathrm{p}$ & $(3 / 2)^{2}[5 / 2]^{\circ}$ & $3 d^{9}\left({ }^{2} D_{3 / 2}\right) 6 p$ & ${ }^{2}[5 / 2]^{\circ}$ & 3 & 141202.628 & 0.006 & & $89 \%+8 \% \mathrm{sp}$ & & 12 \\
\hline $6 \mathrm{p}$ & $(3 / 2)^{2}[5 / 2]^{\circ}$ & $3 d^{9}\left({ }^{2} D_{3 / 2}\right) 6 p$ & ${ }^{2}[5 / 2]^{\circ}$ & 2 & 141244.556 & 0.006 & & $62 \%+22 \% s p+13 \% 6 p(3 / 2)^{2}[3 / 2]^{\circ}$ & $\mathrm{ci}$ & 9 \\
\hline $6 \mathrm{p}$ & $(3 / 2)^{2}[3 / 2]^{\circ}$ & $3 d^{9}\left({ }^{2} D_{3 / 2}\right) 6 p$ & ${ }^{2}[3 / 2]^{\circ}$ & 2 & 141542.001 & 0.005 & & $73 \%+13 \% 6 \mathrm{p}(3 / 2)^{2}[5 / 2]^{\circ}+10 \% \mathrm{sp}$ & & 13 \\
\hline $6 \mathrm{p}$ & $(3 / 2)^{2}[3 / 2]^{\circ}$ & $3 d^{9}\left({ }^{2} D_{3 / 2}\right) 6 p$ & ${ }^{2}[3 / 2]^{\circ}$ & 1 & 141734.175 & 0.005 & & $79 \%+16 \% s p+1 \% 5 p$ & $\mathrm{ci}$ & 10 \\
\hline $\mathrm{sp}$ & $\left({ }^{1} \mathrm{G}\right)^{3} \mathrm{P}^{\circ}{ }^{3} \mathrm{G}^{\circ}$ & $3 d^{8}\left({ }^{1} G\right) 4 s 4 p\left({ }^{3} P^{\circ}\right)$ & ${ }^{3} \mathrm{G}^{\circ}$ & 3 & 143423.319 & 0.020 & & $99 \%$ & $\mathrm{~N}$ & 4 \\
\hline $7 \mathrm{~s}$ & $(5 / 2)^{2}[5 / 2]$ & $3 \mathrm{~d}^{9}\left({ }^{2} \mathrm{D}_{5 / 2}\right) 7 \mathrm{~s}$ & ${ }^{2}[5 / 2]$ & 3 & 144814.9118 & 0.0014 & & $100 \%$ & & 26 \\
\hline $7 \mathrm{~s}$ & $(5 / 2)^{2}[5 / 2]$ & $3 \mathrm{~d}^{9}\left({ }^{2} \mathrm{D}_{5 / 2}\right) 7 \mathrm{~s}$ & $2[5 / 2]$ & 2 & 144882.9859 & 0.0016 & & $100 \%$ & & 26 \\
\hline $5 f$ & $(5 / 2)^{2}[1 / 2]^{\circ}$ & $3 d^{9}\left({ }^{2} D_{5 / 2}\right) 5 f$ & ${ }^{2}[1 / 2]^{\circ}$ & 0 & 145889.334 & 0.004 & & $100 \%$ & & 6 \\
\hline $5 f$ & $(5 / 2)^{2}[1 / 2]^{\circ}$ & $3 d^{9}\left({ }^{2} D_{5 / 2}\right) 5 f$ & ${ }^{2}[1 / 2]^{\circ}$ & 1 & 145900.904 & 0.003 & & $84 \%+16 \% 5 f(5 / 2)^{2}[3 / 2]^{\circ}$ & & 10 \\
\hline $5 f$ & $(5 / 2)^{2}[3 / 2]^{\circ}$ & $3 d^{9}\left({ }^{2} D_{5 / 2}\right) 5 f$ & $2[3 / 2]^{\circ}$ & 2 & 145927.231 & 0.003 & & $100 \%$ & & 13 \\
\hline $5 f$ & $(5 / 2)^{2}[11 / 2]^{\circ}$ & $3 d^{9}\left({ }^{2} D_{5 / 2}\right) 5 f$ & ${ }^{2}[11 / 2]^{\circ}$ & 5 & 145945.5969 & 0.0019 & & $100 \%$ & & 5 \\
\hline $5 f$ & $(5 / 2)^{2}[11 / 2]^{\circ}$ & $3 d^{9}\left({ }^{2} D_{5 / 2}\right) 5 f$ & ${ }^{2}[11 / 2]^{\circ}$ & 6 & 145951.5299 & 0.0014 & & $100 \%$ & & 2 \\
\hline $5 f$ & $(5 / 2)^{2}[3 / 2]^{\circ}$ & $3 d^{9}\left({ }^{2} D_{5 / 2}\right) 5 f$ & ${ }^{2}[3 / 2]^{\circ}$ & 1 & 145955.447 & 0.004 & & $84 \%+16 \% 5 f(5 / 2)^{2}[1 / 2]^{\circ}$ & & 10 \\
\hline $5 f$ & $(5 / 2)^{2}[5 / 2]^{\circ}$ & $3 d^{9}\left({ }^{2} D_{5 / 2}\right) 5 f$ & ${ }^{2}[5 / 2]^{\circ}$ & 3 & 145978.142 & 0.003 & & $98 \%+2 \% 5 f(5 / 2)^{2}[7 / 2]^{\circ}$ & & 12 \\
\hline $5 f$ & $(5 / 2)^{2}[5 / 2]^{\circ}$ & $3 d^{9}\left({ }^{2} D_{5 / 2}\right) 5 f$ & ${ }^{2}[5 / 2]^{\circ}$ & 2 & 145985.199 & 0.003 & & $100 \%$ & & 12 \\
\hline $5 f$ & $(5 / 2)^{2}[7 / 2]^{\circ}$ & $3 d^{9}\left({ }^{2} D_{5 / 2}\right) 5 f$ & ${ }^{2}[7 / 2]^{\circ}$ & 3 & 146021.279 & 0.003 & & $98 \%+2 \% 5 f(5 / 2)^{2}[5 / 2]^{\circ}$ & & 13 \\
\hline $5 f$ & $(5 / 2)^{2}[7 / 2]^{\circ}$ & $3 d^{9}\left({ }^{2} D_{5 / 2}\right) 5 f$ & ${ }^{2}[7 / 2]^{\circ}$ & 4 & 146023.862 & 0.003 & & $86 \%+14 \% 5 f(5 / 2)^{2}[9 / 2]^{\circ}$ & & 13 \\
\hline $5 f$ & $(5 / 2)^{2}[9 / 2]^{\circ}$ & $3 d^{9}\left({ }^{2} D_{5 / 2}\right) 5 f$ & ${ }^{2}[9 / 2]^{\circ}$ & 4 & 146029.350 & 0.003 & & $86 \%+14 \% 5 f(5 / 2)^{2}[7 / 2]^{\circ}$ & & 13 \\
\hline $5 f$ & $(5 / 2)^{2}[9 / 2]^{\circ}$ & $3 d^{9}\left({ }^{2} D_{5 / 2}\right) 5 f$ & ${ }^{2}[9 / 2]^{\circ}$ & 5 & 146032.2635 & 0.0021 & & $100 \%$ & & 7 \\
\hline $5 \mathrm{~g}$ & $(5 / 2)^{2}[3 / 2]$ & $3 d^{9}\left({ }^{2} D_{5 / 2}\right) 5 g$ & ${ }^{2}[3 / 2]$ & 1 & 146051.118 & 0.004 & & $100 \%$ & & 5 \\
\hline $5 g$ & $(5 / 2)^{2}[3 / 2]$ & $3 d^{9}\left({ }^{2} D_{5 / 2}\right) 5 g$ & ${ }^{2}[3 / 2]$ & 2 & 146051.2431 & 0.003 & & $100 \%$ & & 4 \\
\hline $5 g$ & $(5 / 2)^{2}[13 / 2]$ & $3 d^{9}\left({ }^{2} D_{5 / 2}\right) 5 g$ & ${ }^{2}[13 / 2]$ & 6 & 146068.85092 & 0.0019 & & $100 \%$ & & 1 \\
\hline $5 g$ & $(5 / 2)^{2}[13 / 2]$ & $3 d^{9}\left({ }^{2} D_{5 / 2}\right) 5 g$ & ${ }^{2}[13 / 2]$ & 7 & 146068.90547 & 0.0012 & & $100 \%$ & & 1 \\
\hline $5 g$ & $(5 / 2)^{2}[5 / 2]$ & $3 \mathrm{~d}^{9}\left({ }^{2} \mathrm{D}_{5 / 2}\right) 5 \mathrm{~g}$ & ${ }^{2}[5 / 2]$ & 3 & 146072.7739 & 0.0020 & & $100 \%$ & & 5 \\
\hline $5 g$ & $(5 / 2)^{2}[5 / 2]$ & $3 d^{9}\left({ }^{2} D_{5 / 2}\right) 5 g$ & ${ }^{2}[5 / 2]$ & 2 & 146072.8134 & 0.0018 & & $100 \%$ & & 6 \\
\hline
\end{tabular}


Table A2. Cont.

\begin{tabular}{|c|c|c|c|c|c|c|c|c|c|c|}
\hline & Label $^{\text {a }}$ & Configuration & Term & $J$ & Level $^{\mathrm{b}}, \mathrm{cm}^{-1}$ & Unc. ${ }^{c}, \mathrm{~cm}^{-1}$ & Landé $g$ & Leading Percentages ${ }^{\mathrm{e}}$ & Note $^{\mathrm{f}}$ & $N_{\text {lines }} \mathrm{g}$ \\
\hline $5 \mathrm{~g}$ & $(5 / 2)^{2}[7 / 2]$ & $3 \mathrm{~d}^{9}\left({ }^{2} \mathrm{D}_{5 / 2}\right) 5 \mathrm{~g}$ & $2[7 / 2]$ & 3 & 146093.9312 & 0.0014 & & $100 \%$ & & 5 \\
\hline $5 g$ & $(5 / 2)^{2}[7 / 2]$ & $3 d^{9}\left({ }^{2} D_{5 / 2}\right) 5 g$ & ${ }^{2}[7 / 2]$ & 4 & 146093.9535 & 0.0012 & & $100 \%$ & & 2 \\
\hline $5 g$ & $(5 / 2)^{2}[11 / 2]$ & $3 d^{9}\left({ }^{2} D_{5 / 2}\right) 5 g$ & ${ }^{2}[11 / 2]$ & 5 & 146103.2223 & 0.0018 & & $100 \%$ & & 7 \\
\hline $5 \mathrm{~g}$ & $(5 / 2)^{2}[11 / 2]$ & $3 d^{9}\left({ }^{2} D_{5 / 2}\right) 5 g$ & ${ }^{2}[11 / 2]$ & 6 & 146103.25704 & 0.0011 & & $100 \%$ & & 3 \\
\hline $5 g$ & $(5 / 2)^{2}[9 / 2]$ & $3 d^{9}\left({ }^{2} D_{5 / 2}\right) 5 g$ & ${ }^{2}[9 / 2]$ & 4 & 146107.1016 & 0.0016 & & $100 \%$ & & 7 \\
\hline $5 \mathrm{~g}$ & $(5 / 2)^{2}[9 / 2]$ & $3 d^{9}\left({ }^{2} D_{5 / 2}\right) 5 g$ & ${ }^{2}[9 / 2]$ & 5 & 146107.1288 & 0.0010 & & $100 \%$ & & 6 \\
\hline $6 \mathrm{~d}$ & $(5 / 2)^{2}[1 / 2]$ & $3 d^{9}\left({ }^{2} D_{5 / 2}\right) 6 d$ & ${ }^{2}[1 / 2]$ & 1 & 146215.634 & 0.004 & & $98 \%+1 \% 6 d(5 / 2)^{2}[3 / 2]$ & & 7 \\
\hline $6 \mathrm{~d}$ & $(5 / 2)^{2}[9 / 2]$ & $3 d^{9}\left({ }^{2} D_{5 / 2}\right) 6 d$ & ${ }^{2}[9 / 2]$ & 5 & 146402.715 & 0.004 & & $100 \%$ & & 8 \\
\hline $6 \mathrm{~d}$ & $(5 / 2)^{2}[3 / 2]$ & $3 d^{9}\left({ }^{2} D_{5 / 2}\right) 6 d$ & ${ }^{2}[3 / 2]$ & 2 & 146415.599 & 0.004 & & $97 \%+2 \% 6 \mathrm{~d}(5 / 2)^{2}[5 / 2]$ & & 12 \\
\hline $6 \mathrm{~d}$ & $(5 / 2)^{2}[9 / 2]$ & $3 d^{9}\left({ }^{2} D_{5 / 2}\right) 6 d$ & ${ }^{2}[9 / 2]$ & 4 & 146423.201 & 0.003 & & $100 \%$ & & 10 \\
\hline $6 \mathrm{~d}$ & $(5 / 2)^{2}[3 / 2]$ & $3 d^{9}\left({ }^{2} D_{5 / 2}\right) 6 d$ & ${ }^{2}[3 / 2]$ & 1 & 146427.948 & 0.003 & & $99 \%+1 \% 6 \mathrm{~d}(5 / 2)^{2}[1 / 2]$ & & 12 \\
\hline $6 \mathrm{~d}$ & $(5 / 2)^{2}[5 / 2]$ & $3 \mathrm{~d}^{9}\left({ }^{2} \mathrm{D}_{5 / 2}\right) 6 \mathrm{~d}$ & ${ }^{2}[5 / 2]$ & 3 & 146496.100 & 0.003 & & $99 \%+1 \% 6 \mathrm{~d}(5 / 2)^{2}[7 / 2]$ & & 16 \\
\hline $6 \mathrm{~d}$ & $(5 / 2)^{2}[7 / 2]$ & $3 d^{9}\left({ }^{2} D_{5 / 2}\right) 6 d$ & ${ }^{2}[7 / 2]$ & 3 & 146556.381 & 0.003 & & $99 \%+1 \% 6 \mathrm{~d}(5 / 2)^{2}[5 / 2]$ & & 19 \\
\hline $6 \mathrm{~d}$ & $(5 / 2)^{2}[7 / 2]$ & $3 d^{9}\left({ }^{2} D_{5 / 2}\right) 6 d$ & ${ }^{2}[7 / 2]$ & 4 & 146559.703 & 0.003 & & $100 \%$ & & 14 \\
\hline $6 \mathrm{~d}$ & $(5 / 2)^{2}[5 / 2]$ & $3 d^{9}\left({ }^{2} D_{5 / 2}\right) 6 d$ & ${ }^{2}[5 / 2]$ & 2 & 146575.101 & 0.003 & & $97 \%+2 \% 6 \mathrm{~d}(5 / 2)^{2}[3 / 2]+1 \% 7 \mathrm{~s}(3 / 2)^{2}[3 / 2]$ & & 23 \\
\hline $7 \mathrm{~s}$ & $(3 / 2)^{2}[3 / 2]$ & $3 d^{9}\left({ }^{2} D_{3 / 2}\right) 7 \mathrm{~s}$ & ${ }^{2}[3 / 2]$ & 1 & 146886.1667 & 0.0021 & & $100 \%$ & & 20 \\
\hline $7 \mathrm{~s}$ & $(3 / 2)^{2}[3 / 2]$ & $3 \mathrm{~d}^{9}\left({ }^{2} \mathrm{D}_{3 / 2}\right) 7 \mathrm{~s}$ & ${ }^{2}[3 / 2]$ & 2 & 146936.3180 & 0.0018 & & $99 \%$ & & 24 \\
\hline $6 \mathrm{~d}$ & $(5 / 2)^{2}[1 / 2]$ & $3 d^{9}\left({ }^{2} D_{5 / 2}\right) 6 d$ & ${ }^{2}[1 / 2]$ & 0 & 147097.835 & 0.005 & & $60 \%+29 \% 6 d(3 / 2)^{2}[1 / 2]+4 \% 7 d(5 / 2)^{2}[1 / 2]$ & & 6 \\
\hline $7 \mathrm{p}$ & $(5 / 2)^{2}[3 / 2]^{\circ}$ & $3 d^{9}\left({ }^{2} D_{5 / 2}\right) 7 p$ & ${ }^{2}[3 / 2]^{\circ}$ & 2 & 147327.659 & 0.007 & & $94 \%+4 \% s p+1 \% 7 p(5 / 2)^{2}[5 / 2]^{\circ}$ & & 10 \\
\hline $7 \mathrm{p}$ & $(5 / 2)^{2}[7 / 2]^{\circ}$ & $3 d^{9}\left({ }^{2} D_{5 / 2}\right) 7 p$ & ${ }^{2}[7 / 2]^{\circ}$ & 3 & 147525.93 & 0.06 & & $98 \%+1 \% \mathrm{sp}$ & $\mathrm{R}$ & 3 \\
\hline $7 \mathrm{p}$ & $(5 / 2)^{2}[3 / 2]^{\circ}$ & $3 d^{9}\left({ }^{2} D_{5 / 2}\right) 7 p$ & ${ }^{2}[3 / 2]^{\circ}$ & 1 & 147562.672 & 0.006 & & $94 \%+3 \% s p+1 \% 5 f(3 / 2)^{2}[3 / 2]^{\circ}$ & & 8 \\
\hline $7 \mathrm{p}$ & $(5 / 2)^{2}[7 / 2]^{\circ}$ & $3 d^{9}\left({ }^{2} D_{5 / 2}\right) 7 p$ & ${ }^{2}[7 / 2]^{\circ}$ & 4 & 147596.655 & 0.006 & & $100 \%$ & & 4 \\
\hline $7 \mathrm{p}$ & $(5 / 2)^{2}[5 / 2]^{\circ}$ & $3 d^{9}\left({ }^{2} D_{5 / 2}\right) 7 p$ & ${ }^{2}[5 / 2]^{\circ}$ & 2 & 147647.699 & 0.009 & & $94 \%+3 \% s p+2 \% 7 p(5 / 2)^{2}[3 / 2]^{\circ}$ & & 7 \\
\hline $7 \mathrm{p}$ & $(5 / 2)^{2}[5 / 2]^{\circ}$ & $3 d^{9}\left({ }^{2} D_{5 / 2}\right) 7 p$ & ${ }^{2}[5 / 2]^{\circ}$ & 3 & 147762.990 & 0.005 & & $98 \%$ & & 9 \\
\hline $5 \mathrm{f}$ & $(3 / 2)^{2}[3 / 2]^{\circ}$ & $3 d^{9}\left({ }^{2} D_{3 / 2}\right) 5 f$ & ${ }^{2}[3 / 2]^{\circ}$ & 2 & 147987.359 & 0.004 & & $99 \%$ & & 8 \\
\hline $5 \mathrm{f}$ & $(3 / 2)^{2}[3 / 2]^{\circ}$ & $3 d^{9}\left({ }^{2} D_{3 / 2}\right) 5 f$ & ${ }^{2}[3 / 2]^{\circ}$ & 1 & 148016.157 & 0.004 & & $99 \%+1 \% 7 \mathrm{p}(5 / 2)^{2}[3 / 2]^{\circ}$ & & 11 \\
\hline $5 f$ & $(3 / 2)^{2}[9 / 2]^{\circ}$ & $3 d^{9}\left({ }^{2} D_{3 / 2}\right) 5 f$ & ${ }^{2}[9 / 2]^{\circ}$ & 5 & 148028.7360 & 0.0023 & & $100 \%$ & & 5 \\
\hline $5 \mathrm{f}$ & $(3 / 2)^{2}[9 / 2]^{\circ}$ & $3 d^{9}\left({ }^{2} D_{3 / 2}\right) 5 f$ & ${ }^{2}[9 / 2]^{\circ}$ & 4 & 148033.574 & 0.003 & & $100 \%$ & & 6 \\
\hline $5 \mathrm{f}$ & $(3 / 2)^{2}[5 / 2]^{\circ}$ & $3 d^{9}\left({ }^{2} D_{3 / 2}\right) 5 f$ & ${ }^{2}[5 / 2]^{\circ}$ & 3 & 148061.467 & 0.003 & & $97 \%+2 \% 5 f(3 / 2)^{2}[7 / 2]^{\circ}$ & & 9 \\
\hline $5 f$ & $(3 / 2)^{2}[5 / 2]^{\circ}$ & $3 d^{9}\left({ }^{2} D_{3 / 2}\right) 5 f$ & ${ }^{2}[5 / 2]^{\circ}$ & 2 & 148066.011 & 0.003 & & $99 \%$ & & 11 \\
\hline $5 f$ & $(3 / 2)^{2}[7 / 2]^{\circ}$ & $3 d^{9}\left({ }^{2} D_{3 / 2}\right) 5 f$ & ${ }^{2}[7 / 2]^{\circ}$ & 3 & 148102.986 & 0.003 & & $97 \%+3 \% 5 f(3 / 2)^{2}[5 / 2]^{\circ}$ & & 9 \\
\hline $5 f$ & $(3 / 2)^{2}[7 / 2]^{\circ}$ & $3 d^{9}\left({ }^{2} D_{3 / 2}\right) 5 f$ & ${ }^{2}[7 / 2]^{\circ}$ & 4 & 148105.366 & 0.003 & & $100 \%$ & & 5 \\
\hline $5 g$ & $(3 / 2)^{2}[5 / 2]$ & $3 d^{9}\left({ }^{2} D_{3 / 2}\right) 5 g$ & ${ }^{2}[5 / 2]$ & 3 & 148133.777 & 0.003 & & $100 \%$ & & 3 \\
\hline $5 \mathrm{~g}$ & $(3 / 2)^{2}[5 / 2]$ & $3 d^{9}\left({ }^{2} D_{3 / 2}\right) 5 g$ & ${ }^{2}[5 / 2]$ & 2 & 148133.832 & 0.005 & & $100 \%$ & & 3 \\
\hline $5 g$ & $(3 / 2)^{2}[11 / 2]$ & $3 d^{9}\left({ }^{2} D_{3 / 2}\right) 5 g$ & ${ }^{2}[11 / 2]$ & 5 & 148145.7906 & 0.0022 & & $100 \%$ & & 3 \\
\hline $5 g$ & $(3 / 2)^{2}[11 / 2]$ & $3 \mathrm{~d}^{9}\left({ }^{2} \mathrm{D}_{3 / 2}\right) 5 \mathrm{~g}$ & ${ }^{2}[11 / 2]$ & 6 & 148145.8203 & 0.0021 & & $100 \%$ & & 3 \\
\hline $5 g$ & $(3 / 2)^{2}[7 / 2]$ & $3 \mathrm{~d}^{9}\left({ }^{2} \mathrm{D}_{3 / 2}\right) 5 \mathrm{~g}$ & ${ }^{2}[7 / 2]$ & 3 & 148167.557 & 0.003 & & $100 \%$ & & 5 \\
\hline $5 g$ & $(3 / 2)^{2}[7 / 2]$ & $3 \mathrm{~d}^{9}\left({ }^{2} \mathrm{D}_{3 / 2}\right) 5 \mathrm{~g}$ & ${ }^{2}[7 / 2]$ & 4 & 148167.5920 & 0.0017 & & $100 \%$ & & 4 \\
\hline $5 \mathrm{~g}$ & $(3 / 2)^{2}[9 / 2]$ & $3 d^{9}\left({ }^{2} D_{3 / 2}\right) 5 g$ & ${ }^{2}[9 / 2]$ & 4 & 148179.1541 & 0.0019 & & $100 \%$ & & 4 \\
\hline $5 g$ & $(3 / 2)^{2}[9 / 2]$ & $3 \mathrm{~d}^{9}\left({ }^{2} \mathrm{D}_{3 / 2}\right) 5 \mathrm{~g}$ & ${ }^{2}[9 / 2]$ & 5 & 148179.1829 & 0.0016 & & $100 \%$ & & 4 \\
\hline
\end{tabular}


Table A2. Cont.

\begin{tabular}{|c|c|c|c|c|c|c|c|c|c|c|}
\hline & Label $^{\text {a }}$ & Configuration & Term & $J$ & Level $^{\mathrm{b}}, \mathrm{cm}^{-1}$ & Unc. ${ }^{\mathrm{c}}, \mathrm{cm}^{-1}$ & Landé $g^{\mathrm{d}}$ & Leading Percentages $\mathrm{e}$ & Note $^{f}$ & $N_{\text {lines }} \mathrm{g}$ \\
\hline $6 \mathrm{~d}$ & $(3 / 2)^{2}[1 / 2]$ & $3 d^{9}\left({ }^{2} D_{3 / 2}\right) 6 d$ & ${ }^{2}[1 / 2]$ & 1 & 148361.542 & 0.004 & & $99 \%$ & & 10 \\
\hline $6 \mathrm{~d}$ & $(3 / 2)^{2}[7 / 2]$ & $3 d^{9}\left({ }^{2} D_{3 / 2}\right) 6 d$ & ${ }^{2}[7 / 2]$ & 3 & 148481.763 & 0.003 & & $100 \%$ & & 11 \\
\hline $6 \mathrm{~d}$ & $(3 / 2)^{2}[7 / 2]$ & $3 d^{9}\left({ }^{2} D_{3 / 2}\right) 6 d$ & ${ }^{2}[7 / 2]$ & 4 & 148515.532 & 0.004 & & $100 \%$ & & 9 \\
\hline $6 \mathrm{~d}$ & $(3 / 2)^{2}[3 / 2]$ & $3 d^{9}\left({ }^{2} D_{3 / 2}\right) 6 d$ & ${ }^{2}[3 / 2]$ & 1 & 148521.575 & 0.003 & & $100 \%$ & & 12 \\
\hline $6 \mathrm{~d}$ & $(3 / 2)^{2}[3 / 2]$ & $3 d^{9}\left({ }^{2} D_{3 / 2}\right) 6 d$ & $2[3 / 2]$ & 2 & 148559.958 & 0.003 & & $99 \%+1 \% 6 d(3 / 2)^{2}[5 / 2]$ & & 15 \\
\hline $6 \mathrm{~d}$ & $(3 / 2)^{2}[5 / 2]$ & $3 d^{9}\left({ }^{2} D_{3 / 2}\right) 6 d$ & $2[5 / 2]$ & 3 & 148631.096 & 0.003 & & $100 \%$ & & 13 \\
\hline $6 \mathrm{~d}$ & $(3 / 2)^{2}[5 / 2]$ & $3 d^{9}\left({ }^{2} D_{3 / 2}\right) 6 d$ & $2[5 / 2]$ & 2 & 148642.489 & 0.003 & & $99 \%+1 \% 6 \mathrm{~d}(3 / 2)^{2}[3 / 2]$ & & 13 \\
\hline $6 \mathrm{~d}$ & $(3 / 2)^{2}[1 / 2]$ & $3 d^{9}\left({ }^{2} D_{3 / 2}\right) 6 d$ & ${ }^{2}[1 / 2]$ & 0 & 149202.607 & 0.007 & & $51 \%+28 \% 7 d(5 / 2)^{2}[1 / 2]+6 \% 6 d(5 / 2)^{2}[1 / 2]$ & & 3 \\
\hline $7 \mathrm{p}$ & $(3 / 2)^{2}[1 / 2]^{\circ}$ & $3 d^{9}\left({ }^{2} D_{3 / 2}\right) 7 p$ & ${ }^{2}[1 / 2]^{\circ}$ & 0 & 149371.08 & 0.08 & & $94 \%+5 \% \mathrm{sp}$ & $\mathrm{N}$ & 2 \\
\hline $7 \mathrm{p}$ & $(3 / 2)^{2}[1 / 2]^{\circ}$ & $3 d^{9}\left({ }^{2} D_{3 / 2}\right) 7 p$ & ${ }^{2}[1 / 2]^{\circ}$ & 1 & 149484.111 & 0.008 & & $95 \%+4 \% s p+1 \% 7 p(5 / 2)^{2}[3 / 2]^{\circ}$ & $\mathrm{N}$ & 5 \\
\hline $7 \mathrm{p}$ & $(3 / 2)^{2}[5 / 2]^{\circ}$ & $3 d^{9}\left({ }^{2} D_{3 / 2}\right) 7 p$ & ${ }^{2}[5 / 2]^{\circ}$ & 2 & 149525.97 & 0.05 & & $64 \%+26 \% 7 p(3 / 2)^{2}[3 / 2]^{\circ}+8 \% s p$ & $\mathrm{~N}$ & 4 \\
\hline $7 \mathrm{p}$ & $(3 / 2)^{2}[5 / 2]^{\circ}$ & $3 d^{9}\left({ }^{2} D_{3 / 2}\right) 7 p$ & ${ }^{2}[5 / 2]^{\circ}$ & 3 & 149624.47 & 0.05 & & $92 \%+6 \% \mathrm{sp}$ & $\mathrm{N}$ & 2 \\
\hline $7 \mathrm{p}$ & $(3 / 2)^{2}[3 / 2]^{\circ}$ & $3 d^{9}\left({ }^{2} D_{3 / 2}\right) 7 p$ & ${ }^{2}[3 / 2]^{\circ}$ & 2 & 149726.69 & 0.05 & & $63 \%+33 \% 7 p(3 / 2)^{2}[5 / 2]^{\circ}+3 \% s p$ & $\mathrm{~N}$ & 3 \\
\hline $7 \mathrm{p}$ & $(3 / 2)^{2}[3 / 2]^{\circ}$ & $3 d^{9}\left({ }^{2} D_{3 / 2}\right) 7 p$ & ${ }^{2}[3 / 2]^{\circ}$ & 1 & 149765.88 & 0.05 & & $95 \%+4 \% \mathrm{sp}$ & $\mathrm{N}$ & 4 \\
\hline $\mathrm{sp}$ & $\left({ }^{1} \mathrm{D}\right)^{1} \mathrm{P}^{\circ}{ }^{1} \mathrm{D}^{\circ}$ & $3 d^{8}\left({ }^{1} D\right) 4 s 4 p\left({ }^{1} P^{\circ}\right)$ & ${ }^{1} \mathrm{D}^{\circ}$ & 2 & 150249.887 & 0.008 & & $38 \%+28 \%$ sp $\left({ }^{3} \mathrm{P}\right)^{1} \mathrm{P}^{\circ}{ }^{3} \mathrm{P}^{\circ}+11 \% 7 \mathrm{p}^{1} \mathrm{D}^{\circ}$ & & 8 \\
\hline $8 \mathrm{~s}$ & $(5 / 2)^{2}[5 / 2]$ & $3 d^{9}\left({ }^{2} D_{5 / 2}\right) 8 s$ & ${ }^{2}[5 / 2]$ & 3 & 150742.896 & 0.003 & & $100 \%$ & & 13 \\
\hline $8 \mathrm{~s}$ & $(5 / 2)^{2}[5 / 2]$ & $3 d^{9}\left({ }^{2} D_{5 / 2}\right) 8 s$ & ${ }^{2}[5 / 2]$ & 2 & 150782.454 & 0.003 & & $100 \%$ & & 13 \\
\hline $6 f$ & $(5 / 2)^{2}[1 / 2]^{\circ}$ & $3 d^{9}\left({ }^{2} D_{5 / 2}\right) 6 f$ & ${ }^{2}[1 / 2]^{\circ}$ & 1 & 151161.379 & 0.007 & & $63 \%+21 \% s p+5 \% 8 p(5 / 2)^{2}[3 / 2]^{\circ}$ & $\mathrm{N}$ & 6 \\
\hline $6 f$ & $(5 / 2)^{2}[1 / 2]^{\circ}$ & $3 d^{9}\left({ }^{2} D_{5 / 2}\right) 6 f$ & ${ }^{2}[1 / 2]^{\circ}$ & 0 & 151327.262 & 0.008 & & $98 \%+1 \% \mathrm{sp}$ & & 2 \\
\hline $6 f$ & $(5 / 2)^{2}[11 / 2]^{\circ}$ & $3 d^{9}\left({ }^{2} D_{5 / 2}\right) 6 f$ & ${ }^{2}[11 / 2]^{\circ}$ & 5 & 151372.330 & 0.004 & & $100 \%$ & & 4 \\
\hline $6 \mathrm{f}$ & $(5 / 2)^{2}[3 / 2]^{\circ}$ & $3 d^{9}\left({ }^{2} D_{5 / 2}\right) 6 f$ & ${ }^{2}[3 / 2]^{\circ}$ & 1 & 151373.840 & 0.006 & & $96 \%+3 \%$ ff $(5 / 2)^{2}[1 / 2]^{\circ}$ & & 5 \\
\hline $6 f$ & $(5 / 2)^{2}[3 / 2]^{\circ}$ & $3 d^{9}\left({ }^{2} D_{5 / 2}\right) 6 f$ & ${ }^{2}[3 / 2]^{\circ}$ & 2 & 151375.318 & 0.004 & & $52 \%+46 \%$ ff $(5 / 2)^{2}[5 / 2]^{\circ}$ & & 7 \\
\hline $6 f$ & $(5 / 2)^{2}[11 / 2]^{\circ}$ & $3 d^{9}\left({ }^{2} D_{5 / 2}\right) 6 f$ & ${ }^{2}[11 / 2]^{\circ}$ & 6 & 151377.688 & 0.005 & & $100 \%$ & & 2 \\
\hline $6 \mathrm{f}$ & $(5 / 2)^{2}[5 / 2]^{\circ}$ & $3 d^{9}\left({ }^{2} D_{5 / 2}\right) 6 f$ & ${ }^{2}[5 / 2]^{\circ}$ & 3 & 151402.621 & 0.004 & & $71 \%+28 \% 6 f(5 / 2)^{2}[7 / 2]^{\circ}$ & & 7 \\
\hline $6 \mathrm{f}$ & $(5 / 2)^{2}[5 / 2]^{\circ}$ & $3 d^{9}\left({ }^{2} D_{5 / 2}\right) 6 f$ & ${ }^{2}[5 / 2]^{\circ}$ & 2 & 151403.942 & 0.004 & & $53 \%+44 \%$ 6f $(5 / 2)^{2}[3 / 2]^{\circ}$ & & 8 \\
\hline $6 f$ & $(5 / 2)^{2}[7 / 2]^{\circ}$ & $3 d^{9}\left({ }^{2} D_{5 / 2}\right) 6 f$ & $2[7 / 2]^{\circ}$ & 4 & 151419.022 & 0.004 & & $90 \%+10 \%$ 6f $(5 / 2)^{2}[9 / 2]^{\circ}$ & & 8 \\
\hline $6 f$ & $(5 / 2)^{2}[9 / 2]^{\circ}$ & $3 d^{9}\left({ }^{2} D_{5 / 2}\right) 6 f$ & ${ }^{2}[9 / 2]^{\circ}$ & 4 & 151421.791 & 0.004 & & $90 \%+10 \%$ 6f $(5 / 2)^{2}[7 / 2]^{\circ}$ & & 9 \\
\hline $6 f$ & $(5 / 2)^{2}[9 / 2]^{\circ}$ & $3 d^{9}\left({ }^{2} D_{5 / 2}\right) 6 f$ & ${ }^{2}[9 / 2]^{\circ}$ & 5 & 151423.056 & 0.004 & & $100 \%$ & & 4 \\
\hline $8 p$ & $(5 / 2)^{2}[3 / 2]^{\circ}$ & $3 d^{9}\left({ }^{2} D_{5 / 2}\right) 8 p$ & $2[3 / 2]^{\circ}$ & 1 & 151424.241 & 0.004 & & $33 \%$ 6f $(5 / 2)^{2}[1 / 2]^{\circ}+36 \% s p+20 \% 8 p(5 / 2)^{2}[3 / 2]^{\circ}$ & & 8 \\
\hline $6 \mathrm{~g}$ & $(5 / 2)^{2}[3 / 2]$ & $3 \mathrm{~d}^{9}\left({ }^{2} \mathrm{D}_{5 / 2}\right) 6 \mathrm{~g}$ & ${ }^{2}[3 / 2]$ & 1 & 151440.091 & 0.008 & & $100 \%$ & & 2 \\
\hline $6 g$ & $(5 / 2)^{2}[3 / 2]$ & $3 d^{9}\left({ }^{2} D_{5 / 2}\right) 6 g$ & ${ }^{2}[3 / 2]$ & 2 & 151440.178 & 0.006 & & $100 \%$ & & 2 \\
\hline $6 \mathrm{f}$ & $(5 / 2)^{2}[7 / 2]^{\circ}$ & $3 d^{9}\left({ }^{2} D_{5 / 2}\right) 6 f$ & ${ }^{2}[7 / 2]^{\circ}$ & 3 & 151441.899 & 0.006 & & $70 \%+27 \% 6 f(5 / 2)^{2}[5 / 2]^{\circ}+2 \% s p$ & & 7 \\
\hline $6 \mathrm{~g}$ & $(5 / 2)^{2}[13 / 2]$ & $3 d^{9}\left({ }^{2} D_{5 / 2}\right) 6 g$ & ${ }^{2}[13 / 2]$ & 6 & 151450.414 & 0.007 & & $100 \%$ & & 2 \\
\hline 68 & $(5 / 2)^{2}[13 / 2]$ & $3 d^{9}\left({ }^{2} D_{5 / 2}\right) 6 g$ & ${ }^{2}[13 / 2]$ & 7 & 151450.488 & 0.003 & & $100 \%$ & & 1 \\
\hline $6 \mathrm{~g}$ & $(5 / 2)^{2}[5 / 2]$ & $3 d^{9}\left({ }^{2} D_{5 / 2}\right) 6 g$ & ${ }^{2}[5 / 2]$ & 3 & 151452.690 & 0.007 & & $100 \%$ & & 4 \\
\hline $6 g$ & $(5 / 2)^{2}[5 / 2]$ & $3 d^{9}\left({ }^{2} D_{5 / 2}\right) 6 g$ & ${ }^{2}[5 / 2]$ & 2 & 151452.701 & 0.006 & & $100 \%$ & & 4 \\
\hline $6 \mathrm{~h}$ & $(5 / 2)^{2}[5 / 2]^{\circ}$ & $3 d^{9}\left({ }^{2} D_{5 / 2}\right) 6 h$ & ${ }^{2}[5 / 2]^{\circ}$ & 2 & {$[151458.3]$} & 6 & & $100 \%$ & pf & 0 \\
\hline $6 \mathrm{~h}$ & $(5 / 2)^{2}[5 / 2]^{\circ}$ & $3 d^{9}\left({ }^{2} D_{5 / 2}\right) 6 h$ & ${ }^{2}[5 / 2]^{\circ}$ & 3 & {$[151458.4]$} & 6 & & $100 \%$ & $\mathrm{pf}$ & 0 \\
\hline $6 \mathrm{~h}$ & $(5 / 2)^{2}[15 / 2]^{\circ}$ & $3 d^{9}\left({ }^{2} D_{5 / 2}\right) 6 h$ & ${ }^{2}[15 / 2]^{\circ}$ & 7 & {$[151461.7]$} & 6 & & $100 \%$ & $\mathrm{pf}$ & 0 \\
\hline $6 \mathrm{~h}$ & $(5 / 2)^{2}[15 / 2]^{\circ}$ & $3 d^{9}\left({ }^{2} D_{5 / 2}\right) 6 h$ & ${ }^{2}[15 / 2]^{\circ}$ & 8 & [151461.8] & 6 & & $100 \%$ & $\mathrm{pf}$ & 0 \\
\hline $6 \mathrm{~h}$ & $(5 / 2)^{2}[7 / 2]^{\circ}$ & $3 d^{9}\left({ }^{2} D_{5 / 2}\right) 6 h$ & ${ }^{2}[7 / 2]^{\circ}$ & 3 & [151463.9] & 6 & & $100 \%$ & pf & 0 \\
\hline
\end{tabular}


Table A2. Cont.

\begin{tabular}{|c|c|c|c|c|c|c|c|c|c|c|}
\hline & Label $^{\mathrm{a}}$ & Configuration & Term & $\bar{J}$ & Level $^{\mathrm{b}}, \mathrm{cm}^{-1}$ & Unc. ${ }^{\mathrm{c}}, \mathrm{cm}^{-1}$ & Landé $g^{\mathrm{d}}$ & Leading Percentages ${ }^{\mathrm{e}}$ & Note $^{f}$ & $N_{\text {lines }} g$ \\
\hline $6 \mathrm{~h}$ & $(5 / 2)^{2}[7 / 2]^{\circ}$ & $3 d^{9}\left({ }^{2} D_{5 / 2}\right) 6 h$ & ${ }^{2}[7 / 2]^{\circ}$ & 4 & [151464.0] & 6 & & $100 \%$ & $\mathrm{pf}$ & 0 \\
\hline $6 \mathrm{~g}$ & $(5 / 2)^{2}[7 / 2]$ & $3 d^{9}\left({ }^{2} D_{5 / 2}\right) 6 g$ & ${ }^{2}[7 / 2]$ & 3 & 151464.789 & 0.006 & & $100 \%$ & & 4 \\
\hline $6 \mathrm{~g}$ & $(5 / 2)^{2}[7 / 2]$ & $3 d^{9}\left({ }^{2} D_{5 / 2}\right) 6 g$ & ${ }^{2}[7 / 2]$ & 4 & 151464.796 & 0.005 & & $100 \%$ & & 2 \\
\hline $6 \mathrm{~h}$ & $(5 / 2)^{2}[9 / 2]^{\circ}$ & $3 d^{9}\left({ }^{2} D_{5 / 2}\right) 6 h$ & ${ }^{2}[9 / 2]^{\circ}$ & 4 & [151468.8] & 6 & & $100 \%$ & pf & 0 \\
\hline $6 \mathrm{~h}$ & $(5 / 2)^{2}[9 / 2]^{\circ}$ & $3 d^{9}\left(D_{5 / 2}\right) 6 h$ & $2[9 / 2]^{\circ}$ & 5 & [151468.9] & 6 & & $100 \%$ & $\mathrm{pf}$ & 0 \\
\hline $6 \mathrm{~h}$ & $(5 / 2)^{2}[13 / 2]^{\circ}$ & $3 d^{9}\left({ }^{2} D_{5 / 2}\right) 6 h$ & ${ }^{2}[13 / 2]^{\circ}$ & 6 & [151469.8] & 6 & & $100 \%$ & $\mathrm{pf}$ & 0 \\
\hline $6 \mathrm{~h}$ & $(5 / 2)^{2}[13 / 2]^{\circ}$ & $3 d^{9}\left({ }^{2} D_{5 / 2}\right) 6 h$ & ${ }^{2}[13 / 2]^{\circ}$ & 7 & [151470.0] & 6 & & $100 \%$ & $\mathrm{pf}$ & 0 \\
\hline $6 \mathrm{~g}$ & $(5 / 2)^{2}[11 / 2]$ & $3 d^{9}\left({ }^{2} D_{5 / 2}\right) 6 g$ & ${ }^{2}[11 / 2]$ & 5 & 151470.113 & 0.003 & & $100 \%$ & & 5 \\
\hline $6 g$ & $(5 / 2)^{2}[11 / 2]$ & $3 \mathrm{~d}^{9}\left({ }^{2} \mathrm{D}_{5 / 2}\right) 6 \mathrm{~g}$ & $2[11 / 2]$ & 6 & 151470.130 & 0.005 & & $100 \%$ & & 3 \\
\hline $6 \mathrm{~h}$ & $(5 / 2)^{2}[11 / 2]^{\circ}$ & $3 d^{9}\left({ }^{2} D_{5 / 2}\right) 6 h$ & ${ }^{2}[11 / 2]^{\circ}$ & 5 & [151471.4] & 6 & & $100 \%$ & $\mathrm{pf}$ & 0 \\
\hline $6 \mathrm{~h}$ & $(5 / 2)^{2}[11 / 2]^{\circ}$ & $3 d^{9}\left({ }^{2} D_{5 / 2}\right) 6 h$ & ${ }^{2}[11 / 2]^{\circ}$ & 6 & [151471.5] & 6 & & $100 \%$ & $\mathrm{pf}$ & 0 \\
\hline $6 \mathrm{~g}$ & $(5 / 2)^{2}[9 / 2]$ & $3 d^{9}\left({ }^{2} D_{5 / 2}\right) 6 g$ & ${ }^{2}[9 / 2]$ & 4 & 151472.264 & 0.005 & & $100 \%$ & & 5 \\
\hline $6 \mathrm{~g}$ & $(5 / 2)^{2}[9 / 2]$ & $3 d^{9}\left({ }^{2} D_{5 / 2}\right) 6 g$ & ${ }^{2}[9 / 2]$ & 5 & 151472.278 & 0.005 & & $100 \%$ & & 3 \\
\hline $7 \mathrm{~d}$ & $(5 / 2)^{2}[1 / 2]$ & $\left.3 \mathrm{~d}^{9}{ }^{2} \mathrm{D}_{5 / 2}\right) 7 \mathrm{~d}$ & $2[1 / 2]$ & 1 & 151552.191 & 0.007 & & $98 \%+1 \% 7 d(5 / 2)^{2}[3 / 2]$ & & 2 \\
\hline $7 d$ & $(5 / 2)^{2}[9 / 2]$ & $3 d^{9}\left({ }^{2} D_{5 / 2}\right) 7 d$ & $2[9 / 2]$ & 5 & 151656.317 & 0.005 & & $100 \%$ & & 5 \\
\hline $7 \mathrm{~d}$ & $(5 / 2)^{2}[3 / 2]$ & $3 d^{9}\left({ }^{2} D_{5 / 2}\right) 7 d$ & $2[3 / 2]$ & 2 & 151662.985 & 0.005 & & $96 \%+4 \% 7 d(5 / 2)^{2}[5 / 2]$ & & 6 \\
\hline $7 \mathrm{~d}$ & $(5 / 2)^{2}[9 / 2]$ & $3 d^{9}\left({ }^{2} D_{5 / 2}\right) 7 d$ & $2[9 / 2]$ & 4 & 151668.328 & 0.005 & & $99 \%$ & & 7 \\
\hline $7 \mathrm{~d}$ & $(5 / 2)^{2}[3 / 2]$ & $3 d^{9}\left({ }^{2} D_{5 / 2}\right) 7 d$ & $2[3 / 2]$ & 1 & 151671.666 & 0.006 & & $99 \%+1 \% 7 d(5 / 2)^{2}[1 / 2]$ & & 4 \\
\hline $7 \mathrm{~d}$ & $(5 / 2)^{2}[5 / 2]$ & $3 \mathrm{~d}^{9}\left(^{2} \mathrm{D}_{5 / 2}\right) 7 \mathrm{~d}$ & $2[5 / 2]$ & 3 & 151708.347 & 0.004 & & $99 \%+1 \% 7 \mathrm{~d}(5 / 2)^{2}[7 / 2]$ & & 8 \\
\hline $7 \mathrm{~d}$ & $(5 / 2)^{2}[7 / 2]$ & $3 d^{9}\left({ }^{2} D_{5 / 2}\right) 7 d$ & $2[7 / 2]$ & 3 & 151743.558 & 0.003 & & $99 \%+1 \% 7 d(5 / 2)^{2}[5 / 2]$ & & 13 \\
\hline $7 \mathrm{~d}$ & $(5 / 2)^{2}[7 / 2]$ & $3 d^{9}\left({ }^{2} D_{5 / 2}\right) 7 d$ & $2[7 / 2]$ & 4 & 151744.916 & 0.005 & & $99 \%$ & & 8 \\
\hline $7 \mathrm{~d}$ & $(5 / 2)^{2}[5 / 2]$ & $3 d^{9}\left({ }^{2} D_{5 / 2}\right) 7 d$ & $2[5 / 2]$ & 2 & 151757.601 & 0.004 & & $96 \%+4 \% 7 d(5 / 2)^{2}[3 / 2]$ & & 8 \\
\hline $8 p$ & $(5 / 2)^{2}[3 / 2]^{\circ}$ & $3 d^{9}\left({ }^{2} D_{5 / 2}\right) 8 p$ & ${ }^{2}[3 / 2]^{\circ}$ & 2 & 152054.78 & 0.03 & & $53 \%+21 \% 8 p(5 / 2)^{2}[5 / 2]^{\circ}+20 \% s p$ & $\mathrm{~N}$ & 5 \\
\hline $7 \mathrm{~d}$ & $(5 / 2)^{2}[1 / 2]$ & $3 \mathrm{~d}^{9}\left({ }^{2} \mathrm{D}_{5 / 2}\right) 7 \mathrm{~d}$ & ${ }^{2}[1 / 2]$ & 0 & 152179.051 & 0.007 & & $52 \%+28 \% 7 \mathrm{~d}(3 / 2)^{2}[1 / 2]+9 \% 8 \mathrm{~d}(5 / 2)^{2}[1 / 2]$ & & 2 \\
\hline $8 \mathrm{p}$ & $(5 / 2)^{2}[5 / 2]^{\circ}$ & $3 d^{9}\left({ }^{2} D_{5 / 2}\right) 8 p$ & $2[5 / 2]^{\circ}$ & 2 & 152580.19 & 0.05 & & $65 \%+27 \% 8 \mathrm{p}(5 / 2)^{2}[3 / 2]^{\circ}+7 \% \mathrm{sp}$ & $\mathrm{N}$ & 2 \\
\hline $\mathrm{sp}$ & $\left({ }^{3} \mathrm{P}\right)^{1} \mathrm{P}^{\circ}{ }^{3} \mathrm{P}^{\circ}$ & $3 \mathrm{~d}^{8}\left({ }^{3} \mathrm{P}\right) 4 \mathrm{~s} 4 \mathrm{p}\left({ }^{1} \mathrm{P}^{\circ}\right)$ & ${ }^{3} \mathrm{P}^{\circ}$ & 0 & 152783.41 & 0.05 & & $77 \%+9 \% 8 \mathrm{p}^{3} \mathrm{P}^{\circ}+5 \% 7 \mathrm{p}^{3} \mathrm{P}^{\circ}$ & $\mathrm{N}$ & 2 \\
\hline $8 \mathrm{~s}$ & $(3 / 2)^{2}[3 / 2]$ & $3 \mathrm{~d}^{9}\left({ }^{2} \mathrm{D}_{3 / 2}\right) 8 \mathrm{~s}$ & ${ }^{2}[3 / 2]$ & 1 & 152814.032 & 0.007 & & $100 \%$ & & 4 \\
\hline $8 \mathrm{~s}$ & $(3 / 2)^{2}[3 / 2]$ & $3 \mathrm{~d}^{9}\left({ }^{2} \mathrm{D}_{3 / 2}\right) 8 \mathrm{~s}$ & $2[3 / 2]$ & 2 & 152840.475 & 0.005 & & $100 \%$ & & 6 \\
\hline sp & $\left({ }^{3} \mathrm{P}\right)^{1} \mathrm{P}^{\circ}{ }^{3} \mathrm{P}^{\circ}$ & $3 d^{8}(3 \mathrm{P}) 4 \mathrm{~s} 4 \mathrm{p}\left({ }^{1} \mathrm{P}^{\circ}\right)$ & ${ }^{1} \mathrm{P}^{\circ}-1$ & 2 & 152944.11 & 0.05 & & $39 \%+19 \% \operatorname{sp}\left({ }^{1} D\right)^{1} \mathrm{p}^{\circ}{ }^{1} \mathrm{D}^{\circ}+16 \% 8 \mathrm{p}^{3} \mathrm{D}^{\circ}$ & $\mathrm{N}$ & 4 \\
\hline $\mathrm{sp}$ & $\left({ }^{1} \mathrm{D}\right)^{1} \mathrm{P}^{\circ}{ }^{1} \mathrm{P}^{\circ}$ & $3 d^{8}\left({ }^{1} \mathrm{D}\right) 4 \mathrm{~s} 4 \mathrm{p}\left({ }^{1} \mathrm{P}^{\circ}\right)$ & ${ }^{1} \mathrm{P}^{\circ}$ & 1 & 153165.24 & 0.04 & & $22 \%+18 \% 6 \mathrm{f}^{3} \mathrm{D}^{\circ}+17 \% 6 \mathrm{f}^{1} \mathrm{P}^{\circ}$ & $\mathrm{N}$ & 5 \\
\hline $6 f$ & $(3 / 2)^{2}[5 / 2]^{\circ}$ & $3 d^{9}\left({ }^{2} D_{3 / 2}\right) 6 f$ & $2[5 / 2]^{\circ}$ & 3 & 153410.212 & 0.006 & & $89 \%+8 \% \mathrm{sp}+1 \% 6 \mathrm{f}(3 / 2)^{2}[7 / 2]^{\circ}$ & & 5 \\
\hline $6 f$ & $(3 / 2)^{2}[3 / 2]^{\circ}$ & $3 d^{9}\left({ }^{2} D_{3 / 2}\right) 6 f$ & $2[3 / 2]^{\circ}$ & 2 & 153426.632 & 0.006 & & $52 \%+42 \% 6 f(3 / 2)^{2}[5 / 2]^{\circ}+5 \% s p$ & & 7 \\
\hline $6 f$ & $(3 / 2)^{2}[9 / 2]^{\circ}$ & $3 d^{9}\left({ }^{2} D_{3 / 2}\right) 6 f$ & $2[9 / 2]^{\circ}$ & 5 & 153449.633 & 0.005 & & $100 \%$ & & 2 \\
\hline $6 \mathrm{f}$ & $(3 / 2)^{2}[9 / 2]^{\circ}$ & $3 \mathrm{~d}^{9}\left({ }^{2} \mathrm{D}_{3 / 2}\right) 6 \mathrm{f}$ & $2[9 / 2]^{\circ}$ & 4 & 153455.178 & 0.005 & & $100 \%$ & & 4 \\
\hline $6 f$ & $(3 / 2)^{2}[3 / 2]^{\circ}$ & $3 \mathrm{~d}^{9}\left({ }^{2} \mathrm{D}_{3 / 2}\right) 6 \mathrm{f}$ & $2[3 / 2]^{\circ}$ & 1 & 153457.747 & 0.009 & & $55 \%+23 \% \mathrm{sp}+15 \% 8 \mathrm{p}(3 / 2)^{2}[1 / 2]^{\circ}$ & & 6 \\
\hline $6 f$ & $(3 / 2)^{2}[5 / 2]^{\circ}$ & $3 d^{9}\left({ }^{2} D_{3 / 2}\right) 6 f$ & ${ }^{2}[5 / 2]^{\circ}$ & 2 & 153487.668 & 0.005 & & $56 \%+37 \% 6 \mathrm{f}(3 / 2)^{2}[3 / 2]^{\circ}+5 \% \mathrm{sp}$ & & 8 \\
\hline $6 f$ & $(3 / 2)^{2}[7 / 2]^{\circ}$ & $3 d^{9}\left({ }^{2} D_{3 / 2}\right) 6 f$ & $2[7 / 2]^{\circ}$ & 4 & 153495.854 & 0.006 & & $100 \%$ & & 3 \\
\hline $6 f$ & $(3 / 2)^{2}[7 / 2]^{\circ}$ & $3 d^{9}\left({ }^{2} D_{3 / 2}\right) 6 f$ & ${ }^{2}[7 / 2]^{\circ}$ & 3 & 153498.834 & 0.006 & & $98 \%+2 \%$ 6f $(3 / 2)^{2}[5 / 2]^{\circ}$ & & 3 \\
\hline $6 \mathrm{~g}$ & $(3 / 2)^{2}[5 / 2]$ & $3 \mathrm{~d}^{9}\left({ }^{2} \mathrm{D}_{3 / 2}\right) 6 \mathrm{~g}$ & ${ }^{2}[5 / 2]$ & 3 & 153517.905 & 0.007 & & $100 \%$ & & 2 \\
\hline $6 g$ & $(3 / 2)^{2}[5 / 2]$ & $3 \mathrm{~d}^{9}\left({ }^{2} \mathrm{D}_{3 / 2}\right) 6 \mathrm{~g}$ & $2[5 / 2]$ & 2 & 153517.985 & 0.008 & & $100 \%$ & & 1 \\
\hline 68 & $(3 / 2)^{2}[11 / 2]$ & $3 \mathrm{~d}^{9}\left({ }^{2} \mathrm{D}_{3 / 2}\right) 6 \mathrm{~g}$ & $2[11 / 2]$ & 5 & 153524.933 & 0.005 & & $100 \%$ & & 2 \\
\hline
\end{tabular}


Table A2. Cont.

\begin{tabular}{|c|c|c|c|c|c|c|c|c|c|c|}
\hline & Label $^{\text {a }}$ & Configuration & Term & $J$ & Level $^{\mathrm{b}}, \mathrm{cm}^{-1}$ & Unc. ${ }^{\mathrm{c}}, \mathrm{cm}^{-1}$ & Landé $g^{\mathrm{d}}$ & Leading Percentages ${ }^{\mathrm{e}}$ & Note $^{f}$ & $N_{\text {lines }} \mathrm{g}$ \\
\hline $6 \mathrm{~g}$ & $(3 / 2)^{2}[11 / 2]$ & $3 d^{9}\left({ }^{2} D_{3 / 2}\right) 6 g$ & ${ }^{2}[11 / 2]$ & 6 & 153524.950 & 0.007 & & $100 \%$ & & 1 \\
\hline $6 \mathrm{~g}$ & $(3 / 2)^{2}[7 / 2]$ & $3 \mathrm{~d}^{9}\left({ }^{2} \mathrm{D}_{3 / 2}\right) 6 \mathrm{~g}$ & ${ }^{2}[7 / 2]$ & 3 & 153537.395 & 0.007 & & $100 \%$ & & 2 \\
\hline $6 \mathrm{~g}$ & $(3 / 2)^{2}[7 / 2]$ & $3 d^{9}\left({ }^{2} D_{3 / 2}\right) 6 g$ & ${ }^{2}[7 / 2]$ & 4 & 153537.414 & 0.006 & & $100 \%$ & & 3 \\
\hline $6 \mathrm{~h}$ & $(3 / 2)^{2}[7 / 2]^{\circ}$ & $3 d^{9}\left({ }^{2} D_{3 / 2}\right) 6 h$ & ${ }^{2}[7 / 2]^{\circ}$ & 3 & [153543.7] & 6 & & $100 \%$ & $\mathrm{pf}$ & 0 \\
\hline $6 \mathrm{~h}$ & $(3 / 2)^{2}[7 / 2]^{\circ}$ & $3 d^{9}\left({ }^{2} D_{3 / 2}\right) 6 h$ & ${ }^{2}[7 / 2]^{\circ}$ & 4 & [153543.8] & 6 & & $100 \%$ & pf & 0 \\
\hline $6 \mathrm{~g}$ & $(3 / 2)^{2}[9 / 2]$ & $3 d^{9}\left({ }^{2} D_{3 / 2}\right) 6 g$ & ${ }^{2}[9 / 2]$ & 4 & 153544.054 & 0.005 & & $100 \%$ & & 3 \\
\hline $6 \mathrm{~g}$ & $(3 / 2)^{2}[9 / 2]$ & $3 d^{9}\left({ }^{2} D_{3 / 2}\right) 6 g$ & ${ }^{2}[9 / 2]$ & 5 & 153544.069 & 0.007 & & $100 \%$ & & 2 \\
\hline $6 \mathrm{~h}$ & $(3 / 2)^{2}[13 / 2]^{\circ}$ & $3 d^{9}\left({ }^{2} D_{3 / 2}\right) 6 h$ & ${ }^{2}[13 / 2]^{\circ}$ & 6 & [153546.0] & 6 & & $100 \%$ & pf & 0 \\
\hline $6 \mathrm{~h}$ & $(3 / 2)^{2}[13 / 2]^{\circ}$ & $3 d^{9}\left({ }^{2} D_{3 / 2}\right) 6 h$ & ${ }^{2}[13 / 2]^{\circ}$ & 7 & [153546.2] & 6 & & $100 \%$ & pf & 0 \\
\hline $6 \mathrm{~h}$ & $(3 / 2)^{2}[9 / 2]^{\circ}$ & $3 d^{9}\left({ }^{2} D_{3 / 2}\right) 6 h$ & ${ }^{2}[9 / 2]^{\circ}$ & 4 & [153552.0] & 6 & & $100 \%$ & $\mathrm{pf}$ & 0 \\
\hline $6 \mathrm{~h}$ & $(3 / 2)^{2}[9 / 2]^{\circ}$ & $3 d^{9}\left({ }^{2} D_{3 / 2}\right) 6 h$ & ${ }^{2}[9 / 2]^{\circ}$ & 5 & [153552.1] & 6 & & $100 \%$ & $\mathrm{pf}$ & 0 \\
\hline $6 \mathrm{~h}$ & $(3 / 2)^{2}[11 / 2]^{\circ}$ & $3 d^{9}\left({ }^{2} D_{3 / 2}\right) 6 h$ & ${ }^{2}[11 / 2]^{\circ}$ & 5 & [153554.3] & 6 & & $100 \%$ & $\mathrm{pf}$ & 0 \\
\hline $6 \mathrm{~h}$ & $(3 / 2)^{2}[11 / 2]^{\circ}$ & $3 d^{9}\left({ }^{2} D_{3 / 2}\right) 6 h$ & ${ }^{2}[11 / 2]^{\circ}$ & 6 & [153554.4] & 6 & & $100 \%$ & pf & 0 \\
\hline $7 \mathrm{~d}$ & $(3 / 2)^{2}[1 / 2]$ & $3 d^{9}\left({ }^{2} D_{3 / 2}\right) 7 d$ & ${ }^{2}[1 / 2]$ & 1 & 153658.567 & 0.006 & & $99 \%$ & & 5 \\
\hline $7 \mathrm{~d}$ & $(3 / 2)^{2}[7 / 2]$ & $3 d^{9}\left({ }^{2} D_{3 / 2}\right) 7 d$ & ${ }^{2}[7 / 2]$ & 3 & 153730.935 & 0.005 & & $100 \%$ & & 5 \\
\hline $7 \mathrm{~d}$ & $(3 / 2)^{2}[7 / 2]$ & $3 \mathrm{~d}^{9}\left({ }^{2} \mathrm{D}_{3 / 2}\right) 7 \mathrm{~d}$ & ${ }^{2}[7 / 2]$ & 4 & 153750.851 & 0.005 & & $100 \%$ & & 3 \\
\hline $7 \mathrm{~d}$ & $(3 / 2)^{2}[3 / 2]$ & $3 d^{9}\left({ }^{2} D_{3 / 2}\right) 7 d$ & ${ }^{2}[3 / 2]$ & 1 & 153753.738 & 0.007 & & $100 \%$ & & 2 \\
\hline $7 \mathrm{~d}$ & $(3 / 2)^{2}[3 / 2]$ & $3 d^{9}\left({ }^{2} D_{3 / 2}\right) 7 d$ & ${ }^{2}[3 / 2]$ & 2 & 153773.884 & 0.006 & & $97 \%+3 \% 7 d(3 / 2)^{2}[5 / 2]$ & & 5 \\
\hline $7 \mathrm{~d}$ & $(3 / 2)^{2}[5 / 2]$ & $3 d^{9}\left({ }^{2} D_{3 / 2}\right) 7 d$ & ${ }^{2}[5 / 2]$ & 3 & 153815.644 & 0.006 & & $100 \%$ & & 5 \\
\hline $7 \mathrm{~d}$ & $(3 / 2)^{2}[5 / 2]$ & $3 d^{9}\left({ }^{2} D_{3 / 2}\right) 7 d$ & $2[5 / 2]$ & 2 & 153821.937 & 0.006 & & $97 \%+3 \% 7 d(3 / 2)^{2}[3 / 2]$ & & 4 \\
\hline $\mathrm{sp}$ & $\left({ }^{3} \mathrm{P}\right)^{1} \mathrm{P}^{\circ}{ }^{3} \mathrm{D}^{\circ}$ & $3 \mathrm{~d}^{8}\left({ }^{3} \mathrm{P}\right) 4 \mathrm{~s} 4 \mathrm{p}\left({ }^{1} \mathrm{P}^{\circ}\right)$ & ${ }^{3} \mathrm{D}^{\circ}$ & 3 & 153850.18 & 0.04 & & $62 \%+9 \% s p\left({ }^{1} \mathrm{D}\right){ }^{1} \mathrm{P}^{\circ}{ }^{1} \mathrm{~F}^{\circ}+8 \% 8 \mathrm{p}^{3} \mathrm{~F}^{\circ}$ & $\mathrm{N}$ & 2 \\
\hline $7 \mathrm{~d}$ & $(3 / 2)^{2}[1 / 2]$ & $3 d^{9}\left({ }^{2} D_{3 / 2}\right) 7 d$ & ${ }^{2}[1 / 2]$ & 0 & 153853.763 & 0.011 & & $49 \%+44 \% 8 \mathrm{~d}(5 / 2)^{2}[1 / 2]+2 \% 8 \mathrm{~d}(3 / 2)^{2}[1 / 2]$ & & 2 \\
\hline $8 p$ & $(3 / 2)^{2}[3 / 2]^{\circ}$ & $3 d^{9}\left({ }^{2} D_{3 / 2}\right) 8 p$ & $2[3 / 2]^{\circ}$ & 1 & 154225.21 & 0.04 & & $38 \%+44 \% \mathrm{sp}+6 \% 7 \mathrm{f}(5 / 2)^{2}[3 / 2]^{\circ}$ & $\mathrm{N}$ & 2 \\
\hline $9 \mathrm{~s}$ & $(5 / 2)^{2}[5 / 2]$ & $3 d^{9}\left({ }^{2} D_{5 / 2}\right) 9 s$ & ${ }^{2}[5 / 2]$ & 3 & 154255.815 & 0.005 & & $100 \%$ & & 4 \\
\hline $9 \mathrm{~s}$ & $(5 / 2)^{2}[5 / 2]$ & $3 d^{9}\left({ }^{2} D_{5 / 2}\right) 9 s$ & $2[5 / 2]$ & 2 & 154281.251 & 0.008 & & $100 \%$ & & 4 \\
\hline $7 f$ & $(5 / 2)^{2}[11 / 2]^{\circ}$ & $3 d^{9}\left({ }^{2} D_{5 / 2}\right) 7 f$ & $2[11 / 2]^{\circ}$ & 5 & 154641.392 & 0.006 & & $99 \%$ & & 2 \\
\hline $7 f$ & $(5 / 2)^{2}[11 / 2]^{\circ}$ & $3 d^{9}\left({ }^{2} D_{5 / 2}\right) 7 f$ & ${ }^{2}[11 / 2]^{\circ}$ & 6 & 154647.056 & 0.005 & & $100 \%$ & & 2 \\
\hline $7 f$ & $(5 / 2)^{2}[3 / 2]^{\circ}$ & $3 d^{9}\left({ }^{2} D_{5 / 2}\right) 7 f$ & ${ }^{2}[3 / 2]^{\circ}$ & 1 & $154653.77 ?$ & 0.07 & & $51 \%+25 \% 8 p(3 / 2)^{2}[3 / 2]^{\circ}+12 \% 8 p(3 / 2)^{2}[1 / 2]^{\circ}$ & $\mathrm{N}$ & 2 \\
\hline $7 f$ & $(5 / 2)^{2}[5 / 2]^{\circ}$ & $3 d^{9}\left({ }^{2} D_{5 / 2}\right) 7 f$ & $2[5 / 2]^{\circ}$ & 3 & 154672.123 & 0.006 & & $62 \%+37 \% 7 f(5 / 2)^{2}[7 / 2]^{\circ}$ & & 4 \\
\hline $7 f$ & $(5 / 2)^{2}[7 / 2]^{\circ}$ & $3 d^{9}\left({ }^{2} D_{5 / 2}\right) 7 f$ & $2[7 / 2]^{\circ}$ & 4 & 154672.766 & 0.006 & & $91 \%+9 \% 7 f(5 / 2)^{2}[9 / 2]^{\circ}$ & & 5 \\
\hline $7 f$ & $(5 / 2)^{2}[9 / 2]^{\circ}$ & $3 d^{9}\left({ }^{2} D_{5 / 2}\right) 7 f$ & ${ }^{2}[9 / 2]^{\circ}$ & 4 & 154674.551 & 0.006 & & $91 \%+9 \% 7 f(5 / 2)^{2}[7 / 2]^{\circ}$ & & 4 \\
\hline $7 f$ & $(5 / 2)^{2}[9 / 2]^{\circ}$ & $3 d^{9}\left({ }^{2} D_{5 / 2}\right) 7 f$ & ${ }^{2}[9 / 2]^{\circ}$ & 5 & 154675.247 & 0.005 & & $100 \%$ & & 4 \\
\hline $7 f$ & $(5 / 2)^{2}[7 / 2]^{\circ}$ & $3 d^{9}\left({ }^{2} D_{5 / 2}\right) 7 f$ & ${ }^{2}[7 / 2]^{\circ}$ & 3 & 154678.877 & 0.008 & & $62 \%+36 \% 7 f(5 / 2)^{2}[5 / 2]^{\circ}+1 \% s p$ & & 3 \\
\hline $7 \mathrm{~g}$ & $(5 / 2)^{2}[3 / 2]$ & $3 d^{9}\left({ }^{2} D_{5 / 2}\right) 7 \mathrm{~g}$ & $2[3 / 2]$ & 1 & 154688.020 & 0.011 & & $100 \%$ & & 2 \\
\hline $7 \mathrm{~g}$ & $(5 / 2)^{2}[3 / 2]$ & $3 \mathrm{~d}^{9}\left({ }^{2} \mathrm{D}_{5 / 2}\right) 7 \mathrm{~g}$ & $2[3 / 2]$ & 2 & 154688.112 & 0.009 & & $100 \%$ & & 3 \\
\hline $7 \mathrm{~g}$ & $(5 / 2)^{2}[13 / 2]$ & $3 d^{9}\left({ }^{2} D_{5 / 2}\right) 7 g$ & ${ }^{2}[13 / 2]$ & 6 & 154694.589 & 0.008 & & $100 \%$ & & 1 \\
\hline $7 \mathrm{~g}$ & $(5 / 2)^{2}[13 / 2]$ & $3 d^{9}\left({ }^{2} D_{5 / 2}\right) 7 g$ & ${ }^{2}[13 / 2]$ & 7 & 154694.619 & 0.008 & & $100 \%$ & & 1 \\
\hline $7 \mathrm{~g}$ & $(5 / 2)^{2}[5 / 2]$ & $3 d^{9}\left({ }^{2} D_{5 / 2}\right) 7 g$ & ${ }^{2}[5 / 2]$ & 3 & 154695.997 & 0.008 & & $100 \%$ & & 4 \\
\hline $7 \mathrm{~g}$ & $(5 / 2)^{2}[5 / 2]$ & $3 d^{9}\left({ }^{2} D_{5 / 2}\right) 7 g$ & ${ }^{2}[5 / 2]$ & 2 & 154696.009 & 0.007 & & $100 \%$ & $\mathrm{~N}$ & 5 \\
\hline $7 f$ & $(5 / 2)^{2}[5 / 2]^{\circ}$ & $3 \mathrm{~d}^{9}\left({ }^{2} \mathrm{D}_{5 / 2}\right) 7 \mathrm{f}$ & ${ }^{2}[5 / 2]^{\circ}$ & 2 & 154698.288 & 0.020 & & $39 \%+28 \% 8 p(3 / 2)^{2}[3 / 2]^{\circ}+15 \% 7 f(5 / 2)^{2}[3 / 2]^{\circ}$ & $\mathrm{N}$ & 3 \\
\hline $7 \mathrm{~h}$ & $(5 / 2)^{2}[5 / 2]^{\circ}$ & $3 d^{9}\left({ }^{2} D_{5 / 2}\right) 7 h$ & ${ }^{2}[5 / 2]^{\circ}$ & 2 & {$[154702.4]$} & 2.0 & & $100 \%$ & pf & 0 \\
\hline
\end{tabular}


Table A2. Cont.

\begin{tabular}{|c|c|c|c|c|c|c|c|c|c|c|}
\hline & Label $^{\text {a }}$ & Configuration & Term & $\bar{J}$ & Level $^{\mathrm{b}}, \mathrm{cm}^{-1}$ & Unc. ${ }^{\mathrm{c}}, \mathrm{cm}^{-1}$ & Landé $g \mathrm{~d}$ & Leading Percentages $\mathrm{e}$ & Note $^{f}$ & $N_{\text {lines }}{ }^{g}$ \\
\hline $7 \mathrm{~h}$ & $(5 / 2)^{2}[5 / 2]^{\circ}$ & $3 d^{9}\left({ }^{2} D_{5 / 2}\right) 7 \mathrm{~h}$ & ${ }^{2}[5 / 2]^{\circ}$ & 3 & [154702.5] & 2.0 & & $100 \%$ & $\mathrm{pf}$ & 0 \\
\hline $7 \mathrm{~g}$ & $(5 / 2)^{2}[7 / 2]$ & $3 d^{9}\left({ }^{2} D_{5 / 2}\right) 7 g$ & ${ }^{2}[7 / 2]$ & 3 & 154703.597 & 0.008 & & $100 \%$ & & 2 \\
\hline $7 \mathrm{~g}$ & $(5 / 2)^{2}[7 / 2]$ & $3 d^{9}\left({ }^{2} D_{5 / 2}\right) 7 g$ & ${ }^{2}[7 / 2]$ & 4 & 154703.625 & 0.008 & & $100 \%$ & & 2 \\
\hline $7 \mathrm{~h}$ & $(5 / 2)^{2}[15 / 2]^{\circ}$ & $3 d^{9}\left({ }^{2} D_{5 / 2}\right) 7 \mathrm{~h}$ & ${ }^{2}[15 / 2]^{\circ}$ & 7 & [154704.6] & 2.0 & & $100 \%$ & pf & 0 \\
\hline $7 \mathrm{~h}$ & $(5 / 2)^{2}[15 / 2]^{\circ}$ & $3 d^{9}\left(D_{5 / 2}\right) 7 \mathrm{~h}$ & ${ }^{2}[15 / 2]^{\circ}$ & 8 & [154704.6] & 2.0 & & $100 \%$ & $\mathrm{pf}$ & 0 \\
\hline $7 \mathrm{~h}$ & $(5 / 2)^{2}[7 / 2]^{\circ}$ & $3 d^{9}\left({ }^{2} D_{5 / 2}\right) 7 \mathrm{~h}$ & ${ }^{2}[7 / 2]^{\circ}$ & 3 & [154706.0] & 2.0 & & $100 \%$ & $\mathrm{pf}$ & 0 \\
\hline $7 \mathrm{~h}$ & $(5 / 2)^{2}[7 / 2]^{\circ}$ & $3 d^{9}\left({ }^{2} D_{5 / 2}\right) 7 \mathrm{~h}$ & ${ }^{2}[7 / 2]^{\circ}$ & 4 & [154706.1] & 2.0 & & $100 \%$ & $\mathrm{pf}$ & 0 \\
\hline $7 \mathrm{~g}$ & $(5 / 2)^{2}[11 / 2]$ & $3 d^{9}\left({ }^{2} D_{5 / 2}\right) 7 g$ & ${ }^{2}[11 / 2]$ & 5 & 154706.911 & 0.007 & & $100 \%$ & & 3 \\
\hline $7 \mathrm{~g}$ & $(5 / 2)^{2}[9 / 2]$ & $3 d^{9}\left({ }^{2} D_{5 / 2}\right) 7 g$ & ${ }^{2}[9 / 2]$ & 4 & 154708.235 & 0.007 & & $100 \%$ & & 5 \\
\hline $7 \mathrm{~g}$ & $(5 / 2)^{2}[9 / 2]$ & $3 d^{9}\left({ }^{2} D_{5 / 2}\right) 7 g$ & ${ }^{2}[9 / 2]$ & 5 & 154708.244 & 0.007 & & $100 \%$ & $\mathrm{~N}$ & 2 \\
\hline $7 \mathrm{~h}$ & $(5 / 2)^{2}[9 / 2]^{\circ}$ & $3 d^{9}\left({ }^{2} D_{5 / 2}\right) 7 \mathrm{~h}$ & ${ }^{2}[9 / 2]^{\circ}$ & 4 & [154709.1] & 2.0 & & $100 \%$ & $\mathrm{pf}$ & 0 \\
\hline $7 \mathrm{~h}$ & $(5 / 2)^{2}[9 / 2]^{\circ}$ & $3 d^{9}\left({ }^{2} D_{5 / 2}\right) 7 \mathrm{~h}$ & ${ }^{2}[9 / 2]^{\circ}$ & 5 & [154709.1] & 2.0 & & $100 \%$ & $\mathrm{pf}$ & 0 \\
\hline $7 \mathrm{~h}$ & $(5 / 2)^{2}[13 / 2]^{\circ}$ & $3 d^{9}\left({ }^{2} D_{5 / 2}\right) 7 \mathrm{~h}$ & ${ }^{2}[13 / 2]^{\circ}$ & 6 & [154709.7] & 2.0 & & $100 \%$ & $\mathrm{pf}$ & 0 \\
\hline $7 \mathrm{~h}$ & $(5 / 2)^{2}[13 / 2]^{\circ}$ & $\left.3 d^{9}{ }^{2} D_{5 / 2}\right) 7 \mathrm{~h}$ & ${ }^{2}[13 / 2]^{\circ}$ & 7 & {$[154709.8]$} & 2.0 & & $100 \%$ & pf & 0 \\
\hline $7 \mathrm{~h}$ & $(5 / 2)^{2}[11 / 2]^{\circ}$ & $3 d^{9}\left({ }^{2} D_{5 / 2}\right) 7 \mathrm{~h}$ & ${ }^{2}[11 / 2]^{\circ}$ & 5 & [154710.7] & 2.0 & & $100 \%$ & $\mathrm{pf}$ & 0 \\
\hline $7 \mathrm{~h}$ & $(5 / 2)^{2}[11 / 2]^{\circ}$ & $3 \mathrm{~d}^{9}\left({ }^{2} \mathrm{D}_{5 / 2}\right) 7 \mathrm{~h}$ & $2[11 / 2]^{\circ}$ & 6 & [154710.8] & 2.0 & & $100 \%$ & pf & 0 \\
\hline $8 p$ & $(3 / 2)^{2}[1 / 2]^{\circ}$ & $3 d^{9}\left({ }^{2} D_{3 / 2}\right) 8 p$ & ${ }^{2}[1 / 2]^{\circ}$ & 1 & 154719.09 & 0.03 & & $29 \%+32 \% 8 p(3 / 2)^{2}[3 / 2]^{\circ}+19 \% s p$ & $\mathrm{~N}$ & 3 \\
\hline $8 \mathrm{~d}$ & $(5 / 2)^{2}[1 / 2]$ & $3 d^{9}\left({ }^{2} D_{5 / 2}\right) 8 d$ & ${ }^{2}[1 / 2]$ & 1 & 154766.034 & 0.022 & & $98 \%+1 \% 8 \mathrm{~d}(5 / 2)^{2}[3 / 2]$ & & 1 \\
\hline $8 \mathrm{~d}$ & $(5 / 2)^{2}[9 / 2]$ & $\left.3 \mathrm{~d}^{9}{ }^{2} \mathrm{D}_{5 / 2}\right) 8 \mathrm{~d}$ & $2[9 / 2]$ & 5 & 154828.718 & 0.008 & & $100 \%$ & & 2 \\
\hline $8 \mathrm{~d}$ & $(5 / 2)^{2}[3 / 2]$ & $3 d^{9}\left({ }^{2} D_{5 / 2}\right) 8 d$ & $2[3 / 2]$ & 2 & 154832.657 & 0.011 & & $95 \%+5 \% 8 d(5 / 2)^{2}[5 / 2]$ & & 1 \\
\hline $8 \mathrm{~d}$ & $(5 / 2)^{2}[9 / 2]$ & $3 d^{9}\left({ }^{2} D_{5 / 2}\right) 8 d$ & $2[9 / 2]$ & 4 & 154836.434 & 0.006 & & $100 \%$ & & 4 \\
\hline $8 \mathrm{~d}$ & $(5 / 2)^{2}[3 / 2]$ & $3 \mathrm{~d}^{9}\left({ }^{2} \mathrm{D}_{5 / 2}\right) 8 \mathrm{~d}$ & $2[3 / 2]$ & 1 & $154838.973 ?$ & 0.013 & & $98 \%+1 \% 8 d(5 / 2)^{2}[1 / 2]$ & & 2 \\
\hline $8 \mathrm{~d}$ & $(5 / 2)^{2}[5 / 2]$ & $3 d^{9}\left({ }^{2} D_{5 / 2}\right) 8 \mathrm{~d}$ & $2[5 / 2]$ & 3 & 154860.741 & 0.006 & & $98 \%+1 \% 8 \mathrm{~d}(5 / 2)^{2}[7 / 2]$ & & 5 \\
\hline $8 \mathrm{~d}$ & $(5 / 2)^{2}[7 / 2]$ & $3 \mathrm{~d}^{9}\left({ }^{2} \mathrm{D}_{5 / 2}\right) 8 \mathrm{~d}$ & $2[7 / 2]$ & 3 & 154883.104 & 0.009 & & $98 \%+1 \% 8 d(5 / 2)^{2}[5 / 2]$ & & 2 \\
\hline $8 \mathrm{~d}$ & $(5 / 2)^{2}[7 / 2]$ & $3 \mathrm{~d}^{9}\left({ }^{2} \mathrm{D}_{5 / 2}\right) 8 \mathrm{~d}$ & $2[7 / 2]$ & 4 & 154883.625 & 0.007 & & $100 \%$ & & 6 \\
\hline $8 \mathrm{~d}$ & $(5 / 2)^{2}[5 / 2]$ & $3 d^{9}\left({ }^{2} D_{5 / 2}\right) 8 d$ & $2[5 / 2]$ & 2 & 154892.916 & 0.020 & & $95 \%+5 \% 8 d(5 / 2)^{2}[3 / 2]$ & & 2 \\
\hline $8 \mathrm{~d}$ & $(5 / 2)^{2}[1 / 2]$ & $3 d^{9}\left({ }^{2} D_{5 / 2}\right) 8 d$ & $2[1 / 2]$ & 0 & $155244.842 ?$ & 0.021 & & $32 \%+29 \% 9 d(5 / 2)^{2}[1 / 2]+20 \% 8 d(3 / 2)^{2}[1 / 2]$ & & 1 \\
\hline $9 \mathrm{~s}$ & $(3 / 2)^{2}[3 / 2]$ & $3 d^{9}\left({ }^{2} D_{3 / 2}\right) 9 s$ & $2[3 / 2]$ & 1 & 156326.913 & 0.018 & & $100 \%$ & & 4 \\
\hline $9 \mathrm{~s}$ & $(3 / 2)^{2}[3 / 2]$ & $3 d^{9}\left({ }^{2} D_{3 / 2}\right) 9 s$ & ${ }^{2}[3 / 2]$ & 2 & 156341.878 & 0.009 & & $99 \%+1 \% 10 \mathrm{~s}(5 / 2)^{2}[5 / 2]$ & & 5 \\
\hline $10 \mathrm{~s}$ & $(5 / 2)^{2}[5 / 2]$ & $3 \mathrm{~d}^{9}\left({ }^{2} \mathrm{D}_{5 / 2}\right) 10 \mathrm{~s}$ & $2[5 / 2]$ & 3 & 156508.501 & 0.022 & & $100 \%$ & & 1 \\
\hline $10 \mathrm{~s}$ & $(5 / 2)^{2}[5 / 2]$ & $3 \mathrm{~d}^{9}\left({ }^{2} \mathrm{D}_{5 / 2}\right) 10 \mathrm{~s}$ & $2[5 / 2]$ & 2 & 156526.436 & 0.015 & & $99 \%+1 \% 9 \mathrm{~s}(3 / 2)^{2}[3 / 2]$ & & 2 \\
\hline $7 \mathrm{f}$ & $(3 / 2)^{2}[9 / 2]^{\circ}$ & $3 d^{9}\left({ }^{2} D_{3 / 2}\right) 7 f$ & ${ }^{2}[9 / 2]^{\circ}$ & 5 & 156711.894 & 0.007 & & $91 \%+6 \% 8 f(5 / 2)^{2}[11 / 2]^{\circ}+3 \% 8 f(5 / 2)^{2}[9 / 2]^{\circ}$ & & 2 \\
\hline $7 \mathrm{f}$ & $(3 / 2)^{2}[9 / 2]^{\circ}$ & $3 d^{9}\left({ }^{2} D_{3 / 2}\right) 7 f$ & $2[9 / 2]^{\circ}$ & 4 & 156721.308 & 0.007 & & $97 \%+3 \% 8 f(5 / 2)^{2}[9 / 2]^{\circ}$ & & 2 \\
\hline $7 f$ & $(3 / 2)^{2}[7 / 2]^{\circ}$ & $3 d^{9}\left({ }^{2} D_{3 / 2}\right) 7 f$ & ${ }^{2}[7 / 2]^{\circ}$ & 4 & {$[156754.9]$} & 7 & & $99 \%+1 \% 8 f(5 / 2)^{2}[7 / 2]^{\circ}$ & sf & 0 \\
\hline $8 f$ & $(5 / 2)^{2}[11 / 2]^{\circ}$ & $3 d^{9}\left({ }^{2} D_{5 / 2}\right) 8 f$ & ${ }^{2}[11 / 2]^{\circ}$ & 5 & 156761.443 & 0.008 & & $94 \%+6 \% 7 f(3 / 2)^{2}[9 / 2]^{\circ}$ & & 3 \\
\hline $7 \mathrm{~g}$ & $(3 / 2)^{2}[5 / 2]$ & $3 \mathrm{~d}^{9}\left({ }^{2} \mathrm{D}_{3 / 2}\right) 7 \mathrm{~g}$ & ${ }^{2}[5 / 2]$ & 3 & 156763.044 & 0.014 & & $99 \%+1 \% 8 g(5 / 2)^{2}[5 / 2]$ & & 1 \\
\hline $7 \mathrm{~g}$ & $(3 / 2)^{2}[5 / 2]$ & $3 d^{9}\left({ }^{2} D_{3 / 2}\right) 7 g$ & $2[5 / 2]$ & 2 & 156763.090 & 0.011 & & $99 \%+1 \% 8 \mathrm{~g}(5 / 2)^{2}[5 / 2]$ & & 1 \\
\hline $8 \mathrm{f}$ & $(5 / 2)^{2}[11 / 2]^{\circ}$ & $3 \mathrm{~d}^{9}\left({ }^{2} \mathrm{D}_{5 / 2}\right) 8 \mathrm{f}$ & $2[11 / 2]^{\circ}$ & 6 & 156767.066 & 0.016 & & $100 \%$ & & 2 \\
\hline $7 g$ & $(3 / 2)^{2}[11 / 2]$ & $3 \mathrm{~d}^{9}\left({ }^{2} \mathrm{D}_{3 / 2}\right) 7 \mathrm{~g}$ & ${ }^{2}[11 / 2]$ & 6 & 156767.609 & 0.011 & & $99 \%+1 \% 8 g(5 / 2)^{2}[11 / 2]$ & $\mathrm{N}$ & 1 \\
\hline $7 \mathrm{~g}$ & $(3 / 2)^{2}[11 / 2]$ & $3 \mathrm{~d}^{9}\left({ }^{2} \mathrm{D}_{3 / 2}\right) 7 \mathrm{~g}$ & $2[11 / 2]$ & 5 & 156767.630 & 0.011 & & $99 \%+1 \% 8 g(5 / 2)^{2}[11 / 2]$ & & $\begin{array}{l}1 \\
2\end{array}$ \\
\hline $7 \mathrm{~g}$ & $(3 / 2)^{2}[7 / 2]$ & $3 \mathrm{~d}^{9}\left({ }^{2} \mathrm{D}_{3 / 2}\right) 7 \mathrm{~g}$ & ${ }^{2}[7 / 2]$ & 3 & 156775.405 & 0.014 & & $99 \%+1 \% 8 \mathrm{~g}(5 / 2)^{2}[7 / 2]$ & & 2 \\
\hline
\end{tabular}


Table A2. Cont.

\begin{tabular}{|c|c|c|c|c|c|c|c|c|c|c|}
\hline & Label $^{\text {a }}$ & Configuration & Term & $J$ & Level $^{\mathrm{b}}, \mathrm{cm}^{-1}$ & Unc. ${ }^{c}, \mathrm{~cm}^{-1}$ & Landé $g^{\mathrm{d}}$ & Leading Percentages ${ }^{\mathrm{e}}$ & Note ${ }^{\mathrm{f}}$ & $N_{\text {lines }} \mathrm{g}$ \\
\hline $7 \mathrm{~g}$ & $(3 / 2)^{2}[7 / 2]$ & $3 d^{9}\left({ }^{2} D_{3 / 2}\right) 7 g$ & ${ }^{2}[7 / 2]$ & 4 & 156775.430 & 0.014 & & $99 \%+1 \% 8 \mathrm{~g}(5 / 2)^{2}[7 / 2]$ & $\mathrm{N}$ & 1 \\
\hline $8 \mathrm{f}$ & $(5 / 2)^{2}[7 / 2]^{\circ}$ & $3 d^{9}\left({ }^{2} D_{5 / 2}\right) 8 f$ & ${ }^{2}[7 / 2]^{\circ}$ & 4 & [156779.7] & 7 & & $94 \%+5 \% 8 \mathrm{f}(5 / 2)^{2}[9 / 2]^{\circ}+1 \% 7 \mathrm{f}(3 / 2)^{2}[7 / 2]^{\circ}$ & sf & 0 \\
\hline $7 \mathrm{~g}$ & $(3 / 2)^{2}[9 / 2]$ & $3 d^{9}\left({ }^{2} D_{3 / 2}\right) 7 g$ & ${ }^{2}[9 / 2]$ & 4 & 156779.847 & 0.014 & & $100 \%$ & & 2 \\
\hline $7 \mathrm{~g}$ & $(3 / 2)^{2}[9 / 2]$ & $3 d^{9}\left({ }^{2} D_{3 / 2}\right) 7 g$ & ${ }^{2}[9 / 2]$ & 5 & 156779.855 & 0.014 & & $100 \%$ & $\mathrm{~N}$ & 1 \\
\hline $8 \mathrm{f}$ & $(5 / 2)^{2}[9 / 2]^{\circ}$ & $3 d^{9}\left({ }^{2} D_{5 / 2}\right) 8 f$ & ${ }^{2}[9 / 2]^{\circ}$ & 4 & [156781.7] & 7 & & $92 \%+5 \% 8 f(5 / 2)^{2}[7 / 2]^{\circ}+2 \% 7 f(3 / 2)^{2}[9 / 2]^{\circ}$ & sf & 0 \\
\hline $8 \mathrm{f}$ & $(5 / 2)^{2}[9 / 2]^{\circ}$ & $3 d^{9}\left({ }^{2} D_{5 / 2}\right) 8 f$ & ${ }^{2}[9 / 2]^{\circ}$ & 5 & [156782.4] & 7 & & $97 \%+3 \% 7 f(3 / 2)^{2}[9 / 2]^{\circ}$ & sf & 0 \\
\hline $8 \mathrm{~g}$ & $(5 / 2)^{2}[3 / 2]$ & $3 d^{9}\left({ }^{2} D_{5 / 2}\right) 8 g$ & ${ }^{2}[3 / 2]$ & 2 & [156797.3] & 2.0 & & $100 \%$ & $\mathrm{pf}$ & 0 \\
\hline $8 g$ & $(5 / 2)^{2}[7 / 2]$ & $3 d^{9}\left({ }^{2} D_{5 / 2}\right) 8 g$ & ${ }^{2}[7 / 2]$ & 3 & [156805.7] & 2.0 & & $99 \%+1 \% 7 \mathrm{~g}(3 / 2)^{2}[7 / 2]$ & $\mathrm{pf}$ & 0 \\
\hline $8 \mathrm{~g}$ & $(5 / 2)^{2}[9 / 2]$ & $3 d^{9}\left({ }^{2} D_{5 / 2}\right) 8 g$ & ${ }^{2}[9 / 2]$ & 5 & [156807.8] & 2.0 & & $100 \%$ & $\mathrm{pf}$ & 0 \\
\hline $8 g$ & $(5 / 2)^{2}[11 / 2]$ & $3 d^{9}\left({ }^{2} D_{5 / 2}\right) 8 g$ & ${ }^{2}[11 / 2]$ & 6 & $156808.189 ?$ & 0.010 & & $99 \%+1 \% 7 \mathrm{~g}(3 / 2)^{2}[11 / 2]$ & $\mathrm{N}$ & 2 \\
\hline $9 \mathrm{~d}$ & $(5 / 2)^{2}[9 / 2]$ & $3 d^{9}\left({ }^{2} D_{5 / 2}\right) 9 d$ & ${ }^{2}[9 / 2]$ & 5 & [156888.5] & 2.0 & & $100 \%$ & sf & 0 \\
\hline $9 \mathrm{~d}$ & $(5 / 2)^{2}[9 / 2]$ & $3 d^{9}\left({ }^{2} D_{5 / 2}\right) 9 d$ & ${ }^{2}[9 / 2]$ & 4 & 156912.740 & 0.011 & & $51 \%+44 \% 8 d(3 / 2)^{2}[7 / 2]+5 \% 9 d(5 / 2)^{2}[7 / 2]$ & & 2 \\
\hline $8 \mathrm{~d}$ & $(3 / 2)^{2}[5 / 2]$ & $3 d^{9}\left({ }^{2} D_{3 / 2}\right) 8 d$ & ${ }^{2}[5 / 2]$ & 2 & $156958.11 ?$ & 0.06 & & $42 \%+38 \% 9 d(5 / 2)^{2}[5 / 2]+16 \% 8 d(3 / 2)^{2}[3 / 2]$ & & 1 \\
\hline $9 \mathrm{~g}$ & $(5 / 2)^{2}[3 / 2]$ & $3 d^{9}\left({ }^{2} D_{5 / 2}\right) 9 g$ & ${ }^{2}[3 / 2]$ & 2 & [158241.1] & 2.0 & & $100 \%$ & $\mathrm{pf}$ & 0 \\
\hline $9 g$ & $(5 / 2)^{2}[7 / 2]$ & $3 d^{9}\left({ }^{2} D_{5 / 2}\right) 9 g$ & ${ }^{2}[7 / 2]$ & 3 & [158246.8] & 2.0 & & $100 \%$ & $\mathrm{pf}$ & 0 \\
\hline $10 \mathrm{~d}$ & $(5 / 2)^{2}[1 / 2]$ & $3 d^{9}\left({ }^{2} D_{5 / 2}\right) 10 d$ & ${ }^{2}[1 / 2]$ & 1 & [158285.3] & 4 & & $98 \%+2 \% 10 \mathrm{~d}(5 / 2)^{2}[3 / 2]$ & sf & 0 \\
\hline $10 \mathrm{~d}$ & $(5 / 2)^{2}[3 / 2]$ & $3 \mathrm{~d}^{9}\left({ }^{2} \mathrm{D}_{5 / 2}\right) 10 \mathrm{~d}$ & ${ }^{2}[3 / 2]$ & 2 & [158306.0] & 5 & & $95 \%+4 \% 10 \mathrm{~d}(5 / 2)^{2}[5 / 2]$ & sf & 0 \\
\hline $10 \mathrm{~d}$ & $(5 / 2)^{2}[9 / 2]$ & $3 \mathrm{~d}^{9}\left({ }^{2} \mathrm{D}_{5 / 2}\right) 10 \mathrm{~d}$ & ${ }^{2}[9 / 2]$ & 5 & [158306.9] & 2.0 & & $100 \%$ & sf & 0 \\
\hline $10 \mathrm{~d}$ & $(5 / 2)^{2}[9 / 2]$ & $3 \mathrm{~d}^{9}\left({ }^{2} \mathrm{D}_{5 / 2}\right) 10 \mathrm{~d}$ & ${ }^{2}[9 / 2]$ & 4 & [158308.9] & 2.0 & & $100 \%$ & sf & 0 \\
\hline $10 \mathrm{~d}$ & $(5 / 2)^{2}[3 / 2]$ & $3 \mathrm{~d}^{9}\left({ }^{2} \mathrm{D}_{5 / 2}\right) 10 \mathrm{~d}$ & ${ }^{2}[3 / 2]$ & 1 & [158310.4] & 4 & & $98 \%+2 \% 10 d(5 / 2)^{2}[1 / 2]$ & sf & 0 \\
\hline $10 \mathrm{~d}$ & $(5 / 2)^{2}[5 / 2]$ & $3 d^{9}\left({ }^{2} D_{5 / 2}\right) 10 d$ & ${ }^{2}[5 / 2]$ & 3 & [158323.3] & 4 & & $98 \%+2 \% 10 \mathrm{~d}(5 / 2)^{2}[7 / 2]$ & sf & 0 \\
\hline $10 \mathrm{~d}$ & $(5 / 2)^{2}[5 / 2]$ & $3 d^{9}\left({ }^{2} D_{5 / 2}\right) 10 d$ & ${ }^{2}[5 / 2]$ & 2 & [158333.4] & 5 & & $95 \%+4 \% 10 d(5 / 2)^{2}[3 / 2]$ & sf & 0 \\
\hline $10 \mathrm{~d}$ & $(5 / 2)^{2}[7 / 2]$ & $3 \mathrm{~d}^{9}\left({ }^{2} \mathrm{D}_{5 / 2}\right) 10 \mathrm{~d}$ & ${ }^{2}[7 / 2]$ & 3 & [158334.1] & 4 & & $98 \%+2 \% 10 \mathrm{~d}(5 / 2)^{2}[5 / 2]$ & sf & 0 \\
\hline $10 \mathrm{~d}$ & $(5 / 2)^{2}[7 / 2]$ & $3 \mathrm{~d}^{9}\left({ }^{2} \mathrm{D}_{5 / 2}\right) 10 \mathrm{~d}$ & ${ }^{2}[7 / 2]$ & 4 & [158334.2] & 2.0 & & $100 \%$ & sf & 0 \\
\hline $10 \mathrm{~s}$ & $(3 / 2)^{2}[3 / 2]$ & $3 \mathrm{~d}^{9}\left({ }^{2} \mathrm{D}_{3 / 2}\right) 10 \mathrm{~s}$ & ${ }^{2}[3 / 2]$ & 1 & 158579.33 & 0.14 & & $100 \%$ & $\mathrm{~N}$ & 1 \\
\hline $8 \mathrm{f}$ & $(3 / 2)^{2}[9 / 2]^{\circ}$ & $3 \mathrm{~d}^{9}\left({ }^{2} \mathrm{D}_{3 / 2}\right) 8 \mathrm{f}$ & $2[9 / 2]^{\circ}$ & 4 & [158851.1] & 8 & & $100 \%$ & sf & 0 \\
\hline $8 \mathrm{f}$ & $(3 / 2)^{2}[7 / 2]^{\circ}$ & $3 d^{9}\left({ }^{2} D_{3 / 2}\right) 8 f$ & ${ }^{2}[7 / 2]^{\circ}$ & 4 & [158864.4] & 8 & & $100 \%$ & sf & 0 \\
\hline $8 \mathrm{~g}$ & $(3 / 2)^{2}[5 / 2]$ & $3 \mathrm{~d}^{9}\left({ }^{2} \mathrm{D}_{3 / 2}\right) 8 \mathrm{~g}$ & ${ }^{2}[5 / 2]$ & 3 & [158869.3] & 2.0 & & $100 \%$ & $\mathrm{pf}$ & 0 \\
\hline $8 g$ & $(3 / 2)^{2}[11 / 2]$ & $3 d^{9}\left({ }^{2} D_{3 / 2}\right) 8 g$ & ${ }^{2}[11 / 2]$ & 6 & [158871.7] & 2.0 & & $100 \%$ & $\mathrm{pf}$ & 0 \\
\hline $8 \mathrm{~g}$ & $(3 / 2)^{2}[7 / 2]$ & $3 d^{9}\left({ }^{2} D_{3 / 2}\right) 8 g$ & ${ }^{2}[7 / 2]$ & 4 & [158875.8] & 2.0 & & $100 \%$ & $\mathrm{pf}$ & 0 \\
\hline $8 \mathrm{~h}$ & $(3 / 2)^{2}[7 / 2]^{\circ}$ & $3 \mathrm{~d}^{9}\left({ }^{2} \mathrm{D}_{3 / 2}\right) 8 \mathrm{~h}$ & ${ }^{2}[7 / 2]^{\circ}$ & 3 & [158876.9] & 2.0 & & $100 \%$ & $\mathrm{pf}$ & 0 \\
\hline $8 \mathrm{~h}$ & $(3 / 2)^{2}[7 / 2]^{\circ}$ & $3 \mathrm{~d}^{9}\left({ }^{2} \mathrm{D}_{3 / 2}\right) 8 \mathrm{~h}$ & ${ }^{2}[7 / 2]^{\circ}$ & 4 & [158877.0] & 2.0 & & $100 \%$ & pf & 0 \\
\hline $8 \mathrm{~h}$ & $(3 / 2)^{2}[13 / 2]^{\circ}$ & $3 \mathrm{~d}^{9}\left({ }^{2} \mathrm{D}_{3 / 2}\right) 8 \mathrm{~h}$ & ${ }^{2}[13 / 2]^{\circ}$ & 6 & [158877.9] & 2.0 & & $100 \%$ & $\mathrm{pf}$ & 0 \\
\hline $8 \mathrm{~g}$ & $(3 / 2)^{2}[9 / 2]$ & $3 d^{9}\left({ }^{2} D_{3 / 2}\right) 8 g$ & ${ }^{2}[9 / 2]$ & 5 & [158878.0] & 2.0 & & $100 \%$ & $\mathrm{pf}$ & 0 \\
\hline $8 \mathrm{~h}$ & $(3 / 2)^{2}[13 / 2]^{\circ}$ & $3 d^{9}\left({ }^{2} D_{3 / 2}\right) 8 h$ & ${ }^{2}[13 / 2]^{\circ}$ & 7 & [158878.0] & 2.0 & & $100 \%$ & $\mathrm{pf}$ & 0 \\
\hline $8 \mathrm{~h}$ & $(3 / 2)^{2}[9 / 2]^{\circ}$ & $3 d^{9}\left({ }^{2} D_{3 / 2}\right) 8 h$ & ${ }^{2}[9 / 2]^{\circ}$ & 4 & [158880.4] & 2.0 & & $100 \%$ & $\mathrm{pf}$ & 0 \\
\hline $8 \mathrm{~h}$ & $(3 / 2)^{2}[9 / 2]^{\circ}$ & $3 \mathrm{~d}^{9}\left({ }^{2} \mathrm{D}_{3 / 2}\right) 8 \mathrm{~h}$ & ${ }^{2}[9 / 2]^{\circ}$ & 5 & [158880.5] & 2.0 & & $100 \%$ & pf & 0 \\
\hline $8 \mathrm{~h}$ & $(3 / 2)^{2}[11 / 2]^{\circ}$ & $3 \mathrm{~d}^{9}\left({ }^{2} \mathrm{D}_{3 / 2}\right) 8 \mathrm{~h}$ & ${ }^{2}[11 / 2]^{\circ}$ & 5 & [158881.4] & 2.0 & & $100 \%$ & $\mathrm{pf}$ & 0 \\
\hline $8 \mathrm{~h}$ & $(3 / 2)^{2}[11 / 2]^{\circ}$ & $3 d^{9}\left({ }^{2} D_{3 / 2}\right) 8 h$ & ${ }^{2}[11 / 2]^{\circ}$ & 6 & [158881.5] & 2.0 & & $100 \%$ & $\mathrm{pf}$ & 0 \\
\hline $9 \mathrm{~d}$ & $(3 / 2)^{2}[1 / 2]$ & $3 \mathrm{~d}^{9}\left({ }^{2} \mathrm{D}_{3 / 2}\right) 9 \mathrm{~d}$ & ${ }^{2}[1 / 2]$ & 1 & [158935.9] & 4 & & $99 \%$ & sf & 0 \\
\hline
\end{tabular}


Table A2. Cont.

\begin{tabular}{|c|c|c|c|c|c|c|c|c|c|c|}
\hline & Label $^{\text {a }}$ & Configuration & Term & $J$ & Level $^{\mathrm{b}}, \mathrm{cm}^{-1}$ & Unc. ${ }^{\mathrm{c}}, \mathrm{cm}^{-1}$ & Landé $g^{\mathrm{d}}$ & Leading Percentages ${ }^{\mathrm{e}}$ & Note $^{f}$ & $N_{\text {lines }} \mathrm{g}$ \\
\hline $9 \mathrm{~d}$ & $(3 / 2)^{2}[7 / 2]$ & $3 d^{9}\left({ }^{2} D_{3 / 2}\right) 9 d$ & ${ }^{2}[7 / 2]$ & 3 & [158961.1] & 2.0 & & $100 \%$ & sf & 0 \\
\hline $9 \mathrm{~d}$ & $(3 / 2)^{2}[7 / 2]$ & $3 \mathrm{~d}^{9}\left({ }^{2} \mathrm{D}_{3 / 2}\right) 9 \mathrm{~d}$ & ${ }^{2}[7 / 2]$ & 4 & [158966.7] & 2.0 & & $100 \%$ & sf & 0 \\
\hline $9 \mathrm{~d}$ & $(3 / 2)^{2}[3 / 2]$ & $3 d^{9}\left({ }^{2} D_{3 / 2}\right) 9 d$ & ${ }^{2}[3 / 2]$ & 1 & [158970.8] & 2.0 & & $100 \%$ & sf & 0 \\
\hline $9 \mathrm{~d}$ & $(3 / 2)^{2}[3 / 2]$ & $3 d^{9}\left({ }^{2} D_{3 / 2}\right) 9 d$ & $2[3 / 2]$ & 2 & [158977.0] & 5 & & $96 \%+4 \% 9 d(3 / 2)^{2}[5 / 2]$ & sf & 0 \\
\hline $9 \mathrm{~d}$ & $(3 / 2)^{2}[5 / 2]$ & $3 d^{9}\left({ }^{2} D_{3 / 2}\right) 9 d$ & $2[5 / 2]$ & 3 & [158999.4] & 2.0 & & $100 \%$ & sf & 0 \\
\hline $9 \mathrm{~d}$ & $(3 / 2)^{2}[5 / 2]$ & $3 d^{9}\left({ }^{2} D_{3 / 2}\right) 9 d$ & $2[5 / 2]$ & 2 & [159000.4] & 5 & & $96 \%+4 \% 9 d(3 / 2)^{2}[3 / 2]$ & sf & 0 \\
\hline $10 \mathrm{~g}$ & $(5 / 2)^{2}[3 / 2]$ & $3 d^{9}\left({ }^{2} D_{5 / 2}\right) 10 g$ & ${ }^{2}[3 / 2]$ & 2 & [159273.3] & 2.0 & & $100 \%$ & $\mathrm{pf}$ & 0 \\
\hline $10 \mathrm{~g}$ & $(5 / 2)^{2}[7 / 2]$ & $3 d^{9}\left({ }^{2} D_{5 / 2}\right) 10 g$ & ${ }^{2}[7 / 2]$ & 3 & [159277.4] & 2.0 & & $100 \%$ & $\mathrm{pf}$ & 0 \\
\hline $9 \mathrm{~g}$ & $(3 / 2)^{2}[5 / 2]$ & $3 d^{9}\left({ }^{2} D_{3 / 2}\right) 9 g$ & ${ }^{2}[5 / 2]$ & 3 & [160312.5] & 2.0 & & $100 \%$ & $\mathrm{pf}$ & 0 \\
\hline $9 \mathrm{~g}$ & $(3 / 2)^{2}[11 / 2]$ & $3 \mathrm{~d}^{9}\left({ }^{2} \mathrm{D}_{3 / 2}\right) 9 \mathrm{~g}$ & ${ }^{2}[11 / 2]$ & 6 & [160314.1] & 2.0 & & $100 \%$ & $\mathrm{pf}$ & 0 \\
\hline $9 \mathrm{~g}$ & $(3 / 2)^{2}[7 / 2]$ & $3 \mathrm{~d}^{9}\left({ }^{2} \mathrm{D}_{3 / 2}\right) 9 \mathrm{~g}$ & ${ }^{2}[7 / 2]$ & 4 & [160317.0] & 2.0 & & $100 \%$ & $\mathrm{pf}$ & 0 \\
\hline $9 \mathrm{~g}$ & $(3 / 2)^{2}[9 / 2]$ & $3 d^{9}\left({ }^{2} D_{3 / 2}\right) 9 g$ & $2[9 / 2]$ & 5 & [160318.5] & 2.0 & & $100 \%$ & $\mathrm{pf}$ & 0 \\
\hline $10 \mathrm{~d}$ & $(3 / 2)^{2}[1 / 2]$ & $3 \mathrm{~d}^{9}\left({ }^{2} \mathrm{D}_{3 / 2}\right) 10 \mathrm{~d}$ & ${ }^{2}[1 / 2]$ & 1 & [160361.5] & 4 & & $99 \%$ & sf & 0 \\
\hline $10 \mathrm{~d}$ & $(3 / 2)^{2}[7 / 2]$ & $3 d^{9}\left({ }^{2} D_{3 / 2}\right) 10 d$ & ${ }^{2}[7 / 2]$ & 3 & [160378.4] & 2.0 & & $100 \%$ & sf & 0 \\
\hline $10 \mathrm{~d}$ & $(3 / 2)^{2}[7 / 2]$ & $3 \mathrm{~d}^{9}\left({ }^{2} \mathrm{D}_{3 / 2}\right) 10 \mathrm{~d}$ & ${ }^{2}[7 / 2]$ & 4 & [160382.2] & 2.0 & & $100 \%$ & sf & 0 \\
\hline $10 \mathrm{~d}$ & $(3 / 2)^{2}[3 / 2]$ & $3 d^{9}\left({ }^{2} D_{3 / 2}\right) 10 d$ & $2[3 / 2]$ & 1 & [160385.0] & 2.0 & & $100 \%$ & sf & 0 \\
\hline $10 \mathrm{~d}$ & $(3 / 2)^{2}[3 / 2]$ & $3 \mathrm{~d}^{9}\left({ }^{2} \mathrm{D}_{3 / 2}\right) 10 \mathrm{~d}$ & ${ }^{2}[3 / 2]$ & 2 & [160388.7] & 5 & & $96 \%+3 \% 10 d(3 / 2)^{2}[5 / 2]$ & sf & 0 \\
\hline $10 \mathrm{~d}$ & $(3 / 2)^{2}[5 / 2]$ & $3 d^{9}\left({ }^{2} D_{3 / 2}\right) 10 d$ & $2[5 / 2]$ & 3 & [160404.5] & 2.0 & & $100 \%$ & sf & 0 \\
\hline $10 \mathrm{~d}$ & $(3 / 2)^{2}[5 / 2]$ & $3 \mathrm{~d}^{9}\left({ }^{2} \mathrm{D}_{3 / 2}\right) 10 \mathrm{~d}$ & ${ }^{2}[5 / 2]$ & 2 & [160405.1] & 5 & & $96 \%+3 \% 10 \mathrm{~d}(3 / 2)^{2}[3 / 2]$ & sf & 0 \\
\hline $10 \mathrm{~g}$ & $(3 / 2)^{2}[5 / 2]$ & $3 d^{9}\left({ }^{2} D_{3 / 2}\right) 10 g$ & $2[5 / 2]$ & 3 & [161344.3] & 2.0 & & $100 \%$ & $\mathrm{pf}$ & 0 \\
\hline $10 \mathrm{~g}$ & $(3 / 2)^{2}[11 / 2]$ & $3 d^{9}\left({ }^{2} D_{3 / 2}\right) 10 g$ & ${ }^{2}[11 / 2]$ & 6 & [161345.4] & 2.0 & & $100 \%$ & $\mathrm{pf}$ & 0 \\
\hline $10 \mathrm{~g}$ & $(3 / 2)^{2}[7 / 2]$ & $3 d^{9}\left({ }^{2} D_{3 / 2}\right) 10 g$ & $2[7 / 2]$ & 4 & [161347.6] & 2.0 & & $100 \%$ & $\mathrm{pf}$ & 0 \\
\hline \multirow[t]{2}{*}{$10 \mathrm{~g}$} & $(3 / 2)^{2}[9 / 2]$ & $3 d^{9}\left({ }^{2} D_{3 / 2}\right) 10 g$ & ${ }^{2}[9 / 2]$ & 5 & [161348.6] & 2.0 & & $100 \%$ & $\mathrm{pf}$ & 0 \\
\hline & & $\mathrm{Cu}$ III $\left(3 \mathrm{~d}^{9}{ }^{2} \mathrm{D}_{5 / 2}\right)$ & Limit & & 163669.2 & 0.5 & & & & \\
\hline
\end{tabular}

a Label used in the column of Leading Percentages;

$\mathrm{b}$ Level values were obtained in the least-squares optimization procedure using the LOPT code [39] (see text), except the following: (1) Values in square brackets were obtained using extrapolations along level series (see column "Notes"); (2) The ionization limit is quoted from Ross [2] (see text). A question mark after the value indicates an uncertain identification;

c Uncertainties (one standard deviation) are specified for separations from the $3 \mathrm{~d}^{9} 4 \mathrm{~s}^{3} \mathrm{D}_{2}$ level (at $22847.1176 \mathrm{~cm}^{-1}$ ). To determine uncertainties relative to the ground level, the given values should be combined in quadrature with the uncertainty of the ground level, $0.017 \mathrm{~cm}^{-1}$;

d Experimental Landé $g$-factors are quoted from Sugar and Musgrove [17];

e The three leading contributions to the eigenvector are given, if their rounded value is $\geq 1 \%$. The first percentage refers to the configuration and term given in the second and third columns, unless otherwise specified after the percentage value. For the $3 \mathrm{~d}^{9} n l$ levels designated in the $J_{1} l$ (a.k.a. JK) coupling scheme, the percentage value of the $3 \mathrm{~d}^{8} 4 \mathrm{~s} 4 \mathrm{p}$ configuration is the sum of percentage contributions of all terms of this configuration;

${ }^{f}$ Key to the notes: N—newly identified level; R—revised level value (new identification); RJ—revised J value (was 1 in Sugar and Musgrove [17] and Ross [2]); ci-the two previously known levels, for which the identifications have been interchanged; cd-previously known levels, for which the configuration and/or term designations have been revised; sf, pf-level values found by extrapolation using the Ritz quantum-defect or polarization formulas, respectively (see text in Section 4), in combination with the least-squares parametric fitting (see text);

g Number of connecting lines included in the level optimization procedure for this level. 
Table A3. Least-squares fitting parameters for $\mathrm{Cu}$ II.

\begin{tabular}{|c|c|c|c|c|c|c|}
\hline Configuration & Parameter $^{\mathrm{a}}$ & $\operatorname{LSF}^{b}$ & $\mathrm{STD}^{\mathrm{c}}$ & Group $^{d}$ & HFR $^{\mathrm{e}}$ & LSF/HF \\
\hline \multicolumn{7}{|l|}{ Even parity } \\
\hline $3 d^{10}$ & $E_{\mathrm{av}}$ & 5775.8 & 52 & & 0.0 & \\
\hline \multirow[t]{3}{*}{$3 d^{9} 4 s$} & $E_{\mathrm{av}}$ & 26843.5 & 21 & & 9367.0 & 2.8658 \\
\hline & $\zeta(3 \mathrm{~d})$ & 819.9 & 2.0 & 1 & 814.7 & 1.0064 \\
\hline & $G^{2}(3 d, 4 s)$ & 8519.5 & 132 & 2 & 9945.5 & 0.8566 \\
\hline \multirow[t]{8}{*}{$3 d^{9} 4 d$} & $E_{\mathrm{av}}$ & 119071.8 & 12 & & 98893.7 & 1.2040 \\
\hline & $\zeta(3 d)$ & 827.0 & 2.0 & 1 & 821.7 & 1.0064 \\
\hline & $\zeta(4 \mathrm{~d})$ & 13.7 & fixed & & 13.7 & 1.0000 \\
\hline & $F^{2}(3 \mathrm{~d}, 4 \mathrm{~d})$ & 3237.5 & 96 & & 4085.1 & 0.7925 \\
\hline & $F^{4}(3 \mathrm{~d}, 4 \mathrm{~d})$ & 1109.0 & fixed & & 1386.3 & 0.8000 \\
\hline & $G^{0}(3 d, 4 d)$ & 1426.8 & 47 & & 1440.0 & 0.9908 \\
\hline & $G^{2}(3 d, 4 d)$ & 705.6 & 152 & & 1276.5 & 0.5528 \\
\hline & $G^{4}(3 d, 4 d)$ & 538.0 & 228 & & 890.6 & 0.6041 \\
\hline \multirow[t]{3}{*}{$3 d^{9} 5 s$} & $E_{\mathrm{av}}$ & 109078.5 & 20 & & 92286.1 & 1.1820 \\
\hline & $\zeta(3 \mathrm{~d})$ & 826.4 & 2.0 & 1 & 821.1 & 1.0064 \\
\hline & $G^{2}(3 d, 5 s)$ & 1537.1 & 24 & 2 & 1794.4 & 0.8566 \\
\hline \multirow{8}{*}{$3 d^{9} 5 d$} & $E_{\mathrm{av}}$ & 137813.1 & 10 & & 120253.4 & 1.1460 \\
\hline & $\zeta(3 \mathrm{~d})$ & 827.6 & 2.0 & 1 & 822.3 & 1.0064 \\
\hline & $\zeta(5 d)$ & 6.1 & fixed & & 6.1 & 1.0000 \\
\hline & $F^{2}(3 d, 5 d)$ & 1905.2 & 73 & 5 & 1567.9 & 1.2152 \\
\hline & $F^{4}(3 \mathrm{~d}, 5 \mathrm{~d})$ & 463.5 & fixed & & 579.4 & 0.8000 \\
\hline & $G^{0}(3 d, 5 d)$ & 455.2 & 20 & 8 & 616.8 & 0.7380 \\
\hline & $G^{2}(3 d, 5 d)$ & 412.6 & 18 & 8 & 559.0 & 0.7380 \\
\hline & $G^{4}(3 d, 5 d)$ & 290.1 & 13 & 8 & 393.0 & 0.7380 \\
\hline \multirow[t]{4}{*}{$3 d^{9} 5 g$} & $E_{\mathrm{av}}$ & 146914.9 & 9 & & 129486.8 & 1.1346 \\
\hline & $\zeta(3 \mathrm{~d})$ & 828.0 & 2.0 & 1 & 822.7 & 1.0064 \\
\hline & $F^{2}(3 \mathrm{~d}, 5 \mathrm{~g})$ & 133.4 & fixed & & 166.7 & 0.8000 \\
\hline & $F^{4}(3 d, 5 g)$ & 5.2 & fixed & & 6.5 & 0.8000 \\
\hline \multirow[t]{3}{*}{$3 d^{9} 6 s$} & $E_{\mathrm{av}}$ & 134521.0 & 20 & & 117385.5 & 1.1460 \\
\hline & $\zeta(3 d)$ & 827.4 & 2.0 & 1 & 822.1 & 1.0064 \\
\hline & $G^{2}(3 d, 6 s)$ & 564.8 & 9 & 2 & 659.4 & 0.8566 \\
\hline \multirow[t]{8}{*}{$3 d^{9} 6 d$} & $E_{\mathrm{av}}$ & 147347.9 & 10 & & 129891.5 & 1.1344 \\
\hline & $\zeta(3 d)$ & 827.8 & 2.0 & 1 & 822.5 & 1.0064 \\
\hline & $\zeta(6 \mathrm{~d})$ & 3.2 & fixed & & 3.2 & 1.0000 \\
\hline & $F^{2}(3 \mathrm{~d}, 6 \mathrm{~d})$ & 952.5 & 36 & 5 & 783.9 & 1.2152 \\
\hline & $F^{4}(3 \mathrm{~d}, 6 \mathrm{~d})$ & 240.4 & fixed & & 300.5 & 0.8000 \\
\hline & $G^{0}(3 d, 6 d)$ & 182.2 & 17 & 9 & 320.8 & 0.5680 \\
\hline & $G^{2}(3 d, 6 d)$ & 166.8 & 15 & 9 & 293.6 & 0.5680 \\
\hline & $G^{4}(3 d, 6 d)$ & 117.6 & 11 & 9 & 207.0 & 0.5680 \\
\hline \multirow[t]{4}{*}{$3 d^{9} 6 g$} & $E_{\mathrm{av}}$ & 152289.1 & 9 & & 134856.3 & 1.1293 \\
\hline & $\zeta(3 \mathrm{~d})$ & 828.0 & 2.0 & 1 & 822.7 & 1.0064 \\
\hline & $F^{2}(3 d, 6 g)$ & 77.6 & fixed & & 97.0 & 0.8000 \\
\hline & $F^{4}(3 \mathrm{~d}, 6 \mathrm{~g})$ & 3.9 & fixed & & 4.9 & 0.8000 \\
\hline \multirow{3}{*}{$3 d^{9} 7 s$} & $E_{\mathrm{av}}$ & 145697.1 & 20 & & 128417.0 & 1.1346 \\
\hline & $\zeta(3 \mathrm{~d})$ & 827.7 & 2.0 & 1 & 822.4 & 1.0064 \\
\hline & $G^{2}(3 d, 7 s)$ & 273.2 & 4 & 2 & 318.9 & 0.8566 \\
\hline \multirow[t]{8}{*}{$3 d^{9} 7 d$} & $E_{\mathrm{av}}$ & 152539.1 & 10 & & 135114.4 & 1.1290 \\
\hline & $\zeta(3 \mathrm{~d})$ & 827.9 & 2.0 & 1 & 822.6 & 1.0064 \\
\hline & $\zeta(7 d)$ & 1.9 & fixed & & 1.9 & 1.0000 \\
\hline & $F^{2}(3 \mathrm{~d}, 7 \mathrm{~d})$ & 546.1 & 21 & 5 & 449.4 & 1.2152 \\
\hline & $F^{4}(3 d, 7 d)$ & 140.5 & fixed & & 175.6 & 0.8000 \\
\hline & $G^{0}(3 d, 7 d)$ & 149.8 & fixed & & 187.2 & 0.8000 \\
\hline & $G^{2}(3 d, 7 d)$ & 166.8 & fixed & & 172.4 & 0.8000 \\
\hline & $G^{4}(3 d, 7 d)$ & 117.6 & fixed & & 121.8 & 0.8000 \\
\hline \multirow[t]{4}{*}{$3 d^{9} 7 g$} & $E_{\mathrm{av}}$ & 155529.5 & 10 & & 138096.9 & 1.1262 \\
\hline & $\zeta(3 \mathrm{~d})$ & 828.0 & 2.0 & 1 & 822.7 & 1.0064 \\
\hline & $F^{2}(3 \mathrm{~d}, 7 \mathrm{~g})$ & 49.0 & fixed & & 61.3 & 0.8000 \\
\hline & $F^{4}(3 \mathrm{~d}, 7 \mathrm{~g})$ & 2.7 & fixed & & 3.4 & 0.8000 \\
\hline
\end{tabular}


Table A3. Cont.

\begin{tabular}{|c|c|c|c|c|c|c|}
\hline Configuration & Parameter $^{a}$ & $\operatorname{LSF}^{b}$ & $\operatorname{STD}^{\mathrm{c}}$ & Group $^{d}$ & HFR $^{\mathrm{e}}$ & LSF/HF \\
\hline \multicolumn{7}{|l|}{ Even parity } \\
\hline \multirow[t]{3}{*}{$3 d^{9} 8 s$} & $E_{\mathrm{av}}$ & 151604.1 & 20 & & 134260.1 & 1.1292 \\
\hline & $\zeta(3 \mathrm{~d})$ & 827.8 & 2.0 & 1 & 822.5 & 1.0064 \\
\hline & $G^{2}(3 d, 8 s)$ & 153.2 & 2.0 & 2 & 178.9 & 0.8566 \\
\hline \multirow[t]{8}{*}{$3 d^{9} 8 d$} & $E_{\mathrm{av}}$ & 155694.0 & 14 & & 138268.4 & 1.1260 \\
\hline & $\zeta(3 \mathrm{~d})$ & 827.9 & 2.0 & 1 & 822.6 & 1.0064 \\
\hline & $\zeta(8 \mathrm{~d})$ & 1.2 & fixed & & 1.2 & 1.0000 \\
\hline & $F^{2}(3 \mathrm{~d}, 8 \mathrm{~d})$ & 342.5 & 13 & 5 & 281.9 & 1.2152 \\
\hline & $F^{4}(3 d, 8 d)$ & 89.1 & fixed & & 111.4 & 0.8000 \\
\hline & $G^{0}(3 d, 8 d)$ & 95.0 & fixed & & 118.8 & 0.8000 \\
\hline & $G^{2}(3 d, 8 d)$ & 87.7 & fixed & & 109.6 & 0.8000 \\
\hline & $G^{4}(3 d, 8 d)$ & 62.0 & fixed & & 77.5 & 0.8000 \\
\hline \multirow[t]{4}{*}{$3 d^{9} 8 g$} & $E_{\mathrm{av}}$ & 157632.2 & 17 & & 140200.6 & 1.1243 \\
\hline & $\zeta(3 \mathrm{~d})$ & 828.0 & 2.0 & 1 & 822.7 & 1.0064 \\
\hline & $F^{2}(3 \mathrm{~d}, 8 \mathrm{~g})$ & 32.9 & fixed & & 41.1 & 0.8000 \\
\hline & $F^{4}(3 \mathrm{~d}, 8 \mathrm{~g})$ & 2.0 & fixed & & 2.5 & 0.8000 \\
\hline \multirow[t]{3}{*}{$3 d^{9} 9 s$} & $E_{\mathrm{av}}$ & 155106.1 & 20 & & 137730.5 & 1.1262 \\
\hline & $\zeta(3 \mathrm{~d})$ & 827.9 & 2.0 & 1 & 822.6 & 1.0064 \\
\hline & $G^{2}(3 d, 9 s)$ & 94.7 & 1.0 & 2 & 110.5 & 0.8566 \\
\hline \multirow[t]{8}{*}{$3 d^{9} 9 d$} & $E_{\mathrm{av}}$ & 157752.1 & 14 & & 140319.6 & 1.1242 \\
\hline & $\zeta(3 \mathrm{~d})$ & 828.0 & 2.0 & 1 & 822.7 & 1.0064 \\
\hline & $\zeta(9 \mathrm{~d})$ & 0.8 & fixed & & 0.8 & 1.0000 \\
\hline & $F^{2}(3 d, 9 d)$ & 229.1 & 9 & 5 & 188.5 & 1.2152 \\
\hline & $F^{4}(3 d, 9 d)$ & 60.0 & fixed & & 75.0 & 0.8000 \\
\hline & $G^{0}(3 d, 9 d)$ & 64.0 & fixed & & 80.0 & 0.8000 \\
\hline & $G^{2}(3 d, 9 d)$ & 59.2 & fixed & & 74.0 & 0.8000 \\
\hline & $G^{4}(3 d, 9 d)$ & 41.8 & fixed & & 52.2 & 0.8000 \\
\hline \multirow[t]{4}{*}{$3 d^{9} 9 g$} & $E_{\mathrm{av}}$ & 159073.9 & 17 & & 141643.1 & 1.1231 \\
\hline & $\zeta(3 \mathrm{~d})$ & 828.0 & 2.0 & 1 & 822.7 & 1.0064 \\
\hline & $F^{2}(3 d, 9 g)$ & 23.1 & fixed & & 28.9 & 0.8000 \\
\hline & $F^{4}(3 \mathrm{~d}, 9 \mathrm{~g})$ & 1.4 & fixed & & 1.7 & 0.8000 \\
\hline \multirow{3}{*}{$3 d^{9} 10 s$} & $E_{\mathrm{av}}$ & 157351.8 & 23 & & 139959.4 & 1.1243 \\
\hline & $\zeta(3 \mathrm{~d})$ & 827.9 & 2.0 & 1 & 822.6 & 1.0064 \\
\hline & $G^{2}(3 d, 10 s)$ & 62.5 & 1.0 & 2 & 73.0 & 0.8566 \\
\hline \multirow[t]{8}{*}{$3 d^{9} 10 d$} & $E_{\mathrm{av}}$ & 159159.7 & 10 & & 141729.3 & 1.1230 \\
\hline & $\zeta(3 \mathrm{~d})$ & 828.0 & 2.0 & 1 & 822.7 & 1.0064 \\
\hline & $\zeta(10 \mathrm{~d})$ & 0.6 & fixed & & 0.6 & 1.0000 \\
\hline & $F^{2}(3 \mathrm{~d}, 10 \mathrm{~d})$ & 160.7 & 6 & 5 & 132.3 & 1.2152 \\
\hline & $F^{4}(3 \mathrm{~d}, 10 \mathrm{~d})$ & 42.3 & fixed & & 52.9 & 0.8000 \\
\hline & $G^{0}(3 \mathrm{~d}, 10 \mathrm{~d})$ & 45.1 & fixed & & 56.4 & 0.8000 \\
\hline & $G^{2}(3 \mathrm{~d}, 10 \mathrm{~d})$ & 41.8 & fixed & & 52.2 & 0.8000 \\
\hline & $G^{4}(3 d, 10 d)$ & 29.5 & fixed & & 36.9 & 0.8000 \\
\hline \multirow[t]{4}{*}{$3 d^{9} 10 g$} & $E_{\mathrm{av}}$ & 160104.8 & 17 & & 142674.4 & 1.1222 \\
\hline & $\zeta(3 \mathrm{~d})$ & 828.0 & 2.0 & 1 & 822.7 & 1.0064 \\
\hline & $F^{2}(3 \mathrm{~d}, 10 \mathrm{~g})$ & 16.8 & fixed & & 21.0 & 0.8000 \\
\hline & $F^{4}(3 \mathrm{~d}, 10 \mathrm{~g})$ & 1.1 & fixed & & 1.4 & 0.8000 \\
\hline \multirow[t]{5}{*}{$3 d^{8} 4 s^{2}$} & $E_{\mathrm{av}}$ & 88372.4 & 16 & & 62522.0 & 1.4135 \\
\hline & $F^{2}(3 \mathrm{~d}, 3 \mathrm{~d})$ & 91560.3 & 114 & 3 & 109696.0 & 0.8347 \\
\hline & $F^{4}(3 \mathrm{~d}, 3 \mathrm{~d})$ & 58255.9 & 103 & 4 & 68373.1 & 0.8520 \\
\hline & $\alpha(3 \mathrm{~d})$ & 93.8 & 3 & 7 & 0.0 & \\
\hline & $\zeta(3 d)$ & 892.2 & 3 & 1 & 886.5 & 1.0064 \\
\hline \multirow{10}{*}{$3 d^{8} 4 p^{2}$} & $E_{\mathrm{av}}$ & 188427.0 & fixed & & 162579.7 & 1.1590 \\
\hline & $F^{2}(3 \mathrm{~d}, 3 \mathrm{~d})$ & 92340.0 & 115 & 3 & 110630.1 & 0.8347 \\
\hline & $F^{4}(3 \mathrm{~d}, 3 \mathrm{~d})$ & 58793.9 & 104 & 4 & 69004.6 & 0.8520 \\
\hline & $\alpha(3 \mathrm{~d})$ & 93.8 & 3 & 7 & 0.0 & \\
\hline & $F^{2}(4 \mathrm{p}, 4 \mathrm{p})$ & 28518.8 & fixed & & 35648.5 & 0.8000 \\
\hline & $\zeta(3 d)$ & 898.5 & 3 & 1 & 892.8 & 1.0064 \\
\hline & $\zeta(4 \mathrm{p})$ & 619.0 & fixed & & 619.0 & 1.0000 \\
\hline & $F^{2}(3 \mathrm{~d}, 4 \mathrm{p})$ & 12983.5 & fixed & & 16229.4 & 0.8000 \\
\hline & $G^{1}(3 d, 4 p)$ & 4628.9 & fixed & & 5786.1 & 0.8000 \\
\hline & $G^{3}(3 d, 4 p)$ & 3922.3 & fixed & & 4902.9 & 0.8000 \\
\hline
\end{tabular}


Table A3. Cont.

\begin{tabular}{|c|c|c|c|c|c|c|}
\hline Configuration & Parameter $^{a}$ & $\operatorname{LSF}^{b}$ & $\operatorname{STD}^{\mathrm{c}}$ & Group $^{d}$ & HFR $^{\mathrm{e}}$ & LSF/HF \\
\hline \multicolumn{7}{|l|}{ Even parity } \\
\hline \multirow[t]{7}{*}{$3 d^{8} 4 s 4 d$} & $E_{\mathrm{av}}$ & 186352.1 & fixed & & 160504.8 & 1.1610 \\
\hline & $F^{2}(3 \mathrm{~d}, 3 \mathrm{~d})$ & 92375.3 & 115 & 3 & 110672.4 & 0.8347 \\
\hline & $F^{4}(3 \mathrm{~d}, 3 \mathrm{~d})$ & 58816.8 & 104 & 4 & 69031.5 & 0.8520 \\
\hline & $\alpha(3 \mathrm{~d})$ & 93.8 & 3 & 7 & 0.0 & \\
\hline & $\zeta(3 \mathrm{~d})$ & 898.6 & 3 & 1 & 892.9 & 1.0064 \\
\hline & $F^{2}(3 \mathrm{~d}, 4 \mathrm{~d})$ & 5461.6 & 208 & 5 & 4494.5 & 1.2152 \\
\hline & $G^{2}(3 d, 4 s)$ & 9256.3 & 144 & 2 & 10805.6 & 0.8566 \\
\hline \multirow[t]{7}{*}{$3 d^{8} 4 s 5 s$} & $E_{\mathrm{av}}$ & 178348.4 & fixed & & 152501.1 & 1.1695 \\
\hline & $F^{2}(3 \mathrm{~d}, 3 \mathrm{~d})$ & 92277.5 & 115 & 3 & 110555.2 & 0.8347 \\
\hline & $F^{4}(3 d, 3 d)$ & 58748.9 & 104 & 4 & 68951.7 & 0.8520 \\
\hline & $\alpha(3 \mathrm{~d})$ & 93.8 & 3 & 7 & 0.0 & \\
\hline & $\zeta(3 \mathrm{~d})$ & 897.8 & 3 & 1 & 892.1 & 1.0064 \\
\hline & $G^{2}(3 d, 4 s)$ & 9552.4 & 148 & 2 & 11151.3 & 0.8566 \\
\hline & $G^{2}(3 d, 5 s)$ & 1270.3 & 20 & 2 & 1482.9 & 0.8566 \\
\hline \multirow[t]{6}{*}{$3 d^{8} 4 d^{2}$} & $E_{\mathrm{av}}$ & 307518.0 & fixed & & 281670.7 & 1.0918 \\
\hline & $F^{2}(3 d, 3 d)$ & 93279.6 & 116 & 3 & 111755.9 & 0.8347 \\
\hline & $F^{4}(3 d, 3 d)$ & 59441.2 & 105 & 4 & 69764.2 & 0.8520 \\
\hline & $\alpha(3 \mathrm{~d})$ & 93.8 & 3 & 7 & 0.0 & \\
\hline & $\zeta(3 d)$ & 906.0 & 3 & 1 & 900.2 & 1.0064 \\
\hline & $F^{2}(3 \mathrm{~d}, 4 \mathrm{~d})$ & 7552.1 & 288 & 5 & 6214.9 & 1.2152 \\
\hline \multirow[t]{7}{*}{$3 d^{8} 4 s 5 d$} & $E_{\mathrm{av}}$ & 209861.7 & fixed & & 184014.4 & 1.1405 \\
\hline & $F^{2}(3 d, 3 d)$ & 92407.3 & 115 & 3 & 110710.8 & 0.8347 \\
\hline & $F^{4}(3 d, 3 d)$ & 58838.5 & 104 & 4 & 69056.9 & 0.8520 \\
\hline & $\alpha(3 \mathrm{~d})$ & 93.8 & 3 & 7 & 0.0 & \\
\hline & $\zeta(3 d)$ & 899.0 & 3 & 1 & 893.3 & 1.0064 \\
\hline & $F^{2}(3 \mathrm{~d}, 5 \mathrm{~d})$ & 1997.4 & 76 & 5 & 1643.7 & 1.2152 \\
\hline & $G^{2}(3 d, 4 s)$ & 9429.0 & 147 & 2 & 11007.3 & 0.8566 \\
\hline \multicolumn{7}{|c|}{ Configuration interaction } \\
\hline \multirow[t]{6}{*}{$3 d^{9} 4 d-3 d^{9} 5 d$} & $R_{\mathrm{d}}^{0}(3 \mathrm{~d} 4 \mathrm{~d}, 3 \mathrm{~d} 5 \mathrm{~d})$ & 147.8 & 7 & 6 & 135.3 & 1.0921 \\
\hline & $R_{\mathrm{d}}^{2}(3 \mathrm{~d} 4 \mathrm{~d}, 3 \mathrm{~d} 5 \mathrm{~d})$ & 2400.9 & 108 & 6 & 2198.4 & 1.0921 \\
\hline & $R_{\mathrm{d}}^{4}(3 \mathrm{~d} 4 \mathrm{~d}, 3 \mathrm{~d} 5 \mathrm{~d})$ & 959.9 & 43 & 6 & 879.0 & 1.0921 \\
\hline & $R_{\mathrm{e}}^{0}(3 \mathrm{~d} 4 \mathrm{~d}, 3 \mathrm{~d} 5 \mathrm{~d})$ & 1028.7 & 46 & 6 & 942.0 & 1.0921 \\
\hline & $R_{\mathrm{e}}^{2}(3 \mathrm{~d} 4 \mathrm{~d}, 3 \mathrm{~d} 5 \mathrm{~d})$ & 921.6 & 41 & 6 & 843.9 & 1.0921 \\
\hline & $R_{\mathrm{e}}^{4}(3 \mathrm{~d} 4 \mathrm{~d}, 3 \mathrm{~d} 5 \mathrm{~d})$ & 645.4 & 29 & 6 & 591.0 & 1.0921 \\
\hline \multirow[t]{6}{*}{$3 d^{9} 4 d-3 d^{9} 6 d$} & $R_{\mathrm{d}}^{0}(3 \mathrm{~d} 4 \mathrm{~d}, 3 \mathrm{~d} 6 \mathrm{~d})$ & 106.6 & 5 & 6 & 97.6 & 1.0921 \\
\hline & $R_{\mathrm{d}}^{2}(3 \mathrm{~d} 4 \mathrm{~d}, 3 \mathrm{~d} 6 \mathrm{~d})$ & 1631.1 & 73 & 6 & 1493.6 & 1.0921 \\
\hline & $R_{\mathrm{d}}^{4}(3 \mathrm{~d} 4 \mathrm{~d}, 3 \mathrm{~d} 6 \mathrm{~d})$ & 684.3 & 31 & 6 & 626.6 & 1.0921 \\
\hline & $R_{\mathrm{e}}^{0}(3 \mathrm{~d} 4 \mathrm{~d}, 3 \mathrm{~d} 6 \mathrm{~d})$ & 741.5 & 33 & 6 & 679.0 & 1.0921 \\
\hline & $R_{\mathrm{e}}^{2}(3 \mathrm{~d} 4 \mathrm{~d}, 3 \mathrm{~d} 6 \mathrm{~d})$ & 667.2 & 30 & 6 & 610.9 & 1.0921 \\
\hline & $R_{\mathrm{e}}^{4}(3 \mathrm{~d} 4 \mathrm{~d}, 3 \mathrm{~d} 6 \mathrm{~d})$ & 467.9 & 21 & 6 & 428.4 & 1.0921 \\
\hline \multirow[t]{6}{*}{$3 d^{9} 4 d-3 d^{9} 7 d$} & $R_{\mathrm{d}}^{0}(3 \mathrm{~d} 4 \mathrm{~d}, 3 \mathrm{~d} 7 \mathrm{~d})$ & 81.5 & 4 & 6 & 74.6 & 1.0921 \\
\hline & $R_{\mathrm{d}}^{2}(3 \mathrm{~d} 4 \mathrm{~d}, 3 \mathrm{~d} 7 \mathrm{~d})$ & 1212.2 & 54 & 6 & 1110.0 & 1.0921 \\
\hline & $R_{\mathrm{d}}^{4}(3 \mathrm{~d} 4 \mathrm{~d}, 3 \mathrm{~d} 7 \mathrm{~d})$ & 520.2 & 23 & 6 & 476.3 & 1.0921 \\
\hline & $R_{\mathrm{e}}^{0}(3 \mathrm{~d} 4 \mathrm{~d}, 3 \mathrm{~d} 7 \mathrm{~d})$ & 566.5 & 25 & 6 & 518.7 & 1.0921 \\
\hline & $R_{\mathrm{e}}^{2}(3 \mathrm{~d} 4 \mathrm{~d}, 3 \mathrm{~d} 7 \mathrm{~d})$ & 510.8 & 23 & 6 & 467.7 & 1.0921 \\
\hline & $R_{\mathrm{e}}^{4}(3 \mathrm{~d} 4 \mathrm{~d}, 3 \mathrm{~d} 7 \mathrm{~d})$ & 358.5 & 16 & 6 & 328.3 & 1.0921 \\
\hline \multirow[t]{6}{*}{$3 d^{9} 4 d-3 d^{9} 8 d$} & $R_{\mathrm{d}}^{0}(3 \mathrm{~d} 4 \mathrm{~d}, 3 \mathrm{~d} 8 \mathrm{~d})$ & 64.9 & 3 & 6 & 59.4 & 1.0921 \\
\hline & $R_{\mathrm{d}}^{2}(3 \mathrm{~d} 4 \mathrm{~d}, 3 \mathrm{~d} 8 \mathrm{~d})$ & 950.0 & 43 & 6 & 869.9 & 1.0921 \\
\hline & $R_{\mathrm{d}}^{4}(3 \mathrm{~d} 4 \mathrm{~d}, 3 \mathrm{~d} 8 \mathrm{~d})$ & 412.8 & 18 & 6 & 378.0 & 1.0921 \\
\hline & $R_{\mathrm{e}}^{0}(3 \mathrm{~d} 4 \mathrm{~d}, 3 \mathrm{~d} 8 \mathrm{~d})$ & 451.0 & 20 & 6 & 413.0 & 1.0921 \\
\hline & $R_{\mathrm{e}}^{2}(3 \mathrm{~d} 4 \mathrm{~d}, 3 \mathrm{~d} 8 \mathrm{~d})$ & 407.2 & 18 & 6 & 372.9 & 1.0921 \\
\hline & $R_{\mathrm{e}}^{4}(3 \mathrm{~d} 4 \mathrm{~d}, 3 \mathrm{~d} 8 \mathrm{~d})$ & 286.0 & 13 & 6 & 261.9 & 1.0921 \\
\hline \multirow[t]{6}{*}{$3 d^{9} 4 d-3 d^{9} 9 d$} & $R_{\mathrm{d}}^{0}(3 \mathrm{~d} 4 \mathrm{~d}, 3 \mathrm{~d} 9 \mathrm{~d})$ & 53.2 & 2.0 & 6 & 48.7 & 1.0921 \\
\hline & $R_{\mathrm{d}}^{2}(3 \mathrm{~d} 4 \mathrm{~d}, 3 \mathrm{~d} 9 \mathrm{~d})$ & 771.9 & 35 & 6 & 706.8 & 1.0921 \\
\hline & $R_{\mathrm{d}}^{4}(3 \mathrm{~d} 4 \mathrm{~d}, 3 \mathrm{~d} 9 \mathrm{~d})$ & 338.0 & 15 & 6 & 309.5 & 1.0921 \\
\hline & $R_{\mathrm{e}}^{0}(3 \mathrm{~d} 4 \mathrm{~d}, 3 \mathrm{~d} 9 \mathrm{~d})$ & 370.1 & 17 & 6 & 338.9 & 1.0921 \\
\hline & $R_{\mathrm{e}}^{2}(3 \mathrm{~d} 4 \mathrm{~d}, 3 \mathrm{~d} 9 \mathrm{~d})$ & 334.4 & 15 & 6 & 306.2 & 1.0921 \\
\hline & $R_{\mathrm{e}}^{4}(3 \mathrm{~d} 4 \mathrm{~d}, 3 \mathrm{~d} 9 \mathrm{~d})$ & 234.9 & 11 & 6 & 215.1 & 1.0921 \\
\hline
\end{tabular}


Table A3. Cont.

\begin{tabular}{|c|c|c|c|c|c|c|}
\hline Configuration & Parameter $^{\mathrm{a}}$ & $\operatorname{LSF}^{b}$ & $\mathrm{STD}^{\mathrm{c}}$ & Group $^{d}$ & HFR $^{e}$ & LSF/HF \\
\hline \multicolumn{7}{|c|}{ Configuration interaction } \\
\hline \multirow[t]{6}{*}{$3 d^{9} 4 d-3 d^{9} 10 d$} & $R_{\mathrm{d}}^{0}(3 \mathrm{~d} 4 \mathrm{~d}, 3 \mathrm{~d} 10 \mathrm{~d})$ & 44.8 & 2.0 & 6 & 41.0 & 1.0921 \\
\hline & $R_{\mathrm{d}}^{2}(3 \mathrm{~d} 4 \mathrm{~d}, 3 \mathrm{~d} 10 \mathrm{~d})$ & 643.8 & 29 & 6 & 589.5 & 1.0921 \\
\hline & $R_{\mathrm{d}}^{4}(3 \mathrm{~d} 4 \mathrm{~d}, 3 \mathrm{~d} 10 \mathrm{~d})$ & 283.4 & 13 & 6 & 259.5 & 1.0921 \\
\hline & $R_{\mathrm{e}}^{0}(3 \mathrm{~d} 4 \mathrm{~d}, 3 \mathrm{~d} 10 \mathrm{~d})$ & 310.7 & 14 & 6 & 284.5 & 1.0921 \\
\hline & $R_{\mathrm{e}}^{2}(3 \mathrm{~d} 4 \mathrm{~d}, 3 \mathrm{~d} 10 \mathrm{~d})$ & 281.0 & 13 & 6 & 257.3 & 1.0921 \\
\hline & $R_{\mathrm{e}}^{4}(3 \mathrm{~d} 4 \mathrm{~d}, 3 \mathrm{~d} 10 \mathrm{~d})$ & 197.5 & 9 & 6 & 180.8 & 1.0921 \\
\hline \multirow[t]{5}{*}{$3 d^{9} 5 d-3 d^{9} 6 d$} & $R_{\mathrm{d}}^{2}(3 \mathrm{~d} 5 \mathrm{~d}, 3 \mathrm{~d} 6 \mathrm{~d})$ & 1147.0 & 51 & 6 & 1050.3 & 1.0921 \\
\hline & $R_{\mathrm{d}}^{4}(3 \mathrm{~d} 5 \mathrm{~d}, 3 \mathrm{~d} 6 \mathrm{~d})$ & 454.3 & 20 & 6 & 416.0 & 1.0921 \\
\hline & $R_{\mathrm{e}}^{0}(3 \mathrm{~d} 5 \mathrm{~d}, 3 \mathrm{~d} 6 \mathrm{~d})$ & 485.8 & 22 & 6 & 444.8 & 1.0921 \\
\hline & $R_{\mathrm{e}}^{2}(3 \mathrm{~d} 5 \mathrm{~d}, 3 \mathrm{~d} 6 \mathrm{~d})$ & 442.4 & 20 & 6 & 405.1 & 1.0921 \\
\hline & $R_{\mathrm{e}}^{4}(3 \mathrm{~d} 5 \mathrm{~d}, 3 \mathrm{~d} 6 \mathrm{~d})$ & 311.5 & 14 & 6 & 285.2 & 1.0921 \\
\hline \multirow[t]{5}{*}{$3 d^{9} 5 d_{-3}-3 d^{9} 7 d$} & $R_{\mathrm{d}}^{2}(3 \mathrm{~d} 5 \mathrm{~d}, 3 \mathrm{~d} 7 \mathrm{~d})$ & 850.8 & 38 & 6 & 779.1 & 1.0921 \\
\hline & $R_{\mathrm{d}}^{4}(3 \mathrm{~d} 5 \mathrm{~d}, 3 \mathrm{~d} 7 \mathrm{~d})$ & 346.3 & 16 & 6 & 317.1 & 1.0921 \\
\hline & $R_{\mathrm{e}}^{0}(3 \mathrm{~d} 5 \mathrm{~d}, 3 \mathrm{~d} 7 \mathrm{~d})$ & 371.2 & 17 & 6 & 339.9 & 1.0921 \\
\hline & $R_{\mathrm{e}}^{2}(3 \mathrm{~d} 5 \mathrm{~d}, 3 \mathrm{~d} 7 \mathrm{~d})$ & 339.0 & 15 & 6 & 310.4 & 1.0921 \\
\hline & $R_{\mathrm{e}}^{4}(3 \mathrm{~d} 5 \mathrm{~d}, 3 \mathrm{~d} 7 \mathrm{~d})$ & 238.7 & 11 & 6 & 218.6 & 1.0921 \\
\hline \multirow[t]{5}{*}{$3 d^{9} 5 d-3 d^{9} 8 d$} & $R_{\mathrm{d}}^{2}(3 \mathrm{~d} 5 \mathrm{~d}, 3 \mathrm{~d} 8 \mathrm{~d})$ & 666.6 & 30 & 6 & 610.4 & 1.0921 \\
\hline & $R_{\mathrm{d}}^{4}(3 \mathrm{~d} 5 \mathrm{~d}, 3 \mathrm{~d} 8 \mathrm{~d})$ & 275.3 & 12 & 6 & 252.1 & 1.0921 \\
\hline & $R_{\mathrm{e}}^{0}(3 \mathrm{~d} 5 \mathrm{~d}, 3 \mathrm{~d} 8 \mathrm{~d})$ & 295.5 & 13 & 6 & 270.6 & 1.0921 \\
\hline & $R_{\mathrm{e}}^{2}(3 \mathrm{~d} 5 \mathrm{~d}, 3 \mathrm{~d} 8 \mathrm{~d})$ & 270.3 & 12 & 6 & 247.5 & 1.0921 \\
\hline & $R_{\mathrm{e}}^{4}(3 \mathrm{~d} 5 \mathrm{~d}, 3 \mathrm{~d} 8 \mathrm{~d})$ & 190.6 & 9 & 6 & 174.5 & 1.0921 \\
\hline \multirow[t]{5}{*}{$3 d^{9} 5 d_{-3}-99 d$} & $R_{\mathrm{d}}^{2}(3 \mathrm{~d} 5 \mathrm{~d}, 3 \mathrm{~d} 9 \mathrm{~d})$ & 541.3 & 24 & 6 & 495.7 & 1.0921 \\
\hline & $R_{\mathrm{d}}^{4}(3 \mathrm{~d} 5 \mathrm{~d}, 3 \mathrm{~d} 9 \mathrm{~d})$ & 225.8 & 10 & 6 & 206.8 & 1.0921 \\
\hline & $R_{\mathrm{e}}^{0}(3 \mathrm{~d} 5 \mathrm{~d}, 3 \mathrm{~d} 9 \mathrm{~d})$ & 242.4 & 11 & 6 & 222.0 & 1.0921 \\
\hline & $R_{\mathrm{e}}^{2}(3 \mathrm{~d} 5 \mathrm{~d}, 3 \mathrm{~d} 9 \mathrm{~d})$ & 222.0 & 10 & 6 & 203.3 & 1.0921 \\
\hline & $R_{\mathrm{e}}^{4}(3 \mathrm{~d} 5 \mathrm{~d}, 3 \mathrm{~d} 9 \mathrm{~d})$ & 156.6 & 7 & 6 & 143.4 & 1.0921 \\
\hline \multirow[t]{5}{*}{$3 d^{9} 5 d-3 d^{9} 10 d$} & $R_{\mathrm{d}}^{2}(3 \mathrm{~d} 5 \mathrm{~d}, 3 \mathrm{~d} 10 \mathrm{~d})$ & 451.6 & 20 & 6 & 413.5 & 1.0921 \\
\hline & $R_{\mathrm{d}}^{4}(3 \mathrm{~d} 5 \mathrm{~d}, 3 \mathrm{~d} 10 \mathrm{~d})$ & 189.4 & 8 & 6 & 173.4 & 1.0921 \\
\hline & $R_{\mathrm{e}}^{0}(3 \mathrm{~d} 5 \mathrm{~d}, 3 \mathrm{~d} 10 \mathrm{~d})$ & 203.7 & 9 & 6 & 186.5 & 1.0921 \\
\hline & $R_{\mathrm{e}}^{2}(3 \mathrm{~d} 5 \mathrm{~d}, 3 \mathrm{~d} 10 \mathrm{~d})$ & 186.4 & 8 & 6 & 170.7 & 1.0921 \\
\hline & $R_{\mathrm{e}}^{4}(3 \mathrm{~d} 5 \mathrm{~d}, 3 \mathrm{~d} 10 \mathrm{~d})$ & 131.6 & 6 & 6 & 120.5 & 1.0921 \\
\hline \multirow[t]{5}{*}{$3 d^{9} 6 d^{-3} d^{9} 7 d$} & $R_{\mathrm{d}}^{2}(3 \mathrm{~d} 6 \mathrm{~d}, 3 \mathrm{~d} 7 \mathrm{~d})$ & 631.0 & 28 & 6 & 577.8 & 1.0921 \\
\hline & $R_{\mathrm{d}}^{4}(3 \mathrm{~d} 6 \mathrm{~d}, 3 \mathrm{~d} 7 \mathrm{~d})$ & 250.7 & 11 & 6 & 229.6 & 1.0921 \\
\hline & $R_{\mathrm{e}}^{0}(3 \mathrm{~d} 6 \mathrm{~d}, 3 \mathrm{~d} 7 \mathrm{~d})$ & 267.7 & 12 & 6 & 245.1 & 1.0921 \\
\hline & $R_{\mathrm{e}}^{2}(3 \mathrm{~d} 6 \mathrm{~d}, 3 \mathrm{~d} 7 \mathrm{~d})$ & 245.7 & 11 & 6 & 225.0 & 1.0921 \\
\hline & $R_{\mathrm{e}}^{4}(3 \mathrm{~d} 6 \mathrm{~d}, 3 \mathrm{~d} 7 \mathrm{~d})$ & 173.4 & 8 & 6 & 158.8 & 1.0921 \\
\hline \multirow[t]{5}{*}{$3 d^{9} 6 d-3 d^{9} 8 d$} & $R_{\mathrm{d}}^{2}(3 \mathrm{~d} 6 \mathrm{~d}, 3 \mathrm{~d} 8 \mathrm{~d})$ & 494.0 & 22 & 6 & 452.3 & 1.0921 \\
\hline & $R_{\mathrm{d}}^{4}(3 \mathrm{~d} 6 \mathrm{~d}, 3 \mathrm{~d} 8 \mathrm{~d})$ & 199.6 & 9 & 6 & 182.8 & 1.0921 \\
\hline & $R_{\mathrm{e}}^{0}(3 \mathrm{~d} 6 \mathrm{~d}, 3 \mathrm{~d} 8 \mathrm{~d})$ & 213.3 & 10 & 6 & 195.3 & 1.0921 \\
\hline & $R_{\mathrm{e}}^{2}(3 \mathrm{~d} 6 \mathrm{~d}, 3 \mathrm{~d} 8 \mathrm{~d})$ & 196.0 & 9 & 6 & 179.5 & 1.0921 \\
\hline & $R_{\mathrm{e}}^{4}(3 \mathrm{~d} 6 \mathrm{~d}, 3 \mathrm{~d} 8 \mathrm{~d})$ & 138.5 & 6 & 6 & 126.8 & 1.0921 \\
\hline \multirow[t]{5}{*}{$3 d^{9} 6 d-3 d^{9} 9 d$} & $R_{\mathrm{d}}^{2}(3 \mathrm{~d} 6 \mathrm{~d}, 3 \mathrm{~d} 9 \mathrm{~d})$ & 401.0 & 18 & 6 & 367.2 & 1.0921 \\
\hline & $R_{\mathrm{d}}^{4}(3 \mathrm{~d} 6 \mathrm{~d}, 3 \mathrm{~d} 9 \mathrm{~d})$ & 163.7 & 7 & 6 & 149.9 & 1.0921 \\
\hline & $R_{\mathrm{e}}^{0}(3 \mathrm{~d} 6 \mathrm{~d}, 3 \mathrm{~d} 9 \mathrm{~d})$ & 175.1 & 8 & 6 & 160.3 & 1.0921 \\
\hline & $R_{\mathrm{e}}^{2}(3 \mathrm{~d} 6 \mathrm{~d}, 3 \mathrm{~d} 9 \mathrm{~d})$ & 161.0 & 7 & 6 & 147.4 & 1.0921 \\
\hline & $R_{\mathrm{e}}^{4}(3 \mathrm{~d} 6 \mathrm{~d}, 3 \mathrm{~d} 9 \mathrm{~d})$ & 113.7 & 5 & 6 & 104.1 & 1.0921 \\
\hline \multirow[t]{5}{*}{$3 d^{9} 6 d-3 d^{9} 10 d$} & $R_{\mathrm{d}}^{2}(3 \mathrm{~d} 6 \mathrm{~d}, 3 \mathrm{~d} 10 \mathrm{~d})$ & 334.6 & 15 & 6 & 306.4 & 1.0921 \\
\hline & $R_{\mathrm{d}}^{4}(3 \mathrm{~d} 6 \mathrm{~d}, 3 \mathrm{~d} 10 \mathrm{~d})$ & 137.3 & 6 & 6 & 125.7 & 1.0921 \\
\hline & $R_{\mathrm{e}}^{0}(3 \mathrm{~d} 6 \mathrm{~d}, 3 \mathrm{~d} 10 \mathrm{~d})$ & 146.9 & 7 & 6 & 134.5 & 1.0921 \\
\hline & $R_{\mathrm{e}}^{2}(3 \mathrm{~d} 6 \mathrm{~d}, 3 \mathrm{~d} 10 \mathrm{~d})$ & 135.3 & 6 & 6 & 123.9 & 1.0921 \\
\hline & $R_{\mathrm{e}}^{4}(3 \mathrm{~d} 6 \mathrm{~d}, 3 \mathrm{~d} 10 \mathrm{~d})$ & 95.6 & 4 & 6 & 87.5 & 1.0921 \\
\hline \multirow[t]{5}{*}{$3 d^{9} 7 d-3 d^{9} 8 d$} & $R_{\mathrm{d}}^{2}(3 \mathrm{~d} 7 \mathrm{~d}, 3 \mathrm{~d} 8 \mathrm{~d})$ & 382.7 & 17 & 6 & 350.4 & 1.0921 \\
\hline & $R_{\mathrm{d}}^{4}(3 \mathrm{~d} 7 \mathrm{~d}, 3 \mathrm{~d} 8 \mathrm{~d})$ & 152.8 & 7 & 6 & 139.9 & 1.0921 \\
\hline & $R_{\mathrm{e}}^{0}(3 \mathrm{~d} 7 \mathrm{~d}, 3 \mathrm{~d} 8 \mathrm{~d})$ & 163.0 & 7 & 6 & 149.3 & 1.0921 \\
\hline & $R_{\mathrm{e}}^{2}(3 \mathrm{~d} 7 \mathrm{~d}, 3 \mathrm{~d} 8 \mathrm{~d})$ & 150.2 & 7 & 6 & 137.5 & 1.0921 \\
\hline & $R_{\mathrm{e}}^{4}(3 \mathrm{~d} 7 \mathrm{~d}, 3 \mathrm{~d} 8 \mathrm{~d})$ & 106.0 & 5 & 6 & 97.1 & 1.0921 \\
\hline
\end{tabular}


Table A3. Cont.

\begin{tabular}{|c|c|c|c|c|c|c|}
\hline Configuration & Parameter $^{\mathrm{a}}$ & $\operatorname{LSF}^{b}$ & $\mathrm{STD}^{\mathrm{c}}$ & Group $^{d}$ & HFR $^{\mathrm{e}}$ & LSF/HF \\
\hline \multicolumn{7}{|c|}{ Configuration interaction } \\
\hline \multirow[t]{5}{*}{$3 d^{9} 7 d-3 d^{9} 9 d$} & $R_{\mathrm{d}}^{2}(3 \mathrm{~d} 7 \mathrm{~d}, 3 \mathrm{~d} 9 \mathrm{~d})$ & 310.7 & 14 & 6 & 284.5 & 1.0921 \\
\hline & $R_{\mathrm{d}}^{4}(3 \mathrm{~d} 7 \mathrm{~d}, 3 \mathrm{~d} 9 \mathrm{~d})$ & 125.4 & 6 & 6 & 114.8 & 1.0921 \\
\hline & $R_{\mathrm{e}}^{0}(3 \mathrm{~d} 7 \mathrm{~d}, 3 \mathrm{~d} 9 \mathrm{~d})$ & 133.7 & 6 & 6 & 122.4 & 1.0921 \\
\hline & $R_{\mathrm{e}}^{2}(3 \mathrm{~d} 7 \mathrm{~d}, 3 \mathrm{~d} 9 \mathrm{~d})$ & 123.4 & 6 & 6 & 113.0 & 1.0921 \\
\hline & $R_{\mathrm{e}}^{4}(3 \mathrm{~d} 7 \mathrm{~d}, 3 \mathrm{~d} 9 \mathrm{~d})$ & 87.3 & 4 & 6 & 79.9 & 1.0921 \\
\hline \multirow[t]{5}{*}{$3 d^{9} 7 d-3 d^{9} 10 d$} & $R_{\mathrm{d}}^{2}(3 \mathrm{~d} 7 \mathrm{~d}, 3 \mathrm{~d} 10 \mathrm{~d})$ & 259.2 & 12 & 6 & 237.3 & 1.0921 \\
\hline & $R_{\mathrm{d}}^{4}(3 \mathrm{~d} 7 \mathrm{~d}, 3 \mathrm{~d} 10 \mathrm{~d})$ & 105.3 & 5 & 6 & 96.4 & 1.0921 \\
\hline & $R_{\mathrm{e}}^{0}(3 \mathrm{~d} 7 \mathrm{~d}, 3 \mathrm{~d} 10 \mathrm{~d})$ & 112.3 & 5 & 6 & 102.8 & 1.0921 \\
\hline & $R_{\mathrm{e}}^{2}(3 \mathrm{~d} 7 \mathrm{~d}, 3 \mathrm{~d} 10 \mathrm{~d})$ & 103.6 & 5 & 6 & 94.9 & 1.0921 \\
\hline & $R_{\mathrm{e}}^{4}(3 \mathrm{~d} 7 \mathrm{~d}, 3 \mathrm{~d} 10 \mathrm{~d})$ & 73.3 & 3 & 6 & 67.1 & 1.0921 \\
\hline \multirow[t]{5}{*}{$3 d^{9} 8 d-3 d^{9} 9 d$} & $R_{\mathrm{d}}^{2}(3 \mathrm{~d} 8 \mathrm{~d}, 3 \mathrm{~d} 9 \mathrm{~d})$ & 249.3 & 11 & 6 & 228.3 & 1.0921 \\
\hline & $R_{\mathrm{d}}^{4}(3 \mathrm{~d} 8 \mathrm{~d}, 3 \mathrm{~d} 9 \mathrm{~d})$ & 99.9 & 4 & 6 & 91.5 & 1.0921 \\
\hline & $R_{\mathrm{e}}^{0}(3 \mathrm{~d} 8 \mathrm{~d}, 3 \mathrm{~d} 9 \mathrm{~d})$ & 106.5 & 5 & 6 & 97.5 & 1.0921 \\
\hline & $R_{\mathrm{e}}^{2}(3 \mathrm{~d} 8 \mathrm{~d}, 3 \mathrm{~d} 9 \mathrm{~d})$ & 98.4 & 4 & 6 & 90.1 & 1.0921 \\
\hline & $R_{\mathrm{e}}^{4}(3 \mathrm{~d} 8 \mathrm{~d}, 3 \mathrm{~d} 9 \mathrm{~d})$ & 69.6 & 3 & 6 & 63.7 & 1.0921 \\
\hline \multirow[t]{5}{*}{$3 d^{9} 8 d-3 d^{9} 10 d$} & $R_{\mathrm{d}}^{2}(3 \mathrm{~d} 8 \mathrm{~d}, 3 \mathrm{~d} 10 \mathrm{~d})$ & 207.8 & 9 & 6 & 190.3 & 1.0921 \\
\hline & $R_{\mathrm{d}}^{4}(3 \mathrm{~d} 8 \mathrm{~d}, 3 \mathrm{~d} 10 \mathrm{~d})$ & 83.9 & 4 & 6 & 76.8 & 1.0921 \\
\hline & $R_{\mathrm{e}}^{0}(3 \mathrm{~d} 8 \mathrm{~d}, 3 \mathrm{~d} 10 \mathrm{~d})$ & 89.4 & 4 & 6 & 81.9 & 1.0921 \\
\hline & $R_{\mathrm{e}}^{2}(3 \mathrm{~d} 8 \mathrm{~d}, 3 \mathrm{~d} 10 \mathrm{~d})$ & 82.8 & 4 & 6 & 75.8 & 1.0921 \\
\hline & $R_{\mathrm{e}}^{4}(3 \mathrm{~d} 8 \mathrm{~d}, 3 \mathrm{~d} 10 \mathrm{~d})$ & 58.5 & 3 & 6 & 53.6 & 1.0921 \\
\hline \multirow[t]{5}{*}{$3 d^{9} 9 d-3 d^{9} 10 d$} & $R_{\mathrm{d}}^{2}(3 \mathrm{~d} 9 \mathrm{~d}, 3 \mathrm{~d} 10 \mathrm{~d})$ & 171.3 & 8 & 6 & 156.9 & 1.0921 \\
\hline & $R_{\mathrm{d}}^{4}(3 \mathrm{~d} 9 \mathrm{~d}, 3 \mathrm{~d} 10 \mathrm{~d})$ & 68.8 & 3 & 6 & 63.0 & 1.0921 \\
\hline & $R_{\mathrm{e}}^{0}(3 \mathrm{~d} 9 \mathrm{~d}, 3 \mathrm{~d} 10 \mathrm{~d})$ & 73.3 & 3 & 6 & 67.1 & 1.0921 \\
\hline & $R_{\mathrm{e}}^{2}(3 \mathrm{~d} 9 \mathrm{~d}, 3 \mathrm{~d} 10 \mathrm{~d})$ & 67.9 & 3 & 6 & 62.2 & 1.0921 \\
\hline & $R_{\mathrm{e}}^{4}(3 \mathrm{~d} 9 \mathrm{~d}, 3 \mathrm{~d} 10 \mathrm{~d})$ & 48.1 & 2.0 & 6 & 44.0 & 1.0921 \\
\hline \multicolumn{7}{|l|}{ Odd parity } \\
\hline \multirow[t]{6}{*}{$3 d^{9} 4 p$} & $E_{\mathrm{av}}$ & 73281.0 & 22 & & 53995.6 & 1.3572 \\
\hline & $\zeta(3 \mathrm{~d})$ & 828.8 & 5 & 1 & 818.3 & 1.0128 \\
\hline & $\zeta(4 \mathrm{p})$ & 538.0 & 20 & 9 & 444.2 & 1.2112 \\
\hline & $F^{2}(3 \mathrm{~d}, 4 \mathrm{p})$ & 13098.9 & 152 & & 13901.7 & 0.9423 \\
\hline & $G^{1}(3 d, 4 p)$ & 4368.5 & 65 & & 5338.7 & 0.8183 \\
\hline & $G^{3}(3 \mathrm{~d}, 4 \mathrm{p})$ & 3615.9 & 363 & & 4299.1 & 0.8411 \\
\hline \multirow[t]{2}{*}{$3 d^{9} 4 f$} & $E_{\mathrm{av}}$ & 136895.6 & 17 & & 119289.7 & 1.1476 \\
\hline & $\zeta(3 \mathrm{~d})$ & 833.2 & 5 & 1 & 822.7 & 1.0128 \\
\hline \multirow[t]{6}{*}{$3 d^{9} 5 p$} & $E_{\mathrm{av}}$ & 121911.6 & 24 & & 104871.2 & 1.1625 \\
\hline & $\zeta(3 d)$ & 832.0 & 5 & 1 & 821.5 & 1.0128 \\
\hline & $\zeta(5 p)$ & 159.8 & 6 & 9 & 131.9 & 1.2112 \\
\hline & $F^{2}(3 d, 5 p)$ & 3512.5 & 21 & 2 & 3548.4 & 0.9899 \\
\hline & $G^{1}(3 d, 5 p)$ & 1262.1 & 94 & 3 & 1287.6 & 0.9802 \\
\hline & $G^{3}(3 d, 5 p)$ & 1174.4 & 306 & 10 & 1102.4 & 1.0653 \\
\hline \multirow[t]{2}{*}{$3 d^{9} 5 f$} & $E_{\mathrm{av}}$ & 146833.4 & 16 & & 129308.6 & 1.1355 \\
\hline & $\zeta(3 \mathrm{~d})$ & 833.2 & 5 & 1 & 822.7 & 1.0128 \\
\hline \multirow[t]{6}{*}{$3 d^{9} 6 p$} & $E_{\mathrm{av}}$ & 139748.8 & 29 & & 122652.3 & 1.1394 \\
\hline & $\zeta(3 \mathrm{~d})$ & 832.7 & 5 & 1 & 822.2 & 1.0128 \\
\hline & $\zeta(6 \mathrm{p})$ & 69.9 & 3 & 9 & 57.7 & 1.2112 \\
\hline & $F^{2}(3 \mathrm{~d}, 6 \mathrm{p})$ & 1443.6 & 9 & 2 & 1458.4 & 0.9899 \\
\hline & $G^{1}(3 d, 6 p)$ & 521.8 & 39 & 3 & 532.4 & 0.9802 \\
\hline & $G^{3}(3 d, 6 p)$ & 493.4 & 129 & 10 & 463.1 & 1.0653 \\
\hline \multirow[t]{2}{*}{$3 d^{9} 6 f$} & $E_{\mathrm{av}}$ & 152224.4 & 17 & & 134753.4 & 1.1297 \\
\hline & $\zeta(3 \mathrm{~d})$ & 833.2 & 5 & 1 & 822.7 & 1.0128 \\
\hline \multirow[t]{2}{*}{$3 d^{9} 6 h$} & $E_{\mathrm{av}}$ & 152299.5 & 16 & & 134875.8 & 1.1292 \\
\hline & $\zeta(3 \mathrm{~d})$ & 833.2 & 5 & 1 & 822.7 & 1.0128 \\
\hline \multirow[t]{6}{*}{$3 d^{9} 7 p$} & $E_{\mathrm{av}}$ & 148438.8 & 22 & & 131122.8 & 1.1321 \\
\hline & $\zeta(3 \mathrm{~d})$ & 833.0 & 5 & 1 & 822.5 & 1.0128 \\
\hline & $\zeta(7 p)$ & 36.8 & 1.0 & 9 & 30.4 & 1.2112 \\
\hline & $F^{2}(3 d, 7 p)$ & 738.7 & 4 & 2 & 746.3 & 0.9899 \\
\hline & $G^{1}(3 d, 7 p)$ & 268.6 & 20 & 3 & 274.0 & 0.9802 \\
\hline & $G^{3}(3 d, 7 p)$ & 255.8 & 67 & 10 & 240.1 & 1.0653 \\
\hline
\end{tabular}


Table A3. Cont.

\begin{tabular}{|c|c|c|c|c|c|c|}
\hline Configuration & Parameter $^{a}$ & $\operatorname{LSF}^{b}$ & STD $^{c}$ & Group $^{d}$ & HFR $^{\mathrm{e}}$ & LSF/HF \\
\hline \multicolumn{7}{|l|}{ Odd parity } \\
\hline \multirow[t]{2}{*}{$3 d^{9} 7 f$} & $E_{\mathrm{av}}$ & 155498.8 & 22 & & 138032.9 & 1.1265 \\
\hline & $\zeta(3 \mathrm{~d})$ & 833.2 & 5 & 1 & 822.7 & 1.0128 \\
\hline \multirow[t]{2}{*}{$3 d^{9} 7 \mathrm{~h}$} & $E_{\mathrm{av}}$ & 155540.7 & 21 & & 138110.8 & 1.1262 \\
\hline & $\zeta(3 d)$ & 833.2 & 5 & 1 & 822.7 & 1.0128 \\
\hline \multirow[t]{6}{*}{$3 d^{9} 8 p$} & $E_{\mathrm{av}}$ & 153145.3 & 54 & & 135833.1 & 1.1275 \\
\hline & $\zeta(3 \mathrm{~d})$ & 833.1 & 5 & 1 & 822.6 & 1.0128 \\
\hline & $\zeta(8 \mathrm{p})$ & 21.8 & 1.0 & 9 & 18.0 & 1.2112 \\
\hline & $F^{2}(3 \mathrm{~d}, 8 \mathrm{p})$ & 429.6 & 3 & 2 & 434.0 & 0.9899 \\
\hline & $G^{1}(3 d, 8 p)$ & 156.8 & 12 & 3 & 160.0 & 0.9802 \\
\hline & $G^{3}(3 d, 8 p)$ & 150.0 & 39 & 10 & 140.8 & 1.0653 \\
\hline \multirow[t]{2}{*}{$3 d^{9} 8 f$} & $E_{\mathrm{av}}$ & 157610.4 & 28 & & 140157.8 & 1.1245 \\
\hline & $\zeta(3 \mathrm{~d})$ & 833.2 & 5 & 1 & 822.7 & 1.0128 \\
\hline \multirow[t]{2}{*}{$3 d^{9} 8 h$} & $E_{\mathrm{av}}$ & 157629.5 & 26 & & 140210.6 & 1.1242 \\
\hline & $\zeta(3 \mathrm{~d})$ & 833.2 & 5 & 1 & 822.7 & 1.0128 \\
\hline \multirow[t]{11}{*}{$3 d^{8} 4 s 4 p$} & $E_{\mathrm{av}}$ & 132837.3 & 12 & & 104548.6 & 1.2706 \\
\hline & $F^{2}(3 d, 3 d)$ & 92783.8 & 119 & 4 & 110131.5 & 0.8425 \\
\hline & $F^{4}(3 d, 3 d)$ & 59745.4 & 207 & 5 & 68667.5 & 0.8701 \\
\hline & $\alpha(3 \mathrm{~d})$ & 84.8 & 3 & 8 & 0.0 & \\
\hline & $\zeta(3 d)$ & 900.8 & 5 & 1 & 889.4 & 1.0128 \\
\hline & $\zeta(4 \mathrm{p})$ & 732.4 & 27 & 9 & 604.7 & 1.2112 \\
\hline & $F^{2}(3 \mathrm{~d}, 4 \mathrm{p})$ & 15857.7 & 94 & 2 & 16019.7 & 0.9899 \\
\hline & $G^{2}(3 d, 4 s)$ & 8059.3 & 145 & 7 & 10125.0 & 0.7960 \\
\hline & $G^{1}(3 d, 4 p)$ & 5532.7 & 82 & 11 & 5751.7 & 0.9619 \\
\hline & $G^{3}(3 d, 4 p)$ & 4703.6 & 208 & 12 & 4854.4 & 0.9689 \\
\hline & $G^{1}(4 s, 4 p)$ & 37321.3 & 52 & 6 & 47726.6 & 0.7820 \\
\hline \multirow[t]{6}{*}{$3 d^{8} 4 s 4 f$} & $E_{\mathrm{av}}$ & 211778.8 & fixed & & 183501.6 & 1.1541 \\
\hline & $F^{2}(3 \mathrm{~d}, 3 \mathrm{~d})$ & 93301.4 & 119 & 4 & 110745.9 & 0.8425 \\
\hline & $F^{4}(3 d, 3 d)$ & 60104.6 & 208 & 5 & 69080.2 & 0.8701 \\
\hline & $\alpha(3 d)$ & 84.8 & 3 & 8 & 0.0 & \\
\hline & $\zeta(3 d)$ & 905.0 & 5 & 1 & 893.6 & 1.0128 \\
\hline & $G^{2}(3 d, 4 s)$ & 8816.3 & 158 & 7 & 11076.0 & 0.7960 \\
\hline \multirow[t]{9}{*}{$3 d^{8} 4 p 4 d$} & $E_{\mathrm{av}}$ & 244758.6 & fixed & & 216481.4 & 1.1306 \\
\hline & $F^{2}(3 d, 3 d)$ & 93638.6 & 120 & 4 & 111146.1 & 0.8425 \\
\hline & $F^{4}(3 \mathrm{~d}, 3 \mathrm{~d})$ & 60341.3 & 209 & 5 & 69352.4 & 0.8701 \\
\hline & $\alpha(3 d)$ & 84.8 & 3 & 8 & 0.0 & \\
\hline & $\zeta(3 \mathrm{~d})$ & 907.5 & 5 & 1 & 896.1 & 1.0128 \\
\hline & $\zeta(4 p)$ & 873.4 & 33 & 9 & 721.1 & 1.2112 \\
\hline & $F^{2}(3 \mathrm{~d}, 4 \mathrm{p})$ & 17746.3 & 105 & 2 & 17927.6 & 0.9899 \\
\hline & $G^{1}(3 d, 4 p)$ & 6222.8 & 92 & 11 & 6469.1 & 0.9619 \\
\hline & $G^{3}(3 d, 4 p)$ & 5367.4 & 237 & 12 & 5539.4 & 0.9689 \\
\hline \multirow[t]{11}{*}{$3 d^{8} 4 s 5 p$} & $E_{\mathrm{av}}$ & 195045.6 & fixed & & 166768.4 & 1.1696 \\
\hline & $F^{2}(3 \mathrm{~d}, 3 \mathrm{~d})$ & 93179.2 & 119 & 4 & 110600.7 & 0.8425 \\
\hline & $F^{4}(3 \mathrm{~d}, 3 \mathrm{~d})$ & 60019.6 & 208 & 5 & 68982.6 & 0.8701 \\
\hline & $\alpha(3 \mathrm{~d})$ & 84.8 & 3 & 8 & 0.0 & \\
\hline & $\zeta(3 d)$ & 903.9 & 5 & 1 & 892.5 & 1.0128 \\
\hline & $\zeta(5 p)$ & 182.9 & 7 & 9 & 151.0 & 1.2112 \\
\hline & $F^{2}(3 d, 5 p)$ & 3559.6 & 21 & 2 & 3596.0 & 0.9899 \\
\hline & $G^{2}(3 d, 4 s)$ & 8773.2 & 158 & 7 & 11021.9 & 0.7960 \\
\hline & $G^{1}(3 d, 5 p)$ & 1184.8 & 89 & 3 & 1208.8 & 0.9802 \\
\hline & $G^{3}(3 d, 5 p)$ & 1145.1 & 298 & 10 & 1074.9 & 1.0653 \\
\hline & $G^{1}(4 s, 5 p)$ & 4181.2 & 6 & 6 & 5346.9 & 0.7820 \\
\hline
\end{tabular}


Table A3. Cont.

\begin{tabular}{|c|c|c|c|c|c|c|}
\hline Configuration & Parameter $^{a}$ & $\mathrm{LSF}^{\mathrm{b}}$ & $\operatorname{STD}^{\mathrm{c}}$ & Group $^{d}$ & HFR $^{e}$ & $\mathrm{LSF} / \mathrm{HF}$ \\
\hline \multirow{4}{*}{$3 d^{8} 4 s 5 f$} & $F^{2}(3 \mathrm{~d}, 3 \mathrm{~d})$ & 93300.7 & 119 & 4 & 110745.0 & 0.8425 \\
\hline & $F^{4}(3 \mathrm{~d}, 3 \mathrm{~d})$ & 60104.1 & 208 & 5 & 69079.8 & 0.8701 \\
\hline & $\alpha(3 \mathrm{~d})$ & 84.8 & 3 & 8 & 0.0 & \\
\hline & $G^{2}(3 d, 4 s)$ & 8827.6 & 159 & 7 & 11090.3 & 0.7960 \\
\hline
\end{tabular}

a All omitted single-configuration parameters were fixed at HFR values scaled by a factor of 0.80 for the direct and exchange electrostatic parameters $F^{\mathrm{k}}$ and $G^{\mathrm{k}}$, and 1.0 for spin-orbit parameters $\zeta$. All omitted configuration-interaction parameters were fixed at HFR values scaled by a factor of 0.94 in both parities;

b Parameter values determined in the least-squares fitting procedure (see Section 3);

c Standard deviation of the least-squares fitting;

d Parameters within each numbered group were linked together in the LSF procedure, so that the ratios to ab initio HFR values were the same for each parameter in the group;

e The ab initio Hartree-Fock-Relativistic parameter values as computed by Cowan's codes [52]. In this calculation, we included both relativistic and Breit corrections (in Cowan's codes, the latter affect only the average energies of configurations) and used the scaling factor of 1.0 for the exchange contribution.

\section{References}

1. Shenstone, A.G. The first spark spectrum of copper. Philos. Trans. R. Soc. Lond. Ser. A 1936, 235, $195-243$. [CrossRef]

2. Ross, C.B., Jr. Vacuum Ultraviolet Standards in the Spectrum of Cu II. Ph.D. Thesis, Purdue University, West Lafayette, IN, USA, 1969.

3. Thackeray, A.D. Identifications in the spectra of Eta Carinae and RR Telescopii. Mon. Not. R. Astron. Soc. 1953, 113, 211-236. [CrossRef]

4. Aller, L.H.; Polidan, R.S.; Rhodes, E.J., Jr.; Wares, G.W. The spectrum of RR Telescopii in 1968. Astrophys. Space Sci. 1973, 20, 93-110. [CrossRef]

5. McKenna, F.C.; Keenan, F.P.; Hambly, N.C.; Allende Prieto, C.; Rolleston, W.R.J.; Aller, L.H.; Feibelman, W.A. The optical spectral line list of RR Telescopii. Astrophys. J. Suppl. Ser. 1997, 109, 225-239. [CrossRef]

6. Wallerstein, G.; Gilroy, K.K.; Zethson, T.; Johansson, S.; Hamann, F. Line identifications in the spectrum of $\eta$ Carinae as observed in 1990-1991 with CCD detectors. Publ. Astron. Soc. Pac. 2001, 113, 1210-1214. [CrossRef]

7. Jaschek, J.; Jaschek, M. The Behavior of Chemical Elements in Stars; Cambridge University Press: Cambridge, UK, 1995.

8. Danezis, E.; Theodossiou, E. The UV Spectrum of the Be Star 88 Herculis. Astrophys. Space Sci. 1990, 174, 49-90. [CrossRef]

9. Samain, D. A High Spectral Resolution Atlas of the Balloon Ultraviolet Spectrum of the Sun: 1950-2000 A. Astron. Astrophys. Suppl. Ser. 1995, 113, 237-255.

10. McNeil, J.R.; Collins, G.J.; Persson, K.B.; Franzen, D.L. CW laser oscillation in Cu II. Appl. Phys. Lett. 1975, 27, 595-598. [CrossRef]

11. McNeil, J.R.; Collins, G.J.; Persson, K.B.; Franzen, D.L. Ultraviolet laser action from Cu II in the 2500- $\AA$ region. Appl. Phys. Lett. 1976, 28, 207-209. [CrossRef]

12. Jain, K. New UV and IR transitions in gold, copper, and cadmium hollow cathode lasers. IEEE J. Quantum Electron. 1980, 16, 387-391. [CrossRef]

13. Zinchenko, S.P.; Ivanov, I.G. Pulsed hollow-cathode ion lasers: Pumping and lasing parameters. Quantum Electron. 2012, 42, 518-523. [CrossRef]

14. Reader, J.; Meissner, K.W.; Andrew, K.L. Improved Cu II standard wavelengths in the vacuum ultraviolet. J. Opt. Soc. Am. 1960, 50, 221-227. [CrossRef]

15. Kaufman, V.; Ward, J.F. Measurement and calculation of Cu II, Ge II, Si II, and C I vacuum-ultraviolet lines. J. Opt. Soc. Am. 1966, 56, 1591-1597. [CrossRef] 
16. Ross, C.B. Wavelengths and Energy Levels of Singly Ionized Copper, Cu II; University California Report LA-4498; Los Alamos Scientific Lab: Los Alamos, NM, USA, 1970.

17. Sugar, J.; Musgrove, A. Energy levels of copper, Cu I through Cu XXIX. J. Phys. Chem. Ref. Data 1990, 19, 527-616. [CrossRef]

18. Sansonetti, J.E.; Martin, W.C. Handbook of basic atomic spectroscopic data. J. Phys. Chem. Ref. Data 2005, 34, 1559-2259. [CrossRef]

19. Kramida, A.; Ralchenko, Y.; Reader, J.; NIST ASD Team. NIST Atomic Spectra Database; version 5.4; National Institute of Standards and Technology: Gaithersburg, MD, USA, 2016. Available online: http:/ /physics.nist. gov/asd (accessed on 15 February 2017).

20. Nave, G.; Sansonetti, C.J. Reference wavelengths in the spectra of Fe, Ge, and Pt in the region near $1935 \AA$. J. Opt. Soc. Am. B 2004, 21, 442-453. [CrossRef]

21. Litzén, U.; Brault, J.W.; Thorne, A.P. Spectrum and term system of neutral nickel, Ni I. Phys. Scr. 1993, 47, 628-673. [CrossRef]

22. Wiese, W.L.; Martin, G.A. Transition Probabilities. In Wavelengths and Transition Probabilities for Atoms and Atomic Ions; National Standard Reference Data Series NSRDS-68; National Bureau of Standards: Gaithersburg, DC, USA, 1980; Part II; pp. 359-406.

23. Coursey, J.S.; Schwab, D.J.; Tsai, J.J.; Dragoset, R.A. Atomic Weights and Isotopic Compositions; version 4.0; National Institute of Standards and Technology: Gaithersburg, MD, USA, 2015. Available online: http:/ / physics.nist.gov/Comp (accessed on 15 February 2017).

24. Elbel, M.; Fischer, W. Zur Isotopieverschiebung im Kupfer I- und II-Spektrum. Z. Phys. 1961, 165, $151-170$. [CrossRef]

25. Elbel, M.; Fischer, W.; Hartmann, M. Hyperfeinstruktur und Isotopieverschiebung im Kupfer II-Spektrum. Z. Phys. 1963, 176, 288-292. [CrossRef]

26. Reader, J.; Davis, S.P. Promethium 147 hyperfine structure under high resolution. J. Opt. Soc. Am. 1963, 53, 431-435. [CrossRef]

27. Danzmann, K.; Günther, M.; Fisher, J.; Kock, M.; Kühne, M. High current hollow cathode as a radiometric transfer standard source for the extreme vacuum ultraviolet. Appl. Opt. 1988, 27, 4947-4951. [CrossRef] [PubMed]

28. Nave, G.; Griesmann, U.; Brault, J.W.; Abrams, M.C. XGREMLIN: Interferograms and Spectra from Fourier Transform Spectrometers Analysis. Astrophysics Source Code Library, record ascl:1511.004, 2015. Available online: https:/ /github.com/gnave/Xgremlin (accessed on 9 December 2015).

29. Brault, J.W. High precision Fourier transform spectrometry: The critical role of phase corrections. Microchim. Acta 1987, 93, 215-227. [CrossRef]

30. Kaufman, V.; Andrew, K.L. Germanium vacuum ultraviolet Ritz standards. J. Opt. Soc. Am. 1962, 52, 1223-1237. [CrossRef]

31. Sansonetti, C.J.; Veza, D. Doppler-free measurement of the $546 \mathrm{~nm}$ line of mercury. J. Phys. B 2010, 43, 205003. [CrossRef]

32. Nave, G.; Sansonetti, C.J. Wavelengths of the $3 d^{6}\left({ }^{5} \mathrm{D}\right) 4 \mathrm{~s} \mathrm{a}^{6} \mathrm{D}-3 \mathrm{~d}^{5}\left({ }^{6} \mathrm{~S}\right) 4 \mathrm{~s} 4 \mathrm{p} \mathrm{y}^{6} \mathrm{P}$ multiplet of Fe II (UV 8). J. Opt. Soc. Am. B 2011, 28, 737-745. [CrossRef]

33. Whaling, W.; Anderson, W.H.C.; Carle, M.T.; Brault, J.W.; Zarem, H.A. Argon ion linelist and level energies in the hollow-cathode discharge. J. Quant. Spectrosc. Radiat. Transf. 1995, 53, 1-22. [CrossRef]

34. Kaufman, V. Wavelengths, energy levels, and pressure shifts in mercury 198. J. Opt. Soc. Am. 1962, 52, 866-870. [CrossRef]

35. Kramida, A. Re-optimized energy levels and Ritz wavelengths of ${ }^{198} \mathrm{Hg}$ I. J. Res. Natl. Inst. Stand. Technol. 2011, 116, 599-619. [CrossRef]

36. Wagatsuma, K.; Hirokawa, K. Observation of singly-ionized copper emission lines from a Grimm-type glow discharge plasma with argon-helium gas mixtures in a visible wavelength region. Spectrochim. Acta B 1993, 48, 1039-1044. [CrossRef]

37. Prior, M.H. Radiative decay rates of metastable Ar III and Cu II ions. Phys. Rev. A 1984, 30, 3051-3056. [CrossRef]

38. Peck, E.R.; Reeder, K. Dispersion of air. J. Opt. Soc. Am. 1972, 62, 958-962. [CrossRef]

39. Kramida, A.E. The program LOPT for least-squares optimization of energy levels. Comput. Phys. Commun. 2011, 182, 419-434. [CrossRef] 
40. Andersson, M.; Yao, K.; Hutton, R.; Zou, Y.; Chen, C.Y.; Brage, T. Hyperfine-state-dependent lifetimes along the Ni-like isoelectronic sequence. Phys. Rev. A 2008, 77, 042509. [CrossRef]

41. Biémont, E.; Pinnington, E.H.; Quinet, P.; Zeippen, C.J. Core-polarization effects in Cu II. Phys. Scr. 2000, 61, 567-580. [CrossRef]

42. Brown, M.S.; Federman, S.R.; Irving, R.E.; Cheng, S.; Curtis, L.J. Lifetimes and oscillator strengths for ultraviolet transitions in singly ionized copper. Astrophys. J. 2009, 702, 880-883. [CrossRef]

43. Cederquist, H.; Mannervik, S.; Kisielinski, M.; Forsberg, P.; Martinson, I.; Curtis, L.J.; Ramanujam, P.S. Lifetimes of some excited levels in Cu I and Cu II. Phys. Scr. 1984, T8, 104-106. [CrossRef]

44. Crespo López-Urrutia, J.R.; Kenner, B.; Neger, T.; Jäger, H. Absolute transition probabilities of Cu II lines. J. Quant. Spectrosc. Radiat. Transf. 1994, 52, 111-114. [CrossRef]

45. Dong, C.Z.; Fritzsche, S. Relativistic, relaxation, and correlation effects in spectra of Cu II. Phys. Rev. A 2005, 72, 012507. [CrossRef]

46. Garstang, R.H. Transition probabilities of forbidden lines. J. Res. Natl. Bur. Stand. Sect. A 1964, 68, 61-73. [CrossRef]

47. Hefferlin, R.; Kuhlman, H.; Penz, J.; Wheeler, D. Approximate relative log gf for green lines of Cu ${ }^{+}$. Bull. Am. Phys. Soc. 1971, 16, 106.

48. Kono, A.; Hattori, S. Lifetimes and transition probabilities in Cu II. J. Opt. Soc. Am. 1982, 72, 601-605. [CrossRef]

49. Neger, T.; Jäger, H.Z. Transition probabilities of Cu II lines. Z. Naturforsch. A 1988, 43, 507-508. [CrossRef]

50. Ortiz, M.; Mayo, R.; Biémont, É.; Quinet, P.; Malcheva, G.; Blagoev, K. Radiative parameters for some transitions arising from the $3 \mathrm{~d}^{9} 4 \mathrm{~d}$ and $3 \mathrm{~d}^{8} 4 \mathrm{~s}^{2}$ electronic configurations in Cu II spectrum. J. Phys. B 2007, 40, 167-176. [CrossRef]

51. Pinnington, E.H.; Rieger, G.; Kernahan, J.A.; Biémont, E. Beam-laser measurements and relativistic Hartree-Fock calculations of the lifetimes of the $3 d^{9} 4$ p levels in Cu II. Can. J. Phys. 1997, 75, 1-9. [CrossRef]

52. Cowan, R.D. The Theory of Atomic Structure and Spectra; University California Press: Berkeley, CA, USA, 1981.

53. Azarov, V.I. Formal approach to the solution of the complex-spectra identification problem. 2. Implementation. Phys. Scr. 1993, 48, 656-667. [CrossRef]

54. Roth, C. Odd configurations in singly-ionized copper. J. Res. Natl. Bur. Stand. Sect. A 1969, 73, 599-609. [CrossRef]

55. Sansonetti, C.J.; National Institute of Standards and Technology, Gaithersburg, MD, USA. Fortran computer code RITZPL. Personal communication, 2005.

56. Sansonetti, C.J.; National Institute of Standards and Technology, Gaithersburg, MD, USA. Fortran computer code POLAR. Personal communication, 2005.

57. Kramida, A. Critical evaluation of data on atomic energy levels, wavelengths, and transition probabilities. Fusion Sci. Technol. 2013, 63, 313-323.

58. Kramida, A. Critically evaluated energy levels and spectral lines of singly ionized indium (In II). J. Res. Natl. Inst. Stand. Technol. 2013, 118, 52-104. [CrossRef] [PubMed]

59. Kramida, A.; Fuhr, J.R. NIST Atomic Transition Probability Bibliographic Database; version 9.0; National Institute of Standards and Technology: Gaithersburg, MD, USA, 2010. Available online: http://physics.nist.gov/ Fvalbib (accessed on 15 February 2017).

60. Lux, B. Untersuchungen über den Axialdurchschlag bei der Elektrischen Explosion von Kupferdrähten. Ph.D. Thesis, Universität Kiel, Kiel, Germany, 1973.

61. Beck, D.R. Many-electron effects in and operator forms for electron quadrupole transition probabilities. Phys. Rev. A 1981, 23, 159-171. [CrossRef]

(c) 2017 by the authors. Licensee MDPI, Basel, Switzerland. This article is an open access article distributed under the terms and conditions of the Creative Commons Attribution (CC BY) license (http:/ / creativecommons.org/licenses/by/4.0/). 